ORNL/TM-2018/1073

\title{
Omnibus User Manual
}

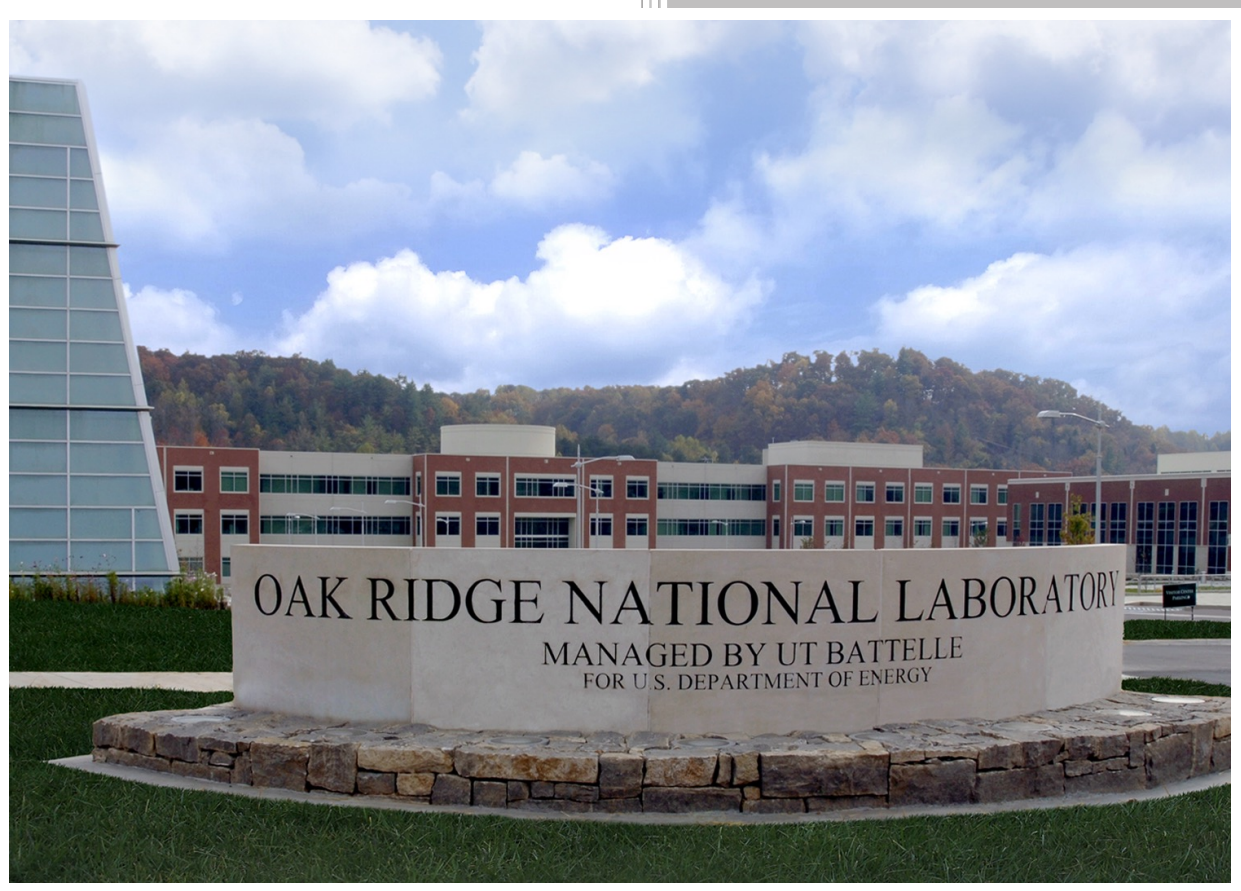

Approved for public release. Distribution is unlimited.
Seth R. Johnson

Thomas M. Evans Gregory G. Davidson Steven P. Hamilton Tara M. Pandya Katherine E. Royston Elliott D. Biondo

Aug. 2020 


\section{DOCUMENT AVAILABILITY}

Reports produced after January 1, 1996, are generally available free via US Department of Energy (DOE) SciTech Connect.

Website osti.gov

Reports produced before January 1, 1996, may be purchased by members of the public from the following source:

National Technical Information Service

5285 Port Royal Road

Springfield, VA 22161

Telephone 703-605-6000 (1-800-553-6847)

TDD 703-487-4639

Fax 703-605-6900

E-mail info@ntis.gov

Website classic.ntis.gov

Reports are available to DOE employees, DOE contractors, Energy Technology Data Exchange representatives, and International Nuclear Information System representatives from the following source:

Office of Scientific and Technical Information

PO Box 62

Oak Ridge, TN 37831

Telephone 865-576-8401

Fax 865-576-5728

E-mail reports@osti.gov

Website osti.gov/contact

This report was prepared as an account of work sponsored by an agency of the United States Government. Neither the United States Government nor any agency thereof, nor any of their employees, makes any warranty, express or implied, or assumes any legal liability or responsibility for the accuracy, completeness, or usefulness of any information, apparatus, product, or process disclosed, or represents that its use would not infringe privately owned rights. Reference herein to any specific commercial product, process, or service by trade name, trademark, manufacturer, or otherwise, does not necessarily constitute or imply its endorsement, recommendation, or favoring by the United States Government or any agency thereof. The views and opinions of authors expressed herein do not necessarily state or reflect those of the United States Government or any agency thereof. 
Reactor and Nuclear Systems Division

\title{
OMNIBUS USER MANUAL
}

\author{
Seth R. Johnson \\ Thomas M. Evans \\ Gregory G. Davidson \\ Steven P. Hamilton \\ Tara M. Pandya \\ Katherine E. Royston \\ Elliott D. Biondo
}

Date Published: Aug. 2020

\author{
Prepared by \\ OAK RIDGE NATIONAL LABORATORY \\ Oak Ridge, TN 37831-6283 \\ managed by \\ UT-Battelle, LLC \\ for the \\ US DEPARTMENT OF ENERGY \\ under contract DE-AC05-00OR22725
}





\section{CONTENTS}

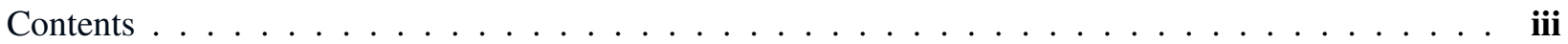

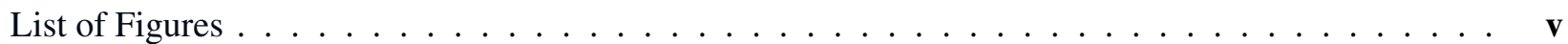

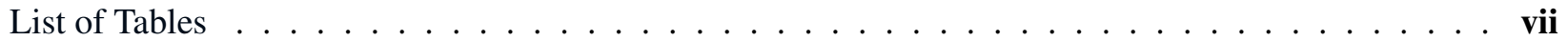

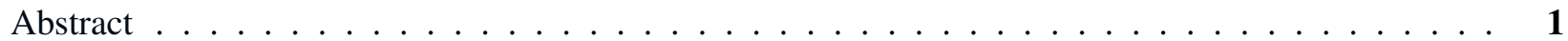

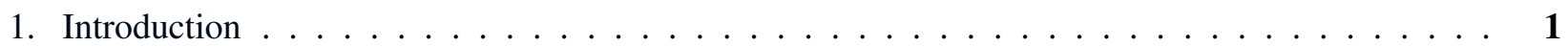

1.1 Omnibus execution $\ldots \ldots \ldots \ldots \ldots \ldots \ldots \ldots \ldots$

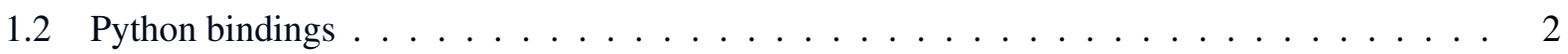

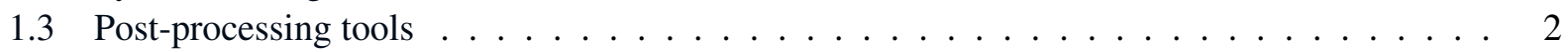

2. Front End Interface $\ldots \ldots \ldots \ldots \ldots \ldots \ldots \ldots$

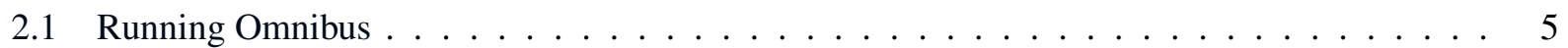

2.2 Omnibus ASCII Input Format $\ldots \ldots \ldots \ldots \ldots \ldots$

2.3 Omnibus input and output . . . . . . . . . . . . . . . . . . 10

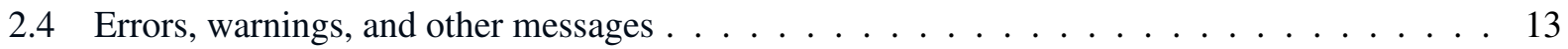

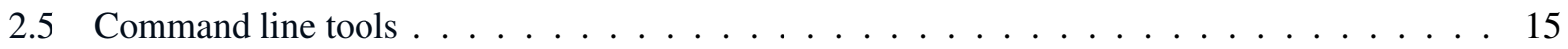

3. Omnibus Input Description $\ldots \ldots \ldots \ldots \ldots \ldots \ldots \ldots$

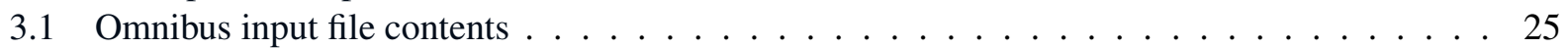

3.2 Problem attributes: $[$ PROBLEM] $\ldots \ldots \ldots \ldots \ldots \ldots \ldots \ldots \ldots$

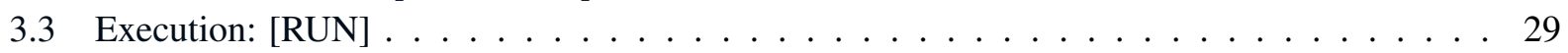

3.4 Output options: $[$ OUTPUT] $\ldots \ldots \ldots \ldots \ldots$

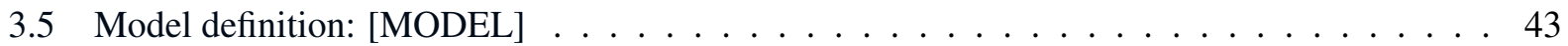

3.6 MCNP input: $[\mathrm{MODEL}=\mathrm{mcnp}] \ldots \ldots \ldots \ldots \ldots \ldots$

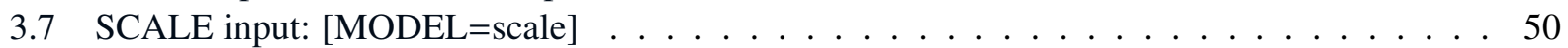

3.8 Geometria input: $[\mathrm{MODEL}=$ geometria $] \ldots \ldots \ldots \ldots \ldots \ldots \ldots$

3.9 Reactor ToolKit input: $[\mathrm{MODEL}=\mathrm{rtk}] \ldots \ldots \ldots \ldots \ldots \ldots$

3.10 Brick mesh input: $[\mathrm{MODEL}=\mathrm{mesh}] \ldots \ldots \ldots \ldots \ldots \ldots$

3.11 Geant 4 input: $[$ MODEL $=$ geant $] \ldots \ldots \ldots \ldots \ldots \ldots \ldots$

3.12 SWORD input: $[$ MODEL $=$ sword $] \ldots \ldots \ldots \ldots \ldots \ldots \ldots$

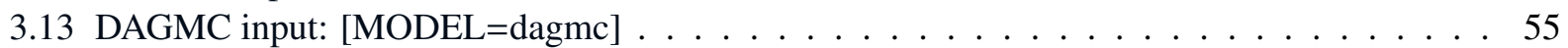

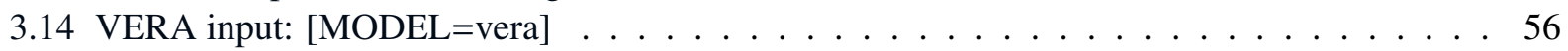

3.15 Particle source definitions: $[$ SOURCE] $\ldots \ldots \ldots \ldots \ldots \ldots$

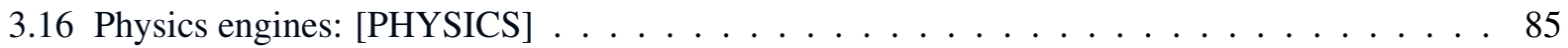

3.17 Continuous-energy physics: $[\mathrm{PHYSICS}=$ ce] $\ldots \ldots \ldots \ldots \ldots$

3.18 Multigroup physics: $[\mathrm{PHYSICS}=\mathrm{mg}] \ldots \ldots \ldots \ldots \ldots$

3.19 Compositions: $[$ COMP] . . . . . . . . . . . . . . . . . . . . . 106

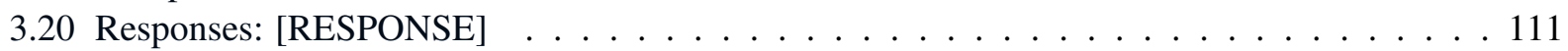

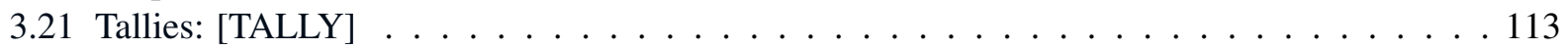

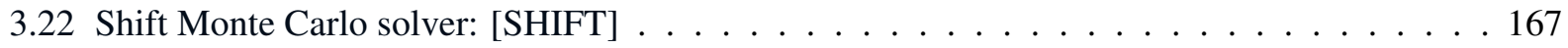

3.23 Denovo deterministic solver: [DENOVO] . . . . . . . . . . . . . . . 176

3.24 ORIGEN depletion solver: $[$ DEPLETION] $\ldots \ldots \ldots$. . . . . . . . . . . . . . . . 212

3.25 Hybrid methodology: [HYBRID] . . . . . . . . . . . . . . . . . . . . . 224

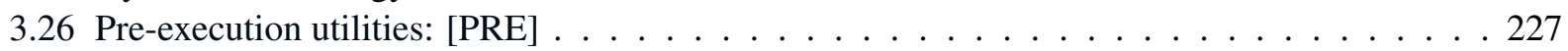

3.27 Post-processing: [POST] . . . . . . . . . . . . . . . . . . . . . . . . 229

4. Geometria Input Description $\ldots \ldots \ldots \ldots \ldots \ldots$

4.1 Geometria input file contents . . . . . . . . . . . . . . . 233

4.2 Universe definitions: [UNIVERSE] . . . . . . . . . . . . . . . . . . . . . . . . . 234

4.3 Shape definitions: $[$ UNIVERSE][SHAPE] . . . . . . . . . . . . . . . . . . . 246 


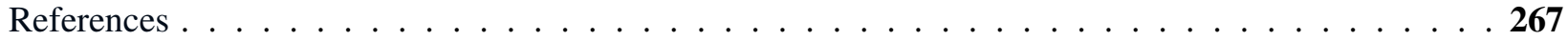

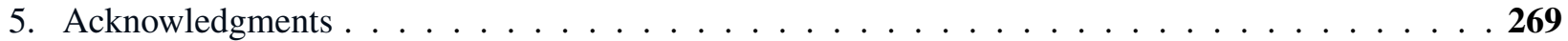

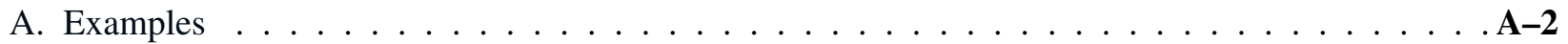

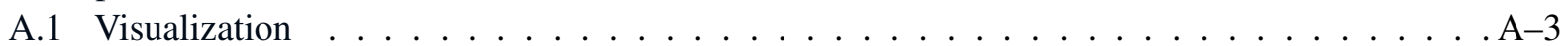

A.2 Denovo . . . . . . . . . . . . . . . . . . . A-9

A.3 Multigroup data exploration $\ldots \ldots \ldots \ldots \ldots \ldots \ldots \ldots$ A . . . . . . . . . . .

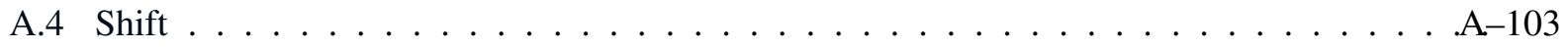

A.5 CE data . . . . . . . . . . . . . . . . . . . . . . . . . . . . . . . . . . . . . . . . . .

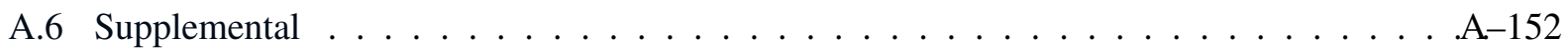

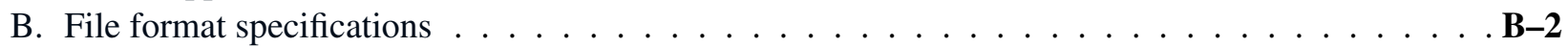

B.1 Denovo Output Specification . . . . . . . . . . . . . . . B-3

B.2 HDF5 Mesh Model Specification . . . . . . . . . . . . . . . . B-4

B.3 RTK XML Input Specification $\ldots \ldots \ldots \ldots \ldots \ldots \ldots$. . . . . . . . . . . . . .

B.4 XS XML Input Specification $\ldots \ldots \ldots \ldots \ldots \ldots \ldots \ldots$ 


\section{LIST OF FIGURES}

1 Execution flow for omnibus-run. The small black boxes are the typical input/output files, blue circles are parts of the Python pre-processor run on the head node, the red circle is the Omnibus executable (run on the compute nodes), and dotted lines denote optional files (e.g.,

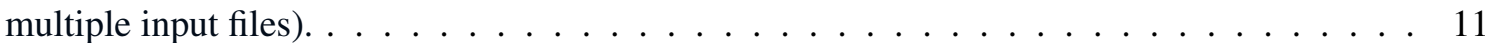





\section{LIST OF TABLES}

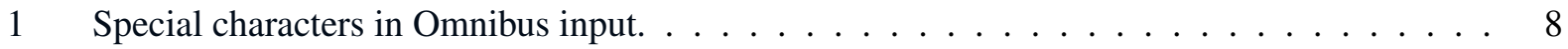

2 Omnibus diagnostic output examples. . . . . . . . . . . . . . . . . . . 14

3 Available types for the $[\mathrm{RUN}]$ database $\ldots \ldots \ldots \ldots \ldots$

4 Feature matrix for the supported models. . . . . . . . . . . . . . . . . . . 43

5 Available types for the $[$ MODEL] database . . . . . . . . . . . . . . . . . . . . . . . . 44

6 Mappings between unusual nuclide IDs. . . . . . . . . . . . . . . . . . . . . 45

7 Mappings between MCNP MT cards and SCALE IDs. . . . . . . . . . . . . . . . 46

8 Available types for the $[$ MOVABLE] database . . . . . . . . . . . . . . . . . . 49

9 MOAB volume properties used in the DAGMC model. . . . . . . . . . . . . . 55

10 Available types for the $[$ SOURCE] database $\ldots \ldots \ldots \ldots \ldots$

11 Available types for the $[$ SHAPE] database $\ldots \ldots \ldots \ldots 6$

12 Available types for the $[$ ENERGY] database $\ldots \ldots \ldots \ldots 6$

13 Available types for the $[$ ANGLE] database $\ldots \ldots \ldots \ldots 6$

14 Available types for the $[$ SPECTRUM] database . . . . . . . . . . . . . . . 66

15 Available types for the $[$ PHYSICS] database $\ldots \ldots \ldots \ldots \ldots$

16 SCALE CE library path aliases in SCALE $6.2 \ldots \ldots \ldots \ldots$

17 Default DBRC data paths in SCALE $6.2 \ldots \ldots \ldots$. . . . . . . . . . 88

18 Available types for the $[$ SPLICE] database . . . . . . . . . . . . . . . . 93

19 Multigroup physics library aliases and filenames in SCALE 6.2 . . . . . . . . . . . . . . 99

20 Possible cross section input formats. . . . . . . . . . . . . . . . . . . . . 104

21 GIP reaction tables. . . . . . . . . . . . . . . . . . . . 105

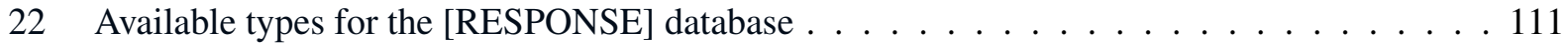

23 Available types for the [DIAGNOSTIC] database . . . . . . . . . . . . . . . 140

24 Available types for the [OUTPUT] database . . . . . . . . . . . . . . . . . . . 166

25 Available types for the $[$ DECOMPOSITION] database $\ldots \ldots \ldots$

26 Available types for the [GENERATIONS] database . . . . . . . . . . . . . . . 175

27 Denovo spatial discretization options. . . . . . . . . . . . . . . . . . 179

28 Available types for the $[$ BOUNDARY] database . . . . . . . . . . . . . . . 183

29 Denovo quadrature construction options. . . . . . . . . . . . . . . . . 185

30 Denovo quadrature availability matrix. . . . . . . . . . . . . . . . . 185

31 Available types for the $[$ SOLVER] database . . . . . . . . . . . . . . . . . 192

32 Denovo solver verbosity options. . . . . . . . . . . . . . . . . . . . . . 198

33 Available types for the $[$ PRECONDITIONER] database . . . . . . . . . . . . . 203

34 Available types for the [PRECONDITIONER] database . . . . . . . . . . . . . . . . . 204

35 Available types for the [PRECONDITIONER] database . . . . . . . . . . . . . . 211

36 Available types for the $[$ HYBRID] database . . . . . . . . . . . . . . . . . . . . 224

37 Weight window input options. . . . . . . . . . . . . . . 226

38 Available types for the $[$ UNIVERSE] database . . . . . . . . . . . . . . . . . . . . . 234

39 Available types for the $[$ SHAPE $]$ database $\ldots \ldots \ldots \ldots$ 



\begin{abstract}
This manual provides instructions for using the Omnibus front end to the Exnihilo code suite, which contains the Denovo and Shift transport solvers.
\end{abstract}

\title{
1. INTRODUCTION
}

Exnihilo is a modern radiation transport framework that implements state-of-the-art algorithms, solvers, and solution methodologies, enabling it to solve a wide variety of nuclear engineering and applications problems with the scalability to run on desktop machines and leadership-class supercomputers. The Omnibus code [3] is a powerful, flexible interface to the extensive functionality of Exnihilo. This manual documents the Exnihilo capabilities exposed by Omnibus and demonstrates their use.

Omnibus provides access to the two core Exnihilo transport solvers, the Denovo deterministic solver (page 176) [4] and the Shift Monte Carlo solver (page 167) [5]. In addition, Omnibus allows the two solvers to be coupled using modern hybrid methods (page 224) that accelerate Shift transport solutions using approximate solutions from Denovo. Omnibus also allows time-dependent Shift depletion calculations using the ORIGEN nuclide depletion solver (page 212) [6].

The Exnihilo transport framework is designed to support computational transport using a combination of input models (page 43) (which define a problem's geometry and compositions) and multiple physics implementations (page 85) (which implement approximations to the Boltzmann transport equation). Many classes of particle sources (page 56) and tallies (page 113) can be defined separately from the model's geometry, and custom compositions (page 106) can also be entered by the user.

The Omnibus interface enables other codes such as ADVANTG [7] and SWORD [8] to create, execute, and post-process Denovo problems, but their use is outside the scope of this document. Other radiation transport codes such as VERAShift [9] and SCALE [2] use the Shift and Denovo codes through a lower-level interface via an internal Omnibus-based API. Although these codes are also outside the scope of this manual, the features and some interfaces presented here may inform the use of downstream codes.

\subsection{OMNIBUS EXECUTION}

At its core, Omnibus is a high-performance, MPI-enabled binary executable, the input of which is a hierarchical problem definition. The Omnibus executable is designed to be launched by a driver, the omnibus Python module, which generates a validated input for the executable before launching it in parallel.

Pre-execution validation, which can be run using the omnibus-pre (page 16) command, is critically important for high-performance computing (HPC) systems in which tens or hundreds of thousands of CPU hours can potentially be lost if an invalid input causes a single process to fail. It is also a tremendous time saver on other shared computing resources such as institutional clusters, ensuring that the input does not need to be queued and launched before it is validated. If a problem input is rejected, then the Python pre-processor can also construct a descriptive, context-sensitive error message such as:

ERROR: From input at ueki-cadis.omn:19:

FATAL ERROR: In /physics/ce, the following unknown inputs were found: 'mood' (did you $\hookrightarrow$ mean 'mode'?)

or 
FATAL ERROR: At ueki-cadis.omn:21: Invalid value 'npe' for keyword 'mode' at /physics/ce/ $\hookrightarrow$ mode:

expected particle transport mode (“n”, “neutron”, “np”, “p”, ”photon”, or $\hookrightarrow$ “pn '), but string is not a particle mode

[mode: Particles to transport]:

npe

Besides validating the user input and providing error messages, the Python input schemas are also used to generate the Omnibus (page 25) and Geometria (page 233) input specifications in this manual.

\subsection{PYTHON BINDINGS}

Exnihilo uses the SWIG ${ }^{1}$ utility to generate Python interfaces to utility classes in Exnihilo. These interfaces power some Omnibus capabilities such as ray tracing, but they may also be used by power users as a high-level interface to many core Exnihilo capabilities, including exploring nuclear data and interacting with problem models.

The following Python modules are installed with Exnihilo ${ }^{2}$

nemesis: Infrastructure components This collection of utilities includes interfaces to MPI and Silo.

robus: Physics data Robus has containers designed to load and store continuous-energy and multigroup cross sections, nuclides, and compositions.

transcore: Transport core components This package includes cross section storage classes, libraries for reading and writing cross sections, etc. See Multigroup data exploration (page A-91) for an example of creating and visualizing multigroup cross sections.

geometria: Geometry interfaces This module has interfaces to the different geometry models used by Shift. It enables ray tracing of the geometry and extraction of compositions.

physica: Physics packages The physics package includes additional interfaces to the CE data. See CE data (page A-132) for examples.

\subsection{POST-PROCESSING TOOLS}

Several tools have been developed to support interacting with, processing, and visualizing Exnihilo input and output. Most of the data are read from and written to HDF5 files, so the workflows rely heavily on the Python-based h5py module.

\subsubsection{OMNIBUS.DATA}

The Omnibus data toolset is an h5py-based interface to Exnihilo HDF5 input and output files. It allows slices of the data to be taken without loading the entire file into memory, and it includes plotting tools based on matplotlib. For examples on how to use this module, see the Denovo (page A-9) and Shift (page A-103) example sections.

Note: The full documentation of the postprocessing tools is outside the scope of this manual. However, if you're using IPython (e.g., through omnibus-analysis) or Jupyter notebook to postprocess the data, tab

\footnotetext{
${ }^{1} \mathrm{http}: / /$ sourceforge.net/projects/swig

${ }^{2}$ SWIG Python bindings will only be available when Exnihilo is built with the SWIG option on and when installing shared libraries.
} 
completion on any of the Omnibus analysis objects will list the available methods for that object. Additionally, adding a question mark symbol after a method or object will provide a help overview showing the arguments that function expects. Finally, the help built-in function provides detailed information about available data members and methods of any object.

\subsection{2 [POST] BLOCK}

When run through omnibus-run (page 15), the post-processing block in the Omnibus input will extract specified data from the HDF5 output in a more human-friendly format. For example, this block will generate an ReST-formatted text file summarizing the run, plot $k_{\text {eff }}$ values for Shift eigenvalue problems, and generate csv-formatted tables of depletion results. More advanced post-processing blocks such as [DENOVO] [SPECTRUM], which will write flux spectra at the given list of points, allow complex extraction of user-specified data.

\subsubsection{H5SH}

The $h 5 \mathrm{sh}$ tool ${ }^{3}$ provides a shell-like interface to browsing HDF5 files. It can be independently downloaded and installed through Python's package manager (pip install h5sh).

It can be cumbersome to use the Omnibus python post-processing tools to quickly examine the contents of an Exnihilo output (or input) file. Some developers have gotten into the habit of using h5ls and h5dump for this, but those tools are impossibly slow for files greater than a few hundred megabytes, and it is inconvenient and slow to use them for multiple consecutive invocations to drill down on a piece of data.

To this end, Exnihilo includes a small but powerful tool that allows the user to browse any HDF5 file as if it were a directory in a shell terminal. HDF5 groups become directories and datasets act as files. It is designed to be intuitive and straightforward. See Using the h5sh tool (page A-152) for an example of its use or the online documentation ${ }^{4}$ for more details.

\footnotetext{
${ }^{3}$ https://pypi.org/project/h5sh/

${ }^{4}$ https://h5sh.readthedocs.io/
} 



\section{FRONT END INTERFACE}

Your Exnihilo installation contains the omnibus executable (which actually drives Denovo and Shift from an XML input file), as well as additional Python scripts that pre-process user input and program output. If using an HPC cluster, then the pre-processing is typically performed on a login node, thus validating and preparing the user input without making it necessary to wait for a job to queue (and without risking the waste of compute hours due to an invalid input being encountered at runtime).

\subsection{RUNNING OMNIBUS}

The omnibus-run (page 15) script creates an Omnibus XML input file, drives and monitors the omnibus (page 17) executable as it is being run, and post-processes the output.

Unlike most code drivers, omnibus-run is meant to be executed on the head node of a cluster rather than on a compute node. Using a machinefile (if the [RUN=mpi] option is being used) or pbs submission (for $[\mathrm{RUN}=\mathrm{pbs}]$ ), it is able to submit the job to other nodes and monitor the application process. To prevent a terminal disconnect from stopping the monitoring (it will not stop the job), it is a good idea to use the screen ${ }^{5}$ utility.

Because the pre-processing, execution, and post-processing steps often generate a dozen files or so, it is highly recommended to create a new working directory for every execution step. The Omnibus pre- and post-processors always generate output in the working directory (as opposed to the directory where the input resides), so a good workflow is

\$ mkdir myrun; cd myrun

\$ omnibus-run .../input.omn

This makes it easier to delete an entire run without accidentally deleting the input, and it also prevents multiple simultaneous Omnibus runs from clobbering each others' files.

Tip: For systems such as Titan that have special filespaces (Lustre) from which the code is executed, the easiest way to ensure that all Omnibus I/O remains on that system is to leave the .omn input file on Lustre and call omnibus-run from that directory.

\subsubsection{EXAMPLE ON A LOCAL MACHINE}

Suppose there is an input file, batman . omn, on a local machine:

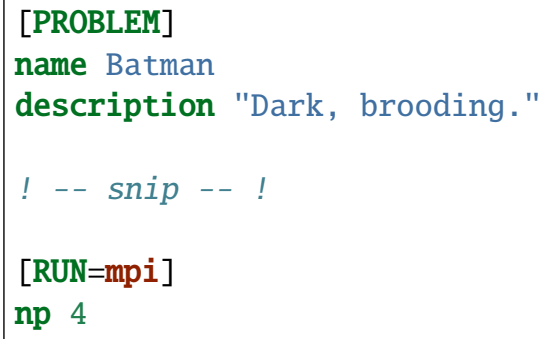

If Omnibus is installed with MPI, then one can simply open a terminal and call:

\footnotetext{
${ }^{5}$ https://kb.iu.edu/d/acuy
} 
\$ omnibus-run batman.omn

This runs the following sequence:

1. The pre-processor will validate the input.

2. After successful validation, the pre-processor will write an xml intermediate file read in by the omnibus binary executable. If necessary, it will also generate other input files (such as the run tape file used for running on Monte Carlo N-Partcle [MCNP] geometry).

3. The script will launch a local process with arguments such as mpirun -np 4 omnibus batman.xml and then will save the output to the current directory and echo it to the screen.

4. When the omnibus execution is complete, it will write out tally data and other program output to several different files.

5. A Python post-processor reads the output and converts it to a human-readable format, leaving the original output files for later post-processing.

\subsubsection{EXAMPLE ON A CLUSTER USING PBS/TORQUE}

In this example, the local machine problem is copied and the run block is changed (see [RUN=pbs] (page 30)):

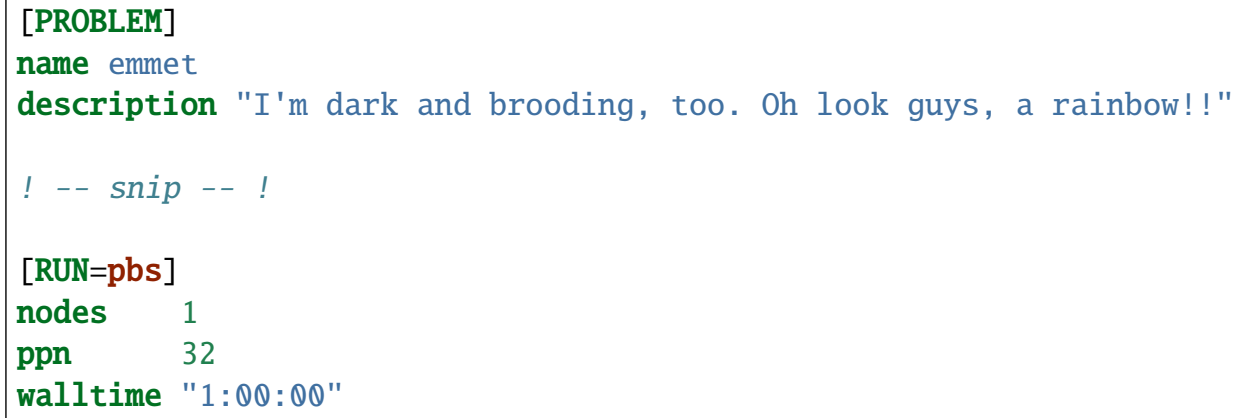

When omnibus-run is executed on the head node, it will launch qsub, monitor the job ID until it begins running on the compute node, and will echo output to the screen over an ssh connection. The process on the head node remains almost entirely idle during the problem run, so the user need not worry about incurring the wrath of the system's administrator.

Note: Be aware that some of the pre-processing may be computationally expensive, specifically when using the MCNP model on a large input deck. In that case, it is recommended that one generate the runtpe file separately and specify the runtpe_path parameter instead of the input command so that Omnibus does not run the MCNP pre-processor on the head node. Alternatively, one may be able to run omnibus-run or omnibus-pre on a compute node.

\subsubsection{RUNNING OMNIBUS MANUALLY}

Separate scripts are provided for pre- and post-processing an Omnibus input. To generate the Omnibus XML input file from an ASCII input, call: 
\$ omnibus-pre my_problem.omn

This will create a Teuchos ParameterList XML input file my_problem.inp.xml. This parameter list is then run with the Omnibus driver:

\$ mpirun -np 16 omnibus my_problem.inp.xml

Post-processing (including plotting keff and Shannon entropy convergence, as well as rendering the XML output into a more human-readable format) is done with the command:

\$ omnibus-post my_problem.pp.json

\subsection{OMNIBUS ASCII INPUT FORMAT}

The Omnibus ASCII input is a human-readable, minimal input syntax for Omnibus. The underlying Omnibus input data structure is hierarchical, and the ASCII input is designed to flatten the hierarchy. The input consists of (1) "blocks" of input data, each of which represents a database, and (2) cards, which consist of parameters and "commands" which generate parameters or perform other functions.

\subsubsection{BLOCKS}

Block titles have the following formats

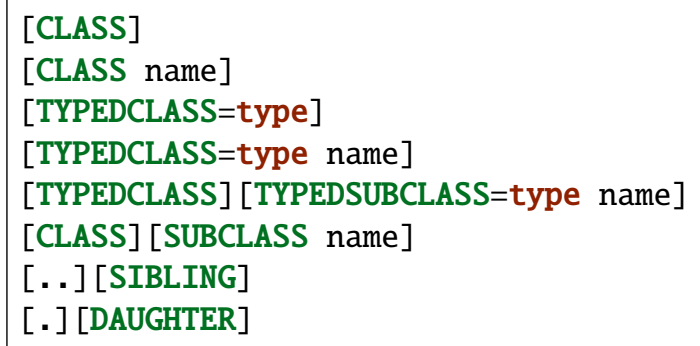

These formats embed the location in the hierarchy, the database class, the database type, and the name of this particular instance of the database class. The "name" (which requires a value with only letters, numbers, and the underscore) is simply a shorthand for declaring the block and adding a "name" parameter. The class type is required for databases with multiple allowed types (e.g., model and physics), but it is disallowed for types that do not. Only the rightmost database can have a type: its parent block types must be omitted.

Relative blocks can be specified using the special [..] and [.] keywords analogous to POSIX paths. The [.. ] block specifies "belonging to two blocks above the current block location." Similarly, [.] means "belonging to one block above the current block location," allowing the easy specification of subdatabases. These specifications simplify deeply nested blocks; in the above example, the full list of blocks is expanded to:

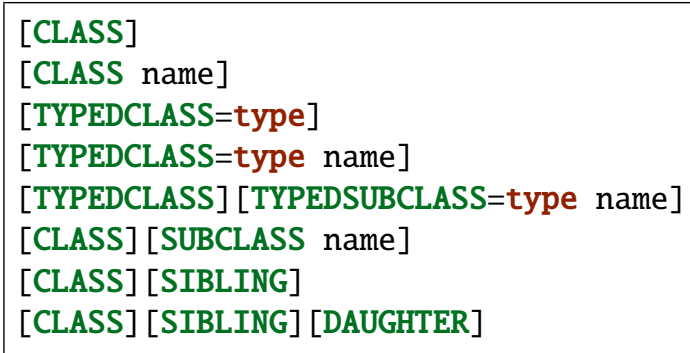

Whitespace in block titles, as well as capitalization for the class and type attributes, is ignored. 


\subsubsection{CARDS}

Cards are started on a new line; an indentation of four or more spaces is treated as a continuation of the previous card. Spaces separate values in a parameter list or arguments in a command. For strings, quotation marks can be used to treat whitespace as standard characters. The backslash can be used to escape quotation marks inside a quoted string.

For example, these two parameters demonstrate the correct usage of whitespace:

param This is a list of seven parameters

param "This is a single parameter with an \" embedded quotation mark."

Tip: One common input error is to mistake a small indentation on the next line for a continuation. This statement declares three parameters inside a block:

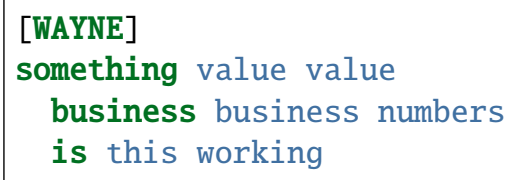

whereas this is one parameter with multiple values:

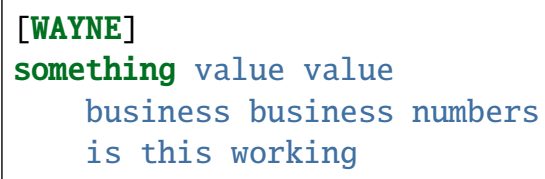

Using the syntax highlighting files for Vim and Emacs provided in the Exnihilo environment or using the omnlexer Pygments lexer will make such errors very obvious.

\subsubsection{OTHER FEATURES}

\subsubsection{Special characters}

The following characters are treated as special tokens in the ASCII input:

Table 1: Special characters in Omnibus input.

\begin{tabular}{|c|c|c|}
\hline Token & Name & Use \\
\hline$!$ & Exclamation & Comment: all following characters on the line are ignored \\
\hline \# & Hash & Used to include other files \\
\hline$\backslash$ & Backslash & Escapes other special characters \\
\hline ' & Single quote & Starts or ends a string \\
\hline$"$ & Quote & Starts or ends a string \\
\hline : & Colon & $\begin{array}{l}\text { Separates variable names in column format, or creates sepa- } \\
\text { rate items in a list }\end{array}$ \\
\hline- & Dash & $\begin{array}{l}\text { A standalone series of dashes is translated to the None } \\
\text { Python token, used in lists or column format to denote the } \\
\text { absence of a value }\end{array}$ \\
\hline$->$ & Arrow & Creates a two-item tuple indicating a mapping \\
\hline$\$$ & Dollar & Encloses a math expression to be evaluated \\
\hline | & Pipe & Encloses units specification \\
\hline
\end{tabular}


Whitespace is generally ignored. The exception is the line continuation described above in which four leading spaces indicate continuation of the previous line. When embedded in a quoted string, whitespace is preserved.

Any text on a line following an exclamation point is ignored.

\subsubsection{Math expressions}

The Omnibus ASCII input format can evaluate ${ }^{6}$ simple math expressions enclosed in a matching pair dollar signs in the input. Like quotations, these signs must be on the same line and separated from other input values by whitespace. An example of a math expression is:

x $\$ 1 / 3 \$ \$ 2 * 5 \$$

\subsubsection{Unit support}

When the Pint ${ }^{8}$ python package is installed, Omnibus can automatically convert units to the correct type needed by an input parameter. Units are surrounded by pipes and modify the previously input value. Like quotations and math expressions, units must be defined on a single line and separated from other input values by whitespace. An example of unit conversion is:

\section{[DEPLETION]}

power $\quad 3.14 \quad$ |Btu / fortnight |

burn_length $1.0 \mathrm{e}-5$ | millenia |

Since the [DEPLETION] database (page 212) expects power in units of megawatts and burn length in units of days, the input quantities will be converted to those types when they are exported for the Omnibus binary driver.

The Pint package provides a comprehensive set of available units 9 .

Warning: Currently, units are only supported for scalar quantities, not parameters that take lists. Trying to use units in that case will cause an error.

\subsubsection{Interpolation}

Inside numerical lists, the MCNP interpolation/repetition shorthand characters "I," "ILOG," "M," and "R" are implemented. For example,

x_coordinates 12 I 4

is interpolated to form

x_coordinates 12334

This feature is tied to the parameter processing itself, so only numeric lists have the ability to interpolate (i.e., the letter I is treated just like that letter in normal parameters). This also means that Python or JSON input can use the interpolation/repetition shorthand characters for convenience.

\footnotetext{
${ }^{6}$ This capability is implemented using the simpleeval ${ }^{7}$ library.

${ }^{7} \mathrm{https}: / /$ github.com/danthedeckie/simpleeval

${ }^{8}$ https://pint.readthedocs.io/en/0.9/

${ }^{9}$ https://github.com/hgrecco/pint/blob/master/pint/default_en.txt
} 
Tip: The vacuum-omnibus-input (page 20) script will read the Omnibus input file, reformat it, and rewrite it. If the input and output are not logically the same, then there may be a subtle syntax error in the input file (e.g., not indenting when continuing). This tool only parses the input file; it does no expansion, validation, or defaulting.

Tip: To view a validated and reformatted ASCII version of input, one can explicitly tell the preprocessor to save an .omn file:

\$ omnibus-pre problem.omn -o problem.validated.omn

This operation is performed automatically when using the omnibus-run (page 15) command.

\subsection{OMNIBUS INPUT AND OUTPUT}

Omnibus accepts multiple input formats, prints output to the terminal screen, writes (possibly multiple) intermediate files, and processes the output into more useful formats.

The following image describes how files are generated and used while driving Omnibus through the front end:

\subsubsection{INPUT FILES}

Omnibus ASCII input files (with the '.omn' extension) are described in ASCII input (page 7):

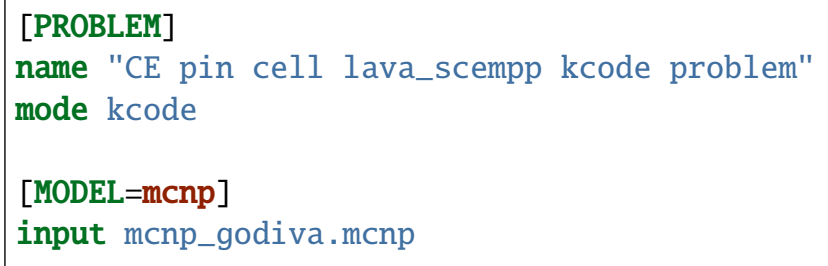

These are unfolded into hierarchical databases and are then converted to XML for the Omnibus executable. YAML and JSON hierarchical databases are also supported, which may appeal more to power users:

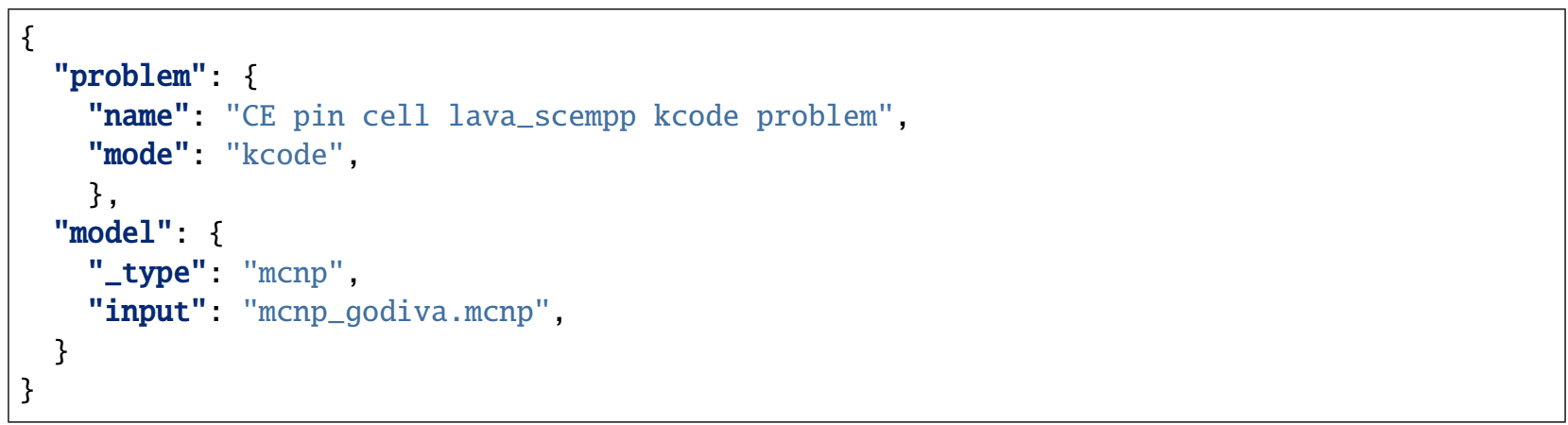

Python files that can modify an existing (e.g., ASCII-created) input definition are also supported. When pre-processing Python input definitions, a local db variable contains the Omnibus input definition hierarchy and can be modified or extended. This Python method is extremely powerful for automating repetitious tallies, as demonstrated in this example that creates five similar cylindrical mesh tallies sharing an energy grid: 


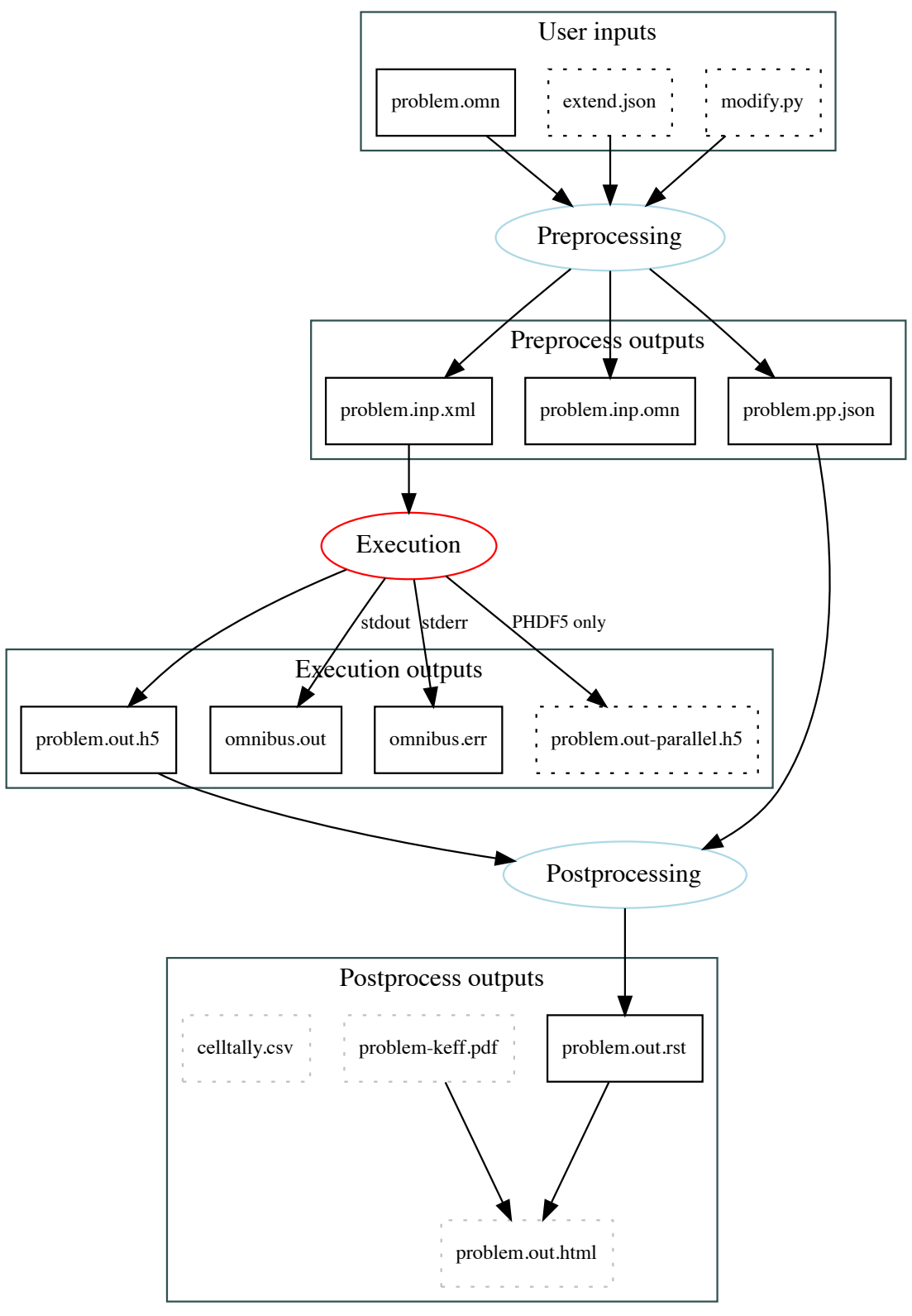

Fig. 1: Execution flow for omnibus-run. The small black boxes are the typical input/output files, blue circles are parts of the Python pre-processor run on the head node, the red circle is the Omnibus executable (run on the compute nodes), and dotted lines denote optional files (e.g., multiple input files). 


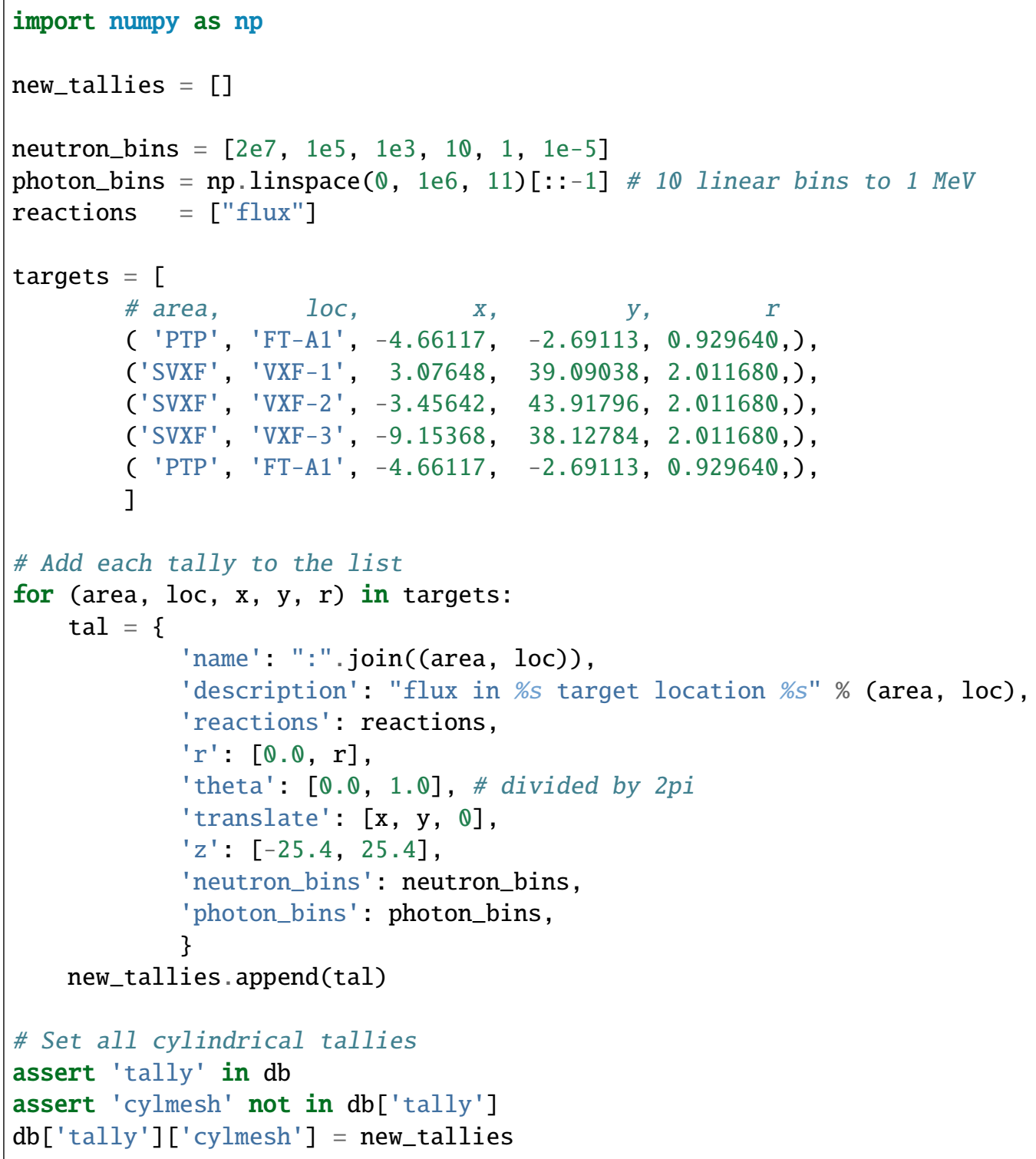

This could be integrated into an Omnibus run file by executing:

omnibus-run hfir.omn hfir-tallies.py

\subsubsection{PRE-PROCESSING OUTPUT FILES}

The pre-processing step will typically create several files for an input problem .omn:

problem.pp.json If using the omnibus-run front end, then this will be created: it is a fully processed version of the problem input, with all default parameters explicitly filled.

problem .inp.omn If using the omnibus-run front end, then this will be created: it is a fully processed and reformatted version of the problem input. It also has all parameters filled.

problem.inp. xml The Teuchos ParameterList XML file is read by the omnibus executable.

Additionally, if an MCNP model is being used, then a runtpe file will be generated. Finally, if a [RUN=pbs] block is present, then a problem .pbs submission script will be generated. 


\subsubsection{EXECUTION OUTPUT FILES}

Running Omnibus will generate one or more output files:

problem.out.h5 Execution results and data will be written using serial HDF5.

problem . out-parallel.h5 If running on a parallel file system and parallel HDF5 is installed, then some datasets will be written to this file and "externally linked" into the serial HDF5 file. Generally, only data that are decomposed across MPI domains are written to this file.

omnibus.out Messages will be written from the executable (and PBS script if applicable) to stdout. Generally, only embedded external code (such as Trilinos solvers and SCALE cross section processing routines) write to stdout.

omnibus.err Messages will be written from the executable (and PBS script if applicable) to stderr. These include logging and diagnostic messages during the program run, as described in the next section.

\subsection{ERRORS, WARNINGS, AND OTHER MESSAGES}

Omnibus can encounter unexpected conditions for a variety of reasons, including:

- problems with the system configuration,

- logic errors in the application code,

- inconsistencies in nuclear data being used, and

- errors in user input.

To the extent possible, Omnibus attempts to detect and gracefully handle these errors to provide feedback to the user that is meant to help in determining the root cause.

\subsubsection{LOG MESSAGES}

The Exnihilo framework has an internal logging system for writing messages of different levels of severity to the screen. The omnibus-run (page 15) process intercepts these messages, as well as all other output text, and will print formatted logging statements. For some statements that are not very important but that provide the user with an idea of the program status, omnibus-run (page 15) will only display the latest statement. Other higher-level statements will remain on the screen (with levels of color, for terminals that support it, indicating their severity).

The different levels of logging messages are:

DEBUG Very fine-grained diagnostic messages that show a level of detail not typically needed for problem execution.

DIAGNOSTIC Progressive output that shows detailed state information about the problem. Example diagnostics include Denovo iteration count and Shift cycle k-effective estimates.

STATUS Traces the flow of Omnibus showing what part of the program is being executed.

INFO Informational messages unique to the particular problem being run, such as "Loaded 123 cross sections" or "Set default for parameter 'foo' to 123."

WARNING Messages about situations that are unusual, unexpected, or possibly inconsistent: something might be wrong. They may indicate the possibility of incorrect solutions, but they may also be totally harmless, depending on the intent of the user. The user should examine these warnings carefully to determine their importance. Examples of warnings are: 
- when nuclear data for a requested nuclide is unavailable and a similar nuclide (e.g., ground state or unbound) was substituted;

- when a user requests Silo output, but Silo support is not compiled;

- when volumes are omitted from cells being tallied, so the solutions change from being normalized by volume to being unnormalized; and

- when statistical checks on Shannon entropy fail.

ERROR Messages indicating a definite inconsistency: something is wrong. Omnibus is built to attempt to recover gracefully from unexpected program input, cross section data, etc. When a recoverable error occurs, an error message is printed. The user should very carefully examine the error to assess its severity. Example error messages include:

- particles being lost while tracking through the geometry;

- CE cross sections not balancing correctly at the particle's energy, suggesting an error in the CE data.

FATAL ERROR This message is the last thing the user will see before the world turns dark: an unrecoverable error (either due to user input or an unexpected program state) has occurred, and the program will shut down. If Omnibus is being monitored inside omnibus-run, then it will attempt to kill the process being run (e.g., by signalling mpiexec or calling qdel).

By default, DIAGNOSTIC and higher levels are printed to the screen and echoed to omnibus. err, and INFO and higher levels are saved to the Omnibus HDF5 output file. There is typically not any output in the omnibus . out file; this usually only contains output from third-party libraries.

Tip: The Omnibus input parameter screen_verbosity (page 42) will change the level of message that is written to the screen and the omnibus.err file.

Note that the warning labels described above correspond to special prefixes in the program output:

Table 2: Omnibus diagnostic output examples.

\begin{tabular}{ll}
\hline Level & Example \\
\hline DIAGNOSTIC & Loading nuclide u-235 a 293K. \\
STATUS & $::$ : Beginning inactive cycles \\
INFO & $>>$ Loading CE library ce_v7.0_endf.h5 \\
WARNING & $* * *$ neutron data for ZAID=1001 is not \\
& available for $\cdots$ MT=301 in the splicing \\
& AMPX library. \\
ERROR & $! ! !$ Geometry error in particle $0: 123: \ldots$ \\
FATAL ERROR & $! * ! * !$ Couldn't find a CE library for ce_v7. \\
& $4 \_$endf \\
\hline
\end{tabular}

\subsubsection{OUT-OF-MEMORY ERRORS}

It is very possible for Omnibus to run out of memory in the middle of execution since large data fields are allocated at different points during the run. On some system configurations, Omnibus will be able to correctly detect and report an out-of-memory (OOM) error ${ }^{10}$.

\footnotetext{
10 The kernel's memory allocation function will correctly return a NULL pointer, indicating a failure to allocate. This will cause the $\mathrm{C}++$ library to throw a std: : bad_alloc exception, which is then caught by Omnibus.
} 
However, the default behavior ${ }^{11}$ on Linux kernels is to overcommit memory. Although overcommitment is practical for most real-world applications, its consequence is that an application cannot know exactly when or why it ran out of memory. Rather than printing a useful message about memory allocation, the offending Omnibus process will immediately be terminated (with SIGKILL, signal 9) without any opportunity to clean up.

Tip: When launched with [RUN=pbs] (page 30), a problem killed due to an OOM error may produce an omnibus.err file that ends with:

Primary job terminated normally, but 1 process returned

a non-zero exit code. Per user-direction, the job has been aborted.

mpiexec noticed that process rank 72 with PID 115153 on node mod-pbs-c62

exited on signal 9 (Killed).

and an output file that may contain:

5 total processes killed (some possibly by mpiexec during cleanup)

!*!*! Omnibus execution failed with error 137

To help diagnose OOM errors, Omnibus provides a print_memory (page 41) parameter that will periodically output local and global memory usage. Additionally, the final status or informational update before the error may provide the context for the failure: if the last message is about constructing a Denovo state vector, then it is likely that the Denovo discretization is too fine to fit on the requested number of processors.

Aside from using a machine with more RAM per core, there are two possible actions to take to mitigate OOM errors. If the allocation failure is about decomposed data (such as the state vector in Denovo), then it will be necessary to use more processors or nodes to decrease the memory requirement per process. However, if the failure is due to replicated data such as material compositions or broadened cross sections in Shift, then the user can reduce the node occupancy (processes per node (page 33)), allowing each process to use more of the available memory on the system.

See Performance considerations (page 178) for a discussion of memory consumption in Denovo.

\subsubsection{MISSING CAPABILITIES}

Although the Omnibus preprocessing validation should encode configuration requirements and feature capabilities through its "applicability" statements and other logic, it is possible that the developers have missed something. If a feature is implemented by SCALE but is not enabled in the installed copy of SCALE (usually due to an unavailable third-party library), then an error message will explain that the build configuration does not support the feature. If a capability is planned for Shift or is only known to work under a limited set of other options, then an error message may explain that the feature is not currently implemented.

\subsection{COMMAND LINE TOOLS}

\subsubsection{OMNIBUS-RUN}

Run the Omnibus pre-processor, run Omnibus, and run the post-processor.

\footnotetext{
${ }^{11}$ https://www.kernel.org/doc/Documentation/vm/overcommit-accounting
} 
Run Omnibus from start to finish.

usage: omnibus-run [-h] [--version] [-g] [-c] [-e ENV] [-v] [-q]

[--very-quiet] [--silent]

[--log \{None, DEBUG, STATUS, INFO, WARNING, ERROR, CRITICAL $\}]$

[inp [inp ...]]

\subsubsection{Positional Arguments}

inp Input file names (omnibus, yaml, and/or python).

\subsubsection{Named Arguments}

--version show program's version number and exit

-g, --debug Enable extended debug assertions

Default: False

-c, --clobber Overwrite exiting output files rather than renaming them

Default: False

-e, --env Update global environment settings with this JSON file

\subsubsection{3 verbosity}

-v, --verbose Print all debug messages

Default: "STATUS"

-q, --quiet Only print informational and warning messages

--very-quiet Only print warning messages

--silent Print messages only on failure

--log Possible choices: None, DEBUG, STATUS, INFO, WARNING, ERROR,

\section{CRITICAL}

Create a $\log$ file with the given verbosity

Exnihilo version (UNKNOWN)

\subsubsection{OMNIBUS-PRE}

Generate an XML input file for Omnibus, validating input along the way.

Preprocess Omnibus input files.

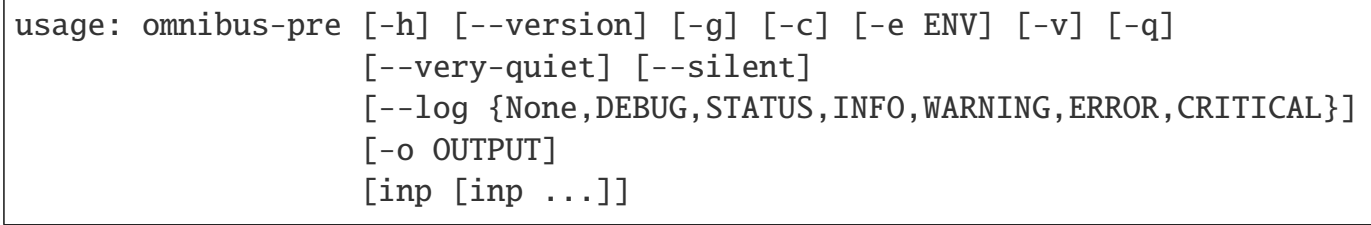

\subsubsection{Positional Arguments}

inp Input file names (omnibus, json, yaml, and/or python). 


\subsubsection{Named Arguments}

$\begin{array}{ll}\text {--version } & \text { show program's version number and exit } \\ \text {-g, --debug } & \text { Enable extended debug assertions } \\ & \text { Default: False } \\ \text {-c, --clobber } & \text { Overwrite exiting output files rather than renaming them } \\ & \text { Default: False } \\ \text {-e, --env } & \text { Update global environment settings with this JSON file } \\ \text {-o, --output } & \text { Output filename (xml, jon, omn, yaml) }\end{array}$

\subsubsection{3 verbosity}

-v, --verbose Print all debug messages

Default: "STATUS"

-q, --quiet Only print informational and warning messages

--very-quiet Only print warning messages

--silent Print messages only on failure

--log Possible choices: None, DEBUG, STATUS, INFO, WARNING, ERROR,

\section{CRITICAL}

Create a log file with the given verbosity

Exnihilo version (UNKNOWN)

\subsubsection{OMNIBUS}

The actual Omnibus binary executable.

usage: omnibus [--version] xml_input

Positional arguments:

xml_input Path to the XML parameter input file.

Options:

--version Show usage information and exit.

\subsubsection{OMNIBUS-POST}

Execute the Omnibus post-processing functions specified in a [POST] block. The argument is the "preprocessed" .pp.json file produced when running omnibus-run.

Post-process Omnibus output. 
usage: omnibus-post [-h] [--version] [-g] [-c] [-e ENV] [-v] [-q]

[--very-quiet] [--silent]

[--log \{None, DEBUG, STATUS, INFO, WARNING , ERROR, CRITICAL $\}]$

$\mathrm{pp}$

\subsubsection{Positional Arguments}

pp Omnibus postprocess file (.pp.json)

\subsubsection{Named Arguments}

--version show program's version number and exit

-g, --debug Enable extended debug assertions

Default: False

-c, --clobber Overwrite exiting output files rather than renaming them

Default: False

-e, --env Update global environment settings with this JSON file

\subsubsection{3 verbosity}

-v, --verbose Print all debug messages

Default: "STATUS"

-q, --quiet Only print informational and warning messages

--very-quiet Only print warning messages

--silent Print messages only on failure

--log Possible choices: None, DEBUG, STATUS, INFO, WARNING, ERROR,

\section{CRITICAL}

Create a $\log$ file with the given verbosity

Exnihilo version (UNKNOWN)

\subsubsection{OMNIBUS-ANALYSIS}

Load an Omnibus output file into an iPython shell

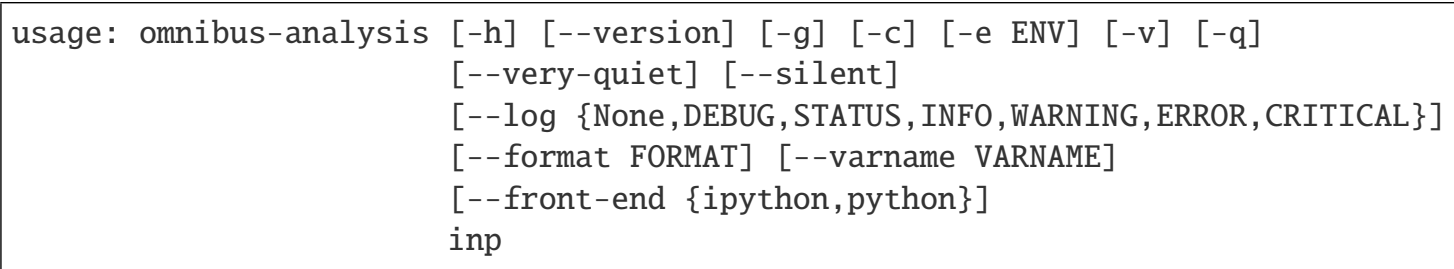

\subsubsection{Positional Arguments}

inp path to Omnibus HDF5 file 


\subsubsection{Named Arguments}

--version show program's version number and exit

-g, --debug Enable extended debug assertions

Default: False

-c, --clobber Overwrite exiting output files rather than renaming them

Default: False

-e, --env

Update global environment settings with this JSON file

--format format of the HDF5 file (e.g. 'output','meshmodel')

Default: "output"

--varname local variable with loaded file wrapper

Default: "f"

--front-end Possible choices: ipython, python

interactive console type

Default: "ipython"

\subsubsection{3 verbosity}

-v, --verbose

Print all debug messages

Default: "STATUS"

-q, --quiet

Only print informational and warning messages

--very-quiet

Only print warning messages

--silent

Print messages only on failure

$--\log$

Possible choices: None, DEBUG, STATUS, INFO, WARNING, ERROR, CRITICAL

Create a $\log$ file with the given verbosity

Exnihilo version (UNKNOWN)

\subsubsection{OMNIBUS-CONF}

Print configuration info from an HDF5 output file or the current Omnibus configuration

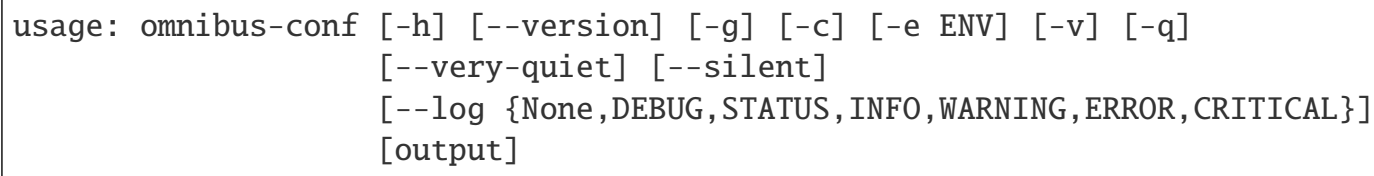

\subsubsection{Positional Arguments}

output Path to HDF5 file, or blank for current configuration 


\subsubsection{Named Arguments}

--version show program's version number and exit

-g, --debug Enable extended debug assertions

Default: False

-c, --clobber Overwrite exiting output files rather than renaming them

Default: False

-e, --env Update global environment settings with this JSON file

\subsubsection{3 verbosity}

-v, --verbose Print all debug messages

Default: "STATUS"

-q, --quiet Only print informational and warning messages

--very-quiet Only print warning messages

--silent Print messages only on failure

--log Possible choices: None, DEBUG, STATUS, INFO, WARNING, ERROR, CRITICAL

Create a log file with the given verbosity

Exnihilo version (UNKNOWN)

\subsubsection{VACUUM-OMNIBUS-INPUT}

Parse an Omnibus input file and write a clean, consistent copy.

usage: vacuum-omnibus-input [-h] [--version] [-g] [-c] [-e ENV] [-v] [-q]

[--very-quiet] [--silent]

[--log \{None, DEBUG, STATUS, INFO, WARNING , ERROR, CRITICAL $\}]$

inp $[$ inp ... ]

\subsubsection{Positional Arguments}

inp omnibus input file name

\subsubsection{Named Arguments}

--version show program's version number and exit

-g, --debug Enable extended debug assertions

Default: False

-c, --clobber Overwrite exiting output files rather than renaming them

Default: False

-e, --env Update global environment settings with this JSON file 


\subsubsection{3 verbosity}

$\begin{array}{ll}\text {-v, --verbose } & \text { Print all debug messages } \\ & \text { Default: "STATUS" } \\ \text {-q, --quiet } & \text { Only print informational and warning messages } \\ \text {--very-quiet } & \text { Only print warning messages } \\ \text {--silent } & \text { Print messages only on failure } \\ \text {--log } & \text { Possible choices: None, DEBUG, STATUS, INFO, WARNING, ERROR, } \\ & \text { CRITICAL }\end{array}$

Create a $\log$ file with the given verbosity

Exnihilo version (UNKNOWN)

\subsubsection{MAKE-DENOVO-MODEL}

Create a Denovo mesh model file from a denovo output file

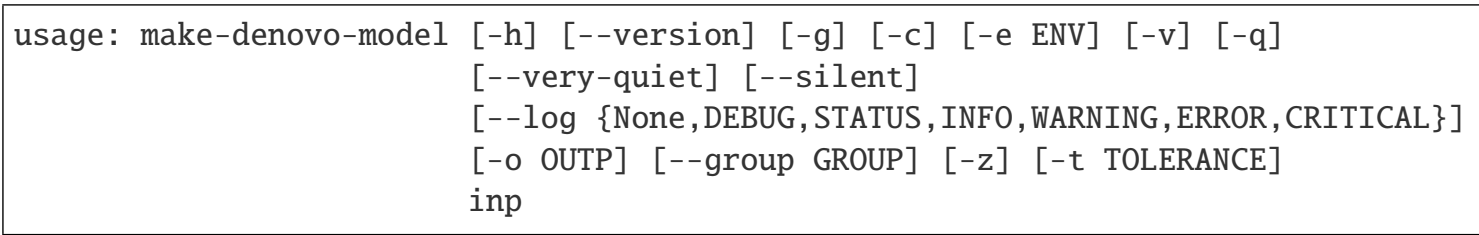

\subsubsection{Positional Arguments}

inp Input file name (.h5).

\subsubsection{Named Arguments}

--version show program's version number and exit

-g, --debug Enable extended debug assertions

Default: False

-c, --clobber Overwrite exiting output files rather than renaming them

Default: False

-e, --env Update global environment settings with this JSON file

-o, --output Mesh model output filename (.h5)

--group HDF5 group that contains the matids

Default: "denovo"

-z, --disable-compression Disable compression of the source term

Default: True

-t, --mix-tolerance Change the threshold for combining similar mixtures

Default: 0.0 


\subsubsection{3 verbosity}

-v, --verbose

Print all debug messages

Default: "STATUS"

-q, --quiet Only print informational and warning messages

--very-quiet Only print warning messages

--silent

Print messages only on failure

$--\log$

Possible choices: None, DEBUG, STATUS, INFO, WARNING, ERROR, CRITICAL

Create a $\log$ file with the given verbosity

Exnihilo version (UNKNOWN)

\subsubsection{DENOVO-POINT-OUTPUT}

Save spectra from a Denovo output file

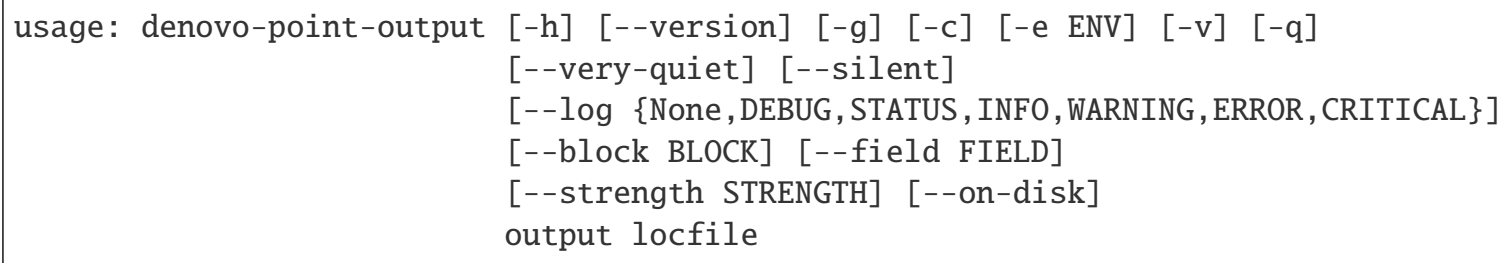

\subsubsection{Positional Arguments}

output Path to Denovo .out.h5 file

locfile Path to point locations, or - for stdin

\subsubsection{Named Arguments}

--version show program's version number and exit

-g, --debug Enable extended debug assertions

Default: False

-c, --clobber Overwrite exiting output files rather than renaming them

Default: False

-e, --env Update global environment settings with this JSON file

--block Name of the Denovo run block to extract

Default: "denovo"

--field $\quad$ Name of the energy-dependent output field (default: flux)

Default: "flux"

--strength, -s Source strength normalization

Default: 1.0

--on-disk, -k Read point data without loading entire file into memory

Default: False 
2.5.9.3 verbosity

$\begin{array}{ll}\text {-v, --verbose } & \text { Print all debug messages } \\ & \text { Default: "STATUS" } \\ \text {-q, --quiet } & \text { Only print informational and warning messages } \\ \text {--very-quiet } & \text { Only print warning messages } \\ \text {--silent } & \text { Print messages only on failure } \\ \text {--log } & \text { Possible choices: None, DEBUG, STATUS, INFO, WARNING, ERROR, } \\ & \text { CRITICAL }\end{array}$

Create a $\log$ file with the given verbosity

Exnihilo version (UNKNOWN) 



\section{OMNIBUS INPUT DESCRIPTION}

The Omnibus input is split into a hierarchy of blocks comprised of databases, sublists, and parameters. The front end also supports "commands" for creating or modifying input parameters, as well as additional pre-processing and post-processing for validation.

Databases, sublists, and parameters all have the following properties:

Name The name of the parameter as it appears in the input. Some parameters have shorter aliases (e.g., nemin for n_energy_min) that appear in the documentation just below the full parameter name.

Description The text immediately below the name should describe what it means and how it is used.

Applicable when A series of rules describing when the parameter may or may not be used. These rules take into account other parameters as well as the build configuration. Bulleted "applicability" items indicate that all the conditions must be met.

Default If present, a default value for the parameter or database. The default may be a fixed value (e.g., 3.0), or it may be a procedure based on the other input parameters, in which case a rough description of the default is given. For databases and sublists, the default may appear as a Python expression (e.g., \{'_type': "none" $\}$ for a database with type "none"). If no default is given, then the parameter or database is required in the given context. However, a few parameters and databases are optional and are marked accordingly.

Export The parameter will be renamed when writing to the .inp. xml file for historical reasons.

Note: This documentation was generated automatically with the following version of Exnihilo:

version 6.3.pre-b13 (branch 'master' on 'upstream', r729: \#9809b44f on 2020JUL16)

date 2020-07-16 22:02:43

\subsection{OMNIBUS INPUT FILE CONTENTS}

Each of the top-level blocks (and the overall problem input file) are described here.

database [COMP]

Composition options and definitions. See [COMP] (page 106).

Default (empty database)

database [DENOVO]

Denovo solver options. See [DENOVO] (page 176).

\section{Applicable when}

- 'Denovo' is enabled in this build; and

- solver is 'denovo'

database [DEPLETION]

ORIGEN nuclide depletion options. See [DEPLETION] (page 212). 


\section{Applicable when}

- 'depletion' is enabled in this build; and

- solver is 'depletion'

deprecated geometry

Deprecated entry geometry has been renamed to model.

Update to model

database [HYBRID]

Monte Carlo acceleration method. See [HYBRID] (page 224).

Applicable when problem mode is hybrid

database [MODEL]

Representation of geometry and compositions. See [MODEL] (page 43).

database [OUTPUT]

Output options. See [OUTPUT] (page 39).

Default (empty database)

sublist [PHYSICS]

Physics treatment. See [PHYSICS] (page 85).

Default void physics when in mode raytrace

database [POST]

Post-processing options. See [POST] (page 229).

Default (empty database)

database [PRE]

Pre-processing options. See [PRE] (page 227).

Default (empty database)

database [PROBLEM]

Problem identifiers and mode. See [PROBLEM] (page 27).

sublist [RESPONSE]

Tally responses. See [RESPONSE] (page 111).

\section{Applicable when}

- using Shift, or in adjoint mode with adjoint_source tally; and

- solver is 'shift'

Default (empty sublist)

database [RUN]

Execution parameter. See [RUN] (page 29).

Default (empty none database)

database [SHIFT]

Shift Monte Carlo solver options. See [SHIFT] (page 167). 


\section{Applicable when}

- 'Shift' is enabled in this build; and

- solver is 'shift'

parameter solvers (advanced)

List of solvers being used.

Default based on presence of Shift/Denovo

Type list in which each element is a string

sublist [SOURCE]

Particle source definition. See [SOURCE] (page 56).

Default model-defined source if applicable, or global fission for kcode

Applicable when using Shift, in forward mode, or in adjoint mode with adjoint_source source

postprocessor

Only a single 'sourcerer' source may be present.

Applicability solver is 'shift'

Applicability solver is 'denovo'

Applicability problem mode is kcode

\section{database [TALLY]}

Tallies and Monte Carlo diagnostics. See [TALLY] (page 113).

Default (empty database)

Applicable when using Shift, or in adjoint mode with adjoint_source tally

\subsection{PROBLEM ATTRIBUTES: [PROBLEM]}

The problem database specifies top-level information about the problem being run. It includes the output file name, a unique problem identifier, and the overall solution technique.

\section{parameter adjoint_source}

Which block to use to construct adjoint source.

Choose whether a cell or mesh tally from the [TALLY] block or a manually defined source from the [SOURCE] block is used as an adjoint source. Currently there are a number of limitations on using tallies as adjoint sources.

Warning: Creating an adjoint source from tallies is experimental: the adjoint source strength and spectrum may unexpected, or the particular type of selected tally might not be implemented. Contact the developers to find out if the latest capabilities meet your needs.

Default source

Type source or tally 
Applicable when mode is adjoint

parameter mode

Problem mode.

Valid modes are:

kcode Solve the $k$-eigenvalue problem for criticality safety or reactor physics analysis.

forward Solve a fixed-source problem for shielding calculations, etc.

adjoint Solve an adjoint fixed-source problem with the [SOURCE] or [TALLY] block interpreted as adjoint source (see adjoint_source (page 27)). Only Denovo can run adjoint problems.

raytrace Use the Denovo ray tracer to generate discretized materials for the problem. No transport will be performed.

hybrid Run a forward transport problem in Shift using deterministic acceleration.

Type adjoint, forward, hybrid, kcode, or raytrace

parameter name

Descriptive problem name.

Default Untitled

Type string

parameter num_threads

Number of OpenMP threads per process.

Currently, only Shift transport supports multithreading. Denovo solution time will not be affected by increasing the number of threads.

Default 1

Type positive integer

Applicable when 'OpenMP' is enabled in this build

preprocessor (advanced)

Ignore manual input of pid, rev.

parameter $\mathbf{p i d}$ (advanced)

Unique identifier automatically set for this problem run.

The problem identifier (pid) is a unique string generated by the Omnibus pre-processor to ensure that input and output files are properly correlated. The problem ID value added to the XML input file is copied to all HDF5 output files. It is comprised of the problem execution date and a randomly generated unique identifier string (UUID).

Default unique problem identifier

Type string

parameter scale_rev(advanced)

SCALE source revision used to generate this file.

Default current Exnihilo revision 


\section{Type integer \\ parameter seed}

Random number generator seed.

The random number generator seed is used for multiple parts of both Denovo and Shift runs:

- Shift particle sourcing and transport

- Denovo uncollided flux (if the MC option is enabled)

- Denovo material ray tracing (if not using the rays_deterministic option)

- Denovo source point sampling

Default 2272013

Type non-negative integer

\subsection{EXECUTION: [RUN]}

The [RUN] database enables support for running the Omnibus executable with the omnibus-run (page 15) command. The auto-run feature will format and echo program output to the screen, and it automatically saves the output and error streams to disk.

If the SCALE and DATA environment variables are not set, omnibus-run will use the values determined at configuration time.

Table 3: Available types for the [RUN] database

\begin{tabular}{lll}
\hline Type & Description & Applicability \\
\hline none (page 29) & Do not run; only perform pre-processing & \\
serial (page 29) & Run on a single CPU core & \\
mpi (page 30) & Run on multiple cores by directly calling MPI & 'MPI' is enabled in this build \\
pbs (page 30) & Run by submitting a PBS job & 'MPI' is enabled in this build \\
cray (page 34) & Run on Cray supercomputers & 'MPI' is enabled in this build \\
titan & Alias to cray type & - \\
\hline
\end{tabular}

\subsection{1 $[$ RUN=NONE $]$}

Do not run; only perform pre-processing.

\subsection{2 [RUN=SERIAL]}

Run as a serial process on the local machine, echoing output to the user. If the omnibus-run process is aborted, the omnibus command will also abort.

parameter hostname (advanced)

Cluster name for automatically determining processor options.

Default based on hostname or PBS_O_HOST environment

Type __unknown_-, apollo, cades, eos, excalibur, falcon, falcon2, oic, poseidon2, remus, romulus, or titan

parameter omnibus (advanced)

Path to the Omnibus executable.

Default ' $/ .$. /omnibus'

Type file path for reading 


\subsection{3 $[R U N=M P I]$}

Run as an MPI process on the local machine, echoing output to the user. If the omnibus-run process is aborted, the mpirun omnibus command will also abort.

parameter hostname(advanced)

Cluster name for automatically determining processor options.

Default based on hostname or PBS_O_HOST environment

Type __unknown_-, apollo, cades, eos, excalibur, falcon, falcon2, oic, poseidon2, remus, romulus, or titan

parameter mpiexec(advanced)

Path to the MPI run command.

Default ' $/ .$. /mpiexec'

Type file path for reading

parameter mpiexec_args

MPI execution arguments passed to mpiexec.

Default Based on CMake configuration and value for $n p$

Type list in which each element is a string

parameter $\mathbf{n p}$

Number of processors to run.

Default PBS_NP if inside a PBS session

Type positive integer

parameter omnibus (advanced)

Path to the Omnibus executable.

Default ' $/ .$. /omnibus'

Type file path for reading

\subsection{4 $[$ RUN=PBS $]$}

Create a PBS run file for this job. An example of a typical PBS run block is

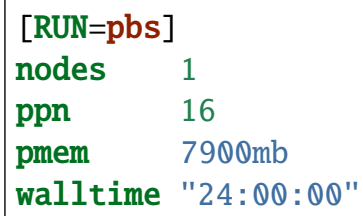

If the omnibus-run command is aborted while the job is run, the job will not be automatically aborted. The qdel command must be invoked independently to abort the job.

The cpp option specifies the number of cores to be used by each MPI task: cpp 2 will use half the cores available on the node. Alternatively, the number of processors per node can be set using the ppn parameter. The product of these two parameters cannot exceed the number of cores available on a compute node.

Additionally, the number of total MPI tasks used to run Exnihilo can be reduced below the requested number of nodes and cores with the np option, which has a default based on the number of requested nodes and cores. Adjusting this parameter may be necessary if, for example, Shift is to be decomposed into a non-power-of-2 number of blocks. 
parameter account

Account number to charge for time (e.g., NFI000, NSED).

Default based on hostname

Type string

parameter attributes

parameter attr

key=value pairs for PBS attributes ( $-\mathrm{W}$ argument).

Default based on hostname

Type list in which each element is a string

parameter bind

Bind processes to hardware tasks.

Type boolean

postprocessor

Ensure that the layout is consistent with the host cluster.

Applicability Host cluster has been detected or specified

parameter $\mathbf{c p p}$

Number of cores to assign to each process.

Type positive integer

parameter detach

Simply submit the job and to not follow it.

Default False

Type boolean

parameter email

Email address of recipient.

Default result of git config author.email (optional)

Type string

parameter environ

Environment variables to export in the PBS script.

Default ---

Type list of variables (each element is a string without special characters)

parameter extra_cmds

Extra commands to run at the beginning of the PBS script.

Default ---

Type list in which each element is a string

parameter hold

Set jobs to 'hold' status when submitting. 


\section{Default False}

Type boolean

Applicable when detach is True

\section{parameter hostname (advanced)}

Cluster name for automatically determining processor options.

Default based on hostname or PBS_O_HOST environment

Type __unknown_-, apollo, cades, eos, excalibur, falcon, falcon2, oic, poseidon2, remus, romulus, or titan

parameter join

Output joining flags.

Default oe

Type string

parameter modules

Modules to load at the beginning of the script execution.

Default based on hostname

Type list in which each element is a string

parameter mpiexec(advanced)

Path to the MPI run command.

Default ' $/ \ldots /$ mpiexec'

Type file path for reading

parameter mpiexec_args

MPI execution arguments passed to mpiexec.

Default Based on host layout

Type list in which each element is a string

parameter name

Job name.

Default base name of problem input file

Type string without special characters

parameter node_kw(advanced)

PBS keyword to specify the number of nodes.

Default usually 'nodes_ppn,' but 'nodes' on Titan and 'select' on others

Type nodes_ppn, nodes, or select

preprocessor (advanced)

Automatically determine the layout from the host and the given arguments.

parameter nodes

Number of compute nodes to use. 
Type positive integer

parameter $\mathbf{n p}$

Total number of MPI processes.

Type positive integer

parameter omnibus (advanced)

Path to the Omnibus executable.

Default ' $/ .$. /omnibus'

Type file path for reading

parameter $\mathbf{p m e m}$

Amount of memory per reserved processor (e.g., '7900mb').

\section{Optional}

Type string

parameter $\mathbf{p p n}$

Number of processes to execute on each node.

Type positive integer

parameter project

Project name used in the $-P$ flag.

Default based on hostname

Type string

parameter qdel

PBS deletion command or path.

Default qdel

Type string

parameter qos

PBS job classification option.

Default based on hostname and job specs

Type string

parameter qstat

PBS status command or path.

Default qstat

Type string

parameter $\mathbf{q s u b}$

PBS submission command or path.

Tip: To generate a PBS file but not actually submit or hold it, set qsub to "echo", and set detach true. With these two options, no PBS commands will be invoked. 


\section{Default qsub \\ Type string \\ parameter queue \\ parameter $\mathbf{q}$ \\ Queue to use. \\ Optional \\ Type string \\ parameter walltime \\ Wall time limit.}

Note: It is common to have colons as part of the wall time. Since colons must be escaped in Omnibus ASCII input, the walltime input parameter will typically need escaping:

[RUN=pbs]

walltime "24:00:00"

Type hh:mm:ss or mm:ss or number of seconds

parameter when_email

When to email.

Default ea

Type string

Applicable when Email is present

\subsection{5 $[R U N=C R A Y]$}

Create a PBS file for this job to run on Cray machines. An example PBS run block is:

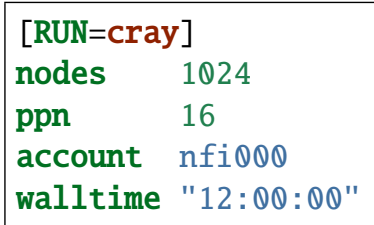

Just like with PBS, if the omnibus-run command is aborted while the job is run, the job will not be automatically aborted. The qdel command must be invoked independently to abort the job.

If the number of cores is less than or equal to 8 (the number of "shared core units"), the $-\mathrm{j} 2$ option will automatically be appended to stride the cores by 1 .

parameter account

Account number to charge for time (e.g., NFI000, NSED).

Default based on hostname

Type string 
parameter aprun(advanced)

Path to the aprun command.

Default ' $/ \ldots /$ mpiexec'

Type file path for reading

parameter attributes

parameter attr

key=value pairs for PBS attributes ( $-\mathrm{W}$ argument).

Default based on hostname

Type list in which each element is a string

parameter bind

Bind processes to hardware tasks.

Type boolean

postprocessor

Ensure that the layout is consistent with the host cluster.

Applicability Host cluster has been detected or specified

parameter $\mathbf{c p p}$

Number of cores to assign to each process.

Type positive integer

parameter debug

Show the aprun layout.

Default False

Type boolean

parameter detach

Simply submit the job and to not follow it.

Default False

Type boolean

parameter email

Email address of recipient.

Default result of git config author.email (optional)

Type string

parameter environ

Environment variables to export in the PBS script.

Default ---

Type list of variables (each element is a string without special characters)

parameter extra_cmds

Extra commands to run at the beginning of the script. 


\section{Default 'ulimit -c unlimited' 'export ATP_ENABLED=1'}

Type list in which each element is a string

parameter hold

Set jobs to 'hold' status when submitting.

\section{Default False}

Type boolean

Applicable when detach is True

parameter hostname(advanced)

Cluster name for automatically determining processor options.

Default based on hostname or PBS_O_HOST environment

Type __unknown_-, apollo, cades, eos, excalibur, falcon, falcon2, oic, poseidon2, remus, romulus, or titan

parameter join

Output joining flags.

\section{Default oe}

Type string

parameter mem

Memory required per MPI task (with $\mathrm{G} / \mathrm{M} / \mathrm{K}$ extension).

Default ' '

Type string

parameter modules

Modules to load at the beginning of the script execution.

Default based on hostname

Type list in which each element is a string

parameter name

Job name.

Default base name of problem input file

Type string without special characters

parameter node_kw(advanced)

PBS keyword to specify the number of nodes.

Default usually 'nodes_ppn,' but 'nodes' on Titan and 'select' on others

Type nodes_ppn, nodes, or select

preprocessor (advanced)

Automatically determine the layout from the host and the given arguments.

parameter nodes

Number of compute nodes to use. 
Type positive integer

parameter $\mathbf{n p}$

Total number of MPI processes.

Type positive integer

parameter omnibus (advanced)

Path to the Omnibus executable.

Default ' /.../omnibus'

Type file path for reading

parameter pin_system

Pin system tasks to a single CPU core.

Default True if not using all cores on a node

Type boolean

Applicable when hostname is titan or eos

parameter $\mathbf{p l a c e}$

How to distribute jobs on the node.

Default 'scatter:excl'

Type string

Applicable when hostname is excalibur

parameter $\mathbf{p p n}$

Number of processes to execute on each node.

Type positive integer

parameter project

Project name used in the $-P$ flag.

Default based on hostname

Type string

parameter $\mathbf{q d e l}$

PBS deletion command or path.

Default qdel

Type string

parameter qos

PBS job classification option.

Default based on hostname and job specs

Type string

parameter qstat

PBS status command or path.

Default qstat 


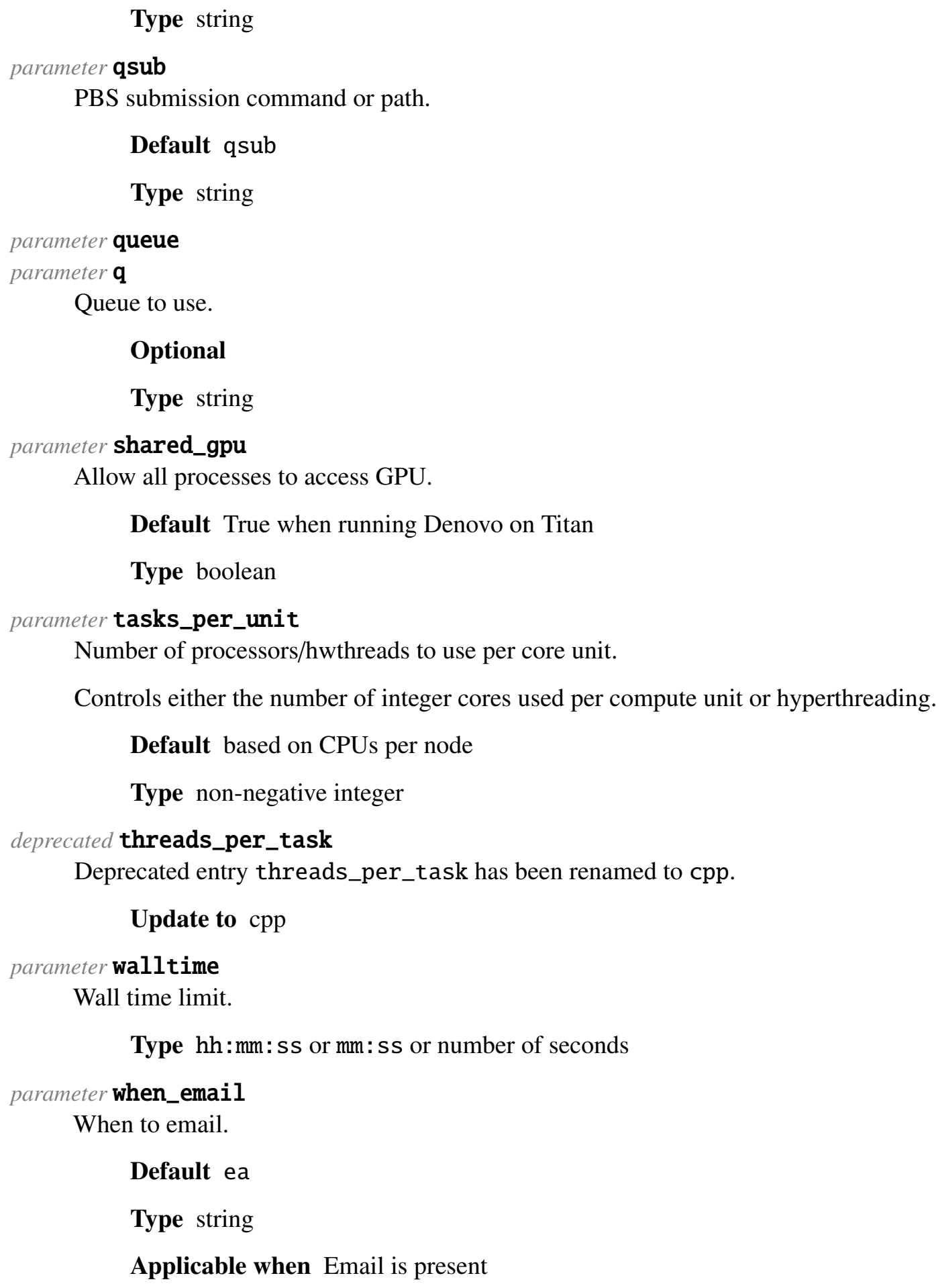

PBS submission command or path.

Default qsub

Type string

parameter queue

parameter $\mathbf{q}$

Queue to use.

\section{Optional}

Type string

parameter shared_gpu

Allow all processes to access GPU.

Default True when running Denovo on Titan

Type boolean

parameter tasks_per_unit

Number of processors/hwthreads to use per core unit.

Controls either the number of integer cores used per compute unit or hyperthreading.

Default based on CPUs per node

Type non-negative integer

deprecated threads_per_task

Deprecated entry threads_per_task has been renamed to cpp.

Update to cpp

parameter walltime

Wall time limit.

Type hh:mm:ss or mm:ss or number of seconds

parameter when_email

When to email.

Default ea

Type string

Applicable when Email is present 


\subsection{OUTPUT OPTIONS: [OUTPUT]}

Omnibus, Denovo, and Shift are used on a multitude of platforms and system configurations and are run at many scales, ranging from single-CPU jobs that produce very little output to $300 \mathrm{k}$-core jobs with hundreds of gigabytes of output. When large amounts of data are written to disk, the I/O performance of HDF5 (the output format used by Omnibus) can make a dramatic difference in write time.

Like Exnihilo, HDF5 supports both desktop and supercomputer platforms. HDF5 also supports a feature found on many larger computational clusters: a parallel file system (PFS). Unlike a networked file system (NFS), in which a file is mirrored or mounted over a network, and updates on one computer will be seen on the remainder of the network, a PFS actually supports writing to and reading from a file concurrently from multiple compute nodes. An example of a PFS is Lustre, on which files are decomposed across multiple separate hard disks. The more disks that a file is stored on, the higher the peak theoretical I/O bandwidth.

Warning: HDF5 will perform extremely poorly when writing in parallel to an NFS drive. (Factor-of-100 slowdowns have been seen.) Install the psutil ${ }^{12}$ python package to allow Omnibus to detect and warn about the file system being written to.

HDF5 can be compiled using MPI to enable "collective" operations, in which each application process can write a subset of the data (e.g., the locally KBA-decomposed Denovo mesh) to a file, and HDF5 will combine the data into a single file automatically. If the HDF5 implementation being used is compiled correctly (for example, configured with --with-io-romio-flags="--with-file-system=nfs+ufs+lustre"), it will be able to interface with the parallel file system and change the file layout when a new HDF5 file is created.

The file layout (number of aggregators and chunk size) is system- and output-dependent; its performance ${ }^{13}$ is tightly coupled to low-level HDF5 parameters, as well. Omnibus attempts to choose the output parameters for some known systems; a lustre (page 42) command is available to change the stripe and aggregator size and to update the HDF5 chunk size to match.

Since parallel HDF5 is not installed on (or performant on) all systems, Exnihilo implements its own collective operations for domain-decomposed data. With this "pseudo-parallel" mode, data from each domain are sent sequentially to processor 0 and then are written out using serial HDF5 calls. The disable_parallel_hdf5 (page 39) option forces this alternative implementation to be enabled, even if HDF5 is available.

When parallel HDF5 is enabled (and the problem is being run on more than one process), a second output file with the extension -parallel.h5 will be created for collective operations. The created fields (such as Denovo flux and depletion number densities) will be linked into the main HDF5 file using the "external link" feature of HDF5, so only the main .h5 file should ever need to be opened, although both files will need to be retained.

parameter disable_parallel_hdf5(advanced)

Disable MPI-I/O and write only from a single process.

Default True unless the file system is parallel

Type boolean

Applicable when 'PARALLEL_HDF5' is enabled in this build

\footnotetext{
${ }^{12}$ http://pythonhosted.org/psutil/

${ }^{13}$ https://support.hdfgroup.org/pubs/papers/howison_hdf5_lustre_iasds2010.pdf
} 
postprocessor

Disallow parallel HDF5 compatibility with non-PFS.

Applicability 'PARALLEL_HDF5' is enabled in this build; disable_parallel_hdf5 is False; and the problem run is parallel

Applicability file_system is __unknown__, $n f s, n f s 3, n f s 4, h f s, n t f s$, ext3, ext4, tmpfs, sysfs, btrfs, hugetlbfs, apfs, or xfs

parameter display_counter(advanced)

Write terminal commands for an interactive counter.

Default True if stderr is a terminal display

Type boolean

Applicable when The 'tqdm' python package is installed

parameter file_system(advanced)

parameter $\mathbf{f} \mathbf{s}$ (advanced)

File system type where output is being written.

Default Based on output of the mount command

Type __unknown_-, _-parallel__, lustre, panasys, nfs, nfs 3, nfs4, hfs, ntfs, ext3, ext4, tmpfs, sysfs, btrfs, hugetlbfs, apfs, or xfs

postprocessor

Disallow NFS output if a PFS is available.

Applicability 'PARALLEL_HDF5' is enabled in this build

Applicability the problem run is parallel

Applicability file_system is __unknown_-, nfs, nfs3, nfs4, hfs, ntfs, ext3, ext4, tmpfs, sysfs, btrfs, hugetlbfs, apfs, or xfs

parameter hdf5_alignment (advanced)

Alignment threshold and alignment value for HDF5.

Default $\theta 0$

Units B

Type list in which each element is a non-negative integer

Applicable when

- 'PARALLEL_HDF5' is enabled in this build; and

- disable_parallel_hdf5 is False; and

- the problem run is parallel

parameter hdf5_chunk(advanced)

Target size of HDF5 chunks; chunks will be this size or smaller.

Default 65536

Units B 
Type positive integer

preprocessor (advanced)

Set default Lustre striping based on hostname.

Applicability 'PARALLEL_HDF5' is enabled in this build

Applicability disable_parallel_hdf5 is False

Applicability the problem run is parallel

parameter hdf5_mpiinfo_key(advanced)

MPI_Info keys for parallel HDF5 file creation.

\section{Default ---}

Type list in which each element is a string

\section{Applicable when}

- 'PARALLEL_HDF5' is enabled in this build; and

- disable_parallel_hdf5 is False; and

- the problem run is parallel

parameter hdf5_mpiinfo_value(advanced)

MPI_Info values for parallel HDF5 file creation.

\section{Default ---}

Type list in which each element is a string

\section{Applicable when}

- 'PARALLEL_HDF5' is enabled in this build; and

- disable_parallel_hdf5 is False; and

- the problem run is parallel

postprocessor

The parameters hdf5_mpiinfo_key and hdf5_mpiinfo_value must have the same length.

Applicability 'PARALLEL_HDF5' is enabled in this build

Applicability disable_parallel_hdf5 is False

Applicability the problem run is parallel

parameter log_memory (advanced)

Periodically print memory usage to screen.

Default True if using Denovo

Type boolean

parameter log_timestamp

Prepend a timestamp with this format to each log message.

The formatting for a time stamp is the standard strftime ${ }^{14}$ formatting. For example, a value of [\%H:\%M:\%S] may produce a log message that looks like:

\footnotetext{
${ }^{14}$ https://en.cppreference.com/w/cpp/chrono/c/strftime
} 
$\gg \gg[11$ [1:29|:43] Time-stamped message

\section{Optional}

Type string

parameter $\mathbf{l o g}$ verbosity

Minimum level of output to save to log file.

Default info

Type debug, diagnostic, status, info, warning, error, or critical

command lustre

Set stripe size, aggregator, and HDF5 chunk size [kB, \#].

Creates hdf5_mpiinfo_key

Creates hdf5_mpiinfo_value

Creates hdf5_alignment

Creates hdf5_chunk

Applicable when file_system is __unknown__,_parallel__, lustre, or panasys

parameter output (advanced)

Destination path for the HDF5 output file.

Default input.out.h5

Type file path to write (extension '.h5')

parameter output_parallel(advanced)

Destination path for parallel HDF5 output.

Default input.out-parallel.h5

Type file path to write (extension '.h5')

Applicable when

- 'PARALLEL_HDF5' is enabled in this build; and

- disable_parallel_hdf5 is False; and

- the problem run is parallel

deprecated print_memory

Deprecated entry print_memory has been renamed to log_memory.

Update to $\log _{-}$memory

parameter screen_verbosity

Minimum level of output to print to screen.

Default diagnostic

Type debug, diagnostic, status, info, warning, error, or critical 


\subsection{MODEL DEFINITION: [MODEL]}

We define a model as the answer to the question: "What is where?" A model can include compositions, spatial geometry, and even sources and tallies. As a radiation transport framework, Exnihilo supports model definitions in several formats.

Every model contains a geometry that can be discretized and solved using Denovo with the built-in ray tracer, and most models can also be used by the Shift Monte Carlo code. Furthermore, material definitions can be converted to multigroup cross sections through the SCALE cross section processing libraries or mixed into multigroup cross sections input in ANISN formats.

Table 4: Feature matrix for the supported models.

\begin{tabular}{llllll}
\hline Model & Denovo & Shift & Compositions & Sources & Tallies \\
\hline DAGMC & Yes & Yes & - & - & - \\
Geant4 & Yes & No & Yes & - & - \\
Geometria & Yes & Yes & - & - & - \\
MCNP & Yes & Yes & Yes & Yes & No \\
Mesh & Yes & Yes & - & Yes & - \\
RTK & Yes & Yes & - & - & - \\
SCALE & Yes & Yes & Yes & No & No \\
SWORD & Yes & No & Yes & Yes & Yes \\
VERA & Yes & Yes & - & - & - \\
\hline
\end{tabular}

If using Shift to obtain volume-averaged reaction rates in geometric cells, the volume of the cells must be obtained a priori. Some models support automatic calculation a subset of cells; only Reactor ToolKit input: [MODEL=rtk] (page 53) supports automatic calculation of all cell volumes due to the restricted simple nature of the geometry. For all other models, the cell volumes can be manually specified with the "volumes" and "volume_cells" keywords:

$\begin{array}{lllll}\text { volumes } & 1.0 & 2.34 & 0.5\end{array}$

volume_cells $1200 \quad 15$

Here, the cells are the "cell labels" (e.g., the cell card IDs in MCNP) and the volumes are the corresponding volumes in $\mathrm{cm}^{3}$. The volumes are used only in the normalization of tallies, including depletion tallies.

\subsubsection{MATERIAL AND CELL IDS}

The model internally defines vectors of "matids" and "cellids" that correspond to user-defined material names and cell labels. Although Omnibus attempts to make these internal identifiers invisible to the user, an occasional advanced option or low-level error message may include references to these.

The material ID is simply the index into the list of compositions (page 106) in the problem; these are written to the comp/compositions dataset in the Omnibus output file. Note that for the MCNP model (page 44), these are usually not the user-assigned $\mathrm{m}$ label.

Cell IDs correspond to identifiable user-created spatial regions in the problem. If arrays or universes are used, a single cell ID might correspond to multiple regions in space. Cell IDs are not written to the output file because

- cells are typically much more numerous than materials; 
- some models such as RTK do not have user-provided identifiers for cells; and

- some models such as Geometria reserve cell IDs as implementation details, so some cell IDs correspond to no physical point in space.

User-provided labels corresponding to cell and material IDs can both be obtained without having to run the Omnibus executable. The following snippet uses the Python bindings (page 2) to print the meanings of all matids and cellids in the model:

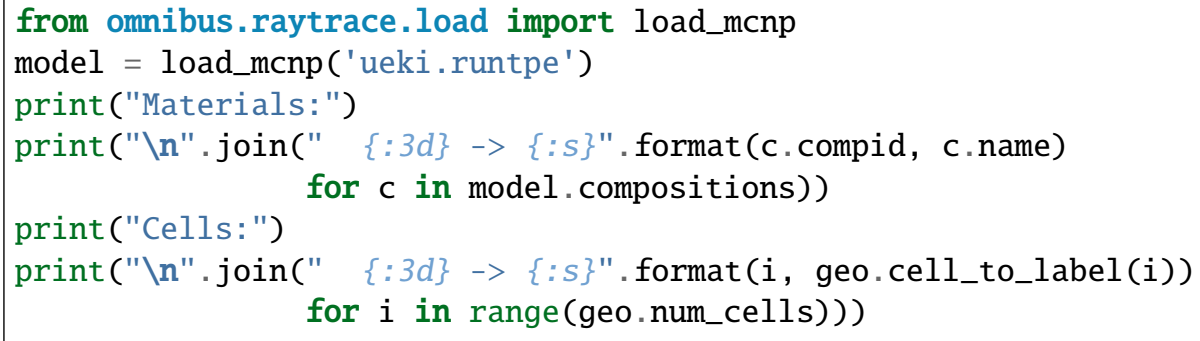

Table 5: Available types for the [MODEL] database

\begin{tabular}{lll}
\hline Type & Description & Applicability \\
\hline mcnp (page 44) & MCNP model definition & 'Lava' is enabled in this build \\
scale (page 50) & SCALE KENO input file & 'SCALE_GEO' is enabled in this build \\
geometria (page 52) & Geometria input definition & 'GG' is enabled in this build \\
gg & Alias to geometria type & \\
rtk (page 53) & RTK input specification & \\
mesh (page 53) & Explicit meshed problem in HDF5 format & 'HDF5' is enabled in this build \\
geant (page 54) & Geant4 GDML model input & 'Geant' is enabled in this build \\
sword (page 54) & SWORD model description & 'SWORD' is enabled in this build \\
dagmc (page 55) & DAGMC CAD geometry definition & 'DAGMC' is enabled in this build \\
vera (page 56) & VERA input specification & 'VERA' is enabled in this build \\
\hline
\end{tabular}

\subsection{MCNP INPUT: [MODEL=MCNP]}

Exnihilo supports reading MCNP [18] input files through ORNL's Lava library [7]. The user's MCNP input file must be processed through MCNP using the monp5 ix inp=\{inp $\}$ command in order to generate the runtpe file that Lava requires. The Omnibus pre-processor does this automatically.

Note: If using Shift cell tallies with an MCNP model, the user must include a cell tally or input volumes for desired cells in the MCNP input to ensure that volumes to automatically be propagated into Shift.

\subsubsection{FEATURES}

- Any MCNP5-compatible geometry is supported.

- MT cards for the materials are automatically converted to their equivalent SCALE IDs, so for example 6000 with a corresponding grph card will be converted to the SCALE ID for graphite, 3006000 .

- In depletion calculations, groups of axis-aligned planes can be moved at each time step without restarting (see [MODEL][MOVABLE=surfaces] (page 50))

- SDEF sources can be transported with Shift using the [SOURCE=mcnp] (page 61) source definition. 


\subsubsection{LIMITATIONS}

- MCNP6 is not supported; MCNPX will not be supported.

- Library suffixes for nuclides in the material block are ignored: only the TMP (temperature) card on each cell is used to determine the composition temperature. The composition temperature is then used when loading CE nuclides.

- Tally definitions are ignored.

- MCNP sources cannot be automatically biased in hybrid modes.

- Only MT cards natively supported by MCNP5 will be interpreted as chemically bound elements.

\subsubsection{NAMING}

Geometry cell names are the same as the numerical cell "names" given in the cell block of the MCNP input. Unlike MCNP, which can tally a single instance of a cell that is present in different arrays or universes with a special input such as $2>4>1$, Shift will lump all instances of a cell into the same tally.

Most material compositions processed from MCNP have the form mNNN, where NNN is the material name specified on the $m$ card. However, if the material is present in the cell definition block at multiple densities and/or temperatures, multiple copies of the composition will be present. Lower-density compositions will have the form mNNN (FF.F\%), where FF.F is the percentage mass density compared to the highest-density instance of the material.

\subsubsection{NUCLIDE MAPPING}

Exnihilo implements a number of hard-coded mappings to convert both bound and unbound MCNP nuclides to their equivalent IDs in SCALE. The following translations are performed for unbound nuclides:

Table 6: Mappings between unusual nuclide IDs.

\begin{tabular}{ll}
\hline MCNP ID & SCALE ID \\
\hline 1001 & 8001001 \\
1002 & 8001002 \\
27458 & 1027058 \\
47510 & 1047110 \\
48515 & 1048115 \\
52527 & 1052127 \\
52529 & 1052129 \\
61548 & 1061148 \\
67566 & 1067166 \\
95642 & 95242 \\
95242 & 1095242 \\
95644 & 1095244 \\
\hline
\end{tabular}

The MT cards in MCNP input mark that atoms in the material are chemically bound, so a $S(\alpha, \beta)$ collision kernel for thermal energies is to be applied. In SCALE, individual nuclides are marked as having bound collision data, so the following nuclides in bound materials in MCNP are translated to special SCALE IDs: 
Table 7: Mappings between MCNP MT cards and SCALE IDs.

\begin{tabular}{|c|c|c|}
\hline MTn & ZAID & SCALE ID \\
\hline al27 & 13027 & 1013027 \\
\hline be & 4009 & 3004009 \\
\hline be/o & 4009 & 5004009 \\
\hline \multirow[t]{3}{*}{ benz } & 1001 & 6001001 \\
\hline & 6000 & 5006000 \\
\hline & 6012 & 5006000 \\
\hline \multirow[t]{2}{*}{ beo } & 4009 & 3004009 \\
\hline & 8016 & 8016 \\
\hline dortho & 1002 & 4001002 \\
\hline dpara & 1002 & 5001002 \\
\hline fe56 & 26056 & 1026000 \\
\hline \multirow[t]{2}{*}{ grph } & 6000 & 3006000 \\
\hline & 6012 & 3006000 \\
\hline $\mathrm{h} / \mathrm{zr}$ & 1001 & 7001001 \\
\hline hortho & 1001 & 4001001 \\
\hline hpara & 1001 & 5001001 \\
\hline hwtr & 1002 & 1002 \\
\hline lmeth & 1001 & 1001001 \\
\hline lwtr & 1001 & 1001 \\
\hline \multirow[t]{3}{*}{ o/be } & 8016 & 5008016 \\
\hline & 8017 & 8017 \\
\hline & 8018 & 8018 \\
\hline \multirow[t]{3}{*}{$\mathrm{o} 2 / \mathrm{u}$} & 8016 & 1008016 \\
\hline & 8017 & 8017 \\
\hline & 8018 & 8018 \\
\hline poly & 1001 & 9001001 \\
\hline \multirow[t]{3}{*}{$\operatorname{sio} 2$} & 14028 & 1014028 \\
\hline & 14029 & 1014029 \\
\hline & 14030 & 1014030 \\
\hline smeth & 1001 & 2001001 \\
\hline $\mathrm{u} / \mathrm{o} 2$ & 92238 & 92238 \\
\hline \multirow[t]{6}{*}{$\mathrm{zr} / \mathrm{h}$} & 40000 & 1040090 \\
\hline & 40090 & 1040090 \\
\hline & 40091 & 1040091 \\
\hline & 40092 & 1040092 \\
\hline & 40094 & 1040094 \\
\hline & 40096 & 1040096 \\
\hline
\end{tabular}

command autoname

Attempt to provide MCNP material names using their compositions.

The autoname command is an experimental tool that loads the MCNP runtpe file into memory using the Exnihilo Python bindings (page 2) and uses the MCNP-defined compositions to construct a "best guess" of their name based on their most abundant elements. 
Tip: After autoname has been run once, a user can extract the created names from the PROBLEM .inp. omn file created in the run directory, replace the autoname command with those names, and modify them as desired.

\section{Creates mat_names \\ Creates mat_name_mno \\ parameter cell_raytrace}

Transform cell labels into material IDs for raytracing.

Default False

Type boolean

Applicable when problem mode is raytrace

\section{parameter extents}

Bounding box for the active region of the geometry.

Setting the model boundary enables certain Exnihilo features that require "global" boundaries. These include:

- Global mesh tallies

- Global initial fission source

- Automatic Shannon entropy mesh

- MCNP source discretization for Denovo

Default $-1 e+100 \quad 1 e+100-1 e+100 \quad 1 e+100-1 e+100 \quad 1 e+100$

Type locations for $-\mathrm{X},+\mathrm{X},-\mathrm{Y},+\mathrm{Y},-\mathrm{Z},+\mathrm{Z}$ (each element is a real number)

\section{command input}

Generate an MCNP runtpe file and set runtpe_path.

This command uses the version of MCNP configured with Exnihilo to generate a run tape. To change the cross section lookup directory xsdir, set the environment variable DATAPATH before running the Omnibus pre-processor. An informational message will acknowledge that the environment variable is being used in the particular MCNP run.

Tip: The MCNP executable path can be overridden by modifying the execution environment with the -e env.j jon argument to omnibus-run (page 15). Use a JSON environment file that looks like

\{"cmake": \{"MCNP_EXECUTABLE": "/path/to/mcnp5"\}\}

Creates runtpe_path 
command $\mathbf{m}$

Map 'mat_name_mno' to 'mat_names' from pairs or arrow-separated items.

Creates mat_name_mno

Creates mat_names

parameter mat_name_mno

MCNP material 'names' corresponding to the given mat_name override.

Default ---

Type Integer material numbers in MCNP (each element is a positive integer (optional leading ' $m$ '))

postprocessor

The parameters mat_names and mat_name_mno must have the same length.

parameter mat_names

parametermat_name

Override names for materials in the geometry.

Custom names can be added to the model that will be reflected in material plots inside VisIT and inside post-processing data elements. MCNP material card numbers are specified alongside new names to use:

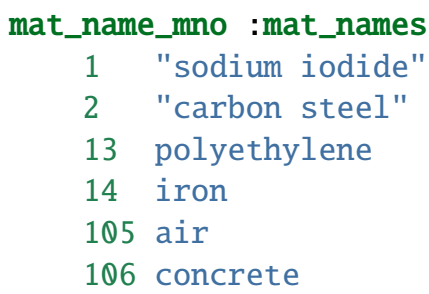

\section{Default ---}

Type list in which each element is a non-empty string

sublist [MOVABLE]

Geometry elements that can be modified during the simulation. See [MODEL][MOVABLE] (page 49).

Default (empty sublist)

\section{database [RAYTRACE]}

Volume calculation. See [MODEL][RAYTRACE] (page 49).

\section{Optional}

Applicable when problem mode is raytrace

parameter runtpe_path (advanced)

Path to the MCNP runtpe file.

Type file path for reading

parameter volume_cells

Cell labels corresponding to the given volume overrides. 


\section{Default ---}

Type list of cell labels (each element is a string)

postprocessor

The parameters volumes and volume_cells must have the same length.

parameter volumes

Provide or override volumes for cells in the geometry.

Default ---

Units $\mathrm{cm}^{3}$

Type list in which each element is a positive real number

\subsection{5 [MODEL][MOVABLE]}

Table 8: Available types for the [MOVABLE] database

\begin{tabular}{lll}
\hline Type & Description & Applicability \\
\hline surfaces (page 50) & Movable surface group & \\
\hline
\end{tabular}

\subsection{6 [MODEL][RAYTRACE]}

Volume calculation.

parameter axes

Axis/axes along which to fire rays for ray trace.

Default $x y z$

Type axis or axes ('x','zy', 'xyz')

parameter error_tolerance

Fraction of lost rays to tolerate before aborting.

Default $1 e-05$

Type real number inside $(0,1)$

parameter max_local_warnings

Max number of lost ray warnings to print per domain.

Default 10

Type non-negative integer

parameter num_batches

Number of batches to use for estimating variance.

Default 8

Type positive integer

parameter ray_spacing

Average spacing between rays in each batch.

Units $\mathrm{cm}^{3}$ 
Type positive real number

parameter rays_deterministic

Use face midpoints rather than stratified sampling.

Default False

Type boolean

parameter trace

Whether to trace materials or cell volumes.

Default cell

Type mat or cell

\subsection{7 [MODEL][MOVABLE=SURFACES]}

The "movable" surface option allows the translation of multiple surfaces simultaneously in the MCNP model. Currently this works only for simple axis-aligned planes.

Warning: Because translations and surface deduplication are applied while generating the runtpe file, moving one surface label may end up affecting other coincident surfaces. Exnihilo will raise an error during setup if this happens: the input deck will need to be modified to remove coincident planes or include all equivalent (coincident) surfaces in the surface group specification.

parameter initial

Movement to apply before the first transport.

Default 0.0

Units $\mathrm{cm}$

Type real number

parameter name

Name of the surface group.

Type string without special characters

parameter surfaces

List of MCNP surface labels to move over time.

Type list in which each element is a positive integer

\subsection{SCALE INPUT: [MODEL=SCALE]}

As an alternative to SCALE's CSAS-Shift sequence, the geometry and composition definitions from a SCALE input file can be processed and run through Omnibus. Any valid SCALE sequence inputs with a single GEOMETRY and COMPOSITION blocks can be transported on automatically.

Details on defining SCALE geometry inputs may be found in the SCALE manual [2].

\subsubsection{FEATURES}

- Supports KENO-V.a and KENO-VI geometry definitions, including geometries defined as part of a sequence (such as MAVRIC or CSAS-Shift).

- Supports all standard composition definitions. 


\subsubsection{LIMITATIONS}

- Only the GEOMETRY and COMP data blocks from the SCALE input will be used. The sequence itself is ignored, as are all other blocks (such as parameters and plotting inputs). This includes cross section processing blocks: see Multigroup physics: [PHYSICS=mg] (page 96) for specifying the physics treatment through Omnibus.

- Only the first one of each of those blocks will be used. If multiple sequences are chained together in the input, all but the first will be ignored.

If no composition block is present, then the user must specify compositions separately; this advanced use case is outside the scope of the manual.

\subsubsection{NAMING}

For KENO-VI geometries, cell names take the form unit . instance, where "unit" is the integer unit number, and "instance" is the index of the media instance in the list of media for that unit.

Composition names are saved as media $\mathrm{N}$, where $\mathrm{N}$ is the media number specified in the composition block.

\subsubsection{GEOMETRY CONVENTIONS}

The legacy geometry implementation in KENO differs from the new Geometria-powered implementation, which leads to confusingly different conventions that will hopefully be resolved. Until then, some of the differences are sketched out here.

- A universe in Geometria generally corresponds to a unit in SCALE. However, each instance of an array placed as a hole (in KENO-VI) is also a universe.

- Numbering in Geometria generally uses zero-based indexing, so the lower-left element in an array has coordinates $\left[\begin{array}{lll}0 & 0 & 0\end{array}\right]$ in the output.

- The "sense" of a surface or shape in Geometria generally takes the opposite sign of an input in a KENO region definition vector. See the [UNIVERSE][CELL] (page 244) section for more details.

parameter input

Path to the KENO VI input file.

Type file path for reading (extension '.inp')

database [RAYTRACE]

Volume calculation. See [MODEL][RAYTRACE] (page 49).

\section{Optional}

Applicable when problem mode is raytrace

parameter volume_cells

Cell labels corresponding to the given volume overrides.

\section{Default ---}

Type list of cell labels (each element is a string)

postprocessor

The parameters volumes and volume_cells must have the same length.

parameter volumes

Provide or override volumes for cells in the geometry.

Default ---

Units $\mathrm{cm}^{3}$

Type list in which each element is a positive real number 


\subsection{GEOMETRIA INPUT: [MODEL=GEOMETRIA]}

The Geometria (internally designated "GG") geometry engine underpins the new SCALE geometry implementation. Geometria supports a less automatic but more rigorous geometry definition than KENO. Its input is an Omnibus-style problem definition (see Geometria Input Description (page 233)) that is translated to an XML file.

This input model only supports geometry definitions; compositions must be input either via an HDF5 input file or in a [COMP] block (page 106).

command input

Generate a Geometria XML representation from an .gg.omn input.

Creates xml_path

database [RAYTRACE]

Volume calculation. See [MODEL][RAYTRACE] (page 49).

\section{Optional}

Applicable when problem mode is raytrace

parameter simplify_max_surfaces(advanced)

Threshold for making a "complex" cell "simple."

In the GG implementation, a "simple" cell is a cell in which crossing a boundary always causes a particle to leave a cell. (An example of a simple cell is the moderator region inside a square and outside a circle.) Some cells, however, have internal surface boundaries that can be crossed while remaining inside the cell. (An example of a "complex" cell is a square region with two non-overlapping squares excluded. Crossing one of the inner planes that comprise the squares does not necessarily put the particle inside the inner squares.) The bookkeeping for a particle inside these cells is more complicated than the simple cell, because all the distances along the particle's path must be calculated and tracked, and every surface crossing requires the cell's logic expression to be reevaluated to determine whether the particle is inside.

If a complex cell is erroneously treated as simple, tracking errors will result; so it is necessary to be conservative in initially calling a cell complex. In GG, a cell is assumed complex if it contains a "positive" shape in its definition (i.e., it is the "outside" of a shape). This has the unfortunate consequence of forcing the assumption that common simple shapes, such as a pin inside a pin cell, are in fact complex. The solution is a piece of code that checks a cell at construction time. It loops over all combinations of the surface IDs connected to the cell to determine if any surface crossing will allow a particle inside the cell to remain inside the cell:

- Fill the "surface sense" vector with the next combination of bits

- If the logic expression evaluates to "false," skip this iteration

- Loop over every surface sense

- Flip this surface's sense

- If the cell logic expression still evaluates to "true," the cell is definitely complex. Return early.

- Restore the flipped sense 
The performance of the above loop scales exponentially with the number of surfaces connected to a cell. (If the cell only has two surfaces, 4 outer logic evaluations must be made; for four surfaces, 16 must be made; for ten surfaces, 1024 must be made.) Therefore, this parameter defaults to checking only cells composed of at most 10 surfaces. Testing results show that this has a negligible build-time penalty but can improve tracking time by almost a factor of two, as in the case of the C5G7 benchmark, in which each pin cell would be marked "complex" without this code.

\section{Default 10}

Type integer in the range $[0,16)$

parameter volume_cells

Cell labels corresponding to the given volume overrides.

Default ---

Type list of cell labels (each element is a string)

postprocessor

The parameters volumes and volume_cells must have the same length.

parameter volumes

Provide or override volumes for cells in the geometry.

Default ---

Units $\mathrm{cm}^{3}$

Type list in which each element is a positive real number

parameter $\mathbf{x m l}$ _path(advanced)

Path to the GG geometry XML input file.

Type file path for reading (extension '.xml')

\subsection{REACTOR TOOLKIT INPUT: [MODEL=RTK]}

RTK is an internal geometry engine used for PWR geometry under the VERA framework produced by CASL. The RTK model implemented by Omnibus reads a geometry from an XML input file. In general, the Insilico front-end should be used for creating reactors with RTK geometries.

parameter input

Path to the RTK geometry XML or HDF5 file.

Type file path for reading (extension '.xml' or '.h5')

\subsection{BRICK MESH INPUT: [MODEL=MESH]}

The "mesh" model type allows a discretized problem to be transported on in Denovo and Shift. It is defined by an external HDF5 file with a field of materials, mixtures, and (optionally) a source description. The file format's specification is described in HDF5 Mesh Model Specification (page B-4)

This file format is primarily intended for Denovo problems, both for analytical benchmarks and allowing the user to "restart" from a previously discretized geometry using the make-denovo-model (page 21) utility. Shift does not support mix tables or the source description.

parameter input

Path to the mesh geometry hdf5 file.

Type file path for reading (extension '.h5')

postprocessor

Check that the mesh model is in the correct format. 


\subsection{GEANT4 INPUT: [MODEL=GEANT]}

Exnihilo supports Geant4 [15] inputs stored as GDML files. The materials and geometry are both read through Exnihilo.

Caution: Because Geant 4 defines a number of nuclides that may be important to high-energy physics but that are not important to neutronics calculations, it may be necessary to use the omit_zaid and orig_zaid/subs_zaid options in the physics if those nuclides are not present on the cross section libraries.

\subsubsection{LIMITATIONS}

- Tracking particles in Shift is not supported. Currently, Geant4 can only be discretized in Denovo or ray-traced in Python.

parameter input

Path to the Geant4 GDML input file.

Type file path for reading (extension '.gdml')

database [RAYTRACE]

Volume calculation. See [MODEL][RAYTRACE] (page 49).

\section{Optional}

Applicable when problem mode is raytrace

parameter volume_cells

Cell labels corresponding to the given volume overrides.

Default ---

Type list of cell labels (each element is a string)

postprocessor

The parameters volumes and volume_cells must have the same length.

\section{parameter volumes}

Provide or override volumes for cells in the geometry.

Default ---

Units $\mathrm{cm}^{3}$

Type list in which each element is a positive real number

\subsection{SWORD INPUT: [MODEL=SWORD]}

The SoftWare for Optimization of Radiation Detectors [8] interface requires files generated by the release (non-beta) version of SWORD 6.0 or higher, as only new versions write the problem's geometry and material definitions as Geant4 GDML files.

In addition to the geometry and material definitions, the SWORD model supports source definitions using [SOURCE=sword] (page 62) and can construct adjoint sources (tallies) using [TALLY][SWORD] (page 141). 


\subsubsection{LIMITATIONS}

- Since SWORD's tracking engine is based on that of Geant (page 54), tracking particles in Shift is not supported. Only Denovo discretization and raytracing are supported.

command input

Generate a binary SWORD representation from a .sword input.

Creates xdr_path

parameter $\mathbf{x d r}$ _path (advanced)

Path to the SWORD binary input file.

Type file path for reading (extension '.xdr')

\subsection{DAGMC INPUT: [MODEL=DAGMC]}

Direct Accelerated Geometry Monte Carlo (DAGMC) is a package in MOAB that has been integrated into Exnihilo to support CAD-based models. This package allows for Monte Carlo transport on complex 3D geometries that have been created by traditional solid modeling software. The model must be pre-processed so that the geometry is a faceted HDF5 file, and volumes must be tagged with specific properties.

Table 9: MOAB volume properties used in the DAGMC model.

\begin{tabular}{ll}
\hline Name & Description \\
\hline mat & Material ID (must be an integer), or the value "graveyard". \\
label & Cell label, ignored for the graveyard volume. \\
implicit_mat & $\begin{array}{l}\text { Specified only in the graveyard cell, the matid that will be } \\
\text { given to cells marked as implicit complements. }\end{array}$ \\
\hline
\end{tabular}

There can only be a single graveyard cell, which will be given the cell label "EXTERIOR," and there can be multiple implicit complements, which all share the label "IMPLICIT_COMPLEMENT".

\subsubsection{FEATURES}

- Any faceted CAD geometry of varying complexity can be used.

- Reflecting boundaries are supported.

\subsubsection{LIMITATIONS}

- Labeling materials by name is not supported. Volumes in the geometry must be tagged with the corresponding material ID.

- Volumes without material labels will be given a matid of 0 .

- The extents of the geometry must be an axis-aligned rectangular prism.

parameter input

Path to the MOAB facet file.

Type file path for reading (extension '.h5m')

parameter mat_input

Path to the material composition file. 


\section{Optional}

Type file path for reading (extension '.h5')

\section{database [RAYTRACE]}

Volume calculation. See [MODEL][RAYTRACE] (page 49).

\section{Optional}

Applicable when problem mode is raytrace

parameter volume_cells

Cell labels corresponding to the given volume overrides.

Default ---

Type list of cell labels (each element is a string)

postprocessor

The parameters volumes and volume_cells must have the same length.

\section{parameter volumes}

Provide or override volumes for cells in the geometry.

Default ---

Units $\mathrm{cm}^{3}$

Type list in which each element is a positive real number

\subsection{VERA INPUT: [MODEL=VERA]}

The model for a Virtual Environment for Reactor Applications (VERA) [9] XML input can be run through the Omnibus front end. The material compositions will be built from the model. Since the VERA geometry can only describe the geometry from the core out through the reactor vessel, the VERA input allows for an Omnibus input file defining the ex-core geometry to be specified. If this ex-core file is given, it will be included in the built geometry.

The [TALLY][VERA] (page 134) can be used to create a tally for the outermost region of the vessel. This tally can then be optimized for when running in hybrid mode.

\section{parameter input}

Path to the VERA XML input file.

Type file path for reading (extension '.xml')

\subsection{PARTICLE SOURCE DEFINITIONS: [SOURCE]}

The source database specifies the source particle distribution for a fixed-source problem, as well as the starting source for an eigenvalue problem. Any combination of types is allowed.

The total strength of all sources is used as a global multiplier for all tallies in fixed-source mode. (The total strength is ignored for the starting source in kcode mode; only the relative strengths of the sources are used.) An alternative to modifying the total source strength is to modify the normalization property of the tallies.

If using Shift with mode kcode, and no source is provided, the default source is a uniform source with global extents isotropically emitting neutrons with a U-235 watt spectrum. Since some geometry types cannot 
determine global extents by default (e.g., MCNP requires an extents keyword), be warned that this default may fail to sample enough particles in fissionable regions.

Table 10: Available types for the [SOURCE] database

\begin{tabular}{|c|c|c|}
\hline Type & Description & Applicability \\
\hline $\begin{array}{l}\text { separable } \\
\text { (page 57) }\end{array}$ & $\begin{array}{l}\text { Source separable in space, energy, an- } \\
\text { gle }\end{array}$ & \\
\hline $\begin{array}{l}\text { fissionmesh } \\
\text { (page 59) }\end{array}$ & $\begin{array}{l}\text { Fixed fission source from a Shift tally } \\
\text { or mesh source }\end{array}$ & \\
\hline mesh (page 61) & $\begin{array}{l}\text { Discretized source from the mesh } \\
\text { model HDF5 file }\end{array}$ & model is 'mesh' and solver is 'denovo' \\
\hline menp (page 61) & Source from MCNP SDEF cards & model is 'monp' \\
\hline sword (page 62) & SWORD source/spectra definitions & model is 'sword' \\
\hline material (page 63) & $\begin{array}{l}\text { Volumetric material composition emis- } \\
\text { sion }\end{array}$ & \\
\hline $\begin{array}{l}\text { surface_census } \\
\text { (page 64) }\end{array}$ & $\begin{array}{l}\text { Emit particles from a pre-computed sur- } \\
\text { face source }\end{array}$ & \\
\hline $\begin{array}{l}\text { sourcerer } \\
\text { (page 65) }\end{array}$ & $\begin{array}{l}\text { Use a Denovo solution as a fission } \\
\text { source }\end{array}$ & $\begin{array}{l}\text { solver is 'shift'; solver is 'denovo'; and } \\
\text { problem mode is kcode }\end{array}$ \\
\hline
\end{tabular}

\subsection{1 [SOURCE=SEPARABLE]}

Exnihilo views the source particle type as being an extension of the energy phase space. This facilitates hybrid methods, since in deterministic solvers the neutron and photon groups are adjacent and treated identically, and it makes some source definitions (such as naturally emitting materials) more straightforward.

\section{parameter allow_biasing}

Enable source biasing for hybrid problem modes.

Default False

Type boolean

\section{database [ANGLE]}

Particle angular distribution. See [SOURCE][ANGLE] (page 66).

Default (empty isotropic database)

parameter biased_distribution_sample_attempts(advanced)

Number of distribution sampling attempts before abandoning the current cell/group phase space.

Default 1000000

Type positive integer

parameter biased_src_sample_attempts (advanced)

Number of sampling attempts of a biased source before declaring a particle lost.

Default 10

Type positive integer

parameter cell_only 
parameter cell

Emit particles only in the given cells.

The cell_only criteria can be used in conjunction with fissionable_only and material_only, where it is combined using an and operation (i.e., emit particles in the given cells and in the given materials).

\section{Optional}

Type list in which each element is a string

parameter description

parameter desc

Optional longer descriptive string.

Default ' '

Type string

database [ENERGY]

Energy spectrum. See [SOURCE][ENERGY] (page 66).

Default Watt spectrum when in 'kcode' mode

parameter fissionable_only

parameter fis

Emit particles only in fissionable regions.

The fissionable_only criteria can be used in conjunction with material_only and cell_only, where it is combined using an and operation (i.e., emit particles in fissionable materials and in the given cells).

Default True if 'kcode' mode

Type boolean

parameter 12_error

Maximum requested source discretization L2 error.

Default 0.01

Type real number inside $(0,1)$

Applicable when using Denovo or hybrid

parameter material_only

parameter mat

Emit particles only in the given materials.

The material_only criteria can be used in conjunction with fissionable_only and cell_only, where it is combined using an and operation (i.e., emit particles in the given materials and in the given cells).

\section{Optional}

Type list in which each element is a non-empty string

parameter max_samples

Maximum number of point samples to use for discretization. 
Default 10000000000.0

Type positive integer

Applicable when using Denovo or hybrid

parameter name

Short title or label for the source.

Default type of the source database

Type string without special characters

parameter num_rejection_samples

parameter $\mathbf{r e j}$

How many samples to try before declaring a particle 'lost.'

Default 50000

Type positive integer

parameter samples_per_batch

parameter batch_samples

Number of point samples per discretization batch.

Default 100000.0

Type positive integer

Applicable when using Denovo or hybrid

database [SHAPE]

Spatial distribution type. See [SOURCE][SHAPE] (page 66).

parameter $\mathbf{s t r e n g t h}$

parameter $\mathbf{q}$

Source strength.

Default 1.0

Units $\frac{\text { particle }}{\mathrm{s}}$

Type positive real number

\subsection{2 [SOURCE $=$ FISSIONMESH]}

The fission mesh source allows a fission neutron production mesh tally from a prior run (kcode or fixed source) to be used as a source. This source could either be a starting source in a kcode calculation (to reduce the number of inactive cycles needed) or a fixed source (so that a coupled neutron-gamma problem can be run separately from the kcode calculation). The input parameter points to the name of the tallies.h5 file; and the mesh_tally_name parameter should be the name of the fission mesh tally.

By default, this source uses a U-235 energy spectrum.

parameter allow_biasing

Enable source biasing for hybrid problem modes.

Default False

Type boolean 
parameter biased_distribution_sample_attempts(advanced)

Number of distribution sampling attempts before abandoning the current cell/group phase space.

Default 1000000

Type positive integer

parameter biased_src_sample_attempts (advanced)

Number of sampling attempts of a biased source before declaring a particle lost.

Default 10

Type positive integer

parameter cell_averaged

parameter avg

Whether the field values are volume-averaged strengths.

Type boolean

Applicable when format is source

parameter description

parameter desc

Optional longer descriptive string.

Default ' '

Type string

preprocessor (advanced)

Default energy spectrum is U-235 Watt spectrum.

database [ENERGY]

Energy spectrum. See [SOURCE][ENERGY] (page 66).

Default Watt spectrum when in 'kcode' mode

parameter field

Path to the source definition in the input file.

Default 'strength' if cell-averaged, else 'pdf'

Type string

Applicable when format is source

parameter format

Format of hdf5 file.

Default tally

Type source or tally

parameter input

Name of file containing source distribution.

Type file path for reading (extension '.h5')

parameter name

Short title or label for the source. 


\section{Default type of the source database}

Type string without special characters

\section{parameter num_rejection_samples}

parameter $\mathbf{r e j}$

How many samples to try before declaring a particle 'lost.'

Default 50000

Type positive integer

parameter strength

parameter $\mathbf{q}$

Source strength.

Default 1.0

Units $\frac{\text { particle }}{\mathrm{s}}$

Type positive real number

parameter tally_name

Name of the fission source rate mesh tally.

Type string without special characters

Applicable when format is tally

\subsection{3 [SOURCE=MESH]}

This "mesh" source is specifically for manually constructed Denovo source strengths and multigroup spectra. To use a field of source strengths in Shift, use the mesh shape (page 76) as part of a separable source.

\section{parameter description \\ parameter desc}

Optional longer descriptive string.

\section{Default ' '}

Type string

\section{parameter name}

Short title or label for the source.

Default type of the source database

Type string without special characters

\subsection{4 [SOURCE $=$ MCNP]}

The MCNP source uses the Lava library to extract and use the source definition from an SDEF card. It supports automatic interpretation of the particle type (defaulting to the problem mode) and the strength (from the WGT parameter) as well as the actual distributions themselves.

The MCNP biasing parameters will be respected when sampling this source. This also applies to creating a discrete Denovo source from an MCNP source: regions where the source is biased to emit more frequently will have better statistical estimates of the source. The weight is accounted for during discretization to ensure that the total source strength is correct as the number of source samples approaches infinity. 
It is important to note that currently the MCNP source is replicated across processors when discretizing. This means that MCNP sources that encompass many cells, especially with a fine-group energy structure, may require more memory than an individual CPU core can allocate.

Note: Currently, the MCNP source is only available if the MCNP geometry is being used.

\section{parameter description \\ parameter desc}

Optional longer descriptive string.

\section{Default ' '}

Type string

\section{parameter extents}

Optional bounding box for MCNP source.

To allow for an MCNP source to be discretized in parallel, it is necessary to replicate the spatial mesh that overlaps the source. By default, this option replicates the entire spatial mesh. On large problems, fully replicating the entire mesh may cause the program to abort for lack of memory.

The solution is to manually define an axis-aligned bounding box that encloses the source region. Thus only the part of the mesh containing the source is replicated.

Tip: When the source discretization takes place, an informational message will print the 'actual' extents (based on sample particles) of the source bounding box. If the source boundaries are not readily available, it may be sufficient to discretize the source on a few-cell mesh problem and use the sampled extents (expanded by some fraction) as the bounding box for the full problem run.

Default $-1 e+100 \quad 1 e+100-1 e+100 \quad 1 e+100-1 e+100 \quad 1 e+100$

Type locations for $-\mathrm{X},+\mathrm{X},-\mathrm{Y},+\mathrm{Y},-\mathrm{Z},+\mathrm{Z}$ (each element is a real number)

parameter name

Short title or label for the source.

Default type of the source database

Type string without special characters

\subsection{5 [SOURCE=SWORD]}

This source option imports source descriptions from the SWORD input model. Currently, it only works with Denovo and is incompatible with other source definitions.

parameter allow_biasing

Enable source biasing for hybrid problem modes.

Default False

Type boolean

parameter biased_distribution_sample_attempts(advanced)

Number of distribution sampling attempts before abandoning the current cell/group phase space. 


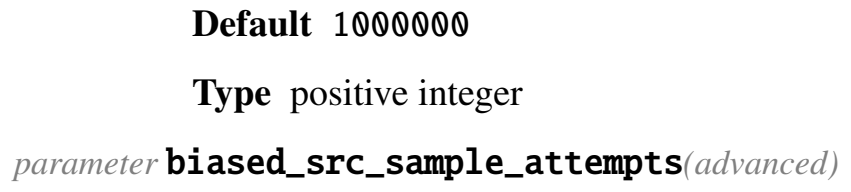

Default 1000000

Type positive integer

parameter biased_src_sample_attempts (advanced)

Number of sampling attempts of a biased source before declaring a particle lost.

Default 10

Type positive integer

parameter description

parameter desc

Optional longer descriptive string.

Default ' '

Type string

parameter name

Short title or label for the source.

Default type of the source database

Type string without special characters

\subsection{6 [SOURCE $=$ MATERIAL]}

Volumetric material composition emission.

parameter allow_biasing

Enable source biasing for hybrid problem modes.

Default False

Type boolean

parameter biased_distribution_sample_attempts(advanced)

Number of distribution sampling attempts before abandoning the current cell/group phase space.

Default 1000000

Type positive integer

parameter biased_src_sample_attempts(advanced)

Number of sampling attempts of a biased source before declaring a particle lost.

Default 10

Type positive integer

parameter description

parameter desc

Optional longer descriptive string.

Default ' '

Type string

sublist [MATERIAL]

Definition for a single source material. See [SOURCE][MATERIAL] (page 67). 
parameter name

Short title or label for the source.

Default type of the source database

Type string without special characters

parameter num_rejection_samples

parameter $\mathbf{r e j}$

How many samples to try before declaring a particle 'lost.'

Default 50000

Type positive integer

database [RAYTRACE]

Material raytrace options. See [SOURCE][RAYTRACE] (page 67).

sublist [SPECTRUM]

Material source spectrum. See [SOURCE][SPECTRUM] (page 66).

\subsection{7 [SOURCE=SURFACE_CENSUS]}

Emit particles from a pre-computed surface source.

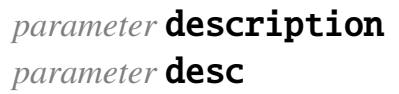

Optional longer descriptive string.

Default ' '

Type string

parameter input

Surface particle input file.

Type file path for reading (extension '.h5')

parameter name

Short title or label for the source.

Default type of the source database

Type string without special characters

parameter surfaces

Surfaces in input to read.

Type list in which each element is a string

parameter translation

Translates the particle by the given transform.

Default 0.00 .00 .0

Type length-3 float vector (each element is a real number) 


\subsection{8 [SOURCE $=$ SOURCERER]}

Use a Denovo solution as a fission source.

parameter $\mathbf{a}$

Value for the 'a' constant in Watt equation.

Units $\mathrm{MeV}$

Type positive real number

Applicable when use_watt_spectrum is True

parameter $\mathbf{b}$

Value for the ' $b$ ' constant in Watt equation.

Units $\frac{1}{\mathrm{MeV}}$

Type positive real number

Applicable when use_watt_spectrum is True

parameter description

parameter desc

Optional longer descriptive string.

Default ' '

Type string

parameter name

Short title or label for the source.

Default type of the source database

Type string without special characters

preprocessor (advanced)

Default Watt spectrum to U-235.

Applicability use_watt_spectrum is True

command nuclide

Produce a Watt spectrum corresponding to the given nuclide.

Creates a

Creates b

parameter num_rejection_samples

parameter $\mathbf{r e j}$

How many samples to try before declaring a particle 'lost.'

Default 50000

Type positive integer

parameter use_watt_spectrum

parameter $\mathbf{u s e \_ w a t t}$

Forces the energy spectrum to be a Watt spectrum.

Default True

Type boolean 


\subsection{9 [SOURCE][SHAPE]}

Table 11: Available types for the [SHAPE] database

\begin{tabular}{lll}
\hline Type & Description & Applicability \\
\hline box (page 68) & Axis-aligned cuboid shape & \\
cos_box (page 69) & Box, cosine distribution in multiple dimensions & \\
flattened_cos_box (page 70) & Box, $1-(1-\cos )^{2}$ distribution in multiple dimensions & \\
squared_cos_box (page 71) & Box, $(1-\cos )^{2}$ distribution in multiple dimensions & \\
cyl (page 72) & Axis-aligned cylinder shape & - \\
cylinder & Alias to cyl type & - \\
cylshell (page 73) & Cylindrical shell shape & \\
cylindershell & Alias to cylshell type & \\
sphere (page 74) & Sphere shape & \\
sphereshell (page 74) & Spherical shell shape & \\
point (page 75) & Single point & \\
multipoint (page 75) & Multiple points \\
global (page 76) & Box covering the geometry extents \\
mesh (page 76) & Discretized mesh source \\
\hline
\end{tabular}

\subsubsection{0 [SOURCE][ENERGY]}

Table 12: Available types for the [ENERGY] database

\begin{tabular}{lll}
\hline Type & Description & Applicability \\
\hline histogram (page 77) & Histogram energy distribution & \\
mono (page 78) & Monoenergetic line energy distribution & \\
lines (page 78) & Multiple line energy distribution & \\
watt (page 79) & Watt fission energy spectrum & \\
origen (page 79) & ORIGEN decay source spectrum input & \\
\hline
\end{tabular}

\subsubsection{1 [SOURCE][ANGLE]}

Table 13: Available types for the [ANGLE] database

\begin{tabular}{lll}
\hline Type & Description & Applicability \\
\hline isotropic (page 80) & Isotopic in angle & \\
mono (page 81) & Monodirectional & \\
\hline
\end{tabular}

\subsubsection{2 [SOURCE][SPECTRUM]}

The [SPECTRUM] energy distributions have exactly the same options as the [ENERGY] (page 66) entries with the same name, except that spectra also have a required per_decay entry and a name for each. This allows a single material source to emit multiple particles with different spectra at different rate per unit of activity.

Table 14: Available types for the [SPECTRUM] database 


\begin{tabular}{lll}
\hline Type & Description & Applicability \\
\hline histogram (page 81) & Histogram energy distribution & \\
mono (page 81) & Monoenergetic line energy distribution & \\
lines (page 82) & Multiple line energy distribution & \\
watt (page 82) & Watt fission energy spectrum & \\
origen (page 83) & ORIGEN decay source spectrum input & \\
\hline
\end{tabular}

\subsubsection{3 [SOURCE][MATERIAL]}

Definition for a single source material.

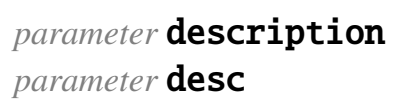

Optional longer descriptive string.

\section{Default ' '}

Type string

parameter name

Name of the material with this source.

Type string

parameter specific_activity

parameter activity

List of specific activity for each spectra.

Units $\frac{\text { becquerel }}{\mathrm{kg}}$

Type list in which each element is a positive real number

postprocessor

The parameters spectra and specific_activity must have the same length.

parameter spectra

List of source spectra names for this material.

Type list in which each element is a string

\subsubsection{4 [SOURCE][RAYTRACE]}

Material raytrace options.

parameter axes

Axis/axes along which to fire rays for ray trace.

Default xyz

Type axis or axes ('x','zy','xyz')

parameter error_tolerance

Fraction of lost rays to tolerate before aborting.

Default $1 \mathrm{e}-05$ 
Type real number inside $(0,1)$

parameter max_local_warnings

Max number of lost ray warnings to print per domain.

Default 10

Type non-negative integer

parameter rays_deterministic

Use face midpoints rather than stratified sampling.

Default False

Type boolean

parameter rays_per_face

Number of ray trace rays to be fired per mesh face.

Default 4

Type positive square integer

parameter $\mathbf{x}$

Raytrace mesh edges along the $\mathrm{X}$ axis.

Type monotonically increasing list with at least two values (each element is a real number)

parameter $\mathbf{y}$

Raytrace mesh edges along the $\mathrm{Y}$ axis.

Type monotonically increasing list with at least two values (each element is a real number)

parameter $\mathbf{Z}$

Raytrace mesh edges along the $\mathrm{Z}$ axis.

Type monotonically increasing list with at least two values (each element is a real number)

\subsubsection{5 [SOURCE][SHAPE $=$ BOX]}

Axis-aligned cuboid shape.

command box

Expand into parameters xmin, xmax, ymin, ymax, zmin, and zmax.

Creates xmin

Creates $x \max$

Creates ymin

Creates ymax

Creates zmin

Creates zmax

parameter $\mathbf{x m a x}$

Maximum $\mathrm{x}$ coordinate of box.

Type real number 


\section{parameter $\mathbf{x m i n}$}

Minimum x coordinate of box.

Type real number

\section{parameter $\mathbf{y m a x}$}

Maximum y coordinate of box.

Type real number

parameter ymin

Minimum y coordinate of box.

Type real number

parameter $\mathbf{z m a x}$

Maximum $\mathrm{z}$ coordinate of box.

Type real number

parameter $\mathbf{z m i n}$

Minimum z coordinate of box.

Type real number

\subsubsection{6 [SOURCE][SHAPE $=$ COS_BOX]}

Box, cosine distribution in multiple dimensions.

command box

Expand into parameters xmin, xmax, ymin, ymax, zmin, and zmax.

Creates xmin

Creates $x \max$

Creates ymin

Creates ymax

Creates zmin

Creates zmax

parameter $\mathbf{c o s}$ _dir

Directions for which the source has a cosine distribution.

Default $z$

Type axis or axes ('x','zy','xyz')

deleted num_cdf_bins

Entry num_cdf_bins has been deleted: The cosine CDF is integrated analytically.

parameter $\mathbf{x m a x}$

Maximum $\mathrm{x}$ coordinate of box.

Type real number

parameter $\mathbf{x m i n}$

Minimum x coordinate of box. 
Type real number

parameter ymax

Maximum y coordinate of box.

Type real number

parameter $\mathbf{y m i n}$

Minimum y coordinate of box.

Type real number

parameter $\mathbf{z m a x}$

Maximum z coordinate of box.

Type real number

parameter $\mathbf{z m i n}$

Minimum $\mathrm{z}$ coordinate of box.

Type real number

\subsubsection{7 [SOURCE][SHAPE=FLATTENED_COS_BOX]}

Box, $1-(1-\cos )^{2}$ distribution in multiple dimensions.

command box

Expand into parameters xmin, xmax, ymin, ymax, zmin, and zmax.

Creates xmin

Creates xmax

Creates ymin

Creates ymax

Creates zmin

Creates zmax

parameter $\mathbf{c o s} \_$dir

Directions for which the source has a flattened cosine distribution.

Default $z$

Type axis or axes ('x','zy', xyz')

deleted num_cdf_bins

Entry num_cdf_bins has been deleted: The cosine CDF is integrated analytically.

parameter $\mathbf{x m a x}$

Maximum x coordinate of box.

Type real number

parameter $\mathbf{x m i n}$

Minimum x coordinate of box.

Type real number 
parameter $\mathbf{y m a x}$

Maximum y coordinate of box.

Type real number

parameter $\mathbf{y m i n}$

Minimum y coordinate of box.

Type real number

parameter $\mathbf{z m a x}$

Maximum $\mathrm{z}$ coordinate of box.

Type real number

parameter $\mathbf{z m i n}$

Minimum z coordinate of box.

Type real number

\subsubsection{8 [SOURCE][SHAPE=SQUARED_COS_BOX]}

Box, $(1-\cos )^{2}$ distribution in multiple dimensions.

command box

Expand into parameters xmin, xmax, ymin, ymax, zmin, and zmax.

Creates xmin

Creates xmax

Creates ymin

Creates ymax

Creates zmin

Creates zmax

parameter $\mathbf{c o s}$ dir

Directions for which the source has a squared cosine distribution.

Default $z$

Type axis or axes ('x','zy','xyz')

parameter $\mathbf{x m a x}$

Maximum $\mathrm{x}$ coordinate of box.

Type real number

parameter $\mathbf{x m i n}$

Minimum x coordinate of box.

Type real number

parameter ymax

Maximum y coordinate of box.

Type real number 


\section{parameter $\mathbf{y m i n}$}

Minimum y coordinate of box.

Type real number

parameter $\mathbf{z m a x}$

Maximum $\mathrm{z}$ coordinate of box.

Type real number

parameter $\mathbf{z m i n}$

Minimum z coordinate of box.

Type real number

\subsubsection{9 [SOURCE][SHAPE $=$ CYL]}

The cylinder shape is defined along one of the three cartesian axes. Its origin (base) is defined as the center point of the lower circular face. The height extends in the positive direction from the origin along the given axis.

parameter axis

Axis of the cylinder.

Type axis ('x','y','z')

parameter height

parameter $\mathbf{h}$

Height of cylinder from the base.

Type real number

command origin

Expand into parameters origin_x, origin_y, and origin_z.

Creates origin_x

Creates origin_y

Creates origin_z

parameter origin_x

parameter $\mathbf{x o}$

$\mathrm{X}$ coordinate of the center of the cylinder's base.

Type real number

parameter origin_y

parameter yo

Y coordinate of the center of the cylinder's base.

Type real number

parameter origin_z

parameter $\mathbf{z o}$

$\mathrm{Z}$ coordinate of the center of the cylinder's base.

Type real number

parameter radius

parameter $\mathbf{r}$

Radius of the cylinder.

Type real number 


\subsubsection{0 [SOURCE][SHAPE $=$ CYLSHELL]}

Cylindrical shell shape.

parameter axis

Axis of the cylinder.

Type axis ('x','y','z')

parameter height

parameter $\mathbf{h}$

Height of cylinder from the base.

Type real number

parameter inner_radius

parameter ir

Inner radius of the cylindrical shell.

Type real number

command origin

Expand into parameters origin_x, origin_y, and origin_z.

Creates origin_x

Creates origin_y

Creates origin_z

parameter origin_x

parameter $\mathbf{x o}$

$\mathrm{X}$ coordinate of the center of the cylinder's base.

Type real number

parameter origin_y

parameter yo

$\mathrm{Y}$ coordinate of the center of the cylinder's base.

Type real number

parameter origin_z

parameter $\mathbf{z o}$

$\mathrm{Z}$ coordinate of the center of the cylinder's base.

Type real number

parameter outer_radius

parameter or

Outer radius of the cylindrical shell.

Type real number 


\subsubsection{1 [SOURCE][SHAPE $=$ SPHERE]}

Sphere shape.

command origin

Expand into parameters origin_x, origin_y, and origin_z.

Creates origin_x

Creates origin_y

Creates origin_z

parameter origin_x

parameter $\mathbf{x o}$

$\mathrm{X}$ coordinate of sphere source origin.

Type real number

parameter origin_y

parameter yo

Y coordinate of sphere source origin.

Type real number

parameter origin_z

parameter $\mathbf{z o}$

$\mathrm{Z}$ coordinate of sphere source origin.

Type real number

parameter radius

parameter $\mathbf{r}$

Radius of sphere source.

Type real number

\subsubsection{2 [SOURCE][SHAPE=SPHERESHELL]}

Spherical shell shape.

parameter inner_radius

parameter ir

Inner radius of spherical shell.

Type real number

command origin

Expand into parameters origin_x, origin_y, and origin_z.

Creates origin_x

Creates origin_y

Creates origin_z

parameter origin_x

parameter $\mathbf{x o}$

$\mathrm{X}$ coordinate of sphere source origin. 


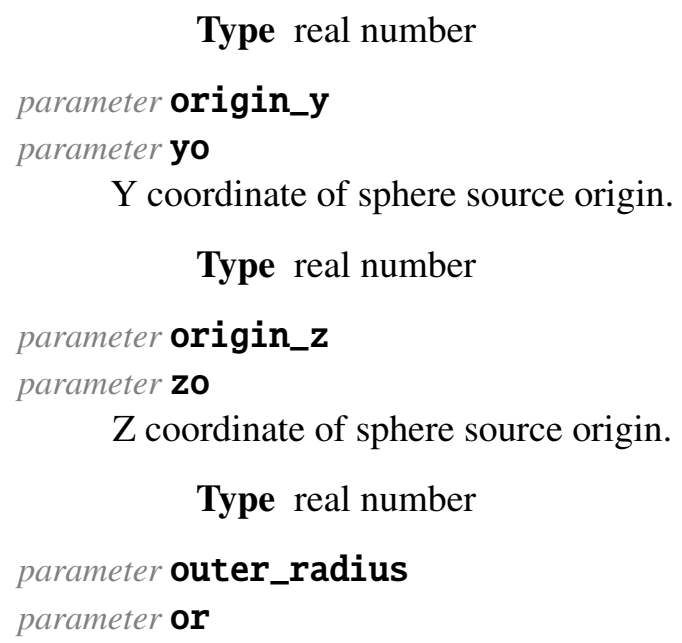

Outer radius of spherical shell.

Type real number

\subsubsection{3 [SOURCE][SHAPE $=$ POINT]}

Single point.

command point

Expand into parameters $\mathrm{x}, \mathrm{y}$, and $\mathrm{z}$.

Creates $\mathrm{x}$

Creates y

\section{Creates $\mathrm{z}$}

parameter $\mathbf{x}$

$\mathrm{X}$ position of point source.

Type real number

parameter $\mathbf{y}$

Y position of point source.

Type real number

parameter $\mathbf{Z}$

$\mathrm{Z}$ position of point source.

Type real number

\subsubsection{4 [SOURCE][SHAPE=MULTIPOINT]}

Multiple points.

parameter probability

Probability of each point being selected.

Type list of non-negative floats (each element is a non-negative real number) postprocessor

The parameters $\mathrm{x}, \mathrm{y}, \mathrm{z}$, and probability must have the same length. 
parameter $\mathbf{x}$

$\mathrm{X}$ positions of point sources.

Type list in which each element is a real number

parameter $\mathbf{y}$

$\mathrm{Y}$ positions of point sources.

Type list in which each element is a real number

parameter $\mathbf{z}$

$\mathrm{Z}$ positions of point sources.

Type list in which each element is a real number

\subsubsection{5 [SOURCE][SHAPE $=$ GLOBAL]}

The "global" source is a labor-saving way to attempt to sample particles inside the geometry. It works by querying the geometry for a bounding box (currently, only RTK and SCALE geometries are supported), and then sampling points uniformly inside that box. Any point outside the geometry will be rejected. The sample_attempts parameter determines how many samples inside the bounding box to attempt before a warning is emitted.

Warning: If the bounding box of the geometry is much different than the underlying geometry, the rejection fraction may be high, and the source sampling may be very slow. This is especially true in the case of fissionable-only sources, for which an additional rejection step is overlaid.

parameter sample_attempts

Number of geometry positions to sample per source particle.

Default 1000

Type positive integer

\subsubsection{6 [SOURCE][SHAPE $=$ MESH]}

Discretized mesh source.

parameter cell_averaged

parameter avg

Whether the field values are volume-averaged strengths. See cell_averaged $\quad($ page $\quad 60)$ in [SOURCE=fissionmesh].

parameter field

Path to the source definition in the input file.

Default 'strength' if cell-averaged, else 'pdf'

Type string

Applicable when format is source

parameter format

Format of hdf5 file. 


\section{Default tally}

Type source or tally

parameter input

Name of file containing source distribution.

Type file path for reading (extension '.h5')

parameter tally_name

Name of the fission source rate mesh tally.

Type string without special characters

Applicable when format is tally

\subsubsection{7 [SOURCE][ENERGY=HISTOGRAM]}

Histogram energy distribution.

parameter energy

parameter $\mathbf{e}$

Histogram energy bin bounds.

\section{Units eV}

Type list of non-negative monotonically increasing floats (each element is a non-negative real number)

parameter particle_type

parameter $\mathbf{p t}$

Particle type to emit.

Type particle type (n, neutron, $\mathrm{p}$, or photon)

parameter probability

parameter $\mathbf{p}$

Probability of an energy bin being selected.

For a discrete source distribution with the following spectrum:

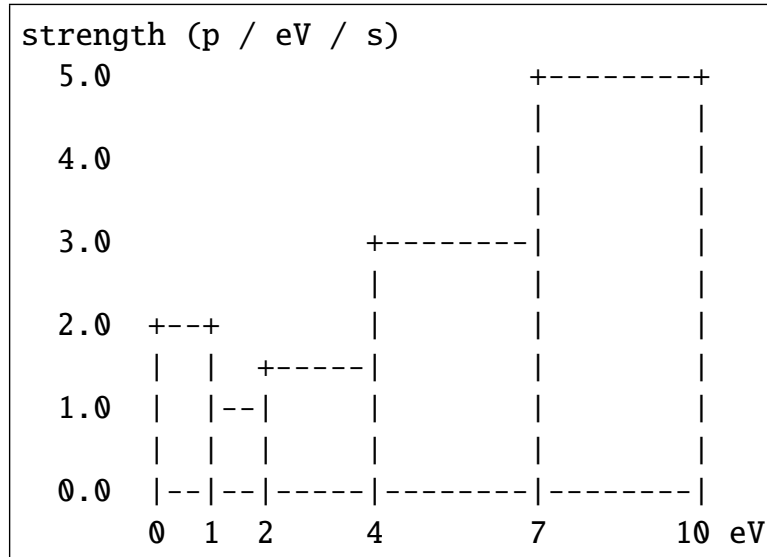

the source strength (page 59) should be set to

$$
q_{\mathrm{tot}}=\int q(E) \mathrm{d} E=30 \mathrm{p} / \mathrm{s}
$$

and the input for the integrated probability for each bin will be: 


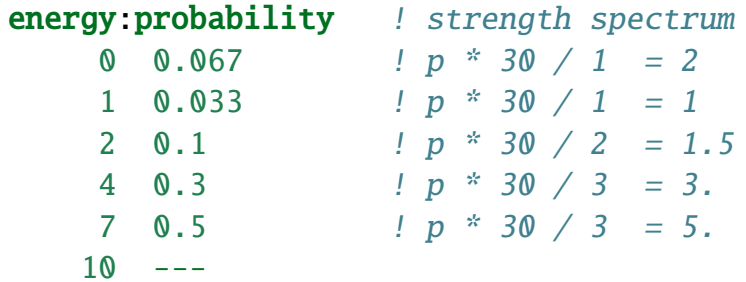

Type list of non-negative floats (each element is a non-negative real number)

\subsubsection{8 [SOURCE][ENERGY=MONO]}

Monoenergetic line energy distribution.

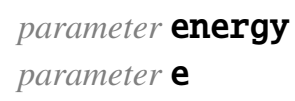

Source energy.

Units $\mathrm{eV}$

Type positive real number

parameter particle_type

parameter $\mathbf{p t}$

Particle type to emit.

Type particle type (n, neutron, $\mathrm{p}$, or photon)

\subsubsection{9 [SOURCE][ENERGY=LINES]}

Multiple line energy distribution.

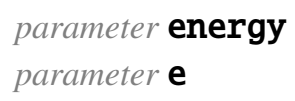

Individual line energies.

\section{Units eV}

Type monotonically increasing list (each element is a positive real number)

parameter particle_type

parameter $\mathbf{p t}$

Particle type to emit.

Type particle type (n, neutron, $\mathrm{p}$, or photon)

parameter probability

parameter $\mathbf{p}$

Probability of each line being selected.

Type list of non-negative floats (each element is a non-negative real number) 


\subsubsection{0 [SOURCE][ENERGY=WATT]}

The Watt distribution samples a fission spectrum:

$$
f(E)=c \exp (-E / a) \sinh (\sqrt{b E})
$$

where $c$ is a normalization constant.

parameter $\mathbf{a}$

Value for the 'a' constant in Watt equation.

\section{Units $\mathrm{MeV}$}

Type positive real number

parameter $\mathbf{b}$

Value for the 'b' constant in Watt equation.

Units $\frac{1}{\mathrm{MeV}}$

Type positive real number

preprocessor (advanced)

Default Watt spectrum to U-235.

command nuclide

Produce a Watt spectrum corresponding to the given nuclide.

Creates a

Creates b

\subsubsection{1 [SOURCE][ENERGY=ORIGEN]}

Reads photon spectra out of an ORIGEN master library.

command activities

Map 'zaid' to 'activity' from pairs or arrow-separated items.

Creates zaid

Creates activity

parameter activity

Activities of each nuclide.

Default (empty sublist)

Type list in which each element is a positive real number

Applicable when

- particle_type is p; and

- using an ORIGEN gamma library

postprocessor

The parameters zaid and activity must have the same length.

Applicability particle_type is p 
Applicability using an ORIGEN gamma library

postprocessor

ORIGEN file type and source definition must be consistent.

parameter apply_origen_strength

Multiply the source strength by the strength of the ORIGEN distribution.

Default True

Type boolean

parameter input

parameter filename

File (f71/mpdkxgam) containing the ORIGEN-generated spectrum.

Default ' /.../origen.rev04.mpdkxgam.data'

Type file path for reading

parameter particle_type

parameter $\mathbf{p t}$

Particle type to emit.

Type particle type (n, neutron, $p$, or photon)

parameter source_type

parameter $\mathbf{s t}$

Indicates origin of the source.

Default based on source type

Type source type (a, alpha, alpha_neutron, an, d, delayed, delayed_neutron, $\mathrm{dn}$, g, gamma, n, neutron, $\mathrm{p}$, photon, sf, sfn, spontaneous_fission, spontaneous_fission_neutron, tn, total_gamma, total_neutron, or total_photon)

parameter statepoint

Statepoint in the file from which to read the source.

Type non-negative integer

Applicable when using an ORIGEN concentration file

parameter zaid

Element/nuclide IDs (MZZZAAA).

Default (empty sublist)

Type list in which each element is a positive integer

Applicable when

- particle_type is $\mathrm{p}$; and

- using an ORIGEN gamma library

\subsubsection{2 [SOURCE][ANGLE=ISOTROPIC]}

Note that when defining a source for Denovo calculations, the only allowable angular discretization is isotropic. 


\subsubsection{3 [SOURCE][ANGLE=MONO]}

Monodirectional.

parameter direction

parameter dir

Direction source particles are emitted.

Type length-3 float vector (each element is a real number)

\subsubsection{4 [SOURCE][SPECTRUM=HISTOGRAM]}

Histogram energy distribution.

parameter energy

parameter $\mathbf{e}$

Histogram energy bin bounds.

Units $\mathrm{eV}$

Type list of non-negative monotonically increasing floats (each element is a non-negative real number)

parameter name

Name of the spectrum.

Type string without special characters

parameter particle_type

parameter $\mathbf{p t}$

Particle type to emit.

Type particle type (n, neutron, $\mathrm{p}$, or photon)

parameter per_decay

Particles emitted per decay.

Type positive real number

parameter probability

parameter $\mathbf{p}$

Probability of an energy bin being selected.

Type list of non-negative floats (each element is a non-negative real number)

\subsubsection{5 [SOURCE][SPECTRUM=MONO]}

Monoenergetic line energy distribution.

parameter energy

parameter $\mathbf{e}$

Source energy.

Units $\mathrm{eV}$

Type positive real number

parameter name

Name of the spectrum. 
Type string without special characters

parameter particle_type

parameter $\mathbf{p t}$

Particle type to emit.

Type particle type (n, neutron, $\mathrm{p}$, or photon)

parameter per_decay

Particles emitted per decay.

Type positive real number

\subsubsection{6 [SOURCE][SPECTRUM=LINES]}

Multiple line energy distribution.

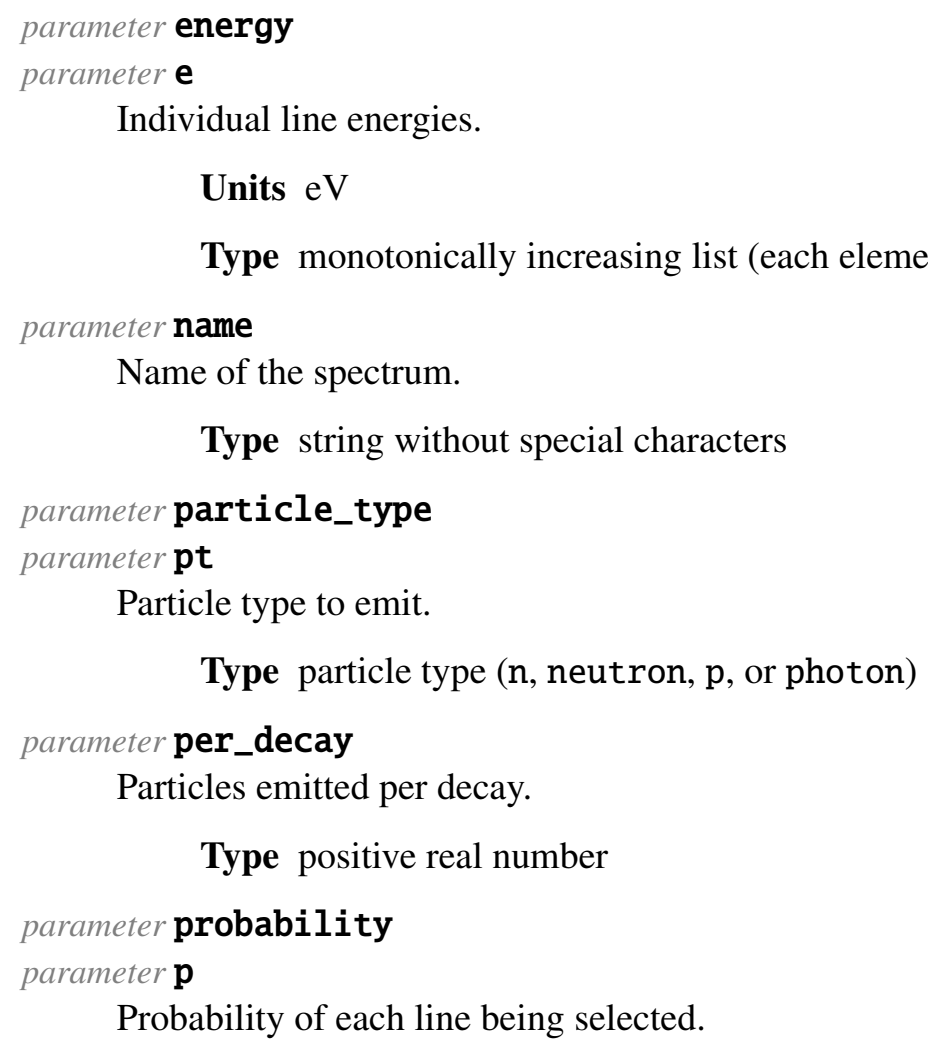

Individual line energies.

Units eV

parameter name

Name of the spectrum.

Type string without special characters

parameter particle_type

parameter $\mathbf{p t}$

Particle type to emit.

Type particle type (n, neutron, $\mathrm{p}$, or photon)

parameter per_decay

Particles emitted per decay.

Type positive real number

parameter probability

parameter $\mathbf{p}$

Probability of each line being selected.

Type monotonically increasing list (each element is a positive real number)

Type list of non-negative floats (each element is a non-negative real number)

\subsubsection{7 [SOURCE][SPECTRUM=WATT]}

Watt fission energy spectrum.

parameter $\mathbf{a}$

Value for the 'a' constant in Watt equation.

Units $\mathrm{MeV}$

Type positive real number

parameter $\mathbf{b}$

Value for the ' $b$ ' constant in Watt equation. 


\section{Units $\frac{1}{\mathrm{MeV}}$}

Type positive real number

\section{parameter name}

Name of the spectrum.

Type string without special characters

preprocessor (advanced)

Default Watt spectrum to U-235.

command nuclide

Produce a Watt spectrum corresponding to the given nuclide.

Creates a

Creates b

parameter per_decay

Particles emitted per decay.

Type positive real number

\subsubsection{8 [SOURCE][SPECTRUM=ORIGEN]}

ORIGEN decay source spectrum input.

command activities

Map 'zaid' to 'activity' from pairs or arrow-separated items.

Creates zaid

Creates activity

parameter activity

Activities of each nuclide.

Default (empty sublist)

Type list in which each element is a positive real number

\section{Applicable when}

- particle_type is $\mathrm{p}$; and

- using an ORIGEN gamma library

postprocessor

The parameters zaid and activity must have the same length.

Applicability particle_type is $p$

Applicability using an ORIGEN gamma library

postprocessor

ORIGEN file type and source definition must be consistent.

parameter apply_origen_strength

Multiply the source strength by the strength of the ORIGEN distribution. 


\section{Default True}

Type boolean

\section{parameter input \\ parameter filename}

File (f71/mpdkxgam) containing the ORIGEN-generated spectrum.

Default ' /. . /origen.rev04.mpdkxgam.data'

Type file path for reading

parameter name

Name of the spectrum.

Type string without special characters

parameter particle_type

parameter $\mathbf{p t}$

Particle type to emit.

Type particle type (n, neutron, $\mathrm{p}$, or photon)

parameter per_decay

Particles emitted per decay.

Type positive real number

parameter source_type

parameter $\mathbf{s t}$

Indicates origin of the source.

Default based on source type

Type source type (a, alpha, alpha_neutron, an, d, delayed, delayed_neutron, $\mathrm{dn}$, g, gamma, n, neutron, $\mathrm{p}$, photon, sf, sfn, spontaneous_fission, spontaneous_fission_neutron, tn, total_gamma, total_neutron, or total_photon)

parameter statepoint

Statepoint in the file from which to read the source.

Type non-negative integer

Applicable when using an ORIGEN concentration file

parameter $\mathbf{z a i d}$

Element/nuclide IDs (MZZZAAA).

Default (empty sublist)

Type list in which each element is a positive integer

\section{Applicable when}

- particle_type is $\mathrm{p}$; and

- using an ORIGEN gamma library 


\subsection{PHYSICS ENGINES: [PHYSICS]}

Omnibus currently supports two coupled-physics packages: multigroup (MG) and continuous energy (CE). Continuous-energy physics is restricted to Shift; multigroup physics is usable by both Shift and Denovo.

Table 15: Available types for the [PHYSICS] database

\begin{tabular}{lll}
\hline Type & Description & Applicability \\
\hline ce (page 85) & SCALE continuous energy & $\begin{array}{l}\text { 'SCALE_CE' is enabled in this build and solver is } \\
\text { 'shift' }\end{array}$ \\
mhysics & SCALE multigroup physics 96) & 'SCALE_MG' is enabled in this build \\
void (page 85) & All materials are replaced with & /problem/mode is forward, adjoint, or \\
& void & raytrace \\
sce & Alias to ce type & - \\
smg & Alias to mg type & - \\
\hline
\end{tabular}

\subsubsection{VOID PHYSICS: [PHYSICS=VOID]}

The special void physics type creates a one-group transport problem with void cross sections for all materials. It is used primarily in conjunction with the raytrace visualization mode, but it can also be used for preliminary model verification.

parameter mode

Particles to transport.

Default Default mode to ' $\mathrm{n}$ ' for kcode, or based on sources if present

Type particle transport mode (n, neutron, np, p, photon, or $\mathrm{pn}$ )

parameter name

Label for the physics.

Default void

Type string without special characters

parameter num_groups (advanced)

Number of energy groups.

Default one for each particle type

Type positive integer

\subsection{CONTINUOUS-ENERGY PHYSICS: [PHYSICS=CE]}

The CE physics implementation in Shift is driven by AMPX-processed cross sections and tabulated physics data. The methodology behind the AMPX cross section processing is documented in the AMPX manual [10], and the physics processes are documented in the KENO section of the SCALE manual [2].

\subsubsection{FEATURES}

- Neutron only, photon only, and coupled neutron-photon problems

- Optional Doppler broadening interpolation between library temperatures at load time

- Optional Doppler-broadened resonance correction (DBRC) enables upscattering for high-Z nuclides 


\subsubsection{LIMITATIONS}

- Photon cross sections do not include Bremsstrahlung reactions, so low-energy photon physics will not perform as expected.

- Data inconsistencies in ENDF are propagated through AMPX and may result in errors or warnings during transport.

- Photonuclear reactions are not supported.

command ampx_kerma

Load KERMA factors from an AMPX library.

Creates splice

parameter balance_tol(advanced)

Tolerance for printing a diagnostic about xs balance errors.

Default 0.005

Type real number inside $(0,1)$

database [BROADEN]

Temperature-corrected cross section Doppler broadening. See [PHYSICS][BROADEN] (page 93).

\section{Optional}

\section{Applicable when}

- mode is $\mathrm{n}$ or $\mathrm{np}$; and

- not using the pole method

command ce_lib

Set the CE library path using SCALE file resolution.

The FileNameAliases.txt file installed to the prefix directory (usually the same as \$SCALE) is used to resolve the data location. The possible values as of SCALE 6.2.3 follow.

Table 16: SCALE CE library path aliases in SCALE 6.2.

\begin{tabular}{ll}
\hline Path & Description \\
\hline ce & ENDF/B-VII.1 \\
ce_v7 & ENDF/B-VII.0 \\
ce_v7.0 & ENDF/B-VII.0 \\
ce_v7.1 & ENDF/B-VII.1 \\
ce_v7_endf & ENDF/B-VII.0 \\
ce_v7.0_endf & ENDF/B-VII.0 \\
ce_v7.1_endf & ENDF/B-VII.1 \\
\hline
\end{tabular}

If the Omnibus build in use has the CMake variable SCALE_HPC_DATA_DIR defined, Shift will preferentially load HDF5 data from that instead of the XML files.

Creates ce_lib_path

parameter ce_lib_path

Path to SCALE CE Library XML or HDF5 file. 
Type file path for reading (extension '.xml' or '.h5')

postprocessor

The SCALE CELibrary package must be enabled to use legacy XML CE data files.

Applicability CE library is the xml legacy format

parameter ce_pole_lib_path

Path to the HDF5 file containing pole and residue data.

\section{Optional}

Type file path for reading (extension '.h5')

\section{Applicable when}

- mode is $n$ or $n p ;$ and

- CE library is in HDF5 format

parameter $\mathbf{d b r c}$

Enable Doppler broadened rejection correction.

DBRC adjusts the free-gas sampling of elastic scattering. In particular, it adjusts the sampling of the target nuclide speed when the target is a heavy nuclide.

This correction improves the accuracy of epithermal neutron scatter off of heavy nuclides. However, because it increases the rejection fraction when sampling the elastic scattering kernel, it may incur a performance penalty.

Default True

Type boolean

Applicable when mode is $n$ or $n p$

\section{parameter dbrc_energy_max}

Maximum incident neutron energy for DBRC.

Default 210.0

Units eV

Type positive real number

Applicable when dbrc is True

\section{postprocessor}

Parameter dbrc_energy_min must be less than dbrc_energy_max.

Applicability dbrc is True

parameter $\mathbf{d b r c}$ _energy_min

Minimum incident neutron energy for DBRC.

Default 0.4

Units $\mathrm{eV}$

Type positive real number

Applicable when dbrc is True 
parameter dbrc_lib_path

Absolute path to the DBRC data directory.

This option can be used to override the default path to the SCALE CE DBRC data. It can only be used with the legacy (xml) format cross sections; HDF5 cross section libraries include the DBRC data as a new temperature-independent reaction type.

Table 17: Default DBRC data paths in SCALE 6.2.

\begin{tabular}{ll}
\hline XML CE library path & DBRC library path \\
\hline \$DATA/ce_v7.0_endf.xml & \$DATA/cekenolib_7.0/dbrc/ \\
\$DATA/ce_v7.1_endf.xml & \$DATA/cekenolib_7.1/dbrc/ \\
\hline
\end{tabular}

Default \$DATA/cekenolib_7.X/dbrc/

Type directory path for reading

\section{Applicable when}

- CE library is the xml legacy format; and

- dbrc is True

\section{parameter disable_collision_yields}

Disables banking photons from all neutron collision reactions, but not from fission.

Default False

Type boolean

Applicable when mode is $\mathrm{np}$

command energy_limits

Expand into parameters n_energy_min and n_energy_max.

Creates n_energy_min

Creates n_energy_max

\section{database [FISSION]}

Fission-kinetics parameters. See [PHYSICS][FISSION] (page 94).

Default (empty database)

Applicable when neutrons are being transported

parameter kappa_library

Read neutron fission and capture heating from the provided libary.

\section{Optional}

Type file path for reading (extension '.h5')

Applicable when mode is $n$ or $n p$

parameter mixture_ordering(advanced)

Internal sorting criterion for nuclides in the mix table. 


\section{Default legacy}

Type legacy, ascending_density, descending_density, ascending_zaid, or descending_zaid

\section{parameter mode}

Particles to transport.

Default Default mode to ' $\mathrm{n}$ ' for kcode, or based on sources if present

Type particle transport mode (n, neutron, np, p, photon, or $\mathrm{pn}$ )

\section{postprocessor}

Kcode problems must be run in mode 'n' or 'np.'

parameter $\mathbf{n} \_$energy_max

parameter nemax

Maximum global neutron_energy cutoff for cross sections.

Default 20000000.0

Units $\mathrm{eV}$

Type positive real number

Applicable when mode is $n$ or $n p$

postprocessor

Parameter n_energy_min must be less than n_energy_max.

Applicability mode is $n$ or $n p$

parameter $\mathbf{n}$ _energy_min

parameter nemin

Minimum global neutron energy cutoff for cross sections.

Default $1 \mathrm{e}-05$

Units $\mathrm{eV}$

Type positive real number

Applicable when mode is $n$ or $n p$

parameter name

Label for the physics.

Default ce

Type string without special characters

parameter num_xsgrid_points

Number of points used in the xs lookup acceleration grid.

Default 16384

Type positive integer

Applicable when xs_accel is True 
parameter omit_zaid_n

Set the neutron cross section to zero for these nuclides.

Default 8018

Type list in which each element is a nuclide specifier (e.g., U-235, 92235, u235, u-235m1)

Applicable when mode is $n$ or $n p$

parameter omit_zaid_p

Set the photon cross section to zero for these nuclides.

Default ---

Type list in which each element is a nuclide specifier (e.g., U-235, 92235, u235, u-235m1)

Applicable when mode is $\mathrm{p}$ or $\mathrm{np}$

parameter orig_zaid_n

Replace CE data for these nuclides in problem materials.

The ZAID remapping options allow a ZAID (or SCALE ID) present in the problem input to be replaced with a different ZAID. These options are most useful when entered in column input form:

orig_zaid_n :subs_zaid_n

$1001 \quad 8001001$

$11022 \quad 11023$

This is usually necessary when the selected library is missing cross sections for a nuclide in the model's compositions.

\section{Default ---}

Type list in which each element is a nuclide specifier (e.g., U-235, 92235, u235, u-235m1)

Applicable when mode is $n$ or $n p$

parameter orig_zaid_p

Replace photon CE data for these nuclides in problem materials.

\section{Default ---}

Type list in which each element is a nuclide specifier (e.g., U-235, 92235, u235, u-235m1)

Applicable when mode is $\mathrm{p}$ or $\mathrm{np}$

parameter otf_elastic_scattering

Use on-the-fly elastic scattering rather than table lookups.

Default False

Type boolean

Applicable when mode is $n$ or $n p$

parameter p_energy_max

parameter pemax

Maximum global photon energy cutoff for cross sections.

Default 25000000.0 
Units $\mathrm{eV}$

Type positive real number

Applicable when mode is $\mathrm{p}$ or $\mathrm{np}$

postprocessor

Parameter p_energy_min must be less than p_energy_max.

Applicability mode is $\mathrm{p}$ or $\mathrm{np}$

parameter $\mathbf{p} \_$energy_min

parameter pemin

Minimum global photon energy cutoff for cross sections.

Default 10000.0

Units $\mathrm{eV}$

Type positive real number

Applicable when mode is $\mathrm{p}$ or $\mathrm{np}$

parameter probability_tables

parameter $\mathbf{p t a b}$

Use the probability table method for the unresolved resonance region (URR).

Default True

Type boolean

Applicable when mode is $n$ or $n p$

parameter reactions (advanced)

Reactions to load (AMPX_MT values).

Default ---

Type list in which each element is a MT number or name (e.g., N_GAMMA, 102)

sublist [SPLICE]

Inject cross sections from a source other than the library. See [PHYSICS][SPLICE] (page 93).

Default (empty sublist)

parameter subs_zaid_n

Substitute ZAID corresponding to 'orig_zaid_n.'

Default ---

Type list in which each element is a nuclide specifier (e.g., U-235, 92235, u235, u-235m1)

Applicable when mode is $n$ or $n p$

postprocessor

The parameters orig_zaid_n and subs_zaid_n must have the same length.

Applicability mode is $n$ or $n p$

parameter subs_zaid_p

Substitute ZAID corresponding to 'orig_zaid_p.' 


\section{Default ---}

Type list in which each element is a nuclide specifier (e.g., U-235, 92235, u235, u-235m1)

Applicable when mode is $\mathrm{p}$ or $\mathrm{np}$

postprocessor

The parameters orig_zaid_p and subs_zaid_p must have the same length.

Applicability mode is $\mathrm{p}$ or $\mathrm{np}$

parameter thermal_energy_cutoff

Thermalization energy cutoff for scattering kernels.

Default 10.0

Units eV

Type positive real number

Applicable when mode is $n$ or $n p$

parameter $\mathbf{x s}$ _accel

Accelerate cross section calculation.

Accelerate total and fission cross section lookup in a material using a log-spaced energy grid. For improved performance, this should always be enabled when neutron transport is enabled.

Default True when neutron transport is enabled

Type boolean

Applicable when xs_cache is tot or totfisnu

parameter $\mathbf{x s}$ _cache

Preserve cross sections between collisions.

This option enables microscopic cross sections to be cached as a particle streams between materials. This is primarily a performance optimization (in many problems it should have no effect on the results), but it subtly changes the physics of streaming through multiple materials when a neutron is in the unresolved resonance regions of the energy spectrum.

In some codes, streaming from one material to another resets the probability band values. As a consequence, if a particle streams through two instances of the same material by traveling through a void, its probability table band will be resampled in the second material.

Unless the xs_cache value is set to none, the physics constructs a hash table of nuclide IDs with probability table data (and in which the particle's energy is inside the URR) encountered since the last collision. When streaming through the same nuclide at different temperatures, the cross section band $[\mathrm{CDF}]$ is preserved, but the cross sections will be updated for the correct temperature. This is more physical than resetting the band at material boundaries and can affect, for example, $k_{\text {eff }}$ of highly voided BWRs.

When xs_cache is set to tot, the total cross sections of all encountered nuclides will be cached. When the option is set to totfisnu, the total, fission, and nu values are all pre-calculated (when a particle enters a nuclide) and cached.

Default totfisnu when problem mode is kcode else tot

Type none, ptab, tot, or totfisnu

postprocessor

Cannot set xs_cache to ptab unless probability_tables is True. 


\subsection{3 [PHYSICS][BROADEN]}

Temperature-corrected cross section Doppler broadening.

parameter delta_t

Finite difference grid spacing for Leal-Hwang temperature interpolation.

Default 1.0

Units $\mathrm{K}$

Type positive real number

Applicable when not using legacy broadener

parameter energy_tol

Relative difference for considering two energy points equal.

Default $1 \mathrm{e}-10$

Type real number inside $(0,1)$

Applicable when not using legacy broadener

parameter $\mathbf{k i n e m a t i c s}$

Interpolate collision data.

Default False

Type boolean

parameter legacy

Use legacy CE Resource interpolation.

Default False

Type boolean

Applicable when CE library is the xml legacy format parameter temperature_tol

Tolerance for reusing existing broadened cross sections.

Default 4.0

Units $\mathrm{K}$

Type positive real number

parameter union_energy

Unionize lower and upper library temperature energy grids.

Default True

Type boolean

Applicable when not using legacy broadener

\subsection{4 [PHYSICS][SPLICE]}

Table 18: Available types for the [SPLICE] database

\begin{tabular}{llc}
\hline Type & Description & Applicability \\
\hline ampx (page 94) & Splice multigroup data from an AMPX library & \\
\hline
\end{tabular}




\subsection{5 [PHYSICS][FISSION]}

Fission-kinetics parameters.

command fission_cells

Generate 'union_fission_cells' and 'union_fission_lengths' from colon-separated unions.

Creates union_fission_cells

Creates union_fission_lengths

parameter fission_gammas

parameter fiss_gam

Generate fission gammas.

Default True if and only if mode is $n p$

Type boolean

Applicable when neutrons and photons are being transported

postprocessor

check fission particle production options.

parameter fission_neutrons

parameter fiss_neut

Generate fission neutrons.

Default True if and only if mode is not kcode

Type boolean

parameter union_fission_cells(advanced)

Flattened list of cells in each union.

Default (empty sublist)

Type list of cell names (each element is a string)

parameter union_fission_lengths(advanced)

Number of cells per union in the above list.

Default (empty sublist)

Type list in which each element is a integer

\subsection{6 [PHYSICS $][$ SPLICE=AMPX]}

The AMPX data splicing option allows multigroup data from an AMPX library to be spliced into the CE transport. This feature allows specialized reactions to be calculated during transport (e.g., KERMA tallies).

Currently, only multigroup reaction rates can be inserted. No probability tables or collision data can be inserted.

If a CE reaction is already present, the AMPX splicer will not override it. To get around this, the user can manually specify the list of reactions to load in the Continuous-energy physics: [PHYSICS=ce] (page 85) block, omitting the reactions to be loaded as multigroup data.

command mg_lib

Set mg_lib to the given value using SCALE DATA resolution. 
Creates xs_library

parameter name

Descriptive name for the data being spliced in.

Type string without special characters

parameter orig_zaid_n

Replace MG data for these nuclides in problem materials.

Default ---

Type list in which each element is a nuclide specifier (e.g., U-235, 92235, u235, u-235m1)

parameter orig_zaid_p

Replace photon MG data for these nuclides in problem materials.

Default ---

Type list in which each element is a nuclide specifier (e.g., U-235, 92235, u235, u-235m1)

parameter reactions (advanced)

Multigroup reactions to splice into the CE data (AMPX_MT values).

Default (empty sublist)

Type list in which each element is a MT number or name (e.g., N_GAMMA, 102)

parameter subs_zaid_n

Substitute ZAID corresponding to 'orig_zaid_n.'

Default ---

Type list in which each element is a nuclide specifier (e.g., U-235, 92235, u235, u-235m1)

postprocessor

The parameters orig_zaid_n and subs_zaid_n must have the same length.

parameter subs_zaid_p

Substitute ZAID corresponding to 'orig_zaid_p.'

Default ---

Type list in which each element is a nuclide specifier (e.g., U-235, 92235, u235, u-235m1)

postprocessor

The parameters orig_zaid_p and subs_zaid_p must have the same length.

parameter $\mathbf{x s}$ _library

Path to AMPX library to load data.

Type file path for reading 


\subsection{MULTIGROUP PHYSICS: [PHYSICS=MG]}

The SCALE multigroup physics package as implemented in Exnihilo can use various multigroup cross section library formats, including:

- AMPX-processed cross sections [10],

- User-defined macroscopic cross sections,

- GIP format macroscopic cross sections, and

- ANISN-formatted multigroup libraries [19].

When compositions (as opposed to user-specified multigroup cross sections) are provided, Omnibus uses SCALE to calculate infinite homogeneous medium cross sections for all materials. No self-shielding is performed. The default cross section processing is primarily intended for generating deterministic solutions for hybrid calculations.

Warning: The default multigroup cross sections generated by Omnibus are only recommended for qualitative transport behavior. Obtaining accurate multigroup cross sections for general problems requires a great deal of experience using SCALE cross section processing that incorporates self-shielding.

The Monte Carlo implementation of MG physics uses the same methods as KENO multigroup physics. Depending on the scattering order of the overall problem and of each particular material, the scattering model takes three forms.

- Isotropic scattering has the standard physical formulation.

- $P_{1}$ scattering forces the particle to scatter with an exiting cosine equal to the mean cosine. That is, instead of sampling the exiting cosine from a linear distribution, the cosine is sampled from a delta function equal to $\mu_{0}=\int_{-1}^{1} \mu \sigma_{s}(\mu) \mathrm{d} \mu$. Thus, in an isotropically scattering medium in a $P_{1}$ problem, the particle's exiting direction will be exactly perpendicular to the particle's incident direction (since isotropic scattering has $\mu_{0}=0$ ). This is not physical, but it is the physics behavior in MG KENO.

- Higher order $\mathrm{P}_{N}$ scattering selects exiting angles from a quadrature.

\subsubsection{FEATURES}

- Neutron only, photon only, and coupled neutron-photon problems

\subsubsection{LIMITATIONS}

- Anisotropic Monte Carlo particle scattering uses the same angular approximations as the multigroup KENO package in SCALE.

parameter ampx_path

Path to an AMPX working library input file.

This input requires a working library with at least the number of materials present in the model. For a library with $N$ materials, the cross sections for matids (page 43) $[0, N)$ are assigned based on the ids of materials in the AMPX file after being sorted in ascending order. For example, if a working library has materials with IDs $[1,21,12]$ (intentionally unsorted); the mapping of material IDs to AMPX library IDs is 
(1) $\rightarrow 1$

$1->12$

$2->21$

There is an additional option implicit_void (page 99) that assigns void cross sections to matid 0 . Thus if the AMPX library has media IDs 1,3,5, and if implicit_void is true, then the mapping will be

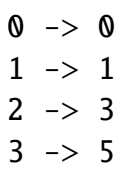

Type file path for reading (extension '.ampx')

Applicable when xsgen is ampx

parameter anisn_info_path

Path to an ADVANTG-format ANISN metadata file.

Type file path for reading (extension '.info')

Applicable when xsgen is anisncomp

command anisn_lib

Set ANISN library and metadata paths using a search path.

Creates anisn_path

Creates anisn_zaid_path

Creates anisn_info_path

parameter anisn_path

Path to an ANISN cross section input.

Type file path for reading (extension '.bin')

Applicable when xsgen is anisncomp

parameter anisn_table

ANISN table IDs corresponding to anisn_zaid.

Default ---

Type list in which each element is a positive integer

Applicable when xsgen is anisncomp

postprocessor

The parameters anisn_zaid and anisn_table must have the same length.

Applicability xsgen is anisncomp

parameter anisn_zaid

Replace the given ZAIDs with corresponding ANISN table IDs.

Default --- 
Type list in which each element is a nuclide specifier (e.g., U-235, 92235, u235, u-235m1)

Applicable when xsgen is anisncomp

parameter anisn_zaid_path

Path to an ADVANTG-format ANISN zaid map.

Type file path for reading (extension '.zaid')

Applicable when xsgen is anisncomp

parameter disable_upscattering

parameter noup

Remove upscattering by altering cross sections.

For shielding calculations, thermal neutrons may contribute little to the detector response. However, converging the distribution of thermal neutrons through upscatter iterations can be expensive.

This option adjusts all material cross sections so that their scattering matrix is strictly lower triangular. The default behavior is to lump upscattering cross sections into within-group scattering, adjusting the outscatter cross sections so that:

$$
\begin{array}{r}
\sigma_{\mathrm{s}, g, g} \leftarrow \sum_{g^{\prime}=1}^{g} \sigma_{\mathrm{s}, g, g^{\prime}} \\
\sigma_{\mathrm{s}, g, g^{\prime}} \leftarrow 0, g^{\prime}=[1, \ldots, g-1]
\end{array}
$$

where the outscatter cross section is

$$
\sigma_{\mathrm{s}}\left(E^{g} \rightarrow E^{g^{\prime}}\right) \equiv \sigma_{\mathrm{s}, g, g^{\prime}}
$$

Default True if only transporting photons or using hybrid solve

Type boolean

command energy_limits

Expand into parameters n_energy_min and n_energy_max.

Creates n_energy_min

Creates n_energy_max

database [GIP]

GIP input file metadata. See [PHYSICS][GIP] (page 104).

Applicable when xsgen is gip

parameter gip_path

Path to a GIP working library file.

Type file path for reading (extension '.gip')

Applicable when xsgen is gip

parameter implicit_capture

Enable implicit capture for Monte Carlo transport.

Default True 
Type boolean

Applicable when solver is 'shift'

parameter implicit_void(advanced)

Insert 'void' material of matid=0 before AMPX-specified mats.

Default False

Type boolean

Applicable when $x s g e n$ is ampx

parameter mc_pn_scattering(advanced)

Enable high-order scattering for MC transport.

Default True when Shift is enabled

Type boolean

Applicable when PN order is greater than 1

postprocessor

Multigroup Monte Carlo transport requires pn_order to be zero or odd.

Applicability mc_pn_scattering is True

command mg_lib

Set mg_lib to the given value using SCALE DATA resolution.

The aliases for the SCALE multigroup libraries can be found at INSTALL/etc/LibraryAliases. txt. The SCALE FileNameAliases. txt file is used to resolve the data files. The current options for multigroup libraries are:

Table 19: Multigroup physics library aliases and filenames in SCALE 6.2. 


\begin{tabular}{ll}
\hline Alias for mg_lib & Filename \\
\hline broad_n & xn56v7.1 \\
broad_ng & xn28g19v7.1 \\
fine_n & xn252v7.1 \\
fine_ng & xn200g47v7.1 \\
ultra_fine_n & xn999v7.1 \\
v7-238 & xn238v7.0 \\
v7-238n & xn238v7.0 \\
v7.0-238n & xn238v7.0 \\
xn238v7 & xn238v7.0 \\
v7-252n & xn252v7.1 \\
v7.1-252 & xn252v7.1 \\
v7.1-252n & xn252v7.1 \\
v7-200n47g & xn200g47v7.1 \\
v7.1-200n47g & xn200g47v7.1 \\
v7.0-200n47g & xn200g47v7.0 \\
v7-27n19g & xn27g19v7.0 \\
v7.0-27n19g & xn27g19v7.0 \\
v7-28n19g & xn28g19v7.1 \\
v7.1-28n19g & xn28g19v7.1 \\
v7-56 & xn56v7.1 \\
v7-56n & xn56v7.1 \\
v7.1-56 & xn56v7.1 \\
v7.1-56n & xn56v7.1 \\
v7-999 & xn999v7.0 \\
v7-999n & xn999v7.0 \\
v7.1-999 & xn999v7.1 \\
v7.1-999n & xn999v7.1 \\
test_n & test8g_v7.1 \\
test-8grp & test8g_v7.1 \\
\hline & \\
\hline
\end{tabular}

Creates xs_library

parameter mode

Particles to transport.

Default Default mode to ' $n$ ' for kcode, or based on sources if present

Type particle transport mode (n, neutron, np, p, photon, or pn)

postprocessor

Kcode problems must be run in mode 'n' or 'np.'

parameter $\mathbf{n} \_$energy_max

parameter nemax

Maximum global neutron_energy cutoff for cross sections.

Default 20000000.0

Units $\mathrm{eV}$ 
Type positive real number

Applicable when mode is $n$ or $n p$

postprocessor

Parameter n_energy_min must be less than n_energy_max.

Applicability mode is $n$ or $n p$

parameter $\mathbf{n}$ _energy_min

parameter nemin

Minimum global neutron energy cutoff for cross sections.

Default $1 e-05$

Units $\mathrm{eV}$

Type positive real number

Applicable when mode is $n$ or $n p$

parameter name

Label for the physics.

Default $\mathrm{mg}$

Type string without special characters

parameter neutron_bounds

parameter nbounds

Neutron group boundaries.

\section{Default ---}

Type positive floats in decreasing order (each element is a positive real number)

Applicable when xsgen is ampx, gip, inline, or xml

parameter num_groups (advanced)

Number of energy groups.

Default based on given user input

Type positive integer

postprocessor

Inline and GIP cross section definitions require group boundaries.

Applicability xsgen is gip or inline

parameter omit_zaid

Omit the provided nuclides from the XS processing.

Default ---

Type list in which each element is a nuclide specifier (e.g., U-235, 92235, u235, u-235m1)

Applicable when xsgen is scalecomp or anisncomp

parameter orig_zaid

Replace MG data for these nuclides in problem materials. 


\section{Default ---}

Type list in which each element is a nuclide specifier (e.g., U-235, 92235, u235, u-235m1)

Applicable when xsgen is scalecomp or anisncomp

\section{parameter $\mathbf{p} \_$energy_max}

parameter $\mathbf{p e m a x}$

Maximum global photon energy cutoff for cross sections.

Default 25000000.0

Units eV

Type positive real number

Applicable when mode is $\mathrm{p}$ or $\mathrm{np}$

postprocessor

Parameter p_energy_min must be less than p_energy_max.

Applicability mode is $\mathrm{p}$ or $\mathrm{np}$

parameter p_energy_min

parameter pemin

Minimum global photon energy cutoff for cross sections.

Default 10000.0

Units $\mathrm{eV}$

Type positive real number

Applicable when mode is $p$ or $n p$

parameter photon_bounds

parameter pbounds

Photon group boundaries.

Default ---

Type positive floats in decreasing order (each element is a positive real number)

Applicable when xsgen is ampx, gip, inline, or xml

parameter pn_order

Scattering order of problem.

Type non-negative integer

parameter processing

Type of cross section processing to apply.

Default fulcrum

Type fulcrum or none

Applicable when xsgen is scalecomp

parameter subs_zaid

Substitute ZAID corresponding to 'orig_zaid.' 


\section{Default ---}

Type list in which each element is a nuclide specifier (e.g., U-235, 92235, u235, u-235m1)

Applicable when xsgen is scalecomp or anisncomp

postprocessor

The parameters orig_zaid and subs_zaid must have the same length.

Applicability xsgen is scalecomp or anisncomp

parameter subtract_upscattering

parameter subup

Remove upscattering by subtracting from the total cross section.

Instead of lumping upscatter cross sections into the self-scattering cross section, delete the upscatter cross sections and reduce the total cross section accordingly.

$$
\begin{array}{r}
\sigma_{\mathrm{t}, g} \leftarrow \sigma_{\mathrm{t}, g}-\sum_{g^{\prime}=1}^{g-1} \sigma_{\mathrm{s}, g, g^{\prime}} \\
\sigma_{\mathrm{s}, g, g^{\prime}} \leftarrow 0, g^{\prime}=[1, \ldots, g-1]
\end{array}
$$

where the outscatter cross section is

$$
\sigma_{\mathrm{S}}\left(E^{g} \rightarrow E^{g^{\prime}}\right) \equiv \sigma_{\mathrm{s}, g, g^{\prime}}
$$

\section{Default False}

Type boolean

Applicable when disable_upscattering is True

parameter transport_correction

Modify the anisotropy of the scattering cross sections to help preserve solution positivity.

Default none

Type diagonal, cesaro, or none

postprocessor

The cesaro transport correction requires pn_order $>=2$.

Applicability transport_correction is cesaro

parameter $\mathbf{x m l}$ _path

Path to an XML cross section input file.

Type file path for reading (extension '.xml')

Applicable when xsgen is xml

sublist [XS]

Manual isotropic cross section input. See [PHYSICS][XS] (page 105).

Applicable when xsgen is inline

parameter $\mathbf{x s}$ _library

SCALE multigroup library name or master library path. 
Type file path for reading

Applicable when xsgen is scalecomp

\section{parameter $\mathbf{x s g e n}$ (advanced)}

Type of cross section data input.

The xsgen parameter specifies in what format the cross sections are defined for multigroup physics. It is defined automatically by the front end (based on what input the user provides) and should not ever have to be set manually.

Table 20: Possible cross section input formats.

\begin{tabular}{|c|c|}
\hline Option & Description \\
\hline inline & $\begin{array}{l}\text { The }[\mathrm{XS}] \text { (page 105) subdatabase is used to define cross } \\
\text { sections for every material. }\end{array}$ \\
\hline ampx & $\begin{array}{l}\text { The cross sections for every material are defined in AMPX } \\
\text { format. This file is specified using the ampx_path parame- } \\
\text { ter. }\end{array}$ \\
\hline gip & $\begin{array}{l}\text { The cross sections for every material are defined in GIP } \\
\text { format. This file is specified using the gip_path parameter. } \\
\text { Other required parameters describing the file must be in the } \\
\text { [GIP] sub-database. }\end{array}$ \\
\hline scalecomp & $\begin{array}{l}\text { SCALE multigroup cross sections will be built from compo- } \\
\text { sition information pulled from the model. }\end{array}$ \\
\hline anisncomp & $\begin{array}{l}\text { Mixed cross sections will be calculated from } \\
\text { ANISN/ADVANTG multigroup libraries using com- } \\
\text { positions pulled from the model. }\end{array}$ \\
\hline $\mathrm{xml}$ & $\begin{array}{l}\text { The cross sections for every material are defined in a separate } \\
\text { XML file format. This file is specified using the xml_path } \\
\text { parameter. }\end{array}$ \\
\hline
\end{tabular}

Default based on the user-given input types

Type scalecomp, anisncomp, ampx, gip, inline, or xml

\subsection{3 [PHYSICS][GIP]}

The GIP file format is supported only for legacy applications. It is essentially a Fortran data dump. Each group of data is written as a single Fortran record comprised of a (num_reactions, num_moments, num_materials) array. The number of reactions here is equal to $\mathrm{ihm}$ plus an extra value if upscattering is present.

parameter $\mathbf{i h m}$

Cross section table length in GIP file.

Type non-negative integer

parameter ihs

Position of within-group scattering in GIP file.

Type non-negative integer 
parameter iht

Postition of total cross section in GIP file.

Type non-negative integer

parameter isct

PN scattering order in GIP file.

Type non-negative integer

parameter iups

Number of thermal (upscatter) groups in GIP file.

Type non-negative integer

parameter nmat

Number of materials in GIP file.

Type non-negative integer

parameter $\mathbf{s t r i c t}$

Whether edit cross sections correspond to the GIP standard.

With the strict option, the "edit" reactions on the GIP file are assumed to have the following interpretation:

Table 21: GIP reaction tables.

\begin{tabular}{ll}
\hline Table & Reaction \\
\hline iht -4 & $\chi$ \\
iht -3 & $\Sigma_{\mathrm{f}}$ \\
iht -2 & $\Sigma_{\mathrm{a}}$ \\
iht -1 & $v \Sigma_{\mathrm{f}}$ \\
\hline
\end{tabular}

Default False

Type boolean

\subsection{4 [PHYSICS][XS]}

This sub-database specifies all cross sections for a single material. If the material is fissionable, all of fission, nu, and chi must be present. If it is not fissionable, then none of them can be present.

parameter chi

Fission spectrum.

Default ---

Type list of non-negative floats (each element is a non-negative real number)

postprocessor

The parameters total, fission, nu, and chi must have the same length. Empty lists are ignored. parameter fission

Fission cross section.

Default --- 
Type list of non-negative floats (each element is a non-negative real number)

parameter matid

Material ID.

Type non-negative integer

parameter name

Label for the material.

Default given matid

Type string without special characters

parameter $\mathbf{n u}$

Neutron production.

Default ---

Type list of non-negative floats (each element is a non-negative real number)

parameter $\mathbf{s} \boldsymbol{\theta}$

Isotropic scattering cross section.

Type list of non-negative floats (each element is a non-negative real number)

parameter total

Total cross section by group.

Type list of non-negative floats (each element is a non-negative real number)

\subsection{COMPOSITIONS: [COMP]}

The $[C O M P]$ block controls output and management of compositions and enables defining them manually. The matids in each block must correspond to the matids in the problem geometry.

command autocolor

Attempt to provide color values using their compositions.

Creates color_key

Creates color_val

Applicable when

- 'ENABLE_PYTHON_WRAPPERS' is enabled in this CMake build; and

- The 'matplotlib' python package is installed

parameter color_key (advanced)

Name of compositions for which colors are being set.

Default ---

Type list in which each element is a string

parameter color_val(advanced)

Compositions color to be set.

Default --- 
Type list in which each element is a X11 color, HTML color like \#FF00ee, tuple, or empty

postprocessor

The parameters color_key and color_val must have the same length.

\section{command colors}

Map 'color_key' to 'color_val' from pairs or arrow-separated items.

VisIT supports custom colors to be associated with each material in its plotting routines. Materials not specified in this list will be filled with grey. Incorrect material names will generate an error:

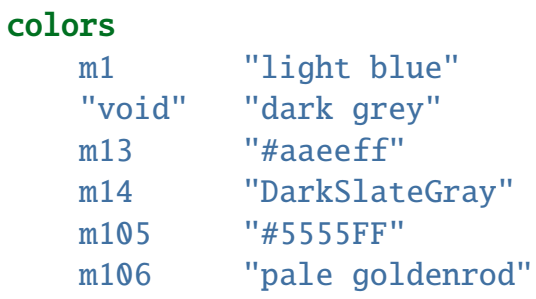

Valid color names are either HTML-style hexadecimal RGB tuples or X11 colors.

For complicated inputs with variable materials, the Python raytracer and coloring modules can be used to automatically assign colors based on materials compositions. (See the omnibus . raytracer . colors module.)

Creates color_key

Creates color_val

parameter compgen (advanced)

Type of composition input.

Default based on the user-given comp types

Type model, hdf5, or inline

sublist [COMPOUND]

Elemental compound definition. See [COMP][COMPOUND] (page 110).

Default (empty sublist)

\section{command elements}

Create compounds from natural abundances of the given elements.

Creates compound

deprecated force_scl

Deprecated entry force_scl has been renamed to load_scl.

Update to load_scl

parameter input

Path to the HDF5 file containing compositions.

Type file path for reading (extension '.h5')

Applicable when compgen is hdf5 
parameter load_scl

Load nuclides from the SCALE Standard Composition Library.

The SCALE standard composition library (SCL) provides a database of nuclides, elements, and compounds that can be used to simplify user composition input.

Exnihilo uses a global database of nuclide data (number densities, names, atomic numbers, etc.) for construction of compositions. This same database is also used to drive SCALE's multigroup cross section builder Fulcrum. If using the SCALE cross section builder (xsgen processed), the names in the database must correspond to the names SCALE expects.

Some model types (Geant4, Lava) are able to load nuclide metadata independently of the SCALE SCL; this can interfere with cross section generation. The load_scl option preferentially loads SCALE's nuclide definitions.

This option is also useful if using a composition source (hdf5 or inline) that has incomplete nuclide data but is being used for depletion. For example, since only nuclides in use by the current composition set are written to the composition output, it may not be sufficient to use those compositions/nuclides in a depletion run (since additional depletion nuclides will be added to the composition and their nuclide data may be missing).

Note that changing the nuclide data source will change the relationship between composition number densities and weight fractions, so output values may change.

Default True when using 'processed' MG physics, CE physics, or HDF5/inline materials

Type boolean

Applicable when 'SCALE_StdCompLib' is enabled in this build

sublist [MATERIAL]

Composition definition. See [COMP][MATERIAL] (page 109).

Applicable when compgen is inline

database [NUCLIDES]

Nuclide definitions. See [COMP][NUCLIDES] (page 110).

Optional

parameter output

Save compositions to the output file.

Default True

Type boolean

parameter Sclib_path

Path to the SCALE standard composition library.

Default ' $/ .$. /scale.rev40.sclib'

Type file path for reading (extension '.sclib')

Applicable when load_scl is True 


\subsection{1 [COMP][MATERIAL]}

The material block is for defining an individual composition. Providing any materials will override all compositions that may be present in the model (page 43).

\section{parameter deplete \\ parameter depl}

Flag the material as depletable.

Marking a material as "depletable" tells Shift to enable ORIGEN depletion for this material unless the user overrides this using the options in the [DEPLETION] database.

The default value of "auto" will set this property to true if and only if it is marked as fissionable.

\section{Default auto}

Type auto, true, or false

\section{parameter fission}

parameter fiss

Flag the material as fissionable.

Marking a material as "fissionable" sets whether (by default) fission gammas and neutrons will be produced during fixed-source transport. In other words, setting this value to false will (again, by default) suppress secondary neutrons or gamma yields from the material.

Note that this option is ignored for fission site sampling in kcode/eigenvalue mode: all fissionable nuclides have a chance to create fission sites.

The default value of "auto" will set this property to true if any fissionable nuclide is present, and false if not.

\section{Default auto}

Type auto, true, or false

\section{parameter matid}

Internal matid number.

The matid is a $[0, N)$-indexed internal numbering system. The matids used by Shift typically differ from the material names used in the problem physics input (for example, $m 10$ in an MCNP input deck might correspond to matid=1). Currently, the only way to guarantee that this corresponds to a particular material in the geometry input is to use an input that specifies matids explicitly (RTK or mesh geometry). However, by viewing the matid-to-label mapping given in an Omnibus post-process output for a SCALE or MCNP input geometry, it is possible to determine what matid corresponds to what material in a particular problem input.

Type non-negative integer

parameter name

Label for the material.

Type non-empty string

parameter $\mathbf{n d}$

Number densities of each nuclide.

$$
\text { Units } \frac{\text { atom }}{(\mathrm{b} \cdot \mathrm{cm})}
$$


Type list in which each element is a positive real number

postprocessor

The parameters nd and zaid must have the same length.

parameter temperature

parameter tmp

Material temperature.

Units $\mathrm{K}$

Type non-negative real number

parameter $\mathbf{z a i d}$

Element/nuclide IDs (MZZZAAA) in this material.

Type list in which each element is a positive integer

\subsection{2 [COMP][NUCLIDES]}

Define properties for a given nuclide ID. This enables custom ZAID values in input files such as multigroup cross section data. These properties will override any existing masses loaded from the SCALE SCL.

parameter mass

Atomic masses of nuclides.

Type list in which each element is a positive real number

postprocessor

The parameters name, zaid, and mass must have the same length.

parameter name

Names of nuclides.

Type list in which each element is a string

parameter zaid

Nuclide IDs (MZZZAAA).

Type list in which each element is a positive integer

\subsection{3 [COMP][COMPOUND]}

Define compounds or natural-abundance elements. Any ZAID in the user model or composition definitions corresponding to the value of czaid will be expanded into the zaid values provided for this compound. These will override any compound defined by the SCALE SCL.

parameter czaid

Compound ZAID (e.g., 1000 for elemental hydrogen).

Type positive integer

parameter name

Descriptive name of the compound.

Type string without special characters

parameter wtfrac

Weight fraction of each constituent. 
Type list in which each element is a positive real number

postprocessor

The parameters wt frac and zaid must have the same length.

parameter zaid

Consitutent nuclide IDs (MZZZAAA).

Type list in which each element is a positive integer

\subsection{RESPONSES: [RESPONSE]}

The RESPONSE block is for defining energy- and particle-dependent responses such as dose conversion factors. Each response can be used multiple times in the tallies.

Table 22: Available types for the [RESPONSE] database

\begin{tabular}{lll}
\hline Type & Description & Applicability \\
\hline histogram (page 111) & Histogram response & \\
interpolated (page 112) & Interpolation between values & \\
xs (page 112) & Microscopic cross section multiplier & \\
\hline
\end{tabular}

\subsection{1 [RESPONSE $=$ HISTOGRAM]}

The "histogram" response is effectively a piecewise constant cross section. The response is the specified value of the response (page 111) parameter inside the corresponding energy (page 111) bin for the given particle type (page 111). The response is zero for other particle types or outside the given energy range.

parameter description

Optional longer descriptive string.

Default ' '

Type string

parameter energy

parameter $\mathbf{e}$

Lowest bound plus upper bounds for the histograms.

Units eV

Type monotonically increasing list (each element is a real number)

parameter name

Short title or label for response.

Type string without special characters

parameter particle_type

parameter $\mathbf{p t}$

Particle type to apply this response.

Type particle type (n, neutron, $\mathrm{p}$, or photon)

parameter response

parameter $\mathbf{r}$

Histogram response values.

Type list in which each element is a real number

postprocessor

Size of energy bounds must be one greater than the number of response values. 


\subsection{2 [RESPONSE=INTERPOLATED]}

Interpolation between values.

parameter description

Optional longer descriptive string.

Default ' '
Type string
parameter energy
parameter e

Energy points.

Units $\mathrm{eV}$

Type monotonically increasing list (each element is a positive real number)

parameter interpolation_type

parameter interp

Type of interpolated response.

Type linear, log_lin, lin_log, or log_log

parameter name

Short title or label for response.

Type string without special characters

parameter particle_type

parameter $\mathbf{p t}$

Particle type to apply this response.

Type particle type (n, neutron, $\mathrm{p}$, or photon)

parameter response

parameter $\mathbf{r}$

Response values at energy points.

Type list in which each element is a real number

postprocessor

Response must be positive for log_log or log_lin.

postprocessor

The parameters energy and response must have the same length.

\subsection{3 $[$ RESPONSE $=X S]$}

Microscopic cross section multiplier.

parameter density

Density multiplier for response calculation.

Default 1.0

Type positive real number 


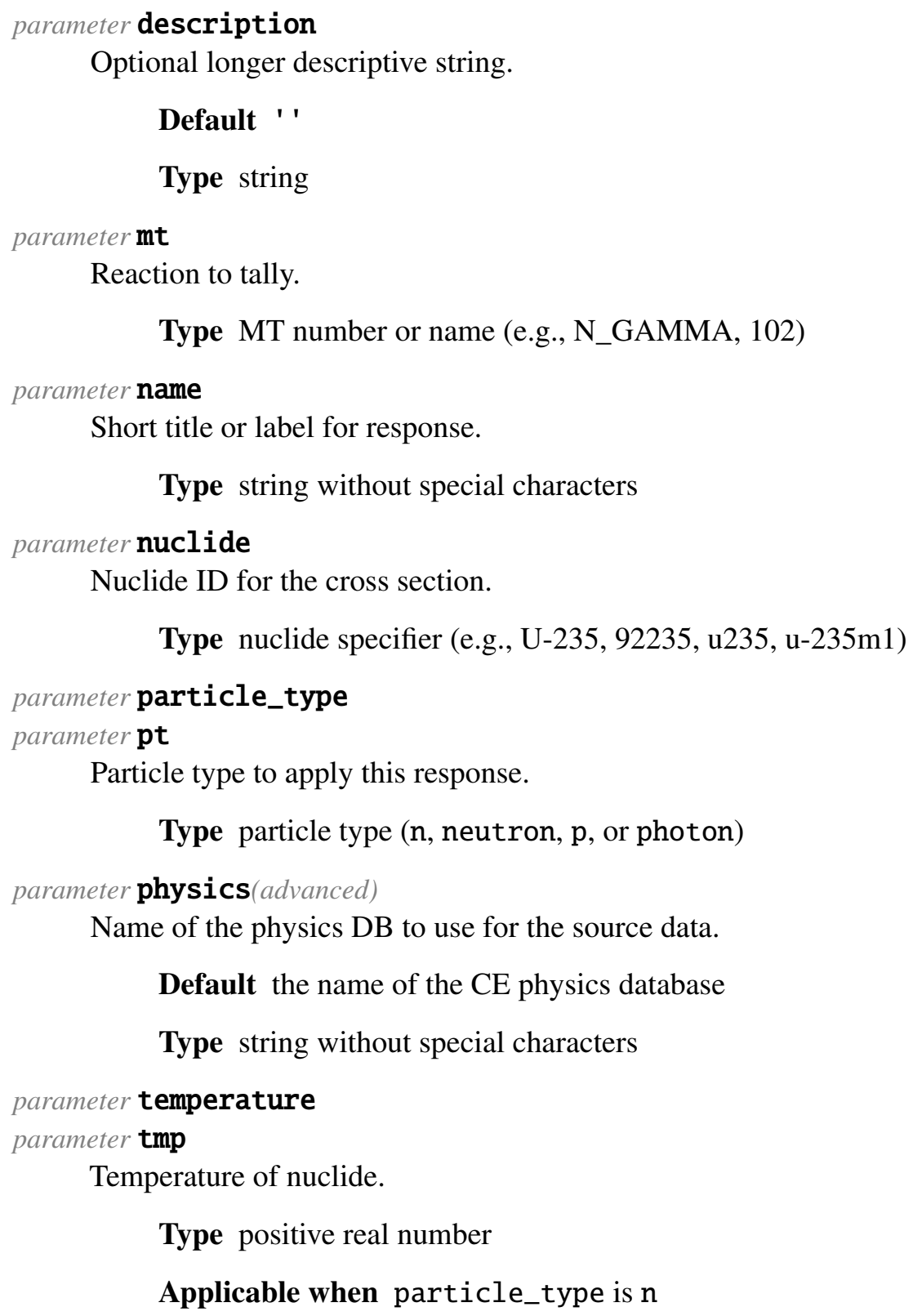

Optional longer descriptive string.

Default ' '

Type string

parameter $\mathbf{m t}$

Reaction to tally.

Type MT number or name (e.g., N_GAMMA, 102)

parameter name

Short title or label for response.

Type string without special characters

parameter nuclide

Nuclide ID for the cross section.

Type nuclide specifier (e.g., U-235, 92235, u235, u-235m1)

parameter particle_type

parameter $\mathbf{p t}$

Particle type to apply this response.

Type particle type (n, neutron, $p$, or photon)

parameter physics(advanced)

Name of the physics DB to use for the source data.

Default the name of the CE physics database

Type string without special characters

parameter temperature

parameter tmp

Temperature of nuclide.

Type positive real number

Applicable when particle_type is $n$

\subsection{TALLIES: [TALLY]}

Be aware that unless otherwise specified, tallies in Shift:

- are replicated, even if domain decomposition is requested;

- use history-based statistics to estimate variance;

- provide volume-averaged reaction rates if the tally region's volume is known;

- are path length estimators. 
Many tallies support a common set of attributes, including a name and optional description, a list of reactions to tally, a list of [RESPONSE] (page 111) objects, and neutron and photon energy bin boundaries.

Note: Unlike other Monte Carlo transport codes, the bin boundaries in Omnibus are truly boundaries, not upper or lower energies. Therefore, the number of energy bins will be one less than the number of bounds. To reproduce the behavior of MCNP and Monaco, which tally from the cutoff energy to the lowest given energy, the user must explicitly add the cutoff energy as the lowest energy bound.

The cell and mesh tallies produce volume averaged quantities when volumes are available for the region being tallied. Therefore, a reaction rate is output as a reaction rate density (units of $\frac{\text { reaction }}{\mathrm{s}-\mathrm{cm}^{3}}$ ). If volumes are not provided (explicitly by the user or automatically by the geometry) for cell tallies, a warning will be issued and the tally result will be cell-integrated.

Warning: Many geometries define the volume of a cell to be the volume for an instance of that cell. If the cell is replicated (e.g., a pin cell in an assembly), then the volume being used to normalize the tally might not correspond to the total volume being tallied. This is because all instances of a cell will contribute to the same tally.

Because of the potential ambiguities that may occur with volume normalization, it is highly recommended that users check the volumes field output alongside cell tallies. A volume entry of $\mathbb{\theta}$ means that the volume was not provided, so that the corresponding tally will have units of $\frac{\text { reaction }}{\mathrm{s}}$ as though the tally region had unit volume.

\section{sublist [BATCH_MESH] (advanced)}

Tally reaction rates using batch statistics (experimental!). See [TALLY][BATCH_MESH] (page 160).

Applicable when problem mode is kcode

\section{Optional}

sublist [BIRTH_SPECTRUM]

Tally the energy distribution of particles at birth in geometry cells. See [TALLY][BIRTH_SPECTRUM] (page 158).

\section{Optional}

Applicable when solver is 'shift'

sublist [CELL]

Tally reaction rates in geometry cells and unions of cells. See [TALLY][CELL] (page 130).

\section{Optional}

sublist [CYLMESH]

Tally reaction rates in cylindrical cells. See [TALLY][CYLMESH] (page 123).

\section{Optional}

\section{sublist [DIAGNOSTIC]}

Singleton tallies used for transport diagnostic purposes. See [TALLY][DIAGNOSTIC] (page 140).

Applicable when solver is 'shift' 


\section{Default (empty sublist) \\ sublist [HEXMESH]}

Tally reaction rates on a hexagonal grid. See [TALLY][HEXMESH] (page 127).

\section{Optional}

\section{sublist [HEXNODAL]}

Tally integral quantities on a hexagonal mesh for nodal calculations. See [TALLY][HEXNODAL] (page 150).

Applicable when solver is 'shift'

\section{Optional}

\section{sublist [MEAN_SCATTER_ANGLE]}

Tally the mean scattering angle in geometry cells. See [TALLY][MEAN_SCATTER_ANGLE] (page 156).

\section{Optional}

Applicable when solver is 'shift'

sublist [MESH]

Tally reaction rates on a domain-decomposed Cartesian mesh. See [TALLY][MESH] (page 116).

\section{Optional}

\section{sublist [MESH_SURFACE]}

Tally reaction rates and partial currents on mesh faces. See [TALLY][MESH_SURFACE] (page 121).

\section{Optional}

sublist [MICRO]

Tally flux and microscopic cross sections in materials. See [TALLY][MICRO] (page 138).

\section{Applicable when}

- solver is 'shift'; and

- physics is CE

\section{Optional}

sublist [MIGRATION_AREA]

Tally particle migration area on a Cartesian mesh. See [TALLY][MIGRATION_AREA] (page 154).

Applicable when solver is 'shift'

\section{Optional}

\section{sublist [NODAL]}

Tally integral quantities on a Cartesian mesh for nodal calculations. See [TALLY][NODAL] (page 146).

Applicable when solver is 'shift'

\section{Optional}

\section{sublist [SCATTERING_PROB_MATRIX]}

Tally the group-to-group scattering probability matrix in geometry cells. See [TALLY][SCATTERING_PROB_MATRIX] (page 157). 


\section{Optional}

Applicable when solver is 'shift'

\section{database [SENSITIVITY]}

Tally sensitivities for use in uncertainty quantification. See [TALLY][SENSITIVITY] (page 144).

\section{Optional}

\section{Applicable when}

- physics is CE; and

- 'Sensitivity' is enabled in this build

sublist [SHADOW]

Tally arbitrary cells in an overlaid but non-interacting geometry. See [TALLY][SHADOW] (page 135).

Applicable when ' $G G$ ' is enabled in this build

\section{Optional}

sublist [SURFACE_CENSUS]

Tally individual particle properties as they cross a geometry surface. See

[TALLY][SURFACE_CENSUS] (page 159).

Applicable when solver is 'shift'

\section{Optional}

database [SWORD]

Include tally definitions from SWORD input. See [TALLY][SWORD] (page 141).

\section{Optional}

Applicable when model is 'sword'

sublist [VERA]

Tally in cells in a VERA model. See [TALLY][VERA] (page 134).

\section{Optional}

\subsection{1 [TALLY][MESH]}

Shift supports multiple structured Cartesian path length mesh tallies. The mesh can be defined over all or part of the physical problem geometry. If domain decomposition is enabled in Shift, only the part of the mesh tally that is present on a spatial domain will be transported on.

\section{parameter cycles}

The kcode problem phase in which this tally is active.

Default active

Type active or inactive

Applicable when problem mode is kcode

\section{parameter delta_x}

Length of mesh tally cell on the $\mathrm{X}$ axis.

\section{Units $\mathrm{cm}$}


Type positive real number

Applicable when type is global

parameter delta_y

Length of mesh tally cell on the Y axis.

Units $\mathrm{cm}$

Type positive real number

Applicable when type is global

parameter delta_z

Length of mesh tally cell on the $\mathrm{Z}$ axis.

Units $\mathrm{cm}$

Type positive real number

Applicable when type is global

command deltas

Expand into parameters delta_x,delta_y, and delta_z.

Creates delta_x

Creates delta_y

Creates delta_z

parameter description

parameter desc

Optional longer descriptive string.

Default ' '

Type string

database [FILTERS]

Tally filtering options. See [TALLY][MESH][FILTERS] (page 161).

\section{Optional}

parameter macro_mt

ENDF reactions for partial macroscopic cross section tallying.

\section{Default ---}

Type list of MT numbers or names (each element is a MT number or name (e.g., N_GAMMA, 102))

Applicable when physics is CE

parameter macro_mt_zaid

Nuclide IDs for partial macroscopic cross section tallying.

Default ---

Type list in which each element is a nuclide specifier (e.g., U-235, 92235, u235, u-235m1)

Applicable when physics is CE 
parameter macro_rxn

Special reactions for macroscopic cross section tallying.

Default ---

Type list in which each element is a flux, pos_partial_current_x, neg_partial_current_x, pos_partial_current_y, neg_partial_current_y, pos_partial_current_z, neg_partial_current_z, total, absorption, scattering, transfer_1n, transfer_2n, transfer_3n, transfer_4n, fission, nu_fission, kappa_sigma, kappa_sigma_f, or kappa_sigma_c

Applicable when physics is CE

parameter macro_rxn_zaid

Nuclide IDs for macroscopic 'special reaction' cross section tallying.

Default ---

Type list in which each element is a nuclide specifier (e.g., U-235, 92235, u235, u-235m1)

Applicable when physics is CE

parameter micro_mt

ENDF reactions for microscopic cross section tallying.

Default ---

Type list of MT numbers or names (each element is a MT number or name (e.g., N_GAMMA, 102))

Applicable when physics is $\mathrm{CE}$

postprocessor

The parameters micro_mt_zaid and micro_mt must have the same length.

Applicability physics is $\mathrm{CE}$

parameter micro_mt_zaid

Nuclide IDs for microscopic cross section tallying.

Default ---

Type list in which each element is a nuclide specifier (e.g., U-235, 92235, u235, u-235m1)

Applicable when physics is $\mathrm{CE}$

parameter micro_rxn

Special reactions for microscopic cross section tallying.

Default ---

Type list in which each element is a flux, pos_partial_current_x, neg_partial_current_x, pos_partial_current_y, neg_partial_current_y, pos_partial_current_z, neg_partial_current_z, total, absorption, scattering, transfer_1n, transfer_2n, transfer_3n, transfer_4n, fission, nu_fission, kappa_sigma, kappa_sigma_f, or kappa_sigma_c

Applicable when physics is CE 
postprocessor

The parameters micro_rxn_zaid and micro_rxn must have the same length.

Applicability physics is CE

parameter micro_rxn_zaid

Nuclide IDs for microscopic 'special reaction' cross section tallying.

Default ---

Type list in which each element is a nuclide specifier (e.g., U-235, 92235, u235, u-235m1)

Applicable when physics is CE

deprecated micro_zaid

Deprecated entry micro_zaid has been renamed to micro_mt_zaid.

Update to micro_mt_zaid

parameter $\mathbf{m t}$

Additional macroscopic reaction rates to tally.

Default ---

Type list of MT numbers or names (each element is a MT number or name (e.g., N_GAMMA, 102))

Applicable when physics is $\mathrm{CE}$

parameter name

Short title or label for the tally.

Type string without special characters

parameter neutron_bins

parameter nbins

Energy bin boundaries for neutrons.

Default ---

Units $\mathrm{eV}$

Type nonnegative floats in decreasing order (each element is a non-negative real number) parameter normalization

Constant multiplicative factor to apply to tally results.

Default 1.0

Type positive real number

parameter photon_bins

parameter $\mathbf{p b i n s}$

Energy bin boundaries for photons.

Default ---

Units $\mathrm{eV}$

Type nonnegative floats in decreasing order (each element is a non-negative real number) 


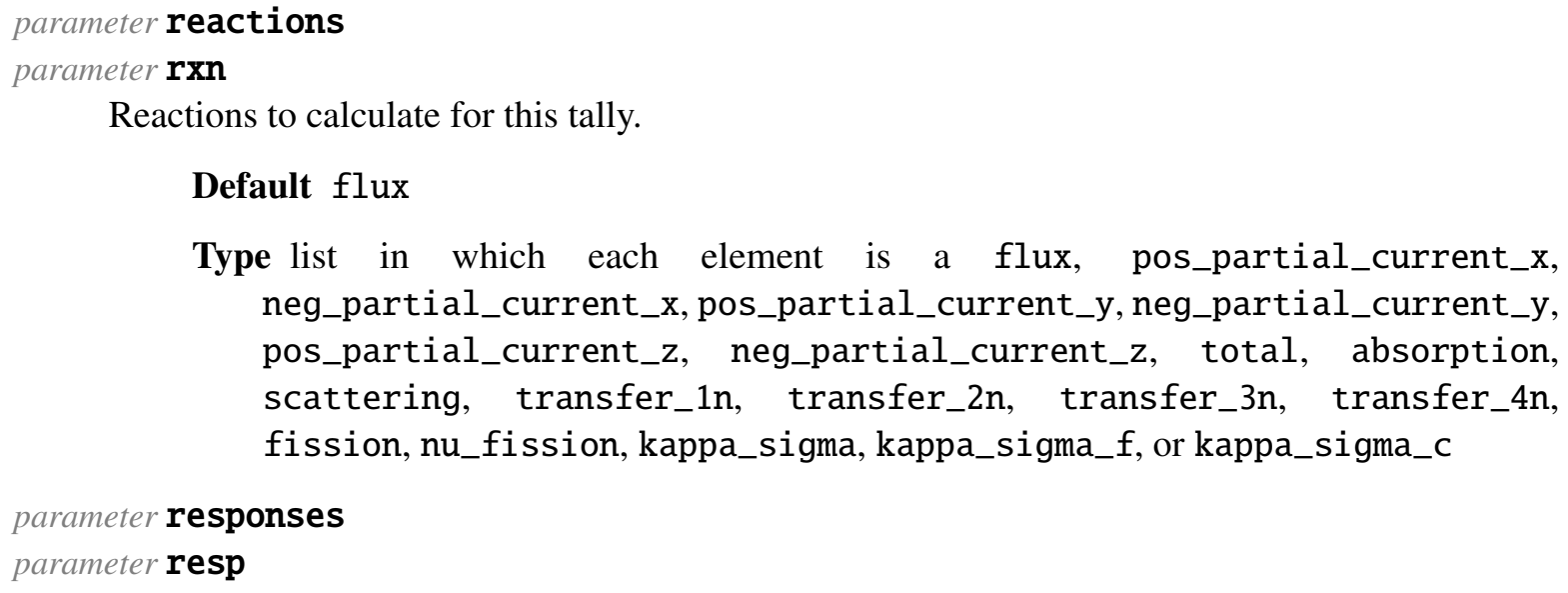

Reactions to calculate for this tally.

Default flux

Type list in which each element is a flux, pos_partial_current_x, neg_partial_current_x,pos_partial_current_y, neg_partial_current_y, pos_partial_current_z, neg_partial_current_z, total, absorption, scattering, transfer_1n, transfer_2n, transfer_3n, transfer_4n, fission, nu_fission, kappa_sigma, kappa_sigma_f, or kappa_sigma_c

parameter responses

parameter resp

Responses for this tally.

Default ---

Type list in which each element is a string

postprocessor

Validate response names against [RESPONSE] blocks.

parameter type

Type of mesh tally.

\section{Default grid}

Type grid or global

parameter $\mathbf{x}$

Tally grid coordinates along the $\mathrm{X}$ axis.

Units $\mathrm{cm}$

Type monotonically increasing list with at least two values (each element is a real number)

Applicable when type is grid

parameter $\mathbf{y}$

Tally grid coordinates along the $\mathrm{Y}$ axis.

Units $\mathrm{cm}$

Type monotonically increasing list with at least two values (each element is a real number)

Applicable when type is grid

parameter $\mathbf{z}$

Tally grid coordinates along the $\mathrm{Z}$ axis.

Units $\mathrm{cm}$

Type monotonically increasing list with at least two values (each element is a real number)

Applicable when type is grid 


\subsection{2 [TALLY][MESH_SURFACE]}

The Cartesian mesh surface tally can calculate surface flux, current, or partial current on each face of the mesh. The allowed reactions for this tally differ from the volumetric mesh tally (page 116) reactions.

\section{parameter cycles}

The kcode problem phase in which this tally is active.

Default active

Type active or inactive

Applicable when solver is 'shift'

parameter delta_x

Length of mesh current tally cell on the $\mathrm{X}$ axis.

Units $\mathrm{cm}$

Type positive real number

Applicable when type is global

parameter delta_y

Length of mesh current tally cell on the Y axis.

Units $\mathrm{cm}$

Type positive real number

Applicable when type is global

parameter delta_z

Length of mesh current tally cell on the $\mathrm{Z}$ axis.

Units $\mathrm{cm}$

Type positive real number

Applicable when type is global

command deltas

Expand into parameters delta_x, delta_y, and delta_z.

Creates delta_x

Creates delta_y

Creates delta_z

parameter description

parameter desc

Optional longer descriptive string.

Default ' '

Type string

database [FILTERS]

Tally filtering options. See [TALLY][MESH][FILTERS] (page 161).

\section{Optional}


parameter name

Short title or label for the tally.

Type string without special characters

parameter neutron_bins

parameter nbins

Energy bin boundaries for neutrons.

\section{Default ---}

Units $\mathrm{eV}$

Type nonnegative floats in decreasing order (each element is a non-negative real number) parameter normalization

Constant multiplicative factor to apply to tally results.

\section{Default 1.0}

Type positive real number

parameter photon_bins

parameter pbins

Energy bin boundaries for photons.

Default ---

Units $\mathrm{eV}$

Type nonnegative floats in decreasing order (each element is a non-negative real number)

parameter reactions

parameter $\mathbf{r x n}$

Type of mesh surface tally.

\section{Default flux}

Type list in which each element is a flux, current, partial_current_negative, or partial_current_positive

parameter type

Type of mesh surface tally.

Default grid

Type grid or global

parameter $\mathbf{x}$

Mesh current tally coordinates along the $\mathrm{X}$ axis.

Units $\mathrm{cm}$

Type monotonically increasing list with at least two values (each element is a real number)

Applicable when type is grid

parameter $\mathbf{y}$

Mesh current tally coordinates along the $\mathrm{Y}$ axis.

Units $\mathrm{cm}$ 
Type monotonically increasing list with at least two values (each element is a real number)

Applicable when type is grid

parameter $\mathbf{z}$

Mesh current tally coordinates along the $\mathrm{Z}$ axis.

Units $\mathrm{cm}$

Type monotonically increasing list with at least two values (each element is a real number)

Applicable when type is grid

\subsection{3 [TALLY][CYLMESH]}

Particles can be tracked on a translated, rotated cylinder broken into $(r, z, \theta)$ mesh cells.

\section{parameter cycles}

The kcode problem phase in which this tally is active.

Default active

Type active or inactive

Applicable when problem mode is kcode

parameter description

parameter desc

Optional longer descriptive string.

Default ' '

Type string

parameter macro_mt

ENDF reactions for partial macroscopic cross section tallying.

Default ---

Type list of MT numbers or names (each element is a MT number or name (e.g., N_GAMMA, 102))

Applicable when physics is CE

parameter macro_mt_zaid

Nuclide IDs for partial macroscopic cross section tallying.

Default ---

Type list in which each element is a nuclide specifier (e.g., U-235, 92235, u235, u-235m1)

Applicable when physics is CE

parameter macro_rxn

Special reactions for macroscopic cross section tallying.

Default ---

Type list in which each element is a flux, pos_partial_current_x, neg_partial_current_x, pos_partial_current_y, neg_partial_current_y, pos_partial_current_z, neg_partial_current_z, total, absorption, scattering, transfer_1n, transfer_2n, transfer_3n, transfer_4n, fission, nu_fission, kappa_sigma, kappa_sigma_f, or kappa_sigma_c 
Applicable when physics is CE

parameter macro_rxn_zaid

Nuclide IDs for macroscopic 'special reaction' cross section tallying.

Default ---

Type list in which each element is a nuclide specifier (e.g., U-235, 92235, u235, u-235m1)

Applicable when physics is CE

parameter micro_mt

ENDF reactions for microscopic cross section tallying.

Default ---

Type list of MT numbers or names (each element is a MT number or name (e.g., N_GAMMA, 102))

Applicable when physics is CE

postprocessor

The parameters micro_mt_zaid and micro_mt must have the same length.

Applicability physics is CE

parameter micro_mt_zaid

Nuclide IDs for microscopic cross section tallying.

Default ---

Type list in which each element is a nuclide specifier (e.g., U-235, 92235, u235, u-235m1)

Applicable when physics is CE

parameter micro_rxn

Special reactions for microscopic cross section tallying.

Default ---

Type list in which each element is a flux, pos_partial_current_x, neg_partial_current_x, pos_partial_current_y, neg_partial_current_y, pos_partial_current_z, neg_partial_current_z, total, absorption, scattering, transfer_1n, transfer_2n, transfer_3n, transfer_4n, fission, nu_fission, kappa_sigma, kappa_sigma_f, or kappa_sigma_c

Applicable when physics is $\mathrm{CE}$

postprocessor

The parameters micro_rxn_zaid and micro_rxn must have the same length.

Applicability physics is CE

parameter micro_rxn_zaid

Nuclide IDs for microscopic 'special reaction' cross section tallying.

Default ---

Type list in which each element is a nuclide specifier (e.g., U-235, 92235, u235, u-235m1)

Applicable when physics is CE 
deprecated micro_zaid

Deprecated entry micro_zaid has been renamed to micro_mt_zaid.

Update to micro_mt_zaid

parameter $\mathbf{m t}$

Additional macroscopic reaction rates to tally.

Default ---

Type list of MT numbers or names (each element is a MT number or name (e.g., N_GAMMA, 102))

\section{Applicable when physics is $\mathrm{CE}$}

parameter name

Short title or label for the tally.

Type string without special characters

parameter neutron_bins

parameter $\mathbf{n b i n s}$

Energy bin boundaries for neutrons.

Default ---

Units $\mathrm{eV}$

Type nonnegative floats in decreasing order (each element is a non-negative real number) parameter normalization

Constant multiplicative factor to apply to tally results.

Default 1.0

Type positive real number

parameter photon_bins

parameter $\mathbf{p b i n s}$

Energy bin boundaries for photons.

Default ---

Units eV

Type nonnegative floats in decreasing order (each element is a non-negative real number)

parameter $\mathbf{r}$

Radial mesh coordinates.

Units $\mathrm{cm}$

Type monotonically increasing list with at least two values, starting with zero (each element is a real number)

parameter reactions

parameter $\mathbf{r x n}$

Reactions to calculate for this tally.

Default flux 
Type list in which each element is a flux, pos_partial_current_x, neg_partial_current_x, pos_partial_current_y, neg_partial_current_y, pos_partial_current_z, neg_partial_current_z, total, absorption, scattering, transfer_1n, transfer_2n, transfer_3n, transfer_4n, fission, nu_fission, kappa_sigma, kappa_sigma_f, or kappa_sigma_c

\section{parameter responses}

parameter resp

Responses for this tally.

Default ---

Type list in which each element is a string

postprocessor

Validate response names against [RESPONSE] blocks.

parameter rotate

parameter rot

Rotation matrix.

Default 1.00 .00 .00 .01 .00 .00 .00 .01 .0

Type length-9 row-major rotation matrix (each element is a real number)

parameter theta

Theta mesh coordinates.

Default 0.01 .0

Units revolution

Type monotonically increasing list with at least two values, starting with zero and ending with one [revolution] (each element is a real number)

parameter translate

parameter trans

Translation vector (applied after rotation).

Default 0.00 .00 .0

Type length-3 float vector (each element is a real number)

postprocessor (advanced)

Squelch identity rotations and null translations.

parameter $\mathbf{z}$

Mesh coordinates along the $\mathrm{Z}$ axis.

Units $\mathrm{cm}$

Type monotonically increasing list with at least two values (each element is a real number) 


\subsection{4 [TALLY][HEXMESH]}

Tally volumetric path lengths on a hexagonal (triangular-pitch) mesh. This tally is meant for nodal data calculations and should generally not be used. The mesh boundaries must encompass the entirety of the transportable problem domain.

\section{parameter apothem}

Distance from hex center to face center.

Units $\mathrm{cm}$

Type positive real number

\section{parameter center_hex}

$(\mathrm{x}, \mathrm{y})$ centroid of center hex.

Units $\mathrm{cm}$

Type length-2 float vector (each element is a real number)

parameter cycles

The kcode problem phase in which this tally is active.

Default active

Type active or inactive

Applicable when problem mode is kcode

parameter description

parameter desc

Optional longer descriptive string.

\section{Default ' '}

Type string

\section{database [FILTERS]}

Tally filtering options. See [TALLY][MESH][FILTERS] (page 161).

\section{Optional}

\section{parameter macro_mt}

ENDF reactions for partial macroscopic cross section tallying.

\section{Default ---}

Type list of MT numbers or names (each element is a MT number or name (e.g., N_GAMMA, 102))

Applicable when physics is $\mathrm{CE}$

parameter macro_mt_zaid

Nuclide IDs for partial macroscopic cross section tallying.

\section{Default ---}

Type list in which each element is a nuclide specifier (e.g., U-235, 92235, u235, u-235m1)

Applicable when physics is $\mathrm{CE}$ 
parameter macro_rxn

Special reactions for macroscopic cross section tallying.

Default ---

Type list in which each element is a flux, pos_partial_current_x, neg_partial_current_x, pos_partial_current_y, neg_partial_current_y, pos_partial_current_z, neg_partial_current_z, total, absorption, scattering, transfer_1n, transfer_2n, transfer_3n, transfer_4n, fission, nu_fission, kappa_sigma, kappa_sigma_f, or kappa_sigma_c

Applicable when physics is CE

parameter macro_rxn_zaid

Nuclide IDs for macroscopic 'special reaction' cross section tallying.

Default ---

Type list in which each element is a nuclide specifier (e.g., U-235, 92235, u235, u-235m1)

Applicable when physics is CE

parameter micro_mt

ENDF reactions for microscopic cross section tallying.

Default ---

Type list of MT numbers or names (each element is a MT number or name (e.g., N_GAMMA, 102))

Applicable when physics is $\mathrm{CE}$

postprocessor

The parameters micro_mt_zaid and micro_mt must have the same length.

Applicability physics is $\mathrm{CE}$

parameter micro_mt_zaid

Nuclide IDs for microscopic cross section tallying.

Default ---

Type list in which each element is a nuclide specifier (e.g., U-235, 92235, u235, u-235m1)

Applicable when physics is $\mathrm{CE}$

parameter micro_rxn

Special reactions for microscopic cross section tallying.

Default ---

Type list in which each element is a flux, pos_partial_current_x, neg_partial_current_x, pos_partial_current_y, neg_partial_current_y, pos_partial_current_z, neg_partial_current_z, total, absorption, scattering, transfer_1n, transfer_2n, transfer_3n, transfer_4n, fission, nu_fission, kappa_sigma, kappa_sigma_f, or kappa_sigma_c

Applicable when physics is CE 
postprocessor

The parameters micro_rxn_zaid and micro_rxn must have the same length.

Applicability physics is $\mathrm{CE}$

parameter micro_rxn_zaid

Nuclide IDs for microscopic 'special reaction' cross section tallying.

Default ---

Type list in which each element is a nuclide specifier (e.g., U-235, 92235, u235, u-235m1)

Applicable when physics is CE

deprecated micro_zaid

Deprecated entry micro_zaid has been renamed to micro_mt_zaid.

Update to micro_mt_zaid

parameter $\mathbf{m t}$

Additional macroscopic reaction rates to tally.

Default ---

Type list of MT numbers or names (each element is a MT number or name (e.g., N_GAMMA, 102))

Applicable when physics is CE

parameter name

Short title or label for the tally.

Type string without special characters

parameter neutron_bins

parameter nbins

Energy bin boundaries for neutrons.

Default ---

Units eV

Type nonnegative floats in decreasing order (each element is a non-negative real number) parameter normalization

Constant multiplicative factor to apply to tally results.

Default 1.0

Type positive real number

parameter num_rings

Number of rings around the central hex.

Type non-negative integer

parameter photon_bins

parameter pbins

Energy bin boundaries for photons.

Default --- 


\section{Units eV}

Type nonnegative floats in decreasing order (each element is a non-negative real number)

\section{parameter reactions}

parameter $\mathbf{r x n}$

Reactions to calculate for this tally.

Default flux

Type list in which each element is a flux, pos_partial_current_x, neg_partial_current_x, pos_partial_current_y, neg_partial_current_y, pos_partial_current_z, neg_partial_current_z, total, absorption, scattering, transfer_1n, transfer_2n, transfer_3n, transfer_4n, fission, nu_fission, kappa_sigma, kappa_sigma_f, or kappa_sigma_c

parameter responses

parameter resp

Responses for this tally.

\section{Default ---}

Type list in which each element is a string

postprocessor

Validate response names against [RESPONSE] blocks.

parameter $\mathbf{z}$

Mesh coordinates along the $\mathrm{Z}$ axis.

Units $\mathrm{cm}$

Type monotonically increasing list with at least two values (each element is a real number)

\subsection{5 [TALLY][CELL]}

Geometry cells and unions of cells are tallied using a hash table, allowing constant-time scaling with respect to the number of total cells being tallied.

The recommended way to tally multiple particle spectra in the same cell is to use a single tally.

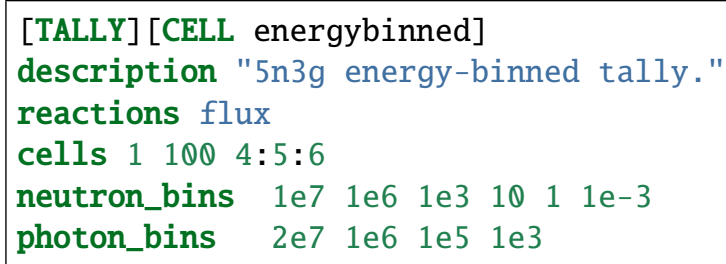

command cells

Generate 'union_cells' and 'union_lengths' from colon-separated unions.

Creates union_cells

Creates union_lengths

parameter cycles

The kcode problem phase in which this tally is active. 


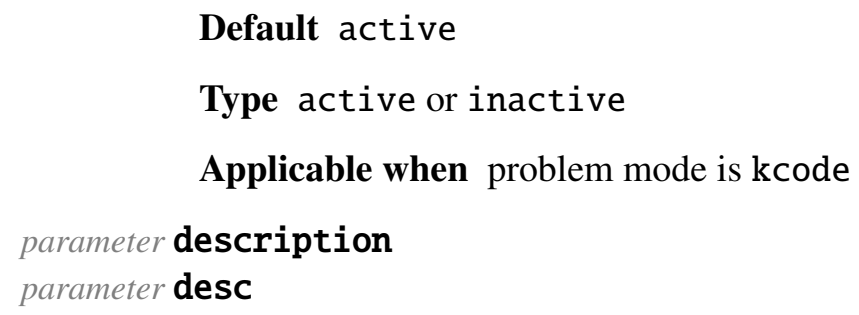

Default active

Type active or inactive

Applicable when problem mode is kcode

parameter description

parameter desc

Optional longer descriptive string.

Default ' '

Type string

database [FILTERS]

Tally filtering options. See [TALLY][MESH][FILTERS] (page 161).

\section{Optional}

parameter macro_mt

ENDF reactions for partial macroscopic cross section tallying.

Default ---

Type list of MT numbers or names (each element is a MT number or name (e.g., N_GAMMA, 102))

Applicable when physics is $\mathrm{CE}$

parameter macro_mt_zaid

Nuclide IDs for partial macroscopic cross section tallying.

Default ---

Type list in which each element is a nuclide specifier (e.g., U-235, 92235, u235, u-235m1)

Applicable when physics is CE

parameter macro_rxn

Special reactions for macroscopic cross section tallying.

Default ---

Type list in which each element is a flux, pos_partial_current_x, neg_partial_current_x, pos_partial_current_y, neg_partial_current_y, pos_partial_current_z, neg_partial_current_z, total, absorption, scattering, transfer_1n, transfer_2n, transfer_3n, transfer_4n, fission, nu_fission, kappa_sigma, kappa_sigma_f, or kappa_sigma_c

Applicable when physics is CE

parameter macro_rxn_zaid

Nuclide IDs for macroscopic 'special reaction' cross section tallying.

Default ---

Type list in which each element is a nuclide specifier (e.g., U-235, 92235, u235, u-235m1)

Applicable when physics is CE 
parameter micro_mt

ENDF reactions for microscopic cross section tallying.

Default ---

Type list of MT numbers or names (each element is a MT number or name (e.g., N_GAMMA, 102))

Applicable when physics is $\mathrm{CE}$

postprocessor

The parameters micro_mt_zaid and micro_mt must have the same length.

Applicability physics is $\mathrm{CE}$

parameter micro_mt_zaid

Nuclide IDs for microscopic cross section tallying.

Default ---

Type list in which each element is a nuclide specifier (e.g., U-235, 92235, u235, u-235m1)

Applicable when physics is CE

parameter micro_rxn

Special reactions for microscopic cross section tallying.

Default ---

Type list in which each element is a flux, pos_partial_current_x, neg_partial_current_x, pos_partial_current_y, neg_partial_current_y, pos_partial_current_z, neg_partial_current_z, total, absorption, scattering, transfer_1n, transfer_2n, transfer_3n, transfer_4n, fission, nu_fission, kappa_sigma, kappa_sigma_f, or kappa_sigma_c

Applicable when physics is $\mathrm{CE}$

postprocessor

The parameters micro_rxn_zaid and micro_rxn must have the same length.

Applicability physics is CE

parameter micro_rxn_zaid

Nuclide IDs for microscopic 'special reaction' cross section tallying.

Default ---

Type list in which each element is a nuclide specifier (e.g., U-235, 92235, u235, u-235m1)

Applicable when physics is CE

deprecated micro_zaid

Deprecated entry micro_zaid has been renamed to micro_mt_zaid.

The micro_zaid and micro_mt parameters are used to tally microscopic reaction rates for nuclides in the material being tallied. That is, if the user requests reaction rates for tungsten absorption in a cell that has pure water, the tally result for that cell will be zero. This allows the user (for example) to input trace nuclides in depletion calculations and only have nonzero reaction rates when the transport nuclide density is nonzero.

Example: 


$\begin{aligned} \text { micro_zaid } & \text { :micro_mt } \\ 1001 & \text { N_TOTAL } \\ 8016 & \text { N_TOTAL } \\ 8016 & \text { N_ELASTIC } \\ 8016 & \text { N_INELASTIC }\end{aligned}$

Update to micro_mt_zaid

parameter $\mathbf{m t}$

Additional macroscopic reaction rates to tally.

Default ---

Type list of MT numbers or names (each element is a MT number or name (e.g., N_GAMMA, 102))

Applicable when physics is $\mathrm{CE}$

parameter name

Short title or label for the tally.

Type string without special characters

parameter neutron_bins

parameter nbins

Energy bin boundaries for neutrons.

Default ---

Units eV

Type nonnegative floats in decreasing order (each element is a non-negative real number)

parameter normalization

Constant multiplicative factor to apply to tally results.

Default 1.0

Type positive real number

parameter photon_bins

parameter $\mathbf{p b i n s}$

Energy bin boundaries for photons.

Default ---

Units $\mathrm{eV}$

Type nonnegative floats in decreasing order (each element is a non-negative real number)

parameter reactions

parameter $\mathbf{r x n}$

Reactions to calculate for this tally. See reactions (page 119) in [TALLY][MESH].

parameter responses

parameter resp

Responses for this tally. 


\section{Default ---}

Type list in which each element is a string

postprocessor

Validate response names against [RESPONSE] blocks.

parameter union_cells(advanced)

Flattened list of cells in each union.

Type list of cell names (each element is a string)

parameter union_lengths (advanced)

Number of cells per union in the above list.

Type list in which each element is a integer

\subsection{6 [TALLY][VERA]}

If using a VERA model input, this will create a cell tally for the outermost cell of the vessel, which can then be optimized for when running in hybrid mode.

\section{parameter description}

parameter desc

Optional longer descriptive string.

\section{Default ' '}

Type string

\section{parameter name}

Short title or label for the tally.

Type string without special characters

parameter normalization

Constant multiplicative factor to apply to tally results.

\section{Default 1.0}

Type positive real number

\section{parameter reactions}

parameter $\mathbf{r x n}$

Reactions to calculate for this tally.

\section{Default flux}

Type list in which each element is a flux, pos_partial_current_x, neg_partial_current_x,pos_partial_current_y, neg_partial_current_y, pos_partial_current_z, neg_partial_current_z, total, absorption, scattering, transfer_1n, transfer_2n, transfer_3n, transfer_4n, fission, nu_fission, kappa_sigma, kappa_sigma_f, or kappa_sigma_c 


\subsection{7 [TALLY][SHADOW]}

"Shadow" path length tallies track through a secondary geometry during normal transport. Particles do not interact with this "shadow geometry," but they are tallied spatially over the cells in the shadow geometry. The shadow geometry is defined by an external Geometria/GG input file, distinct from the primary tracking geometry.

During transport, a fast test is performed at the beginning of every particle track to determine whether the track intersects with the bounding box of the geometry. If so, the particle's location inside the shadow geometry is determined, distances to boundaries are calculated, and the particle's track is tallied on top of the shadow geometry cells.

Every cell in the shadow geometry is tallied; however, due to the underlying geometry implementation, some cells will never receive a score. For example, a "cell" representing the outside of the universe is always created, but since the tally is not accumulated if the particle is outside the shadow geometry, that cell is never scored.

\section{parameter cycles}

The kcode problem phase in which this tally is active.

Default active

Type active or inactive

Applicable when problem mode is kcode

\section{parameter description}

parameter desc

Optional longer descriptive string.

Default ' '

Type string

command input

Generate a Geometria XML representation from an .gg.omn input.

Creates xml_path

parameter macro_mt

ENDF reactions for partial macroscopic cross section tallying.

Default ---

Type list of MT numbers or names (each element is a MT number or name (e.g., N_GAMMA, 102))

Applicable when physics is CE

parameter macro_mt_zaid

Nuclide IDs for partial macroscopic cross section tallying.

Default ---

Type list in which each element is a nuclide specifier (e.g., U-235, 92235, u235, u-235m1)

Applicable when physics is CE 
parameter macro_rxn

Special reactions for macroscopic cross section tallying.

Default ---

Type list in which each element is a flux, pos_partial_current_x, neg_partial_current_x, pos_partial_current_y, neg_partial_current_y, pos_partial_current_z, neg_partial_current_z, total, absorption, scattering, transfer_1n, transfer_2n, transfer_3n, transfer_4n, fission, nu_fission, kappa_sigma, kappa_sigma_f, or kappa_sigma_c

Applicable when physics is CE

parameter macro_rxn_zaid

Nuclide IDs for macroscopic 'special reaction' cross section tallying.

Default ---

Type list in which each element is a nuclide specifier (e.g., U-235, 92235, u235, u-235m1)

Applicable when physics is CE

parameter micro_mt

ENDF reactions for microscopic cross section tallying.

Default ---

Type list of MT numbers or names (each element is a MT number or name (e.g., N_GAMMA, 102))

Applicable when physics is $\mathrm{CE}$

postprocessor

The parameters micro_mt_zaid and micro_mt must have the same length.

Applicability physics is $\mathrm{CE}$

parameter micro_mt_zaid

Nuclide IDs for microscopic cross section tallying.

Default ---

Type list in which each element is a nuclide specifier (e.g., U-235, 92235, u235, u-235m1)

Applicable when physics is $\mathrm{CE}$

parameter micro_rxn

Special reactions for microscopic cross section tallying.

Default ---

Type list in which each element is a flux, pos_partial_current_x, neg_partial_current_x, pos_partial_current_y, neg_partial_current_y, pos_partial_current_z, neg_partial_current_z, total, absorption, scattering, transfer_1n, transfer_2n, transfer_3n, transfer_4n, fission, nu_fission, kappa_sigma, kappa_sigma_f, or kappa_sigma_c

Applicable when physics is CE 
postprocessor

The parameters micro_rxn_zaid and micro_rxn must have the same length.

Applicability physics is CE

parameter micro_rxn_zaid

Nuclide IDs for microscopic 'special reaction' cross section tallying.

Default ---

Type list in which each element is a nuclide specifier (e.g., U-235, 92235, u235, u-235m1)

Applicable when physics is CE

deprecated micro_zaid

Deprecated entry micro_zaid has been renamed to micro_mt_zaid.

Update to micro_mt_zaid

parameter $\mathbf{m t}$

Additional macroscopic reaction rates to tally.

Default ---

Type list of MT numbers or names (each element is a MT number or name (e.g., N_GAMMA, 102))

Applicable when physics is $\mathrm{CE}$

parameter name

Short title or label for the tally.

Type string without special characters

parameter neutron_bins

parameter nbins

Energy bin boundaries for neutrons.

Default ---

Units $\mathrm{eV}$

Type nonnegative floats in decreasing order (each element is a non-negative real number) parameter normalization

Constant multiplicative factor to apply to tally results.

Default 1.0

Type positive real number

parameter photon_bins

parameter $\mathbf{p b i n s}$

Energy bin boundaries for photons.

Default ---

Units $\mathrm{eV}$

Type nonnegative floats in decreasing order (each element is a non-negative real number) 
parameter reactions

parameter $\mathbf{r x n}$

Reactions to calculate for this tally.

Default flux

Type list in which each element is a flux, pos_partial_current_x, neg_partial_current_x, pos_partial_current_y, neg_partial_current_y, pos_partial_current_z, neg_partial_current_z, total, absorption, scattering, transfer_1n, transfer_2n, transfer_3n, transfer_4n, fission, nu_fission, kappa_sigma, kappa_sigma_f, or kappa_sigma_c

parameter responses

parameter resp

Responses for this tally.

\section{Default ---}

Type list in which each element is a string

postprocessor

Validate response names against [RESPONSE] blocks.

parameter $\mathbf{x m l}$ _path (advanced)

Path to the Geometria XML input file.

Type file path for reading (extension '. $x \mathrm{ml}^{\prime}$ ')

\subsection{8 [TALLY][MICRO]}

The "micro" tally is primarily used for depletion coupling. It calculates material-averaged, flux-weighted cross sections for multiple materials, nuclides, and reactions. The user specifies a list of materials to tally, as well as a list of nuclide/reaction pairs. During the tally setup phase in Shift, a list is constructed of all the possible nuclide/reaction pairs in each mix table, and nuclides or reactions that are absent in each mixture are elided.

Note that the volumes of all cells that contain the given materials must be specified. If any of these are absent, an error will be issued that enumerates the needed cells with missing volumes.

If no particles are sampled in one of the requested materials, the flux is zero and the microscopic cross section will be set to the sentinel flag of -1 .

\section{parameter description \\ parameter desc}

Optional longer descriptive string.

\section{Default ' '}

Type string

parameter materials

parameter mats

Materials in which to tally.

Type list in which each element is a non-empty string

postprocessor

At least one micro_mt_zaid:micro_mt pair must be set. 
parameter micro_mt

ENDF reactions for microscopic cross section tallying.

Default ---

Type list of MT numbers or names (each element is a MT number or name (e.g., N_GAMMA, 102))

Applicable when physics is $\mathrm{CE}$

postprocessor

The parameters micro_mt_zaid and micro_mt must have the same length.

Applicability physics is CE

parameter micro_mt_zaid

Nuclide IDs for microscopic cross section tallying.

Default ---

Type list in which each element is a nuclide specifier (e.g., U-235, 92235, u235, u-235m1)

Applicable when physics is $\mathrm{CE}$

parameter micro_rxn

Special reactions for microscopic cross section tallying.

Default ---

Type list in which each element is a flux, pos_partial_current_x, neg_partial_current_x,pos_partial_current_y, neg_partial_current_y, pos_partial_current_z, neg_partial_current_z, total, absorption, scattering, transfer_1n, transfer_2n, transfer_3n, transfer_4n, fission, nu_fission, kappa_sigma, kappa_sigma_f, or kappa_sigma_c

Applicable when physics is $\mathrm{CE}$

postprocessor

The parameters micro_rxn_zaid and micro_rxn must have the same length.

Applicability physics is CE

parameter micro_rxn_zaid

Nuclide IDs for microscopic 'special reaction' cross section tallying.

Default ---

Type list in which each element is a nuclide specifier (e.g., U-235, 92235, u235, u-235m1)

Applicable when physics is CE

deprecated micro_zaid

Deprecated entry micro_zaid has been renamed to micro_mt_zaid.

Update to micro_mt_zaid

parameter name

Short title or label for the tally.

Type string without special characters 


\section{parameter neutron_bins \\ parameter nbins}

Energy bin boundaries for neutrons.

Default ---

Units eV

Type nonnegative floats in decreasing order (each element is a non-negative real number) parameter normalization

Constant multiplicative factor to apply to tally results.

\section{Default 1.0}

Type positive real number

parameter photon_bins

parameter $\mathbf{p b i n s}$

Energy bin boundaries for photons.

Default -- -

Units eV

Type nonnegative floats in decreasing order (each element is a non-negative real number)

\subsection{9 [TALLY][DIAGNOSTIC]}

Table 23: Available types for the [DIAGNOSTIC] database

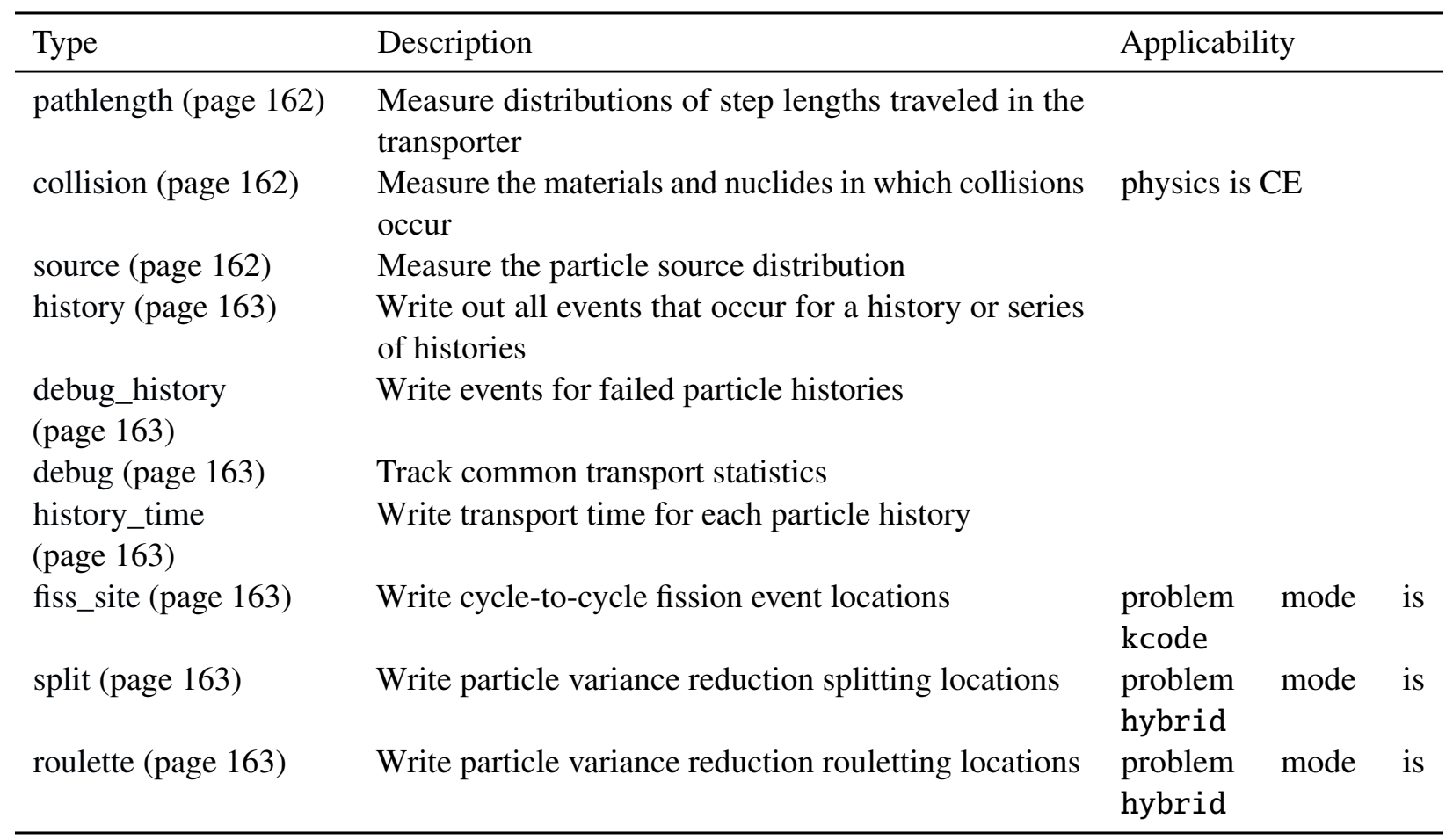




\subsubsection{0 [TALLY][SWORD]}

When using a SWORD model input, this will import SWORD tally cells into the problem.

parameter cycles

The kcode problem phase in which this tally is active.

Default active

Type active or inactive

Applicable when problem mode is kcode

parameter description

parameter desc

Optional longer descriptive string.

Default ' '

Type string

parameter macro_mt

ENDF reactions for partial macroscopic cross section tallying.

Default ---

Type list of MT numbers or names (each element is a MT number or name (e.g., N_GAMMA, 102))

Applicable when physics is CE

parameter macro_mt_zaid

Nuclide IDs for partial macroscopic cross section tallying.

Default ---

Type list in which each element is a nuclide specifier (e.g., U-235, 92235, u235, u-235m1)

Applicable when physics is CE

parameter macro_rxn

Special reactions for macroscopic cross section tallying.

Default ---

Type list in which each element is a flux, pos_partial_current_x, neg_partial_current_x, pos_partial_current_y, neg_partial_current_y, pos_partial_current_z, neg_partial_current_z, total, absorption, scattering, transfer_1n, transfer_2n, transfer_3n, transfer_4n, fission, nu_fission, kappa_sigma, kappa_sigma_f, or kappa_sigma_c

Applicable when physics is $\mathrm{CE}$

parameter macro_rxn_zaid

Nuclide IDs for macroscopic 'special reaction' cross section tallying.

Default ---

Type list in which each element is a nuclide specifier (e.g., U-235, 92235, u235, u-235m1)

Applicable when physics is CE 
parameter micro_mt

ENDF reactions for microscopic cross section tallying.

Default ---

Type list of MT numbers or names (each element is a MT number or name (e.g., N_GAMMA, 102))

Applicable when physics is $\mathrm{CE}$

postprocessor

The parameters micro_mt_zaid and micro_mt must have the same length.

Applicability physics is CE

parameter micro_mt_zaid

Nuclide IDs for microscopic cross section tallying.

Default ---

Type list in which each element is a nuclide specifier (e.g., U-235, 92235, u235, u-235m1)

Applicable when physics is CE

parameter micro_rxn

Special reactions for microscopic cross section tallying.

Default ---

Type list in which each element is a flux, pos_partial_current_x, neg_partial_current_x, pos_partial_current_y, neg_partial_current_y, pos_partial_current_z, neg_partial_current_z, total, absorption, scattering, transfer_1n, transfer_2n, transfer_3n, transfer_4n, fission, nu_fission, kappa_sigma, kappa_sigma_f, or kappa_sigma_c

Applicable when physics is CE

postprocessor

The parameters micro_rxn_zaid and micro_rxn must have the same length.

Applicability physics is CE

parameter micro_rxn_zaid

Nuclide IDs for microscopic 'special reaction' cross section tallying.

Default ---

Type list in which each element is a nuclide specifier (e.g., U-235, 92235, u235, u-235m1)

Applicable when physics is $\mathrm{CE}$

deprecated micro_zaid

Deprecated entry micro_zaid has been renamed to micro_mt_zaid.

Update to micro_mt_zaid

parameter $\mathbf{m t}$

Additional macroscopic reaction rates to tally.

Default --- 
Type list of MT numbers or names (each element is a MT number or name (e.g., N_GAMMA, 102))

Applicable when physics is CE

\section{parameter name}

Short title or label for the tally.

Type string without special characters

parameter neutron_bins

parameter nbins

Energy bin boundaries for neutrons.

Default ---

Units eV

Type nonnegative floats in decreasing order (each element is a non-negative real number) parameter normalization

Constant multiplicative factor to apply to tally results.

Default 1.0

Type positive real number

parameter photon_bins

parameter pbins

Energy bin boundaries for photons.

Default ---

Units eV

Type nonnegative floats in decreasing order (each element is a non-negative real number)

parameter reactions

parameter $\mathbf{r x n}$

Reactions to calculate for this tally.

Default flux

Type list in which each element is a flux, pos_partial_current_x, neg_partial_current_x, pos_partial_current_y, neg_partial_current_y, pos_partial_current_z, neg_partial_current_z, total, absorption, scattering, transfer_1n, transfer_2n, transfer_3n, transfer_4n, fission, nu_fission, kappa_sigma, kappa_sigma_f, or kappa_sigma_c

parameter responses

parameter resp

Responses for this tally.

Default ---

Type list in which each element is a string

postprocessor

Validate response names against [RESPONSE] blocks. 


\subsubsection{1 [TALLY][SENSITIVITY]}

The sensitivity tally calculates eigenvalue sensitivities to cross sections and to ratios of cross sections.

parameter binned

Store fully binned tallies.

\section{Default False}

Type boolean

parameter constrained_chi

Calculate constrained chi values.

\section{Default True}

Type boolean

parameter covariance

Use the covariance between tallies and the normalization factor when calculating final sensitivity variance.

\section{Default True}

Type boolean

parameter cycles_per_batch

Number of kcode cycles in a statistical batch.

\section{Default 1}

Type positive integer

parameter eint

Store energy-integrated tallies.

Default False

Type boolean

parameter latent_generations

parameter $\mathbf{c f p}$

Number of latent generations.

\section{Default 5}

Type positive integer

Applicable when method is ifp or gpt

parameter materials

parameter mats

Materials in which to tally sensitivities.

A single asterisk (as a wild card) will cause sensitivities for all materials to be tallied:

materials *

\section{Default $' *$ '}


Type list in which each element is a non-empty string

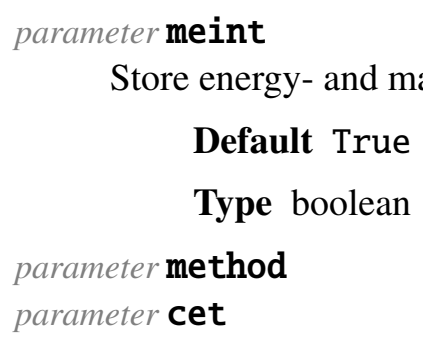

\section{Default True}

Type boolean

parameter method

parameter cet

Store energy- and material-integrated tallies.

Sensitivity coefficient calculation mode.

Default clutch

Type clutch, ifp, or gpt

parameter mint

Store material-integrated tallies.

Default True

Type boolean

parameter $\mathbf{m t s}$

parameter $\mathbf{m t}$

MT reactions to tally.

Default -1

Type list in which each element is a MT name or ZAID, or '*' for default reactions

parameter neutron_bins

parameter nbins

Energy bin boundaries for neutrons.

Default ---

Units eV

Type nonnegative floats in decreasing order (each element is a non-negative real number)

parameter nuclides

parameter nucl

Nuclide IDs for microscopic cross section tallying.

A single asterisk (as a wild card) will cause sensitivities for all nuclides to be tallied:

nuclides *

Default -1

Type list in which each element is a Nuclide name or ZAID, or '*' for all nuclides

sublist [RATIO]

Define a ratio of two responses to calculate. See [TALLY][SENSITIVITY][RATIO] (page 165).

Applicable when method is gpt

sublist [RESPONSE]

Define a sensitivity response. See [TALLY][SENSITIVITY][RESPONSE] (page 164).

Applicable when method is gpt 


\subsubsection{2 [TALLY][NODAL]}

Tally integral quantities on a Cartesian mesh for nodal calculations.

parameter birth_spectrum

Tally birth spectrum of particles.

Type boolean

parameter cycles

The kcode problem phase in which this tally is active.

Default active

Type active or inactive

Applicable when problem mode is kcode

parameter delta_x

Length of mesh tally cell on the $\mathrm{X}$ axis.

Units $\mathrm{cm}$

Type positive real number

Applicable when type is global

parameter delta_y

Length of mesh tally cell on the Y axis.

Units $\mathrm{cm}$

Type positive real number

Applicable when type is global

parameter delta_z

Length of mesh tally cell on the $\mathrm{Z}$ axis.

Units $\mathrm{cm}$

Type positive real number

Applicable when type is global

command deltas

Expand into parameters delta_x, delta_y, and delta_z.

Creates delta_x

Creates delta_y

Creates delta_z

parameter description

parameter desc

Optional longer descriptive string.

Default ' '

Type string 
parameter diffusion_coefficients

Tally diffusion coefficients.

Type boolean

parameter eddington_factors

Tally the eddington factors.

Type boolean

database [FILTERS]

Tally filtering options. See [TALLY][MESH][FILTERS] (page 161).

Optional

parameter macro_mt

ENDF reactions for partial macroscopic cross section tallying.

Default ---

Type list of MT numbers or names (each element is a MT number or name (e.g., N_GAMMA, 102))

Applicable when physics is CE

parameter macro_mt_zaid

Nuclide IDs for partial macroscopic cross section tallying.

Default ---

Type list in which each element is a nuclide specifier (e.g., U-235, 92235, u235, u-235m1)

Applicable when physics is $\mathrm{CE}$

\section{parameter macro_rxn}

Special reactions for macroscopic cross section tallying.

\section{Default ---}

Type list in which each element is a flux, pos_partial_current_x, neg_partial_current_x,pos_partial_current_y, neg_partial_current_y, pos_partial_current_z, neg_partial_current_z, total, absorption, scattering, transfer_1n, transfer_2n, transfer_3n, transfer_4n, fission, nu_fission, kappa_sigma, kappa_sigma_f, or kappa_sigma_c

Applicable when physics is $\mathrm{CE}$

parameter macro_rxn_zaid

Nuclide IDs for macroscopic 'special reaction' cross section tallying.

\section{Default ---}

Type list in which each element is a nuclide specifier (e.g., U-235, 92235, u235, u-235m1)

Applicable when physics is $\mathrm{CE}$

parameter mean_transfer_angle

Tally mean transfer angle.

Type boolean 
parameter micro_mt

ENDF reactions for microscopic cross section tallying.

Default ---

Type list of MT numbers or names (each element is a MT number or name (e.g., N_GAMMA, 102))

Applicable when physics is $\mathrm{CE}$

postprocessor

The parameters micro_mt_zaid and micro_mt must have the same length.

Applicability physics is CE

parameter micro_mt_zaid

Nuclide IDs for microscopic cross section tallying.

Default ---

Type list in which each element is a nuclide specifier (e.g., U-235, 92235, u235, u-235m1)

Applicable when physics is CE

parameter micro_rxn

Special reactions for microscopic cross section tallying.

Default ---

Type list in which each element is a flux, pos_partial_current_x, neg_partial_current_x, pos_partial_current_y, neg_partial_current_y, pos_partial_current_z, neg_partial_current_z, total, absorption, scattering, transfer_1n, transfer_2n, transfer_3n, transfer_4n, fission, nu_fission, kappa_sigma, kappa_sigma_f, or kappa_sigma_c

Applicable when physics is CE

postprocessor

The parameters micro_rxn_zaid and micro_rxn must have the same length.

Applicability physics is CE

parameter micro_rxn_zaid

Nuclide IDs for microscopic 'special reaction' cross section tallying.

Default ---

Type list in which each element is a nuclide specifier (e.g., U-235, 92235, u235, u-235m1)

Applicable when physics is $\mathrm{CE}$

deprecated micro_zaid

Deprecated entry micro_zaid has been renamed to micro_mt_zaid.

Update to micro_mt_zaid

parameter $\mathbf{m t}$

Additional macroscopic reaction rates to tally.

Default --- 
Type list of MT numbers or names (each element is a MT number or name (e.g., N_GAMMA, 102))

Applicable when physics is CE

\section{parameter name}

Short title or label for the tally.

Type string without special characters

parameter neutron_bins

parameter nbins

Energy bin boundaries for neutrons.

Default ---

Units eV

Type nonnegative floats in decreasing order (each element is a non-negative real number) parameter normalization

Constant multiplicative factor to apply to tally results.

Default 1.0

Type positive real number

parameter photon_bins

parameter pbins

Energy bin boundaries for photons.

Default ---

Units eV

Type nonnegative floats in decreasing order (each element is a non-negative real number)

parameter reactions

parameter $\mathbf{r x n}$

Reactions to calculate for this tally.

Default flux

Type list in which each element is a flux, pos_partial_current_x, neg_partial_current_x, pos_partial_current_y, neg_partial_current_y, pos_partial_current_z, neg_partial_current_z, total, absorption, scattering, transfer_1n, transfer_2n, transfer_3n, transfer_4n, fission, nu_fission, kappa_sigma, kappa_sigma_f, or kappa_sigma_c

parameter responses

parameter resp

Responses for this tally.

Default ---

Type list in which each element is a string

postprocessor

Validate response names against [RESPONSE] blocks. 
parameter transfer_matrices

Tally the P0 and P1 transfer matrices.

Type boolean

parameter type

Type of mesh tally.

Default grid

Type grid or global

parameter $\mathbf{x}$

Tally grid coordinates along the $\mathrm{X}$ axis.

Units $\mathrm{cm}$

Type monotonically increasing list with at least two values (each element is a real number)

Applicable when type is grid

parameter $\mathbf{y}$

Tally grid coordinates along the $\mathrm{Y}$ axis.

Units $\mathrm{cm}$

Type monotonically increasing list with at least two values (each element is a real number)

Applicable when type is grid

parameter $\mathbf{z}$

Tally grid coordinates along the $\mathrm{Z}$ axis.

Units $\mathrm{cm}$

Type monotonically increasing list with at least two values (each element is a real number)

Applicable when type is grid

\subsubsection{3 [TALLY][HEXNODAL]}

Tally integral quantities on a hexagonal mesh for nodal calculations.

parameter apothem

Distance from hex center to face center.

Units $\mathrm{cm}$

Type positive real number

parameter birth_spectrum

Tally birth spectrum of particles.

Type boolean

parameter center_hex

$(\mathrm{x}, \mathrm{y})$ centroid of center hex.

Units $\mathrm{cm}$

Type length-2 float vector (each element is a real number) 
parameter cycles

The kcode problem phase in which this tally is active.

Default active

Type active or inactive

Applicable when problem mode is kcode

parameter description

parameter desc

Optional longer descriptive string.

Default ' '

Type string

parameter diffusion_coefficients

Tally diffusion coefficients.

Type boolean

parameter eddington_factors

Tally the eddington factors.

Type boolean

database [FILTERS]

Tally filtering options. See [TALLY][MESH][FILTERS] (page 161).

Optional

parameter macro_mt

ENDF reactions for partial macroscopic cross section tallying.

\section{Default ---}

Type list of MT numbers or names (each element is a MT number or name (e.g., N_GAMMA, 102))

Applicable when physics is CE

parameter macro_mt_zaid

Nuclide IDs for partial macroscopic cross section tallying.

Default ---

Type list in which each element is a nuclide specifier (e.g., U-235, 92235, u235, u-235m1)

Applicable when physics is CE

parameter macro_rxn

Special reactions for macroscopic cross section tallying.

Default ---

Type list in which each element is a flux, pos_partial_current_x, neg_partial_current_x,pos_partial_current_y, neg_partial_current_y, pos_partial_current_z, neg_partial_current_z, total, absorption, scattering, transfer_1n, transfer_2n, transfer_3n, transfer_4n, fission, nu_fission, kappa_sigma, kappa_sigma_f, or kappa_sigma_c 
Applicable when physics is CE

parameter macro_rxn_zaid

Nuclide IDs for macroscopic 'special reaction' cross section tallying.

Default ---

Type list in which each element is a nuclide specifier (e.g., U-235, 92235, u235, u-235m1)

Applicable when physics is CE

parameter mean_transfer_angle

Tally mean transfer angle.

Type boolean

parameter micro_mt

ENDF reactions for microscopic cross section tallying.

Default ---

Type list of MT numbers or names (each element is a MT number or name (e.g., N_GAMMA, 102))

Applicable when physics is CE

postprocessor

The parameters micro_mt_zaid and micro_mt must have the same length.

Applicability physics is CE

parameter micro_mt_zaid

Nuclide IDs for microscopic cross section tallying.

Default ---

Type list in which each element is a nuclide specifier (e.g., U-235, 92235, u235, u-235m1)

Applicable when physics is $\mathrm{CE}$

parameter micro_rxn

Special reactions for microscopic cross section tallying.

Default ---

Type list in which each element is a flux, pos_partial_current_x, neg_partial_current_x,pos_partial_current_y, neg_partial_current_y, pos_partial_current_z, neg_partial_current_z, total, absorption, scattering, transfer_1n, transfer_2n, transfer_3n, transfer_4n, fission, nu_fission, kappa_sigma, kappa_sigma_f, or kappa_sigma_c

Applicable when physics is $\mathrm{CE}$

postprocessor

The parameters micro_rxn_zaid and micro_rxn must have the same length.

Applicability physics is CE

parameter micro_rxn_zaid

Nuclide IDs for microscopic 'special reaction' cross section tallying. 


\section{Default ---}

Type list in which each element is a nuclide specifier (e.g., U-235, 92235, u235, u-235m1)

Applicable when physics is CE

deprecated micro_zaid

Deprecated entry micro_zaid has been renamed to micro_mt_zaid.

Update to micro_mt_zaid

parameter $\mathbf{m t}$

Additional macroscopic reaction rates to tally.

Default ---

Type list of MT numbers or names (each element is a MT number or name (e.g., N_GAMMA, 102))

Applicable when physics is CE

parameter name

Short title or label for the tally.

Type string without special characters

parameter neutron_bins

parameter nbins

Energy bin boundaries for neutrons.

\section{Default ---}

Units $\mathrm{eV}$

Type nonnegative floats in decreasing order (each element is a non-negative real number) parameter normalization

Constant multiplicative factor to apply to tally results.

\section{Default 1.0}

Type positive real number

parameter num_rings

Number of rings around the central hex.

Type non-negative integer

parameter photon_bins

parameter $\mathbf{p b i n s}$

Energy bin boundaries for photons.

\section{Default ---}

Units eV

Type nonnegative floats in decreasing order (each element is a non-negative real number)

parameter reactions

parameter $\mathbf{r x n}$

Reactions to calculate for this tally. 


\section{Default flux}

Type list in which each element is a flux, pos_partial_current_x, neg_partial_current_x, pos_partial_current_y, neg_partial_current_y, pos_partial_current_z, neg_partial_current_z, total, absorption, scattering, transfer_1n, transfer_2n, transfer_3n, transfer_4n, fission, nu_fission, kappa_sigma, kappa_sigma_f, or kappa_sigma_c

parameter responses

parameter resp

Responses for this tally.

Default ---

Type list in which each element is a string

postprocessor

Validate response names against [RESPONSE] blocks.

parameter transfer_matrices

Tally the P0 and P1 transfer matrices.

Type boolean

parameter $\mathbf{z}$

Mesh coordinates along the $\mathrm{Z}$ axis.

Units $\mathrm{cm}$

Type monotonically increasing list with at least two values (each element is a real number)

\subsubsection{4 [TALLY][MIGRATION_AREA]}

Tally particle migration area on a Cartesian mesh.

parameter $\mathbf{d e l t} \mathbf{t a} \_\mathbf{x}$

Length of mesh tally cell on the $\mathrm{X}$ axis.

Units $\mathrm{cm}$

Type positive real number

Applicable when type is global

parameter delta_y

Length of mesh tally cell on the Y axis.

Units $\mathrm{cm}$

Type positive real number

Applicable when type is global

parameter delta_z

Length of mesh tally cell on the $\mathrm{Z}$ axis.

Units $\mathrm{cm}$

Type positive real number 


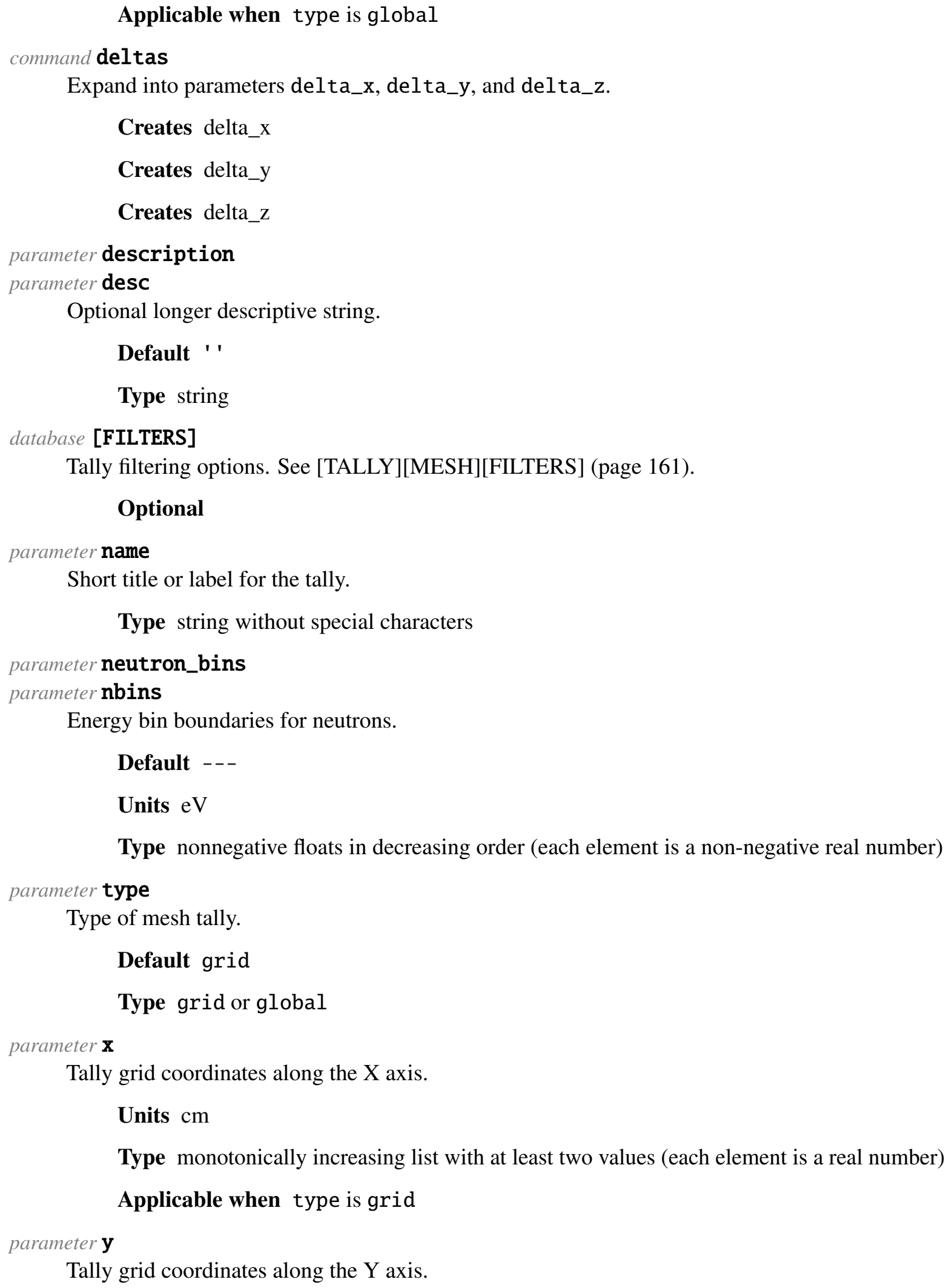

Optional

parameter name

Short title or label for the tally.

Type string without special characters

parameter neutron_bins

parameter $\mathbf{n b i n s}$

Energy bin boundaries for neutrons.

\section{Default ---}

Units eV

Type nonnegative floats in decreasing order (each element is a non-negative real number)

parameter type

Type of mesh tally.

Default grid

Type grid or global

parameter $\mathbf{x}$

Tally grid coordinates along the $\mathrm{X}$ axis.

Units $\mathrm{cm}$

Type monotonically increasing list with at least two values (each element is a real number)

\section{Applicable when type is grid}

parameter $\mathbf{y}$

Tally grid coordinates along the $\mathrm{Y}$ axis.

Units $\mathrm{cm}$

Type monotonically increasing list with at least two values (each element is a real number) 


\section{Applicable when type is grid}

parameter $\mathbf{Z}$

Tally grid coordinates along the $\mathrm{Z}$ axis.

Units $\mathrm{cm}$

Type monotonically increasing list with at least two values (each element is a real number)

Applicable when type is grid

\subsubsection{5 [TALLY][MEAN_SCATTER_ANGLE]}

Tally the mean scattering angle in geometry cells.

\section{command cells}

Generate 'union_cells' and 'union_lengths' from colon-separated unions.

Creates union_cells

Creates union_lengths

parameter description

parameter desc

Optional longer descriptive string.

\section{Default ' '}

Type string

parameter name

Short title or label for the tally.

Type string without special characters

parameter neutron_bins

parameter nbins

Energy bin boundaries for neutrons.

Default ---

Units eV

Type nonnegative floats in decreasing order (each element is a non-negative real number) parameter normalization

Constant multiplicative factor to apply to tally results.

Default 1.0

Type positive real number

parameter photon_bins

parameter $\mathbf{p b i n s}$

Energy bin boundaries for photons.

Default ---

Units $\mathrm{eV}$

Type nonnegative floats in decreasing order (each element is a non-negative real number) 
postprocessor

The parameters “neutron_bins and photon_bins ’ must not all be empty.

parameter union_cells (advanced)

Flattened list of cells in each union.

Type list of cell names (each element is a string)

parameter union_lengths (advanced)

Number of cells per union in the above list.

Type list in which each element is a integer

\subsubsection{6 [TALLY][SCATTERING_PROB_MATRIX]}

Tally the group-to-group scattering probability matrix in geometry cells.

\section{command cells}

Generate 'union_cells' and 'union_lengths' from colon-separated unions.

Creates union_cells

Creates union_lengths

parameter description

parameter desc

Optional longer descriptive string.

Default ' '

Type string

parameter name

Short title or label for the tally.

Type string without special characters

parameter neutron_bins

parameter nbins

Energy bin boundaries for neutrons.

\section{Default ---}

Units eV

Type nonnegative floats in decreasing order (each element is a non-negative real number) parameter normalization

Constant multiplicative factor to apply to tally results.

Default 1.0

Type positive real number

parameter photon_bins

parameter $\mathbf{p b i n s}$

Energy bin boundaries for photons.

Default --- 


\section{Units eV}

Type nonnegative floats in decreasing order (each element is a non-negative real number)

postprocessor

The parameters “neutron_bins and photon_bins ’ must not all be empty.

parameter union_cells (advanced)

Flattened list of cells in each union.

Type list of cell names (each element is a string)

parameter union_lengths (advanced)

Number of cells per union in the above list.

Type list in which each element is a integer

\subsubsection{7 [TALLY][BIRTH_SPECTRUM]}

Tally the energy distribution of particles at birth in geometry cells.

command cells

Generate 'union_cells' and 'union_lengths' from colon-separated unions.

Creates union_cells

Creates union_lengths

parameter description

parameter desc

Optional longer descriptive string.

Default ' '

Type string

parameter name

Short title or label for the tally.

Type string without special characters

parameter neutron_bins

parameter nbins

Energy bin boundaries for neutrons.

Default ---

Units $\mathrm{eV}$

Type nonnegative floats in decreasing order (each element is a non-negative real number)

parameter normalization

Constant multiplicative factor to apply to tally results.

Default 1.0

Type positive real number

parameter photon_bins 
parameter pbins

Energy bin boundaries for photons.

Default ---

Units eV

Type nonnegative floats in decreasing order (each element is a non-negative real number)

postprocessor

The parameters ' neutron_bins and photon_bins “ must not all be empty.

parameter union_cells (advanced)

Flattened list of cells in each union.

Type list of cell names (each element is a string)

parameter union_lengths (advanced)

Number of cells per union in the above list.

Type list in which each element is a integer

\subsubsection{8 [TALLY][SURFACE_CENSUS]}

The surface census tally records a particle's state (position, direction, type, energy) when crossing from the exiting cell to the target cell. The resulting data can be used for using a Shift calculation to generate a starting source for another transport code such as Geant 4 .

command cells

Map 'exiting_cells' to 'target_cells' from pairs or arrow-separated items.

Creates exiting_cells

Creates target_cells

parameter description

parameter desc

Optional longer descriptive string.

Default ' '

Type string

command exiting_cells

Generate 'union_exiting_cells' and 'union_exiting_lengths' from colon-separated unions.

Creates union_exiting_cells

Creates union_exiting_lengths

parameter kill_particle

Kill particle when tallied.

Default True

Type boolean

parameter name

Short title or label for the tally. 
Type string without special characters

database [TAGS]

Particle tagging options. See [TALLY][SURFACE_CENSUS][TAGS] (page 165).

Optional

command target_cells

Generate 'union_target_cells' and 'union_target_lengths' from colon-separated unions.

Creates union_target_cells

Creates union_target_lengths

parameter union_exiting_cells (advanced)

Flattened list of exiting cells in each union.

Type list of cell names (each element is a string)

parameter union_exiting_lengths(advanced)

Number of cells per union in the exiting surface list.

Type list in which each element is a integer

parameter union_target_cells(advanced)

Flattened list of target cells in each union.

Type list of cell names (each element is a string)

parameter union_target_lengths(advanced)

Number of cells per union in the target surface list.

Type list in which each element is a integer

\subsubsection{9 [TALLY][BATCH_MESH]}

Tally reaction rates using batch statistics (experimental!).

\section{parameter description \\ parameter desc}

Optional longer descriptive string.

Default ' '

Type string

parameter name

Short title or label for the tally.

Type string without special characters

parameter normalization

Constant multiplicative factor to apply to tally results.

Default 1.0

Type positive real number

database [OUTPUT]

Output method for the batch tallies. See [TALLY][BATCH_MESH][OUTPUT] (page 166). 


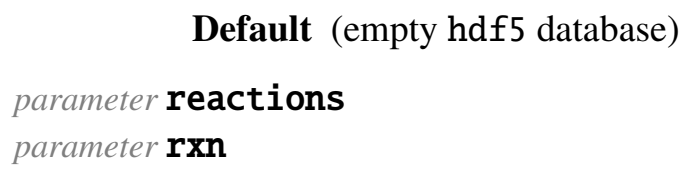

Default (empty hdf5 database)

parameter reactions

parameter $\mathbf{r x n}$

Reactions to calculate for this tally.

Default flux nu_fission

Type list in which each element is a flux, pos_partial_current_x, neg_partial_current_x, pos_partial_current_y, neg_partial_current_y, pos_partial_current_z, neg_partial_current_z, total, absorption, scattering, transfer_1n, transfer_2n, transfer_3n, transfer_4n, fission, nu_fission, kappa_sigma, kappa_sigma_f, or kappa_sigma_c

parameter $\mathbf{x}$

Mesh tally coordinates along the $\mathrm{X}$ axis.

Units $\mathrm{cm}$

Type monotonically increasing list with at least two values (each element is a real number)

parameter $\mathbf{y}$

Mesh tally coordinates along the $\mathrm{Y}$ axis.

Units $\mathrm{cm}$

Type monotonically increasing list with at least two values (each element is a real number)

parameter $\mathbf{z}$

Mesh tally coordinates along the $\mathrm{Z}$ axis.

Units $\mathrm{cm}$

Type monotonically increasing list with at least two values (each element is a real number)

\subsubsection{0 [TALLY][MESH][FILTERS]}

Tally filtering options.

parameter birth_cells

Only tallies particles born in the given cells.

Default ---

Type list in which each element is a string

parameter $\mathbf{b i r t h}$ _energy_range

parameter be_range

Lower and upper birth energy of particles to tally.

\section{Default ---}

Units $\mathrm{eV}$

Type monotonically increasing list (each element is a positive real number)

\section{parameter birth_matids}

Only tallies particles born in the given materials.

\section{Default ---}


Type list in which each element is a positive integer

parameter particle_type

parameter $\mathbf{p t}$

Only tallies particles of the given type.

Default ---

Type list in which each element is a particle type (n, neutron, $\mathrm{p}$, or photon)

parameter source_names

Only tallies particles born in the given sources.

Default ---

Type list in which each element is a string

\subsubsection{1 [TALLY][DIAGNOSTIC $=$ PATHLENGTH]}

The path length diagnostic produces a distribution of the path length traveled by a particle between events. Note that this is not the same as the true path length distribution (distance between collisions), as the events considered by Shift include material boundary crossings, problem boundary crossings, etc.

parameter event_bins

Lower bin boundaries for the number of events per history.

Type monotonically increasing list (each element is a non-negative integer)

parameter $\mathbf{p l}$ l_bins

Lower bin boundaries for the traversed path lengths in each event.

Type monotonically increasing list (each element is a positive real number)

\subsubsection{2 [TALLY][DIAGNOSTIC $=$ COLLISION]}

The collision diagnostic tallies the number of collisions per history as a function of material, nuclide, and reaction ID.

\subsubsection{3 [TALLY][DIAGNOSTIC $=$ SOURCE]}

This diagnostic tally is provided to calculate the source density binned into spatial cells. It also calculates the particle source density (i.e., binning $n(\vec{r})$ rather than $w n(\vec{r})$ ) to assist in the construction of biased sources.

parameter $\mathbf{x}$

Mesh tally coordinates along the $\mathrm{X}$ axis.

Units $\mathrm{cm}$

Type monotonically increasing list with at least two values (each element is a real number)

parameter $\mathbf{y}$

Mesh tally coordinates along the $\mathrm{Y}$ axis.

Units $\mathrm{cm}$

Type monotonically increasing list with at least two values (each element is a real number)

parameter $\mathbf{z}$

Mesh tally coordinates along the $\mathrm{Z}$ axis.

Units $\mathrm{cm}$

Type monotonically increasing list with at least two values (each element is a real number) 


\subsubsection{4 [TALLY][DIAGNOSTIC $=$ HISTORY]}

The history diagnostic tallies every event in a particle's lifetime. Currently, all particle histories are tallied, and only one processor writes the histories.

\section{parameter begin_history}

First history for which events will be saved.

Default 0

Type non-negative integer

parameter domain

Domain on which history events will be saved.

Default $\theta$

Type non-negative integer

parameter end_history

Last +1 history for which events will be saved.

Default $\theta$

Type non-negative integer

\subsubsection{5 [TALLY][DIAGNOSTIC=DEBUG_HISTORY]}

Write events for failed particle histories.

\subsubsection{6 [TALLY][DIAGNOSTIC=DEBUG]}

The debug diagnostic currently records the number of events per particle history, but it will be extended to provide other useful high-level debug information.

\subsubsection{7 [TALLY][DIAGNOSTIC=HISTORY_TIME]}

Write transport time for each particle history.

\subsubsection{8 [TALLY][DIAGNOSTIC=FISS_SITE]}

The fission site diagnostic records the locations where fission occurs for every kcode cycle provided by write_cycles. These are written to the

parameter $\mathbf{w r i t e}$ _cycles

parameter $\mathbf{w C}$

List of cycles in which to write the fission source.

Default ---

Type list in which each element is a non-negative integer

\subsubsection{9 [TALLY][DIAGNOSTIC $=$ SPLIT]}

Write particle variance reduction splitting locations.

\subsubsection{0 [TALLY][DIAGNOSTIC $=$ ROULETTE]}

Write particle variance reduction rouletting locations. 


\subsubsection{1 [TALLY][SENSITIVITY][RESPONSE]}

Define a sensitivity response.

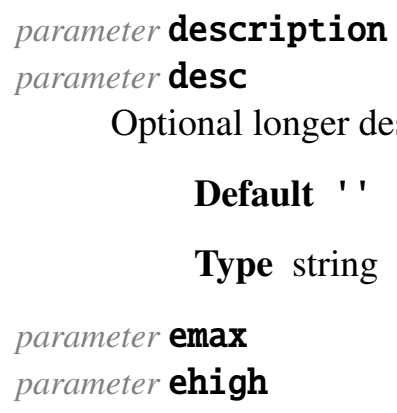

Default ' '

Type string

parameter emax

parameter ehigh

Optional longer descriptive string.

Upper energy threshold for this response.

\section{Optional}

Units $\mathrm{eV}$

Type positive real number

parameter emax_out

Upper exiting energy window for this response.

Optional

Units $\mathrm{eV}$

Type positive real number

parameter emin

parameter elow

Lower energy threshold for this response.

\section{Optional}

Units eV

Type positive real number

parameter emin_out

Lower exiting energy window for this response.

\section{Optional}

Units eV

Type positive real number

parameter materials

parameter mats

Material names to include in this response.

Default $' *$ '

Type list in which each element is a string

parameter name

Short title or label for the response. 
Type string without special characters

parameter nuclide

Nuclide to include in this response.

Type Nuclide name or ZAID, or '*' for all nuclides

parameter reaction

Nuclide reaction MTs to include in this response.

Default 18

Type MT number or name, or 0 for FLUX (e.g., N_GAMMA, 102)

\subsubsection{2 [TALLY][SENSITIVITY][RATIO]}

Define a ratio of two responses to calculate.

\section{parameter denom}

Denominator's response name.

Type string without special characters

parameter description

parameter desc

Optional longer descriptive string.

Default ' '

Type string

parameter name

Short title or label for the system response.

Type string without special characters

parameter numer

Numerator's response name.

Type string without special characters

\subsubsection{3 [TALLY][SURFACE_CENSUS][TAGS]}

Particle tagging options.

\section{parameter birth_angle \\ parameter brth_dir}

Tag each particle with its birth angle.

Default False

Type boolean

parameter $\mathbf{b i r t h}$ _cell_label

parameter $\mathbf{b r t h}$ _cell

Tag each particle with its birth cell label.

Default False

Type boolean 


\section{parameter $\mathbf{b i r t h}$ _energy \\ parameter $\mathbf{b r t h}$ _e}

Tag each particle with its birth energy.

Default False

Type boolean

parameter birth_mat_label

parameter brth_mat

Tag each particle with its birth material label.

Default False

Type boolean

parameter birth_position

parameter $\mathbf{b r t h}$ _pos

Tag particles with its birth position.

Default False

Type boolean

parameter source_name

parameter src_name

Tag each particle with the name of its originating source.

Default False

Type boolean

\subsubsection{4 [TALLY][BATCH_MESH][OUTPUT]}

Table 24: Available types for the [OUTPUT] database

\begin{tabular}{lll}
\hline Type & Description & Applicability \\
\hline $\begin{array}{l}\text { hdf5 (page 166) } \\
\text { adios (page 166) }\end{array}$ & Write to the standard serial HDF5 file & \\
\hline
\end{tabular}

\subsubsection{5 [TALLY][BATCH_MESH][OUTPUT=HDF5]}

Write to the standard serial HDF5 file.

\subsubsection{6 [TALLY][BATCH_MESH][OUTPUT=ADIOS]}

Write to an independent ADIOS file.

parameter bufsize

ADIOS buffer size.

Default 4194304

Units $\mathrm{kB}$

Type positive integer 
parameter output

Output filename.

Type file path to write (extension '.bp')

parameter time_bufsize

ADIOS time aggregation size.

\section{Optional}

Units $\mathrm{kB}$

Type positive integer

parameter transport

Aggregation and write method.

Default MPI, or if using a Lustre PFS, MPI_LUSTRE

Type string

parameter transport_params

Parameters to pass to the transport method.

Default ' '

Type string

parameter verbosity

ADIOS log verbosity.

Default warning

Type silent, error, warning, info, or debug

\subsection{SHIFT MONTE CARLO SOLVER: [SHIFT]}

Shift is the Monte Carlo radiation transport code in Exnihilo. Like all Monte Carlo methods, the output is an estimation of the true solution (the mean of a tally), and a reported error bound provides an estimation of the error in this estimate. In accordance with the central limit theorem, as the number of transported particles increases, the output will converge to the true solution, and the variance will diminish proportionally to the square root of the number of particles.

Two classes of problems can be solved with Shift: eigenvalue ("kcode") solutions for steady-state fissionable systems, and fixed-source solutions for problems with a constant source of particles. The eigenvalue solution technique is described in [SHIFT][KCODE] (page 169). The only option in Shift that does not apply to kcode mode is num_histories (page 169). However, fixed-source problems are also the only ones that support hybrid methods.

The [SHIFT][DECOMPOSITION] (page 169) block enables Shift's domain decomposition feature for large problems. Currently, only mesh tallies are decomposed; compositions, cell tallies, and the problem geometry are all replicated. Some diagnostic tallies and other features may not be usable when domain decomposition is enabled.

Shift also supports an experimental GPU mode when built with CUDA support. It supports a limited subset of Shift features, restricting the available tallies, models, and physics options. 
The behavior of Shift is strongly affected by the [HYBRID] (page 224) block corresponding to the hybrid fixed-source problem mode. Hybrid methods provide weight windows for variance reduction in Shift, and they can additionally bias the sources (page 56) to improve the chance that an emitted particle contributes to the user's tallies.

With the exception of the automatic $k$-effective tally and associated diagnostics in eigenvalue mode, Shift's tallies are specified in the [TALLY] (page 113) database.

parameter arch

Architecture type.

Default $\mathrm{cpu}$

Type cpu or gpu

Applicable when 'SHIFT_CUDA' is enabled in this build

parameter batch_size

Number of particles per batch for computing tally statistics.

\section{Optional}

Type positive integer

\section{Applicable when}

- arch is gpu; and

- problem mode is adjoint, forward, or hybrid

\section{database [DECOMPOSITION]}

Domain decomposition options. See [SHIFT][DECOMPOSITION] (page 169).

Default (empty none database)

\section{parameter device_id}

Device id of GPU.

\section{Default 0}

Type non-negative integer

Applicable when arch is gpu

parameter do_transport (advanced)

Transport particles instead of merely setting up the problem.

Setting do_transport to false is a way to ensure problem integrity without running an expensive transport calculation. It disables transport itself but allows the rest of the code (including building sources, tallies, and physics) to run. This is similar to parm=check in SCALE.

\section{Default True}

Type boolean

\section{parameter gpu_vector_size}

Maximum size of GPU particle vector.

\section{Optional}

Type positive integer 
database [KCODE]

Eigenvalue solution options. See [SHIFT][KCODE] (page 169).

Applicable when problem mode is kcode

parameter num_histories

parameter $\mathbf{n p}$

Number of particle histories.

Type positive integer

Applicable when problem mode is adjoint, forward, or hybrid

parameter physics(advanced)

Name of the physics DB to use.

Default the name of the last physics database

Type string without special characters

\section{database [TRANSPORTER]}

Transport communication options. See [SHIFT][TRANSPORTER] (page 171).

Default (empty database)

database [VR]

Variance reduction options. See [SHIFT][VR] (page 172).

Default (empty database)

\subsection{1 [SHIFT][DECOMPOSITION]}

This database determines how the Shift Monte Carlo problem is spatially decomposed. If not present, the problem will be fully replicated.

Table 25: Available types for the [DECOMPOSITION] database

\begin{tabular}{lll}
\hline Type & Description & Applicability \\
\hline none (page 174) & Fully domain-replicated & \\
bmesh (page 175) & Boundary mesh for domain decomposition & \\
\hline
\end{tabular}

\subsection{2 [SHIFT][KCODE]}

Criticality (or eigenvalue, or kcode) calculations involve iteratively sampling and transporting "generations" of fission neutrons. Several different parameters are needed to specify these generations.

The power iteration method used by Shift (and traditional Monte Carlo codes) may require many particle generations before the fission source converges. The generation at which the fission source converges can be estimated with the help of two diagnostics that Shift tallies by default:

- Shannon entropy, which provides a scalar value summarizing the distribution of occupied spatial cells in the problem, and

- Spatial moments, which provide a low-order approximation of the global particle distribution and shape. 
parameter convergence_method

Kcode cycle convergence criteria for inactive and active cycles.

Shift supports different convergence criteria for switching from inactive to active cycles and for completing the kcode solve. Currently, two traditional methods are supported. The number of inactive cycles is always fixed by the user and specified with the num_inactive_cycles parameter; this number should be high enough that the fission source distribution is converged before tallying begins.

If the convergence method is set to count, then the number of active cycles is also set by the user with the num_cycles parameter, which is the total of inactive plus active cycles. This method should be used if a certain number of particles is needed to be run before finishing the problem (e.g., if a mesh tally is to be used).

The second implemented option is count_ksig, in which a counted number of inactive cycles is run, but the batch-estimated standard deviation of $k_{\text {eff }}$ is used to stop the active cycles.

Default count

Type count or count_ksig

parameter entropy_mesh(advanced)

How the entropy mesh is specified.

The default entropy grid uses the Shift decomposition (which by default is the problem's extents, which are automatically calculated in some geometry types) as its outer extents. If unspecified, Shift tries to build a mesh with:

$$
N_{\text {cells }}=\max \left(N_{\mathrm{p}} / 10,64\right),
$$

where $N_{\mathrm{p}}$ is the number of particles per cycle.

Default manual when entropy mesh input is given

Type automatic or manual

parameter initial_keff

parameter initk

Initial keff value.

Default 1.0

Type positive real number

parameter keff_sigma_active

parameter $\mathbf{s i g}$

Standard deviation of keff at which to stop active cycles.

Type positive real number

Applicable when convergence_method is count_ksig

parameter num_cycles

parameter $\mathbf{n k}$

Number of active + inactive kcode cycles.

Type integer

parameter num_histories_per_cycle 


\section{parameter $\mathbf{n p k}$}

Number of histories per cycle.

Type positive integer

parameter num_inactive_cycles

parameter nik

Number of inactive kcode cycles.

Type integer

parameter $\mathbf{x} \_$entropy

Boundaries in $\mathrm{x}$ for the Shannon entropy mesh.

Units $\mathrm{cm}$

Type monotonically increasing list with at least two values (each element is a real number)

Applicable when entropy_mesh is manual

parameter $\mathbf{y}$ entropy

Boundaries in y for the Shannon entropy mesh.

Units $\mathrm{cm}$

Type monotonically increasing list with at least two values (each element is a real number)

Applicable when entropy_mesh is manual

parameter $\mathbf{z}$ entropy

Boundaries in $\mathrm{z}$ for the Shannon entropy mesh.

Units $\mathrm{cm}$

Type monotonically increasing list with at least two values (each element is a real number)

Applicable when entropy_mesh is manual

\subsection{3 [SHIFT][TRANSPORTER]}

Transport communication options.

parameter bank_size_warnings

Initial bank size warnings to print per domain.

Default 2000

Type non-negative integer

parameter error_tolerance

Fraction of lost particles to tolerate before aborting.

Some especially complex models occasionally have nearly undetectable geometry errors. However, when running enough particles, inevitably some will be lost. To support transport on these models despite any errors, the tolerance parameter can be set to a higher value. This number is multiplied into the total number of particles to be run (in the case of an eigenvalue problem, the number of particles in a single generation) and divided amongst all the parallel processes. When that number is reached, the transport run will be aborted. The tolerance is rounded upward so that each domain will accept at least one lost particle.

Setting the error tolerance too high (a statistically significant number of particles in an important part of the problem) will result in erroneous tally results.

Finally, note that because of particle splitting, multiple particles could be lost per actual source particle. 
Default $1 \mathrm{e}-06$

Type real number inside $(0,1)$

database [GENERATIONS]

Synchronous DD control. See [SHIFT][TRANSPORTER][GENERATIONS] (page 175).

Default (empty constant database)

Applicable when Shift spatial partitioning is domain decomposed parameter max_local_warnings

Max number of lost particle warnings to print per domain.

On complex inputs with known modeling errors, it may be infeasible to correct the model for a test run, but running a trillion particles may produce a million error messages, which (on large parallel systems) will slow down program execution significantly due to the increased I/O.

Setting this value will suppress warnings after the counter is reached.

Default 10

Type non-negative integer

parameter method

Domain-decomposed transport method.

Default sync

Type sync

Applicable when Shift spatial partitioning is domain decomposed

parameter verbosity

How often to print about particles being transported.

Default none if kcode, low otherwise

Type none, low, or high

\subsection{4 [SHIFT][VR]}

Variance reduction is key to making Monte Carlo calculations tractable. Although Shift supports a fully analog mode, in which particles have a weight of either one or zero to mimic the physical event-by-event behavior, the Monte Carlo solution is almost exclusively used with some form of variance reduction.

The key concept behind variance reduction is adding a dimension to the phase space of the transport equation: weight. The weight-averaged Monte Carlo particle density is an estimate of the true particle density because a single Monte Carlo particle can now represent an average of multiple physical particles.

The simplest form of variance reduction, implicit capture, prevents particles from being physically absorbed. Instead, the weight of the particle is reduced proportionally to the chance that it would have been absorbed during its step. When a particle reaches a sufficiently small weight, it no longer contributes meaningfully to the solution but still requires equal computational resources as an important (high-weight) particle. To reduce the number of low-weight particles in the problem, the Russian roulette (or simply roulette) method will probabilistically kill particles below a specified weight. The weight of particles that survive the roulette increases proportionally to the inverse of the fraction of particles that survive the roulette process, maintaining a "fair game" and the resulting solution. 
A more complex variance reduction method is that of weight windows, which introduces a spatially dependent "target weight" rather than a constant value as with Russian roulette. Besides killing particles of low weight, weight windows will also split particles that have high weight into multiple particles closer to the target weight so that multiple potential pathways for a particle's behavior can be averaged into the final result. Weight windows can be provided as input or generated automatically by Denovo. The weight window options are provided in the Hybrid methodology: [HYBRID] (page 224) documentation.

The Consistent Adjoint Driven Importance Sampling (CADIS) family of methods extends the weight window method by biasing particle sources so that the weight of a particle at birth corresponds to the weight window at its point in phase space. Consequently, particles are more likely to be born in the important regions of a problem rather than being rouletted or split immediately at birth. CADIS was originally designed for importance maps generated by a single tally of interest, but biased sources can be constructed "consistently" for any provided weight window map. Source biasing can be enabled for each source using the Particle source definitions: [SOURCE] (page 56) options.

\section{deleted apply_ww}

Entry apply_ww has been deleted: Weight windows are now applied at a set of events specified by the vr_events parameter.

parameter method

parameter $\mathbf{v r}$

Variance reduction method.

Default ' $w w$ ' when hybrid, otherwise 'roulette'

Type ww, analog, or roulette

parameter minimum_thickness

parameter min_thick

Minimum window optical thickness above which to perform VR.

Default 0.01

Type non-negative real number

Applicable when Weight window lookup vr_events has enabled tracking

parameter output

Write weight window centers to the output file.

Default True

Type boolean

Applicable when method is ww

\section{parameter vr_events}

When to apply weight windows.

This entry specifies which transport events should trigger a weight window lookup, thereby potentially initiating a particle splitting or rouletting. A weight window lookup is always performed after a collision. Additional lookup events are precollision, which causes a weight window lookup before a collision, surface, which causes a weight window lookup every time a geometric surface is crossed, $m f p$, which causes a weight window lookup every time a particle streams a mean free path, and tracking, which causes a particle to track through the weight window grid and look up the weight windows every time a weight window grid plane is crossed. These different lookup events may be used in any combination. 


\section{Default tracking}

Type list in which each element is a precollision, $\mathrm{mfp}$, surface, or tracking

Applicable when method is ww

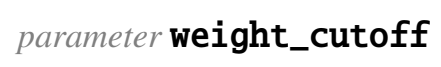

Particle weight cutoff for roulette.

Default 0.25

Type non-negative real number

Applicable when method is roulette

parameter weight_survival

parameter ws

Particle weight survival for roulette.

\section{Default 0.5}

Type non-negative real number

Applicable when method is roulette

\section{parameter $\mathbf{w w}$ _decomp}

Whether the weight window adjoint flux should be decomposed.

Default full

Type full or separable

Applicable when method is ww

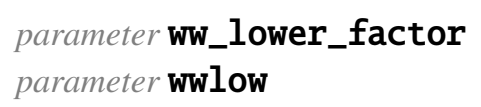

Lower weight window ratio.

\section{Default 0.5}

Type real number inside $(0,1)$

Applicable when method is ww

parameter $\mathbf{w w}$ upper_factor

parameter whigh

Upper weight window ratio.

Default 2.5

Type real number greater than 1

Applicable when method is ww

\subsection{5 [SHIFT][DECOMPOSITION=NONE]}

Fully domain-replicated. 


\subsection{6 [SHIFT][DECOMPOSITION=BMESH]}

Boundary mesh for domain decomposition.

parameter boundary_condition

Boundary condition for each side of the boundary mesh.

Default vacuum vacuum vacuum vacuum vacuum vacuum

Type list of boundary conditions on $-\mathrm{X},+\mathrm{X},-\mathrm{Y},+\mathrm{Y},-\mathrm{Z},+\mathrm{Z}$ (each element is a vacuum, reflect, rotate, or periodic)

parameter overlap

Fraction of DD domain overlap.

Default 0.0

Type real number inclusive [0.0, 1.0]

Applicable when Shift spatial partitioning is domain decomposed

parameter subblock_procs

Processor allocation for blocks of the boundary mesh.

Default ---

Type list in which each element is a positive integer

postprocessor

Ensure that the Shift spatial decomposition is compatible with the number of processors if using a

[RUN] block.

postprocessor

Check to make sure that Tpetra is enabled in build for DD decompositions.

Applicability Shift spatial partitioning is domain decomposed

parameter $\mathbf{x}$ _partition

parameter $\mathbf{x}$

Boundary mesh along the $\mathrm{X}$ axis.

Type monotonically increasing list with at least two values (each element is a real number)

parameter $\mathbf{y}$ _partition

parameter $\mathbf{y}$

Boundary mesh along the $\mathrm{Y}$ axis.

Type monotonically increasing list with at least two values (each element is a real number)

parameter $\mathbf{z}$ _partition

parameter $\mathbf{Z}$

Boundary mesh along the $\mathrm{Z}$ axis.

Type monotonically increasing list with at least two values (each element is a real number)

\subsection{7 [SHIFT][TRANSPORTER][GENERATIONS]}

Table 26: Available types for the [GENERATIONS] database

\begin{tabular}{lll}
\hline Type & Description & Applicability \\
\hline constant (page 176) & Same number of iterations per cycle & \\
three_component (page 176) & Advanced iteration flexibility & \\
\hline
\end{tabular}




\subsection{8 [SHIFT][TRANSPORTER][GENERATIONS=CONSTANT]}

Same number of iterations per cycle.

parameter num_iterations

Number of iterations per generation.

Default 100

Type positive integer

\subsection{9 [SHIFT][TRANSPORTER][GENERATIONS=THREE_COMPONENT]}

Advanced iteration flexibility.

parameter bound 1

First boundary of three-component generations.

Default 20.0

Type non-negative real number

parameter bound2

Second boundary of three-component generations.

Default 50.0

Type positive real number

parameter exponent

Exponential decay constant of three-component generations.

Default 0.025

Type non-negative real number

parameter intercept

Intercept of first component of three-component generations.

Default 100.0

Type positive real number

parameter minimum

Minimum iterations of three-component generations.

Default 100.0

Type positive real number

parameter slope

Slope of first component of three-component generations.

Default 5.0

Type non-negative real number

\subsection{DENOVO DETERMINISTIC SOLVER: [DENOVO]}

Denovo is the parallel deterministic solver inside the Exnihilo code suite. It solves the steady-state Boltzmann transport equation by discretizing it in space, angle, and energy. The [DENOVO] database specifies these discretization parameters as well as numerical solver parameters. 


\subsubsection{DENOVO APPLICATIONS}

Denovo is often used to generate importance maps for variance reduction, typically using the [HYBRID] (page 224) block. The figure of merit of a tally,

$$
\mathrm{FOM}=\frac{1}{\sigma^{2} T},
$$

where $\sigma^{2}$ is the variance of the tally in question and $T$ is the time to solution, can improve by orders of magnitude given a good approximation to the importance map. However, even with a bad approximation to an adjoint transport solution for an importance map, the expected value of the Shift solution will not change as a result of the Denovo solver parameters. The figure of merit will simply be lowered, and the estimation of the variance for an unconverged solution may also be affected. This principle of hybrid methods means that coarse and fast Denovo solves can be adequate to improve the convergence rate of a Shift calculation.

In contrast to hybrid applications, using any discrete ordinates code to generate final results for an analysis usually requires a great deal of care and experience to produce accurate solutions. Significant errors can be introduced by poor approximations to:

- the spatial grid (e.g., by not capturing a streaming pathway);

- the spatial integration method (e.g., by using a difference method for optically thick cells) (see the method parameter (page 180));

- the energy discretization (e.g., by failing to capture self-shielding effects) (see Multigroup physics: [PHYSICS=mg] (page 96)); and

- the angular discretization (e.g., by ray effects in optically thin regions) (see [DENOVO][QUADRATURE] (page 184)).

Warning: The default settings for Denovo are generally for obtaining qualitatively accurate answers for use in hybrid schemes or for initial problem scoping. Obtaining accurate answers for most realistic problems typically requires a good deal of experience, parametric studies, and finesse.

Denovo has two categories of options for the angular discretization: discrete ordinates $\left(\mathrm{S}_{N}\right)$, in which particles are constrained to travel along specific angular directions; and spherical harmonics $\left(\mathrm{SP}_{N}\right)$, which is similar in treatment to the diffusion approximation. The method parameter (page 180) chooses between the two. The various $S_{N}$ discretization closure equations are described in the equations parameter (page 179).

\subsubsection{SOLVER OVERVIEW}

The linear algebraic formulation of these deterministic approximations to the transport equation require numerical methods to solve. (Analytically solving the exact representation of the discretized transport equation is intractable except for toy problems.) The solution methodology will vary depending on whether the input is a "fixed source" problem

$$
A x=b
$$

or an $k$-eigenvalue problem

$$
F A x=\frac{1}{k} x
$$


Fixed-source problems without upscattering can be solved exactly with a series of consecutive within-group solutions: the solution vector is the flux (and higher-order moments) whose components are coupled by the spatial and angular behavior of the problem. This solution is the within-group (page 200) solve and can be performed with several numerical methods.

Upscattering introduces a dependency between multiple within-group solutions and therefore generally requires an outer level of iteration. The solvers that converge this multigroup solution are described in [DENOVO][SOLVER][UPSCATTER] (page 198). It is important to note that to support more advanced methods and to improve the parallelism of Denovo solves, there is an option to treat all groups as upscatter groups (even if the cross sections physically prevent upscatter) in order to solve the groups simultaneously. This option is discussed in the upscatter_groups parameter (page 194).

Finally, the convergence of an eigenvalue problem generally requires an additional level of iteration. The options for converging the eigenvalue (a measure of criticality of the problem) and eigenvector (the distribution of neutrons at a critical state) are described in [DENOVO][SOLVER=eigenvalue] (page 195).

For a more detailed discussion of Denovo's solution methodology and discrete ordinate methodology in general, see [4] and [11] and the references therein.

\subsubsection{PERFORMANCE CONSIDERATIONS}

The number of degrees of freedom $D$ in a Denovo $S_{N}$ calculation is

$$
D=N_{u} \times N_{c} \times N_{m} \times N_{a} \times N_{g},
$$

where $N_{u}$ is the number of spatial unknowns (cf. the equations parameter (page 179)), $N_{c}$ is the number of cells (determined by the spacing of $\mathrm{x}, \mathrm{y}$, and $\mathrm{z}$ ), $N_{m}$ is the number of moments (cf. the pn_order physics parameter (page 102)), $N_{a}$ is the number of discrete angles (see [DENOVO][QUADRATURE] (page 184)), and $N_{g}$ is the number of energy groups. This value is proportional to the number of floating point operations per transport sweep when all groups are being swept simultaneously (upscatter_groups (page 194) is set to "all").

A more important figure for some users will be the memory consumption of Denovo rather than the time to solution. The Denovo state vector (or solution vector) does not store angular fluxes; rather, it stores angular moments up to the specified maximum scattering order. It therefore contains $M$ elements, with

$$
M=N_{u} \times N_{c} \times N_{m} \times N_{g} .
$$

This state vector is distributed across all MPI processes in a parallel run (see [DENOVO][DECOMPOSITION] (page 183)). In most problems, the state vector dominates memory usage. However, in problems with an unusually large number of groups, it is important to be aware of the memory footprint of scattering matrices:

$$
M_{\mathrm{xs}} \propto N_{g}^{2} N_{\text {mixtures }}
$$

The number of mixtures depends both on the original compositions of the input model and on the mix tolerance (page 187) used in the model ray tracing. Also critically important is that the number of mixtures on each domain depends on the complexity of the spatial region local to that domain! Since Denovo spatially partitions the problem to equally distribute the number of cells along the $x y$ plane, some complex domains may end up with a hundred times as many mixtures as the simpler domains, and that memory requirement could contribute significantly to the total Denovo memory usage.

parameter arch

Architecture type. 


\section{Default $\mathrm{cpu}$}

Type cpu or gpu

\section{Applicable when}

- 'KBA_CUDA' is enabled in this build; and

- problem mode is forward or kcode; and

- Denovo discretization method is $1 \mathrm{~d}$ or sc

\section{database [BOUNDARY]}

Boundary condition specification. See [DENOVO][BOUNDARY] (page 183).

Default (empty vacuum database)

\section{database [DECOMPOSITION]}

Denovo space-energy decomposition. See [DENOVO][DECOMPOSITION] (page 183).

Default (empty database)

parameter dimension (advanced)

Spatial dimension of the problem.

Default based on spatial discretization equations

Type integer 2 or 3

\section{parameter disable}

Choose to skip the 'solve' step or both 'solve' and construction.

\section{Default none}

Type none, transport, or state

\section{parameter equations}

\section{parameter eq}

Closure equations for the $\mathrm{S}_{N}$ approximation in a cell.

Like most options for deterministic methods, the choice of spatial discretization (closure equations for the $S_{N}$ approximation) involves a trade-off between performance and accuracy. In this case, inaccuracy can result in numerical instability and negativities in the computed solution. The performance varies by discretization partly due to the solution method (e.g., the exponential function evaluations in step characteristics) and the increased memory costs of discretizations that have more unknowns per spatial cell.

The following spatial discretizations are available for 3D problems:

Table 27: Denovo spatial discretization options.

\begin{tabular}{lll}
\hline Value & Discretization & Unknowns \\
\hline sc & Step characteristics & 1 \\
ld & Linear discontinuous finite element & 4 \\
tld & Trilinear discontinuous finite element & 8 \\
twd & Theta-weighted diamond difference & 1 \\
wdd & Weighted diamond difference & 1 \\
wdd_ff & Weighted diamond difference with flux fixup & 1 \\
\hline
\end{tabular}


Additionally, for 2D problems, a step characteristics sc_2d (1 unknown) option and a bilinear discontinuous bld_2d (4 unknowns) finite element discretization are available.

The step characteristics (SC) equations guarantee positive solutions and offer good accuracy for a wide range of problems. This should be the preferred method for difficult shielding problems and for problems in which a coarse spatial mesh must be used.

The linear discontinuous (LD) finite element method (FEM) is often more accurate than SC, but it does not guarantee positivity of the solution. The trilinear discontinuous (TLD) FEM is more accurate and rigorous in the asymptotic limit of diffusive cells, but it still does not guarantee positivity. For this reason, the FEM group of methods is not usually recommended for difficult shielding problems or problems where the spatial mesh does not adequately resolve the physics of the problem. They are most appropriate for problems that are not prone to negative solutions (such as reactors) and problems in which a finely resolved spatial mesh is possible.

The diamond difference methods, twd, wdd, and wdd_ff, are available primarily for direct comparisons to legacy radiation transport solvers and are not recommended.

Default sC

Type sc, sc_2d, bld_2d, ld, tld, twd, wdd, or wdd_ff

Applicable when /denovo/disable is none or transport and /denovo/method is sn

parameter first_group

The first energy group to solve.

\section{Optional}

Type non-negative integer

parameter last_group

The last energy group to solve.

\section{Optional}

Type non-negative integer

postprocessor

Check first_group and last_group for consistency.

parameter log_memory (advanced)

Periodically print memory usage to screen.

Default value of log_memory in [OUTPUT]

Type boolean

command logically_uniform_grid

Generate a grid (approximately uniform in num cells) from the given extents.

Creates $x$

Creates y

Creates $\mathrm{z}$

preprocessor (advanced)

Change deprecated SN 'method' values to 'equations.' 
parameter method

Deterministic solution method.

This parameter chooses between the discrete ordinates $\left(\mathrm{S}_{N}\right)$ and simplified spherical harmonics $\left(\mathrm{SP}_{N}\right)$ methods.

The discrete ordinates method is suitable for a variety of shielding applications and for accelerating Monte Carlo transport by estimating the global (space and energy) importance field with the adjoint flux. It is subject to discretization error not only by the spatial grid but also by the angular quadrature. Converging to a true monoenergetic transport solution requires simultaneous refinement in both angle and space.

The Simplified $\mathrm{P}_{N}$ finite volume discretization approach $\left(\mathrm{SP}_{N}\right)$ provides fast, accurate solutions primarily for light water reactor eigenvalue problems. The $\mathrm{SP}_{N}$ equations introduce approximations not present in other discretizations and are not recommended for general purpose (i.e., non-reactor) problems. In particular, problems with void or near-void regions cannot be handled accurately by this method. It should also be noted that, unlike the more rigorous $\mathrm{P}_{N}$ approximation, solutions with increasing $\mathrm{SP}_{N}$ order will not converge to the actual transport solution.

Full details on the mathematical methods used in Denovo can be found in the Exnihilo methods manual.

Default sn

Type spn or sn

Applicable when transporter state is constructed

database [OUTPUT]

HDF5 output options. See [DENOVO][OUTPUT] (page 189).

Default (empty database)

parameter output_spn_matrices(advanced)

Output $\mathrm{SP}_{N}$ matrices for analysis.

Default False

Type boolean

Applicable when /denovo/disable is none or transport and /denovo/method is spn

\section{preprocessor (advanced)}

Set disable state for mode raytrace.

parameter physics

Name of the associated MG physics database.

Default the name of the MG physics database

Type string without special characters

\section{database [QUADRATURE]}

Discrete ordinates quadrature. See [DENOVO][QUADRATURE] (page 184).

Default (empty database)

Applicable when /denovo/disable is none or transport and /denovo/method is sn 
database [RAYTRACE]

Problem discretization options. See [DENOVO][RAYTRACE] (page 187).

Default (empty database)

Applicable when model type is not 'mesh'

database [SILO]

Silo output options. See [DENOVO][SILO] (page 190).

Default (empty database)

Applicable when 'silo' is enabled in this build

\section{database [SOLVER]}

Linear algebraic solver options. See [DENOVO][SOLVER] (page 192).

Default (empty database)

Applicable when transporter state is constructed

database [SOURCE]

Source discretization options. See [DENOVO][SOURCE] (page 188).

Default (empty database)

Applicable when problem mode is adjoint, forward, or hybrid

parameter spn_order

Discretization order of $\mathrm{SP}_{N}$.

Default 1

Type positive integer

Applicable when /denovo/disable is none or transport and /denovo/method is spn

command uniform_grid

Generate a grid (approximately uniform in cell size) from the given extents.

This command is primarily for scalability testing. It will generate a mesh with approximately uniform cells, limited by the provided number of cells. An optional parameter snaps the grid lengths to the nearest multiple (i.e., the number of cells in each direction will be divisible by this number).

It accepts the following sequence of arguments

xmin, $x \max , y \min , y \max , z \min , z \max$, num_cells [, multiple]

\section{Creates $\mathrm{x}$}

Creates y

Creates $\mathrm{z}$

\section{parameter $\mathbf{x}$}

Mesh coordinates along the $\mathrm{X}$ axis.

Type monotonically increasing list with at least two values (each element is a real number) 
Applicable when model type is not 'mesh'

parameter $\mathbf{y}$

Mesh coordinates along the $\mathrm{Y}$ axis.

Type monotonically increasing list with at least two values (each element is a real number)

Applicable when model type is not 'mesh'

parameter $\mathbf{Z}$

Mesh coordinates along the $\mathrm{Z}$ axis.

Type monotonically increasing list with at least two values (each element is a real number)

Applicable when

- model type is not 'mesh'; and

- Problem is 3-D

postprocessor

Print the number of cells in the mesh.

Applicability model type is not 'mesh'

Applicability Problem is 3-D

\subsection{4 [DENOVO][BOUNDARY]}

Table 28: Available types for the [BOUNDARY] database

\begin{tabular}{lll}
\hline Type & Description & Applicability \\
\hline vacuum (page 192) & Vacuum boundaries & \\
reflect (page 193) & Reflecting and vacuum boundaries & \\
isotropic (page 193) & One or more isotropic incident boundaries & \\
\hline
\end{tabular}

\subsection{5 [DENOVO][DECOMPOSITION]}

The Denovo decomposition determines how the solution is distributed across the computational resources. The KBA decomposition splits the Denovo mesh into columns along an $x y$ grid specified by dividing the number

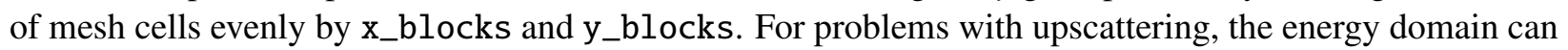
also be decomposed into an orthogonal dimension using energy_sets. The number of processors being used must equal the product of these three parameters:

$$
N_{p}=N_{x} N_{y} N_{E}
$$

If using a [RUN] block (page 29), Omnibus will automatically provide reasonable values for the decomposition.

parameter energy_sets

The number of energy sets.

\section{Default 1}

Type non-negative integer 
preprocessor (advanced)

If a [RUN] block is present, come up with a logically square KBA decomposition.

parameter $\mathbf{x} \_$blocks

parameter $\mathbf{x}$

The number of spatial partitions along the $\mathrm{x}$ axis.

Type non-negative integer

parameter $\mathbf{y}$ blocks

parameter $\mathbf{y}$

The number of spatial partitions along the y axis.

Type non-negative integer

postprocessor

Ensure Denovo decomposition is compatible with number of processors.

parameter $\mathbf{z}$ blocks

parameter $\mathbf{z}$

The number of pipelining blocks along the $\mathrm{z}$ axis.

Default Default z_blocks to min(nx,ny)

Type non-negative integer

\section{Applicable when}

- Problem is 3-D; and

- /denovo/disable is none or transport and /denovo/method is sn

\subsection{6 [DENOVO][QUADRATURE]}

The runtime of an $\mathrm{S}_{N}$ calculation is proportional to the number of quadrature angles, in the limit of a large number of angles. To summarize from the ADVANTG 3.0 manual [7]:

- Level-symmetric quadratures have historically been used most often. These consist of rotationally symmetric quadratures that have positive weights up to $S_{20}$. In three dimensions, there are $N \times(N+2) / 8$ angles per octant, where $N$ is the order of the quadrature. Therefore, the order of these quadratures must be even. These quadratures tend to exhibit the most ray effects.

- Gauss-Legendre product quadratures are formed by taking the Cartesian product of a set of uniformly distributed azimuthal angles and a 1D GaussLegendre quadrature in the polar angle.

- Quadruple range $(\mathrm{QR})$ product quadratures exactly integrate maximal-order products of sines and cosines of the polar and azimuthal angles [12]. These quadratures generally perform well across a broad range of transport problems and tend to exhibit far less ray effects than level-symmetric quadratures.

- Linear-discontinuous finite element (LDFE) quadratures approximate the angular flux using direction cosines [13]. They are determined by requiring that the integration of the basis functions is equal to the surface area of a unit sphere. The advantage of these LDFE sets is that they have positive weights and are rotationally symmetric about the $\mathrm{x}, \mathrm{y}$, and $\mathrm{z}$ axes. For a quadrature of order $N$, there are $4^{(N+1)}$ angles per octant, so the order can be even or odd. 
parameter construction

Construction methodology for the quadrature set.

Table 29: Denovo quadrature construction options.

\begin{tabular}{ll}
\hline Construction & Description \\
\hline levelsym & $\begin{array}{l}\text { Level-symmetric quadrature set, which features rotationally } \\
\text { invariant angular positions and weights }\end{array}$ \\
product & $\begin{array}{l}\text { Product quadrature set, which has a specified number of } \\
\text { azimuthal angles per polar angle and a specified number of } \\
\text { polar angles. }\end{array}$ \\
product_vec & $\begin{array}{l}\text { A specialization of the product quadrature set that allows } \\
\text { the number of azimuthal angles to be different at each polar } \\
\text { angle. }\end{array}$ \\
\hline
\end{tabular}

Each quadrature set only supports a limited subset of construction options.

Table 30: Denovo quadrature availability matrix.

\begin{tabular}{llll}
\hline Quadrature & levelsym & product & product_vec \\
\hline levelsym & $\times$ & & \\
glproduct & & $\times$ & \\
qr & $\times$ & $\times$ & $\times$ \\
\hline
\end{tabular}

Default based on quadrature type

Type levelsym, product, or product_vec

Applicable when quadrature is levelsym, glproduct, or qr

postprocessor

Validate construction of quadrature sets.

parameter input

User-specified quadrature set file.

The Denovo quadrature may be manually input as an ASCII file. The format is:

- Optional comments have a \# at the beginning of the line

- Each line contains the whitespace-separated tuple $(\mu, \eta, \xi, w)$, which is the $x y z$ projection of the angle tied to the angle's associated weight.

- Angles for just a single octant (or quadrant if 2D) may be defined, or all angles in all octants may be defined. (This is checked by summing the weights over the input angles.)

An example input for $S_{2}$ is

\# S2 quadrature input

0.577350269190 .577350269190 .577350269191 .0

Type file path for reading 
Applicable when quadrature is userdefined

parameter $\mathbf{l d f e}$ _order

Order for the LDFE quadrature set.

Default 1

Type positive integer

Applicable when quadrature is $l \mathrm{dfe}$

parameter num_azi

Number of azimuthal angles per level per octant.

Default 4

Type positive integer

Applicable when construction is product

parameter num_azi_vec

List of the number of azimuthal angles per polar angle per octant, ordered from pole to equator.

Default ---

Type list in which each element is a positive integer

Applicable when construction is product_vec

parameter num_polar

Number of polar angles per level per octant.

Default 4

Type positive integer

Applicable when construction is product or product_vec

parameter order

Level-symmetric quadrature set order.

\section{Default 10}

Type non-negative integer

Applicable when construction is levelsym

parameter polar_axis

Axis of rotation for product quadrature sets.

Default $z$

Type $\mathrm{x}, \mathrm{y}$, or $\mathrm{z}$

Applicable when construction is product or product_vec parameter quadrature

Discrete ordinates quadrature set class.

Default ' $q r$ ' unless input or ldfe_order are given

Type levelsym, glproduct, qr, ldfe, or userdefined 


\subsection{7 [DENOVO][RAYTRACE]}

Denovo's Cartesian "brick mesh" spatial discretization requires cross sections to be homogenized within each spatial cell (a rectangular prism). Whereas legacy solvers required the user to specify a material for each spatial cell, Denovo can assist by using the same particle tracking engine as Shift (page 167) to map the continuous-in-space geometry specification of a model (page 43) to the Denovo mesh.

This raytracing is performed in parallel, with each local Denovo KBA decomposition generating an independent set of local materials and mixtures.

The options in this block determine how ray tracing is performed.

parameter axes

Axis/axes along which to fire rays for ray trace.

Default $x y z$

Type axis or axes ('x','zy','xyz')

parameter error_tolerance

Fraction of lost rays to tolerate before aborting.

Default $1 \mathrm{e}-05$

Type real number inside $(0,1)$

parameter max_local_warnings

Max number of lost ray warnings to print per domain.

Default 10

Type non-negative integer

parameter $\mathbf{m i x}$ _tolerance

Tolerance for collapsing similar mixed materials into one.

To reduce memory requirements (see Performance considerations (page 178)), mixed cells with similar contents are collapsed to a single mixture on the fly [14]. A higher "mix tolerance" will reduce both memory consumption and accuracy for coarsely discretized problems. "Coarse" is relative to the length scale of the finest features of the input model.

Warning: Because the mixture collapsing is done locally on each KBA domain and not globally, different KBA decompositions will result in different discretized Denovo solutions! Since hybrid applications are relatively insensitive to weight windows (the estimated mean should converge to the same value), this is generally ok for hybrid mode. However, the on-the-fly discretization provided by the [DENOVO][RAYTRACE] (page 187) capability might not be appropriate for standalone solutions. The make-denovo-model (page 21) tool can be used to convert an initial raytraced Denovo run into a reproducible, more efficient model for comparison between multiple Denovo runs.

\section{Default 0.05}

Type real number inside $(0,1)$

parameter rays_deterministic

Use face midpoints rather than stratified sampling. 


\section{Default False \\ Type boolean \\ parameter rays_per_face}

Number of ray trace rays to be fired per mesh face.

\section{Default 4}

Type positive square integer

parameter void_matid(advanced)

Material ID to be used when raytrace is outside the problem.

\section{Default 0}

Type integer

parameter $\mathbf{z}$ _loc

The $\mathrm{z}$ coordinate for the ray trace.

Type real number

Applicable when Problem is 2-D

\subsection{8 [DENOVO][SOURCE]}

The [SOURCE] database controls discretization of Shift sources. Source strengths, including the WGT card for MCNP sources, are taken into account when creating the discrete source.

parameter mc_num_particles

Number of uncollided source particles to sample.

Type positive integer

Applicable when uncflux is mc

parameter mc_uncf_sets

Number of Denovo blocks per uncollided flux decomposition.

Default 1

Type positive integer

\section{Applicable when}

- uncflux is mc; and

- 'Tpetra' is enabled in this build

\section{preprocessor}

Sources must be the same type when using Denovo.

parameter uncflux

parameter uncf

Uncollided flux treatment.

The uncollided flux treatment can reduce ray effects from point sources or other small sources. It currently applies only to separable sources, mesh problem sources (defined in an HDF5 input file), and SWORD sources. 
The default is to treat all sources by discretizing them onto the Denovo grid as $\mathrm{S}_{N}$ sources.

If the "analytic" option is chosen, all point sources will be used as analytic uncollided flux sources. Ray traces are performed through the underlying geometry from each point source to quadrature points on each Denovo mesh cell to determine the uncollided flux contribution inside that cell.

The "mc" option is only valid when a single source is present. If selected, the source will be converted into a Monte Carlo uncollided flux source.

Default analytic

Type none, analytic, or mc

\section{Applicable when}

- not using an MCNP source; and

-.../decomposition/energy_sets is 1

\subsection{9 [DENOVO][OUTPUT]}

HDF5 output options.

parameter angular_flux

Write the full angular flux.

Default False

Type boolean

Applicable when /denovo/disable is none or transport and /denovo/method is sn

postprocessor

Flux output is not automatically disabled when transport is disabled.

Applicability /denovo/disable is state or transport

parameter angular_mesh

Write the quadrature angles and weights.

Default True

Type boolean

Applicable when /denovo/disable is none or transport and /denovo/method is sn parameter $\mathbf{b l o c k}$

Write the KBA domain for each cell.

Default True

Type boolean

parameter current

Write the current (first angular moment).

Default False

Type boolean

Applicable when 
- scattering order is not isotropic (pn_order $>0$ ); and

- transporter state is constructed

deprecated $\mathbf{f c}$ _source

Deprecated entry fc_source has been renamed to uncflux.

Update to uncflux

parameter $\mathbf{f l u x}$

Write the scalar flux.

Default True when performing transport

Type boolean

Applicable when transporter state is constructed

parameter mat

Write materials and mix tables.

Default True when the model type is not 'mesh'

Type boolean

parameter source

Write the energy-dependent source term.

Default True when performing transport and model is not 'mesh'

Type boolean

Applicable when problem mode is adjoint, forward, or hybrid

parameter uncflux

Write the uncollided flux if present.

Default True when performing transport

Type boolean

Applicable when

- /denovo/disable is none or transport and /denovo/method is sn; and

- problem mode is adjoint, forward, or hybrid; and

- when uncflux is enabled

\subsubsection{0 [DENOVO][SILO]}

Silo output options.

\section{parameter blocks_per_file}

Number of KBA blocks written to each SILO output file.

Increasing this number will reduce the number of files in the _silo output directory, and each file will be correspondingly larger. The number of files can affect the output bandwidth, depending on the parallel file system being used.

\section{Default 1}




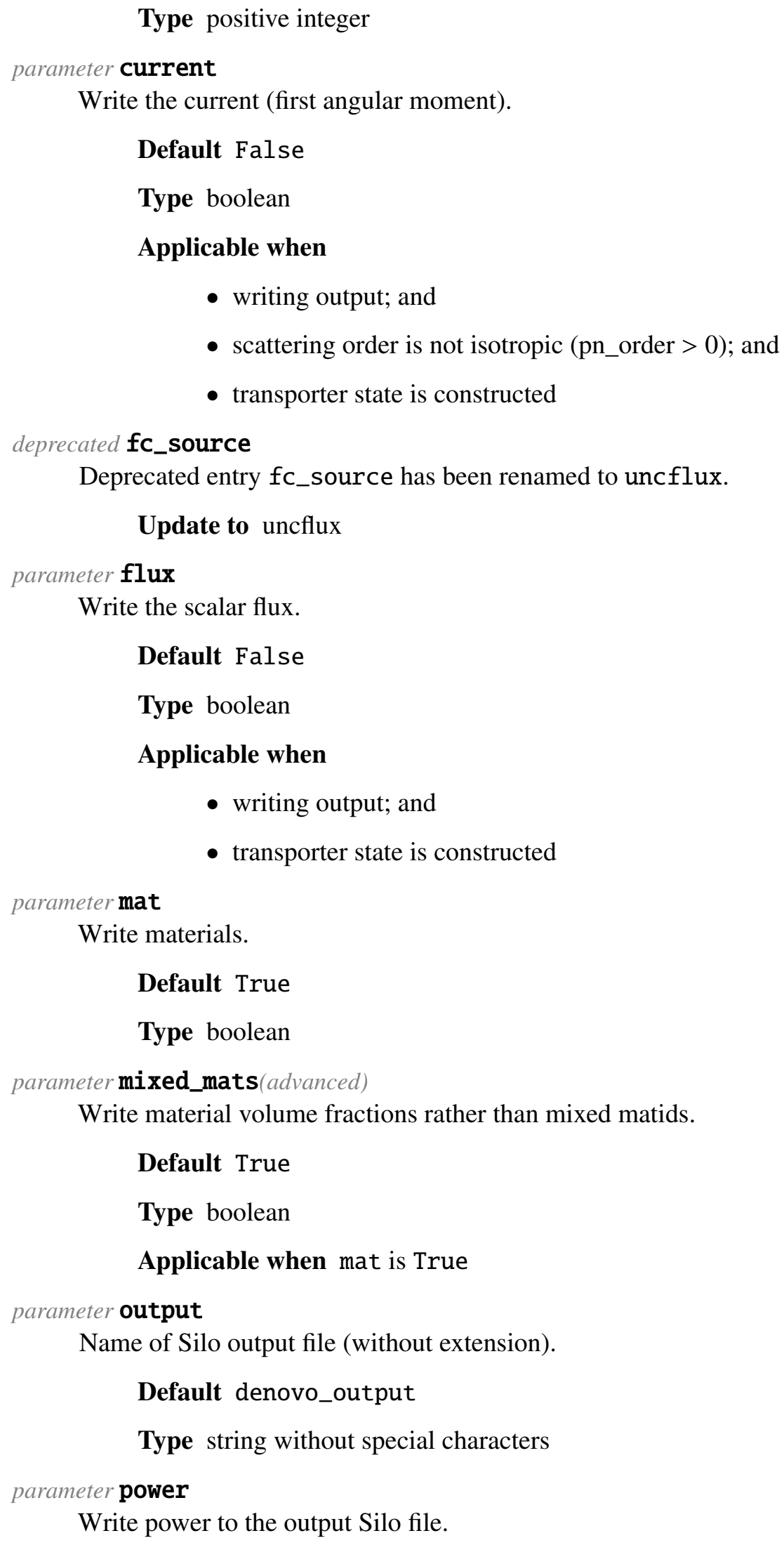

Deprecated entry fc_source has been renamed to uncflux.

Update to uncflux

parameter $\mathbf{f l u x}$

Write the scalar flux.

Default False

Type boolean

Applicable when

- writing output; and

- transporter state is constructed

parameter mat

Write materials.

Default True

Type boolean

parameter mixed_mats(advanced)

Write material volume fractions rather than mixed matids.

Default True

Type boolean

Applicable when mat is True

parameter output

Name of Silo output file (without extension).

Default denovo_output

Type string without special characters

parameter power

Write power to the output Silo file.

Default False 
Type boolean

\section{Applicable when}

- writing output; and

- problem mode is kcode; and

- transporter state is constructed

parameter source

Write the energy-dependent source term.

\section{Default False}

Type boolean

\section{Applicable when}

- writing output; and

- problem mode is adjoint, forward, or hybrid; and

- transporter state is constructed

parameter uncflux

Write the uncollided flux if present.

Default False

Type boolean

\section{Applicable when}

- writing output; and

- /denovo/disable is none or transport and /denovo/method is sn; and

- problem mode is adjoint, forward, or hybrid; and

- transporter state is constructed

\subsubsection{1 [DENOVO][SOLVER]}

Table 31: Available types for the [SOLVER] database

\begin{tabular}{lll}
\hline Type & Description & Applicability \\
\hline fixed (page 194) & Fixed-source solver options & $\begin{array}{l}\text { problem mode is adjoint, forward, or } \\
\text { hybrid }\end{array}$ \\
$\begin{array}{l}\text { eigenvalue } \\
\text { (page 195) }\end{array}$ & $\begin{array}{l}\text { Solver options for eigenvalue prob- } \\
\text { problem mode is kcode }\end{array}$ \\
\hline
\end{tabular}

\subsubsection{2 [DENOVO][BOUNDARY=VACUUM]}

Vacuum boundaries. 


\subsubsection{3 [DENOVO][BOUNDARY $=$ REFLECT]}

Reflecting and vacuum boundaries.

parameter reflect

Mark each exterior face of the problem as reflecting.

Default reflecting on all dimensions

Type list of boundary conditions for $-\mathrm{X},+\mathrm{X},-\mathrm{Y},+\mathrm{Y},[-\mathrm{Z},+\mathrm{Z}]$ (each element is a integer 0 or 1)

postprocessor

Reflect array length must be compatible with dimension.

\subsubsection{4 [DENOVO][BOUNDARY=ISOTROPIC]}

One or more isotropic incident boundaries.

parameter minus_x

Flux for each energy group on the $-\mathrm{X}$ boundary surface.

\section{Optional}

Type list in which each element is a real number

parameter minus_y

Flux for each energy group on the -Y boundary surface.

\section{Optional}

Type list in which each element is a real number

parameter minus_z

Flux for each energy group on the $-Z$ boundary surface.

\section{Optional}

Type list in which each element is a real number

parameter $\mathbf{p l u s} \mathbf{x}$

Flux for each energy group on the $+\mathrm{X}$ boundary surface.

\section{Optional}

Type list in which each element is a real number

parameter $\mathbf{p l u s} \mathbf{y}$

Flux for each energy group on the $+\mathrm{Y}$ boundary surface.

\section{Optional}

Type list in which each element is a real number

parameter plus_z

Flux for each energy group on the $+\mathrm{Z}$ boundary surface.

\section{Optional}

Type list in which each element is a real number

postprocessor

Check number of groups and for one non-empty surface. 


\subsubsection{5 [DENOVO][SOLVER=FIXED]}

The fixed solver database type specifies the solvers and options to use when solving a forward, adjoint, or hybrid problem through Denovo. The [DENOVO][SOLVER][WITHIN_GROUP] (page 200) database controls the solver options for solving within each energy group. The upscatter database controls the solver options for solving multiple coupled groups; some of the upscatter solution methods additionally have an inner iteration of within-group solves.

The tolerance parameter sets an overall desired convergence tolerance. The tolerances of any embedded solvers will be set accordingly to achieve a solution to this given accuracy. (The upscatter and within-group databases default to the same tolerance as the overall solver.)

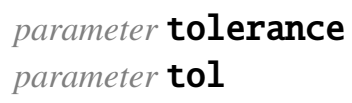

Convergence tolerance to govern solver accuracy.

The default tolerance in most cases is to converge to a factor of ten tighter than the parent database. However, in the case of fixed-source problems, the tolerance of the within-group and upscatter databases is set to the value of the fixed-source solver database (with no additional multiplier). These default values are always written to the screen as a diagnostic.

\section{Default 0.001}

Type real number inside $(0,1)$

\section{database [UPSCATTER]}

Upscatter block solver options. See [DENOVO][SOLVER][UPSCATTER] (page 198).

Default (empty database)

Applicable when /denovo/disable is none or transport and /denovo/method is spn; upscattering is enabled; or upscatter_groups is all

\section{parameter upscatter_groups}

Which groups to treat as upscatter groups during solve.

This parameter controls which energy groups to treat as upscatter when solving. If set to all, all energy groups are treated as upscatter during the solve. If set to thermal, only the thermal groups are treated as upscatter during the solve.

The upscatter_groups parameter has dependencies on other solver options:

- If the method (page 180) is spn, this parameter is not used as $\mathrm{SP}_{N}$ always solves simultaneously over all groups.

- If the eigenvalue solver (page 195) is rayleigh_quotient, which must simultaneously solve all groups, it must be set to all.

- If use_cuda is True, it must be all. Increasing the amount of simultaneous work by transporting over all groups at once is critical to performant GPU code.

- If the number of energy set decompositions (page 183) is one, the default is to solve only thermal groups. Otherwise, increased parallelism is needed and the default changes to all.

Default thermal unless arch is gpu, eigenvalue solver is rayleigh_quotient, energy_sets $>1$, "method" is spn, or upscatter preconditioner is multilevel 
Type all or thermal

postprocessor

CUDA sweeper, rayleigh_quotient solver, SPN, and multilevel upscatter preconditioner require all upscatter groups.

postprocessor

Warn if using downscatter-only physics with upscatter all unless needed.

Applicability upscatter_groups is all

database [WITHIN_GROUP]

One-group transport solver options. See [DENOVO][SOLVER][WITHIN_GROUP] (page 200).

Default (empty database)

Applicable when upscatter_groups is thermal

\subsubsection{6 [DENOVO][SOLVER=EIGENVALUE]}

The eigenvalue database specifies the solvers and options to use for solving an eigenvalue problem (mode kcode) through Denovo. Along with the within-group (page 200) and upscatter (page 198) databases described below, this database also sets the solver and options for the eigenvalue solve.

The tolerance parameter which controls the $l_{2}$ convergence tolerance of the eigenvalue solve can be used to set an overall desired convergence tolerance. Inner iteration tolerances will be set accordingly to achieve a solution with this accuracy.

The verbosity (page 198) setting determines the output level within the linear algebraic solver.

parameter acceleration_method

Type of power iteration acceleration.

Default none

Type none or rebalance

Applicable when solver is power_iteration

parameter calculate_flux_moments

Calculate the flux moments during Arnoldi iteration.

Default True

Type boolean

Applicable when solver is arnoldi

parameter fission_tolerance

Tolerance for infinity norm of flux.

Default 1.0

Type real number inclusive $[0.0,1.0]$

Applicable when solver is power_iteration

parameter $\mathbf{k}$ _tolerance

Tolerance for relative error of k-eff eigenvalue. 


\section{Default $1 e-05$}

Type real number inclusive $[0.0,1.0]$

Applicable when solver is power_iteration or rayleigh_quotient

\section{parameter max_iterations}

parameter maxitr

Max number of iterations for this solver.

\section{Default 1000}

Type non-negative integer

parameter shift

Shift parameter for RQI.

Default -1.0

Type real number

Applicable when solver is rayleigh_quotient

parameter solver

Solver used for eigenvalue iteration.

The Arnoldi solver offers fast, robust convergence for almost all problems. Like the GMRES (Generalized Minimal RESidual method) linear solver, Arnoldi is a subspace solver and therefore incurs some extra memory usage. To reduce memory usage, it is possible to use a variation of this solver that turns off energy dependence, which will reduce the memory used by the solver in exchange for some extra computational work at the end of the solve.

Power Iteration is a robust eigenvalue solver, but it suffers from extremely slow convergence and long computational times for many problems. It has minimal memory requirements; however, using the energy independent Arnoldi solver typically results in only a small extra memory cost and should be preferred for most problems.

Rayleigh Quotient Iteration (RQI) offers superior performance to Arnoldi for certain very challenging problems. However, this solver often results in very high memory usage, and the stability is not as well understood as for Arnoldi and Power Iteration. RQI should be considered experimental and is not recommended for most users.

The Davidson solver offers extremely fast convergence, but it is currently only available when using the $\mathrm{SP}_{N}$ discretization. Although all of the above solvers are also available with $\mathrm{SP}_{N}$, use of the Davidson solver is always preferred.

With the exception of the Davidson solver, all eigenvalue solvers require the solution of a fixed-source sub-problem as part of the iterative solution process. See the multigroup (page 198) and within-group (page 200) sections for details on the inner loops of the solution process.

Default arnoldi for SN, davidson for SPN

Type power_iteration, arnoldi, rayleigh_quotient, or davidson

Cannot set solver to arnoldi, davidson, or rayleigh_quotient unless 'equations' discretization is linear (not 'twd' or 'wdd_ff'). 
postprocessor

Cannot set solver to davidson unless /denovo/disable is none or transport and /denovo/ method is spn.

parameter subspace_size

Max subspace size for this solver.

Default 20

Type non-negative integer

Applicable when solver is gmres, arnoldi, or davidson

parameter tolerance

parameter tol

L2 convergence tolerance for this solver.

Default 0.001

Type real number inside $(0,1)$

parameter tolerance_relax_factor

Inner tolerance relaxation factor for Arnoldi iteration.

Default 1.0

Type real number inclusive [1.0, 10.0]

\section{Applicable when}

- solver is arnoldi; and

- tolerance_relaxer is constant

parameter tolerance_relaxer

Type of inner tolerance relaxer for Arnoldi iteration.

Default none

Type none or constant

Applicable when solver is arnoldi

database [UPSCATTER]

Upscatter block solver options. See [DENOVO][SOLVER][UPSCATTER] (page 198).

Default (empty database)

Applicable when /denovo/disable is none or transport and /denovo/method is spn; upscattering is enabled; or upscatter_groups is all

parameter upscatter_groups

Which groups to treat as upscatter groups during solve.

Default thermal unless arch is gpu, eigenvalue solver is rayleigh_quotient, energy_sets $>1$, "method" is spn, or upscatter preconditioner is multilevel

Type all or thermal 
postprocessor

CUDA sweeper, rayleigh_quotient solver, SPN, and multilevel upscatter preconditioner require all upscatter groups.

postprocessor

Warn if using downscatter-only physics with upscatter all unless needed.

Applicability upscatter_groups is all

parameter use_energy_dependent

Use energy-dependent Arnoldi iteration.

Default True

Type boolean

Applicable when solver is arnoldi

parameter verbosity

Solver verbosity.

Table 32: Denovo solver verbosity options.

\begin{tabular}{ll}
\hline Level & Description \\
\hline none & Output only warnings from the solver. \\
low & $\begin{array}{l}\text { Additionally, output a diagnostic summary of each (typically } \\
\text { within-group) transport solution. }\end{array}$ \\
medium & $\begin{array}{l}\text { Additionally, output information about the convergence tests } \\
\text { (residual, iteration count) for each solve. }\end{array}$ \\
high & $\begin{array}{l}\text { Additionally, output diagnostic information about each } \\
\text { solver iteration. }\end{array}$ \\
\hline
\end{tabular}

Default low

Type none, low, medium, or high

database [WITHIN_GROUP]

One-group transport solver options. See [DENOVO][SOLVER][WITHIN_GROUP] (page 201).

Default (empty database)

Applicable when upscatter_groups is thermal

\subsubsection{7 [DENOVO][SOLVER][UPSCATTER]}

The parameters and sub-databases in this database control the solve options for the multigroup solve. If method is spn, this database is required and is used to determine all solve options since the $\mathrm{SP}_{N}$ representation used by Denovo is an explicit multigroup sparse matrix rather than an implicit operator such as $S_{N}$.

The available multigroup solvers in Denovo are GMRES (the default) and Gauss-Seidel. These solvers are only used to converge inter-group coupling. When the upscatter (page 194) parameter is set to all, all energy groups are coupled. For problems that have no upscatter and use the thermal coupling option, this database is not used. If the upscatter option is set to thermal, the within-group (page 200) solver settings determine how the high-energy (uncoupled) groups are solved, and this database applies only to the coupled low-energy groups. 
Gauss-Seidel, the traditional multigroup iterative solution method, offers the smallest possible memory footprint of the upscatter solvers at a cost of significantly degraded convergence behavior for many problems. Gauss-Seidel is essentially an extra level of iteration over the upscattering groups.

The GMRES upscatter convergence option typically offers the most rapid, robust convergence. Its solution vector comprises the entire upscatter flux. Consequently, since multiple Krylov vectors are necessary for the method, its memory requirements are higher than Gauss-Seidel. It should also be noted that since the GMRES option is currently incompatible with other features of Denovo such as adjoint solutions and firstcollision sources. The Omnibus input processor should fail informatively if these incompatible conditions are requested.

\section{parameter max_iterations \\ parameter maxitr}

Max number of iterations for this solver.

Default 1000, or 3 when SPN + multilevel

Type non-negative integer

\section{database [PRECONDITIONER]}

Upscatter preconditioner type. See [DENOVO][SOLVER][UPSCATTER][PRECONDITIONER] (page 203).

Default (empty none database)

parameter solver

Solver used for the multigroup block solve.

Default 'gmres' unless mode is adjoint or a nonlinear spatial discretization is being used

Type gauss_seidel or gmres

postprocessor

Cannot set solver to gmres unless 'equations' discretization is linear (not 'twd' or 'wdd_ff').

postprocessor

Cannot set solver to gmres unless problem mode is forward, hybrid, kcode, or raytrace or

/denovo/disable is none or transport and /denovo/method is spn.

postprocessor

Cannot set solver to gauss_seidel unless CUDA sweeper is disabled.

parameter subspace_size

Max subspace size for this solver.

Default 20

Type non-negative integer

Applicable when solver is gmres, arnoldi, or davidson

parameter tolerance

parameter tol

Convergence tolerance for this solver.

Default typically $0.1 *$ parent database tolerance

Type real number inside $(0,1)$ 
postprocessor

Tolerance must be less than or equal to tolerance of parent solver database.

parameter verbosity

Solver verbosity.

Default low, or none when SPN + multilevel

Type none, low, medium, or high

database [WITHIN_GROUP]

One-group transport solver options. See [DENOVO][SOLVER][UPSCATTER][WITHIN_GROUP] (page 203).

Default (empty database)

Applicable when solver is gauss_seidel

\subsubsection{8 [DENOVO][SOLVER][WITHIN_GROUP]}

The parameters and sub-databases in this database control the solver options for single-group solves embedded in the multigroup iteration schemes. The available within-group solvers in Denovo are GMRES (the default), BiCGStab, and source iteration (SI).

GMRES (Generalized Minimal RESidual method) offers fast, robust convergence for most problems. GMRES is a subspace solver, so multiple vectors must be stored simultaneously which results in additional memory requirements. Because a downscatter-only problem solves only a single energy group at a time, the extra memory required is typically small compared to the cost of storing the solution vector over all energy groups.

The performance of BiCGStab (BiConjugate Gradient Stabilized method) is generally similar to that of GMRES. Memory requirements for BiCGStab are smaller than for GMRES, at a cost of typically slightly slower convergence leading to longer runtimes. BiCGStab may be suitable for problems where memory usage must be minimized.

Source iteration offers minimal memory requirements at a cost of significantly worse convergence behavior. It is only recommended for problems in which memory usage is at an absolute premium; it should be expected that run times will be significantly longer when using this method.

parameter matrix_output(advanced)

Matrix output file for Krylov solver.

Default A.h5

Type file path to write (extension '.h5')

Applicable when solver is matrixwriter

parameter max_iterations

parameter maxitr

Max number of iterations for this solver.

Default 1000

Type non-negative integer

database [PRECONDITIONER]

Within-group preconditioner type. See [DENOVO][SOLVER][WITHIN_GROUP][PRECONDITIONER] (page 204). 
Default (empty none database)

parameter solver

Solver used in each within_group solve.

Default gmres if using a linear discretization, otherwise $s i$

Type gmres, gmres_r, si, bicgstab, stratimikos, or matrixwriter

postprocessor

Cannot set solver to bicgstab, gmres, gmres_r, matrixwriter, or stratimikos unless 'equations' discretization is linear (not 'twd' or 'wdd_ff').

parameter subspace_size

Max subspace size for this solver.

\section{Default 20}

Type non-negative integer

Applicable when solver is gmres, arnoldi, or davidson

parameter tolerance

parameter tol

Convergence tolerance for this solver.

Default typically $0.1 *$ parent database tolerance

Type real number inside $(0,1)$

postprocessor

Tolerance must be less than or equal to tolerance of parent solver database.

unvalidated-database [TRILINOS]

Advanced Trilinos solver options. See [DENOVO][SOLVER][WITHIN_GROUP][TRILINOS]

(page 204).

\section{Optional}

parameter verbosity

Solver verbosity.

Default low

Type none, low, medium, or high

\subsubsection{9 [DENOVO][SOLVER][WITHIN_GROUP]}

One-group transport solver options.

parameter matrix_output (advanced)

Matrix output file for Krylov solver.

Default A.h5

Type file path to write (extension '.h5')

Applicable when solver is matrixwriter

parameter max_iterations 
parameter maxitr

Max number of iterations for this solver.

Default 1000

Type non-negative integer

database [PRECONDITIONER]

Within-group preconditioner type. See [DENOVO][SOLVER][WITHIN_GROUP][PRECONDITIONER] (page 204).

Default (empty none database)

parameter solver

Solver used in each within_group solve.

Default gmres if using a linear discretization, otherwise $s i$

Type gmres, gmres_r, si, bicgstab, stratimikos, or matrixwriter

postprocessor

Cannot set solver to bicgstab, gmres, gmres_r, matrixwriter, or stratimikos unless 'equations' discretization is linear (not 'twd' or 'wdd_ff').

parameter subspace_size

Max subspace size for this solver.

\section{Default 20}

Type non-negative integer

Applicable when solver is gmres, arnoldi, or davidson

parameter tolerance

parameter tol

Convergence tolerance for this solver.

Default typically $0.1 *$ parent database tolerance

Type real number inside $(0,1)$

postprocessor

Tolerance must be less than or equal to tolerance of parent solver database.

unvalidated-database [TRILINOS]

Advanced Trilinos solver options. See [DENOVO][SOLVER][WITHIN_GROUP][TRILINOS]

(page 204).

\section{Optional}

parameter verbosity

Solver verbosity.

Default low

Type none, low, medium, or high 


\subsubsection{0 ... [SOLVER][UPSCATTER][PRECONDITIONER]}

Table 33: Available types for the [PRECONDITIONER] database

\begin{tabular}{lll}
\hline Type & Description & Applicability \\
\hline $\begin{array}{l}\text { none (page 204) } \\
\text { multilevel } \\
\text { (page 205) }\end{array}$ & $\begin{array}{l}\text { No preconditioner } \\
\text { Multilevel preconditioner }\end{array}$ & $\begin{array}{l}\text { solver is gmres } \\
\text { twogrid (page 206) }\end{array}$ \\
$\begin{array}{l}\text { Two-grid energy precondi- } \\
\text { tioner }\end{array}$ & $\begin{array}{l}\text { /denovo/disable is none or transport } \\
\text { and /denovo/method is sn and solver is } \\
\text { gauss_seidel } \\
\text { ifpack (page 206) }\end{array}$ & ifpack energy preconditioner \\
ml (page 206) & ml energy preconditioner & $\begin{array}{l}\text { denovo/method is spn } \\
\text { /denovo/disable is none or transport and / } \\
\text { denovo/method is spn }\end{array}$ \\
\hline
\end{tabular}

\subsubsection{1 ...[SOLVER][UPSCATTER][WITHIN_GROUP]}

One-group transport solver options.

parameter matrix_output (advanced)

Matrix output file for Krylov solver.

Default A.h5

Type file path to write (extension '.h5')

Applicable when solver is matrixwriter

parameter $\max \_$iterations

parameter maxitr

Max number of iterations for this solver.

Default 1000

Type non-negative integer

database [PRECONDITIONER]

Within-group preconditioner type. See [DENOVO][SOLVER][WITHIN_GROUP][PRECONDITIONER] (page 204).

Default (empty none database)

parameter solver

Solver used in each within_group solve.

Default gmres if using a linear discretization, otherwise $s i$

Type gmres, gmres_r, si, bicgstab, stratimikos, or matrixwriter

postprocessor

Cannot set solver to bicgstab, gmres, gmres_r, matrixwriter, or stratimikos unless 'equations' discretization is linear (not 'twd' or 'wdd_ff').

parameter subspace_size

Max subspace size for this solver. 


\section{Default 20}

Type non-negative integer

Applicable when solver is gmres, arnoldi, or davidson

\section{parameter tolerance}

parameter tol

Convergence tolerance for this solver.

Default typically $0.1 *$ parent database tolerance

Type real number inside $(0,1)$

postprocessor

Tolerance must be less than or equal to tolerance of parent solver database.

unvalidated-database [TRILINOS]

Advanced Trilinos solver options. See [DENOVO][SOLVER][WITHIN_GROUP][TRILINOS] (page 204).

\section{Optional}

parameter verbosity

Solver verbosity.

Default low

Type none, low, medium, or high

\subsubsection{2 ...[SOLVER][WITHIN_GROUP][PRECONDITIONER]}

Table 34: Available types for the [PRECONDITIONER] database

\begin{tabular}{lll}
\hline Type & Description & Applicability \\
\hline none (page 206) & No preconditioner & \\
\hline
\end{tabular}

\subsubsection{3 ...[SOLVER][WITHIN_GROUP][TRILINOS]}

Advanced Trilinos solver options.

Note: This database passes its input through exactly as given. If it is defined in an ASCII (.omn) input file, all embedded parameters will be propagated as strings, which is usually not the desired behavior. Users should only include this database when the input file is a Python script or JSON file (or alternatively using the advanced Python interface to modify an ASCII input).

\subsubsection{4 ...[SOLVER][UPSCATTER][PRECONDITIONER=NONE]}

No preconditioner. 


\subsubsection{5 ...[SOLVER][UPSCATTER][PRECONDITIONER=MULTILEVEL]}

Multilevel preconditioner.

parameter depth_v_cycle

Level of depth.

Default $\log _{2}\left(N_{g}-1\right)+2$

Type positive integer

Applicable when /denovo/disable is none or transport and /denovo/method is sn parameter num_v_cycles

Number of V-cycles.

Default 1

Type positive integer

Applicable when /denovo/disable is none or transport and /denovo/method is sn database [QUADRATURE]

Discrete ordinates quadrature. See [DENOVO][QUADRATURE] (page 184).

Default (empty database)

Applicable when /denovo/disable is none or transport and /denovo/method is sn parameter relax_count

Number of rounds of weighted richardson iterations.

Default 1

Type positive integer

Applicable when /denovo/disable is none or transport and /denovo/method is sn parameter relax_weight

Weight for richardson iteration.

Default 1.0

Type positive real number

Applicable when /denovo/disable is none or transport and /denovo/method is sn database [SMOOTHER]

Multigrid smoother. See [DENOVO][SOLVER][UPSCATTER][PRECONDITIONER][SMOOTHER] (page 207).

\section{Optional}

Applicable when /denovo/disable is none or transport and /denovo/method is spn 


\subsubsection{6 ...[SOLVER][UPSCATTER][PRECONDITIONER=TWOGRID]}

Two-grid energy preconditioner.

\section{database [QUADRATURE]}

Discrete ordinates quadrature. See [DENOVO][QUADRATURE] (page 184).

Default (empty database)

Applicable when /denovo/disable is none or transport and /denovo/method is sn

database [WITHIN_GROUP]

One-group transport solver options. See [DENOVO][SOLVER][UPSCATTER][PRECONDITIONER][WITHIN_GROL (page 207).

Default (empty database)

\subsubsection{7 ...[SOLVER][UPSCATTER][PRECONDITIONER=IFPACK]}

ifpack energy preconditioner.

\section{database [QUADRATURE]}

Discrete ordinates quadrature. See [DENOVO][QUADRATURE] (page 184).

Default (empty database)

Applicable when /denovo/disable is none or transport and /denovo/method is sn

\section{database [WITHIN_GROUP]}

One-group transport solver options. See [DENOVO][SOLVER][UPSCATTER][PRECONDITIONER][WITHIN_GROL (page 209).

Default (empty database)

\subsubsection{8 $\ldots$.. [SOLVER][UPSCATTER][PRECONDITIONER=ML]}

$\mathrm{ml}$ energy preconditioner.

\section{database [QUADRATURE]}

Discrete ordinates quadrature. See [DENOVO][QUADRATURE] (page 184).

Default (empty database)

Applicable when /denovo/disable is none or transport and /denovo/method is sn

\section{database [WITHIN_GROUP]}

One-group transport solver options. See [DENOVO][SOLVER][UPSCATTER][PRECONDITIONER][WITHIN_GROL (page 210).

Default (empty database)

\subsubsection{9 ...[SOLVER][WITHIN_GROUP][PRECONDITIONER=NONE]}

No preconditioner. 


\subsubsection{0 ...[UPSCATTER][PRECONDITIONER][SMOOTHER]}

Multigrid smoother.

parameter $\max \_$iterations

parameter maxitr

Max number of iterations for this solver.

Default 1000

Type non-negative integer

database [PRECONDITIONER]

Smoother preconditioner type. See [DENOVO][SOLVER][UPSCATTER][PRECONDITIONER][SMOOTHER][PREC (page 211).

Default (empty none database)

parameter solver

Solver used for smoother solve.

Default gmres

Type gmres, si, bicgstab, or stratimikos

parameter subspace_size

Max subspace size for this solver.

Default 20

Type non-negative integer

Applicable when solver is gmres, arnoldi, or davidson

parameter tolerance

parameter tol

Convergence tolerance for this solver.

Default typically $0.1 *$ parent database tolerance

Type real number inside $(0,1)$

postprocessor

Tolerance must be less than or equal to tolerance of parent solver database.

parameter verbosity

Solver verbosity.

Default low

Type none, low, medium, or high

\subsubsection{1 ...[UPSCATTER][PRECONDITIONER][WITHIN_GROUP]}

One-group transport solver options.

parameter matrix_output(advanced)

Matrix output file for Krylov solver.

Default A.h5 
Type file path to write (extension '.h5')

Applicable when solver is matrixwriter

parameter max_iterations

parameter maxitr

Max number of iterations for this solver.

Default 1000

Type non-negative integer

database [PRECONDITIONER]

Within-group preconditioner type. See [DENOVO][SOLVER][WITHIN_GROUP][PRECONDITIONER] (page 204).

Default (empty none database)

parameter solver

Solver used in each within_group solve.

Default gmres if using a linear discretization, otherwise $s i$

Type gmres, gmres_r, si, bicgstab, stratimikos, or matrixwriter

postprocessor

Cannot set solver to bicgstab, gmres, gmres_r, matrixwriter, or stratimikos unless 'equa-

tions' discretization is linear (not 'twd' or 'wdd_ff').

parameter subspace_size

Max subspace size for this solver.

Default 20

Type non-negative integer

Applicable when solver is gmres, arnoldi, or davidson

parameter tolerance

parameter tol

Convergence tolerance for this solver.

Default typically $0.1 *$ parent database tolerance

Type real number inside $(0,1)$

postprocessor

Tolerance must be less than or equal to tolerance of parent solver database.

unvalidated-database [TRILINOS]

Advanced Trilinos solver options. See [DENOVO][SOLVER][WITHIN_GROUP][TRILINOS] (page 204).

\section{Optional}

parameter verbosity

Solver verbosity.

Default low

Type none, low, medium, or high 


\subsubsection{2 ...[UPSCATTER][PRECONDITIONER][WITHIN_GROUP]}

One-group transport solver options.

parameter matrix_output(advanced)

Matrix output file for Krylov solver.

Default A.h5

Type file path to write (extension '.h5')

Applicable when solver is matrixwriter

parameter max_iterations

parameter maxitr

Max number of iterations for this solver.

Default 1000

Type non-negative integer

database [PRECONDITIONER]

Within-group preconditioner type. See [DENOVO][SOLVER][WITHIN_GROUP][PRECONDITIONER]

(page 204).

Default (empty none database)

parameter solver

Solver used in each within_group solve.

Default gmres if using a linear discretization, otherwise $s i$

Type gmres, gmres_r, si, bicgstab, stratimikos, or matrixwriter

postprocessor

Cannot set solver to bicgstab, gmres, gmres_r, matrixwriter, or stratimikos unless 'equations' discretization is linear (not 'twd' or 'wdd_ff').

parameter subspace_size

Max subspace size for this solver.

Default 20

Type non-negative integer

Applicable when solver is gmres, arnoldi, or davidson

parameter tolerance

parameter tol

Convergence tolerance for this solver.

Default typically $0.1 *$ parent database tolerance

Type real number inside $(0,1)$

postprocessor

Tolerance must be less than or equal to tolerance of parent solver database.

unvalidated-database [TRILINOS]

Advanced Trilinos solver options. See [DENOVO][SOLVER][WITHIN_GROUP][TRILINOS]

(page 204). 


\section{Optional \\ parameter verbosity}

Solver verbosity.

Default low

Type none, low, medium, or high

\subsubsection{3 ...[UPSCATTER][PRECONDITIONER][WITHIN_GROUP]}

One-group transport solver options.

parameter matrix_output(advanced)

Matrix output file for Krylov solver.

Default A.h5

Type file path to write (extension '.h5')

Applicable when solver is matrixwriter

parameter $\max \_$iterations

parameter maxitr

Max number of iterations for this solver.

Default 1000

Type non-negative integer

database [PRECONDITIONER]

Within-group preconditioner type. See [DENOVO][SOLVER][WITHIN_GROUP][PRECONDITIONER]

(page 204).

Default (empty none database)

parameter solver

Solver used in each within_group solve.

Default gmres if using a linear discretization, otherwise $s i$

Type gmres, gmres_r, si, bicgstab, stratimikos, or matrixwriter

postprocessor

Cannot set solver to bicgstab, gmres, gmres_r, matrixwriter, or stratimikos unless 'equations' discretization is linear (not 'twd' or 'wdd_ff').

parameter subspace_size

Max subspace size for this solver.

Default 20

Type non-negative integer

Applicable when solver is gmres, arnoldi, or davidson

parameter tolerance

parameter tol

Convergence tolerance for this solver. 
Default typically $0.1 *$ parent database tolerance

Type real number inside $(0,1)$

postprocessor

Tolerance must be less than or equal to tolerance of parent solver database.

unvalidated-database [TRILINOS]

Advanced Trilinos solver options. See [DENOVO][SOLVER][WITHIN_GROUP][TRILINOS] (page 204).

\section{Optional}

parameter verbosity

Solver verbosity.

Default low

Type none, low, medium, or high

\subsubsection{4 ...[PRECONDITIONER][SMOOTHER][PRECONDITIONER]}

Table 35: Available types for the [PRECONDITIONER] database

\begin{tabular}{|c|c|c|}
\hline Type & Description & Applicability \\
\hline none (page 211) & No preconditioner & \\
\hline $\begin{array}{l}\text { ifpack } \\
\text { (page 211) }\end{array}$ & $\begin{array}{l}\text { IFPACK algebraic precondi- } \\
\text { tioner }\end{array}$ & $\begin{array}{l}\text { /denovo/disable is none or transport and / } \\
\text { denovo/method is spn }\end{array}$ \\
\hline $\mathrm{ml}$ (page 211) & ML multi-level preconditioner & $\begin{array}{l}\text { /denovo/disable is none or transport and / } \\
\text { denovo/method is spn }\end{array}$ \\
\hline
\end{tabular}

\subsubsection{5 ...[PRECONDITIONER][SMOOTHER][PRECONDITIONER=NONE]}

No preconditioner.

\subsubsection{6 ...[PRECONDITIONER][SMOOTHER][PRECONDITIONER=IFPACK]}

IFPACK algebraic preconditioner.

unvalidated-database [TRILINOS]

Advanced Trilinos solver options. See [DENOVO][SOLVER][WITHIN_GROUP][TRILINOS] (page 204).

\section{Optional}

\subsubsection{7 ...[PRECONDITIONER][SMOOTHER][PRECONDITIONER=ML]}

ML multi-level preconditioner.

\section{unvalidated-database [TRILINOS]}

Advanced Trilinos solver options. See [DENOVO][SOLVER][WITHIN_GROUP][TRILINOS] (page 204).

\section{Optional}




\subsection{ORIGEN DEPLETION SOLVER: [DEPLETION]}

Omnibus couples Shift and ORIGEN, a depletion/transmutation analysis code, using ORIGEN's new C++ API and CRAM solver [20]. This new in-memory API avoids the prohibitive cost (on HPC systems) of writing to disk between transport and depletion steps, and it also enables each depletion step to be performed on each depletable region in parallel. Shift uses the same approach as the VESTA code to obtain microscopic per-nuclide reaction rates. This approach tallies ultra-fine-group fluxes in each depletion region and then uses these fluxes to collapse the microscopic continuous-energy cross sections into one-group reaction rates. Shift then sends these reaction rates to ORIGEN for a depletion calculation.

In order to optimize the parallel efficiency of the depletion calculation, the depletable regions on each block are distributed among the available processors, thereby minimizing the number of depletion solves performed on each core. After every core has calculated the new concentrations for its depletion regions, the results are broadcast to every other core on the block.

Shift provides several different transport-depletion coupling schemes and renormalization methods for the burnup calculations, with additional parameters to fine tune the coupling. Only coupling_method should need to be selected in everyday applications. The other coupling parameters (depletion_method, renormalization_method, predictor_substeps, corrector-substeps, predictor_renormalization, calculate_depletion_energy, and cram_internal_substeps) get reasonable defaults, some of which depend on the coupling scheme. Altering them should be considered an advanced feature.

Note: Depletion is currently supported only when using Shift with continuous energy. Deterministic and multigroup depletion are not implemented.

parameter always_transport

Always run transport for each timestep, even during decay.

Default True

Type boolean

parameter burn_length

parameter burn

Burnup lengths for each input step.

Units day

Type list of non-negative floats (each element is a non-negative real number)

parameter calculate_depletion_energy

parameter calc_de

Calculate energy released in depletion to get accurate burnups.

Default True

Type boolean

parameter constant_flux_per_step

parameter $\mathbf{f l u x}$

Constant flux to be applied per burnup step.

Default --- 


\section{Units $\frac{\mathrm{n}}{\mathrm{cm}^{2}}$}

Type list of non-negative floats (each element is a non-negative real number)

\section{parameter corrector_substeps}

Number of substeps on the corrector.

The corrector_substeps parameter specifies the number of depletion substeps to use on the corrector (if applicable). See predictor_substeps for more details. The default value of this parameter depends on the depletion solver, coupling scheme, and renormalization.

Default heuristic values based on coupling method and solver

Type positive integer

Applicable when coupling_method is middlestep, ce/li, le/li, le/qi, triton, or polaris

postprocessor

Cannot set renormalization_method to energy unless calculate_depletion_energy is True. postprocessor

Cannot set renormalization_method to origen unless coupling_method is fully_explicit, middlestep, ce, triton, or polaris.

\section{postprocessor}

Energy-based renormalization is unreliable with the MATREX depletion solver.

\section{parameter coupling_method}

parameter method

Method used to solve the coupled depletion-transport problem.

The coupling_method parameter specifies which method to use for selecting the cross sections over each depletion step or predicting their behavior over the step (when not assumed constant). The same treatment is used for the spatial distribution of the neutron flux. The behavior of the methods is further controlled by the predictor_substeps, corrector_substeps and predictor_renormalization parameters. The options (methods) and their descriptions are the following:

fully_explicit On the predictor, transport is solved at the beginning of step (BOS), and the BOS cross sections are used for the entire step. No corrector.

middlestep A two-transport-solutions-per-step version of the middlestep method. Predictor uses BOS cross sections and flux to obtain middle-of-step compositions. Corrector uses MOS cross sections and flux to deplete for the entire step.

ce A newer implementation of the fully_explicit method.

le On the predictor, transport is solved at BOS and the cross sections are linearly interpolated through the previous step values and the BOS values. No corrector.

ce/li Predictor works as ce. On the corrector, transport is solved at the end of step (EOS), and the cross sections are interpolated linearly through the BOS and EOS values.

le/li Predictor works as le. On the corrector, transport is solved at the end of step (EOS), and the cross sections are interpolated linearly through the BOS and EOS values. 
le/qi Predictor works as le. On the corrector, transport is solved at the end of step (EOS), and the cross sections are interpolated quadratically through the previous step, BOS, and EOS values.

triton As middlestep except that after the first step, there is no transport calculation on the predictor. Instead, the cross sections and flux from the middle of the previous step are used. Note that this method outputs on a different time grid than the others.

polaris Predictor works as ce. On the corrector, transport is solved at the end of step (EOS), and the EOS cross sections and flux are used to re-deplete the materials. The average of the EOS compositions obtained on the predictor and corrector becomes the initial composition for the next step.

The labels ce, le, li, and qi come from constant extrapolation, linear extrapolation, linear interpolation, and quadratic interpolation, respectively. They stand for the approximation used for the time development of the cross sections and flux on the predictor or corrector.

The methods le, le/li, and le/qi are more accurate than the rest but also sensitive to large (factor of 35 or more) changes in step lengths. These methods will default to lower-order methods when previous step data is not available, or is not applicable due to changed normalization. le/li and le/qi are the most accurate, but if steps need to be short due to desired output, le might be preferable as it takes half as long per step (but needs more then twice as many steps to reach given accuracy).

Regardless of the choice of coupling_method, pure decay steps with zero flux will always be solved with ce, as it is the simplest method and still exact in the absence of neutron flux.

\section{Default fully_explicit}

Type fully_explicit, middlestep, ce, le, ce/li, le/li, le/qi, triton, or polaris

parameter cram_internal_substeps

Number of internal substeps in the CRAM depletion solver.

This option is only applied when there are fewer coupling substeps.

The cram_internal_substeps parameter specifies the minimum number of substeps that CRAM depletion solver should use on each step for depletion accuracy purposes. If this amount is larger than the number of regular substeps, the CRAM solver uses a feature called internal substeps to meet the amount specified here.

Internal substeps do not involve different cross sections or renormalization, but they are much faster than regular substeps. The number of internal substeps to apply on each regular substep is selected so that the total number of internal substeps over all substeps adds up to at least cram_internal_substeps.

The default value is 2 .

\section{Default 2}

Type positive integer

Applicable when depletion_solver is cram

parameter cram_order

Order of the CRAM depletion solver.

\section{Default 16}


Type positive integer

Applicable when depletion_solver is cram

parameter decay_length

parameter decay

Decay lengths for each input step.

Default ---

Units day

Type list of non-negative floats (each element is a non-negative real number)

parameter deplete_cells

parameter cells

Labels of cells to deplete instead of all fissionable cells, or * to indicate all cells.

By default, when depletion is enabled, all "depletable" cells will be tracked and depleted. (Depletable cells correspond to materials with fissionable materials, or if a [COMP] block is used, materials with the depletable flag set.) This parameter overrides this default: cells with the listed labels will have their materials depleted.

Default ---

Type list of cell names (each element is a string)

parameter deplete_nuclides

parameter nuclides

List of nuclides to deplete. All cells containing these nuclides will be depleted. Can be used together with 'deplete_cells.'

\section{Default ---}

Type list of nuclides in the TRITON nuclide set (each element is a nuclide specifier (e.g., $\mathrm{U}-235,92235, \mathrm{u} 235, \mathrm{u}-235 \mathrm{~m} 1)$ )

parameter depletion_solver

Depletion solver type.

Default cram

Type cram or matrex

parameter group_bounds

Energy bin boundaries for depletion tally.

\section{Default ---}

Units $\mathrm{eV}$

Type nonnegative floats in decreasing order (each element is a non-negative real number) parameter jeff_library

Filepath to an ORIGEN JEFF multigroup file.

Default ' $/$. /origen.rev 01 . jeff252g'

Type library path 
parameter kappa_library

Filepath to an HDF5 file containing kappa values.

\section{Optional}

Type file path for reading (extension '.h5')

parameter max_burn_substep_size

Maximum substep size for an ORIGEN time step of non-zero power/flux.

Default 40.0

Units day

Type non-negative real number

parameter max_decay_substep_size

Maximum substep size for an ORIGEN time step of zero power/flux.

Default 75.0

Units day

Type non-negative real number

parameter max_step

Maximum time before automatically increasing the number of steps.

Default 400.0

Units day

Type non-negative real number

database [MICRO]

Tally microscopic cross sections. See [DEPLETION][MICRO] (page 223).

\section{Optional}

sublist [MOVE]

Time-dependent geometry movement. See [DEPLETION][MOVE] (page 223).

\section{Optional}

parameter nuclide_filter_threshold

Threshold at which nuclides below are removed from transport.

Default appropriate filtering threshold for the filter type

Type non-negative real number

Applicable when filtering nuclides

parameter nuclide_filter_type

How to filter nuclides for transport calculations.

Default none

Type none, number_density, absorption, or total 
parameter num_burn_steps

Number of steps to take for each burn length entry.

The num_burn_steps parameter must have the same length as burn_length and decay_length. It is the number of constant-flux calculations per entry. Increasing the number will increase the accuracy of the answer by having better approximations of the depleted concentrations during the transport step, but it will increase the computational cost because more transport calculations must be performed.

\section{Default ---}

Type list in which each element is a non-negative integer

parameter num_decay_steps

Number of steps to take for each decay length entry.

\section{Default ---}

Type list in which each element is a non-negative integer

parameter origen_library

Filepath to an ORIGEN library file.

Default ' $/ \ldots /$ pwr.rev03.orglib'

Type library path

parameter power

Constant power to be applied per burnup step.

\section{Default ---}

\section{Units MW}

Type list of non-negative floats (each element is a non-negative real number)

postprocessor

The parameters burn_length, decay_length, power, num_burn_steps, and num_decay_steps must have the same length. Empty lists are ignored.

parameter predictor_renormalization

Perform renormalization on the predictor.

Default true only if using Polaris coupling

Type boolean

Applicable when coupling_method is middlestep, ce/li, le/li, le/qi, triton, or polaris

\section{parameter predictor_substeps}

Number of substeps on the predictor.

The predictor_substeps parameter specifies the number of depletion substeps to use on the predictor. Substeps are always equidistant except for long zero- flux steps with MATREX, in which case each substep will be thrice as long as the previous.

Substeps serve two roles:

1. The depletion for each substep uses cross sections and flux averaged over that substeps from the prediction made in the coupling scheme. 
2. The neutron flux is renormalized at each substep using the compositions, cross sections, and flux/power distribution for that substep.

In addition, the number of substeps affects the depletion solver's accuracy. The MATREX depletion solver requires a sufficient number of substeps that are not too long. The number of substeps is automatically incremented on a step-by-step basis to meet these requirements. Decay steps with the CRAM solver always use one only substep. See the parameter cram_internal_substeps.

The default value of this parameter depends on the depletion solver, coupling scheme, and renormalization.

Default heuristic values based on coupling method and solver

Type positive integer

parameter print_filtering

Print statistics about nuclide filtering.

Default False

Type boolean

parameter renormalization_method

How to renormalize the flux at each substep.

Which renormalization method (if any) to use on each substep of depletion calculations. The following options are available:

none No renormalization is performed.

boss Beginning-of-substep cross sections and compositions are used for the renormalization. This method is inaccurate and should not be used.

moss Middle-of-substep cross sections and compositions are used for the renormalization.

energy Renormalization is based on the energy released during depletion. This method is very accurate with CRAM, although the difference to moss has no practical significance if substeps are used. There is no proof that this would always be accurate with MATREX.

origen ORIGEN-style renormalization where power distribution is assumed to remain constant through the step.

The default is energy if using CRAM and moss if using MATREX or if calculate_depletion_energy is false.

Default energy if allowable, else moss

Type none, boss, moss, energy, or origen

parameter reset_inactive_cycles

Number of inactive cycles to run for all transport calculations except the initial calculation.

Default -1

Type integer

parameter tracking_nuclides

Nuclides that will be tracked for depletion. 


\section{Default ---}

Type list of nuclides in the TRITON nuclide set (each element is a nuclide specifier (e.g., $\mathrm{U}-235,92235, \mathrm{u} 235, \mathrm{u}-235 \mathrm{~m} 1)$ )

\section{command tracking_set}

append a set of TRITON nuclides to tracking_nuclides.

The tracking_set command exposes the TRITON addnux option to the user.

tracking_set none adds no extra nuclides to track (the default).

tracking_set addnux 1 corresponds to addnux $=1$ and adds:

U-234, U-235, U-236, U-238, Np-237, Pu-238, Pu-239, Pu-240, Pu-241, Pu-242, Am-241, Am-242, Am-243, Cm-242, Cm-243

tracking_set addnux -2 corresponds to addnux $=-2$ and adds the above nuclides as well as:

H-1, B-10, B-11, N-14, O-16, Kr-83, Zr-94, Nb-93, Mo-95, Tc-99, Ru-106, Rh-103, Rh-105, Ag-109, Sn-126, I-135, Xe-131, Xe-135, Cs-133, Cs-134, Cs-135, Cs-137, Ce-144, Pr-143, Nd-143, Nd-145, Nd-146, Nd-147, Nd-148, Pm-147, Pm-148, Pm-149, Sm-147, Sm-149, Sm-150, Sm-151, Sm-152, Eu-151, Eu-153, Eu-154, Eu-155, Gd-152, Gd-154, Gd-155, Gd-156, Gd-157, Gd-158, Gd-160, Cm-244

tracking_set addnux 2 corresponds to addnux $=2$ and adds the above nuclides as well as:

Zr-91, Zr-93, Zr-95, Zr-96, Nb-95, Mo-97, Mo-98, Mo-99, Mo-100, Ru-101, Ru-102, Ru-103, Ru-104, Pd-105, Pd-107, Pd-108, Cd-113, In-115, I-127, I-129, Xe-133, Ba-140, La-139, Ce-141, Ce-142, Ce-143, Pr-141, Nd-144, Sm-153, Eu-156

tracking_set addnux 3 corresponds to addnux $=3$ and adds the above nuclides as well as:

Ge-72, Ge-73, Ge-74, Ge-76, As-75, Se-76, Se-77, Se-78, Se-80, Se-82, Br-79, Br-81, Kr-80, Kr-82, Kr-84, Kr-85, Kr-86, Rb-85, Rb-86, Rb-87, Sr-84, Sr-86, Sr-87, Sr-88, Sr-89, Sr-90, Y-89, Y-90, Y-91, Zr-90, Zr-92, Nb-94, Mo-92, Mo-94, Mo-96, Ru-96, Ru-98, Ru-99, Ru-100, Ru-105, Pd-102, Pd-104, Pd-106, Pd-110, Ag-107, Ag-111, Cd-106, Cd-108, Cd-110, Cd-111, Cd-112, Cd-114, Cd-115m, Cd-116, In-113, Sn-112, Sn-114, Sn-115, Sn-116, Sn-117, Sn-118, Sn-119, Sn-120, Sn-122, Sn-123, Sn-124, Sn-125, Sb-121, Sb123, Sb-124, Sb-125, Sb-126, Te-120, Te-122, Te-123, Te-124, Te-125, Te-126, Te-127m, Te-128, Te-129m, Te-130, Te-132, I-130, I-131, Xe-124, Xe-126, Xe-128, Xe-129, Xe-130, Xe-132, Xe-134, Xe-136, Cs-136, Ba-134, Ba-135, Ba-136, Ba-137, Ba-138, La-140, Ce140, Pr-142, Nd-142, Nd-150, Pm-151, Sm-144, Sm-148, Sm-154, Eu-152, Eu-157, Tb-159, Tb-160, Dy-160, Dy-161, Dy-162, Dy-163, Dy-164, Ho-165, Er-166, Er-167, Lu-175, Lu-176, Ta-181, W-182, W-183, W-184, W-186, Re-185, Re-187, Au-197, Th-230, Th-232, Pa-231, Pa-233, U-232, U-233

tracking_set addnux 4 corresponds to addnux $=4$ and adds the above nuclides as well as:

H-2, H-3, He-3, He-4, Li-6, Li-7, Be-7, Be-9, N-15, O-17, F-19, Na-23, Mg-24, Mg-25, Mg-26, Al-27, Si-28, Si-29, Si-30, P-31, S-32, S-33, S-34, S-36, Cl-35, Cl-37, Ar-36, Ar-38, Ar-40, Ka-39, Ka-40, Ka-41, Ca-40, Ca-42, Ca-43, Ca-44, Ca-46, Ca-48, Sc-45, Ti-46, Ti-47, Ti-48, Ti-49, Ti-50, Cr-50, Cr-52, Cr-53, Cr-54, Mn-55, Fe-54, Fe-56, Fe-57, Fe-58, Co-58m, Co-58, Co-59, Ni-58, Ni-59, Ni-60, Ni-61, Ni-62, Ni-64, Cu-63, Cu-65, Ga-69, Ga-71, Ge-70, As-74, Se-74, Se-79, Kr-78, Ag-110m, Sn-113, Xe-123, Ba-130, Ba-132, 
Ba-133, La-138, Ce-136, Ce-138, Ce-139, Pm-148m, Gd-153, Dy-156, Dy-158, Ho-166m, Er-162, Er-164, Er-168, Er-170, Hf-174, Hf-176, Hf-177, Hf-178, Hf-179, Hf-180, Ta-182, Ir-191, Ir-193, Hg-196, Hg-198, Hg-199, Hg-200, Hg-201, Hg-202, Hg-204, Pb-204, Pb206, Pb-207, Pb-208, Bi-209, Ra-223, Ra-224, Ra-225, Ra-226, Ac-225, Ac-226, Ac-227, Th-227, Th-228, Th-229, Th-233, Th-234, Pa-232, U-237, U-239, U-240, U-241, Np-235, Np-236, Np-238, Np-239, Pu-236, Pu-237, Pu-243, Pu-244, Pu-246, Am-242m, Am-244, Am-244m, Cm-241, Cm-245, Cm-246, Cm-247, Cm-248, Cm-249, Cm-250, Bk-249, Bk-250, Cf-249, Cf-250, Cf-251, Cf-252, Cf-253, Cf-254, Es-253, Es-254, Es-255

tracking_set all contains all the nuclides available in ORIGEN, which are the above nuclides as well as:

H-4, He-5, He-6, He-8, Li-8, Li-9, Be-8, Be-10, Be-11, Be-12, B-12, C-0, C-12, N-13, N-16, O-18, O-19, F-20, Ne-20, Ne-21, Ne-22, Ne-23, Na-22, Na-24, Na-24m, Na-25, Mg-27, Mg-28, Al-26, Al-28, Al-29, Al-30, Si-31, Si-32, P-32, P-33, P-34, S-25, S-35, S-37, Cl-36, Cl-38, Cl-38m, Ar-37, Ar-39, Ar-41, Ar-42, Ka-42, Ka-43, Ka-44, Ca-41, Ca-45, Ca-47, Ca-49, Sc-44, Sc-44m, Sc-45m, Sc-46, Sc-46m, Sc-47, Sc-48, Sc-49, Sc-50, Ti-44, Ti-45, Ti-51, V-48, V-49, V-50, V-51, V-52, V-53, V-54, Cr-48, Cr-49, Cr-51, Cr-55, Cr-66, Cr-67, Mn-52, Mn-53, Mn-54, Mn-56, Mn-57, Mn-58, Mn-66, Mn-67, Mn-68, Mn-69, Fe-55, Fe-59, Fe-60, Fe-65, Fe-66, Fe-67, Fe-68, Fe-69, Fe-70, Fe-71, Fe-72, Co-55, Co-56, Co-57, Co-60, Co-60m, Co-61, Co-62, Co-65, Co-66, Co-67, Co-68, Co-69, Co-70, Co-71, Co-72, Co-73, Co-74, Co-75, Ni-56, Ni-57, Ni-63, Ni-65, Ni-66, Ni-67, Ni-68, Ni-69, Ni-70, Ni-71, Ni-72, Ni-73, Ni-74, Ni-75, Ni-76, Ni-77, Ni-78, Cu-62, Cu-64, Cu-66, Cu-67, Cu-68, Cu-68m, Cu-69, Cu-70, Cu-70m, Cu-71, Cu-72, Cu-73, Cu-74, Cu-75, Cu-76, Cu-77, Cu-78, Cu-79, Cu-80, Cu-81, Zn-63, Zn-64, Zn-65, Zn-66, Zn-67, Zn-68, Zn-69, Zn-69m, Zn-70, Zn-71, Zn-71m, Zn-72, Zn-73, Zn-74, Zn-75, Zn-76, Zn-77, Zn-78, Zn-79, Zn-80, Zn-81, Zn-82, Zn-83, Ga-66, Ga-67, Ga-68, Ga-70, Ga-72, Ga-72m, Ga-73, Ga-74, Ga-74m, Ga-75, Ga-76, Ga-77, Ga-78, Ga-79, Ga-80, Ga-81, Ga-82, Ga-83, Ga-84, Ga-85, Ga-86, Ge-66, Ge-67, Ge-68, Ge-69, Ge-71, Ge-71m, Ge-73m, Ge-75, Ge-75m, Ge-77, Ge-77m, Ge-78, Ge-79, Ge-79m, Ge-80, Ge-81m, Ge-81, Ge-82, Ge-83, Ge-84, Ge-85, Ge-86, Ge-87, Ge-88, Ge-89, As-69, As-71, As-72, As-73, As-75m, As76, As-77, As-78, As-79, As-80, As-81, As-82, As-82m, As-83, As-84, As-84m, As-85, As-86, As-87, As-88, As-89, As-90, As-91, As-92, Se-72, Se-73, Se-73m, Se-75, Se-77m, Se-79m, Se-81, Se-81m, Se-83m, Se-83, Se-84, Se-85, Se-86, Se-87, Se-88, Se-89, Se-90, Se-91, Se-92, Se-93, Se-94, Br-75, Br-76, Br-77, Br-77m, Br-78, Br-79m, Br-80, Br-80m, Br-82, Br-82m, Br-83, Br-84, Br-84m, Br-85, Br-86, Br-87, Br-88, Br-89, Br-90, Br-91, Br-92, Br-93, Br-94, Br-95, Br-96, Br-97, Kr-76, Kr-77, Kr-79m, Kr-79, Kr-81, Kr-81m, Kr-83m, Kr-85m, Kr-87, Kr-88, Kr-89, Kr-90, Kr-91, Kr-92, Kr-93, Kr-94, Kr-95, Kr-96, Kr-97, Kr-98, Kr-99, Kr-100, Rb-79, Rb-81, Rb-82, Rb-83, Rb-84, Rb-86m, Rb-88, Rb89, Rb-90, Rb-90m, Rb-91, Rb-92, Rb-93, Rb-94, Rb-95, Rb-96, Rb-97, Rb-98, Rb-99, Rb-100, Rb-101, Rb-102, Sr-82, Sr-83, Sr-85, Sr-85m, Sr-87m, Sr-91, Sr-92, Sr-93, Sr-94, Sr-95, Sr-96, Sr-97, Sr-98, Sr-99, Sr-100, Sr-101, Sr-102, Sr-103, Sr-104, Sr-105, Y-85, Y-86, Y-87, Y-87m, Y-88, Y-89m, Y-90m, Y-91m, Y-92, Y-93, Y-93m, Y-94, Y-95, Y-96, Y-96m, Y-97, Y-97m, Y-98, Y-98m, Y-99, Y-100, Y-101, Y-102, Y-103, Y-104, Y-105, Y-106, Y-107, Y-108, Zr-86, Zr-87, Zr-88, Zr-89, Zr-89m, Zr-90m, Zr-97, Zr-98, Zr-99, Zr-100, Zr-101, Zr-102, Zr-103, Zr-104, Zr-105, Zr-106, Zr-107, Zr-108, Zr-109, Zr-110, Nb-89, Nb-90, Nb-91, Nb-91m, Nb-92, Nb-92m, Nb-93m, Nb-94m, Nb-95m, Nb-96, Nb-97, Nb-97m, Nb-98, Nb-98m, Nb-99, Nb-99m, Nb-100, Nb-100m, Nb-101, Nb-102, Nb-102m, Nb-103, Nb-104, Nb-104m, Nb-105, Nb-106, Nb-107, Nb-108, Nb-109, Nb-110, 
Nb-111, Nb-112, Nb-113, Mo-90, Mo-91, Mo-93, Mo-93m, Mo-101, Mo-102, Mo-103, Mo-104, Mo-105, Mo-106, Mo-107, Mo-108, Mo-109, Mo-110, Mo-111, Mo-112, Mo-113, Mo-114, Mo-115, Tc-93, Tc-95, Tc-95m, Tc-96, Tc-97, Tc-97m, Tc-98, Tc-99m, Tc-100, Tc-101, Tc-102, Tc-102m, Tc-103, Tc-104, Tc-105, Tc-106, Tc-107, Tc-108, Tc-109, Tc-110, Tc-111, Tc-112, Tc-113, Tc-114, Tc-115, Tc-116, Tc-117, Tc-118, Ru-95, Ru-97, Ru-107, Ru-108, Ru-109, Ru-109m, Ru-110, Ru-111, Ru-112, Ru-113, Ru-114, Ru-115, Ru-116, Ru-117, Ru-118, Ru-119, Ru-120, Rh-99, Rh-99m, Rh-100, Rh-101, Rh-101m, Rh-102, Rh-102m, Rh-103m, Rh-104, Rh-104m, Rh-105m, Rh-106, Rh-106m, Rh-107, Rh-108, Rh-108m, Rh-109, Rh-109m, Rh-110, Rh-110m, Rh-111, Rh-112, Rh-113, Rh114, Rh-115, Rh-116, Rh-117, Rh-118, Rh-119, Rh-120, Rh-121, Rh-122, Rh-123, Pd-99, Pd-100, Pd-101, Pd-103, Pd-107m, Pd-109, Pd-109m, Pd-111, Pd-111m, Pd-112, Pd-113, Pd-114, Pd-115, Pd-116, Pd-117, Pd-118, Pd-119, Pd-120, Pd-121, Pd-122, Pd-123, Pd124, Pd-125, Pd-126, Ag-103, Ag-105, Ag-105m, Ag-106, Ag-106m, Ag-107m, Ag-108, Ag-108m, Ag-109m, Ag-110, Ag-111m, Ag-112, Ag-113, Ag-113m, Ag-114, Ag-115, Ag-115m, Ag-116, Ag-116m, Ag-117, Ag-117m, Ag-118, Ag-118m, Ag-119, Ag-120, Ag-120m, Ag-121, Ag-122, Ag-122m, Ag-123, Ag-124, Ag-125, Ag-126, Ag-127, Ag-128, Ag-129, Ag-130, Cd-105, Cd-107, Cd-109, Cd-111m, Cd-113m, Cd-115, Cd-117, Cd117m, Cd-118, Cd-119, Cd-119m, Cd-120, Cd-121, Cd-121m, Cd-122, Cd-123, Cd-123m, Cd-124, Cd-125, Cd-126, Cd-127, Cd-128, Cd-129, Cd-130, Cd-131, Cd-132, In-107, In-109, In-111m, In-111, In-112, In-112m, In-113m, In-114m, In-114, In-115m, In-116m, In-116, In-117m, In-117, In-118m, In-118, In-119m, In-119, In-120m, In-120, In-121m, In-121, In-122m, In-122, In-123m, In-123, In-124m, In-124, In-125m, In-125, In-126m, In-126, In-127m, In-127, In-128m, In-128, In-129m, In-129, In-130m, In-130, In-131m, In-131, In-132, In-133, In-134, In-135, Sn-111, Sn-113m, Sn-117m, Sn-119m, Sn-121, Sn-121m, Sn-123m, Sn-125m, Sn-127, Sn-127m, Sn-128, Sn-128m, Sn-129, Sn-129m, Sn-130, Sn-130m, Sn-131, Sn-131m, Sn-132, Sn-133, Sn-134, Sn-135, Sn-136, Sn-137, Sb-113, Sb-115m, Sb-115, Sb-117, Sb-118m, Sb-118, Sb-119, Sb-120m, Sb-120, Sb-122m, Sb-122, Sb-124m, Sb-126m, Sb-127, Sb-128m, Sb-128, Sb-129, Sb-130m, Sb-130, Sb-131, Sb-132m, Sb-132, Sb-133, Sb-134m, Sb-134, Sb-135, Sb-136, Sb-137, Sb-138, Sb-139, Te-115, Te-117, Te-118, Te-119m, Te-119, Te-121, Te-121m, Te-123m, Te-125m, Te-127, Te-129, Te-131, Te-131m, Te-133, Te-133m, Te-134, Te-135, Te-136, Te-137, Te-138, Te-139, Te-140, Te-141, Te-142, I-121, I-122, I-123, I-124, I-125, I-126, I-128, I-130m, I-132, I-132m, I-133, I-133m, I-134m, I-134, I-136m, I-136, I-137, I-138, I-139, I-140, I-141, I-142, I-143, I-144, I-145, Xe-122, Xe-125, Xe-125m, Xe-127m, Xe-127, Xe-129m, Xe-131m, Xe-133m, Xe-134m, Xe-135m, Xe-137, Xe-138, Xe-139, Xe-140, Xe-141, Xe142, Xe-143, Xe-144, Xe-145, Xe-145m, Xe-146, Xe-147, Cs-127, Cs-128, Cs-129, Cs-130, Cs-131, Cs-132, Cs-134m, Cs-135m, Cs-136m, Cs-138m, Cs-138, Cs-139, Cs-140, Cs-141, Cs-142, Cs-143, Cs-144, Cs-145, Cs-146, Cs-147, Cs-148, Cs-149, Cs-150, Cs-151, Ba-128, Ba-129, Ba-131, Ba-131m, Ba-133m, Ba-135m, Ba-136m, Ba-137m, Ba-139, Ba-141, Ba142, Ba-143, Ba-144, Ba-145, Ba-146, Ba-147, Ba-148, Ba-149, Ba-150, Ba-151, Ba-152, Ba-153, La-133, La-134, La-135, La-136, La-137, La-141, La-142, La-143, La-144, La145, La-146m, La-146, La-147, La-148, La-149, La-150, La-151, La-152, La-153, La-154, La-155, Ce-134, Ce-135, Ce-137m, Ce-137, Ce-139m, Ce-145, Ce-146, Ce-147, Ce-148, Ce-149, Ce-150, Ce-151, Ce-152, Ce-153, Ce-154, Ce-155, Ce-156, Ce-157, Pr-139, Pr140, Pr-142m, Pr-144m, Pr-144, Pr-145, Pr-146, Pr-147, Pr-148m, Pr-148, Pr-149, Pr-150, Pr-151, Pr-152, Pr-153, Pr-154, Pr-155, Pr-156, Pr-157, Pr-158, Pr-159, Nd-140, Nd-141, Nd-149, Nd-151, Nd-152, Nd-153, Nd-154, Nd-155, Nd-156, Nd-157, Nd-158, Nd-159, Nd-160, Nd-161, Pm-141, Pm-143, Pm-144, Pm-145, Pm-146, Pm-150, Pm-152m, Pm-152, 
Pm-153, Pm-154m, Pm-154, Pm-155, Pm-156, Pm-157, Pm-158, Pm-159, Pm-160, Pm161, Pm-162, Pm-163, Sm-143, Sm-143m, Sm-145, Sm-146, Sm-155, Sm-156, Sm-157, Sm-158, Sm-159, Sm-160, Sm-161, Sm-162, Sm-163, Sm-164, Sm-165, Eu-145, Eu-146, Eu-147, Eu-148, Eu-149, Eu-150m, Eu-150, Eu-152m, Eu-154m, Eu-158, Eu-159, Eu-160, Eu-161, Eu-162, Eu-163, Eu-164, Eu-165, Eu-166, Eu-167, Gd-146, Gd-147, Gd-148, Gd-149, Gd-150, Gd-151, Gd-153m, Gd-155m, Gd-159, Gd-161, Gd-162, Gd-163, Gd-164, Gd-165, Gd-166, Gd-167, Gd-168, Gd-169, Tb-151, Tb-152, Tb-153, Tb-154m, Tb-154, Tb-155, Tb-156m, Tb-156, Tb-157, Tb-158m, Tb-158, Tb-161, Tb-162, Tb-163, Tb-164, Tb-165, Tb-166, Tb-167, Tb-168, Tb-169, Tb-170, Tb-171, Dy-154, Dy-155, Dy-157, Dy-159, Dy-165, Dy-165m, Dy-166, Dy-167, Dy-168, Dy-169, Dy-170, Dy-171, Dy-172, Ho-159m, Ho-159, Ho-160m, Ho-160, Ho-161m, Ho-161, Ho-162m, Ho-162, Ho-163m, Ho-163, Ho-164m, Ho-164, Ho-166, Ho-167, Ho-168, Ho-169, Ho-170m, Ho-170, Ho-171, Ho-172, Er-160, Er-161, Er-163, Er-165, Er-167m, Er-169, Er-171, Er-172, Tm-165, Tm166, Tm-167, Tm-168, Tm-169, Tm-170m, Tm-170, Tm-171, Tm-172, Tm-173, Yb-166, Yb-167, Yb-168, Yb-169m, Yb-169, Yb-170, Yb-171, Yb-172, Yb-173, Yb-174, Yb-175m, Yb-175, Yb-176, Yb-177, Lu-169m, Lu-169, Lu-170, Lu-171m, Lu-171, Lu-172m, Lu-172, Lu-173, Lu-174m, Lu-174, Lu-176m, Lu-177m, Lu-177, Hf-170, Hf-171, Hf-172, Hf-173, Hf-175, Hf-178m, Hf-179m, Hf-180m, Hf-181, Hf-182, Ta-177, Ta-178, Ta-179, Ta-180m, Ta-180, Ta-182m, Ta-183, W-178, W-180, W-181, W-183m, W-185, W-185m, W-187, W-188, W-189, Re-181, Re-182, Re-182m, Re-183, Re-184, Re-184m, Re-186, Re-186m, Re-188, Re-188m, Re-189, Os-182, Os-183, Os-184, Os-185, Os-186, Os-187, Os-188, Os-189, Os-189m, Os-190, Os-190m, Os-191, Os-191m, Os-192, Os-193, Os-194, Ir-185, Ir-186, Ir-188, Ir-189, Ir-189m, Ir-190, Ir-191m, Ir-192, Ir-192m, Ir-193m, Ir-194, Ir-194m, Ir-196, Ir-196m, Pt-188, Pt-189, Pt-190, Pt-191, Pt-192, Pt-193, Pt-193m, Pt-194, Pt-195, Pt-195m, Pt-196, Pt-197, Pt-197m, Pt-198, Pt-199, Pt-199m, Pt-200, Au-193, Au-194, Au-195, Au-195m, Au-196, Au-198, Au-198m, Au-199, Au-199m, Au-200, Au-200m, Hg-193m, Hg-193, Hg-194, Hg-195m, Hg-195, Hg-197, Hg-197m, Hg-199m, Hg-203, Hg-205, Hg-206, Tl-200, Tl-201, Tl-202, Tl-203, Tl-204, Tl-205, Tl-206, Tl-207, Tl-208, Tl-209, Tl-210, Pb-200, Pb-202, Pb-203, Pb-205, Pb-205m, Pb-207m, Pb-209, Pb-210, Pb-211, Pb-212, Pb-214, Bi-205, Bi-206, Bi-207, Bi-208, Bi-210, Bi-210m, Bi-211, Bi-212, Bi-212m, Bi-213, Bi-214, Po-206, Po-207, Po-208, Po-209, Рo-210, Po-211, Po-211m, Po-212, Po-213, Po-214, Po-215, Po-216, Po-218, At-216, At-217, At-218, Rn-216, Rn-217, Rn-218, Rn-219, Rn-220, Rn-222, Fr-220, Fr-221, Fr-222, Fr-223, Ra-220, Ra-222, Ra-227, Ra-228, Ac-224, Ac-228, Th-226, Th-231, Pa-228, Pa-229, Pa-230, Pa-234, Pa-234m, Pa-235, U-230, U-231, Np-234, Np-236m, Np-240, Np-240m, Np-241, Pu-237m, Pu-245, Pu-247, Am-239, Am-240, Am-245, Am-246, Am-247, Cm-240, Cm-251, Bk-245, Bk-246, Bk-247, Bk-248, Bk-248m, Bk-251, Cf-246, Cf-248, Cf-255, Es-251, Es-252, Es-254m

These tables are available in the SCALE 6.1 manual.

Creates tracking_nuclides

parameter $\mathbf{w r i t e \_ m i x t a b l e s}$ (advanced)

Write the transport mixtables.

Default False

Type boolean

parameter write_predictor_data

parameter $\mathbf{w r i t e \_ p}$

Write predictor data. 


\section{Default False}

Type boolean

Applicable when coupling_method is middlestep, ce/li, le/li, le/qi, triton, or polaris

parameter write_xs

Write the collapsed origen XS.

Default False

Type boolean

parameter yield_library

Filepath to an ORIGEN fission yields library file.

Default '/.../origen.rev05.yields.data'

Type library path

\subsection{1 [DEPLETION][MOVE]}

Time-dependent geometry movement.

parameter delta

Distance to move surfaces at the beginning of each step.

Type list in which each element is a real number

postprocessor

Number of movement steps should match depletion steps.

parameter name

Name of the surface group.

Type string without special characters

postprocessor

Move name must correspond to a movable geometry name.

\subsection{2 [DEPLETION][MICRO]}

This creates a "micro" tally to calculate reaction rates for depletion and calculates the material-averaged, flux-weighted cross sections for multiple materials, nuclides, and reactions. The user specifies a list of materials to tally, as well as a list of nuclide/reaction pairs.

The multibinning method is used to calculate any additional rates which are not specified in this tally.

Note: Micro tallies can currently only calculate reaction rates for nuclides that are present in the material.

parameter materials

parameter mats

Materials in which to tally.

Type list in which each element is a non-empty string 
parameter micro_mt

ENDF reactions for microscopic cross section tallying.

Type list in which each element is a MT number or name (e.g., N_GAMMA, 102)

postprocessor

The parameters micro_mt_zaid and micro_mt must have the same length.

parameter micro_mt_zaid

Nuclide IDs for microscopic cross section tallying.

Type list in which each element is a nuclide specifier (e.g., U-235, 92235, u235, u-235m1)

\subsection{HYBRID METHODOLOGY: [HYBRID]}

In computational nuclear engineering, the term "hybrid" describes methods that couple two different classes of approximate methods to obtain a more accurate or faster solution. The primary hybrid method in Exnihilo is to use an approximate Denovo deterministic adjoint solution to generate an important map that reduces the variance of a Shift Monte Carlo calculation using weight windows.

Table 36: Available types for the [HYBRID] database

\begin{tabular}{lll}
\hline Type & Description & Applicability \\
\hline cadis (page 224) & CADIS hybrid method & solver is 'denovo' \\
fwcadis (page 224) & FW-CADIS hybrid method & solver is 'denovo' \\
ww (page 226) & Manually input weight windows & Shift is enabled and Denovo is not \\
\hline
\end{tabular}

\subsection{1 [HYBRID=CADIS]}

Consistent Adjoint Driven Importance Sampling (CADIS) goes beyond the use of weight windows by also applying source biasing. Full details on the theory of this method can be found in [16]. This variance reduction technique is useful when considering local quantities of interest.

In Shift, a Denovo adjoint calculation is first performed to generate the adjoint flux. This adjoint flux is used to automatically generate weight windows and also to consistently bias the source used in the subsequent Shift fixed source calculation. See Shift Omnibus hybrid cadis input section (page 224) for specific input options available when running CADIS with Shift. Also see the Denovo Omnibus input section (page 176) for the input options for Denovo adjoint calculations.

parameter tallies

Tallies to optimize.

Type list in which each element is a string

postprocessor

Validate tally names against [TALLY] blocks.

\subsection{2 [HYBRID=FWCADIS]}

Forward-Weighted Consistent Adjoint Driven Importance Sampling (FW-CADIS) takes the CADIS method a step further by weighting the adjoint source by the inverse of the forward flux. Full details on the theory of this method can be found in [17]. This variance reduction technique is useful for reducing variance of global quantities of interest. 
In Shift, first, a Denovo forward calculation is performed to optimize a response. The forward flux solution is used to determine the adjoint source for the following Denovo adjoint calculation. The procedure then follows the same as that used for CADIS. The adjoint source can be built to optimize for both energy-binned and energy-integrated responses using the energy_treatment (page 225) parameter. Furthermore, two types of weightings are applied: global and pathlength, as determined by the tally_weighting (page 226) parameter. The adjoint source construction for each weighting and energy treatment is as follows:

global weighting, integrated energy treatment

$$
q^{\dagger}(\mathbf{r}, E)=\frac{g(\mathbf{r}) \sigma_{\mathrm{d}}(E)}{\int_{E} \sigma_{\mathrm{d}}(E) \phi(\mathbf{r}, E) d E}
$$

global weighting, binned energy treatment

$$
q^{\dagger}(\mathbf{r}, E)=\frac{g(\mathbf{r})}{\phi(\mathbf{r}, E)}
$$

pathlength weighting, integrated energy treatment

$$
q^{\dagger}(\mathbf{r}, E)=\frac{g(\mathbf{r}) \sigma_{\mathrm{d}}(E)}{\int_{E} \sigma_{\mathrm{d}}(E) \int_{V} \phi(\mathbf{r}, E) d V d E}
$$

pathlength weighting, binned energy treatment

$$
q^{\dagger}(\mathbf{r}, E)=\frac{g(\mathbf{r})}{\int_{V} \phi(\mathbf{r}, E) d V}
$$

In the pathlength weightings, the volume integrals are over the cell tally volume (in the case of cell tallies) or over each tally mesh cell volume (for mesh tallies). Also, $g(\mathbf{r})$ is the volume fraction of a Denovo mesh cell subtended by the tally region. Thus, Denovo cells that are completely enclosed by $V$ have $g=1$, whereas cells completely outside the tally region have $g=0$. For flux tally optimization (see multipliers (page 225)), the detector response is $\sigma_{\mathrm{d}}=1$.

See Shift Omnibus hybrid cadis input section (page 224) for specific input options that are available when running FW-CADIS with Shift along with the input options for Denovo adjoint and forward calculations in the Denovo Omnibus input section (page 176).

parameter energy_treatment

Optimize for energy-integrated or -binned responses.

Default integrated

Type integrated or binned

parameter multipliers

Multipliers to optimize for each tally.

Default Flux for each tally

Type list in which each element is a string

postprocessor

Validate tally multipliers. 
parameter tallies

Tallies to optimize.

If a cell tally is specified, each cell union will be treated as a separate tally to optimize.

Type list in which each element is a string

postprocessor

Validate tally names against [TALLY] blocks.

parameter tally_weighting

parameter weighting

Flux weighting to use for each tally.

The tally weighting can be specified as default, global, or pathlength. The default option is replaced by global for mesh tallies and by pathlength for cell tallies.

Default Default flux weighting for each tally

Type list in which each element is a default, global, or pathlength

postprocessor

Validate tally weighting.

\subsection{3 [HYBRID=WW]}

Importance maps to accelerate Shift calculations can be set manually from a previous Omnibus output file. The field_type parameter selects a field to read from that input file.

\section{deleted field}

Entry field has been deleted: Use 'field_type' instead.

\section{parameter field_type}

Dataset from which to calculate weight windows.

Table 37: Weight window input options.

\begin{tabular}{ll}
\hline Option & Description \\
\hline auto & $\begin{array}{l}\text { Automatically select from the following options based on the } \\
\text { contents of the HDF5 file. This will prefer ww to adjoint } \\
\text { to forward. }\end{array}$ \\
ww & $\begin{array}{l}\text { Use previously calculated weight windows in the hybrid/ } \\
\text { ww group. }\end{array}$ \\
adjoint & $\begin{array}{l}\text { Use a Denovo solution in denovo-adjoint/flux as an im- } \\
\text { portance map, setting weight window centers to the inverse } \\
\text { of the adjoint flux. }\end{array}$ \\
forward & $\begin{array}{l}\text { Use a Denovo solution in denovo-forward/flux directly } \\
\text { as weight window centers, the Cooper-Larsen method. }\end{array}$ \\
\hline
\end{tabular}

The default field type is auto, which will build from previously built weight windows if they are present in the file. If not, the weight windows will be built from the adjoint flux in the file. If neither weight windows nor an adjoint flux is present, the weight windows will be built proportional to the forward flux.

Default auto 
Type auto, forward, adjoint, or ww

parameter input

Manual weight window HDF5 file.

Type file path for reading (extension '.h5')

deleted method

Entry method has been deleted: Use 'field_type' instead.

parameter normalization

parameter norm

Multiplicative normalization for weight windows.

Default 1.0

Type positive real number

Applicable when normalization_method is manual

parameter normalization_method

parameter norm_method

How to normalize weight window centers based on source responses.

Default cadis

Type manual or cadis

\subsection{PRE-EXECUTION UTILITIES: [PRE]}

These utilities are run via omnibus-run on the local system before the Omnibus executable is launched.

sublist [PLOT]

Generate a raytraced slice of the model. See [PRE][PLOT] (page 227).

Default (empty sublist)

\section{Applicable when}

- 'ENABLE_PYTHON_WRAPPERS' is enabled in this CMake build; and

- The 'matplotlib' python package is installed

\subsection{1 [PRE][PLOT]}

This experimental feature uses the Exnihilo python bindings to generate raytraced images of the problem geometry. To generate more customized outputs, the interested user should use the direct Python bindings (see the examples).

Tip: For example, this entry in the front end will generate three slices showing the materials at $z=-10,-5,0$, with the lower left coordinate at $(x, y)=(-15,-20)$ and the upper right coordinate at $(15,20)$ :

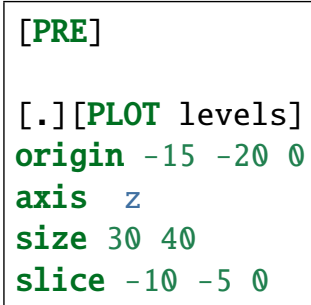


parameter axis

Axis orthogonal to the image.

Default 2

Type axis ('x','y','z')

parameter check

Use error checking during raytrace.

Default False

Type boolean

parameter $\mathbf{c m a p}$ (advanced)

Color map for plotting.

Default rainbow if trace cell else Set3

Type string without special characters

parameter name

Base name of the output file.

Default plotNNN

Type string without special characters

parameter origin

Lower-left corner of the image.

Units $\mathrm{cm}$

Type length-3 float vector (each element is a real number)

parameter render

Save as a standalone rendered PNG.

Default False

Type boolean

Applicable when The 'PIL' python package is installed

parameter resolution

Maximum number of pixels along one axis of the image.

Default 1024

Type positive integer

parameter size

Width and height of the image slice.

Units $\mathrm{cm}$

Type pair of floats (each element is a positive real number)

parameter $\mathbf{s l i c e}$

Positions along 'axis' to take slices, relative to 'origin.' 


\section{Default 0.0}

Type non-empty float list (each element is a real number)

parameter trace

Model property to raytrace.

Default mat

Type cell or mat

\subsection{POST-PROCESSING: [POST]}

The [POST] block controls post-processing of Omnibus output. It extracts data from the HDF5 output file and formats it to be more human-accessible.

database [DENOVO]

Denovo post-processing options. See [POST][DENOVO] (page 230).

Default (empty database)

Applicable when solver is 'denovo'

database [DEPLETION]

Depletion post-processing options. See [POST][DEPLETION] (page 231).

Default (empty database)

Applicable when solver is 'depletion'

parameter $\mathbf{h t m l}$

Convert the ReStructured Text problem summary to HTML.

Default False

Type boolean

Applicable when rst is True

database [HYBRID]

Shift post-processing options. See [POST][HYBRID] (page 231).

Default (empty database)

Applicable when problem mode is hybrid

parameter $\mathbf{r s t}$

Write a ReStructured Text problem summary.

Default True

Type boolean

database [TALLY]

Tally post-processing options. See [POST][TALLY] (page 230).

Default (empty database)

Applicable when

- solver is 'shift'; and

- /shift/do_transport is True 


\subsection{1 [POST][TALLY]}

Tally post-processing options.

parameter $\mathbf{c e l l}$ _csv

Generate CSV files from cell tallies.

Default True

Type boolean

parameter $\mathbf{k c o d e \_ p l o t s}$

Generate keff and convergence plots.

Default True

Type boolean

Applicable when problem mode is kcode

parameter max_csv_size(advanced)

Maximum allowed size of average CSV output file.

Default 500.0

Units kB

Type positive real number

Applicable when cell_csv is True

parameter mesh_xdmf

Generate XDMF files from mesh tallies.

Default True

Type boolean

\subsection{2 [POST][DENOVO]}

Denovo post-processing options.

sublist [SPECTRUM]

Save spectra from a list of $\mathrm{x} / \mathrm{y} / \mathrm{z}$ points. See [POST][DENOVO][SPECTRUM] (page 231).

Default (empty sublist)

Applicable when /denovo/disable is none

parameter $\mathbf{x d m f}$

Create an XDMF file for visualization.

Default True

Type boolean

Applicable when /denovo/disable is none 


\subsection{3 [POST][HYBRID]}

Shift post-processing options.

parameter $\mathbf{w w} \_\mathbf{x d m f}$

Create an XDMF file for weight windows.

Default True

Type boolean

Applicable when /shift/vr/output is True

\subsection{4 [POST][DEPLETION]}

Depletion post-processing options.

parameter $\mathbf{m a x}$ _csv_size(advanced)

Maximum allowed size of average CSV output file.

Default 500.0

Units $\mathrm{kB}$

Type positive real number

Applicable when nd_csv is True or xs_csv is True

parameter $\mathbf{n d}$ _csv

Write Excel-compatible CSV files with number densities.

Default True

Type boolean

parameter $\mathbf{x S}$ _csv

Write CSV files with cross sections.

Default True

Type boolean

Applicable when /depletion/write_xs is True

\subsection{5 [POST][DENOVO][SPECTRUM]}

Save spectra from a list of $\mathrm{x} / \mathrm{y} / \mathrm{z}$ points.

parameter field

Output field from which to save spectra.

Default flux

Type flux, source, or uncflux

postprocessor

The requested field must be enabled in the Denovo output block.

parameter name

Short title or label for the source.

Default spectrumNNN 
Type string without special characters

parameter normalization

Constant multiplicative factor to apply to spectra.

Default 1.0

Type positive real number

parameter on_disk(advanced)

Refrain from loading the solution into memory.

Default False

Type boolean

parameter points

List of $\mathrm{x} / \mathrm{y} / \mathrm{z}$ points.

Type List of space-separated $\mathrm{x} / \mathrm{y} / \mathrm{z}$ tuples (each element is a real number) 


\section{GEOMETRIA INPUT DESCRIPTION}

The Geometria input format is identical to the Omnibus input format and is split into a hierarchy of blocks. All input blocks are described in the following sections.

Note: This documentation was generated automatically with the following version of Exnihilo:

version 6.3.pre-b13 (branch 'master' on 'upstream', r729: \#9809b44f on 2020JUL16)

date 2020-07-16 22:02:43

\subsection{GEOMETRIA INPUT FILE CONTENTS}

Each of the top-level blocks (and the overall problem input file) are described here.

database [GEOMETRY]

Global geometry options. See [GEOMETRY] (page 233).

sublist [UNIVERSE]

Universes. See [UNIVERSE] (page 234).

postprocessor

Universe names in /geometry/global must already have been defined.

\subsection{1 [GEOMETRY]}

Global geometry options.

parameter check_overlapping_volumes

Perform extra (and slow) geometry validation checks during transport.

Default False

Type boolean

parameter composition

parameter comp

Provide an optional explicit mapping for composition names.

Default ---

Type list in which each element is a string

command comps

Map 'comp' to 'matid' from pairs or arrow-separated items.

Creates comp

Creates matid

parameter deduplication_warning

parameter dedupe_warn

Warn about duplicate surfaces being elided.

Default False 
Type boolean

parameter global

Name of the global universe.

Type string without special characters

parameter length_scale

Characteristic length scale of the problem.

Default 1.0

Type positive real number

parameter matid

Matids corresponding to the given commposition names.

Default ---

Type list in which each element is a non-negative integer

postprocessor

The parameters composition and matid must have the same length.

parameter tolerance

parameter tol

Global tolerance for geometry construction and particle bumping.

Default $1 e-08$

Type real number inside $(0,1)$

parameter $\mathbf{w r i t e \_ k d t r e e ( a d v a n c e d )}$

Output the k-D tree representation for each universe.

Default False

Type boolean

\subsection{UNIVERSE DEFINITIONS: [UNIVERSE]}

Universes are analogous to 'units' in KENO: an independent, complete definition of the problem within some region of space. Universes can be reused multiple times via holes (page 243) and arrays (page 237).

Table 38: Available types for the [UNIVERSE] database

\begin{tabular}{lll}
\hline Type & Description & Applicability \\
\hline general (page 235) & General universe & \\
keno6 (page 235) & KENO6 universe & \\
random (page 237) & Randomly constructed universe & \\
array (page 237) & Rectangular array & \\
hexarray (page 239) & Hexagonal array & \\
dodarray (page 241) & Dodecahedral array & Insert an RTK geometry from an external file \\
rtk (page 242) & Insert a VERA-defined reactor core & - \\
core (page 243) & Alias to rtk type & \\
rtkarray & &
\end{tabular}




\subsection{1 [UNIVERSE $=$ GENERAL]}

The "general" universe is a constructive solid geometry universe, essentially equivalent to a "unit" in KENO. Its components are "cells", solid bodies with a single fill material, and "holes", which are other universes embedded into this general universe.

command boundary

Create an interior parameter for a single bounding shape.

Creates interior

sublist [CELL]

Cell definition. See [UNIVERSE][CELL] (page 244).

Default (empty sublist)

sublist [HOLE]

Inserted sub-universes. See [UNIVERSE][HOLE] (page 243).

\section{Optional}

parameter interior

Senses and shape names defining the interior of this universe.

Type list in which each element is a shape with optional leading +- sense

postprocessor

Check that interior shapes have been defined.

parameter name

Name of the universe.

Type string without special characters

sublist [SHAPE]

Geometry shapes. See [UNIVERSE][SHAPE] (page 246).

Default (empty sublist)

\subsection{2 [UNIVERSE $=$ KENO6]}

The "KENO" universe activates features necessary to build KENO geometry definitions using GG. Unlike GG, KENO continually tracks on many geometry layers simultaneously, so that the "topmost" layer's cells are used. This allows holes and arrays to be defined without integrating them into the daughter universes they simply override whatever is there.

GG's tracking engine operates differently: it tracks on only one universe at a time, so that in any universe, a single point in space corresponds to exactly one logical position in that universe, or else it is in the "exterior" of that universe. Particles hitting the exterior are transported to another universe (except in the outermost universe where the boundary condition is applied).

To unify these two tracking types, this special universe uses intersection tests to automatically modify the cell definitions. It uses shape-to-shape intersection tests to determine whether any of the specified holes, arrays, or external boundaries modify the cells interior to the problem. The test for shape $A$ intersecting shape $B$ can return one of five results:

Separate $A$ and $B$ do not intersect. 
Identical $A$ and $B$ are exactly the same shape: their logical definition and surfaces are identical.

Encloses The region in $A$ is a superset of the region of $B$

Enclosed by The region in $B$ is a superset of the region of $A$

Overlaps The two regions may intersect (or one may even enclose the other). This result increases geometry complexity but is the most conservative.

Some of the shape-to-shape intersection tests are more accurate than others. Spheres, aligned cylinders, cuboids, and planes can tell their exact relationship to shapes of the same kind, but it's not always possible to

tell the exact intersection result for some shapes. The conservative case extends cell definitions to exclude the shapes in question.

sublist [ARRAY]

Implicitly truncated arrays. See [UNIVERSE][ARRAY] (page 244).

\section{Optional}

\section{command boundary}

Create an interior parameter for a single bounding shape.

Creates interior

sublist [CELL]

Cell definition. See [UNIVERSE][CELL] (page 244).

Default (empty sublist)

sublist [HOLE]

Inserted sub-universes. See [UNIVERSE][HOLE] (page 243).

\section{Optional}

\section{parameter interior}

Senses and shape names defining the interior of this universe.

Type list in which each element is a shape with optional leading +- sense

postprocessor

Check that interior shapes have been defined.

parameter name

Name of the universe.

Type string without special characters

\section{sublist [SHAPE]}

Geometry shapes. See [UNIVERSE][SHAPE] (page 246).

Default (empty sublist) 


\subsection{3 [UNIVERSE=RANDOM]}

The "random" universe is a procedurally generated universe filled with a given volume fraction of spheres. It is used for HTGRs to model pebbles and TRISO particles.

It is defined by specifying one or more previously defined spherical universes (e.g. particles with different compositions) that will be replicated inside the universe. The number of embedded particles is determined by specifying a volume fraction $v$ such that $N_{i}=\frac{\mathrm{vf}_{i} V_{T}}{V_{i}}$ for particle type $i$ which has volume $V_{i}$ inside an object of volume $V_{t}$.

The region outside the sampled particles is filled with the single composition composition. (If the random universe is a fuel pebble and the particles are TRISO particles, this composition will be the graphite matrix.) Currently, the only shapes supported as bounding shapes are a single cuboid, sphere, and cylinder without any rotations or translations applied.

\section{parameter composition \\ parameter comp}

Composition outside of the particles (the matrix).

Type non-empty string

parameter fill

Names of particle universes to emplace.

Type list in which each element is a string without special characters

postprocessor

Universe names in fill must already have been defined.

parameter name

Name of the universe.

Type string without special characters

database [OPTIONS]

Random universe construction options. See [UNIVERSE][OPTIONS] (page 245).

database [SHAPE]

Geometry shapes. See [UNIVERSE][SHAPE] (page 246).

parameter volume_fraction

parameter $\mathbf{v f}$

Names of particle universes to emplace.

Type fractions that sum to less than one (each element is a real number inside $(0,1)$ )

\subsection{4 [UNIVERSE=ARRAY]}

Rectangular array.

command boundary

Create an interior parameter for a single bounding shape.

Creates interior

parameter $\mathbf{c l i p}$ _composition 
parameter clip

Composition that will replace any clipped array cell.

Optional

Type non-empty string

parameter $\mathbf{f i l l}$

Names of array universe fills, indexed as ZUV.

Type list in which each element is a string without special characters

postprocessor

Universe names in fill must already have been defined.

sublist [HOLE]

Inserted sub-universes. See [UNIVERSE][HOLE] (page 243).

\section{Optional}

parameter interior

Sense/shapes defining the interior of the array.

\section{Optional}

Type list in which each element is a shape with optional leading +- sense

postprocessor

Check that interior shapes have been defined.

parameter name

Name of the universe.

Type string without special characters

parameter $\mathbf{n} \mathbf{x}$

Number of units in the $\mathrm{X}$ direction.

Type positive integer

parameter ny

Number of units in the Y direction.

Type positive integer

parameter $\mathbf{n z}$

Number of units in the $\mathrm{Z}$ direction.

\section{Default 1}

Type positive integer

postprocessor

Check array sizes.

parameter origin

Location of the lower-left corner of the array bounds.

Default 0.00 .00 .0

Type length-3 float vector (each element is a real number) 


\section{parameter origin_is}

Whether origin is the array lower-left or a unit origin.

Default 'array' unless the 'place' parameter is present

Type array or unit

parameter $\mathbf{p l a c e}$

Location of the lower-left corner of the array bounds.

Type length-3 logical position vector (each element is a non-negative integer)

Applicable when origin_is is unit

sublist [SHAPE]

Geometry shapes. See [UNIVERSE][SHAPE] (page 246).

Default (empty sublist)

\subsection{5 [UNIVERSE $=$ HEXARRAY]}

Hexagonal array.

\section{command boundary}

Create an interior parameter for a single bounding shape.

Creates interior

parameter clip_composition

parameter clip

Composition that will replace any clipped array cell.

\section{Optional}

Type non-empty string

parameter fill

Names of array universe fills, indexed as ZUV.

Type list in which each element is a string without special characters

postprocessor

Universe names in fill must already have been defined.

sublist [HOLE]

Inserted sub-universes. See [UNIVERSE][HOLE] (page 243).

\section{Optional}

parameter interior

Sense/shapes defining the interior of the array.

Type list in which each element is a shape with optional leading +- sense

postprocessor

Check that interior shapes have been defined.

parameter layout

Layout of the hex array elements. 


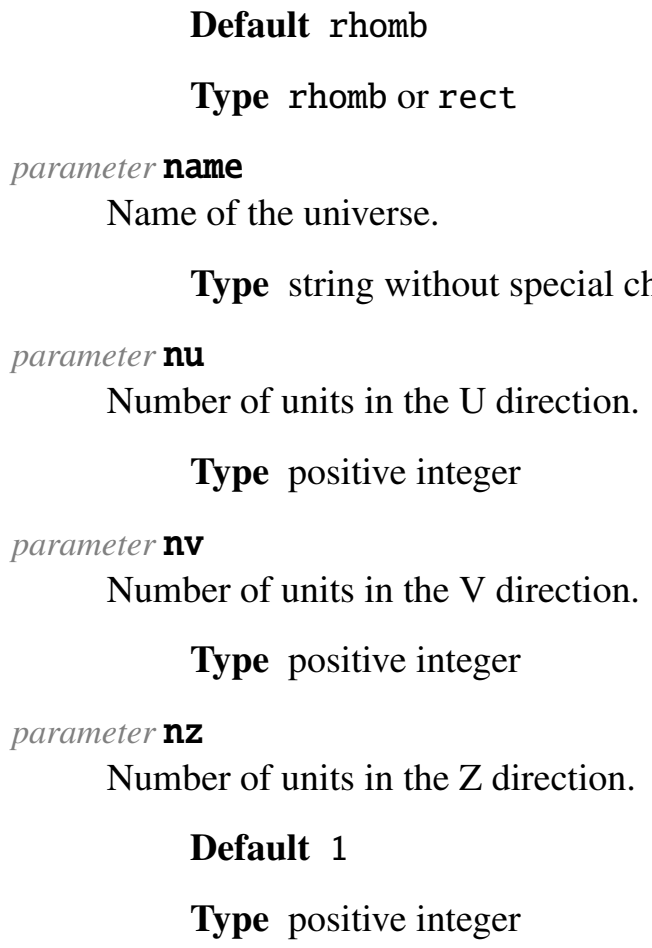

Default rhomb

Type rhomb or rect

parameter name

Name of the universe.

parameter $\mathbf{n u}$

Number of units in the $\mathrm{U}$ direction.

Type positive integer

parameter $\mathbf{n v}$

Number of units in the $\mathrm{V}$ direction.

Type positive integer

parameter $\mathbf{n z}$

Number of units in the $\mathrm{Z}$ direction.

Default 1

Type positive integer

Type string without special characters

postprocessor

Check array sizes.

parameter origin

Location of the lower-left corner of the array bounds.

Default 0.00 .00 .0

Type length-3 float vector (each element is a real number)

parameter origin_is

Whether origin is the array lower-left or a unit origin.

Default 'array' unless the 'place' parameter is present

Type array or unit

parameter $\mathbf{p l a c e}$

Location of the lower-left corner of the array bounds.

Type length-3 logical position vector (each element is a non-negative integer)

Applicable when origin_is is unit

sublist [SHAPE]

Geometry shapes. See [UNIVERSE][SHAPE] (page 246).

Default (empty sublist) 


\subsection{6 [UNIVERSE=DODARRAY]}

Dodecahedral array.

command boundary

Create an interior parameter for a single bounding shape.

Creates interior

parameter clip_composition

parameter $\mathbf{c l i p}$

Composition that will replace any clipped array cell.

\section{Optional}

Type non-empty string

parameter $\mathbf{f i l l}$

Names of array universe fills, indexed as ZUV.

Type list in which each element is a string without special characters

postprocessor

Universe names in fill must already have been defined.

sublist [HOLE]

Inserted sub-universes. See [UNIVERSE][HOLE] (page 243).

\section{Optional}

parameter interior

Sense/shapes defining the interior of the array.

Type list in which each element is a shape with optional leading +- sense

postprocessor

Check that interior shapes have been defined.

parameter name

Name of the universe.

Type string without special characters

parameter $\mathbf{n} \mathbf{x}$

Number of units in the $\mathrm{X}$ direction.

Type positive integer

parameter ny

Number of units in the $\mathrm{Y}$ direction.

Type positive integer

parameter $\mathbf{n z}$

Number of units in the $\mathrm{Z}$ direction.

\section{Default 1}

Type positive integer 
postprocessor

Check array sizes.

parameter origin

Location of the lower-left corner of the array bounds.

Default $0.0 \quad 0.0 \quad 0.0$

Type length-3 float vector (each element is a real number)

parameter origin_is

Whether origin is the array lower-left or a unit origin.

Default 'array' unless the 'place' parameter is present

Type array or unit

parameter $\mathbf{p l a c e}$

Location of the lower-left corner of the array bounds.

Type length-3 logical position vector (each element is a non-negative integer)

Applicable when origin_is is unit

sublist [SHAPE]

Geometry shapes. See [UNIVERSE][SHAPE] (page 246).

Default (empty sublist)

\subsection{7 [UNIVERSE $=$ RTK]}

Insert an RTK geometry from an external file.

command boundary

Create an interior parameter for a single bounding shape.

Creates interior

sublist [HOLE]

Inserted sub-universes. See [UNIVERSE][HOLE] (page 243).

\section{Optional}

parameter input

Path to the RTK geometry XML file.

Type file path for reading (extension '.xml')

parameter interior

Sense/shapes defining the interior of the array.

Type list in which each element is a shape with optional leading +- sense

postprocessor

Check that interior shapes have been defined.

parameter name

Name of the universe.

Type string without special characters

\section{sublist [SHAPE]}

Geometry shapes. See [UNIVERSE][SHAPE] (page 246).

Default (empty sublist) 


\subsection{8 [UNIVERSE $=\mathrm{CORE}]$}

The core universe can only be used when running a VERA (Virtual Environment for Reactor Applications) model in Omnibus or when run externally through the VERA code suite. It inserts the analyst-defined reactor design into a Geometria input for external dosimetry applications.

parameter name

Name of universe.

Type string without special characters

\subsection{9 [UNIVERSE][HOLE]}

Inserted sub-universes.

command euler

Perform an Euler rotation about the $z, x^{\prime}, z^{\prime \prime}$ axes.

Units revolution

Creates rotate

parameter $\mathbf{f i l l}$

Name of the universe to fill this hole with.

Type string without special characters

postprocessor

Universe names in fill must already have been defined.

parameter name

Name of the shape that this hole creates.

Type string without special characters

parameter rotate

Rotation matrix.

Default $1.0 \quad 0.0 \quad 0.0 \quad 0.01 .0 \quad 0.0 \quad 0.0 \quad 0.01 .0$

Type length-9 row-major rotation matrix (each element is a real number)

postprocessor (advanced)

Squelch identity rotations and null translations.

parameter translate

Local-to-global translation vector.

Default 0.00 .00 .0

Type length-3 float vector (each element is a real number) 


\subsubsection{0 [UNIVERSE][CELL]}

Cells are defined as the intersection of one or more regions defined by shapes and holes. Each shape separates space into two half-spaces: "inside" the shape, with a "negative" sense, and "outside" the shape with a "positive" sense. For a shape called sphere, these two senses are written as -sphere (inside) and +sphere (outside). When another universe is placed into this general universe via a hole, it implicitly creates a shape the external boundary of the embedded universe - that can be referenced when defining cells.

The choice for the signs is based on the standard form of the quadric equations, such as the surface of a sphere:

$$
x^{2}+y^{2}+z^{2}-R^{2}=0
$$

When the left-hand side of this equation is positive $(+)$, the point $(x, y, z)$ is outside the sphere; when negative $(-)$, the point is inside. We think of surfaces and shapes as having outward-facing normals; when the projection of a point onto the surface is negative, the point is inside.

parameter composition

parameter comp

Name of the composition that fills this cell.

Type non-empty string

deleted matid

Entry matid has been deleted: 'matid' has been replaced with 'comp', the name of the composition.

parameter name

Name of the cell.

Type string without special characters

parameter shapes

Senses and shape names defining this cell.

Type list in which each element is a shape with optional leading +- sense

postprocessor

Check that shapes have been defined.

parameter volume

Add a pre-calculated volume for this cell.

\section{Optional}

Type real number

\subsubsection{1 [UNIVERSE][ARRAY]}

Implicitly truncated arrays.

command euler

Perform an Euler rotation about the $z, x^{\prime}, z^{\prime \prime}$ axes.

Units revolution

Creates rotate 
parameter fill

Name of the universe to fill this hole with.

Type string without special characters

postprocessor

Universe names in fill must already have been defined.

parameter name

Name of the shape that this hole creates.

Type string without special characters

parameter rotate

Rotation matrix.

Default $1.0 \quad 0.0 \quad 0.0 \quad 0.01 .0 \quad 0.0 \quad 0.0 \quad 0.01 .0$

Type length-9 row-major rotation matrix (each element is a real number)

postprocessor (advanced)

Squelch identity rotations and null translations.

parameter translate

Local-to-global translation vector.

Default $0.0 \quad 0.0 \quad 0.0$

Type length-3 float vector (each element is a real number)

\subsubsection{2 [UNIVERSE][OPTIONS]}

Random universe construction options.

parameter failure_batch_size

Number of samples per batch to test.

Default 100000

Type positive integer

parameter failure_tolerance

Fraction of samples per batch that must be rejected to fail.

Default 0.99999

Type real number inside $(0,1)$

\section{parameter insert_method}

Method for attempting to insert particles into a pebble.

The naive method is the simplest and slowest way to instantiate a loosely packed particle universe. It samples random points inside the universe and rejects them if the sphere around that particle intersects with any particle previously sampled. The naive collision detection scales with $O\left(N^{2}\right)$ for $N$ particles, and the rejection fraction approaches 1 very quickly.

When sampling with the naive method, batches of failure_batch_size samples are tallied; if the fraction of failed samples exceeds failure_tolerance then an error will be raised.

Default naive 


\section{Type naive \\ parameter seed}

Seed value for instantiating this universe.

The seed is used to choose an independent random number stream when instantiating a universe. Different universes' particle placements will be uncorrelated even if they share a seed, but changing the universe's name will change the particle placements, as the actual computational seed value is a combination of the seed given by this parameter and the name of this universe.

\section{Default 0}

Type non-negative integer

\subsection{SHAPE DEFINITIONS: [UNIVERSE][SHAPE]}

Shapes are primitive solid bodies, the constituents of cells. Although this manual does not yet include helpful images for the shape descriptions, the KENO-VI shape description section of the SCALE user manual [2] is essentially equivalent to the input quantities in Geometria. For example, the wedge construction example gives wedge label xbase xpt ypt zlng, and the wedge input block (page 256) defines zlng as an alias for height, xbase as an alias for width, and xy as an alias for corner_pt.

Each shape is the intersection of half-spaces defined by primitive quadric surfaces. When a geometry is instantiated, the shapes are decomposed into their surfaces and volumes are expressed by a logical representation of these surfaces using boolean logic as is typical with a constructive solid geometry definition.

The coordinate system of the shape is defined using a rotation matrix and translation vector: rotations are applied to the shape about its origin, then translations moves the daughter shape relative to the parent universe. The transformation from a point in the shape's coordinate system into the universe's system is

$$
\mathbf{x}_{u}=\mathbf{R} \mathbf{x}_{s}+\mathbf{t}
$$

Where the subscripts $u, s$ refer to the universe and shape coordinate systems, respectively. The vector $\mathbf{t}$ is a translation vector. To transform from the parent into the daughter system we apply the inverse:

$$
\mathbf{x}_{s}=\mathbf{R}^{T}\left(\mathbf{x}_{u}-\mathbf{t}\right) .
$$

(Note that because $\mathbf{R}$ is unitary, the transpose is equal to the inverse.)

The surfaces created by each shape have unique names that can be used to reference them later, or to create reflecting outer boundaries during construction.

Most shapes share many of the same parameters such as "reflect" and "rotate". To reduce duplication, the more detailed documentation is provided for the "cuboid" shape:

- euler (page 247)

- rotate (page 248)

- reflect (page 248)

Table 39: Available types for the [SHAPE] database 


\begin{tabular}{lll}
\hline Type & Description & Applicability \\
\hline cuboid (page 247) & Box shape & - \\
box & Alias to cuboid type & \\
sphere (page 249) & Sphere shape & \\
cyl (page 250) & Cylinder shape & - \\
cylsegment (page 251) & Cylinder segment shape & \\
pad & Alias to cylsegment type & \\
ring (page 252) & Cylindrical shell shape & \\
cylshell & Alias to ring type & \\
prism (page 253) & Regular prism shape & \\
slab (page 255) & Infinite slab shape \\
plane (page 255) & Infinite half-space \\
wedge (page 256) & Wedge shape & \\
cone (page 257) & Cone shape & \\
ellipsoid (page 258) & Ellipsoid shape \\
hopper (page 259) & Hopper shape \\
righttet (page 260) & Right tetrahedron shape \\
triprism (page 261) & Triangular prism shape \\
ecylinder (page 261) & Elliptical cylinder shape \\
rhombdod (page 262) & Rhombic dodecahedron shape \\
ppiped (page 263) & Parallelepiped \\
quadric (page 264) & General quadric \\
\hline
\end{tabular}

\subsection{1 [UNIVERSE][SHAPE $=$ CUBOID $]$}

The possible reflecting faces for the cuboid (chosen via reflect) are:

\begin{tabular}{ll}
\hline Face & Description \\
\hline $\mathrm{mx}$ & lower $x$ face \\
$\mathrm{px}$ & upper $\mathrm{x}$ face \\
$\mathrm{my}$ & lower y face \\
$\mathrm{py}$ & upper y face \\
$\mathrm{mz}$ & lower $\mathrm{z}$ face \\
$\mathrm{pz}$ & upper $\mathrm{z}$ face \\
\hline
\end{tabular}

command euler

Perform an Euler rotation about the $z, x^{\prime}, z^{\prime \prime}$ axes.

See [the Euler angles wikipedia page](https://en.wikipedia.org/wiki/Euler_angles\#Definition_by_ intrinsic_rotations) for a graphic representation of the $z, x^{\prime}, z^{\prime \prime}$ rotation. This command simply generates a rotation matrix.

Units revolution

Creates rotate

command faces

Expand into parameters xmin, xmax, ymin, ymax, zmin, and zmax. 
Creates xmin

Creates xmax

Creates ymin

Creates ymax

Creates zmin

Creates zmax

parameter name

Name of the shape.

Type string without special characters

parameter reflect

Reflecting boundary surfaces.

The reflect option allows a subset of faces on a shape to specularly reflect particles. Enter the single value * to reflect all faces for the given shape.

\section{Default ---}

Type list of shape surfaces (e.g. mx, co) (each element is a string without special characters)

\section{parameter rotate}

Rotation matrix.

The rotation matrix

$$
\mathbf{R}=\left[\begin{array}{lll}
a & b & c \\
d & e & f \\
g & h & i
\end{array}\right]
$$

is applied to a point in the shape's coordinate system as a column vector

$$
\mathbf{R} \mathbf{x}_{s}=\mathbf{R}\left[\begin{array}{l}
x \\
y \\
z
\end{array}\right]
$$

The input to the code is the row-major flattened list $[a, b, c, d, e, f, g, h, i]$.

Default $1.00 .00 .0 \quad 0.01 .00 .00 .0 \quad 0.01 .0$

Type length-9 row-major rotation matrix (each element is a real number)

postprocessor (advanced)

Squelch identity rotations and null translations.

parameter translate

parameter origin

Local-to-global translation vector.

Default $0.0 \quad 0.0 \quad 0.0$

Type length-3 float vector (each element is a real number) 
parameter $\mathbf{x m a x}$

Maximum x-coordinate of box source.

Type real number

postprocessor

Parameter xmin must be less than xmax.

parameter $\mathbf{x m i n}$

Minimum x-coordinate of box source.

Type real number

parameter ymax

Maximum y-coordinate of box source.

Type real number

postprocessor

Parameter ymin must be less than ymax.

parameter $\mathbf{y m i n}$

Minimum y-coordinate of box source.

Type real number

parameter $\mathbf{z m a x}$

Maximum z-coordinate of box source.

Type real number

postprocessor

Parameter zmin must be less than zmax.

parameter zmin

Minimum z-coordinate of box source.

Type real number

\subsection{2 [UNIVERSE][SHAPE=SPHERE]}

Sphere shape.

command euler

Perform an Euler rotation about the $z, x^{\prime}, z^{\prime \prime}$ axes.

Units revolution

Creates rotate

parameter name

Name of the shape.

Type string without special characters

parameter radius

parameter $\mathbf{r}$

Radius of sphere.

Type real number 
parameter reflect

Reflecting boundary surfaces.

Default ---

Type list of shape surfaces (e.g. mx, co) (each element is a string without special characters)

parameter rotate

Rotation matrix.

Default $1.0 \quad 0.0 \quad 0.0 \quad 0.01 .0 \quad 0.0 \quad 0.0 \quad 0.01 .0$

Type length- 9 row-major rotation matrix (each element is a real number)

postprocessor (advanced)

Squelch identity rotations and null translations.

parameter translate

parameter origin

Local-to-global translation vector.

Default $0.00 .0 \quad 0.0$

Type length-3 float vector (each element is a real number)

\subsection{3 [UNIVERSE][SHAPE $=$ CYL]}

Cylinder shape.

parameter axis

Axis along the cylinder.

Type axis (' $\mathrm{x}$ ','y','z')

command euler

Perform an Euler rotation about the $z, x^{\prime}, z^{\prime \prime}$ axes.

Units revolution

Creates rotate

parameter extents

Negative and positive position along the given axis.

Type (min, max) extent values (each element is a real number)

parameter name

Name of the shape.

Type string without special characters

parameter radius

parameter $\mathbf{r}$

Radius of cylinder.

Type real number

parameter reflect

Reflecting boundary surfaces. 


\section{Default ---}

Type list of shape surfaces (e.g. mx, co) (each element is a string without special characters)

parameter rotate

Rotation matrix.

Default $1.0 \quad 0.0 \quad 0.0 \quad 0.01 .0 \quad 0.0 \quad 0.0 \quad 0.01 .0$

Type length-9 row-major rotation matrix (each element is a real number)

postprocessor (advanced)

Squelch identity rotations and null translations.

parameter translate

parameter origin

Local-to-global translation vector.

Default $0.0 \quad 0.0 \quad 0.0$

Type length-3 float vector (each element is a real number)

\subsection{4 [UNIVERSE][SHAPE $=$ CYLSEGMENT $]$}

Cylinder segment shape.

parameter angle

parameter $\mathbf{a}$

Beginning angle CCW from $x=0$.

Units revolution

Type real number inclusive $[0.0,1.0]$

parameter arc

parameter da

Angle subtended by cylindrical segment.

Units revolution

Type real number in $[0.0,0.5]$

deleted begin_angle

deleted $\mathbf{b a}$

Entry begin_angle has been deleted: Replaced by 'angle' in turns not radians.

command euler

Perform an Euler rotation about the $z, x^{\prime}, z^{\prime \prime}$ axes.

Units revolution

Creates rotate

parameter extents

Negative and positive position along the $\mathrm{z}$-axis.

Type (min, max) extent values (each element is a real number)

parameter inner_radius 
parameter $\mathbf{r i}$

Inner radius.

Type positive real number

parameter name

Name of the shape.

Type string without special characters

parameter outer_radius

parameter ro

Outer radius.

Type positive real number

postprocessor

Parameter inner_radius must be less than outer_radius.

parameter reflect

Reflecting boundary surfaces.

Default ---

Type list of shape surfaces (e.g. mx, co) (each element is a string without special characters)

parameter rotate

Rotation matrix.

Default $1.0 \quad 0.0 \quad 0.0 \quad 0.01 .0 \quad 0.0 \quad 0.0 \quad 0.01 .0$

Type length-9 row-major rotation matrix (each element is a real number)

postprocessor (advanced)

Squelch identity rotations and null translations.

deleted solid_angle

deleted sa

Entry solid_angle has been deleted: Replaced by 'arc' in turns not radians.

parameter translate

parameter origin

Local-to-global translation vector.

Default 0.00 .00 .0

Type length-3 float vector (each element is a real number)

\subsection{5 [UNIVERSE][SHAPE $=$ RING]}

Cylindrical shell shape.

command euler

Perform an Euler rotation about the $z, x^{\prime}, z^{\prime \prime}$ axes.

Units revolution

Creates rotate 
parameter extents

Negative and positive position along the $\mathrm{Z}$ axis.

Type (min, max) extent values (each element is a real number)

parameter inner_radius

parameter $\mathbf{r i}$

Inner radius.

Type real number

parameter name

Name of the shape.

Type string without special characters

parameter outer_radius

parameter ro

Outer radius.

Type real number

postprocessor

Parameter inner_radius must be less than outer_radius.

parameter reflect

Reflecting boundary surfaces.

Default ---

Type list of shape surfaces (e.g. mx, co) (each element is a string without special characters)

parameter rotate

Rotation matrix.

Default $1.0 \quad 0.0 \quad 0.0 \quad 0.01 .0 \quad 0.0 \quad 0.0 \quad 0.01 .0$

Type length-9 row-major rotation matrix (each element is a real number)

postprocessor (advanced)

Squelch identity rotations and null translations.

parameter translate

parameter origin

Local-to-global translation vector.

Default 0.00 .00 .0

Type length-3 float vector (each element is a real number)

\subsection{6 [UNIVERSE][SHAPE $=$ PRISM]}

The possible reflecting faces for a prism depend on the number of sides:

\begin{tabular}{ll}
\hline Face & Description \\
\hline $\mathrm{pN}$ & for integer $\mathrm{N}$ in $[0$, num_sides $), \mathrm{CCW}$ from $\mathrm{x}=0$ \\
$\mathrm{mz}$ & lower $\mathrm{z}$ face \\
$\mathrm{pz}$ & upper $\mathrm{z}$ face \\
\hline
\end{tabular}




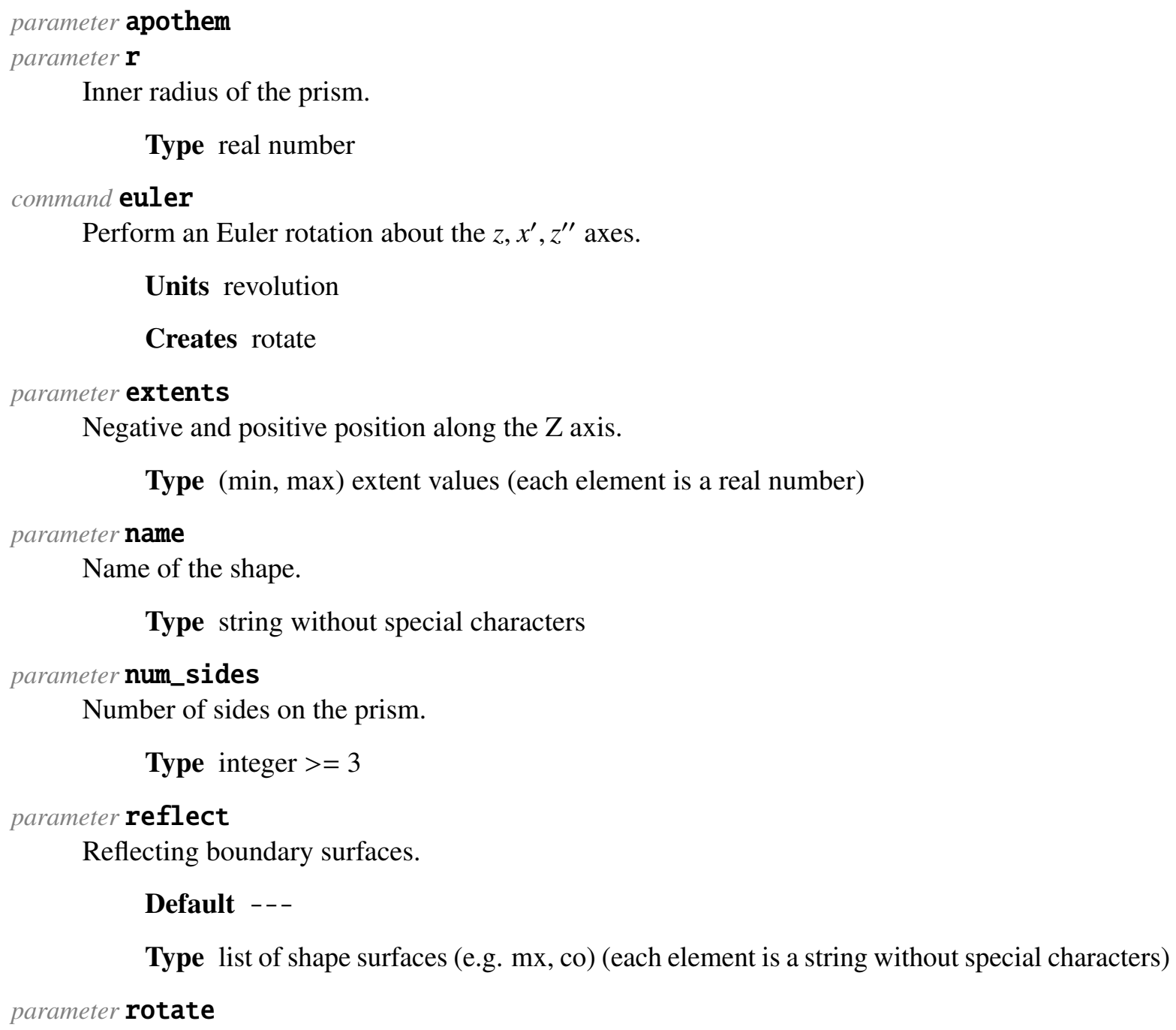

Inner radius of the prism.

Type real number

command euler

Perform an Euler rotation about the $z, x^{\prime}, z^{\prime \prime}$ axes.

Units revolution

Creates rotate

parameter extents

Negative and positive position along the $\mathrm{Z}$ axis.

Type (min, max) extent values (each element is a real number)

parameter name

Name of the shape.

Type string without special characters

parameter num_sides

Number of sides on the prism.

Type integer $>=3$

parameter reflect

Reflecting boundary surfaces.

Default ---

Type list of shape surfaces (e.g. mx, co) (each element is a string without special characters)

parameter rotate

Rotation matrix.

Default $1.0 \quad 0.0 \quad 0.0 \quad 0.01 .0 \quad 0.0 \quad 0.0 \quad 0.01 .0$

Type length-9 row-major rotation matrix (each element is a real number)

postprocessor (advanced)

Squelch identity rotations and null translations.

parameter rotfrac

Angle (fraction of the angle spanned by one face) to rotate.

Default 0.0

Type real number inclusive $[0.0,1.0]$

parameter translate

parameter origin

Local-to-global translation vector.

Default 0.00 .00 .0

Type length-3 float vector (each element is a real number) 


\subsection{7 [UNIVERSE][SHAPE $=$ SLAB]}

Infinite slab shape.

parameter axis

Axis along the slab.

Type axis ('x','y','z')

command euler

Perform an Euler rotation about the $z, x^{\prime}, z^{\prime \prime}$ axes.

Units revolution

Creates rotate

parameter extents

Negative and positive position along the slab axis.

Type (min, max) extent values (each element is a real number)

parameter name

Name of the shape.

Type string without special characters

parameter reflect

Reflecting boundary surfaces.

Default ---

Type list of shape surfaces (e.g. mx, co) (each element is a string without special characters)

parameter rotate

Rotation matrix.

Default $1.0 \quad 0.0 \quad 0.0 \quad 0.01 .0 \quad 0.0 \quad 0.0 \quad 0.01 .0$

Type length-9 row-major rotation matrix (each element is a real number)

postprocessor (advanced)

Squelch identity rotations and null translations.

parameter translate

parameter origin

Local-to-global translation vector.

Default 0.00 .00 .0

Type length-3 float vector (each element is a real number)

\subsection{8 [UNIVERSE][SHAPE $=$ PLANE]}

Infinite half-space.

command euler

Perform an Euler rotation about the $z, x^{\prime}, z^{\prime \prime}$ axes.

Units revolution

Creates rotate 
parameter name

Name of the shape.

Type string without special characters

parameter normal

Vector (possibly unnormalized) point outward from the plane.

Type length-3 float vector (each element is a real number)

parameter point

A point somewhere on the plane.

Type length-3 float vector (each element is a real number)

parameter reflect

Reflecting boundary surfaces.

Default ---

Type list of shape surfaces (e.g. mx, co) (each element is a string without special characters)

parameter rotate

Rotation matrix.

Default $1.0 \quad 0.0 \quad 0.0 \quad 0.01 .0 \quad 0.0 \quad 0.0 \quad 0.01 .0$

Type length-9 row-major rotation matrix (each element is a real number)

postprocessor (advanced)

Squelch identity rotations and null translations.

parameter translate

parameter origin

Local-to-global translation vector.

Default 0.00 .00 .0

Type length-3 float vector (each element is a real number)

\subsection{9 [UNIVERSE][SHAPE $=$ WEDGE]}

Wedge shape.

parameter corner_pt

parameter $\mathbf{x y}$

$\mathrm{XY}$ location of the right-angle corner of the wedge.

Type length-2 (x,y) position (each element is a positive real number)

command euler

Perform an Euler rotation about the $z, x^{\prime}, z^{\prime \prime}$ axes.

Units revolution

Creates rotate

parameter height

parameter $\mathbf{z l n g}$

Height of the wedge (along $\mathrm{Z}$ axis). 
Type positive real number

parameter name

Name of the shape.

Type string without special characters

parameter reflect

Reflecting boundary surfaces.

Default ---

Type list of shape surfaces (e.g. mx, co) (each element is a string without special characters)

parameter rotate

Rotation matrix.

Default $1.0 \quad 0.0 \quad 0.0 \quad 0.01 .0 \quad 0.0 \quad 0.0 \quad 0.01 .0$

Type length-9 row-major rotation matrix (each element is a real number)

postprocessor (advanced)

Squelch identity rotations and null translations.

parameter translate

parameter origin

Local-to-global translation vector.

Default $0.0 \quad 0.0 \quad 0.0$

Type length-3 float vector (each element is a real number)

parameter width

parameter $\mathbf{x b a s e}$

Length of the hypotenuse of the wedge (along the $\mathrm{X}$ axis).

Type positive real number

\subsubsection{0 [UNIVERSE] [SHAPE $=$ CONE]}

Cone shape.

parameter axis

Axis along the centerline of the cone.

Type axis ('x','y','z')

command euler

Perform an Euler rotation about the $z, x^{\prime}, z^{\prime \prime}$ axes.

Units revolution

Creates rotate

parameter extents

Negative and positive base positions along the given axis.

Type (min, max) extent values (each element is a real number)

parameter name

Name of the shape. 
Type string without special characters

parameter radii

parameter $\mathbf{r}$

Radii at the top and bottom.

Type 2 positive floats (each element is a positive real number)

parameter reflect

Reflecting boundary surfaces.

Default ---

Type list of shape surfaces (e.g. mx, co) (each element is a string without special characters)

parameter rotate

Rotation matrix.

Default $1.00 .0 \quad 0.0 \quad 0.01 .0 \quad 0.00 .00 .01 .0$

Type length-9 row-major rotation matrix (each element is a real number)

postprocessor (advanced)

Squelch identity rotations and null translations.

parameter translate

parameter origin

Local-to-global translation vector.

Default $0.0 \quad 0.0 \quad 0.0$

Type length-3 float vector (each element is a real number)

\subsubsection{1 [UNIVERSE][SHAPE=ELLIPSOID]}

Ellipsoid shape.

command euler

Perform an Euler rotation about the $z, x^{\prime}, z^{\prime \prime}$ axes.

Units revolution

Creates rotate

parameter name

Name of the shape.

Type string without special characters

parameter radii

parameter $\mathbf{r}$

Radii in the $\mathrm{x}, \mathrm{y}$, and $\mathrm{z}$ directions.

Type 3 positive floats (each element is a positive real number)

parameter reflect

Reflecting boundary surfaces.

Default --- 
Type list of shape surfaces (e.g. mx, co) (each element is a string without special characters)

parameter rotate

Rotation matrix.

Default $1.0 \quad 0.0 \quad 0.0 \quad 0.01 .0 \quad 0.0 \quad 0.0 \quad 0.01 .0$

Type length-9 row-major rotation matrix (each element is a real number)

postprocessor (advanced)

Squelch identity rotations and null translations.

parameter translate

parameter origin

Local-to-global translation vector.

Default 0.00 .00 .0

Type length-3 float vector (each element is a real number)

\subsubsection{2 [UNIVERSE][SHAPE $=$ HOPPER]}

Hopper shape.

command euler

Perform an Euler rotation about the $z, x^{\prime}, z^{\prime \prime}$ axes.

Units revolution

Creates rotate

parameter extents

Negative and positive base positions along the $\mathrm{Z}$ axis.

Type (min, max) extent values (each element is a real number)

parameter lower_pt

parameter $\mathbf{l o}$

$\mathrm{X}$ and $\mathrm{Y}$ half-lengths on the low side of the hopper.

Type length-2 (x,y) position (each element is a positive real number)

parameter name

Name of the shape.

Type string without special characters

parameter reflect

Reflecting boundary surfaces.

Default ---

Type list of shape surfaces (e.g. mx, co) (each element is a string without special characters)

parameter rotate

Rotation matrix.

Default $1.00 .0 \quad 0.0 \quad 0.01 .0 \quad 0.0 \quad 0.0 \quad 0.01 .0$

Type length-9 row-major rotation matrix (each element is a real number) 
postprocessor (advanced)

Squelch identity rotations and null translations.

parameter translate

parameter origin

Local-to-global translation vector.

Default $0.0 \quad 0.0 \quad 0.0$

Type length-3 float vector (each element is a real number)

parameter upper_pt

parameter hi

$\mathrm{X}$ and $\mathrm{Y}$ half-lengths on the high side of the hopper.

Type length-2 (x,y) position (each element is a positive real number)

\subsubsection{3 [UNIVERSE][SHAPE $=$ RIGHTTET]}

Right tetrahedron shape.

command euler

Perform an Euler rotation about the $z, x^{\prime}, z^{\prime \prime}$ axes.

Units revolution

Creates rotate

parameter lengths

Length of the tetrahedron edges along the $\mathrm{x}, \mathrm{y}$, and $\mathrm{z}$ axes.

Type 3 positive floats (each element is a positive real number)

parameter name

Name of the shape.

Type string without special characters

parameter reflect

Reflecting boundary surfaces.

Default ---

Type list of shape surfaces (e.g. mx, co) (each element is a string without special characters)

parameter rotate

Rotation matrix.

Default $1.0 \quad 0.0 \quad 0.0 \quad 0.01 .0 \quad 0.0 \quad 0.0 \quad 0.01 .0$

Type length-9 row-major rotation matrix (each element is a real number)

postprocessor (advanced)

Squelch identity rotations and null translations.

parameter translate

parameter origin

Local-to-global translation vector.

Default 0.00 .00 .0

Type length-3 float vector (each element is a real number) 


\subsubsection{4 [UNIVERSE][SHAPE $=$ TRIPRISM]}

Triangular prism shape.

command euler

Perform an Euler rotation about the $z, x^{\prime}, z^{\prime \prime}$ axes.

Units revolution

Creates rotate

parameter lengths

Length of the triangular prism's bounding box.

Type 3 positive floats (each element is a positive real number)

parameter name

Name of the shape.

Type string without special characters

parameter reflect

Reflecting boundary surfaces.

Default ---

Type list of shape surfaces (e.g. mx, co) (each element is a string without special characters)

parameter rotate

Rotation matrix.

Default $1.0 \quad 0.0 \quad 0.0 \quad 0.01 .0 \quad 0.0 \quad 0.0 \quad 0.01 .0$

Type length-9 row-major rotation matrix (each element is a real number)

postprocessor (advanced)

Squelch identity rotations and null translations.

parameter translate

parameter origin

Local-to-global translation vector.

Default 0.00 .00 .0

Type length-3 float vector (each element is a real number)

\subsubsection{5 [UNIVERSE][SHAPE=ECYLINDER]}

Elliptical cylinder shape.

command euler

Perform an Euler rotation about the $z, x^{\prime}, z^{\prime \prime}$ axes.

Units revolution

Creates rotate

parameter extents

Negative and positive position along the given axis.

Type (min, max) extent values (each element is a real number) 
parameter name

Name of the shape.

Type string without special characters

parameter radii

parameter $\mathbf{r}$

Radii in the $\mathrm{x}$ and $\mathrm{y}$ directions.

Type length-2 (x,y) position (each element is a positive real number)

parameter reflect

Reflecting boundary surfaces.

Default ---

Type list of shape surfaces (e.g. mx, co) (each element is a string without special characters)

parameter rotate

Rotation matrix.

Default $1.00 .0 \quad 0.0 \quad 0.01 .0 \quad 0.0 \quad 0.0 \quad 0.01 .0$

Type length-9 row-major rotation matrix (each element is a real number)

postprocessor (advanced)

Squelch identity rotations and null translations.

parameter translate

parameter origin

Local-to-global translation vector.

Default 0.00 .00 .0

Type length-3 float vector (each element is a real number)

\subsubsection{6 [UNIVERSE][SHAPE=RHOMBDOD]}

The faces of a rhombic dodecahedron are ordered so that face $n$ connects to $n+6 \bmod 12$ of an adjacent lattice cell. For a dodecahedron with interior radius 1, these faces are:

\begin{tabular}{ll}
\hline Face & Description \\
\hline px & Plane: $\mathrm{x}=1$ \\
py & Plane: $\mathrm{y}=1$ \\
p0 & Plane: $\mathrm{n}=(0.50 .50 .707107), \mathrm{d}=1$ \\
p1 & Plane: $\mathrm{n}=(0.5-0.5-0.707107), \mathrm{d}=-1$ \\
p2 & Plane: $\mathrm{n}=(0.5-0.50 .707107), \mathrm{d}=1$ \\
p3 & Plane: $\mathrm{n}=(0.50 .5-0.707107), \mathrm{d}=-1$ \\
mx & Plane: $\mathrm{x}=-1$ \\
my & Plane: $\mathrm{y}=-1$ \\
p4 & Plane: $\mathrm{n}=(0.50 .50 .707107), \mathrm{d}=-1$ \\
p5 & Plane: $\mathrm{n}=(0.5-0.5-0.707107), \mathrm{d}=1$ \\
p6 & Plane: $\mathrm{n}=(0.5-0.50 .707107), \mathrm{d}=-1$ \\
p7 & Plane: $\mathrm{n}=(0.50 .5-0.707107), \mathrm{d}=1$ \\
\hline
\end{tabular}




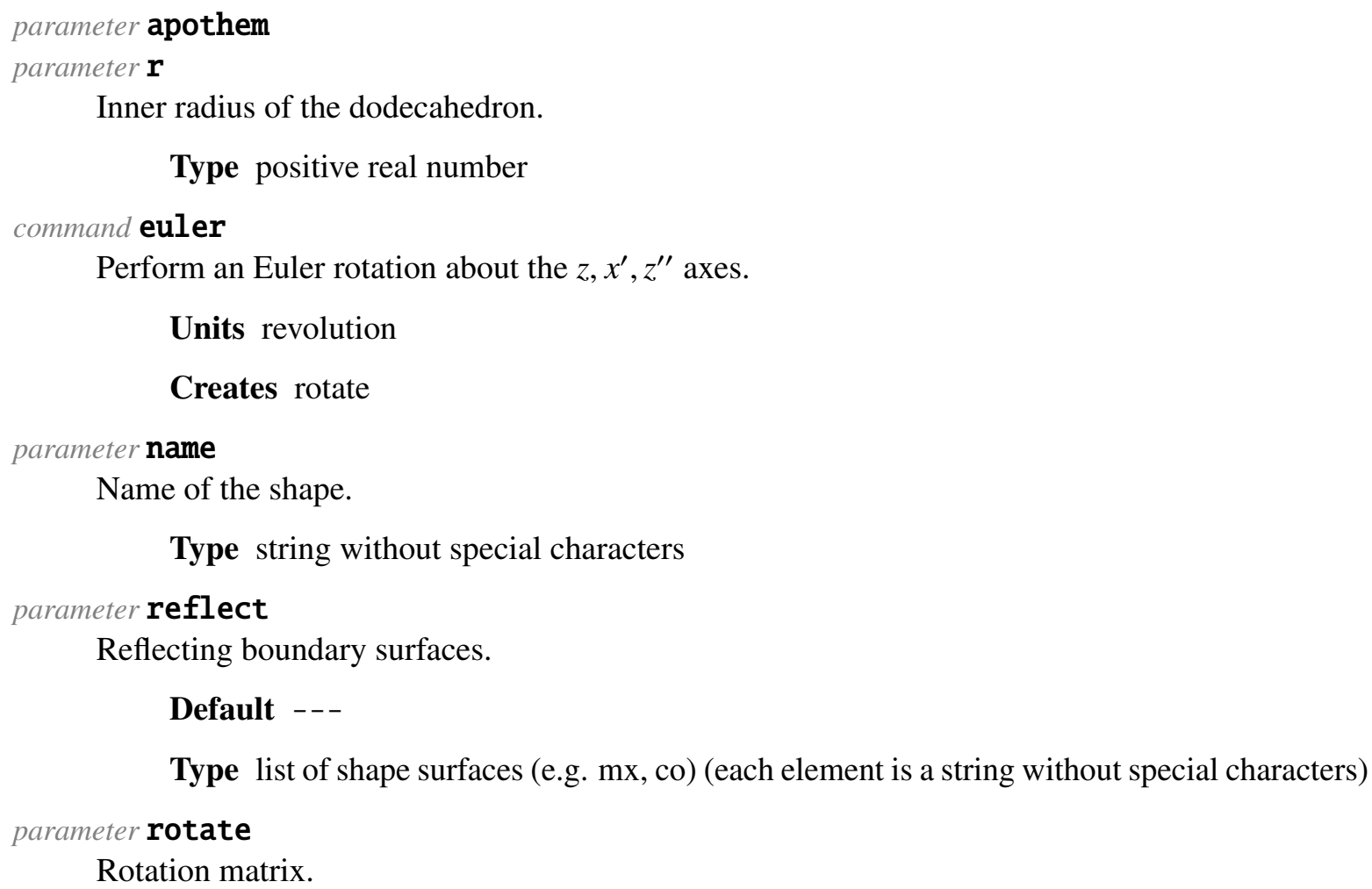

Inner radius of the dodecahedron.

Type positive real number

command euler

Perform an Euler rotation about the $z, x^{\prime}, z^{\prime \prime}$ axes.

Units revolution

Creates rotate

parameter name

Name of the shape.

Type string without special characters

parameter reflect

Reflecting boundary surfaces.

Default ---

Type list of shape surfaces (e.g. mx, co) (each element is a string without special characters)

parameter rotate

Rotation matrix

Default $1.0 \quad 0.0 \quad 0.0 \quad 0.01 .0 \quad 0.0 \quad 0.0 \quad 0.01 .0$

Type length-9 row-major rotation matrix (each element is a real number)

postprocessor (advanced)

Squelch identity rotations and null translations.

parameter translate

parameter origin

Local-to-global translation vector.

Default 0.00 .00 .0

Type length-3 float vector (each element is a real number)

\subsubsection{7 [UNIVERSE][SHAPE=PPIPED]}

Parallelepiped.

command euler

Perform an Euler rotation about the $z, x^{\prime}, z^{\prime \prime}$ axes.

Units revolution

Creates rotate

parameter lengths

Length of the $\mathrm{x}, \mathrm{xy}$, and $\mathrm{xyz}$ edges.

Type 3 positive floats (each element is a positive real number)

parameter name

Name of the shape. 


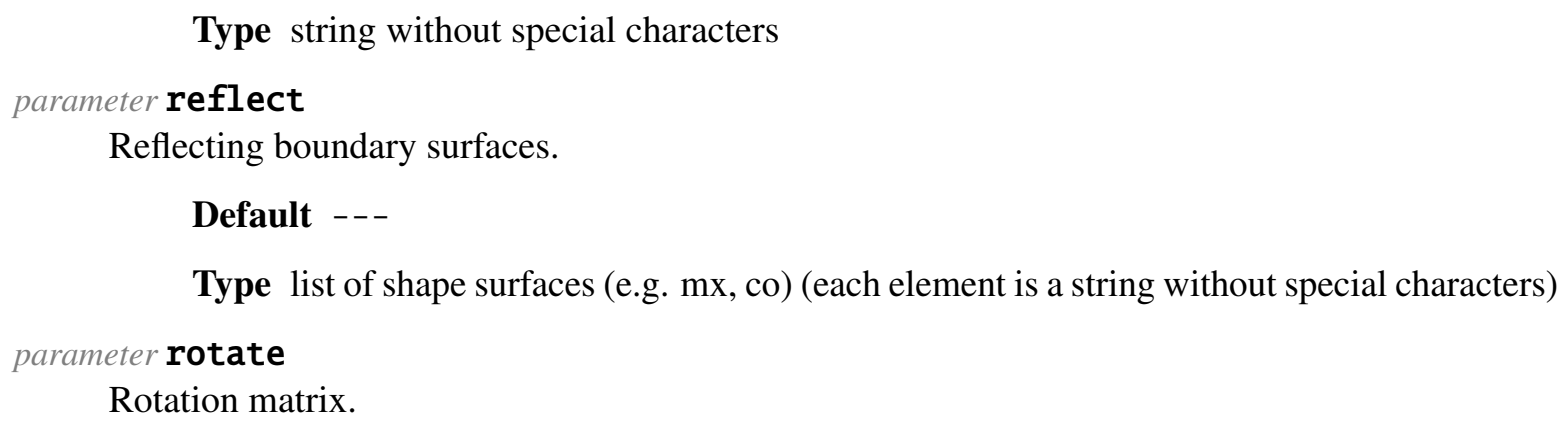

Reflecting boundary surfaces.

Default ---

Type list of shape surfaces (e.g. mx, co) (each element is a string without special characters)

parameter rotate

Rotation matrix.

Default $1.0 \quad 0.0 \quad 0.0 \quad 0.01 .0 \quad 0.0 \quad 0.0 \quad 0.01 .0$

Type length-9 row-major rotation matrix (each element is a real number)

postprocessor (advanced)

Squelch identity rotations and null translations.

parameter translate

parameter origin

Local-to-global translation vector.

Default $0.0 \quad 0.0 \quad 0.0$

Type length-3 float vector (each element is a real number)

parameter $\mathbf{x y}$ _angle

parameter phi

Angle between xy component of xyz edge and $\mathrm{x}$ axis (phi).

Units revolution

Type real number in $[0, .25)$

\section{parameter $\mathbf{x y z}$ _angle \\ parameter theta}

Angle between xyz edge and $z$ axis (theta).

Units revolution

Type real number in $[0, .25)$

parameter $\mathbf{y}$ angle

parameter psi

Angle between xy edge and y axis (psi).

Units revolution

Type real number in $[0, .25)$

\subsubsection{8 [UNIVERSE][SHAPE $=$ QUADRIC]}

General quadric.

parameter cross

parameter def

Second-order cross coefficients $d X Y+e Y Z+f X Z$.

Default $0.00 .0 \quad 0.0$ 
Type length-3 float vector (each element is a real number)

command euler

Perform an Euler rotation about the $z, x^{\prime}, z^{\prime \prime}$ axes.

Units revolution

Creates rotate

parameter $\mathbf{f i r s t}$

parameter $\mathbf{g h i}$

First-order coefficients $g X+h Y+i Z$.

Default $0.00 .0 \quad 0.0$

Type length-3 float vector (each element is a real number)

parameter name

Name of the shape.

Type string without special characters

parameter reflect

Reflecting boundary surfaces.

Default ---

Type list of shape surfaces (e.g. mx, co) (each element is a string without special characters)

parameter rotate

Rotation matrix.

Default $1.00 .0 \quad 0.0 \quad 0.01 .0 \quad 0.0 \quad 0.0 \quad 0.01 .0$

Type length-9 row-major rotation matrix (each element is a real number)

postprocessor (advanced)

Squelch identity rotations and null translations.

parameter scalar

parameter $\mathbf{j}$

Scalar coefficient $j$.

Default 0.0

Type real number

parameter second

parameter abc

Second-order coefficients $a X^{2}+b Y^{2}+c Z^{2}$.

Default $0.00 .0 \quad 0.0$

Type length-3 float vector (each element is a real number)

parameter translate

parameter origin

Local-to-global translation vector.

Default 0.00 .00 .0

Type length-3 float vector (each element is a real number) 



\section{REFERENCES}

[1] Kobayashi, K., Sugimura, N., and Nagaya, Y. 3-D Radiation Transport Benchmark Problems and Results for Simple Geometries with Void Regions. Nuclear Energy Agency, 2000.

[2] Rearden (ed.), B. T. and Jessee (ed.), M. A. "SCALE code system." Technical Report ORNL/TM2005/39, Version 6.2.3, Oak Ridge National Laboratory, March 2018.

[3] Johnson, S. R. "Omnibus: A New Front End to Denovo and Shift." Transactions of the American Nuclear Society, 117:4, 2017.

[4] Evans, T. M., Stafford, A. S., Slaybaugh, R. N., and Clarno, K. T. "DENOVO: a new three-dimensional parallel discrete ordinates code in SCALE." Nuclear Technology, 171:171-200, 2010.

[5] Pandya, T. M., Johnson, S. R., Evans, T. M., Davidson, G. G., et al. "Implementation, capabilities, and benchmarking of Shift, a massively parallel Monte Carlo radiation transport code." Journal of Computational Physics, 308:239-272, March 2016. URL: http://linkinghub.elsevier.com/retrieve/pii/ S0021999115008566, doi:10.1016/j.jcp.2015.12.03715.

[6] Wieselquist, W. A. "The SCALE 6.2 ORIGEN API for High Performance Depletion." In ANS MC2015-Joint International Conference on Mathematics and Computation (MEEC), Supercomputing in Nuclear Applications (SNA) and the Monte Carlo (MC) Method, 11. LaGrange Park, IL, April 2015.

[7] Mosher, S. W., Johnson, S. R., Bevill, A. M., Ibrahim, A. M., et al. "ADVANTG-an automated variance reduction parameter generator." Technical Report ORNL/TM-2013/416-rev1, Oak Ridge National Laboratory, 2013.

[8] Gwon, C. S., Novikova, E. I., Phlips, B. F., Strickman, M. S., et al. "Interacting with the SWORD package (SoftWare for the Optimization of Radiation Detectors)." In 2007 IEEE Nuclear Science Symposium Conference Record, 1130-1133. Honolulu, HI, USA, 2007. IEEE. URL: http://ieeexplore. ieee.org/document/4437206/, doi:10.1109/NSSMIC.2007.4437206 ${ }^{16}$.

[9] Pandya, T. M., Evans, T. M., Clarno, K. T., and Collins, B. S. "Excore Modeling with VERA.” Technical Report CASL-U-2017-1311-001, CASL, 2017.

[10] Wiarda, D., Dunn, M. E., Greene, N. M., Williams, M. L., et al. "AMPX-6: a modular code system for processing ENDF/B.” Technical Report ORNL/TM-2016/43, Oak Ridge National Laboratory, April 2016.

[11] Davidson, G., Evans, T., Jarrell, J., Hamilton, S., et al. "Massively Parallel, Three-Dimensional Transport Solutions for the k-Eigenvalue Problem." Nuclear Science and Engineering, 177(2):111-125, June 2014. URL: http://www.ans.org/pubs/journals/nse/a_35675, doi:10.13182/NSE12-101 ${ }^{17}$.

[12] Abu-Shumays, I. K. "Angular quadratures for improved transport computations." Transp. Theory Stat. Phys., 30:169-204, 2001.

[13] Jarrell, J. J. An Adaptive Angular Discretization method for Neutral-Particle Transport in ThreeDimensional Geometries. PhD thesis, Texas A\&M University, 2010.

\footnotetext{
15 https://doi.org/10.1016/j.jcp.2015.12.037

${ }^{16}$ https://doi.org/10.1109/NSSMIC.2007.4437206

${ }^{17}$ https://doi.org/10.13182/NSE12-101
} 
[14] Johnson, S. R. "Fast mix table construction for material discretization." In International Conference on Mathematics and Computational Methods Applied to Nuclear Science and Engineering (MESC 2013). Sun Valley, ID, May 2013.

[15] Agostinelli, S., Allison, J., Amako, K., Apostolakis, J., et al. "Geant4—a simulation toolkit." Nuclear Instruments and Methods in Physics Research Section A: Accelerators, Spectrometers, Detectors and Associated Equipment, 506(3):250-303, July 2003. URL: http://linkinghub.elsevier.com/retrieve/pii/ S0168900203013688, doi:10.1016/S0168-9002(03)01368-8 ${ }^{18}$.

[16] Wagner, J. C. and Haghighat, A. "Automated variance reduction of Monte Carlo shielding calculations using the discrete ordinates adjoint function.” Nuclear Science and Engineering, 128(2):186-208, 1998.

[17] Wagner, J. C., Peplow, D. E., Mosher, S. W., and Evans, T. M. "Review of hybrid (deterministic/Monte Carlo) radiation transport methods, codes, and applications at Oak Ridge National Laboratory." Proc. Joint Intl. Conf. Supercomputing in Nucl. Appl. and Monte Carlo, 2010.

[18] X-5 Monte Carlo Team. "MCNP - a general Monte Carlo n-particle transport code, version 5." Technical Report LA-UR-03-1987, Los Alamos National Laboratory, 2008.

[19] Engle, Jr., W. W. "A user's manual for ANISN: a one dimensional discrete ordinates code with anisotropic scattering." Technical Report K-1693, 4448708, Oak Ridge National Laboratory, January 1967. URL: http://www.osti.gov/servlets/purl/4448708/, doi:10.2172/4448708 ${ }^{19}$.

[20] Davidson, G. G., Pandya, T. M., Johnson, S. R., Evans, T. M., et al. "Nuclide depletion capabilities in the Shift Monte Carlo code." Annals of Nuclear Energy, 114:259-276, April 2018. URL: https: //linkinghub.elsevier.com/retrieve/pii/S0306454917304322, doi:10.1016/j.anucene.2017.11.04220.

\footnotetext{
${ }^{18}$ https://doi.org/10.1016/S0168-9002(03)01368-8

${ }^{19}$ https://doi.org/10.2172/4448708

${ }^{20}$ https://doi.org/10.1016/j.anucene.2017.11.042
} 


\section{ACKNOWLEDGMENTS}

This research used resources of the Oak Ridge Leadership Computing Facility, which is a DOE Office of Science User Facility supported under Contract DE-AC05-00OR22725. 


Appendix A. EXAMPLES

A-2 


\section{Appendix A. EXAMPLES}

The following examples demonstrate many of the capabilities in Exnihilo, such as:

- creating and running a problem in Omnibus,

- postprocessing the data with the included Python tools, and

- interacting with nuclear data using the Exnihilo Python bindings.

Caution: This LaTeX version of the documentation is not able to render some of the postprocessed output datasets. The HTML version of this document includes the missing inline tables. A future revision to this manual will improve the typesetting to better distinguish user input, system output, and Jupyter notebook results. Also, since the figures are the result of inline computations, they are presented as inline text rather than figures with captions.

\section{A.1 VISUALIZATION}

These examples demonstrate basic problem visualization techniques for Denovo and Shift input.

\section{A.1.1 OMNIBUS GEOMETRY VISUALIZATION}

This example shows how to process and visualize an MCNP input and its compositions, using both the interactive Python 2-D ray tracer and the executable 3-D Silo-based ray tracer (using mode=raytrace). We also save the compositions to a separate HDF5 input file; they can later be used to override the compositions provided by MCNP.

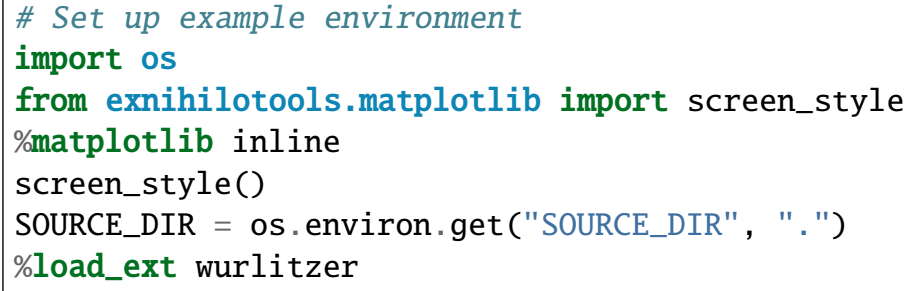

Visualizing the MCNP input requires first loading the geometry. The load_mcnp command accepts either an existing runtpe filename or the path to an MCNP input (which must have a .inp, .i, or .mcnp extension). Generating a runtpe file requires an MCNP5 executable, since it invokes monp5 ix. The resulting model stores the geometry and a vector of loaded compositions.

from omnibus.raytrace.load import load_mcnp

model = load_mcnp(os.path.join(SOURCE_DIR, "data", "dv1a.mcnp"))

Generating MCNP runtpe file...

...finished generating MCNP runtpe file in 4.5 seconds

$\gg>$ Loading nuclide data from processed MCNP libraries

>> Loading compositions from MCNP input 


\section{A.1.1.1 Construct a color table}

Material visualization is more easily interpreted when the chosen color scheme associates physical properties with the materials.

The model stores a vector of wrapped $\mathrm{C}++$ Composition objects. Their contents can be extracted through accessors such as name and density, or converted into a dictionary using the _asdict method. Omnibus includes a convenience class that mimics the $\mathrm{C}++$ composition object but uses native python datatypes. Since this class's constructor accepts keyword arguments, it can be constructed by expanding each composition using _asdict and using the Python keyword argument expansion $* *$.

from omnibus.raytrace. composition import Composition

comps $=$ [Composition $(* *$ c.asdict ()$)$ for $\mathrm{c}$ in model.compositions]

comps [1]

Each composition's name is constructed by default from the MCNP material numbers in the input deck. These can be changed to more descriptive names by using a convenience function that returns the MCNP M card identifiers that correspond to each composition.

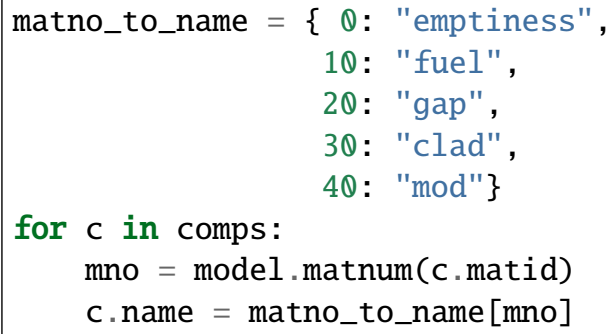

The ColorMap class automatically applies colors for common reactor materials such as fuel, clad, control rods, and water. Colors from unknown materials are assigned from the matplotlib "Set3" color map ${ }^{21}$.

from omnibus.raytrace. colors import ColorMap

colors = ColorMap.from_compositions (comps)

colors [colors . unassigned] = 'Set 3 '

The raytracing Imager class renders the geometry to an image. The lower and upper coordinates are the 3-D points corresponding to the lower-left and upper-right corner of the visualization window. The basis vector completes the window specification by defining the rightward direction ( $\mathrm{x}$ axis in the resulting plot) of the window. Increasing the pixel count increases rendering time but provides a sharper-resolution image when exporting. The trace option can be set to cell (plot the cell names obtained from the MCNP input) or mat (for plotting materials, the default). Colors and names can be assigned from the compositions and color map constructed above.

The raytracing engine uses the same particle tracking engine as Shift, so unlike the quadric-intersection-based method in MCNP's visualizer, it will catch actual tracking errors and is typically faster to render.

The Imager's plot command returns a matplotlib plot displaying the cells or materials present along the ray-traced slice. Regions not encountered in this specific trace are not displayed, increasing the zoom level will decrease the number of materials.

\footnotetext{
${ }^{21}$ https://matplotlib.org/3.1.0/gallery/color/colormap_reference.html\#sphx-glr-gallery-color-colormap-reference-py
} 

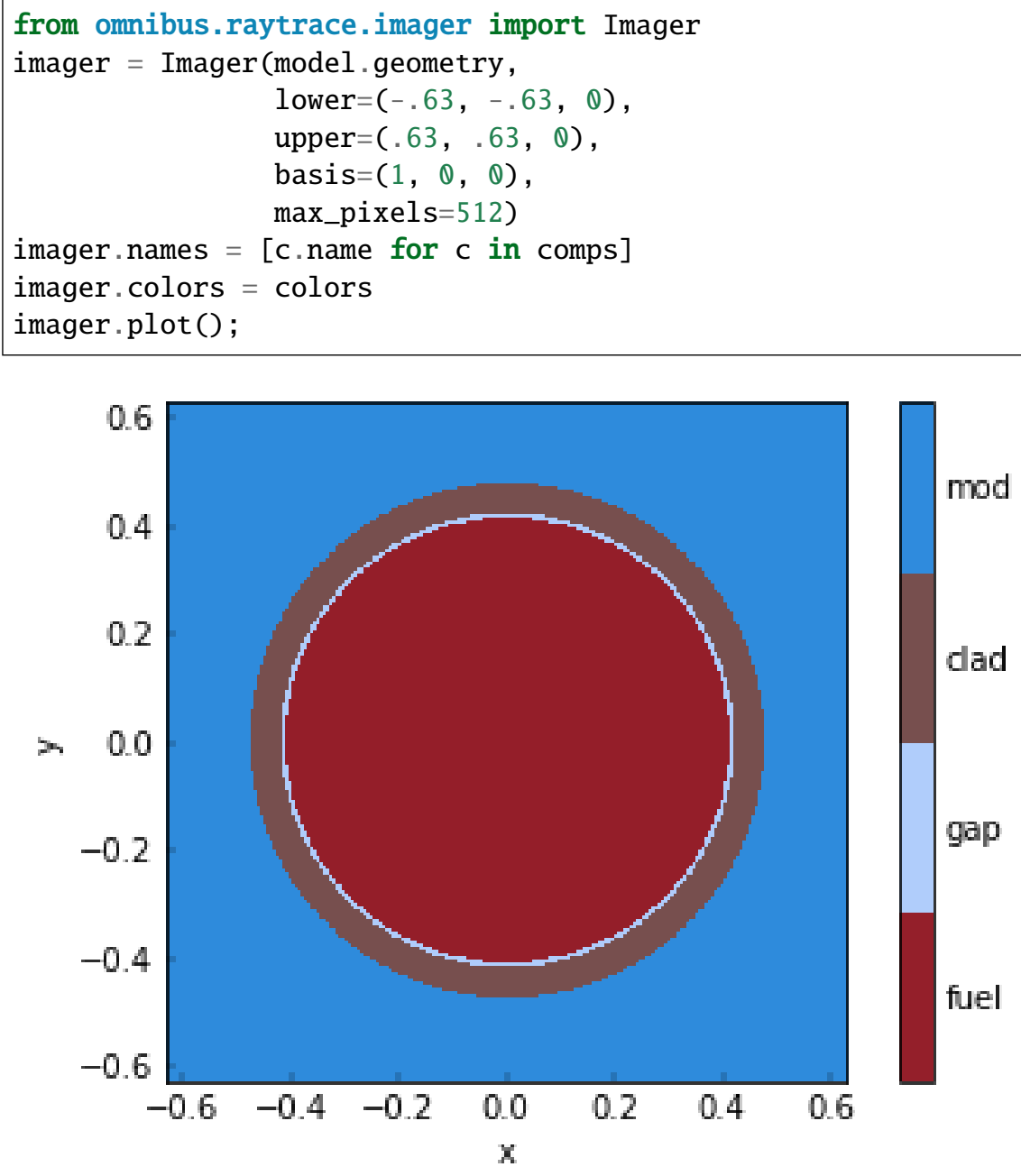

Here, input files that contain the colors and the name mappings are written out so they can be imported into the raytrace input.

from omnibus. raytrace.colors import dump_colors

dump_colors ("dv1a-colors.omn", comps, colors)

\%cat "dv1a-colors.omn"

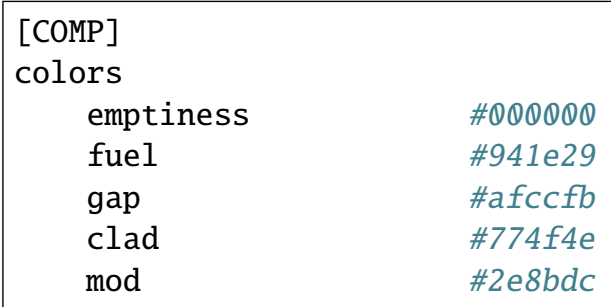




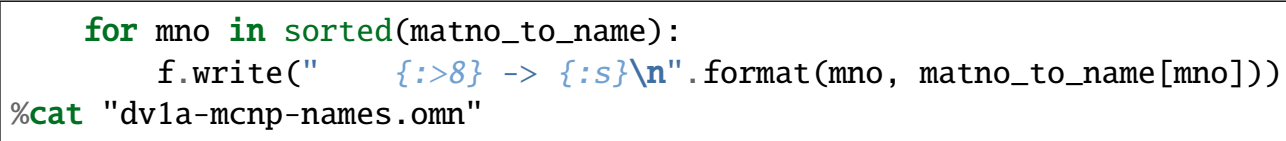

\section{A.1.1.2 Save compositions to HDF5}

It is possible to manually load HDF5 compositions into Omnibus: composition input may be needed for some geometry types like GG or RTK, or it can be used to override compositions present in the model. For example, one could perturb the MCNP nuclide densities without modifying the original MCNP input file. The following block of code saves a composition HDF5 file from the compositions that were loaded from the model.

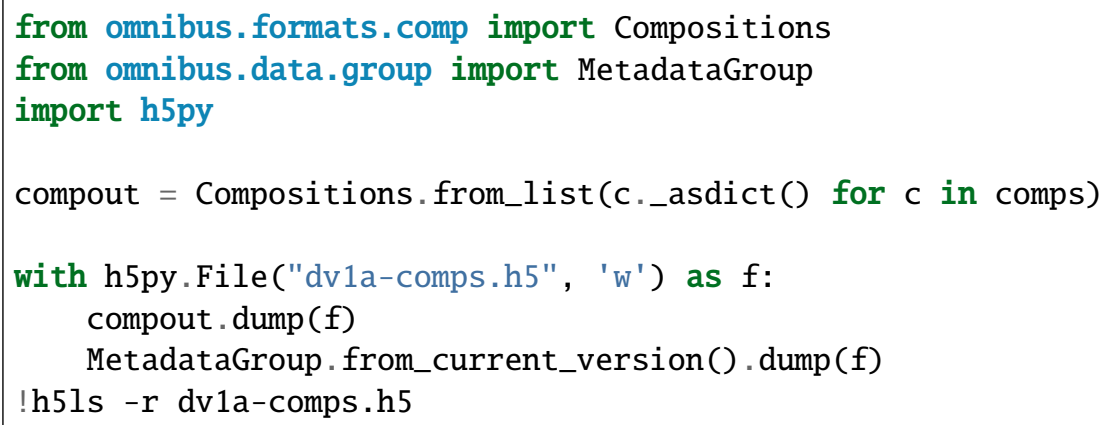

\section{A.1.1.3 Run the Omnibus 3D ray tracer}

Now let's execute the Omnibus raytracer (which uses the Shift geometry tracking engine to generate a Silo visualization file). Our input file uses \#include to import the material names and composition colors we generated above.

! cp $\{$ SOURCE_DIR $\} /$ data/dv1a* .

\%cat "dv1a-raytrace.omn"

!omnibus-run dv1a-raytrace.omn

[PROBLEM]

name "Pin cell ray trace example" 


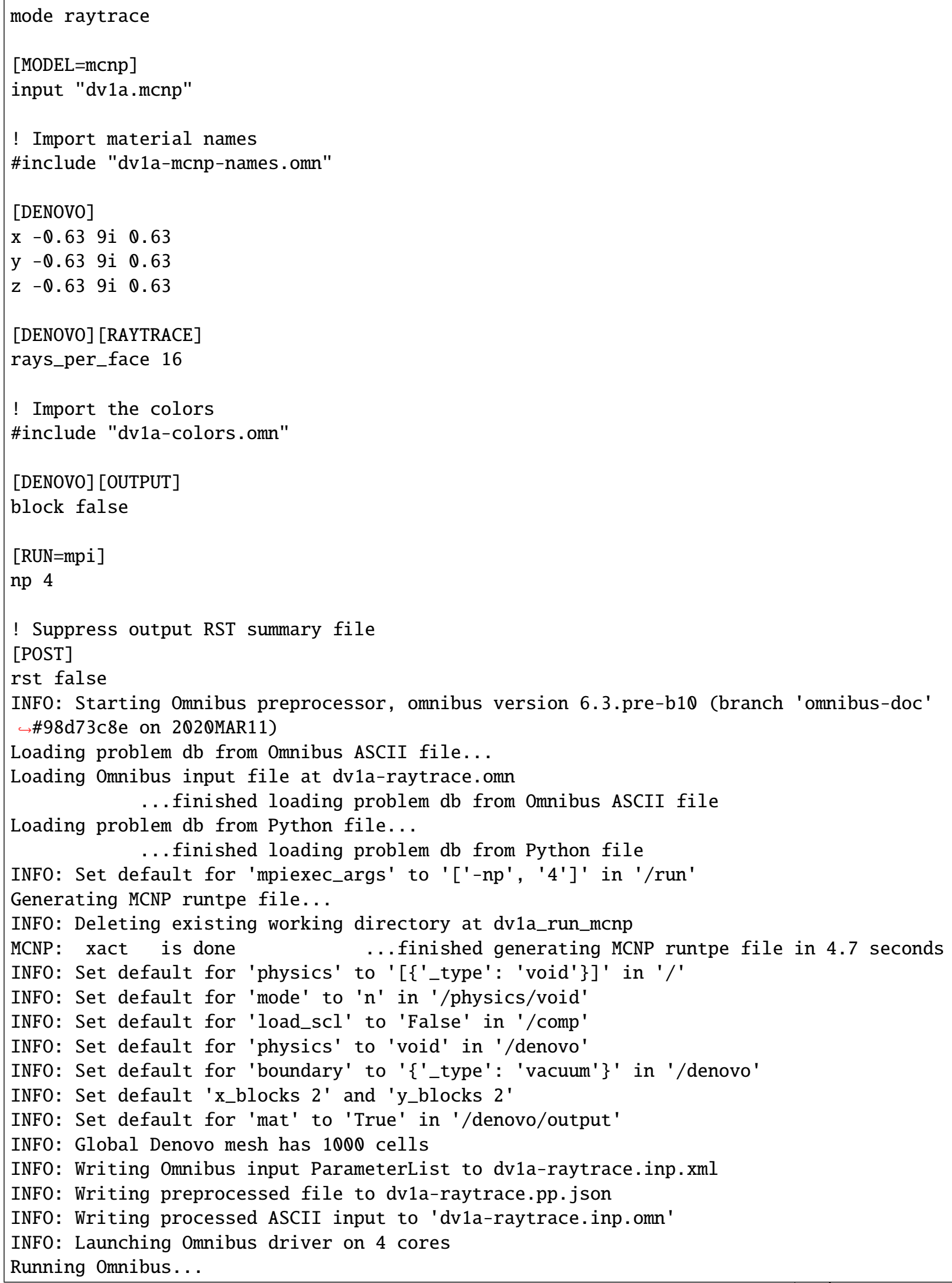


WARNING: The Exnihilo software revision ( 5400 ) used to generate the input file differs $\hookrightarrow$ from this version being used to run it ( $r 539$ ).

WARNING: This could cause internal consistency checks to unexpectedly fail, and it could ${ }_{\lrcorner}$ seven lead to unexpected database value changes.

WARNING: Please check your output very carefully after this run to make sure the $\sqcup$

$\hookrightarrow$ interpreted values match your input values.

Building model

INFO: Loading nuclide data from processed MCNP libraries

INFO: Loading compositions from MCNP input

INFO: Applying 5 user-provided composition colors

WARNING: Extents of MCNP geometry are extremely large or unknown; this may adversely

$\rightarrow$ affect 'global' tallies, entropy mesh, etc.

Building physics 'void'

Building Denovo solver internals

Ray tracing Denovo mesh

Mixing Denovo cross sections

Building Denovo sources

Initializing Denovo solver

INFO: Skipping Denovo state construction due to user option

Skipping transport due to user option

Writing Denovo HDF5 output

Run complete

Cleaning up

Running Omnibus postprocessing

Loading HDF 5 file...

INFO: Loaded Omnibus output data from 'dv1a-raytrace.out.h5', problem name 'Pin cell ray

$\rightarrow$ trace example', created on 2020MAR11 22:36 using SCALE version 6.3.pre-b10 (branch

$\hookrightarrow$ 'omnibus-doc' \#17579cc6 on 2020MAR10)

...finished loading HDF5 file

Loading HDF5 file...

INF0: Loaded Omnibus output data from 'dv1a-raytrace.out.h5', problem name 'Pin cell ray

$\hookrightarrow$ trace example', created on 2020MAR11 22:36 using SCALE version 6.3.pre-b10 (branch

$\hookrightarrow$ 'omnibus-doc' \#17579cc6 on 2020MAR10)

...finished loading HDF5 file

Omnibus created one silo file (inside a subdirectory) for each KBA block (execution core), as well as one "master" silo file, which is what we'll open in VisIT.

! ls *silo

\# ! visit denovo_output.silo

denovo_output.silo

denovo_output_silo:

denovo_output.0.silo denovo_output.2.silo

denovo_output.1.silo denovo_output.3.silo

A filled boundary plot lets us visualize the ray traced output. Note that the material interface reconstruction is imprecise because we used a relatively coarse mesh. Also note that the material colors and names have been correctly propagated. 


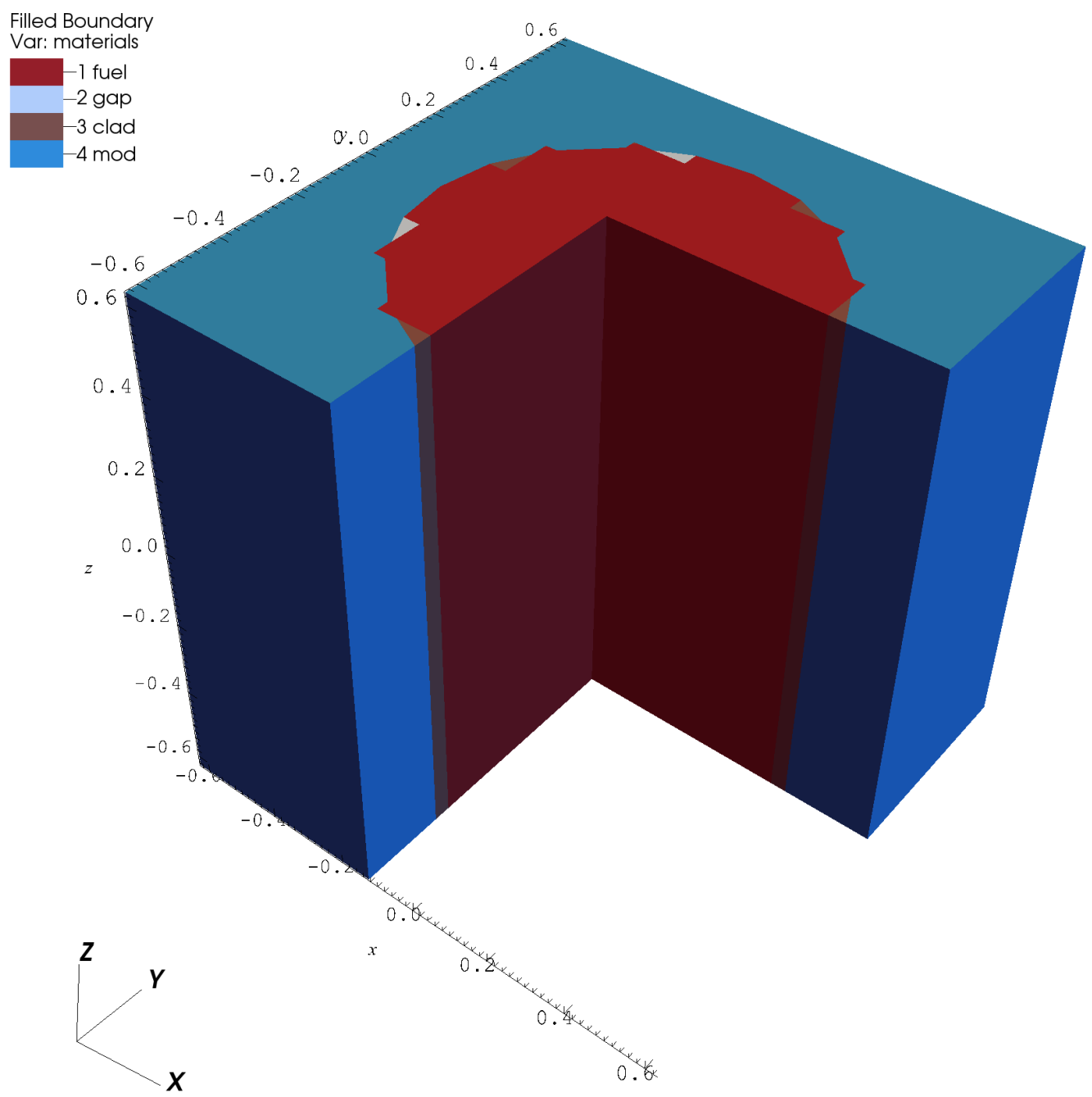

\section{A.2 DENOVO}

This section provides examples, each of which demonstrates a different way to visualize, input, run, and/or post-process typical problems for Denovo to solve.

\section{A.2.1 DISCRETIZED MCNP GODIVA}

This example demonstrates post-processing and visualization for a simple criticality problem using the Denovo deterministic solver. 
\# Set up example environment

import os

from exnihilotools.matplotlib import screen_style

\%matplotlib inline

screen_style()

SOURCE_DIR = os.environ.get ("SOURCE_DIR", ".")

The model and its cell IDs are previsualized using the Omnibus Python tools described in the geometry visualization example.
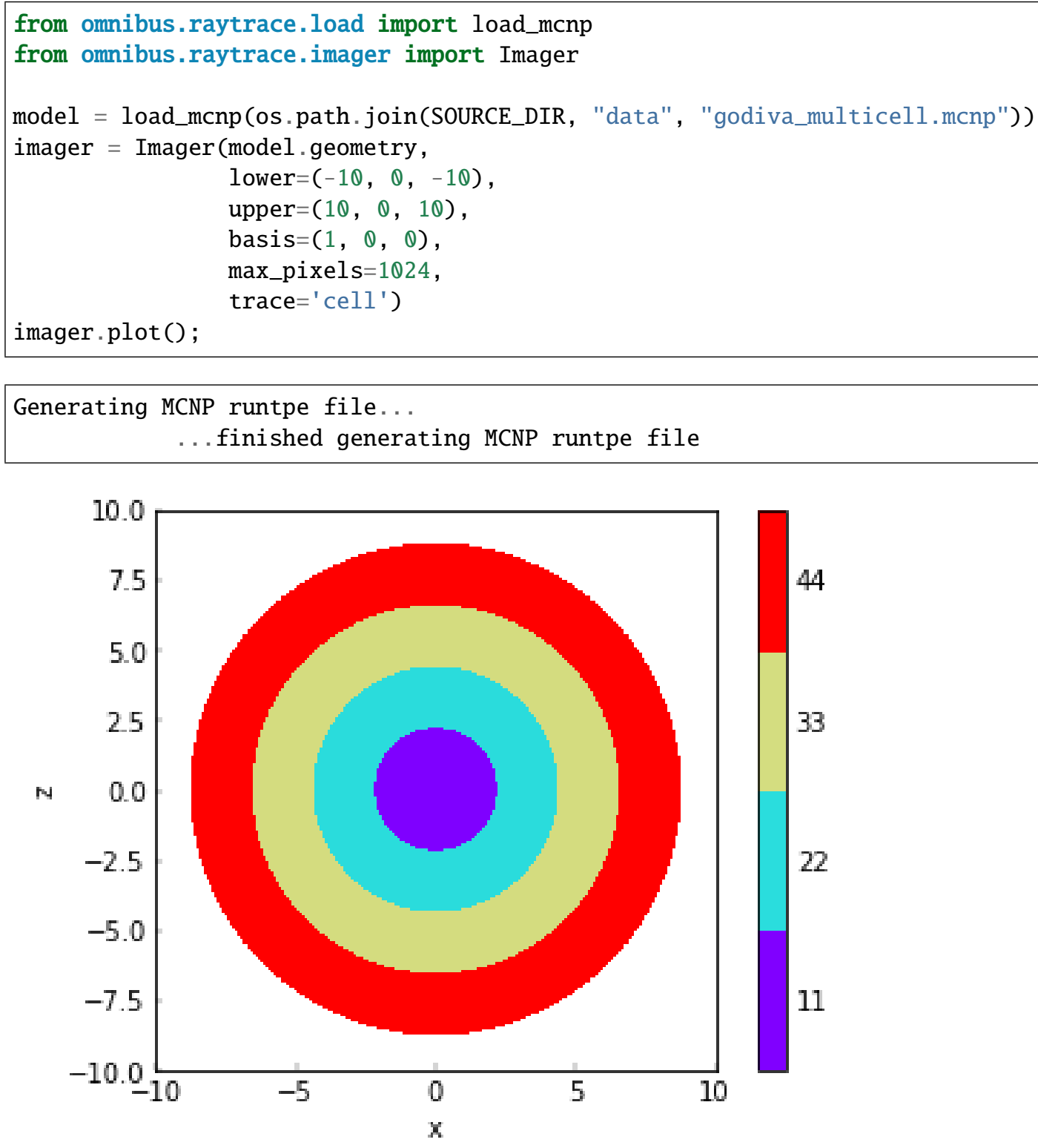

\section{A.2.1.1 Execute Omnibus}

Running Denovo through Omnibus requires an Omnibus input with SCALE multigroup physics. The kcode mode specifies that this is an eigenvalue problem; the [SOLVER=eigenvalue] block specifies the numerical accuracy of the solver result. 
\%cat \{SOURCE_DIR\}/data/denovo-godiva.omn

! omnibus-run \{SOURCE_DIR\}/data/denovo-godiva.omn

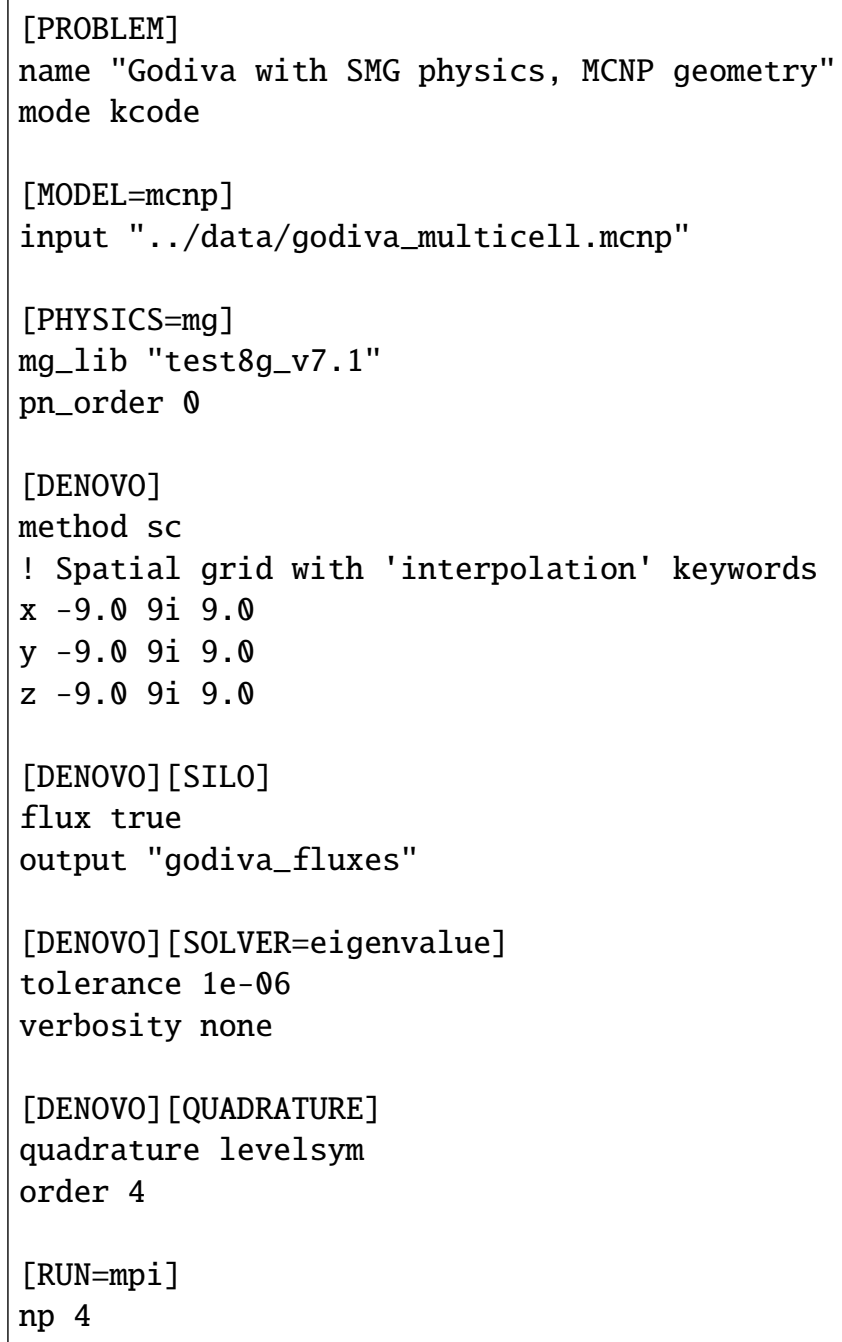


(continued from previous page)

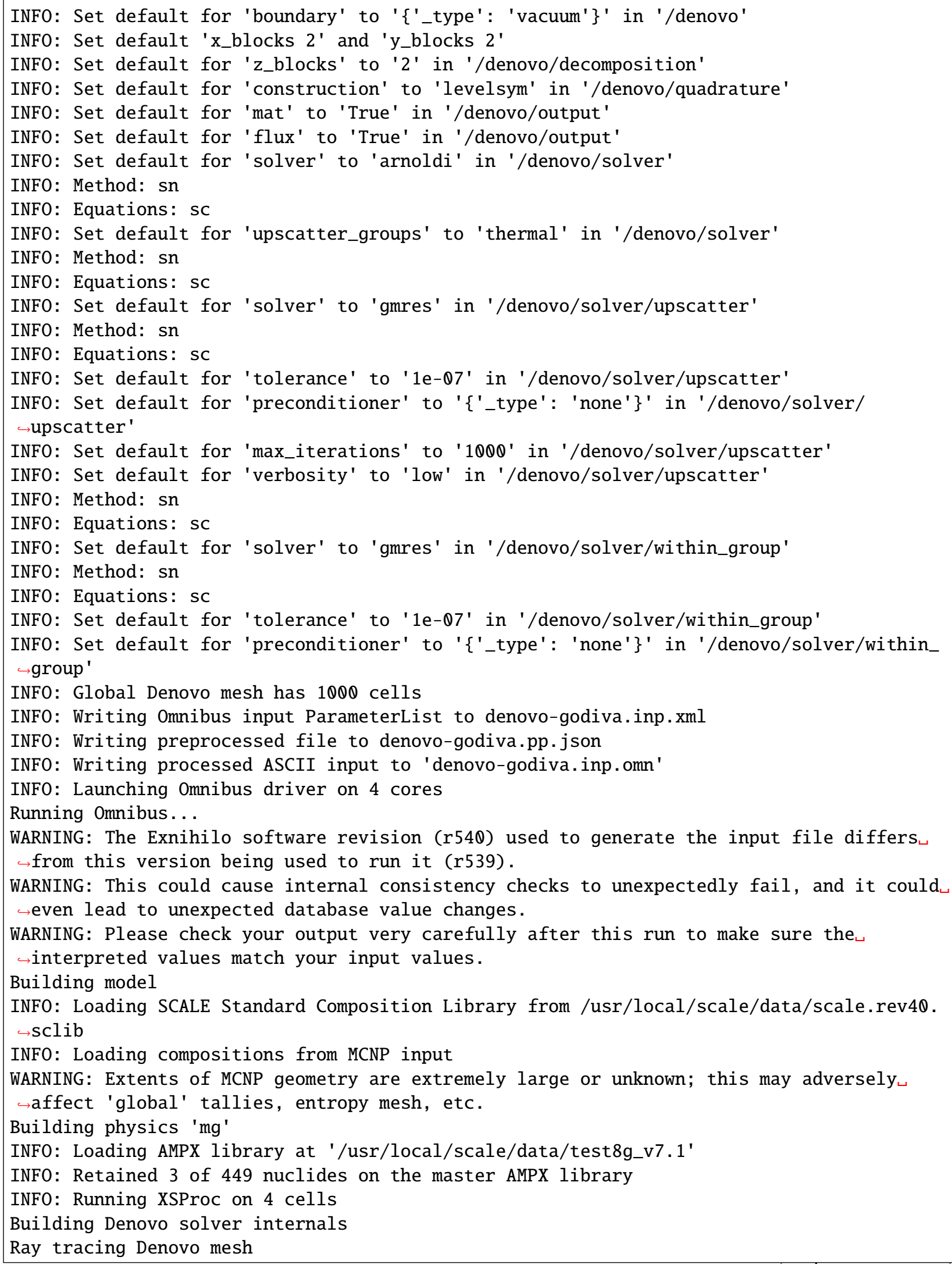


(continued from previous page)

Mixing Denovo cross sections

Initializing Denovo solver

INFO: Constructing Denovo state vector with 8 groups, 1000 cells, 1 moments, 1 unknowns $\hookrightarrow$ per cell

Running Denovo transport calculation

INFO: Inner Krylov iterations in replicated group $0: 7$ on node $\theta$

INFO: Inner Krylov iterations in replicated group 1: 9 on node $\theta$

INFO: Inner Krylov iterations in replicated group 2: 7 on node 0

INFO: Inner Krylov iterations in replicated group 3: 5 on node 0

The following inner iteration counts are for Arnoldi solve(wg tol $=1 \mathrm{e} .$.

Belos Block GMRES converged after 7 iterations.

>> Inner Krylov iterations in replicated group $0: 7$ on node $\theta$

Belos Block GMRES converged after 9 iterations.

>> Inner Krylov iterations in replicated group 1: 9 on node $\theta$

Belos Block GMRES converged after 7 iterations.

>> Inner Krylov iterations in replicated group 2: 7 on node $\theta$

Belos Block GMRES converged after 5 iterations.

>> Inner Krylov iterations in replicated group 3: 5 on node 0

INFO: Inner Krylov iterations in partitioned groups 4-7 on set $\theta$ and node $\theta$ : 5

INFO: Inner Krylov iterations in replicated group 0: 7 on node 0

INF0: Inner Krylov iterations in replicated group 1: 9 on node 0

INFO: Inner Krylov iterations in replicated group 2: 7 on node $\theta$

INFO: Inner Krylov iterations in replicated group 3: 5 on node 0

INFO: Inner Krylov iterations in partitioned groups $4-7$ on set $\theta$ and node $\theta$ : 5

INFO: Inner Krylov iterations in replicated group $0: 7$ on node $\theta$

INFO: Inner Krylov iterations in replicated group 1: 9 on node 0

INFO: Inner Krylov iterations in replicated group 2: 7 on node 0

INFO: Inner Krylov iterations in replicated group 3: 5 on node 0

INFO: Inner Krylov iterations in partitioned groups 4-7 on set $\theta$ and node $\theta$ : 5

INFO: Inner Krylov iterations in replicated group $0: 7$ on node $\theta$

INFO: Inner Krylov iterations in replicated group 1: 9 on node $\theta$

INFO: Inner Krylov iterations in replicated group 2: 7 on node 0

Belos Block GMRES converged after 5 iterations.

>> Inner Krylov iterations in partitioned groups 4-7 on set $\theta$ and nod...

The following inner iteration counts are for Arnoldi solve(wg tol $=1 \mathrm{e} .$.

Belos Block GMRES converged after 7 iterations.

>> Inner Krylov iterations in replicated group $0: 7$ on node $\theta$

Belos Block GMRES converged after 9 iterations.

>> Inner Krylov iterations in replicated group 1: 9 on node $\theta$

Belos Block GMRES converged after 7 iterations.

>> Inner Krylov iterations in replicated group 2: 7 on node 0

Belos Block GMRES converged after 5 iterations.

>> Inner Krylov iterations in replicated group 3: 5 on node $\theta$

Belos Block GMRES converged after 5 iterations.

>> Inner Krylov iterations in partitioned groups 4-7 on set $\theta$ and nod...

The following inner iteration counts are for Arnoldi solve(wg tol $=1 \mathrm{e} .$.

Belos Block GMRES converged after 7 iterations.

>> Inner Krylov iterations in replicated group $0: 7$ on node $\theta$

Belos Block GMRES converged after 9 iterations.

>> Inner Krylov iterations in replicated group 1: 9 on node $\theta$

Belos Block GMRES converged after 7 iterations.

>> Inner Krylov iterations in replicated group 2: 7 on node $\theta$ 
(continued from previous page)

Belos Block GMRES converged after 5 iterations.

>> Inner Krylov iterations in replicated group 3: 5 on node $\theta$

Belos Block GMRES converged after 5 iterations.

>> Inner Krylov iterations in partitioned groups 4-7 on set $\theta$ and nod...

The following inner iteration counts are for Arnoldi solve(wg tol $=1 \mathrm{e} .$.

Belos Block GMRES converged after 7 iterations.

>> Inner Krylov iterations in replicated group $0: 7$ on node $\theta$

Belos Block GMRES converged after 9 iterations.

>> Inner Krylov iterations in replicated group 1: 9 on node $\theta$

Belos Block GMRES converged after 7 iterations.

\> Inner Krylov iterations in replicated group 2: 7 on node 0

INFO: Inner Krylov iterations in replicated group 3: 5 on node 0

INFO: Inner Krylov iterations in partitioned groups $4-7$ on set $\theta$ and node 0 : 5

INFO: Inner Krylov iterations in replicated group 0 : 7 on node 0

INFO: Inner Krylov iterations in replicated group 1: 9 on node 0

INFO: Inner Krylov iterations in replicated group 2: 7 on node 0

INFO: Inner Krylov iterations in replicated group 3: 5 on node 0

INFO: Inner Krylov iterations in partitioned groups $4-7$ on set $\theta$ and node 0 : 5

INFO: Inner Krylov iterations in replicated group $0: 8$ on node 0

INFO: Inner Krylov iterations in replicated group 1: 10 on node $\theta$

INFO: Inner Krylov iterations in replicated group 2: 7 on node $\theta$

INFO: Inner Krylov iterations in replicated group 3: 5 on node 0

INFO: Inner Krylov iterations in partitioned groups $4-7$ on set $\theta$ and node 0 : 5

INFO: Inner Krylov iterations in replicated group $0: 8$ on node $\theta$

INFO: Inner Krylov iterations in replicated group 1: 10 on node $\theta$

Belos Block GMRES converged after 5 iterations.

>> Inner Krylov iterations in replicated group 3: 5 on node $\theta$

Belos Block GMRES converged after 5 iterations.

>> Inner Krylov iterations in partitioned groups 4-7 on set $\theta$ and nod...

The following inner iteration counts are for Arnoldi solve(wg tol $=1 \mathrm{e} .$.

Belos Block GMRES converged after 7 iterations.

>> Inner Krylov iterations in replicated group $0: 7$ on node $\theta$

Belos Block GMRES converged after 9 iterations.

>> Inner Krylov iterations in replicated group 1: 9 on node $\theta$

Belos Block GMRES converged after 7 iterations.

>> Inner Krylov iterations in replicated group 2: 7 on node $\theta$

Belos Block GMRES converged after 5 iterations.

>> Inner Krylov iterations in replicated group 3: 5 on node $\theta$

Belos Block GMRES converged after 5 iterations.

>> Inner Krylov iterations in partitioned groups 4-7 on set $\theta$ and nod...

The following inner iteration counts are for Arnoldi solve(wg tol $=1 \mathrm{e} .$.

Belos Block GMRES converged after 8 iterations.

>> Inner Krylov iterations in replicated group $0: 8$ on node $\theta$

Belos Block GMRES converged after 10 iterations.

>> Inner Krylov iterations in replicated group 1: 10 on node $\theta$

Belos Block GMRES converged after 7 iterations.

>> Inner Krylov iterations in replicated group 2: 7 on node $\theta$

Belos Block GMRES converged after 5 iterations.

>> Inner Krylov iterations in replicated group 3: 5 on node $\theta$

Belos Block GMRES converged after 5 iterations.

>> Inner Krylov iterations in partitioned groups 4-7 on set $\theta$ and nod...

The following inner iteration counts are for Arnoldi solve(wg tol $=1 \mathrm{e} .$. 
(continued from previous page)

Belos Block GMRES converged after 8 iterations.

>> Inner Krylov iterations in replicated group $0: 8$ on node $\theta$

Belos Block GMRES converged after 10 iterations.

>> Inner Krylov iterations in replicated group 1: 10 on node $\theta$

INF0: Inner Krylov iterations in replicated group 2: 8 on node 0

INFO: Inner Krylov iterations in replicated group 3: 5 on node 0

INFO: Inner Krylov iterations in partitioned groups $4-7$ on set $\theta$ and node 0 : 5

INFO: Inner Krylov iterations in replicated group $0: 8$ on node 0

INFO: Inner Krylov iterations in replicated group 1: 10 on node $\theta$

INFO: Inner Krylov iterations in replicated group 2: 8 on node $\mathbb{0}$

INFO: Inner Krylov iterations in replicated group 3: 5 on node 0

INFO: Inner Krylov iterations in partitioned groups $4-7$ on set $\theta$ and node $\theta$ : 5

INF0: $\mathrm{k}$-eff $=1.035583673$

INFO: Writing Silo file to 4 concurrent files using material volume fractions

Writing Denovo HDF5 output

Run complete

Cleaning up

Belos Block GMRES converged after 8 iterations.

>> Inner Krylov iterations in replicated group 2: 8 on node 0

Belos Block GMRES converged after 5 iterations.

>> Inner Krylov iterations in replicated group 3: 5 on node $\theta$

Belos Block GMRES converged after 5 iterations.

〉> Inner Krylov iterations in partitioned groups 4-7 on set $\theta$ and nod...

The following inner iteration counts are for Arnoldi solve(wg tol $=1 \mathrm{e} .$.

Belos Block GMRES converged after 8 iterations.

>> Inner Krylov iterations in replicated group $0: 8$ on node $\theta$

Belos Block GMRES converged after 10 iterations.

>> Inner Krylov iterations in replicated group 1: 10 on node $\theta$

Belos Block GMRES converged after 8 iterations.

>> Inner Krylov iterations in replicated group 2: 8 on node 0

Belos Block GMRES converged after 5 iterations.

>> Inner Krylov iterations in replicated group 3: 5 on node $\theta$

Belos Block GMRES converged after 5 iterations.

>> Inner Krylov iterations in partitioned groups 4-7 on set $\theta$ and nod...

...finished running Omnibus in 2.1 seconds

Running Omnibus postprocessing

Loading HDF 5 file...

INFO: Loaded Omnibus output data from 'denovo-godiva.out.h5', problem name 'Godiva with

$\hookrightarrow$ SMG physics, MCNP geometry', created on 2020MAR11 22:36 using SCALE version 6.3.pre-

$\hookrightarrow$ b10 (branch 'omnibus-doc' \#17579cc6 on 2020MAR10)

Loading HDF5 file...

...finished loading HDF5 file

INFO: Loaded Omnibus output data from 'denovo-godiva.out.h5', problem name 'Godiva with

$\hookrightarrow$ SMG physics, MCNP geometry', created on 2020MAR11 22:36 using SCALE version 6.3.pre-

$\hookrightarrow$ b10 (branch 'omnibus-doc' \#17579cc6 on 2020MAR10)

...finished loading HDF 5 file

Writing Denovo visualization file...

INF0: Wrote Denovo visualization file to denovo-godiva.denovo.xmf

...finished writing Denovo visualization file

Building RST summary...

INFO: Wrote summary file to denovo-godiva.rst

...finished building RST summary 
files it may be impossible to load the entire data file into memory, in which case the extract method should not be called. Instead, each time the output data is accessed, an HDF5 read operation will be performed on only the requested data (e.g., a single energy group). Thus these post-processing tools can be used efficiently even for very large files by processing data in smaller chunks.

The eigenvalue is stored in the Denovo block of the output file.

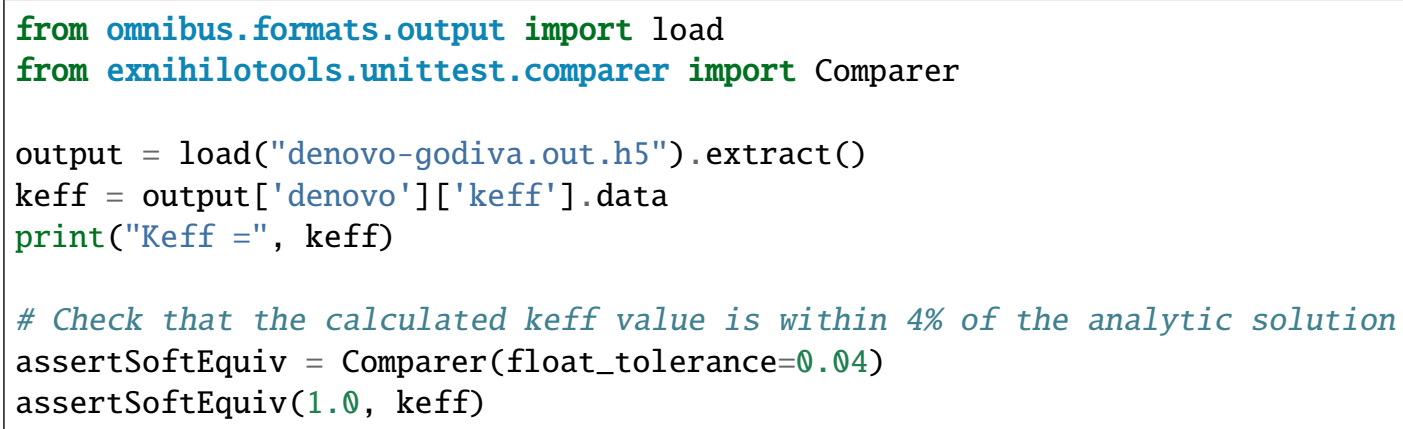

Keff $=1.0355836734048962$

The entire set of denovo results are readily available:

dnv $=$ output ['denovo']

dnv

The above table shows all the datasets and groups present in the original file. Note that since the data reside in memory, the hdf_file attribute is blank. The other attributes are blank because, by default, they are not written to the output. Additional entries to the [DENOVO] [OUTPUT] block will cause them to be written to disk.

The omnibus. field.Field data wrappers allow easy indexing and slicing of the data: it is not necessary to manually determine integer indices in the output: the spatial or energy values can be used directly. Slicing is usually done by taking a "cross sectional" view (hence, xs) of the data. The selection attribute of a slice shows where the slice was taken from (i.e., what part of the original data is selected).

All the axes remain part of the sliced field, so each dataset always retains knowledge of its dimensions and name. The names of the sliced fields are used by omnibus. plot to automatically determine the kind of plot to produce.

The following command plots the flux along the $\mathrm{z}$ axis in cells where $x=y=0$ in energy group $g=1$ :

from omnibus.data import plot

plots $=\operatorname{plot}\left(\operatorname{dnv}\left[\right.\right.$ 'flux' $\left.\left.^{\prime}\right] \cdot \mathrm{xs}(\mathrm{x}=0, \mathrm{y}=0, \mathrm{~g}=1)\right)$

$\mathrm{ax}=$ plots ['ax']

ax.set_ylim( $\theta$, None)

ax.set_title(ax.get_ylabel())

ax.set_ylabel("Particle flux $\left.\left(\mathrm{p} / \mathrm{cm}^{\wedge} 2-\mathrm{s}\right) "\right)$; 
flux: group 1: $(\mathrm{n}: 2.000 \mathrm{e}+04,8.200 \mathrm{e}+05) \mathrm{eV}$; $\mathrm{y}$ in $(0,1.8) ; \mathrm{x}$ in $(0,1.8)$

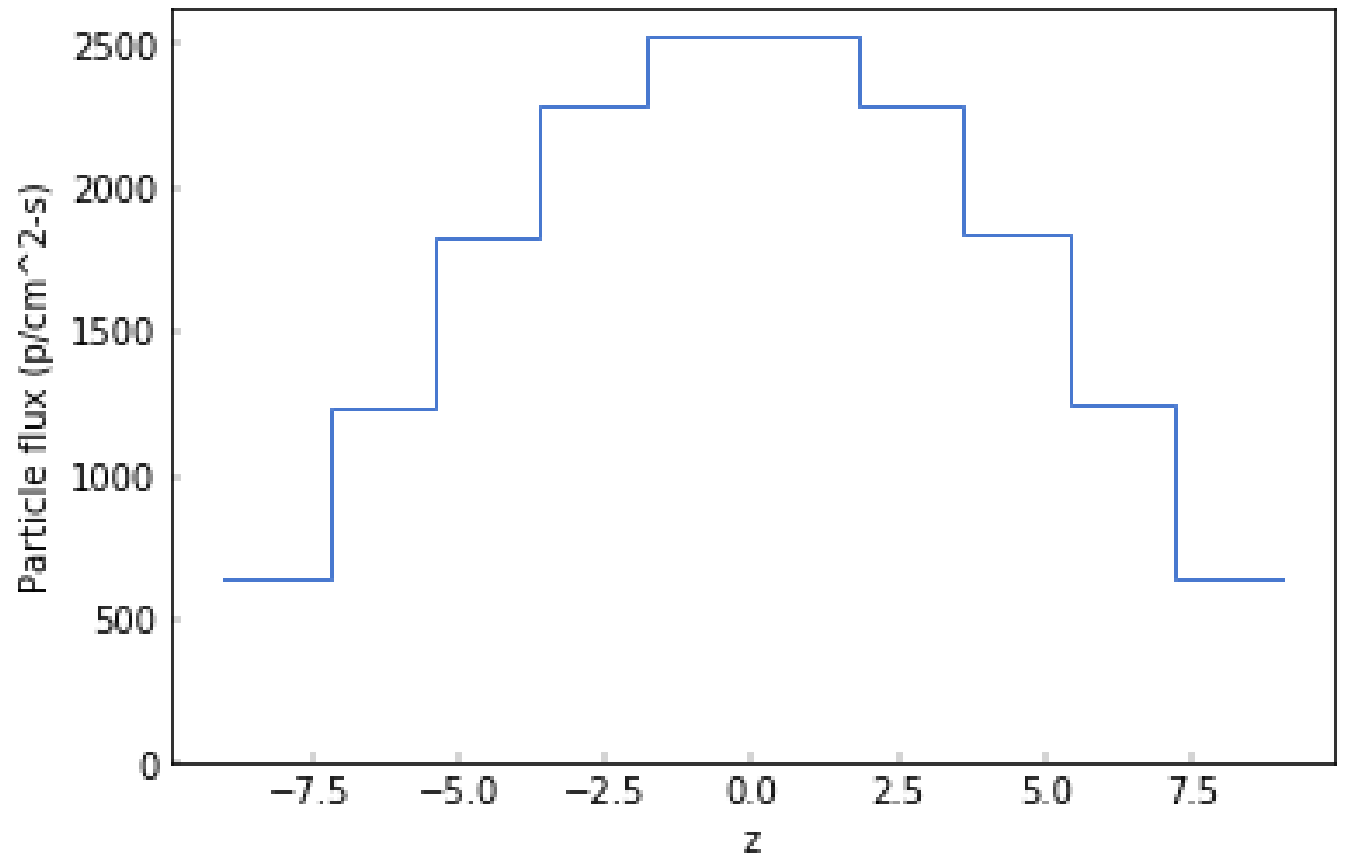

Rendering a slice of the flux as a pseudocolor plot is similarly trivial. The options on the matplotlib LogNorm ${ }^{22}$ class constrain the range of the color scale.

print("Max flux:", dnv.flux.xs(g=3, z=1.0).data.max())

print ("Min flux:", dnv.flux.xs $(g=4, z=1.0) \cdot \operatorname{data} \cdot \min ())$

Max flux: 1.5522386128007388

Min flux: 0.003584260291936911

from matplotlib.colors import LogNorm

norm $=\operatorname{LogNorm}(\operatorname{vmin}=1 \mathrm{e}-3, \operatorname{vmax}=1)$

plot (dnv.flux.xs ( $g=3, z=1.0)$, norm=norm)

plot (dnv . flux .xs (g=4, z=5.0), norm=norm);

22 http://matplotlib.org/api/colors_api.html\#matplotlib.colors.LogNorm 

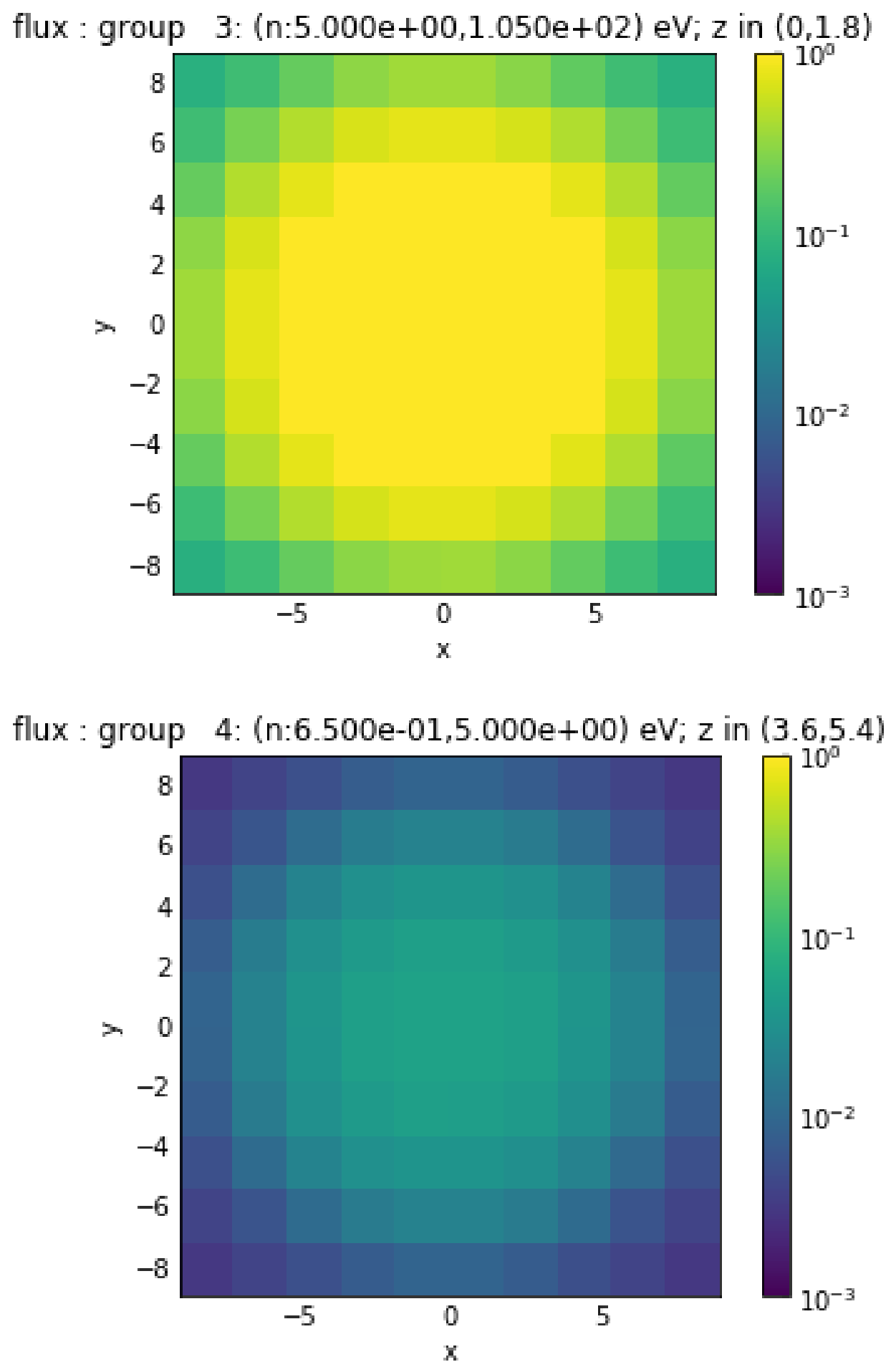

\section{A.2.1.3 Visualize 3D data}

The denovo_godiva.omn input is configured with a [SILO] block to create a Silo-format visualization file, godiva_fluxes.silo, containing a voxelized representation of the scalar flux and the materials. This is an alternate way to view and manipulate data produced from Omnibus.

Launch VisIt and open godiva_fluxes.silo to view this data. An image of the scalar flux has been pre-generated 
in VisIt an example:

from IPython.display import Image

Image (os.path.join(SOURCE_DIR, "data", "godiva_scalar_flux_1.png"), width=600)

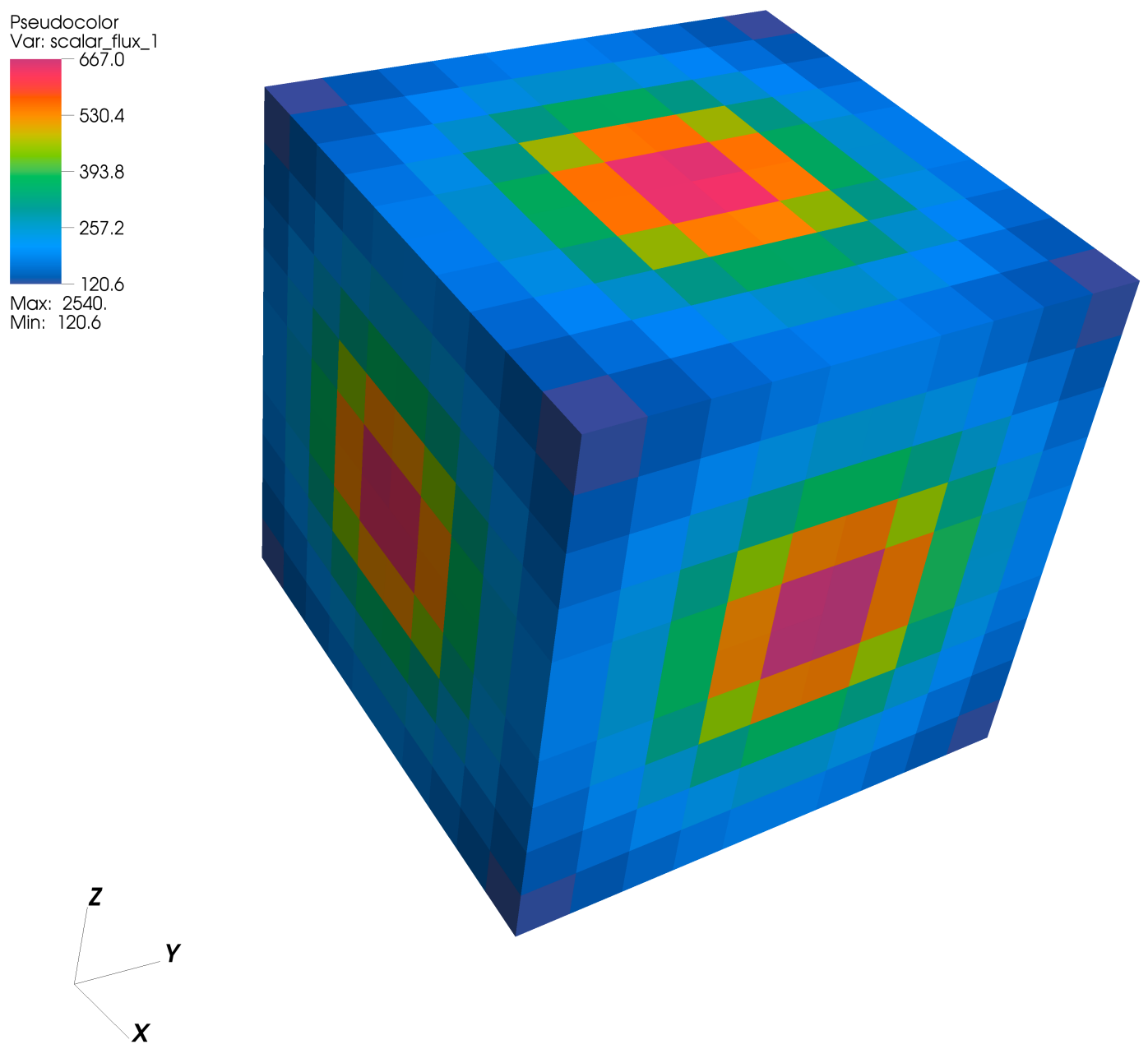

\section{A.2.2 A TOY SHIELDING PROBLEM}

This example demonstrates:

- Denovo's capability to directly discretize and use an MCNP source definition,

- the various Denovo spatial discretization methods, and

- post-processing/flux visualization for a criticality problem.

Three spatial discretization methods will be explored:

- a first order method, Step Characteristics (SC),

- a second order method, Linear Discontinuous (LD), and 
- a diffusion-based method, the Simplified PN $\left(\mathrm{SP}_{N}\right)$ finite volume discretization.

The Denovo method parameter in this manual provides guidance on the choice of discretization scheme:

- SC guarantees positive solutions. It is the preferred method for difficult shielding problems and for problems where a coarse spatial mesh must be used.

- LD methods are often more accurate than SC methods, but they do not guarantee positivity of the solution. They are most appropriate for problems that are not prone to negative solutions (such as reactors).

- $\mathrm{SP}_{N}$ provides fast, accurate solutions primarily for light water reactor eigenvalue problems.

This example uses the expedients of a reduced mesh resolution and a smaller multigroup library to decrease run time. The parameters here are not characteristic of a reliable analysis and should not be construed as such.

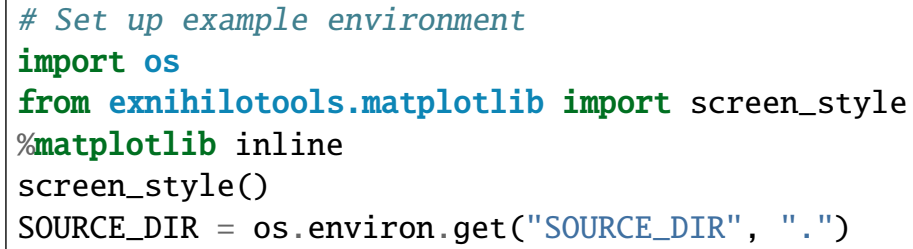

\section{A.2.2.1 Visualize the geometry}

The $[\mathrm{PRE}]$ block in the input file generates images of the geometry before the Omnibus executable is run. It is automatically run when omnibus-run is called, but it can also be used with the standalone omnibus-pre.

In this input file, the autoname command is used to automatically set MCNP names based on the material compositions. (It substitutes "ss" for "m1" and "water" for "m2").

!grep -A 4 "\[PRE $\backslash] "$ \{SOURCE_DIR\}/data/denovo-ironsphere.omn

! omnibus-pre \{SOURCE_DIR\}/data/denovo-ironsphere.omn

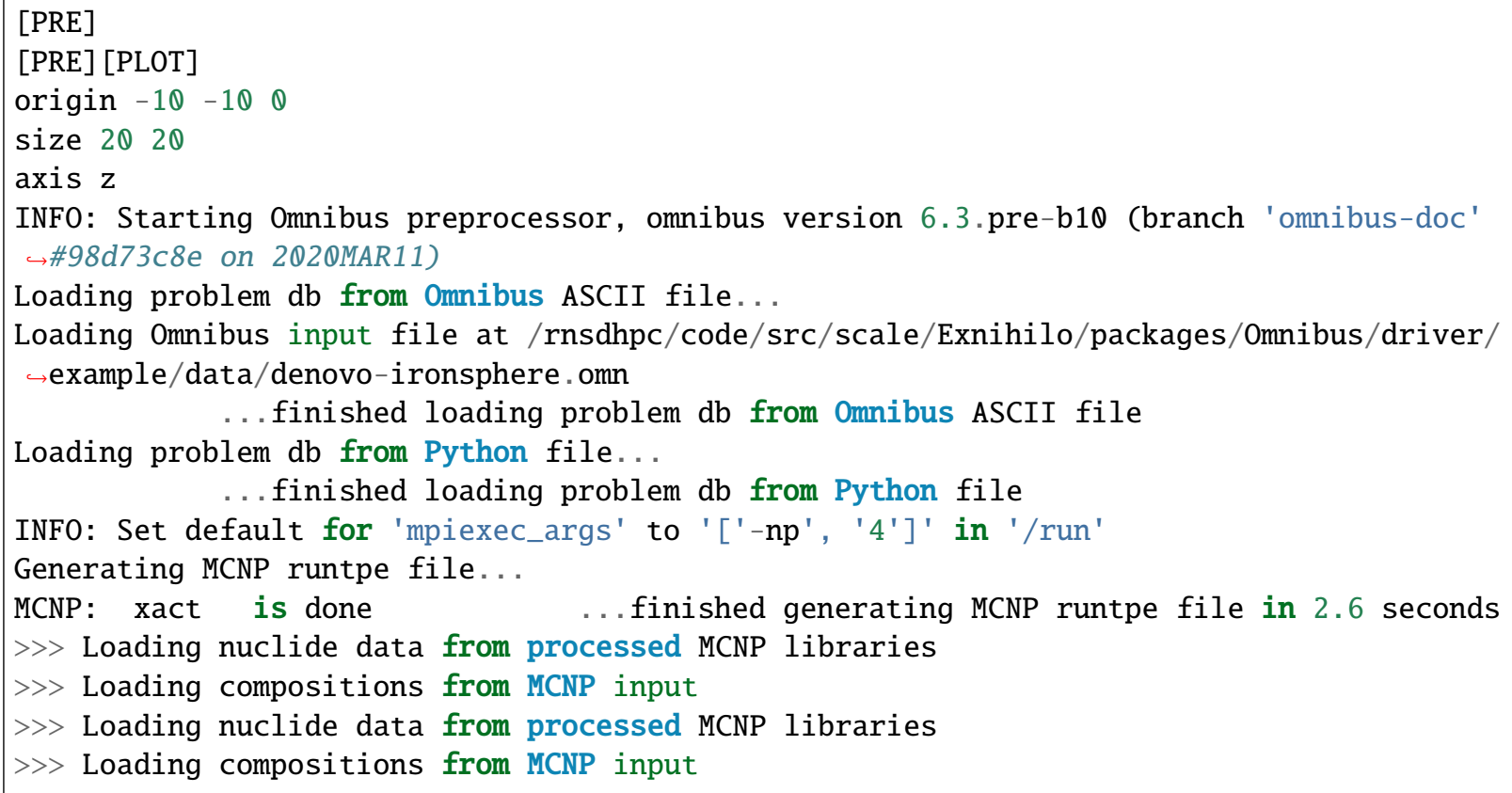


(continued from previous page)

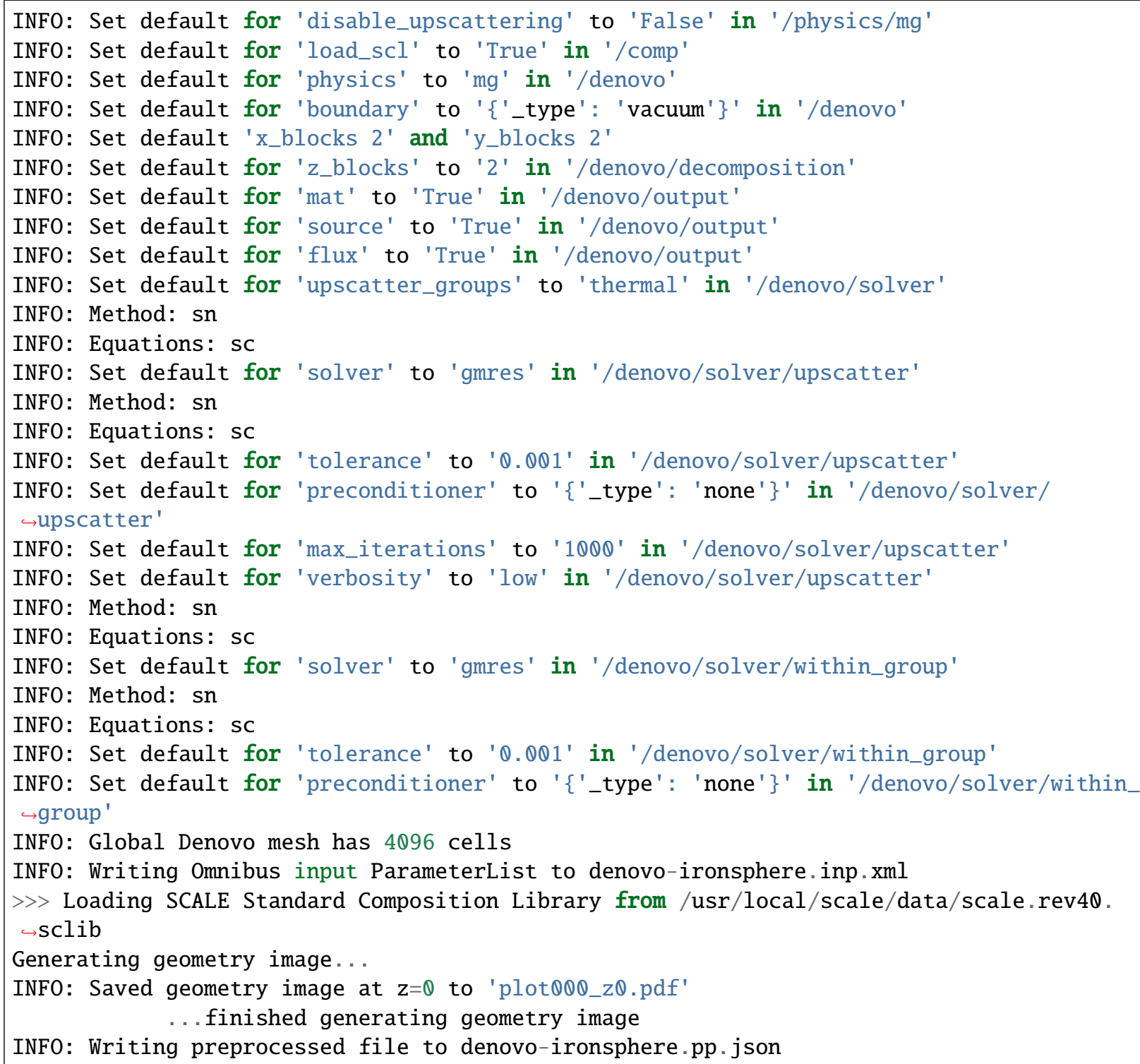

\section{A.2.2.2 SC results}

The following Omnibus input uses SCALE multigroup physics for the Denovo calculation. The forward mode specifies a fixed source problem for this shielding calculation; the [SOURCE=mcnp] uses the MCNP SDEF card to generate the source term; the DENOVO method specifies the spatial discretization; and the [SOLVER=fixed] block specifies an overall desired convergence tolerance for this fixed source problem.

To avoid repeating the same input file for the three different methods in this example, a "base" input file stores the common parameters. Each individual method uses a Python snippet to tweak the input. Calling omnibus-run on multiple input arguments allows them to modify a common input. 
\%cat \{SOURCE_DIR\}/data/denovo-ironsphere.omn \{SOURCE_DIR\}/data/denovo-ironsphere-sc.py

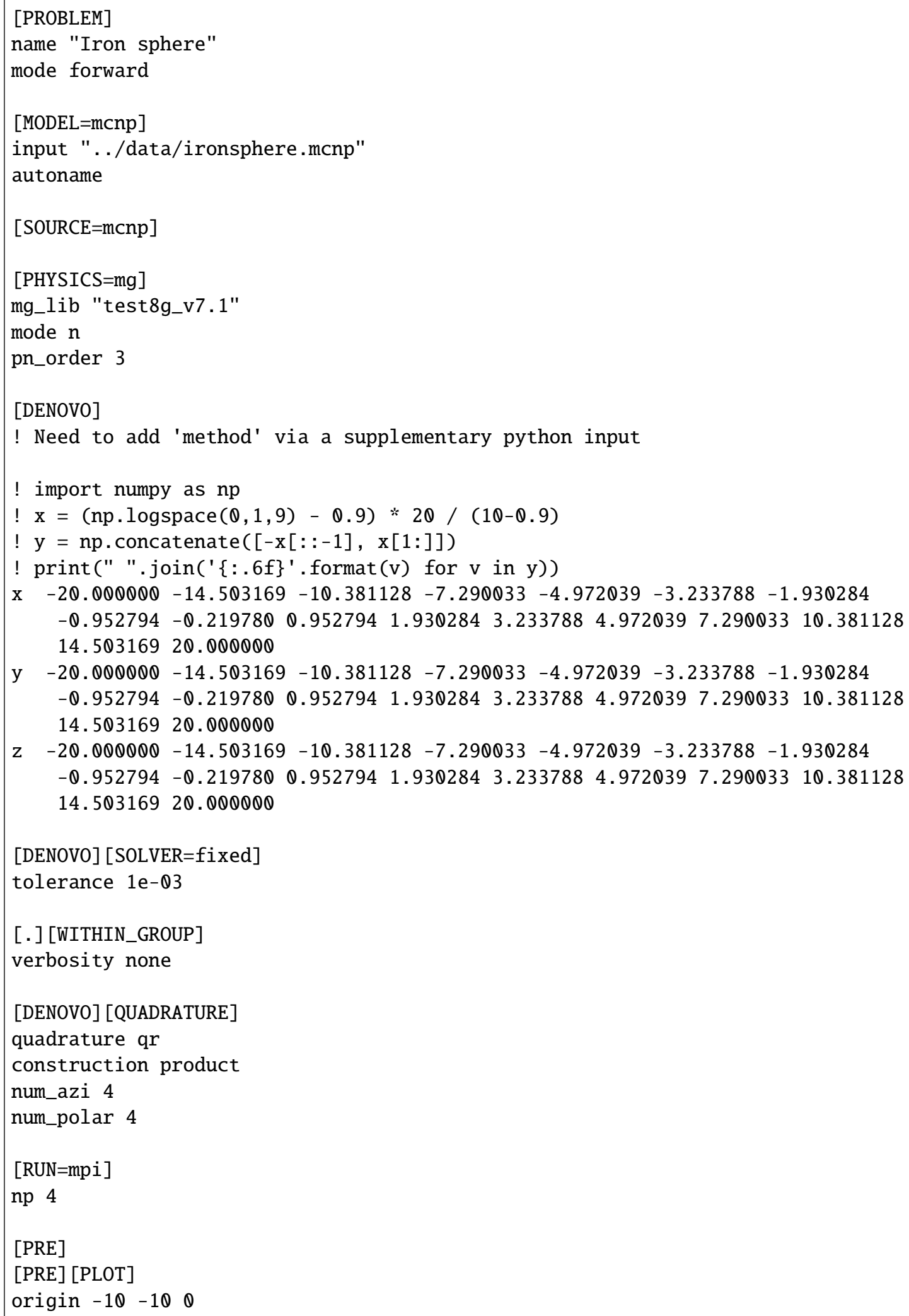


(continued from previous page)

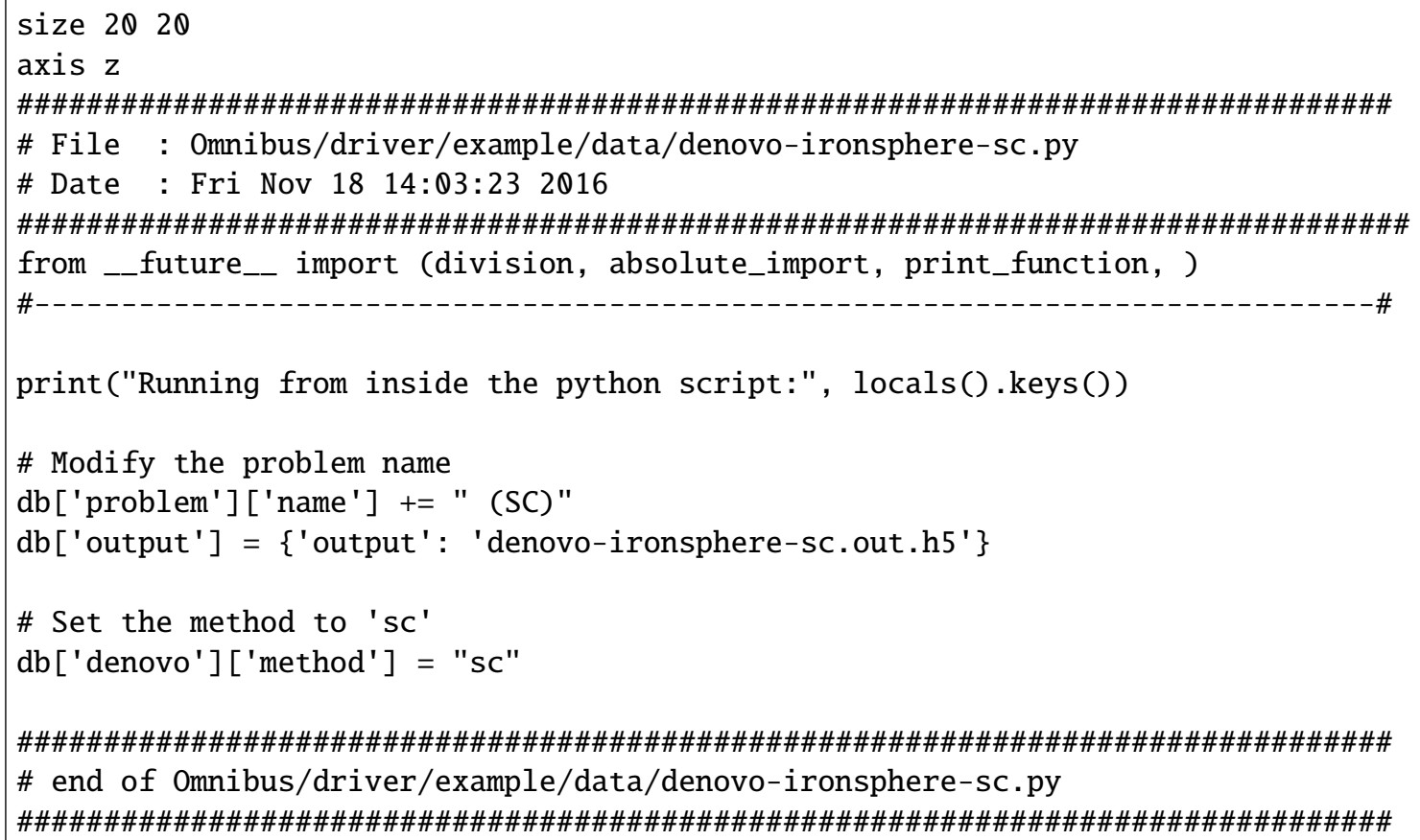

! omnibus-run \{SOURCE_DIR\}/data/denovo-ironsphere.omn \{SOURCE_DIR\}/data/denovo-ironsphere$\hookrightarrow$ SC.py

INF0: Starting Omnibus preprocessor, omnibus version 6.3.pre-b10 (branch 'omnibus-doc' $\hookrightarrow$ \#98d73c8e on 2020MAR11)

Loading problem db from Omnibus ASCII file...

Loading Omnibus input file at/rnsdhpc/code/src/scale/Exnihilo/packages/Omnibus/driver/

$\hookrightarrow$ example/data/denovo-ironsphere.omn ...finished loading problem db from Omnibus ASCII file

Loading problem db from Python file...

Running from inside the python script: dict_keys(['db', '__builtins_-', 'division', $\hookrightarrow$ 'absolute_import', 'print_function']) ...finished loading problem db from Python file

Loading problem db from Python file... ...finished loading problem db from Python file

INFO: Set default for 'mpiexec_args' to '['-np', '4']' in '/run'

Generating MCNP runtpe file...

...finished generating MCNP runtpe file

$\gg>$ Loading nuclide data from processed MCNP libraries

$\gg>$ Loading compositions from MCNP input

$\gg>$ Loading nuclide data from processed MCNP libraries

>> Loading compositions from MCNP input

INFO: Set default for 'disable_upscattering' to 'False' in '/physics/mg'

INFO: Set default for 'load_scl' to 'True' in '/comp'

INFO: Set default for 'physics' to ' $\mathrm{mg}$ ' in '/denovo'

WARNING: The 'method' keyword is now 'equations' for SN discretization types. Changing $\hookrightarrow$ 'method $\mathrm{sc}^{\prime}$ to 'equations $\mathrm{sc}$

INFO: Set default for 'boundary' to '\{'_type': 'vacuum'\}' in '/denovo'

INFO: Set default 'x_blocks 2' and 'y_blocks 2' 
(continued from previous page)

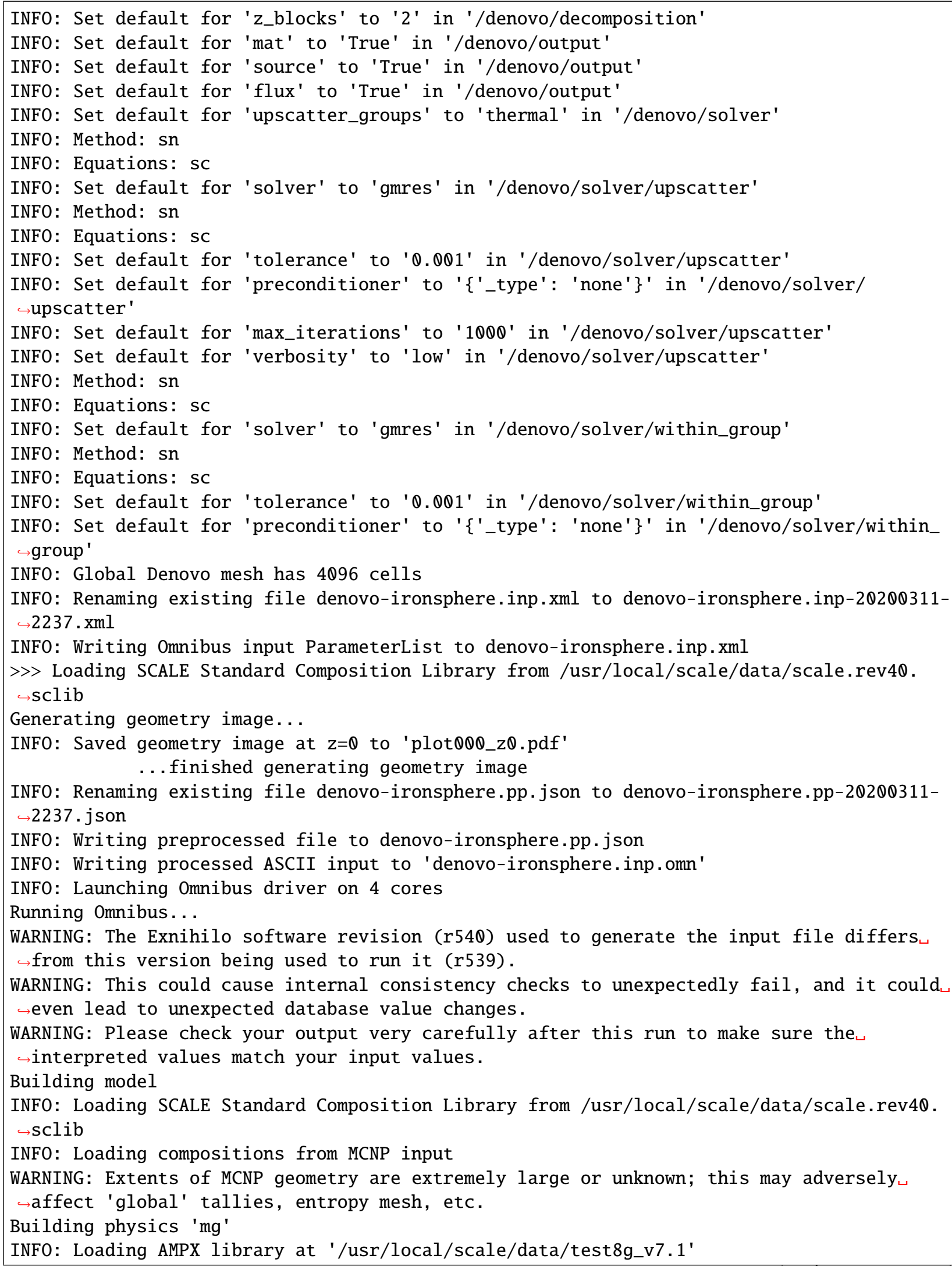


INFO: Retained 21 of 449 nuclides on the master AMPX library

INFO: Running XSProc on 2 cells

WARNING: Remapped 2 nuclide IDs: $6012->6000,6013->6000$

Building sources

Building Denovo solver internals

Ray tracing Denovo mesh

Mixing Denovo cross sections

Building Denovo sources

Constructing forward sources for Denovo

WARNING: MCNP source discretization is currently experimental: only a single isotropic

$\rightarrow$ volumetric source will be treated correctly.

WARNING: Discretization of source (type MCNP) was undersampled (undersampling fraction 0 . $\rightarrow$ 999329).

WARNING: Consider increasing the number of samples per batch or decreasing the source ${ }_{\sqcup}$

$\rightarrow$ bounding box to $(-0.952794,-0.952794,-0.952794)-(0.952794,0.952794,0.952794)$

Initializing Denovo solver

INFO: Constructing Denovo state vector with 8 groups, 4096 cells, 16 moments, 1 unknowns $\hookrightarrow$ per cell

Running Denovo transport calculation

Forward group 0 finished in 3 Belos Block GMRES iterations in $1 \mathrm{~s} .$.

Forward group 1 finished in 4 Belos Block GMRES iterations in $1 \mathrm{~s} \ldots$

Forward group 2 finished in 5 Belos Block GMRES iterations in $1 \mathrm{~s} .$.

Forward group 3 finished in 3 Belos Block GMRES iterations in $1 \mathrm{~s} \ldots$

INFO: Forward groups 4-7 finished in 25 Belos Block GMRES iterations.

INFO: Writing Silo file to 4 concurrent files using material volume fractions

Writing Denovo HDF5 output

Run complete

Cleaning up

Belos Block GMRES converged after 25 iterations.

>> Forward groups 4-7 finished in 25 Belos Block GMRES iterations.

...finished running Omnibus in 3.3 seconds

Running Omnibus postprocessing

Loading HDF5 file...

INFO: Loaded Omnibus output data from 'denovo-ironsphere-sc.out.h5', problem name 'Iron $\hookrightarrow$ sphere (SC)', created on 2020MAR11 22:37 using SCALE version 6.3.pre-b10 (branch

$\hookrightarrow$ 'omnibus-doc' \#17579cc6 on 2020MAR10) ...finished loading HDF5 file

Loading HDF 5 file...

INFO: Loaded Omnibus output data from 'denovo-ironsphere-sc.out.h5', problem name 'Iron

$\rightarrow$ sphere (SC)', created on 2020MAR11 22:37 using SCALE version 6.3.pre-b10 (branch

$\hookrightarrow$ 'omnibus-doc' \#17579cc6 on 2020MAR10)

...finished loading HDF5 file

Writing Denovo visualization file...

INF0: Wrote Denovo visualization file to denovo-ironsphere-sc.denovo.xmf ...finished writing Denovo visualization file

Building RST summary...

INFO: Wrote summary file to denovo-ironsphere-sc.rst ...finished building RST summary

After executing Omnibus, the data can be extracted and visualized: 
from omnibus. formats.output import load

dnv_sc = load("denovo-ironsphere-sc.out.h5") ['denovo'].extract()

dnv_sc

Loading HDF5 file...

INFO: Loaded Omnibus output data from 'denovo-ironsphere-sc.out.h5', problem name 'Iron $\sqcup$ $\hookrightarrow$ sphere (SC)', created on 2020MAR11 22:37 using SCALE version 6.3.pre-b10 (branch

$\hookrightarrow$ 'omnibus-doc' \#17579cc6 on 2020MAR10)

...finished loading HDF5 file

print (dnv_sc ['flux']. shape)

print (dnv_sc['flux'].dims)

$(8,16,16,16)$

$\left[{ }^{\prime}{ }^{\prime},{ }^{\prime} z\right.$ ', 'y', 'x']

\# Print group-5 flux

flux_sc $=$ dnv_sc $\cdot f l u x \cdot x s(g=5)$

flux_sc

from omnibus.data import plot

def plot_x_flux(flux, groups):

"""Plot multigroup wise fluxes along the $x$ axis"""

$\mathrm{ax}=$ None

for $g$ in groups:

plots $=\operatorname{plot}(f \operatorname{lux} \cdot \mathrm{xs}(\mathrm{y}=0.0, \mathrm{z}=0.0, \mathrm{~g}=\mathrm{g})$, logy $=$ True, $\mathrm{ax}=\mathrm{ax})$

$\mathrm{ax}=\operatorname{plots}\left[\mathrm{ax}^{\prime}\right]$

plots['plot'][0].set_label(flux.axis('g').describe_index(g))

ax.legend (loc=' lower right')

ax.set_title("')

ax.set_xlabel("x $[\mathrm{cm}] ")$

ax.set_ylabel ("Neutron flux $[\mathrm{n} / \mathrm{cm} \$ \wedge 2 \$-\mathrm{s}] ")$

plot_x_flux(dnv_sc['flux'], $\operatorname{range}(3,6))$ 


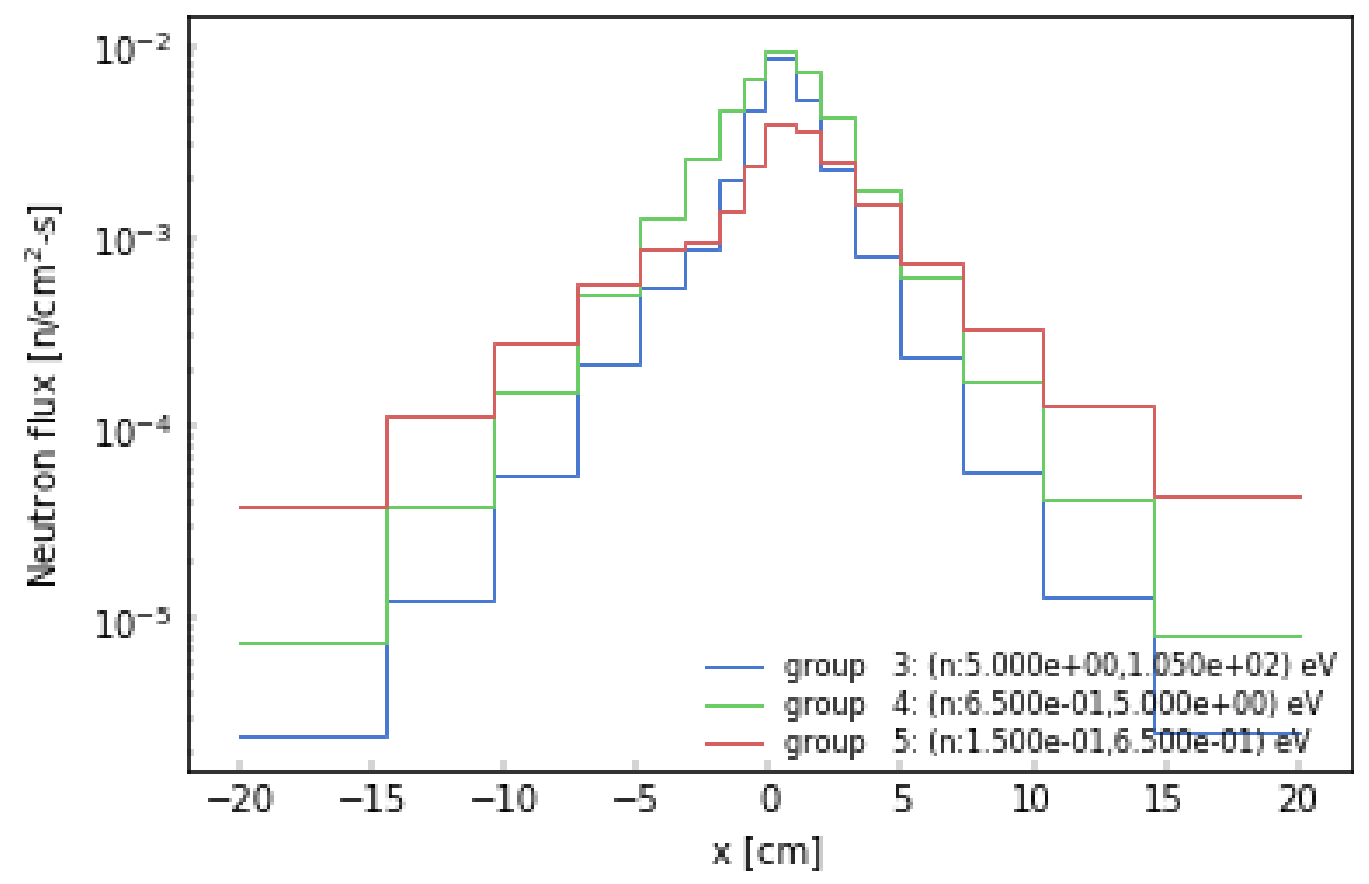

Two-dimensional pseudocolor plots of the flux are easy to render. Note that the ray effects seen in the following plot are characteristic of any discrete ordinate method, including SC.

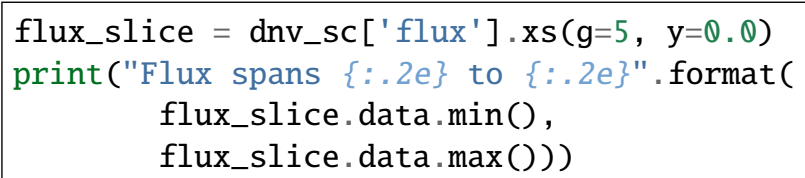

Flux spans $3.72 \mathrm{e}-06$ to $3.88 \mathrm{e}-03$

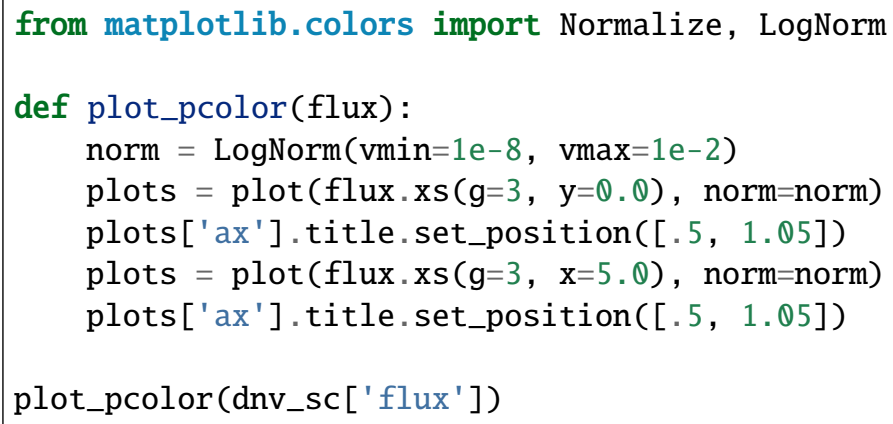


flux: group 3: (n:5.000e+00,1.050e+02) eV; y in $(-0.21978,0.952794)$

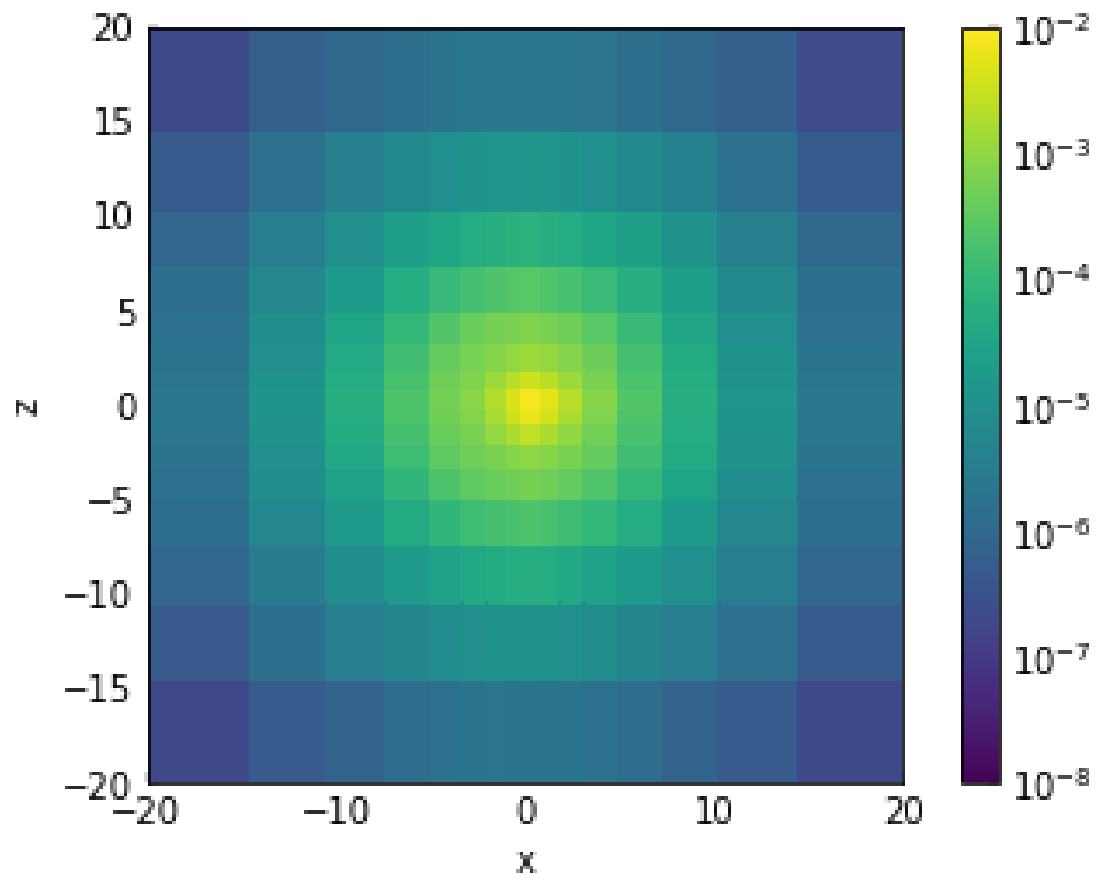

flux: group 3: $(n: 5.000 e+00,1.050 e+02) e V ; x$ in $(4.97204,7.29003)$

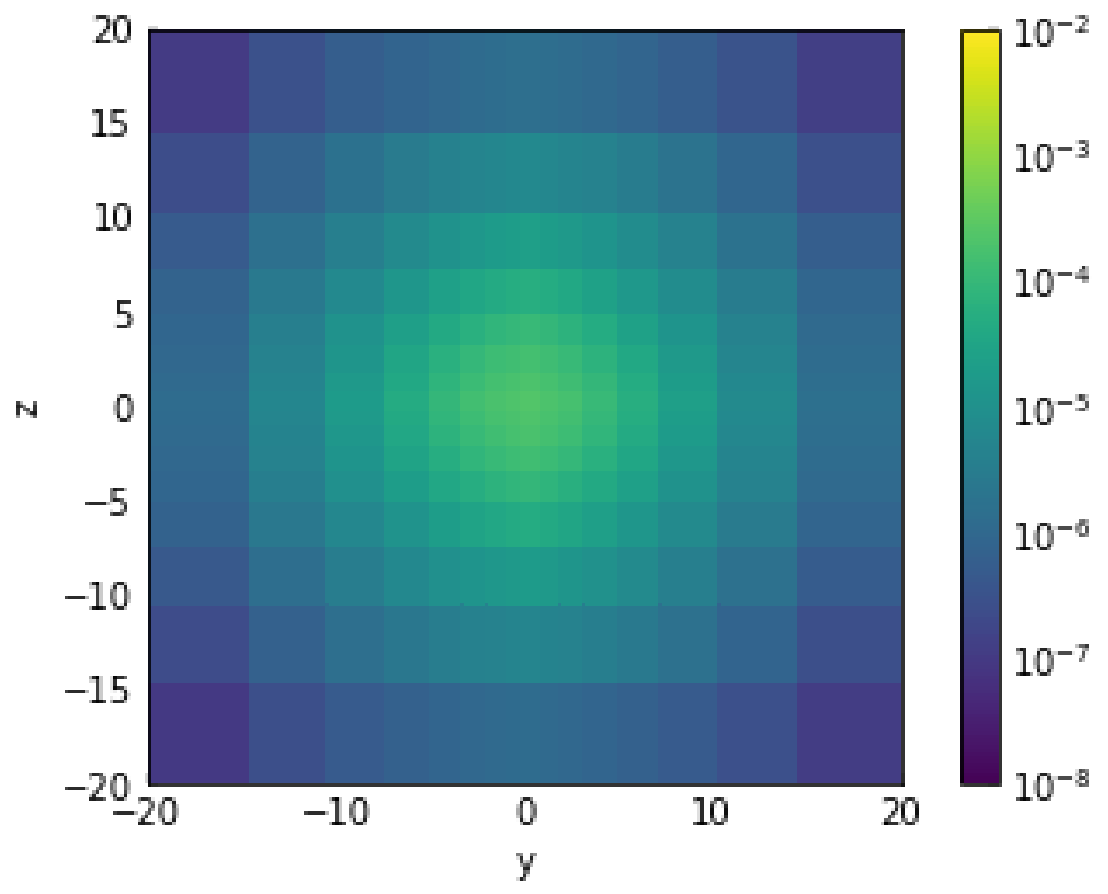

\section{A.2.2.3 LD results}

The following changes to the base input run Omnibus with LD instead of SC. 
cat: /rnsdhpc/code/src/scale/Exnihilo/packages/Omnibus/driver/example/data/denovo-

$\hookrightarrow$ ironsphere.ld.py: No such file or directory

! omnibus-run \{SOURCE_DIR\}/data/denovo-ironsphere.omn \{SOURCE_DIR\}/data/denovo-ironsphere$\leftrightarrow l$ d.py

INF0: Starting Omnibus preprocessor, omnibus version 6.3.pre-b10 (branch 'omnibus-doc' $\hookrightarrow \# 98 \mathrm{~d} 73 \mathrm{c} 8 \mathrm{e}$ on 2020MAR11)

Loading problem db from Omnibus ASCII file...

Loading Omnibus input file at/rnsdhpc/code/src/scale/Exnihilo/packages/Omnibus/driver/

$\hookrightarrow$ example/data/denovo-ironsphere.omn

...finished loading problem db from Omnibus ASCII file

Loading problem db from Python file...

...finished loading problem db from Python file

Loading problem db from Python file...

...finished loading problem db from Python file

INFO: Set default for 'mpiexec_args' to '['-np', '4']' in '/run'

Generating MCNP runtpe file...

...finished generating MCNP runtpe file

>> Loading nuclide data from processed MCNP libraries

$\gg>$ Loading compositions from MCNP input

>> Loading nuclide data from processed MCNP libraries

>> Loading compositions from MCNP input

INFO: Set default for 'disable_upscattering' to 'False' in '/physics/mg'

INFO: Set default for 'load_scl' to 'True' in '/comp'

INFO: Set default for 'physics' to 'mg' in '/denovo'

WARNING: The 'method' keyword is now 'equations' for SN discretization types. Changing

$\hookrightarrow$ 'method $l d$ ' to 'equations $l d$ '

INFO: Set default for 'boundary' to '\{'_type': 'vacuum'\}' in '/denovo'

INFO: Set default 'x_blocks 2' and 'y_blocks 2'

INFO: Set default for 'z_blocks' to ' 2 ' in '/denovo/decomposition'

INFO: Set default for 'mat' to 'True' in '/denovo/output'

INFO: Set default for 'source' to 'True' in '/denovo/output'

INFO: Set default for 'flux' to 'True' in '/denovo/output'

INFO: Set default for 'upscatter_groups' to 'thermal' in '/denovo/solver'

INFO: Method: sn

INFO: Equations: ld

INFO: Set default for 'solver' to 'gmres' in '/denovo/solver/upscatter'

INF0: Method: sn

INFO: Equations: ld

INFO: Set default for 'tolerance' to ' 0.001 ' in '/denovo/solver/upscatter'

INFO: Set default for 'preconditioner' to '\{'_type': 'none'\}' in '/denovo/solver/

$\rightarrow$ upscatter'

INFO: Set default for 'max_iterations' to '1000' in '/denovo/solver/upscatter'

INFO: Set default for 'verbosity' to 'low' in '/denovo/solver/upscatter'

INFO: Method: sn

INFO: Equations: $1 \mathrm{~d}$

INF0: Set default for 'solver' to 'gmres' in '/denovo/solver/within_group'

INFO: Method: sn 
(continued from previous page)

INF0: Equations: ld

INFO: Set default for 'tolerance' to ' 0.001 ' in '/denovo/solver/within_group'

INFO: Set default for 'preconditioner' to '\{'_type': 'none'\}' in '/denovo/solver/within_ $\rightarrow$ group '

INFO: Global Denovo mesh has 4096 cells

INFO: Renaming existing file denovo-ironsphere.inp.xml to denovo-ironsphere.inp-20200311$\hookrightarrow 2237 \mathrm{a} . \mathrm{xml}$

INFO: Writing Omnibus input ParameterList to denovo-ironsphere.inp.xml

\> Loading SCALE Standard Composition Library from/usr/local/scale/data/scale.rev40.

$\hookrightarrow$ Sclib

Generating geometry image...

INFO: Saved geometry image at $z=0$ to 'plot000_zQ.pdf'

...finished generating geometry image

INFO: Renaming existing file denovo-ironsphere.pp.json to denovo-ironsphere.pp-20200311$\hookrightarrow 2237 \mathrm{a}$.json

INFO: Writing preprocessed file to denovo-ironsphere.pp.json

INFO: Renaming existing file denovo-ironsphere.inp.omn to denovo-ironsphere.inp-20200311$\hookrightarrow 2237$. omn

INFO: Writing processed ASCII input to 'denovo-ironsphere.inp.omn'

INFO: Launching Omnibus driver on 4 cores

Running Omnibus...

INFO: Renaming existing file omnibus.out to omnibus-20200311-2237.out

INFO: Renaming existing file omnibus.err to omnibus-20200311-2237.err

WARNING: The Exnihilo software revision ( $(540)$ used to generate the input file differs $\rightarrow$ from this version being used to run it ( $r 539)$.

WARNING: This could cause internal consistency checks to unexpectedly fail, and it could seven lead to unexpected database value changes.

WARNING: Please check your output very carefully after this run to make sure the

$\hookrightarrow$ interpreted values match your input values.

Building model

INFO: Loading SCALE Standard Composition Library from/usr/local/scale/data/scale.rev40.

$\hookrightarrow$ Sclib

INFO: Loading compositions from MCNP input

WARNING: Extents of MCNP geometry are extremely large or unknown; this may adversely

$\rightarrow$ affect 'global' tallies, entropy mesh, etc.

Building physics 'mg'

INFO: Loading AMPX library at '/usr/local/scale/data/test8g_v7.1'

INFO: Retained 21 of 449 nuclides on the master AMPX library

INFO: Running XSProc on 2 cells

WARNING: Remapped 2 nuclide IDs: 6012->6000, 6013->6000

Building sources

Building Denovo solver internals

Ray tracing Denovo mesh

Mixing Denovo cross sections

Building Denovo sources

Constructing forward sources for Denovo

WARNING: MCNP source discretization is currently experimental: only a single isotropic ${ }_{\sqcup}$

$\hookrightarrow$ volumetric source will be treated correctly.

WARNING: Discretization of source (type MCNP) was undersampled (undersampling fraction 0 . $\hookrightarrow$ 999329).

WARNING: Consider increasing the number of samples per batch or decreasing the source $\rightarrow$ bounding box to $(-0.952794,-0.952794,-0.952794)-(0.952794,0.952794,0.952794)$ 
(continued from previous page)

Initializing Denovo solver

INFO: Constructing Denovo state vector with 8 groups, 4096 cells, 16 moments, 4 unknowns $\sqcup$ $\hookrightarrow$ per cell

Running Denovo transport calculation

Forward group 0 finished in 3 Belos Block GMRES iterations in $1 \mathrm{~s} .$.

Forward group 1 finished in 4 Belos Block GMRES iterations in $1 \mathrm{~s} .$.

Forward group 2 finished in 5 Belos Block GMRES iterations in $1 \mathrm{~s} .$.

Forward group 3 finished in 4 Belos Block GMRES iterations in $1 \mathrm{~s} .$.

INFO: Forward groups 4-7 finished in 37 Belos Block GMRES iterations.

INFO: Writing Silo file to 4 concurrent files using material volume fractions

INFO: Removing SILO directory denovo_output_silo

Writing Denovo HDF5 output

Run complete

Cleaning up

Belos Block GMRES converged after 37 iterations.

>> Forward groups 4-7 finished in 37 Belos Block GMRES iterations. ...finished running Omnibus in 6.1 seconds

Running Omnibus postprocessing

Loading HDF5 file...

INFO: Loaded Omnibus output data from 'denovo-ironsphere-ld.out.h5', problem name 'Iron

$\hookrightarrow$ sphere (LD)', created on 2020MAR11 22:37 using SCALE version 6.3.pre-b10 (branch

$\hookrightarrow$ 'omnibus-doc' \#17579cc6 on 2020MAR10) ...finished loading HDF5 file

Loading HDF5 file...

INF0: Loaded Omnibus output data from 'denovo-ironsphere-ld.out.h5', problem name 'Iron $\hookrightarrow$ sphere (LD)', created on 2020MAR11 22:37 using SCALE version 6.3.pre-b10 (branch

$\hookrightarrow$ 'omnibus-doc' \#17579cc6 on 2020MAR10) ...finished loading HDF5 file

Writing Denovo visualization file...

INFO: Wrote Denovo visualization file to denovo-ironsphere-ld.denovo.xmf ...finished writing Denovo visualization file

Building RST summary...

INFO: Wrote summary file to denovo-ironsphere-ld.rst ...finished building RST summary

dnv_ld = load("denovo-ironsphere-ld.out.h5") ['denovo'].extract()

plot_x_flux (dnv_ld['flux'], $\operatorname{range}(3,6))$

plot_pcolor(dnv_ld['flux'])

Loading HDF5 file...

INFO: Loaded Omnibus output data from 'denovo-ironsphere-ld.out.h5', problem name 'Iron $\sqcup$ $\hookrightarrow$ sphere (LD)', created on 2020MAR11 22:37 using SCALE version 6.3.pre-b10 (branch

$\hookrightarrow$ 'omnibus-doc' \#17579cc6 on 2020MAR10)

... finished loading HDF5 file 


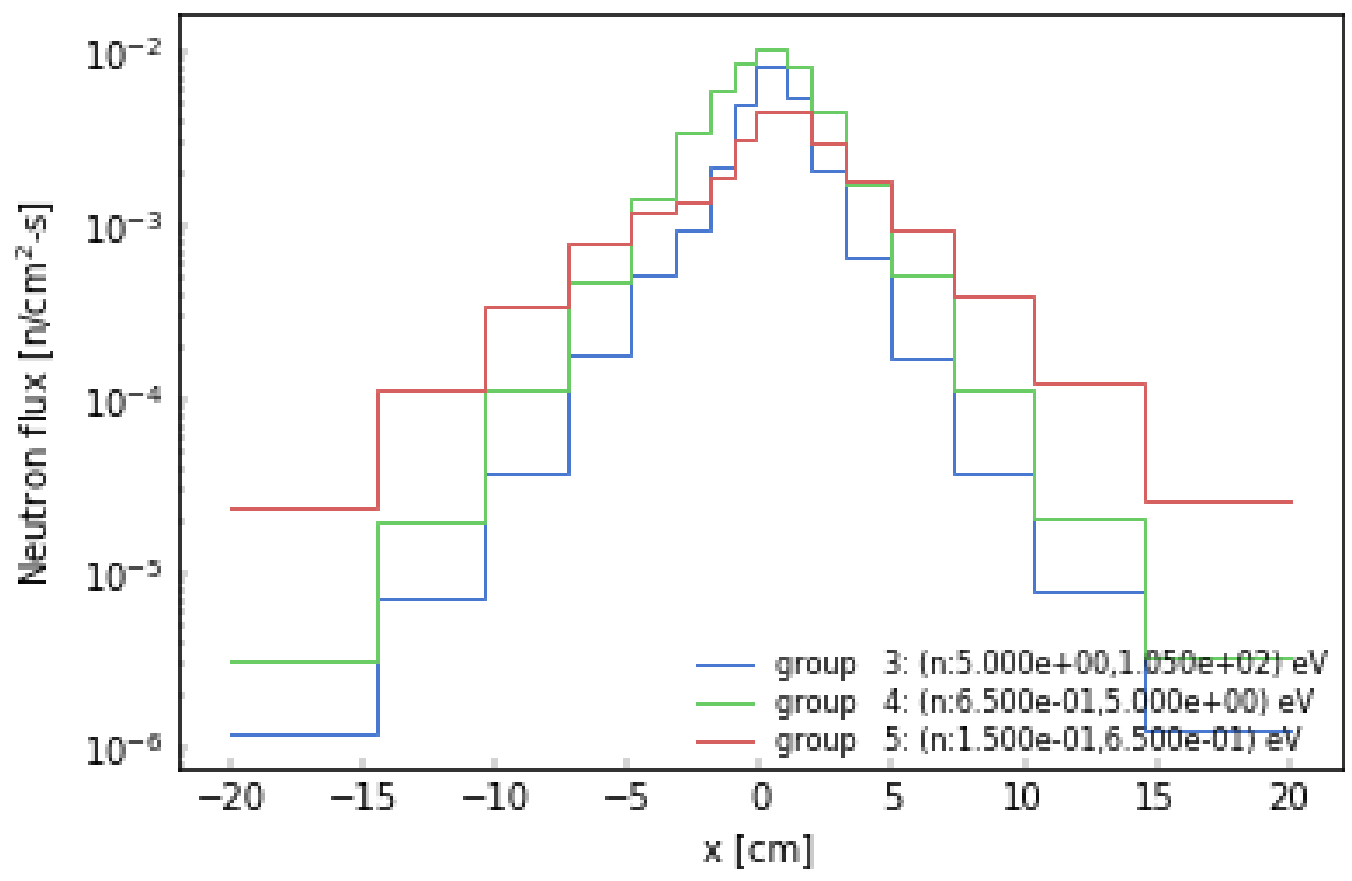

flux: group 3: $(\mathrm{n}: 5.000 \mathrm{e}+00,1.050 \mathrm{e}+02) \mathrm{eV}$; $\mathrm{y}$ in $(-0.21978,0.952794)$

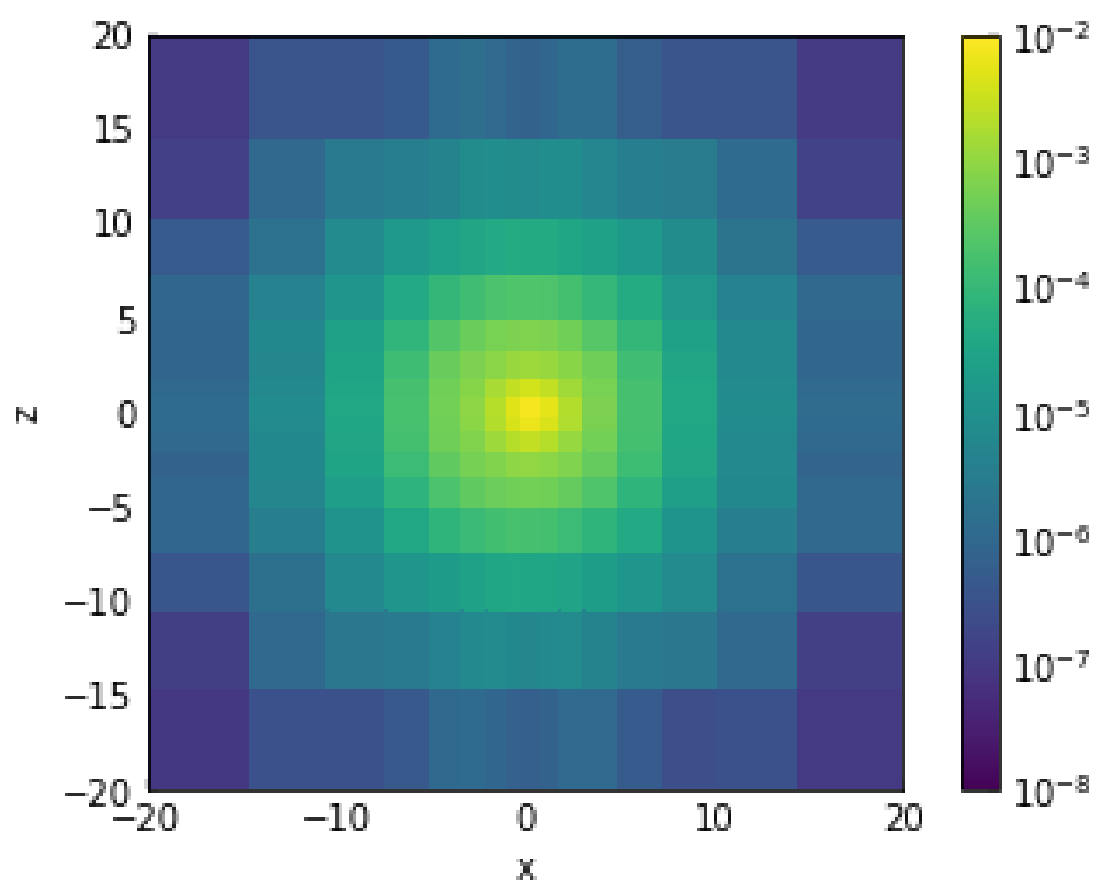




\section{flux: group 3: $(n: 5.000 e+00,1.050 e+02) e V ; x$ in $(4.97204,7.29003)$}

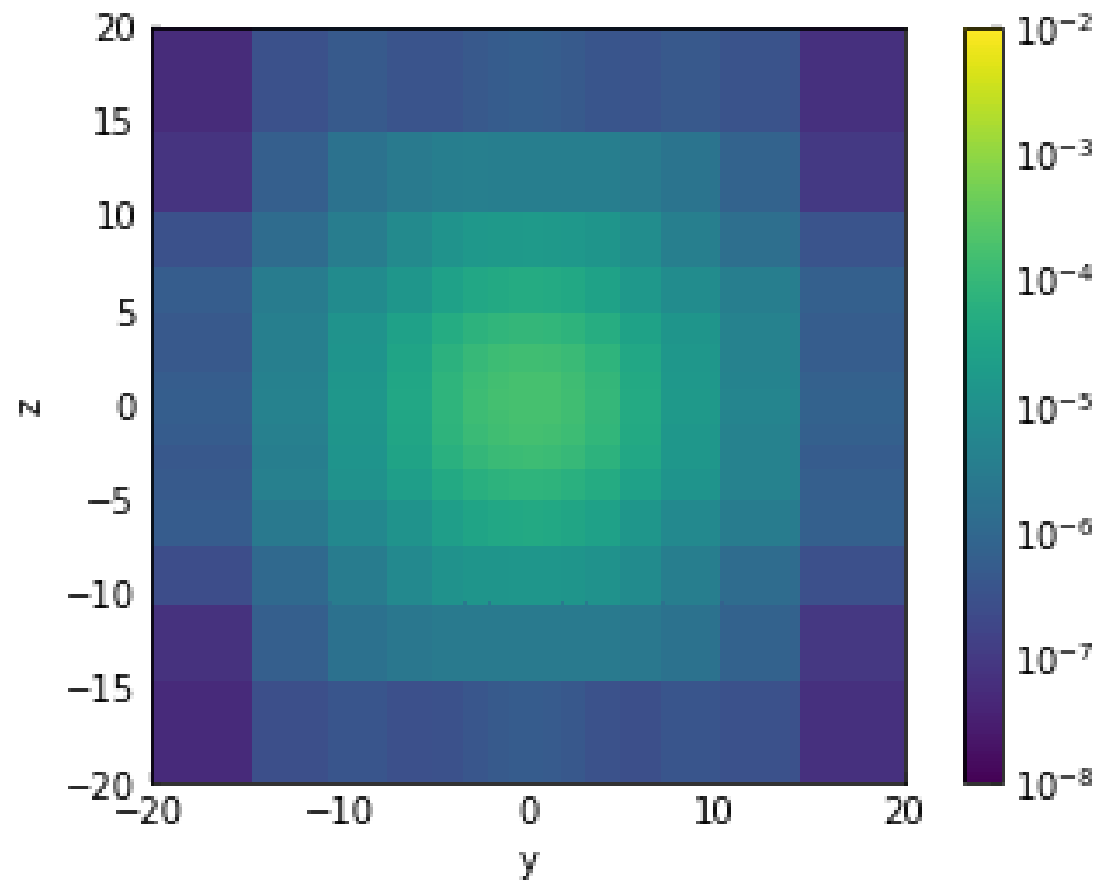

Again, notice the ray effects in the scalar flux plot below due to LD being a discrete ordinates method.

\section{A.2.2.4 Run with $\mathrm{SP}_{N}$}

A final set of changes to the base input runs Omnibus with $\mathrm{SP}_{3}$.

\%cat \{SOURCE_DIR\}/data/denovo-ironsphere-spn.py

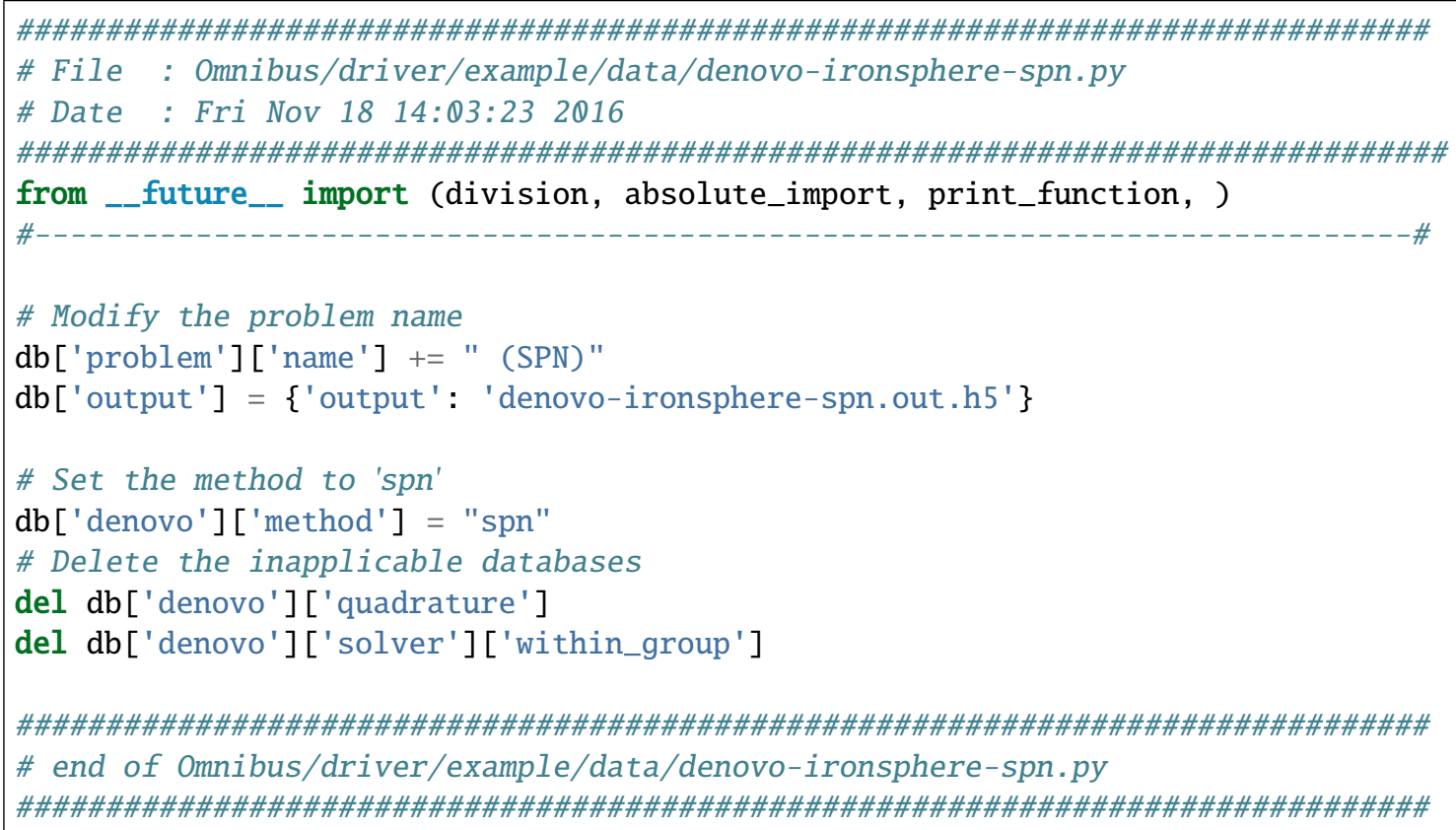


! omnibus-run \{SOURCE_DIR\}/data/denovo-ironsphere.omn \{SOURCE_DIR\}/data/denovo-ironsphere$\hookrightarrow$ spn.py

INF0: Starting Omnibus preprocessor, omnibus version 6.3.pre-b10 (branch 'omnibus-doc' $\hookrightarrow$ ↔98d73c8e on 2020MAR11)

Loading problem db from Omnibus ASCII file...

Loading Omnibus input file at/rnsdhpc/code/src/scale/Exnihilo/packages/Omnibus/driver/

$\rightarrow$ example/data/denovo-ironsphere.omn

...finished loading problem db from Omnibus ASCII file

Loading problem db from Python file...

... finished loading problem db from Python file

Loading problem db from Python file...

...finished loading problem db from Python file

INFO: Set default for 'mpiexec_args' to '['-np', '4']' in '/run'

Generating MCNP runtpe file...

...finished generating MCNP runtpe file

$\gg>$ Loading nuclide data from processed MCNP libraries

>> Loading compositions from MCNP input

$\gg>$ Loading nuclide data from processed MCNP libraries

>>> Loading compositions from MCNP input

INFO: Set default for 'disable_upscattering' to 'False' in '/physics/mg'

INFO: Set default for 'load_scl' to 'True' in '/comp'

INFO: Set default for 'physics' to 'mg' in '/denovo'

INFO: Set default for 'boundary' to '\{'_type': 'vacuum'\}' in '/denovo'

INFO: Set default 'x_blocks 2' and 'y_blocks 2'

INFO: Set default for 'mat' to 'True' in '/denovo/output'

INFO: Set default for 'source' to 'True' in '/denovo/output'

INFO: Set default for 'flux' to 'True' in '/denovo/output'

INFO: Set default for 'upscatter_groups' to 'all' in '/denovo/solver'

INFO: Method: spn

INFO: Set default for 'solver' to 'gmres' in '/denovo/solver/upscatter'

INFO: Method: spn

INFO: Set default for 'tolerance' to ' 0.001 ' in '/denovo/solver/upscatter'

INFO: Set default for 'preconditioner' to '\{'_type': 'none'\}' in '/denovo/solver/

$\rightarrow$ upscatter'

INF0: Set default for 'max_iterations' to '1000' in '/denovo/solver/upscatter'

INF0: Set default for 'verbosity' to 'low' in '/denovo/solver/upscatter'

INF0: Global Denovo mesh has 4096 cells

INFO: Renaming existing file denovo-ironsphere.inp.xml to denovo-ironsphere.inp-20200311$\hookrightarrow 2237 \mathrm{~b} . \mathrm{xml}$

INFO: Writing Omnibus input ParameterList to denovo-ironsphere.inp.xml

>> Loading SCALE Standard Composition Library from/usr/local/scale/data/scale.rev40.

$\hookrightarrow$ Sclib

Generating geometry image...

INFO: Saved geometry image at $\mathrm{z}=0$ to 'plot000_z0.pdf'

...finished generating geometry image

INFO: Renaming existing file denovo-ironsphere.pp.json to denovo-ironsphere.pp-20200311$\hookrightarrow 2237 b$. json

INF0: Writing preprocessed file to denovo-ironsphere.pp.json

INFO: Renaming existing file denovo-ironsphere.inp.omn to denovo-ironsphere.inp-20200311$\hookrightarrow 2237 \mathrm{a}$.omn

INFO: Writing processed ASCII input to 'denovo-ironsphere.inp.omn' 
(continued from previous page)

INFO: Launching Omnibus driver on 4 cores

Running Omnibus...

INFO: Renaming existing file omnibus.out to omnibus-20200311-2237a.out

INF0: Renaming existing file omnibus.err to omnibus-20200311-2237a.err

WARNING: The Exnihilo software revision ( $r 540$ ) used to generate the input file differs $\hookrightarrow$ from this version being used to run it (r539).

WARNING: This could cause internal consistency checks to unexpectedly fail, and it could $\hookrightarrow$ even lead to unexpected database value changes.

WARNING: Please check your output very carefully after this run to make sure the

$\hookrightarrow$ interpreted values match your input values.

Building model

INFO: Loading SCALE Standard Composition Library from/usr/local/scale/data/scale.rev40. $\hookrightarrow$ Sclib

INFO: Loading compositions from MCNP input

WARNING: Extents of MCNP geometry are extremely large or unknown; this may adversely $\hookrightarrow$ affect 'global' tallies, entropy mesh, etc.

Building physics 'mg'

INFO: Loading AMPX library at '/usr/local/scale/data/test8g_v7.1'

INF0: Retained 21 of 449 nuclides on the master AMPX library

INFO: Running XSProc on 2 cells

WARNING: Remapped 2 nuclide IDs: 6012->6000, 6013->6000

Building sources

Building Denovo solver internals

Ray tracing Denovo mesh

Mixing Denovo cross sections

Building Denovo sources

Constructing forward sources for Denovo

WARNING: MCNP source discretization is currently experimental: only a single isotropic $\sqcup$ $\hookrightarrow$ volumetric source will be treated correctly.

WARNING: Discretization of source (type MCNP) was undersampled (undersampling fraction 0. $\hookrightarrow$ 999329).

WARNING: Consider increasing the number of samples per batch or decreasing the source $\sqcup$

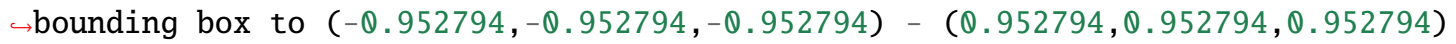

Initializing Denovo solver

INFO: Built SPN FV Element LHS Matrix with 1333248 nonzero entries.

Running Denovo transport calculation

INFO: Writing Silo file to 4 concurrent files using material volume fractions

INFO: Removing SILO directory denovo_output_silo

Writing Denovo HDF5 output

Run complete

Cleaning up

Belos Block GMRES converged after 274 iterations.

...finished running Omnibus in 2.1 seconds

Running Omnibus postprocessing

Loading HDF5 file...

INFO: Loaded Omnibus output data from 'denovo-ironsphere-spn.out.h5', problem name 'Iron $\hookrightarrow$ Sphere (SPN)', created on 2020MAR11 22:37 using SCALE version 6.3.pre-b10 (branch

$\hookrightarrow$ 'omnibus-doc' \#17579cc6 on 2020MAR10) ... finished loading HDF5 file

Loading HDF5 file...

INFO: Loaded Omnibus output data from 'denovo-ironsphere-spn.out.h5', problem name 'Iron $\hookrightarrow$ Sphere (SPN)', created on 2020MAR11 22:37 using SCALE version 6.3.pre-b10 (branch

$\hookrightarrow$ 'omnibus-doc' \#17579cc6 on 2020MAR10) (continues on next page) 
Writing Denovo visualization file...

INFO: Wrote Denovo visualization file to denovo-ironsphere-spn.denovo.xmf ...finished writing Denovo visualization file

Building RST summary...

INFO: Wrote summary file to denovo-ironsphere-spn.rst

... finished building RST summary

dnv_spn = load("denovo-ironsphere-spn.out.h5") ['denovo'].extract()

plot_x_flux (dnv_spn ['flux'], $\operatorname{range}(3,6))$

plot_pcolor(dnv_spn['flux'])

Loading HDF5 file...

INFO: Loaded Omnibus output data from 'denovo-ironsphere-spn.out.h5', problem name 'Iron $\hookrightarrow$ Sphere (SPN)', created on 2020MAR11 22:37 using SCALE version 6.3.pre-b10 (branch

$\hookrightarrow$ 'omnibus-doc' \#17579cc6 on 2020MAR10)

... finished loading HDF5 file

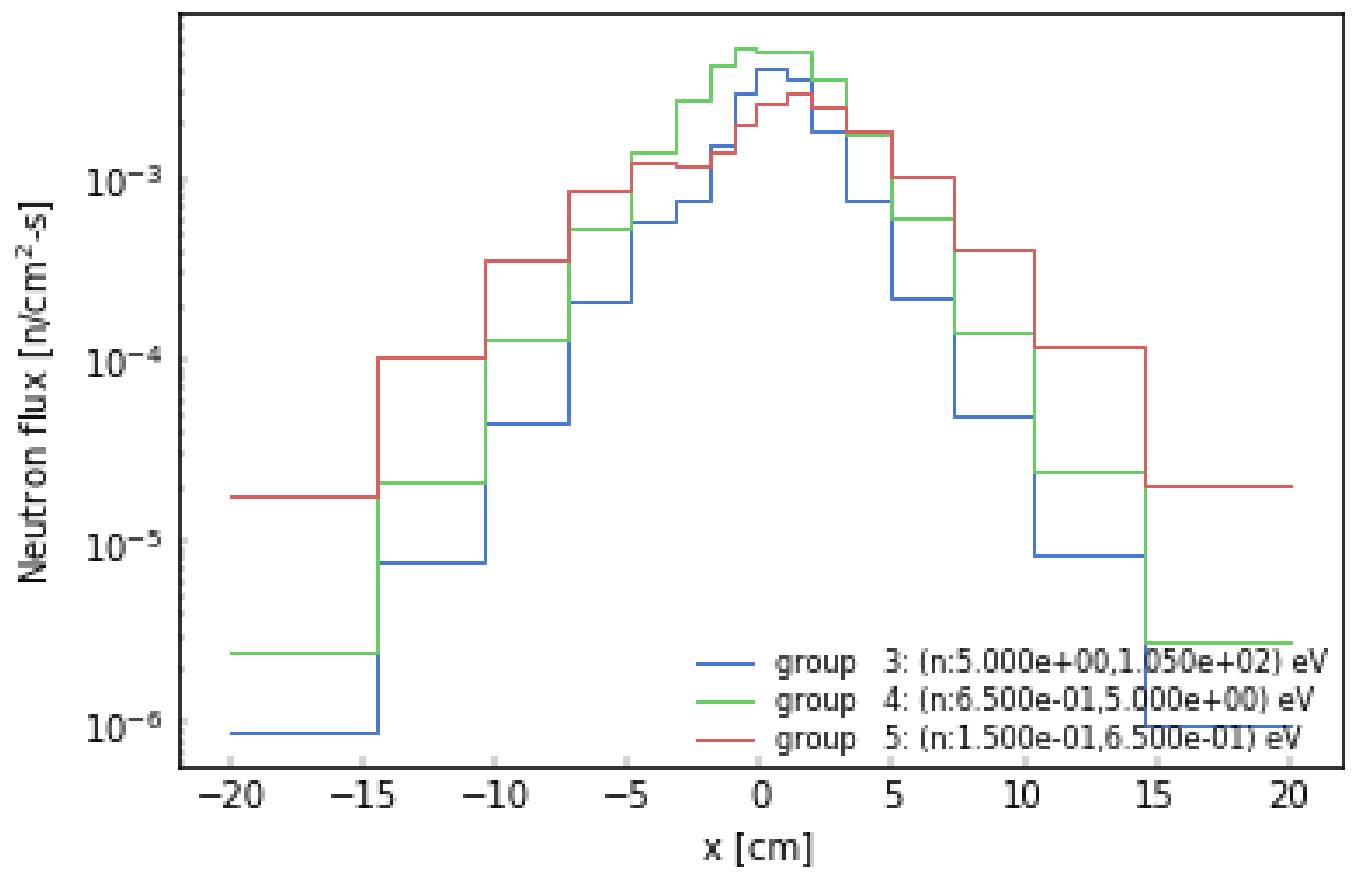


flux: group 3: (n:5.000e+00,1.050e+02) eV; y in $(-0.21978,0.952794)$

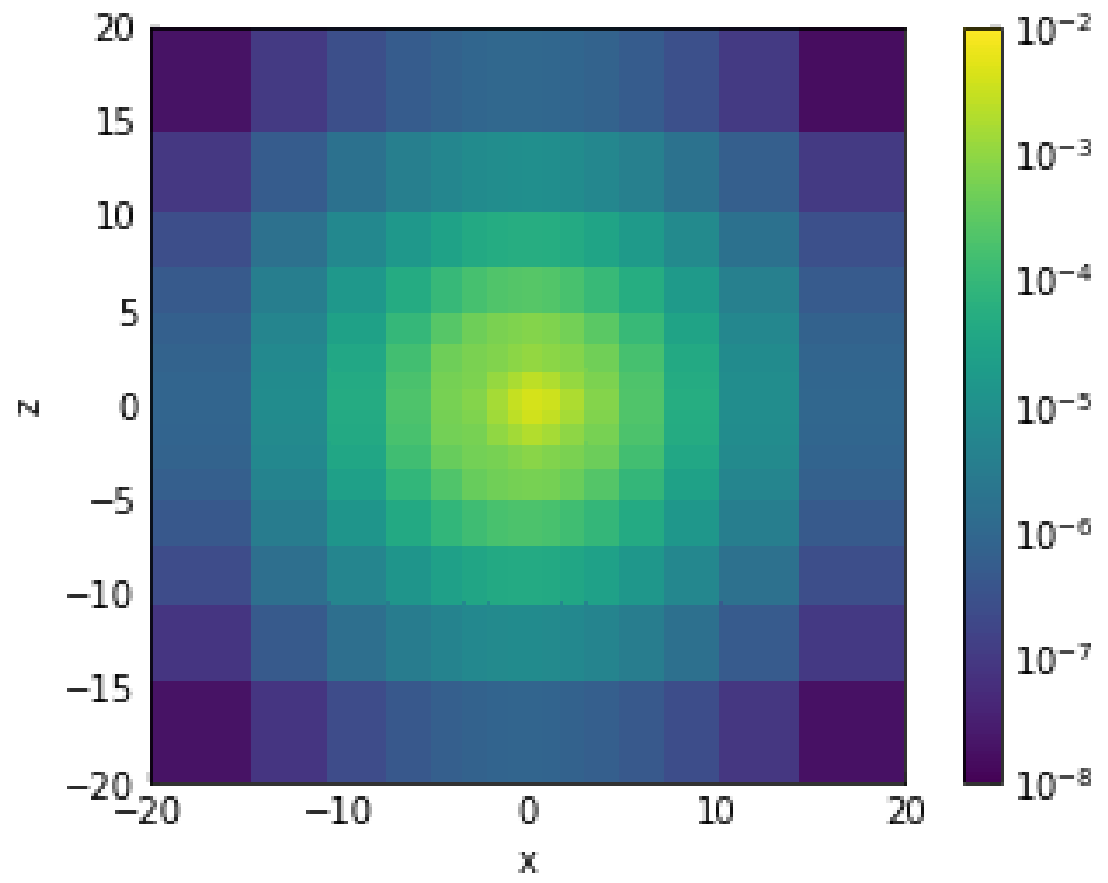

flux: group 3: (n:5.000e+00,1.050e+02) eV; $x$ in $(4.97204,7.29003)$

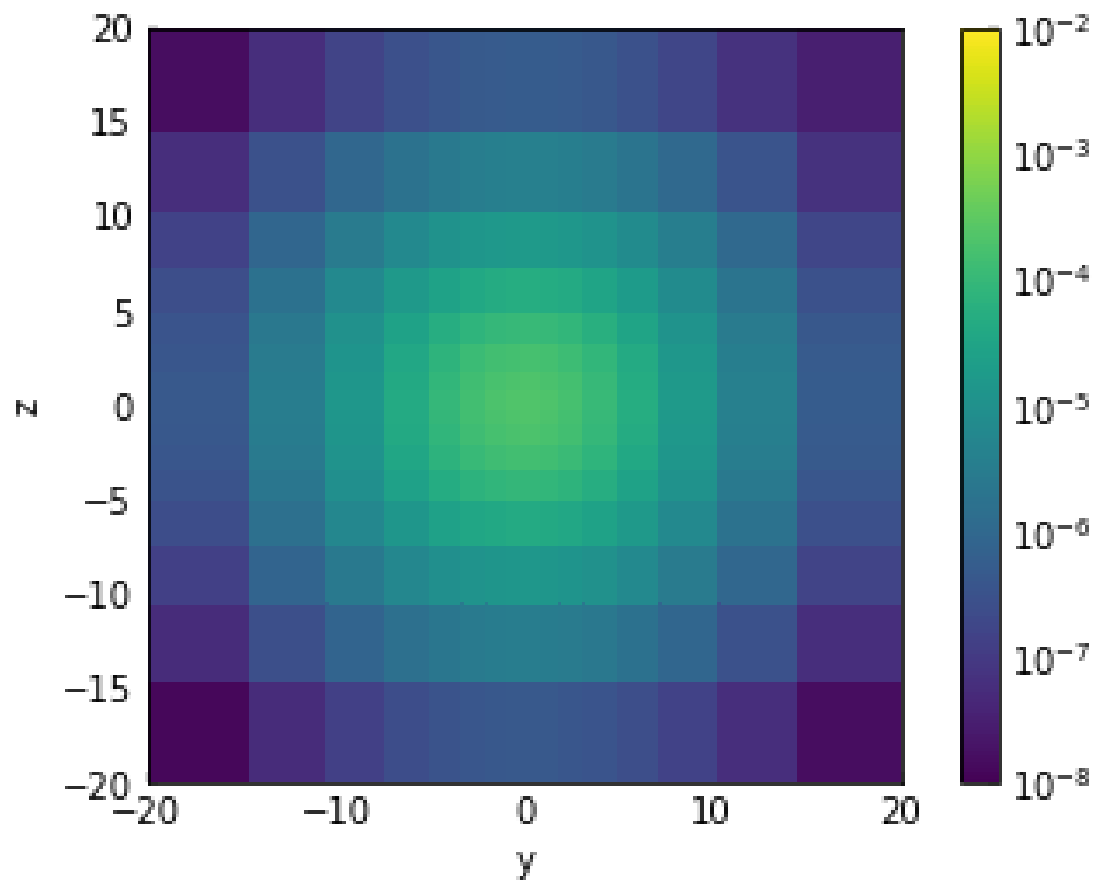

Note that the $\mathrm{SP}_{N}$ method demonstrates no ray effects since it is a spherical harmonics method rather than a discrete ordinates method. 


\section{A.2.2.5 Compare the three methods}

A final plot summarizes results from the three spatial discretization methods, plotting along the $x$ axis (where $y=0$ and $z=0)$.

The $\mathrm{SP}_{3}$ solution is depressed at the source material bounary as expected.

All solutions agree well farther from the source.

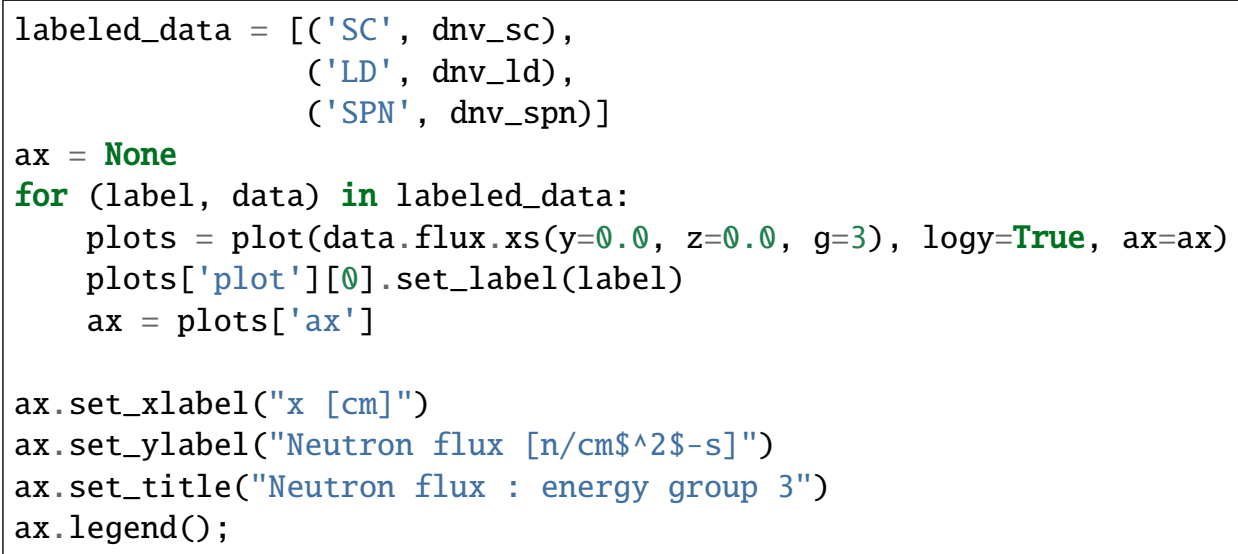

Neutron flux : energy group 3

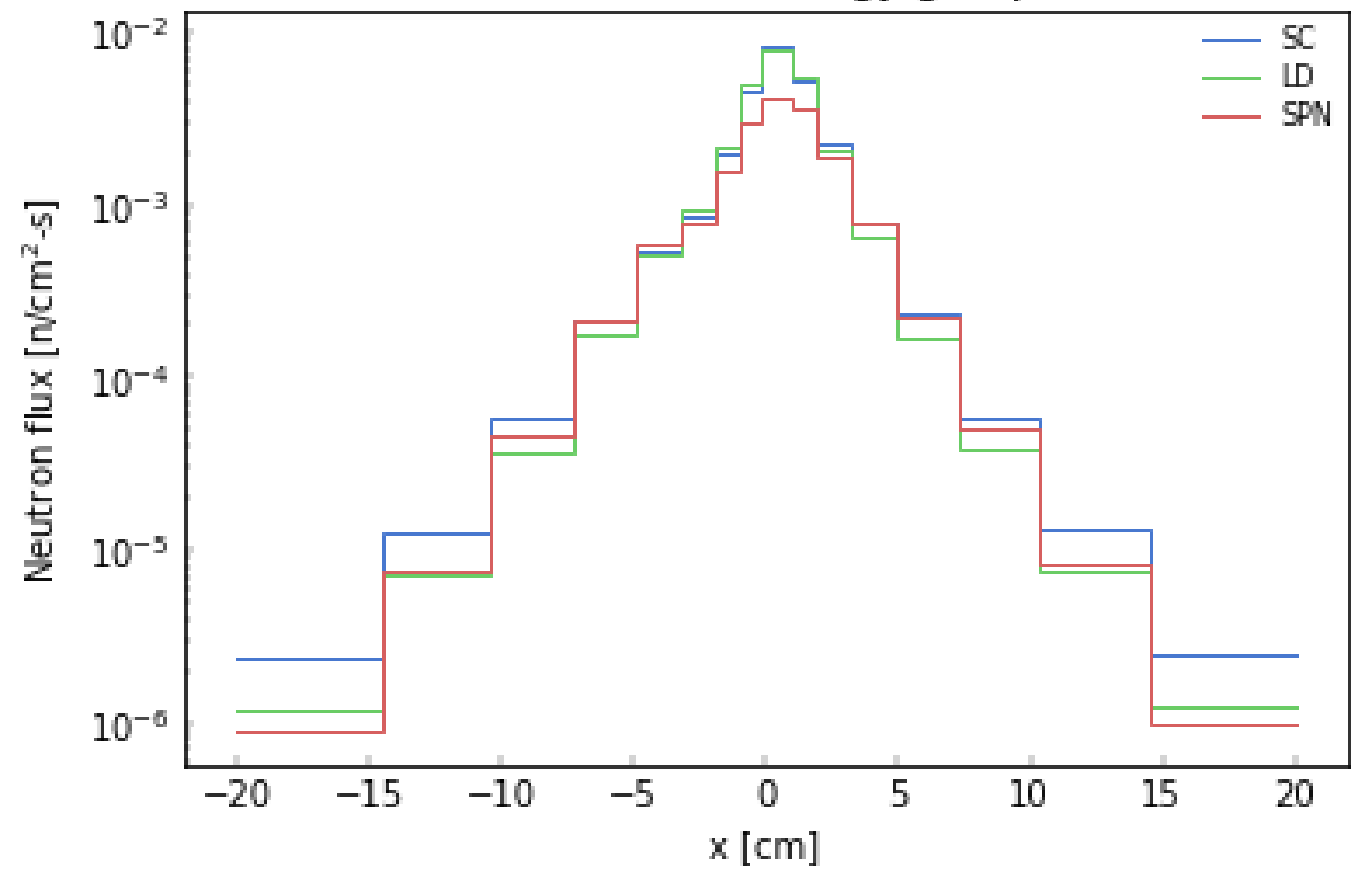

\section{A.2.3 KOBAYASHI PROBLEM 1.II}

This example compares Denovo results to published transport benchmark results [1].

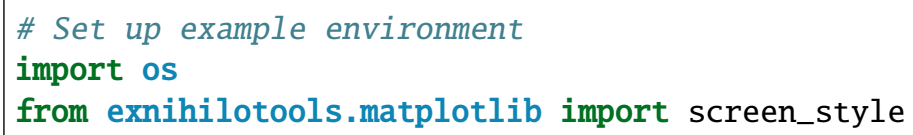


\%matplotlib inline

screen_style()

SOURCE_DIR = os.environ.get ("SOURCE_DIR", ".")

\section{A.2.3.1 Visualizing geometry}

The geometry is loaded from the GG input file to create a raytrace imager for visualizing the geometry. The from_extents method works for geometries that have bounding boxes (e.g., GG, SCALE, SWORD)..

from geometria import GG_Geometry

from omnibus. raytrace.imager import Imager

from omnibus.raytrace.load import load_gg

model = load_gg(os.path.join(SOURCE_DIR, "data", "kobp1.gg.omn"))

imager $=$ Imager.from_extents (model.geometry, $\mathrm{x}=0$, trace $\left.={ }^{\prime} \operatorname{cell}{ }^{\prime}\right)$

imager . plot ();

Generating Geometria XML input file from .gg.omn...

INF0: Starting Geometria preprocessor, omnibus version 6.3.pre-b10 (branch 'omnibus-doc' $\hookrightarrow \# 98 d 73 c 8$ e on 2020MAR11)

Loading problem db from Omnibus ASCII file...

Loading Omnibus input file at/rnsdhpc/code/src/scale/Exnihilo/packages/Omnibus/driver/

$\hookrightarrow$ example/data/kobp1.gg.omn

...finished loading problem db from Omnibus ASCII file

INF0: Writing Geometria input ParameterList to kobp1.gg.xml

...finished generating Geometria XML input file from .gg.omn

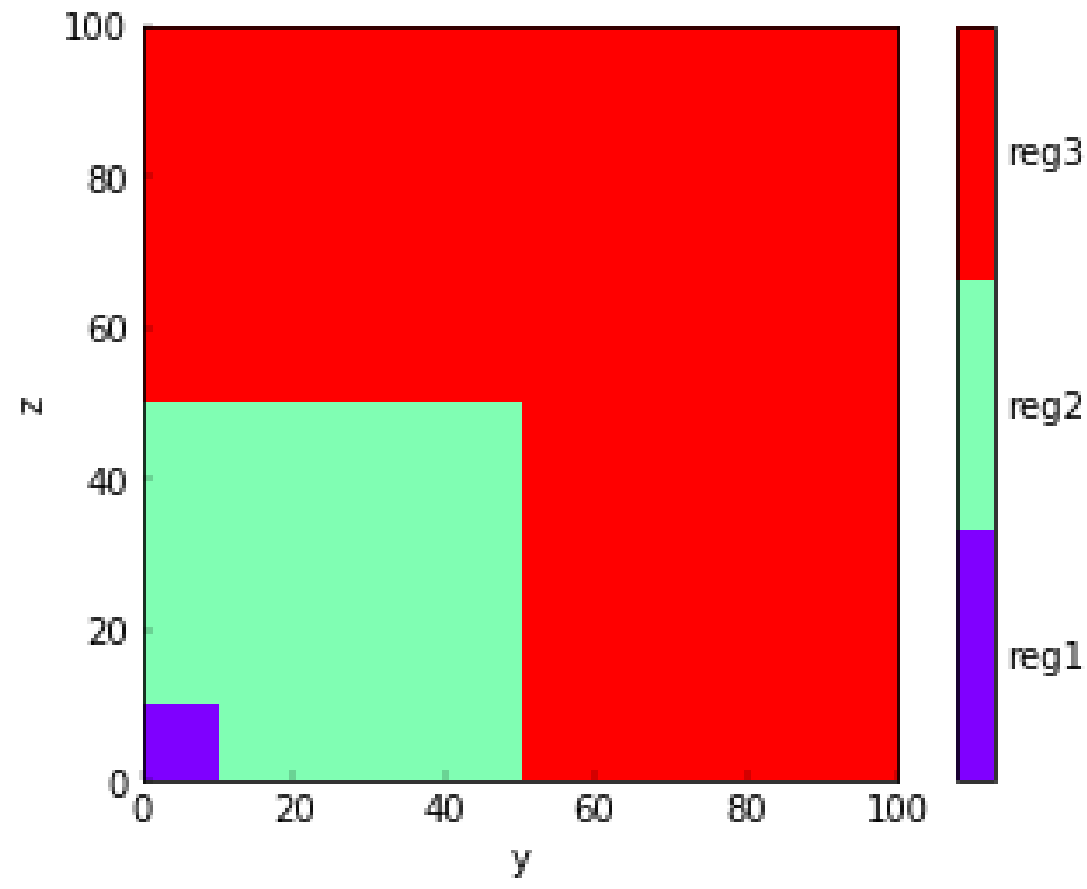




\section{A.2.3.2 Kobayashi Cross Sections}

Now the Exnihilo python wrappers are used to generate analytic 1-group cross sections for the Kobayashi problem. The Materials class is a simple working multigroup cross section library container.

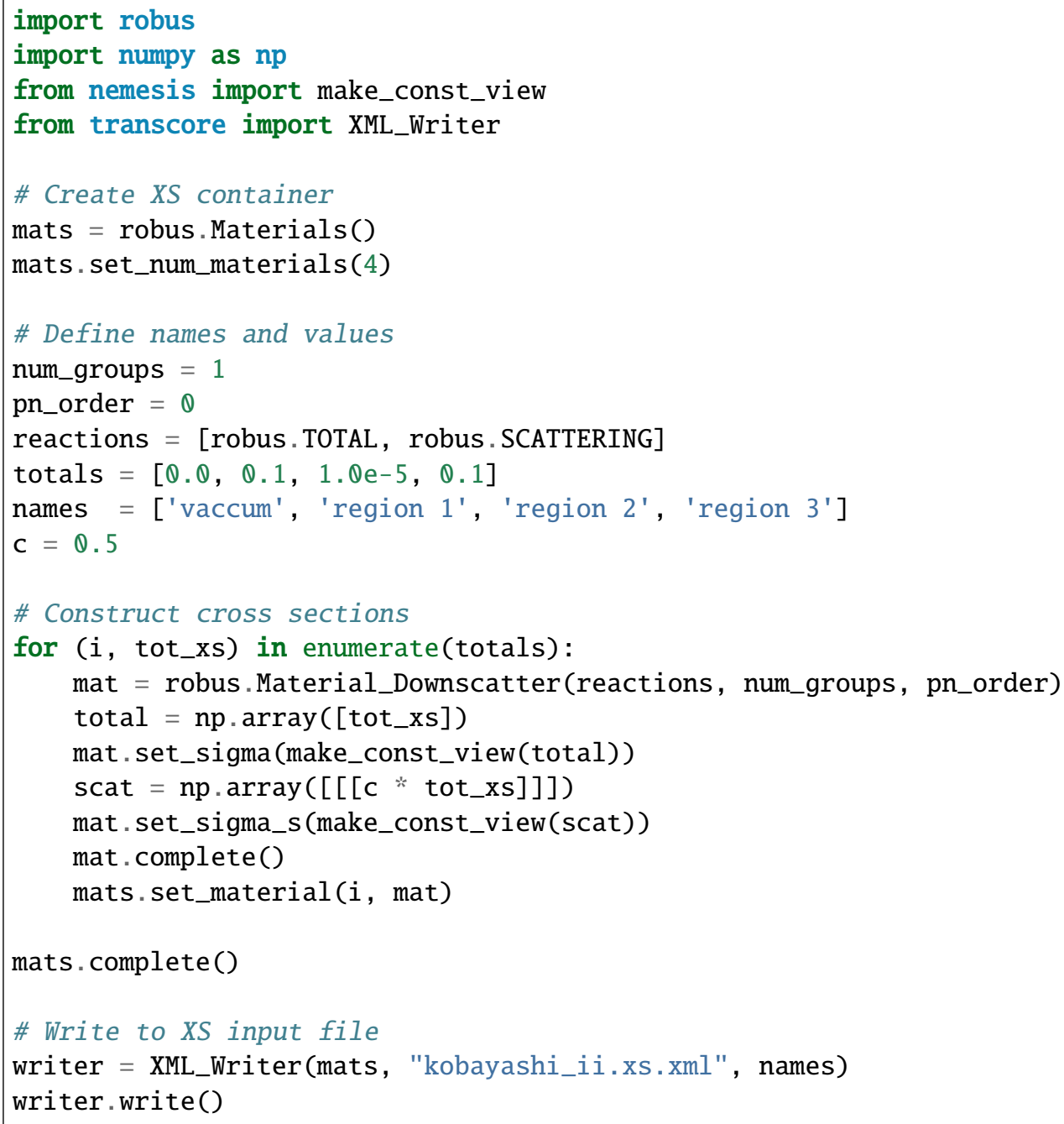

\section{A.2.3.3 Execute Omnibus}

Because the cross sections we generated are in the working directory, the Omnibus input files must also be copied the working directory too, since paths in the input file are relative to the input file.

$\%$ cp \{SOURCE_DIR\}/data/denovo-kobayashi.omn .

$\%$ cp \{SOURCE_DIR\}/data/kobp1.gg.omn .

\%cat denovo-kobayashi.omn

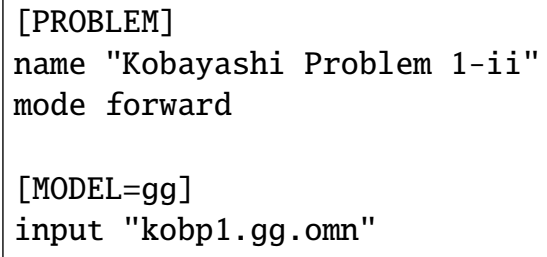




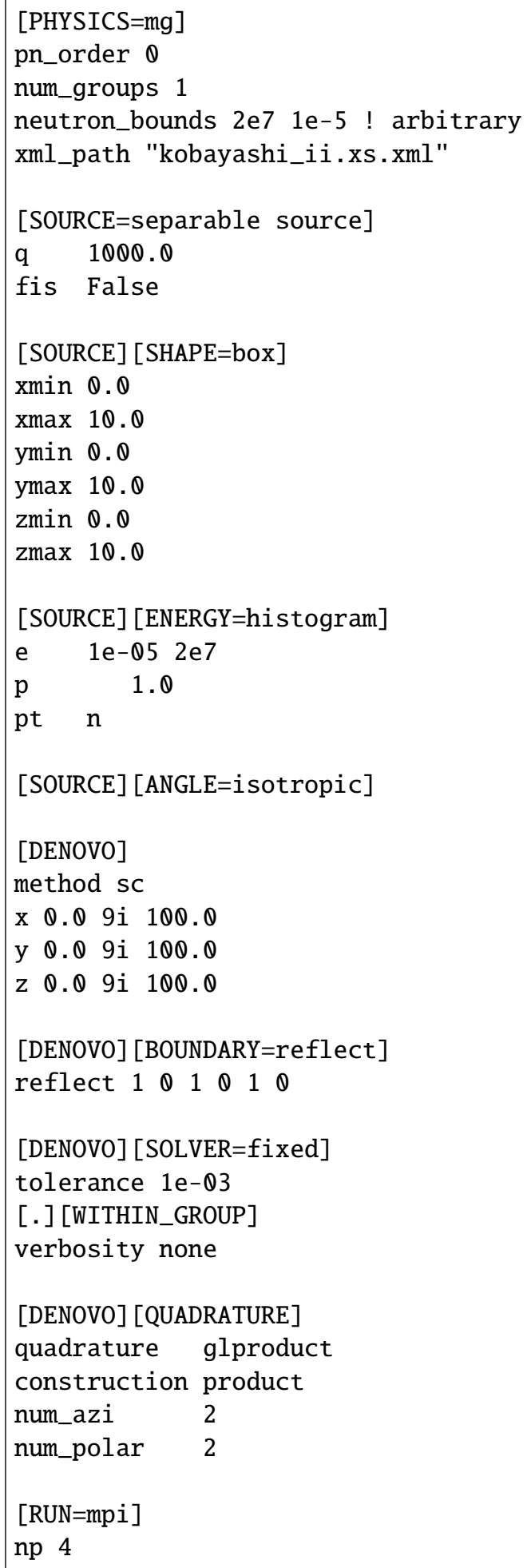


INF0: Starting Omnibus preprocessor, omnibus version 6.3.pre-b10 (branch 'omnibus-doc' $\hookrightarrow \# 98 \mathrm{~d} 73 \mathrm{c} 8 \mathrm{e}$ on 2020MAR11)

Loading problem db from Omnibus ASCII file...

Loading Omnibus input file at denovo-kobayashi.omn

...finished loading problem db from Omnibus ASCII file

Loading problem db from Python file...

...finished loading problem db from Python file

INFO: Set default for 'mpiexec_args' to '['-np', '4']' in '/run'

Generating Geometria XML input file from .gg.omn...

INF0: Starting Geometria preprocessor, omnibus version 6.3.pre-b10 (branch 'omnibus-doc' $\hookrightarrow$ \#98d73c8e on 2020MAR11)

Loading problem db from Omnibus ASCII file...

Loading Omnibus input file at /rnsdhpc/code/build/Exnihilo-examples/Exnihilo/packages/

$\hookrightarrow$ Omnibus/driver/example/denovo-kobayashi/kobp1.gg.omn

...finished loading problem db from Omnibus ASCII file

INF0: Renaming existing file kobp1.gg.xml to kobp1.gg-20200311-2236.xml

INF0: Writing Geometria input ParameterList to kobp1.gg.xml

...finished generating Geometria XML input file from .gg.omn

INFO: Set default for 'mode' to ' $\mathrm{n}$ ' in '/physics/mg'

INFO: Set default for 'disable_upscattering' to 'False' in '/physics/mg'

INFO: Set default for 'load_scl' to 'False' in '/comp'

INFO: Set default for 'physics' to ' $\mathrm{mg}$ ' in '/denovo'

WARNING: The 'method' keyword is now 'equations' for SN discretization types. Changing

$\hookrightarrow$ 'method $\mathrm{sc}^{\prime}$ to 'equations $\mathrm{sc}$

INF0: Set default 'x_blocks 2' and 'y_blocks 2'

INFO: Set default for 'z_blocks' to ' 2 ' in '/denovo/decomposition'

INFO: Set default for 'mat' to 'True' in '/denovo/output'

INFO: Set default for 'source' to 'True' in '/denovo/output'

INF0: Set default for 'uncflux' to 'True' in '/denovo/output'

INF0: Set default for 'flux' to 'True' in '/denovo/output'

INFO: Set default for 'upscatter_groups' to 'thermal' in '/denovo/solver'

INFO: Method: sn

INFO: Equations: sc

INFO: Set default for 'solver' to 'gmres' in '/denovo/solver/upscatter'

INF0: Method: sn

INF0: Equations: sc

INFO: Set default for 'tolerance' to ' 0.001 ' in '/denovo/solver/upscatter'

INFO: Set default for 'preconditioner' to '\{'_type': 'none'\}' in '/denovo/solver/

$\rightarrow$ upscatter'

INFO: Set default for 'max_iterations' to '1000' in '/denovo/solver/upscatter'

INFO: Set default for 'verbosity' to 'low' in '/denovo/solver/upscatter'

INF0: Method: sn

INFO: Equations: sc

INFO: Set default for 'solver' to 'gmres' in '/denovo/solver/within_group'

INFO: Method: sn

INFO: Equations: sc

INFO: Set default for 'tolerance' to ' 0.001 ' in '/denovo/solver/within_group'

INF0: Set default for 'preconditioner' to '\{'_type': 'none'\}' in '/denovo/solver/within_ $\rightarrow$ group'

INFO: Global Denovo mesh has 1000 cells

INFO: Writing Omnibus input ParameterList to denovo-kobayashi.inp.xml

INFO: Writing preprocessed file to denovo-kobayashi.pp.json 
(continued from previous page)

INFO: Writing processed ASCII input to 'denovo-kobayashi.inp.omn'

INFO: Launching Omnibus driver on 4 cores

Running Omnibus...

WARNING: The Exnihilo software revision ( 540 ) used to generate the input file differs $\rightarrow$ from this version being used to run it (r539).

WARNING: This could cause internal consistency checks to unexpectedly fail, and it could - even lead to unexpected database value changes.

WARNING: Please check your output very carefully after this run to make sure the

$\hookrightarrow$ interpreted values match your input values.

Building model

INFO: No composition data is present in the GG model at $/ /$ rnsdhpc/code/build/Exnihilo๑examples/Exnihilo/packages/Omnibus/driver/example/denovo-kobayashi/kobp1.gg.xml '

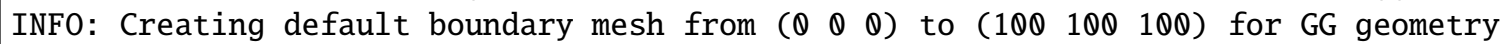
Building physics ' $\mathrm{mg}$ '

Building sources

Building Denovo solver internals

Ray tracing Denovo mesh

Mixing Denovo cross sections

Building Denovo sources

Constructing forward sources for Denovo

Initializing Denovo solver

INF0: Constructing Denovo state vector with 1 groups, 1000 cells, 1 moments, 1 unknowns $\hookrightarrow$ per cell

Running Denovo transport calculation

INFO: Writing Silo file to 4 concurrent files using material volume fractions

Writing Denovo HDF5 output

Run complete

Cleaning up

Forward group 0 finished in 6 Belos Block GMRES iterations in $1 \mathrm{~s} \ldots$

...finished running Omnibus in 1.2 seconds

Running Omnibus postprocessing

Loading HDF5 file...

INFO: Loaded Omnibus output data from 'denovo-kobayashi.out.h5', problem name 'Kobayashi $\rightarrow$ Problem 1-ii', created on 2020MAR11 22:36 using SCALE version 6.3.pre-b10 (branch

$\hookrightarrow$ 'omnibus-doc' \#17579cc6 on 2020MAR10) ...finished loading HDF5 file

Loading HDF5 file...

INFO: Loaded Omnibus output data from 'denovo-kobayashi.out.h5', problem name 'Kobayashi $\hookrightarrow$ Problem 1-ii', created on 2020MAR11 22:36 using SCALE version 6.3.pre-b10 (branch

$\hookrightarrow$ 'omnibus-doc' \#17579cc6 on 2020MAR10)

...finished loading HDF5 file

Writing Denovo visualization file...

INFO: Wrote Denovo visualization file to denovo-kobayashi.denovo.xmf

...finished writing Denovo visualization file

Building RST summary...

INFO: Wrote summary file to denovo-kobayashi.rst

...finished building RST summary

The run generates several files, including an HDF5 file with the denovo solution data and a ReStructured Text file that summarizes the problem run. 
assert os.path.exists("denovo-kobayashi.out.h5")

\section{A.2.3.4 Check the source term}

Verifying the computational input is an important step in analysis. Plotting the source region (again only in group zero) reveals that it has a single nonzero source mesh cell.

Since this is a one-group problem, it is easier to extract group zero at the beginning. Clearing the hyperslice field reduces clutter by discarding the metadata that this source object is group zero.

from omnibus. formats.output import load

dnv = load("denovo-kobayashi.out.h5") ['denovo'].extract()

source $=$ dnv. source $\cdot x s(g=0)$

source.hyperslice $=[]$

source

Loading HDF5 file...

INFO: Loaded Omnibus output data from 'denovo-kobayashi.out.h5', problem name 'Kobayashi॰ $\hookrightarrow$ Problem 1-ii', created on 2020MAR11 22:36 using SCALE version 6.3.pre-b10 (branch

$\hookrightarrow$ 'omnibus-doc' \#17579cc6 on 2020MAR10)

... finished loading HDF5 file

from omnibus.data import plot

print (np.count_nonzero(source))

plot (source $x s(x=5.0)$ );

1 


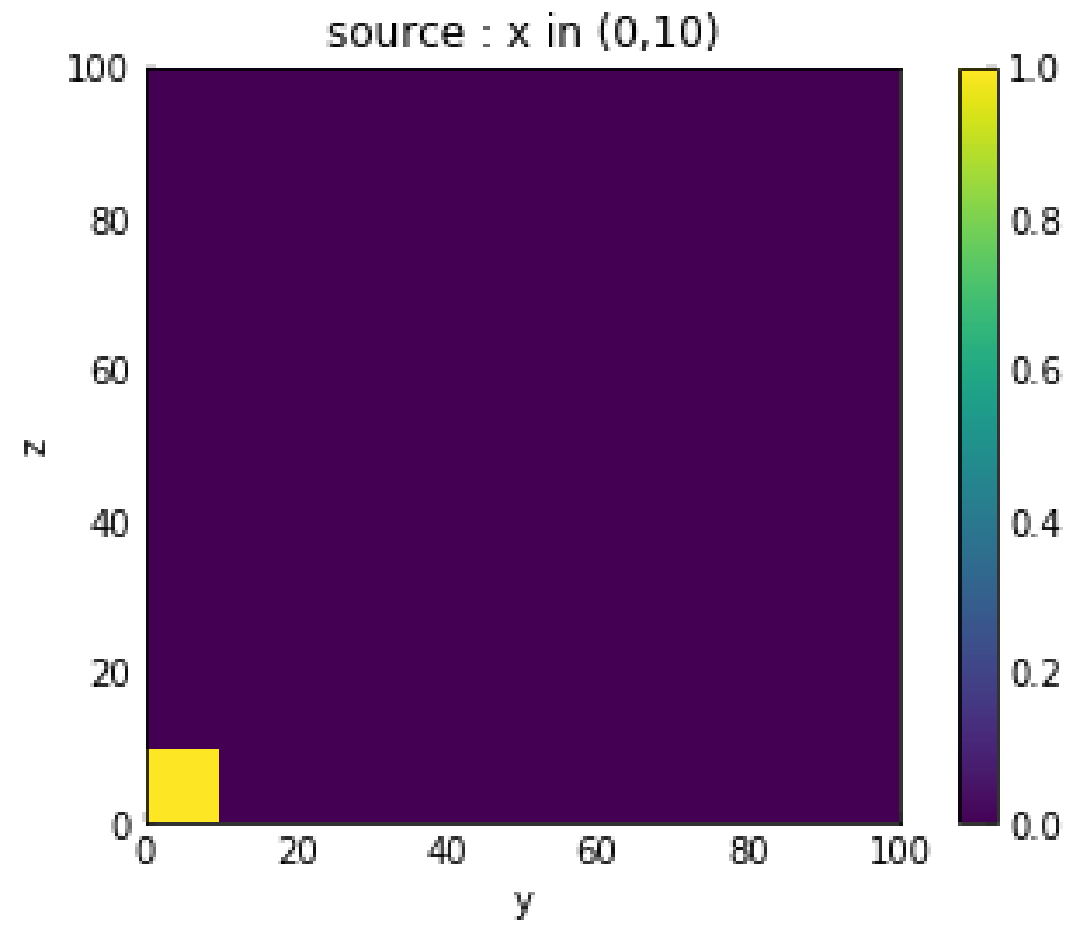

A.2.3.5 Visualize the flux

flux $=$ dnv $\cdot$ flux $\cdot x s(g=0)$

flux.hyperslice $=[]$

flux

This code plots a lineout along the $z$ axis in the cell where $x=y=5$ :

plots $=\operatorname{plot}(f l u x \cdot x s(x=5, y=5)$, logy=True $)$

$\mathrm{ax}=\operatorname{plots}\left[\mathrm{ax}^{\prime}\right]$

ax.set_title(ax.get_ylabel())

ax.set_ylabel("Particle flux (p/cm^2-s)"); 


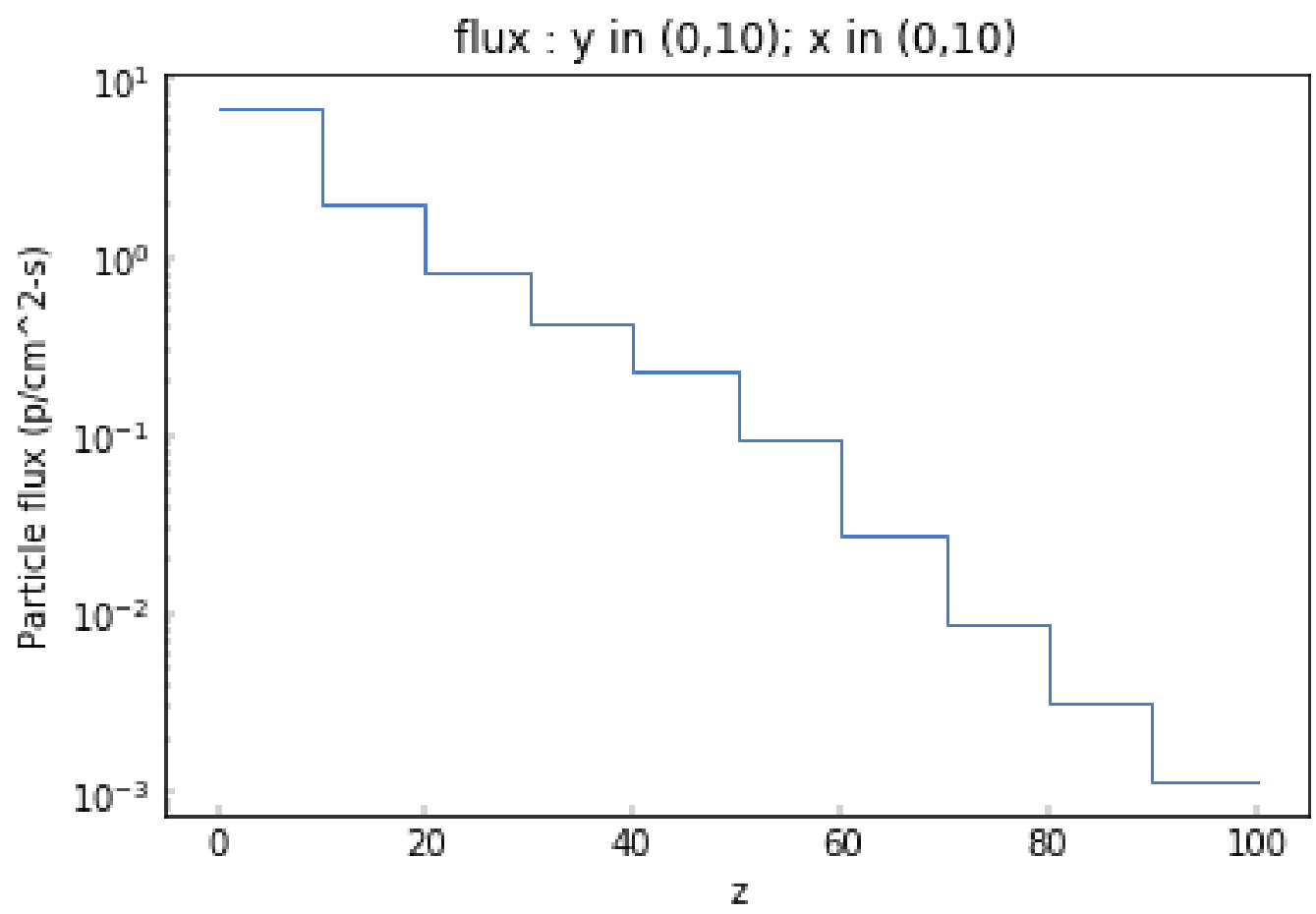

A pseudocolor plot can visualize the flux. The options on the matplotlib LogNorm class constrain the range of the color scale.

from matplotlib.colors import LogNorm

norm $=\operatorname{LogNorm}(\operatorname{vmin}=1 \mathrm{e}-6, \quad \operatorname{vmax}=1)$

plot (flux.xs $(z=5.0)$, norm=norm);

$\operatorname{plot}(f l u x \cdot x s(x=5.0)$, norm $=$ norm $)$; 

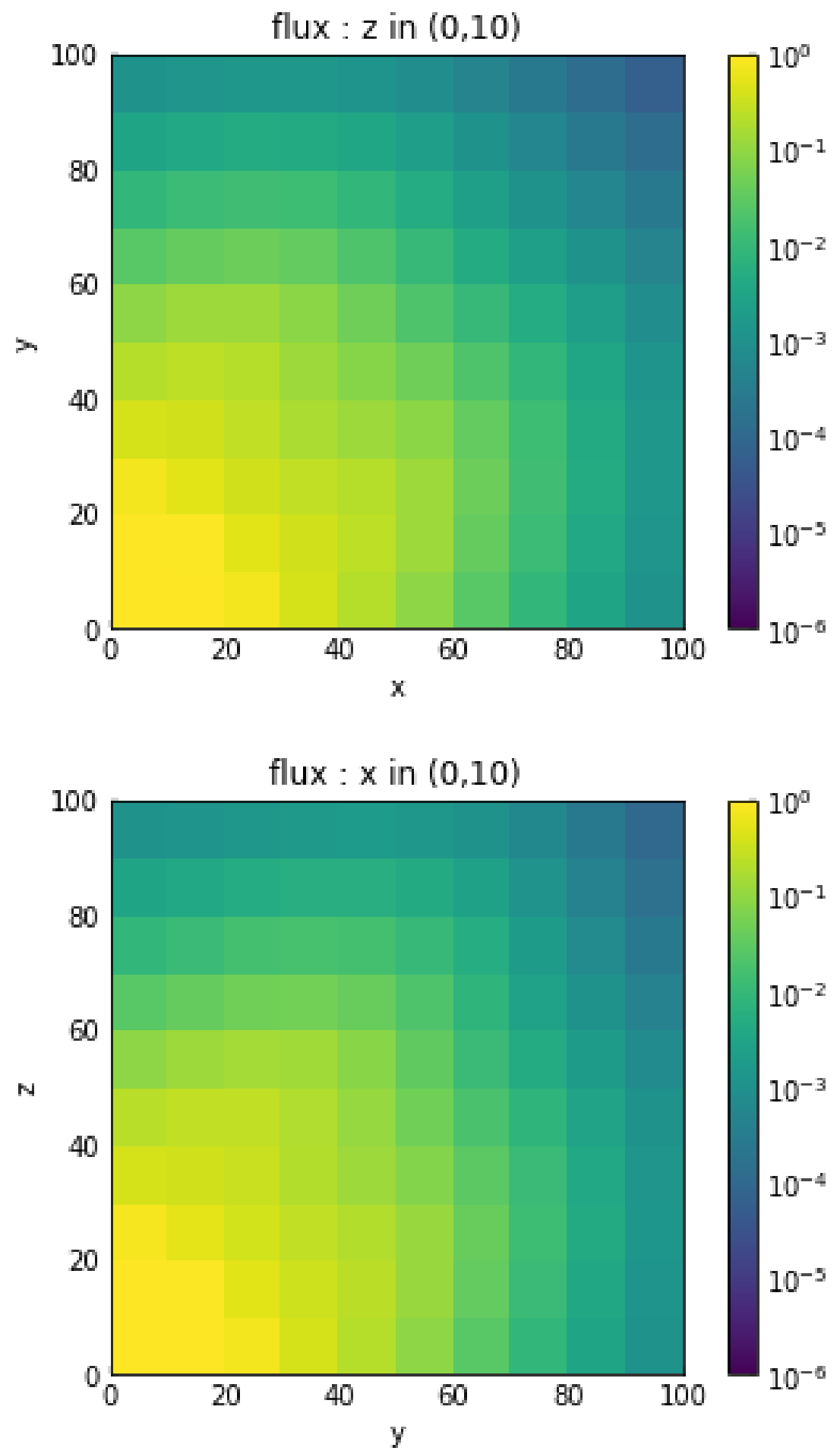

\section{Compare against reference solutions}

The reference data in the Kobayashi paper are point samples formatted as $(x, y, z, \phi)$. They comprise three lineouts: one along $y$, one along $x=y=z$, and one along $x$. 


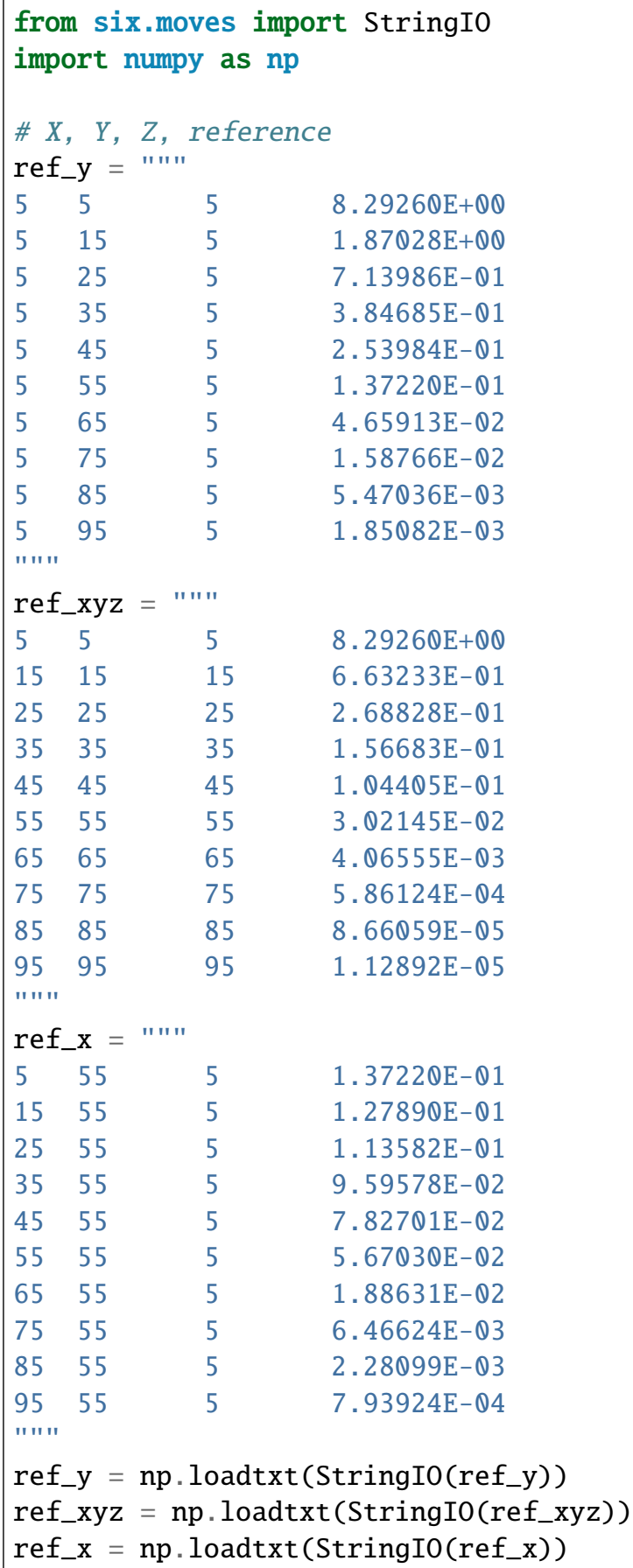

Since these are all physical points, it is easy to use the meshes to look up the points where the reference data is using the xs method.

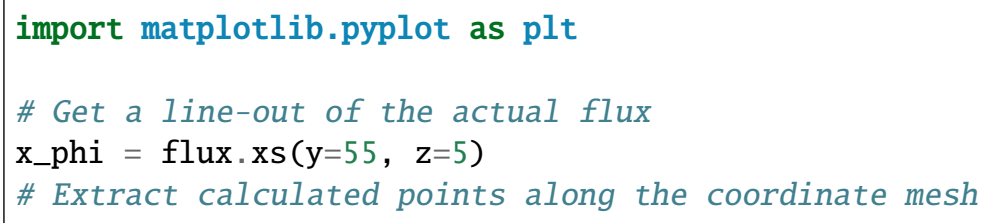


(continued from previous page)
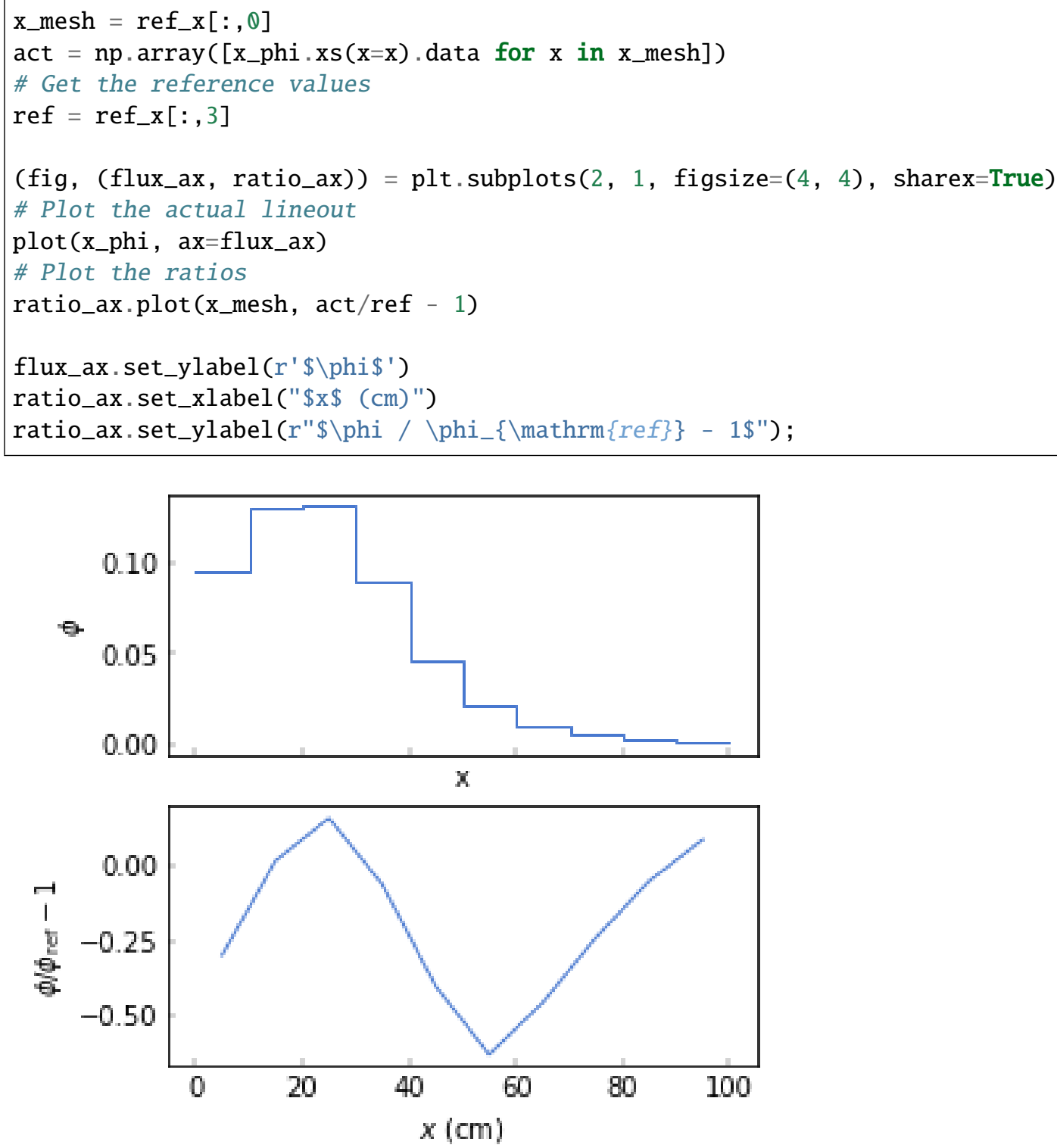

axis $=$ ref_y $[:, 1]$

y_phi $=$ flux.xs $(x=5, z=5)$

act $=n p \cdot \operatorname{array}\left(\left[y \_p h i \cdot x s(y=y) \cdot \operatorname{data}\right.\right.$ for $y$ in axis] $)$

ref $=$ ref_y $[:, 3]$

(fig, ax $)=p l t \cdot \operatorname{subplots}()$

ax.plot (axis, act/ref - 1)

ax.set_xlabel ("\$y $(\mathrm{cm}) ")$

ax.set_ylabel ("\$\phi / \phi_\{\mathrm $\{r e f\}\}-1 \$ ")$; 


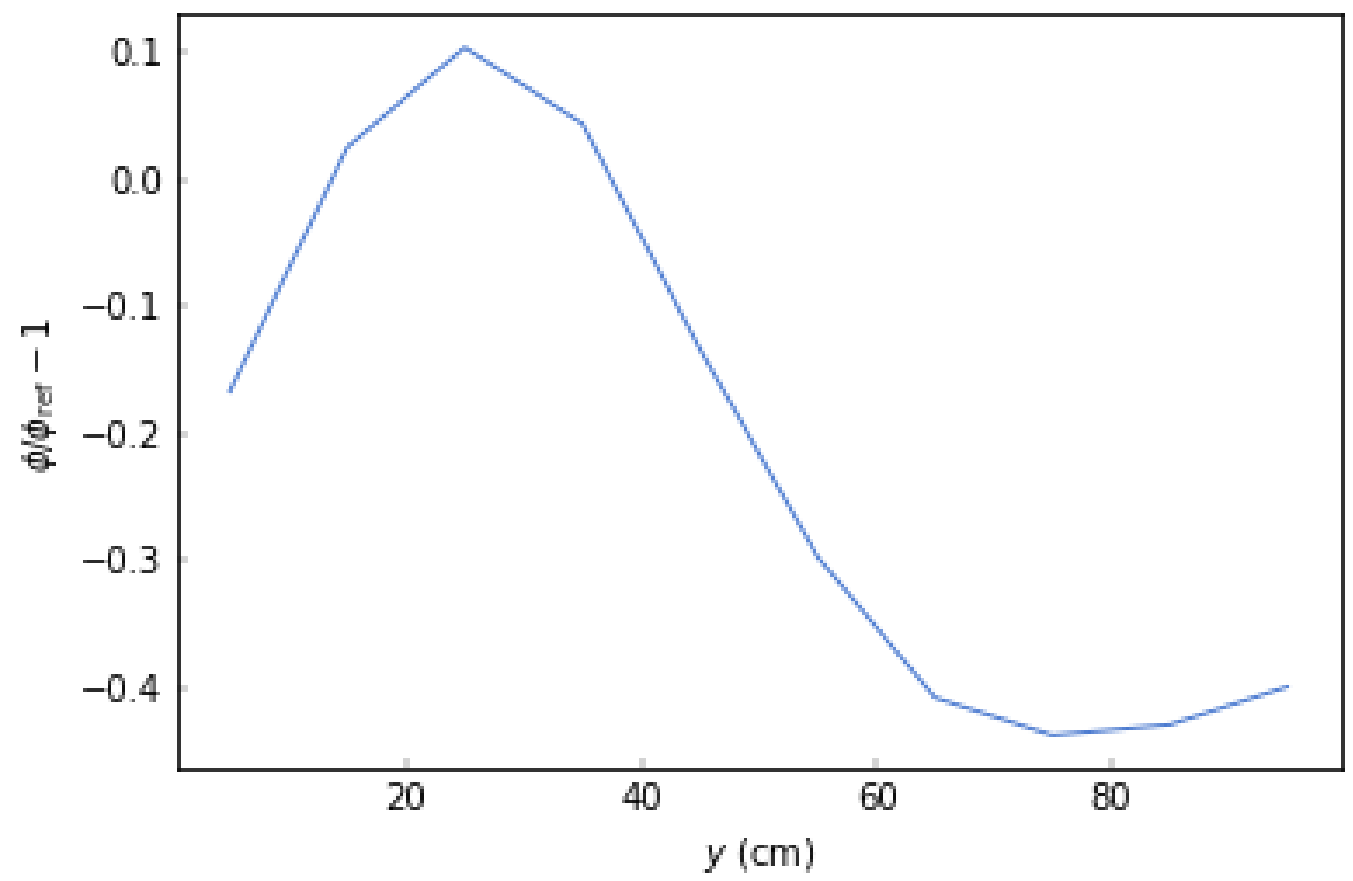

axis $=$ ref_xyz $[:, 0]$

act $=\mathrm{np} \cdot \operatorname{array}([$ flux. $\mathrm{xs}(\mathrm{x}=\mathrm{v}, \mathrm{y}=\mathrm{v}, \mathrm{z}=\mathrm{v})$. data for $\mathrm{v}$ in axis $])$

ref $=$ ref_xyz $[:, 3]$

(fig, ax $)=$ plt. $\operatorname{subplots}()$

ax.plot (axis, act/ref - 1)

ax.set_xlabel ("\$xyz\$ (cm)")

ax.set_ylabel("\$\phi / \phi_\{\mathrm\{ref\}\}-1\$");

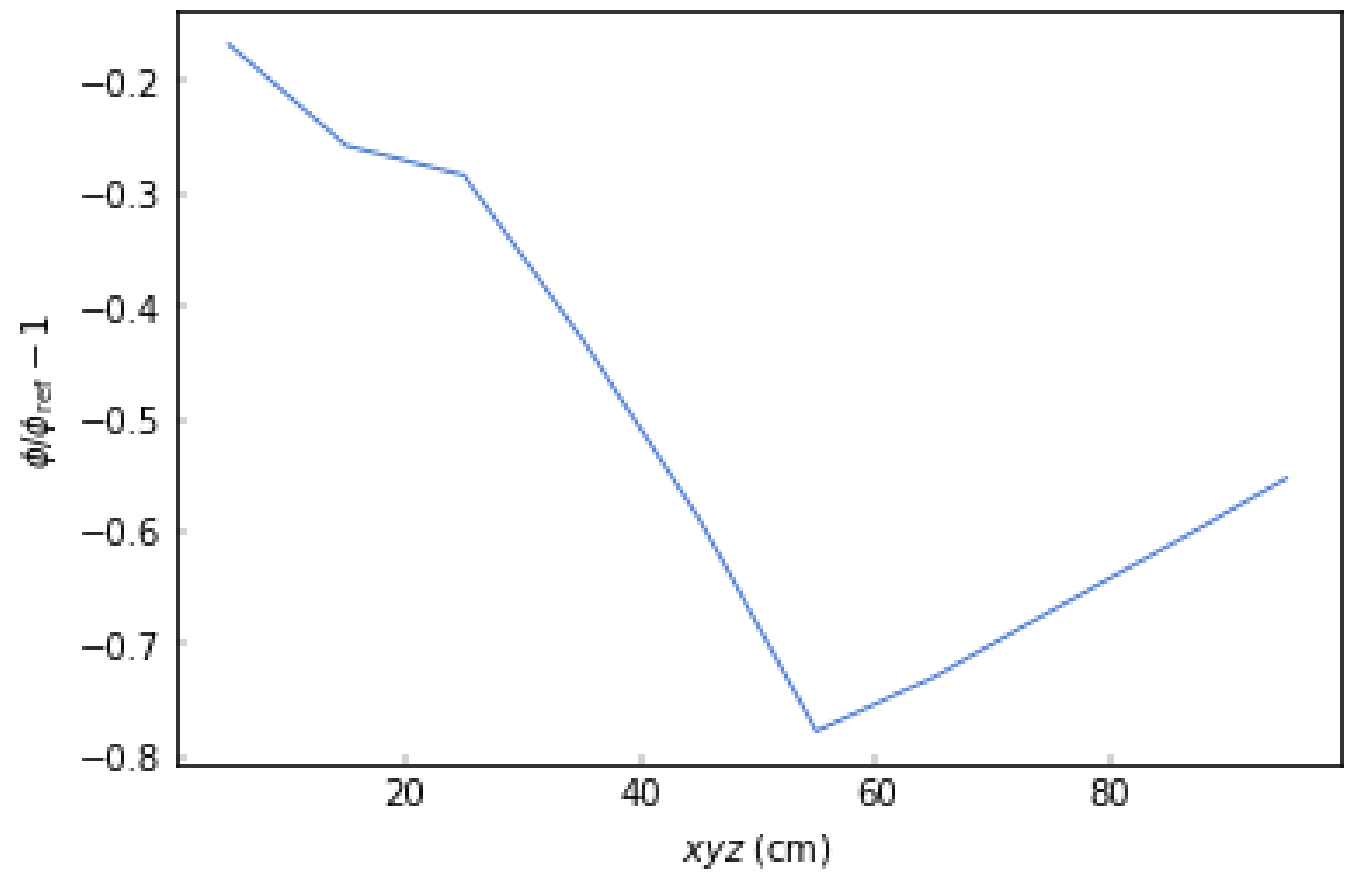




\section{A.2.4 UEKI BENCHMARK PROBLEM}

This example uses Denovo to determine a dose rate to the detector, and it demonstrates using the adjoint solution capability to obtain an importance function. Since this problem also contains a point source, it shows how an "uncollided flux" treatment can reduce ray effects. Two approximation methods for the uncollided flux source (uncf) will be explored: the "analytic" approximation, where the source is physically raytraced to every computational cell in the geometry; and a Monte Carlo approximation, where numerous samples from a stochastic point source generate the deterministic source term.

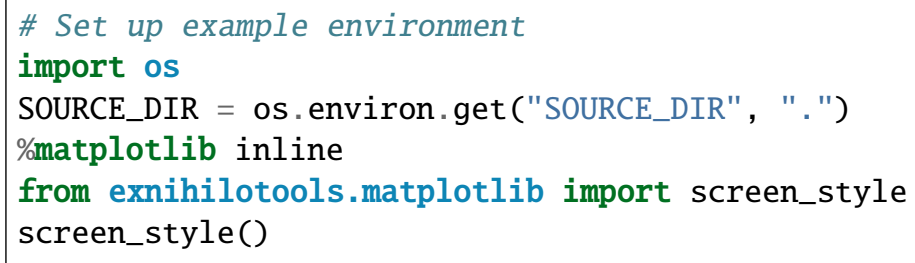

\section{A.2.4.1 Pre-visualize the geometry}

The $[\mathrm{PLOT}][\mathrm{PRE}]$ block in the input file generates a 2D image of the problem geometry for visualizing before actually executing the problem.

The Ueki problem consists of a neutron source is placed at the center of a block of paraffin with a 45 degree cone-shaped opening at the front. This opening points toward a graphite shield of varying thickness, with an ideal detector on the far side.

! grep -A 5 "\[PRE $\backslash$ " \{SOURCE_DIR\}/data/denovo-ueki-adj.omn

!omnibus-pre \{SOURCE_DIR\}/data/denovo-ueki-adj.omn

\section{$[\mathrm{PRE}]$}

[PRE] [PLOT lateral]

origin $-25 \quad 0 \quad-40$

size 127.580

axis y

render true

INF0: Starting Omnibus preprocessor, omnibus version 6.3.pre-b10 (branch 'omnibus-doc' $\hookrightarrow \# 98 \mathrm{~d} 73 \mathrm{c} 8 \mathrm{e}$ on 2020MAR11)

Loading problem db from Omnibus ASCII file...

Loading Omnibus input file at/rnsdhpc/code/src/scale/Exnihilo/packages/Omnibus/driver/

$\hookrightarrow$ example/data/denovo-ueki-adj.omn

...finished loading problem db from Omnibus ASCII file

Loading problem db from Python file...

...finished loading problem db from Python file

INFO: Set default for 'mpiexec_args' to '['-np', '4']' in '/run'

Generating MCNP runtpe file...

MCNP: xact is done ...finished generating MCNP runtpe file

$\gg>$ Loading nuclide data from processed MCNP libraries

$\gg>$ Loading compositions from MCNP input

$\gg>$ Loading nuclide data from processed MCNP libraries

$\gg>$ Loading compositions from MCNP input

INF0: Set default for 'load_scl' to 'True' in '/comp'

INFO: Set default for 'physics' to ' $\mathrm{mg}$ ' in '/denovo'

WARNING: The 'method' keyword is now 'equations' for SN discretization types. Changing $\hookrightarrow$ 'method $\mathrm{Sc}^{\prime}$ to 'equations $\mathrm{sc}$ ' 
(continued from previous page)

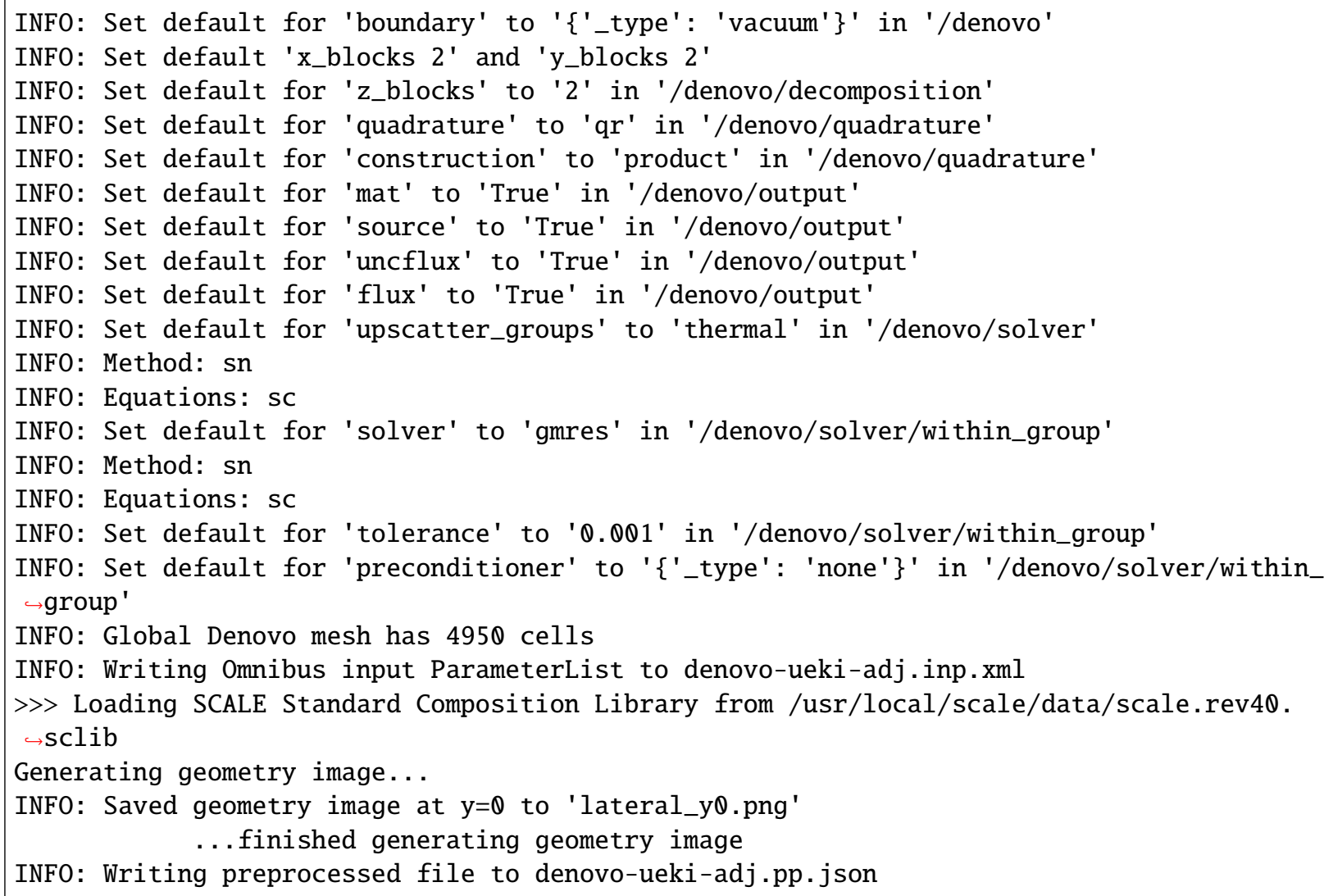




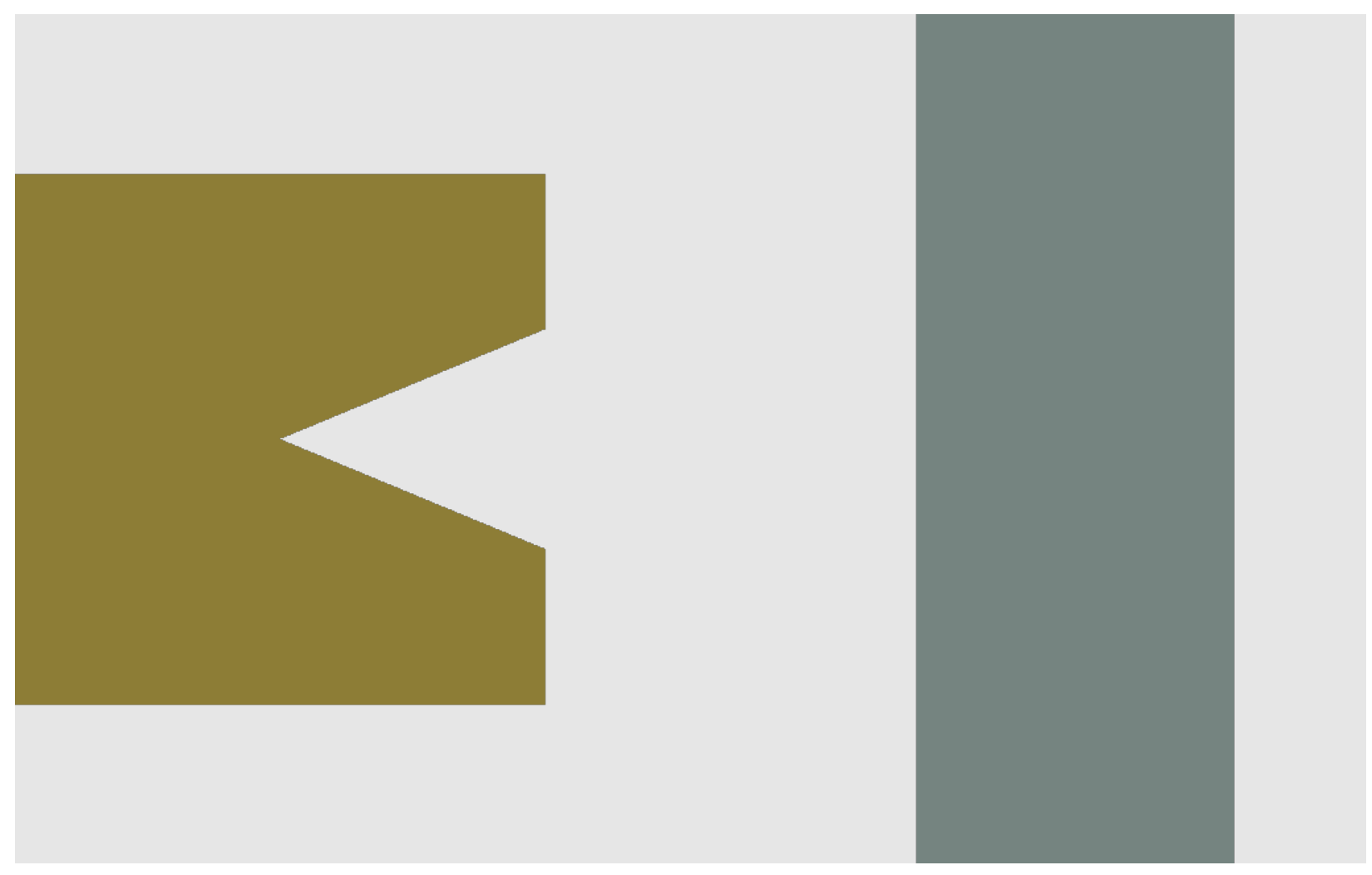

\section{A.2.4.2 Analytic uncollided flux results}

The uncollided flux from a point source is the solution to a purely absorbing transport equation in each group:

$$
\Omega \cdot \nabla \hat{\psi}_{g}+\sigma_{g} \hat{\psi}_{g}=\frac{q_{g}}{4 \pi} \delta\left(r-r_{0}\right) .
$$

This equation has a simple Green's function solution:

$$
\hat{\psi}_{g}=\delta\left(\Omega-\Omega_{0 \rightarrow r}\right) \frac{q_{g}}{4 \pi\left|r-r_{0}\right|^{2}} \mathrm{e}^{-\tau\left(r_{0}, r\right)} .
$$

Denovo calculates the total optical thickness $\tau$ between the point source and every point on the grid cell, and using the source spectrum, constructs an analytic solution to the first-collision source. The uncf analytic option in the [DENOVO] [SOURCE] block enables this feature.

\%cat \{SOURCE_DIR\}/data/denovo-ueki-fwd-uncf-analytic.omn

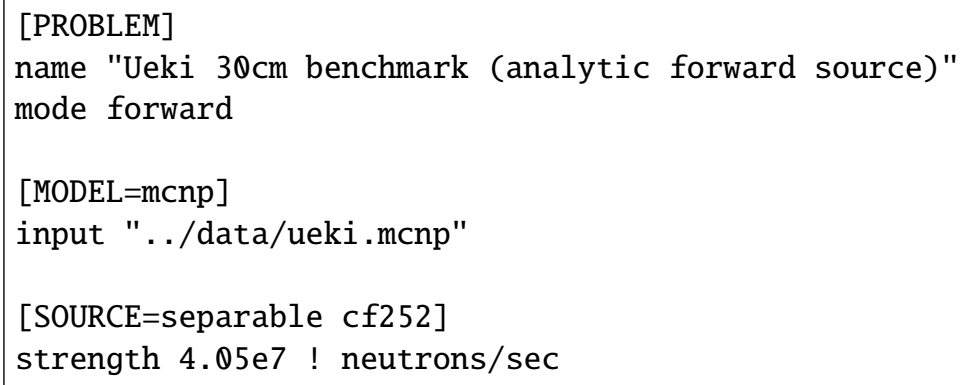




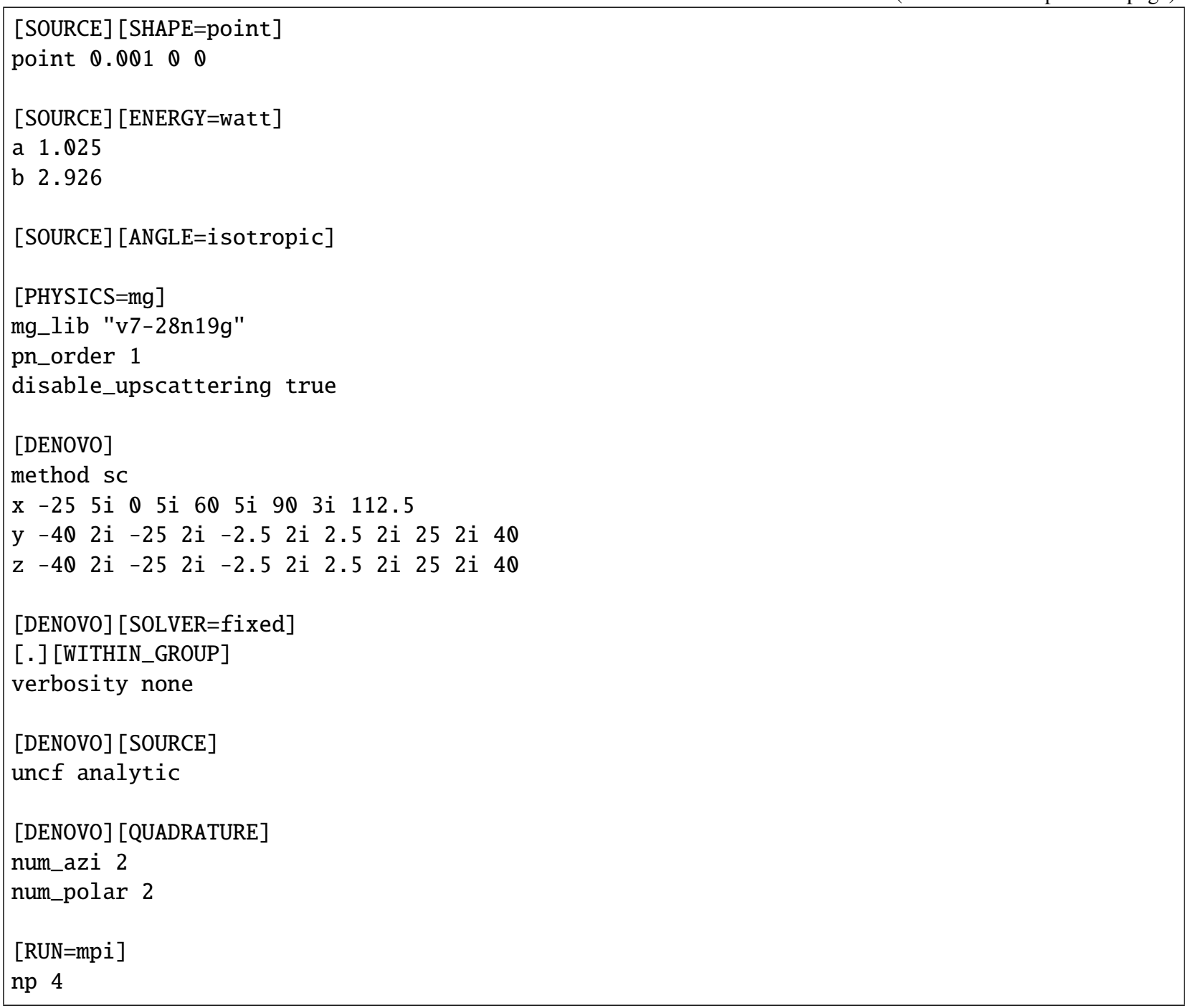

! omnibus-run \{SOURCE_DIR\}/data/denovo-ueki-fwd-uncf-analytic.omn

INF0: Starting Omnibus preprocessor, omnibus version 6.3.pre-b10 (branch 'omnibus-doc' $\hookrightarrow \# 98 \mathrm{~d} 73 \mathrm{c} 8 \mathrm{e}$ on 2020MAR11)

Loading problem db from Omnibus ASCII file...

Loading Omnibus input file at/rnsdhpc/code/src/scale/Exnihilo/packages/Omnibus/driver/

↔example/data/denovo-ueki-fwd-uncf-analytic.omn

...finished loading problem db from Omnibus ASCII file

Loading problem db from Python file...

...finished loading problem db from Python file

INFO: Set default for 'mpiexec_args' to '['-np', '4']' in '/run'

Generating MCNP runtpe file...

...finished generating MCNP runtpe file

INFO: Set default for 'fissionable_only' to 'False' in '/source/cf252'

INFO: Set default for 'mode' to ' $\mathrm{n}$ ' in '/physics/mg'

INFO: Set default for 'load_scl' to 'True' in '/comp'

INFO: Set default for 'physics' to 'mg' in '/denovo' 
(continued from previous page)

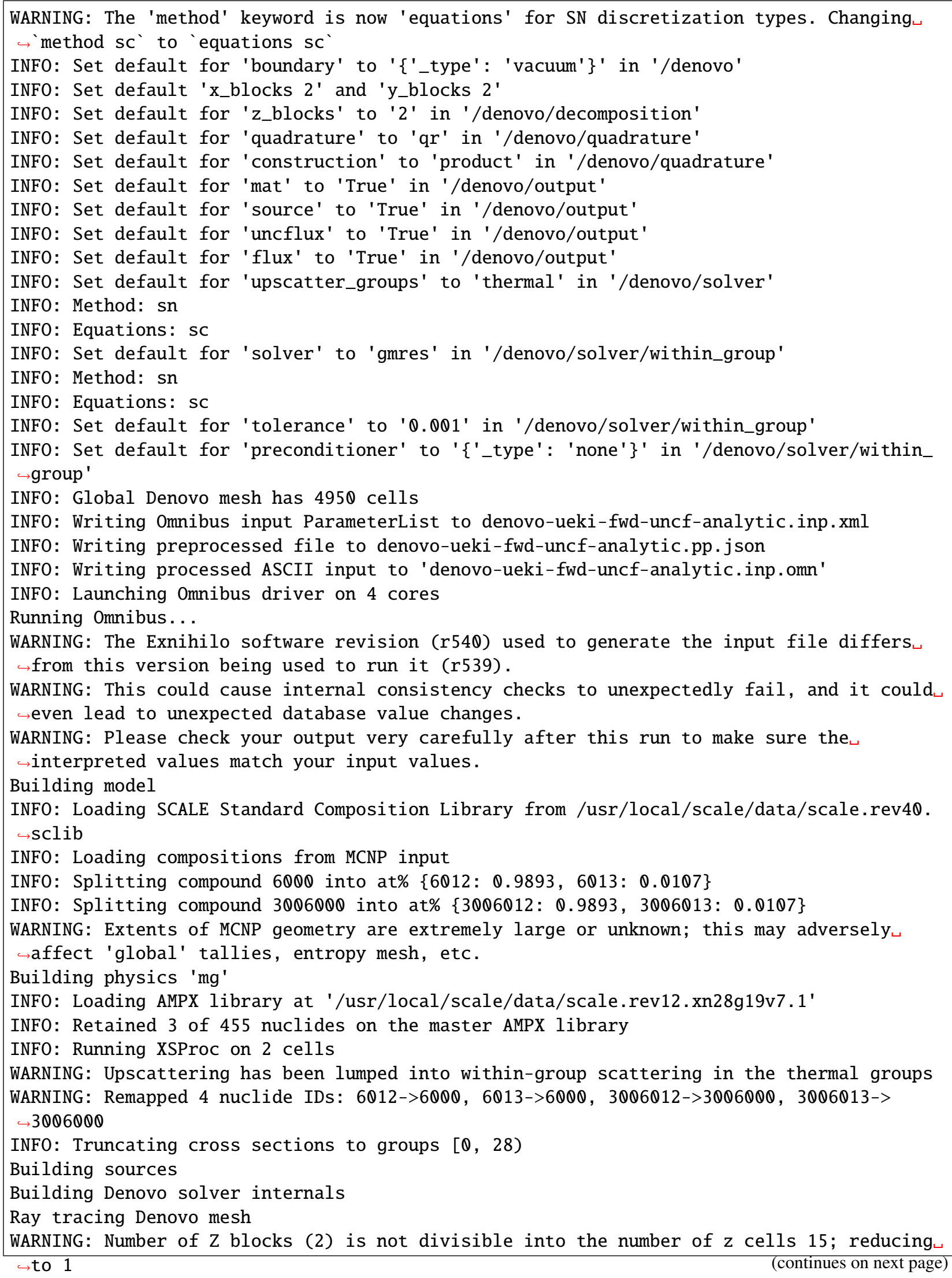


(continued from previous page)

Mixing Denovo cross sections

Building Denovo sources

Constructing forward sources for Denovo

Initializing Denovo solver

INFO: Constructing Denovo state vector with 28 groups, 4950 cells, 4 moments, 1 unknowns $\hookrightarrow$ per cell

Performing analytic first-collision source calculation on 1 sources

Running Denovo transport calculation

Forward group $\theta$ finished in 4 Belos Block GMRES iterations.

Forward group 1 finished in 4 Belos Block GMRES iterations.

Forward group 2 finished in 4 Belos Block GMRES iterations.

Forward group 3 finished in 3 Belos Block GMRES iterations.

Forward group 4 finished in 4 Belos Block GMRES iterations.

Forward group 5 finished in 5 Belos Block GMRES iterations.

Forward group 6 finished in 5 Belos Block GMRES iterations.

Forward group 7 finished in 6 Belos Block GMRES iterations.

Forward group 8 finished in 6 Belos Block GMRES iterations.

Forward group 9 finished in 6 Belos Block GMRES iterations.

Forward group 10 finished in 6 Belos Block GMRES iterations.

Forward group 11 finished in 5 Belos Block GMRES iterations.

Forward group 12 finished in 4 Belos Block GMRES iterations.

Forward group 13 finished in 4 Belos Block GMRES iterations.

Forward group 14 finished in 4 Belos Block GMRES iterations.

Forward group 15 finished in 4 Belos Block GMRES iterations.

Forward group 16 finished in 3 Belos Block GMRES iterations.

Forward group 17 finished in 3 Belos Block GMRES iterations.

Forward group 18 finished in 3 Belos Block GMRES iterations.

Forward group 19 finished in 3 Belos Block GMRES iterations.

Forward group 20 finished in 4 Belos Block GMRES iterations.

Forward group 21 finished in 4 Belos Block GMRES iterations.

Forward group 22 finished in 4 Belos Block GMRES iterations.

Forward group 23 finished in 6 Belos Block GMRES iterations.

Forward group 24 finished in 8 Belos Block GMRES iterations.

Forward group 25 finished in 9 Belos Block GMRES iterations.

Forward group 26 finished in 13 Belos Block GMRES iterations.

INFO: Writing Silo file to 4 concurrent files using material volume fractions

Writing Denovo HDF5 output

Run complete

Cleaning up

Forward group 27 finished in 39 Belos Block GMRES iterations.

...finished running Omnibus in 3.3 seconds

Running Omnibus postprocessing

Loading HDF5 file...

INFO: Loaded Omnibus output data from 'denovo-ueki-fwd-uncf-analytic.out.h5', problem $\sqcup$ $\hookrightarrow$ name 'Ueki $30 \mathrm{~cm}$ benchmark (analytic forward source)', created on 2020MAR11 22:36 using

$\hookrightarrow$ SCALE version 6.3.pre-b10 (branch 'omnibus-doc' \#17579cc6 on 2020MAR10) ...finished loading HDF5 file

Loading HDF5 file...

INFO: Loaded Omnibus output data from 'denovo-ueki-fwd-uncf-analytic.out.h5', problem $\sqcup$ $\hookrightarrow$ name 'Ueki $30 \mathrm{~cm}$ benchmark (analytic forward source)', created on 2020MAR11 22:36 using $\hookrightarrow$ SCALE version 6.3.pre-b10 (branch 'omnibus-doc' \#17579cc6 on 2020MAR10) ...finished loading HDF5 file 
(continued from previous page)

Writing Denovo visualization file...

INFO: Wrote Denovo visualization file to denovo-ueki-fwd-uncf-analytic.denovo.xmf ...finished writing Denovo visualization file

Building RST summary...

INF0: Wrote summary file to denovo-ueki-fwd-uncf-analytic.rst

...finished building RST summary

The first-collision method works by splitting the transport equation into uncollided and collided flux components. The uncollided flux is solved using an analytic approximation. Visualizing the uncollided flux term helps in verifying the problem setup.

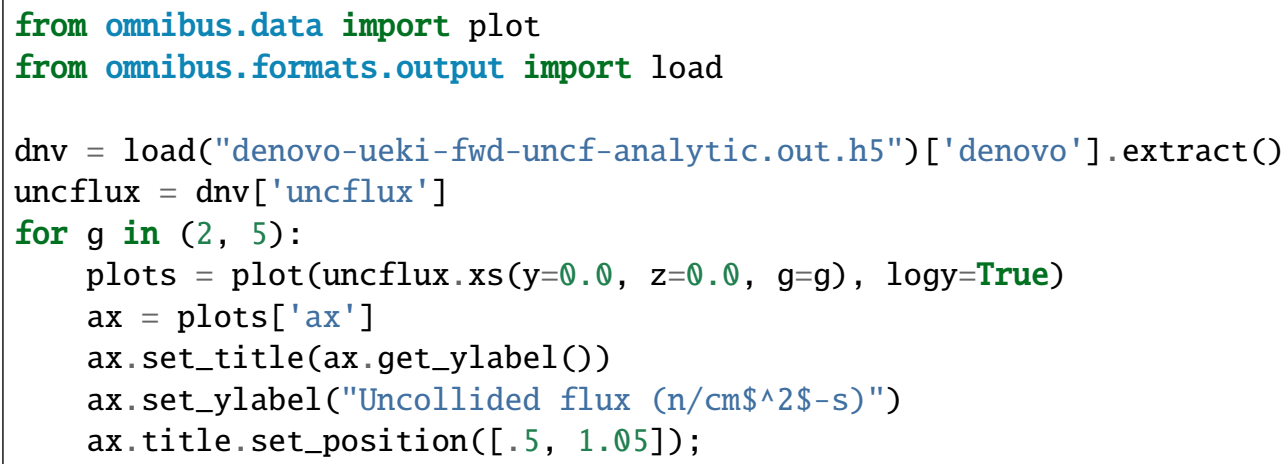

uncflux : group 2: (n:1.827e+06,3.012e+06) eV; $z$ in $(-0.833333,0.833333)$; y in $(-0.833333,0.833333)$

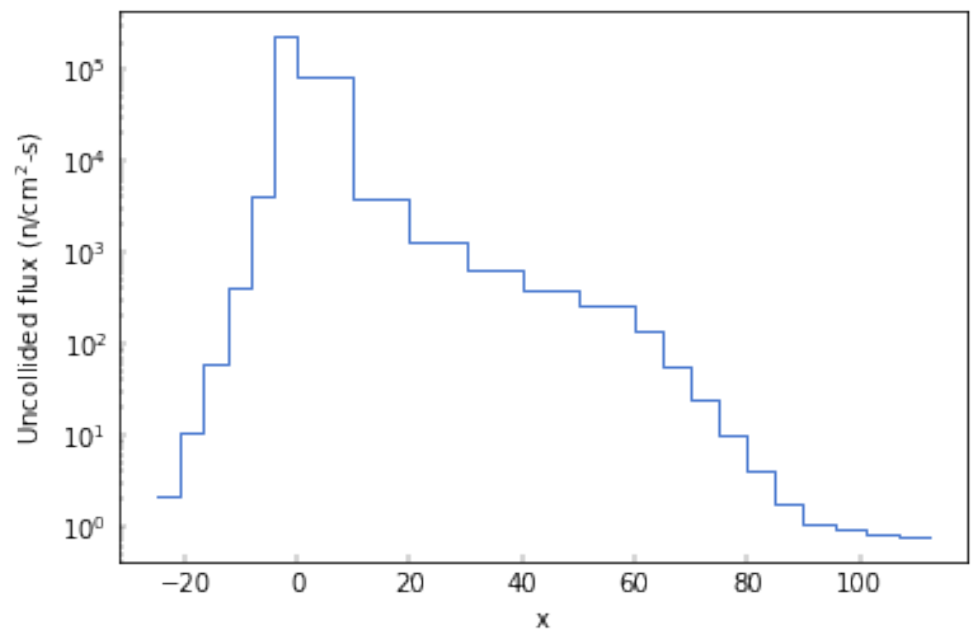


uncflux : group $5:(n: 4.076 e+05,9.072 e+05) e V ; z$ in $(-0.833333,0.833333)$; $y$ in $(-0.833333,0.833333)$

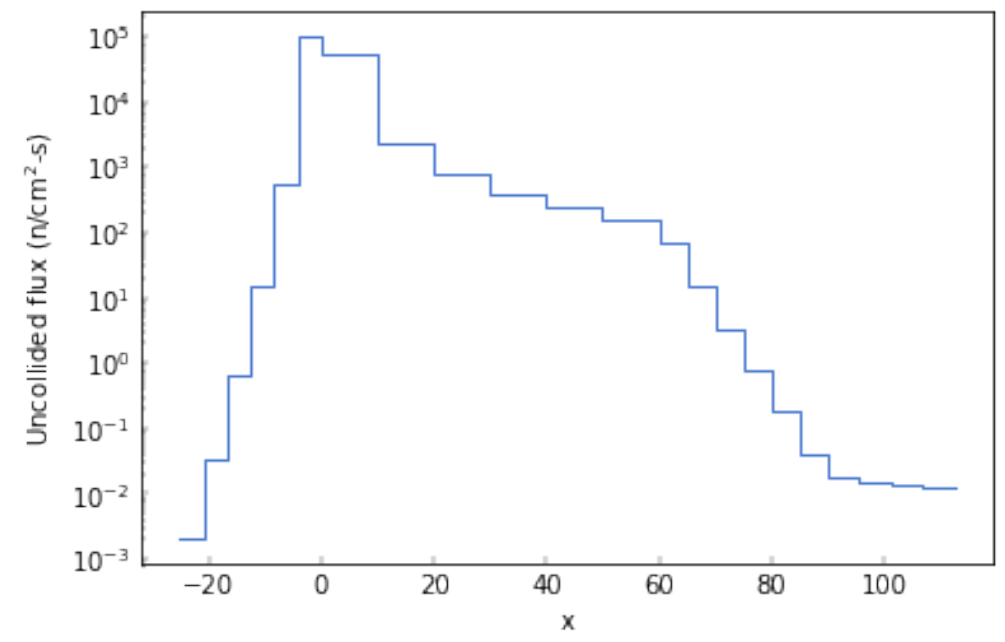

uncf_slice $=$ uncflux $\cdot \mathrm{xs}(\mathrm{g}=2, \mathrm{z}=0.0)$

print("Flux spans $\{: 2 e\}$ to $\{: 2 e\}$ ". format (

uncf_slice.data.min(),

uncf_slice.data.max()))

Flux spans $8.63 \mathrm{e}-04$ to $2.39 \mathrm{e}+05$

Pseudocolor plots show the attenuation of the source term through the material and through space. Since the material cross sections depend on energy group, the shape of the uncollided flux terms is different in groups 2 and 5 .

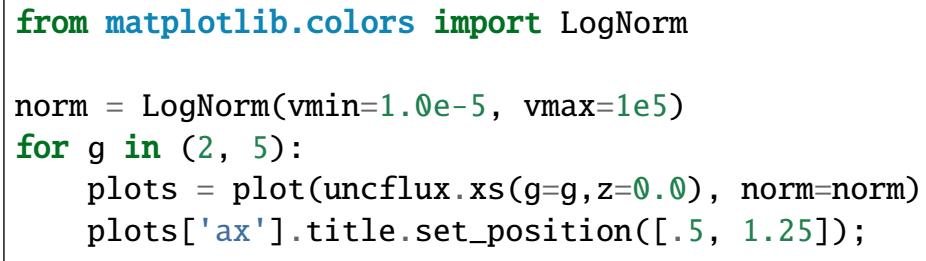




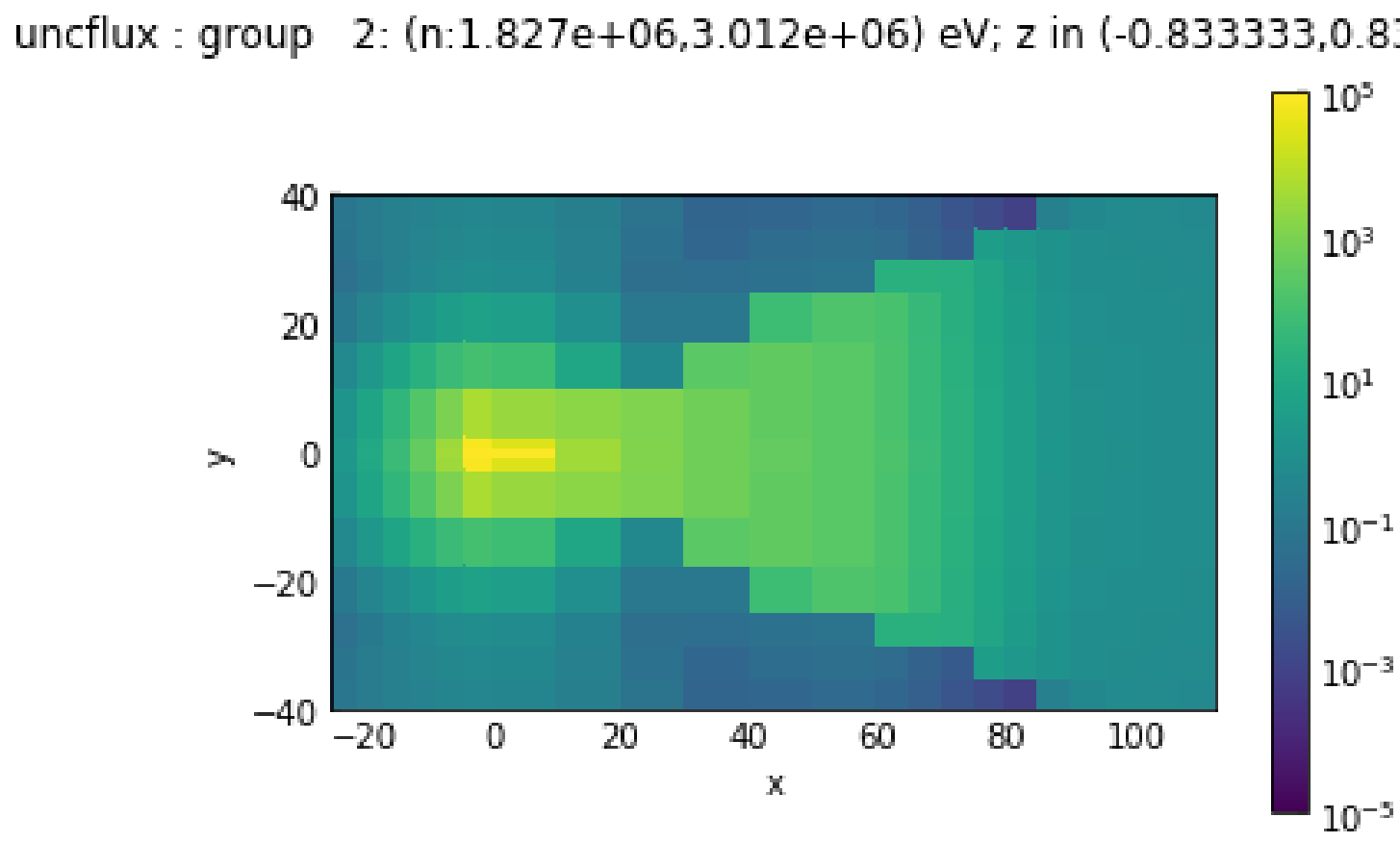

uncflux: group 5: (n:4.076e+05,9.072e+05) eV; $z$ in $(-0.833333,0.833333)$

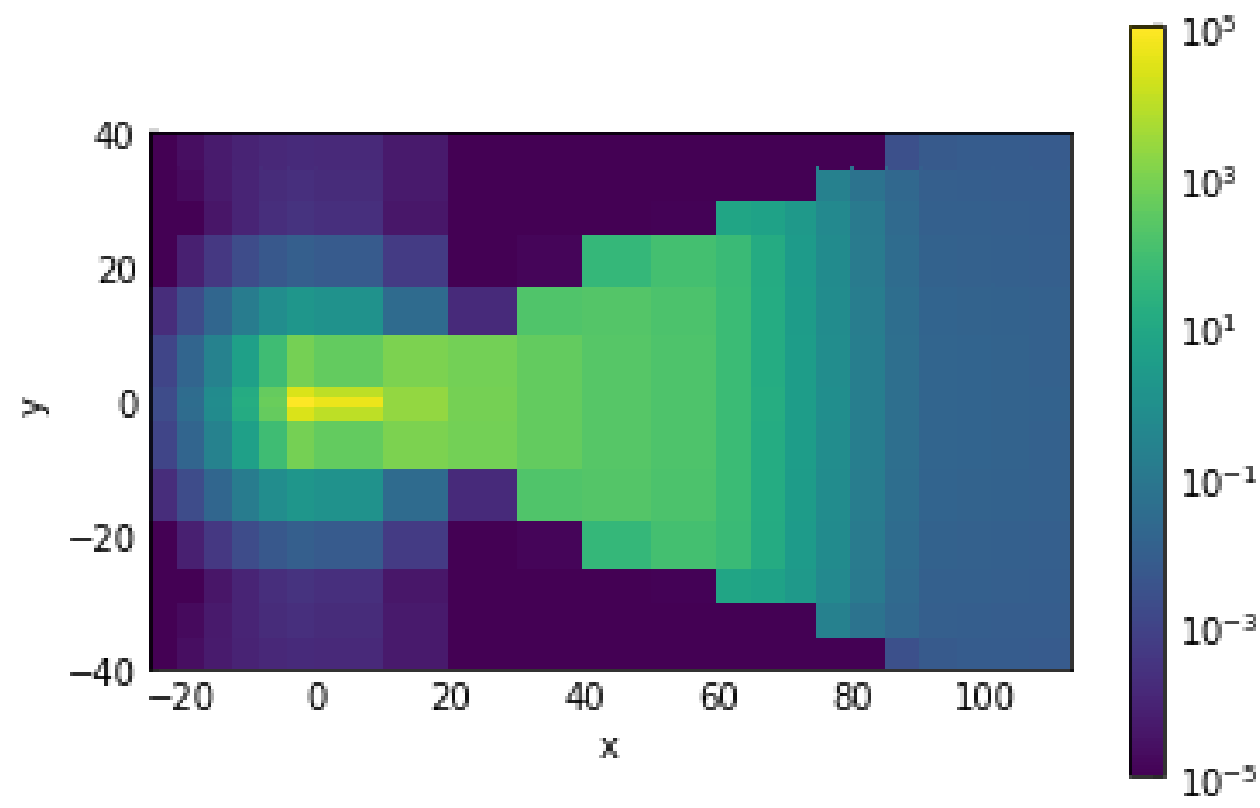

The flux along the $x$ axis in energy group 2 follows the uncollided flux contribution but also includes inscattering in the material from the higher-energy groups.

plots $=\operatorname{plot}\left(\operatorname{dnv}\left[\right.\right.$ 'flux' $^{\prime} \cdot \mathrm{xs}(\mathrm{g}=2, \mathrm{y}=0, \mathrm{z}=0), \log =$ True $)$

$\mathrm{ax}=\operatorname{plots}[$ 'ax']

ax.set_title(ax.get_ylabel())

ax.set_ylabel("Neutron flux (n/cm\$^2\$-s)")

ax.title.set_position $([.5,1.05])$; 
flux: group 2: $(n: 1.827 e+06,3.012 e+06) e V ; z$ in $(-0.833333,0.833333)$; $y$ in $(-0.833333,0.833333)$

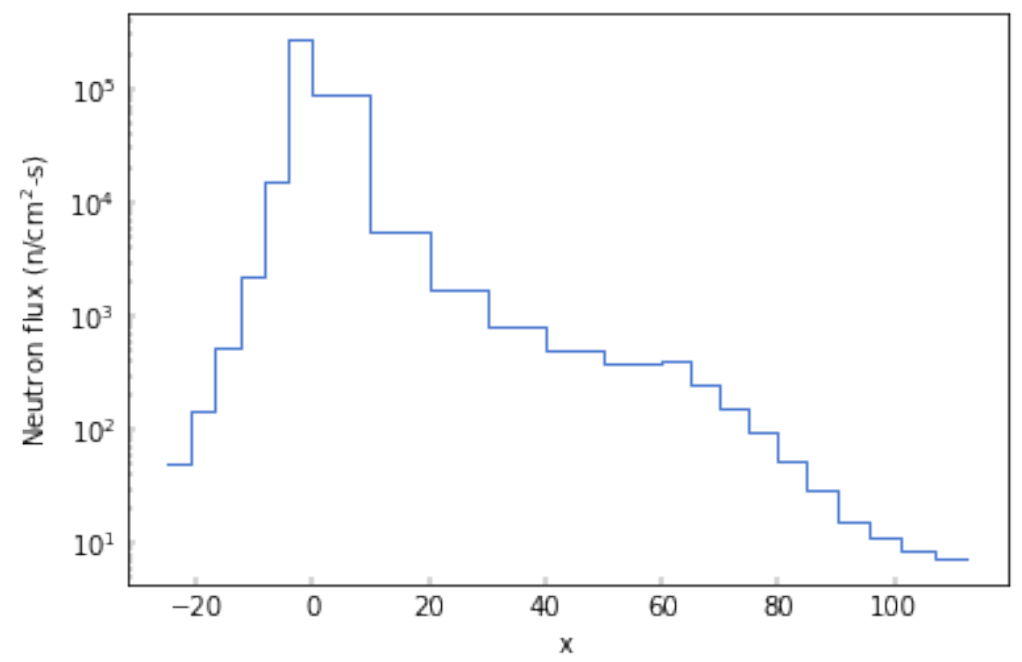

\section{A.2.4.3 Calculate dose rates}

The SCALE master library contains American National Standards Institute (ANSI) standard (1977) neutron flux-to-dose conversion factors ( $\mathrm{rem} / \mathrm{hr}) /\left(\mathrm{n} / \mathrm{cm}^{\wedge} 2-\mathrm{s}\right)(\mathrm{MT} 9029)$. The PALEALE code module can be used to print out these conversion factors from the SCALE AMPX library.

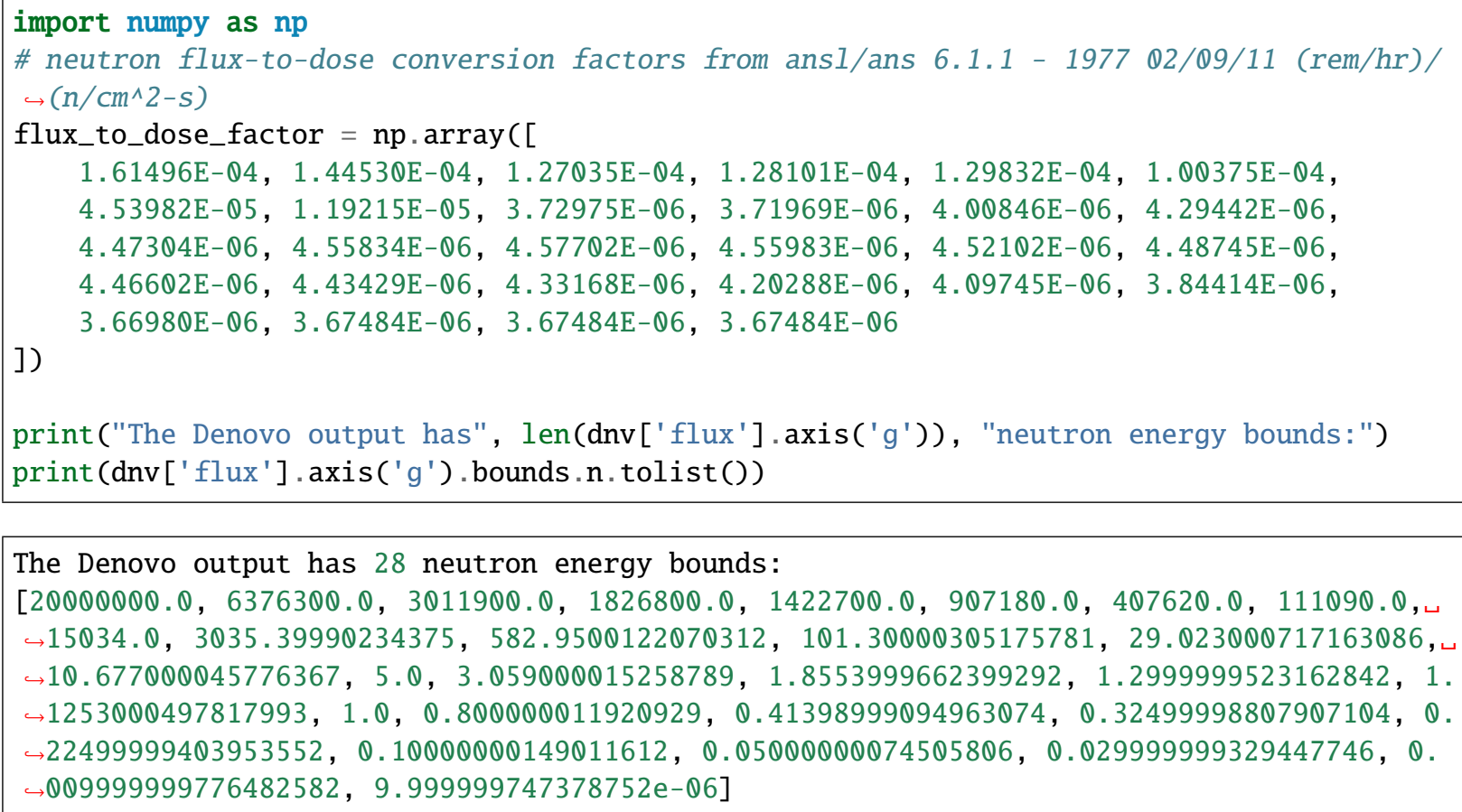

The Denovo output has 28 neutron energy bounds:

$[20000000.0,6376300.0,3011900.0,1826800.0,1422700.0,907180.0,407620.0,111090.0 . \sqcup$ $\hookrightarrow 15034.0,3035.39990234375,582.9500122070312,101.30000305175781,29.023000717163086$, ᄂ $\hookrightarrow 10.677000045776367,5.0,3.059000015258789,1.8553999662399292,1.2999999523162842,1$. $\hookrightarrow 1253000497817993,1.0,0.800000011920929,0.41398999094963074,0.32499998807907104,0$. $\hookrightarrow 22499999403953552,0.10000000149011612$, 0.05000000074505806, 0.029999999329447746, Q. $\hookrightarrow 009999999776482582,9.999999747378752 \mathrm{e}-06]$

from omnibus.data import Field

def weighted_energy_integrate(flux, weights, **kwargs):

assert len(flux.axis ('g')) == len(weights)

assert flux.dims[0] == ' $g$ ' 
(continued from previous page)

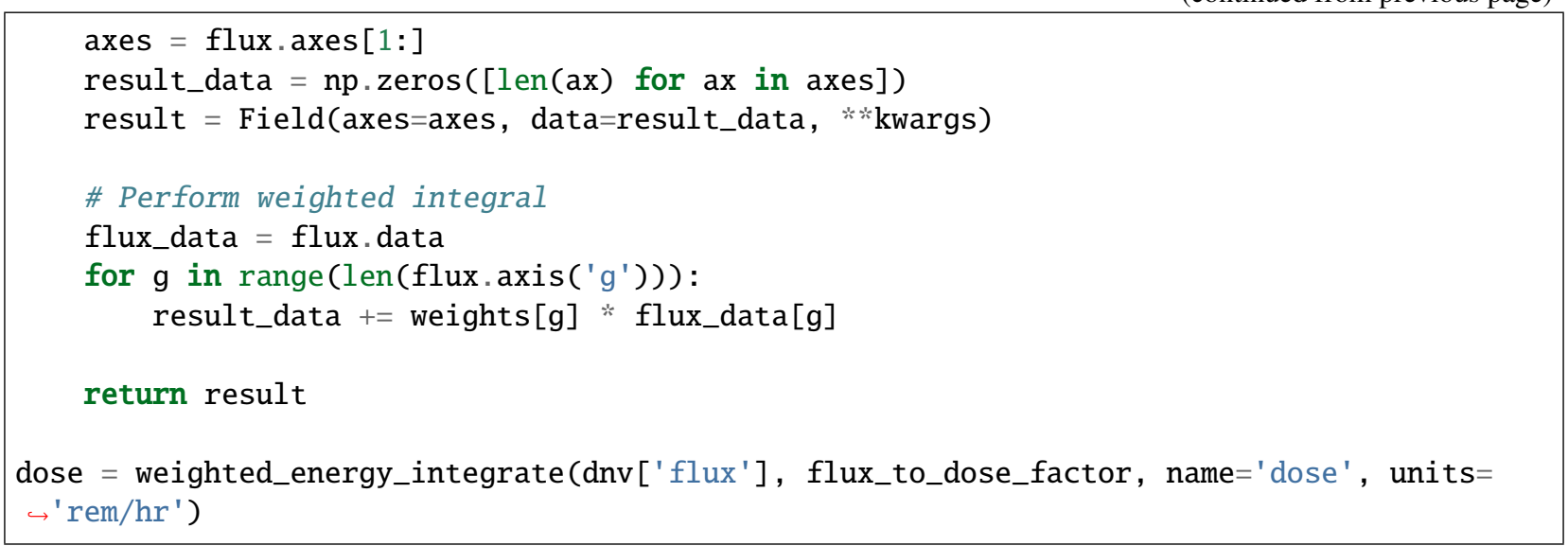

The 3D field of doses can be visualized by extracting lineouts or by coloring slices.

plots $=\operatorname{plot}(\operatorname{dose} \cdot x s(y=0, z=0), \log y=$ True $)$

$\mathrm{ax}=\operatorname{plots}\left[\mathrm{Cx}^{\prime}\right]$

ax.set_ylabel ("Dose [rem/hr]")

plots $=\operatorname{plot}(\operatorname{dose} \cdot x s(z=0.0), \operatorname{norm}=\operatorname{LogNorm}())$

plots['ax'].title.set_position([.5, 1.25]);

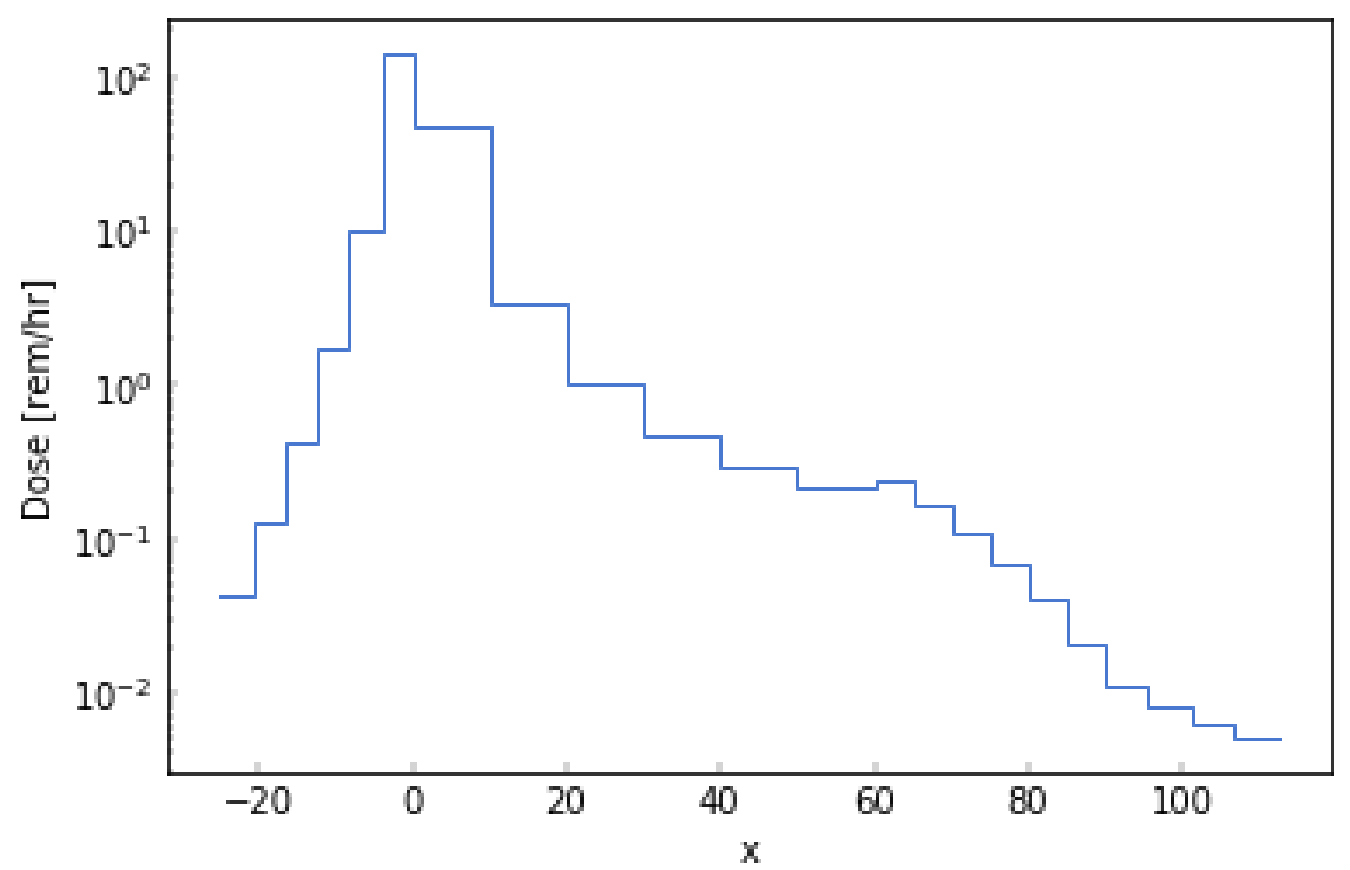


dose $[\mathrm{rem} / \mathrm{hr}]: \mathrm{z}$ in $(-0.833333,0.833333)$

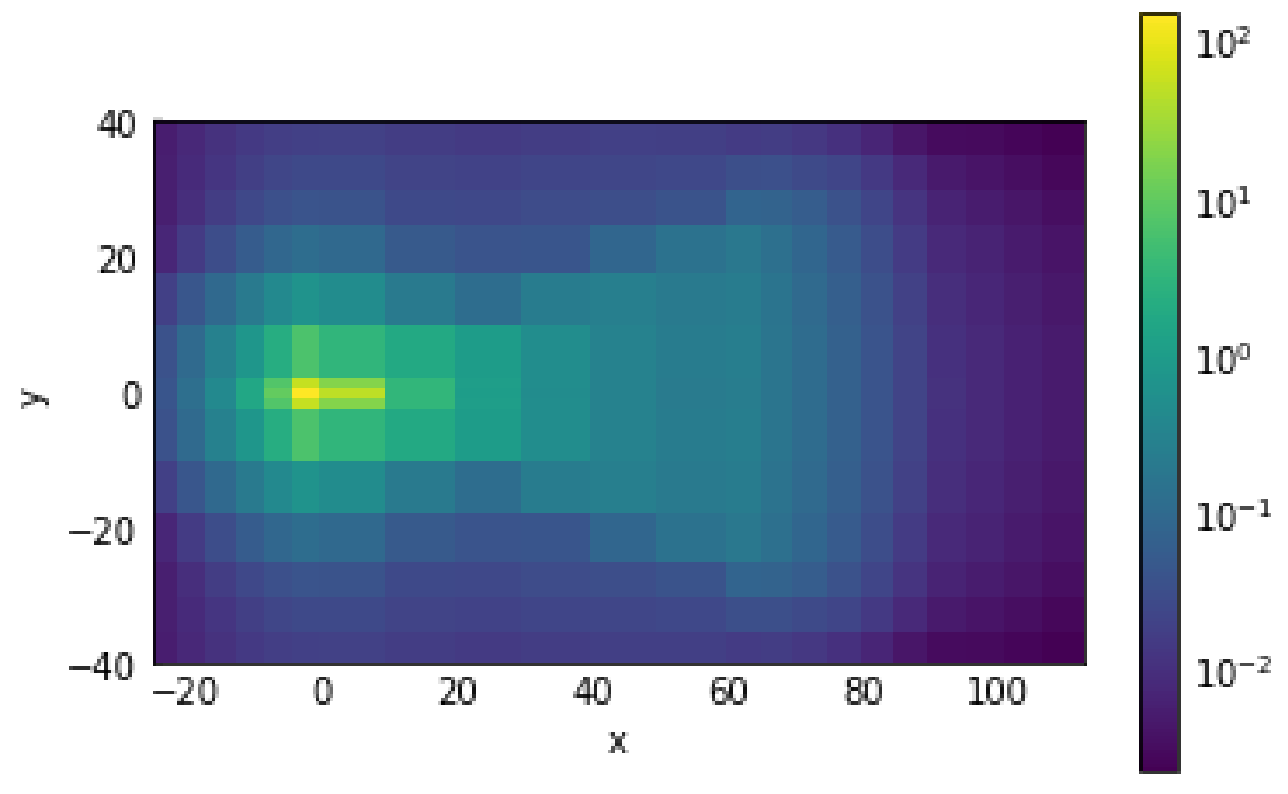

\section{A.2.4.4 Monte Carlo uncollided flux results}

With the Monte Carlo approach, the first collision source (uncollided flux) is obtained by direct Monte Carlo sampling. Thus the resulting uncollided flux fluctuates around the mean. As the number of Monte Carlo particles simulated increases, the uncollided flux obtained from the Monte Carlo approach should approach the analytic uncollided flux. The uncf mc option in the [DENOVO] [SOURCE] block enables this feature.

\%cat \{SOURCE_DIR\}/data/denovo-ueki-fwd-uncf-mc.omn

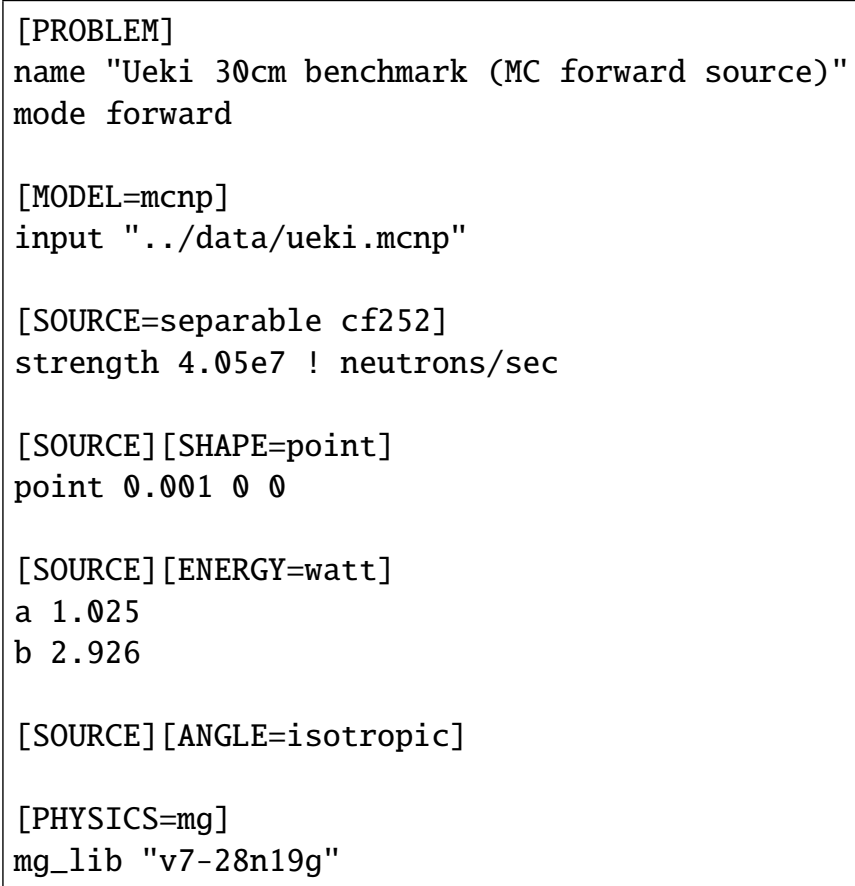


(continued from previous page)

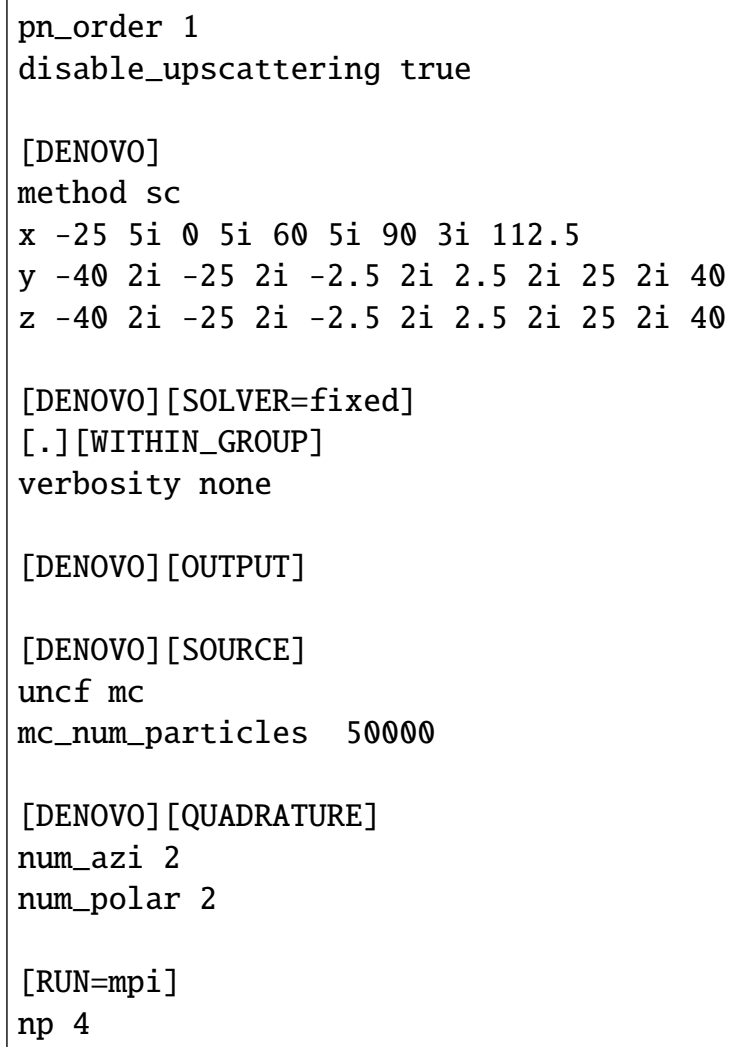

! omnibus-run \{SOURCE_DIR\}/data/denovo-ueki-fwd-uncf-mc.omn

INFO: Starting Omnibus preprocessor, omnibus version 6.3.pre-b10 (branch 'omnibus-doc' $\hookrightarrow \# 98 \mathrm{~d} 73 \mathrm{c} 8 \mathrm{e}$ on 2020MAR11)

Loading problem db from Omnibus ASCII file...

Loading Omnibus input file at /rnsdhpc/code/src/scale/Exnihilo/packages/Omnibus/driver/ -example/data/denovo-ueki-fwd-uncf-mc.omn ...finished loading problem db from Omnibus ASCII file

Loading problem db from Python file... ...finished loading problem db from Python file

INFO: Set default for 'mpiexec_args' to '['-np', '4']' in '/run'

Generating MCNP runtpe file... ...finished generating MCNP runtpe file

INFO: Set default for 'fissionable_only' to 'False' in '/source/cf252'

INFO: Set default for 'mode' to ' $\mathrm{n}$ ' in '/physics/mg'

INFO: Set default for 'load_scl' to 'True' in '/comp'

INFO: Set default for 'physics' to ' $\mathrm{mg}$ ' in '/denovo'

WARNING: The 'method' keyword is now 'equations' for SN discretization types. Changing $\hookrightarrow$ 'method $\mathrm{Sc}^{\prime}$ to 'equations $\mathrm{Sc}$ '

INFO: Set default for 'boundary' to '\{'_type': 'vacuum'\}' in '/denovo'

INFO: Set default 'x_blocks 2 ' and 'y_blocks 2'

INFO: Set default for 'z_blocks' to ' 2 ' in '/denovo/decomposition'

INFO: Set default for 'quadrature' to 'qr' in '/denovo/quadrature'

INFO: Set default for 'construction' to 'product' in '/denovo/quadrature'

INFO: Set default for 'mat' to 'True' in '/denovo/output' 
(continued from previous page)

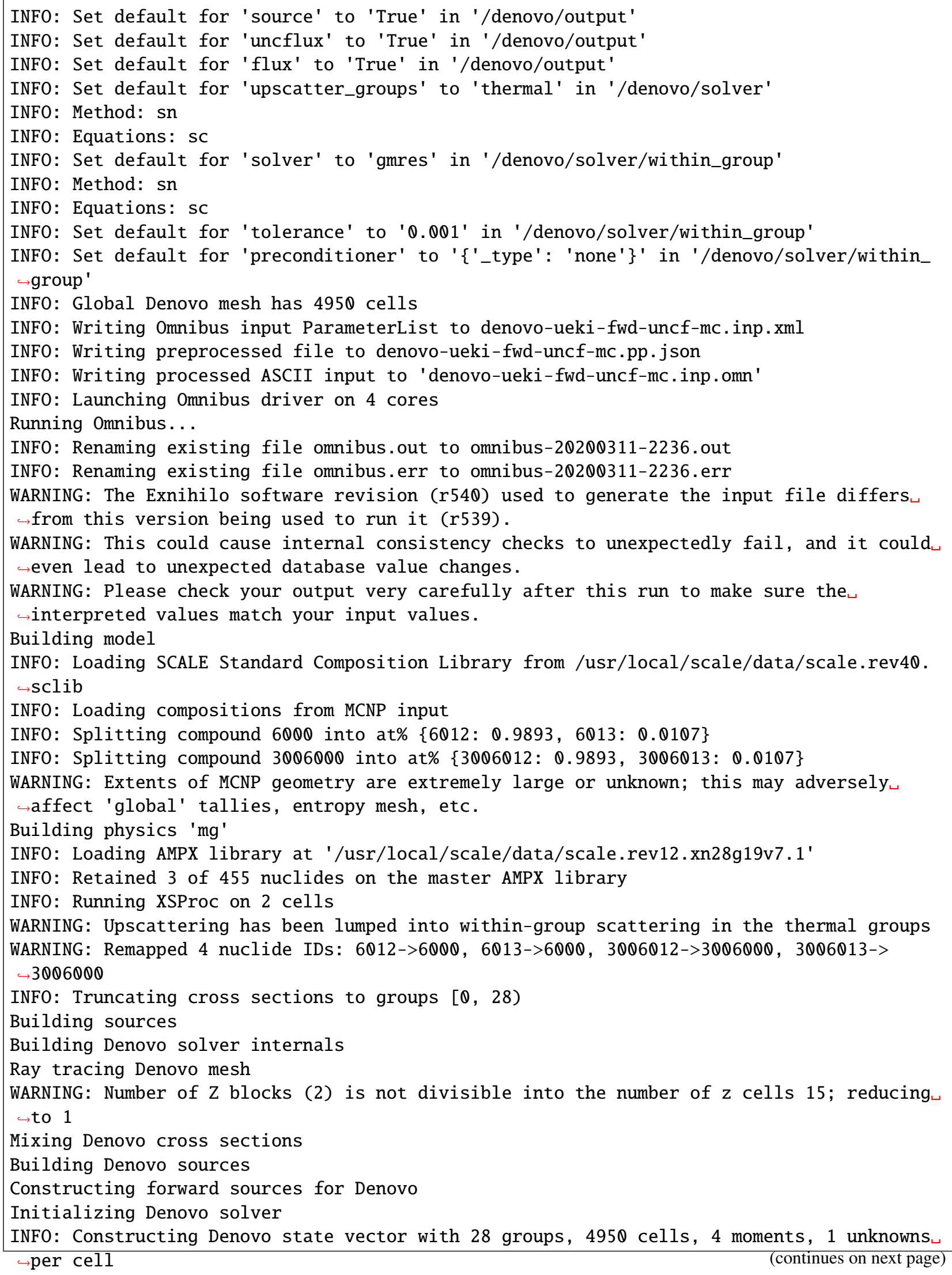


(continued from previous page)

$\begin{array}{llll}\text { Performing Monte Carlo first-collision source calculation } \\ \text { Running Denovo transport calculation } \\ \text { Forward group } & 0 \text { finished in } & 3 \text { Belos Block GMRES iterations. } \\ \text { Forward group } & 1 \text { finished in } & 4 \text { Belos Block GMRES iterations. } \\ \text { Forward group } & 2 \text { finished in } & 4 \text { Belos Block GMRES iterations. } \\ \text { Forward group } & 3 \text { finished in } & 3 \text { Belos Block GMRES iterations. } \\ \text { Forward group } & 4 \text { finished in } & 4 \text { Belos Block GMRES iterations. } \\ \text { Forward group } & 5 \text { finished in } & 4 \text { Belos Block GMRES iterations. } \\ \text { Forward group } & 6 \text { finished in } & 5 \text { Belos Block GMRES iterations. } \\ \text { Forward group } & 7 \text { finished in } & 6 \text { Belos Block GMRES iterations. } \\ \text { Forward group } & 8 \text { finished in } & 6 \text { Belos Block GMRES iterations. } \\ \text { Forward group } & 9 \text { finished in } & 6 \text { Belos Block GMRES iterations. } \\ \text { Forward group } & 10 \text { finished in } & 5 \text { Belos Block GMRES iterations. } \\ \text { Forward group } & 11 \text { finished in } & 5 \text { Belos Block GMRES iterations. } \\ \text { Forward group } & 12 \text { finished in } & 4 \text { Belos Block GMRES iterations. } \\ \text { Forward group } & 13 \text { finished in } & 4 \text { Belos Block GMRES iterations. } \\ \text { Forward group } & 14 \text { finished in } & 4 \text { Belos Block GMRES iterations. } \\ \text { Forward group } & 15 \text { finished in } & 4 \text { Belos Block GMRES iterations. } \\ \text { Forward group } & 16 \text { finished in } & 3 \text { Belos Block GMRES iterations. } \\ \text { Forward group } & 17 \text { finished in } & 3 \text { Belos Block GMRES iterations. } \\ \text { Forward group } & 18 \text { finished in } & 3 \text { Belos Block GMRES iterations. } \\ \text { Forward group } & 19 \text { finished in } & 3 \text { Belos Block GMRES iterations. } \\ \text { Forward group } & 20 \text { finished in } & 4 \text { Belos Block GMRES iterations. } \\ \text { Forward group } & 21 \text { finished in } & 4 \text { Belos Block GMRES iterations. } \\ \text { Forward group } & 22 \text { finished in } & 4 \text { Belos Block GMRES iterations. } \\ \text { Forward group } & 23 \text { finished in } & 6 \text { Belos Block GMRES iterations. } \\ \text { Forward group } & 24 \text { finished in } & 7 \text { Belos Block GMRES iterations. } \\ \text { Forward group } & 25 \text { finished in } & 8 \text { Belos Block GMRES iterations. } \\ \text { Forward group } & 26 \text { finished in } & 13 \text { Belos Block GMRES iterations. } \\ \text { INF: }\end{array}$

INFO: Writing Silo file to 4 concurrent files using material volume fractions

INFO: Removing SILO directory denovo_output_silo

Writing Denovo HDF5 output

Forward group 27 finished in 39 Belos Block GMRES iterations.

Run complete

Cleaning up

...finished running Omnibus in 3.7 seconds

Running Omnibus postprocessing

Loading HDF5 file...

INFO: Loaded Omnibus output data from 'denovo-ueki-fwd-uncf-mc.out.h5', problem name $\hookrightarrow$ 'Ueki $30 \mathrm{~cm}$ benchmark (MC forward source)', created on 2020MAR11 22:36 using SCALE

$\hookrightarrow$ version 6.3.pre-b10 (branch 'omnibus-doc' \#17579cc6 on 2020MAR10) ...finished loading HDF5 file

Loading HDF5 file...

INFO: Loaded Omnibus output data from 'denovo-ueki-fwd-uncf-mc.out.h5', problem name $\hookrightarrow$ 'Ueki $30 \mathrm{~cm}$ benchmark (MC forward source)', created on 2020MAR11 22:36 using SCALE

$\hookrightarrow$ version 6.3.pre-b10 (branch 'omnibus-doc' \#17579cc6 on 2020MAR10) ...finished loading HDF5 file

Writing Denovo visualization file...

INFO: Wrote Denovo visualization file to denovo-ueki-fwd-uncf-mc.denovo.xmf ...finished writing Denovo visualization file

Building RST summary...

INF0: Wrote summary file to denovo-ueki-fwd-uncf-mc.rst 
dnv_mc = load("denovo-ueki-fwd-uncf-mc.out.h5") ['denovo'].extract()

Loading HDF5 file...

INFO: Loaded Omnibus output data from 'denovo-ueki-fwd-uncf-mc.out.h5', problem name $\hookrightarrow$ 'Ueki 30cm benchmark (MC forward source)', created on 2020MAR11 22:36 using SCALE

$\hookrightarrow$ version 6.3.pre-b10 (branch 'omnibus-doc' \#17579cc6 on 2020MAR10) ...finished loading HDF5 file

Here the uncollided flux is solved using the Monte Carlo approximation. Note that unlike the smooth exponential shape calculated by the analytic method, the MC approximation has statistical error (the uncollided term abruptly drops to zero in undersampled regions far from the source). The shapes for each group uncollided flux are similar because the energy discretization on the source is performed separately from the spatial tracking.

fc_source_mc $=$ dnv_mc ['uncflux']

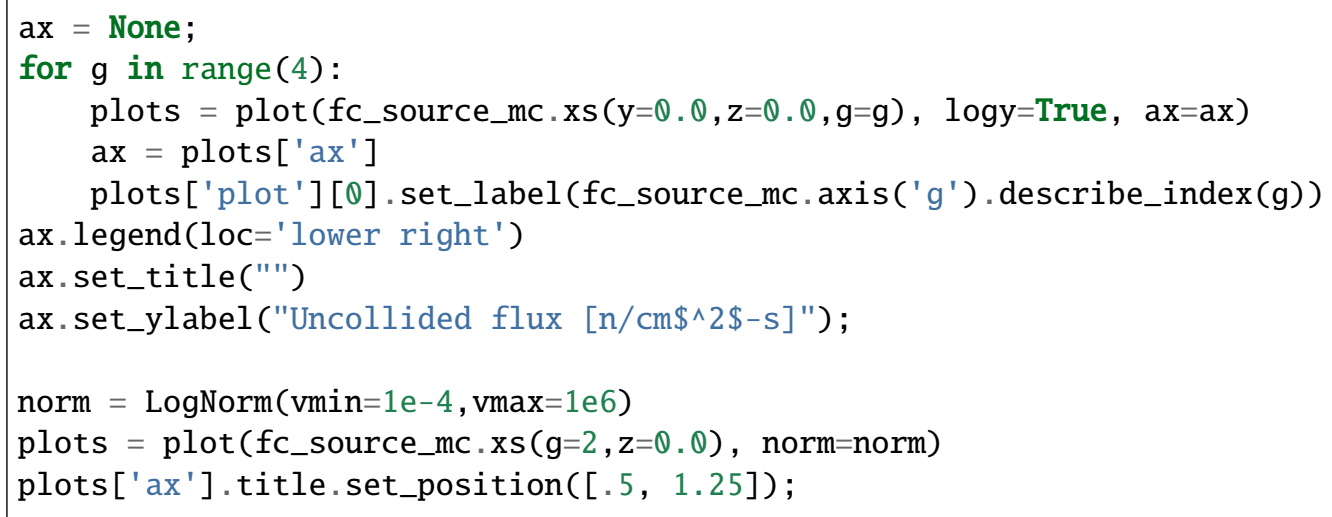




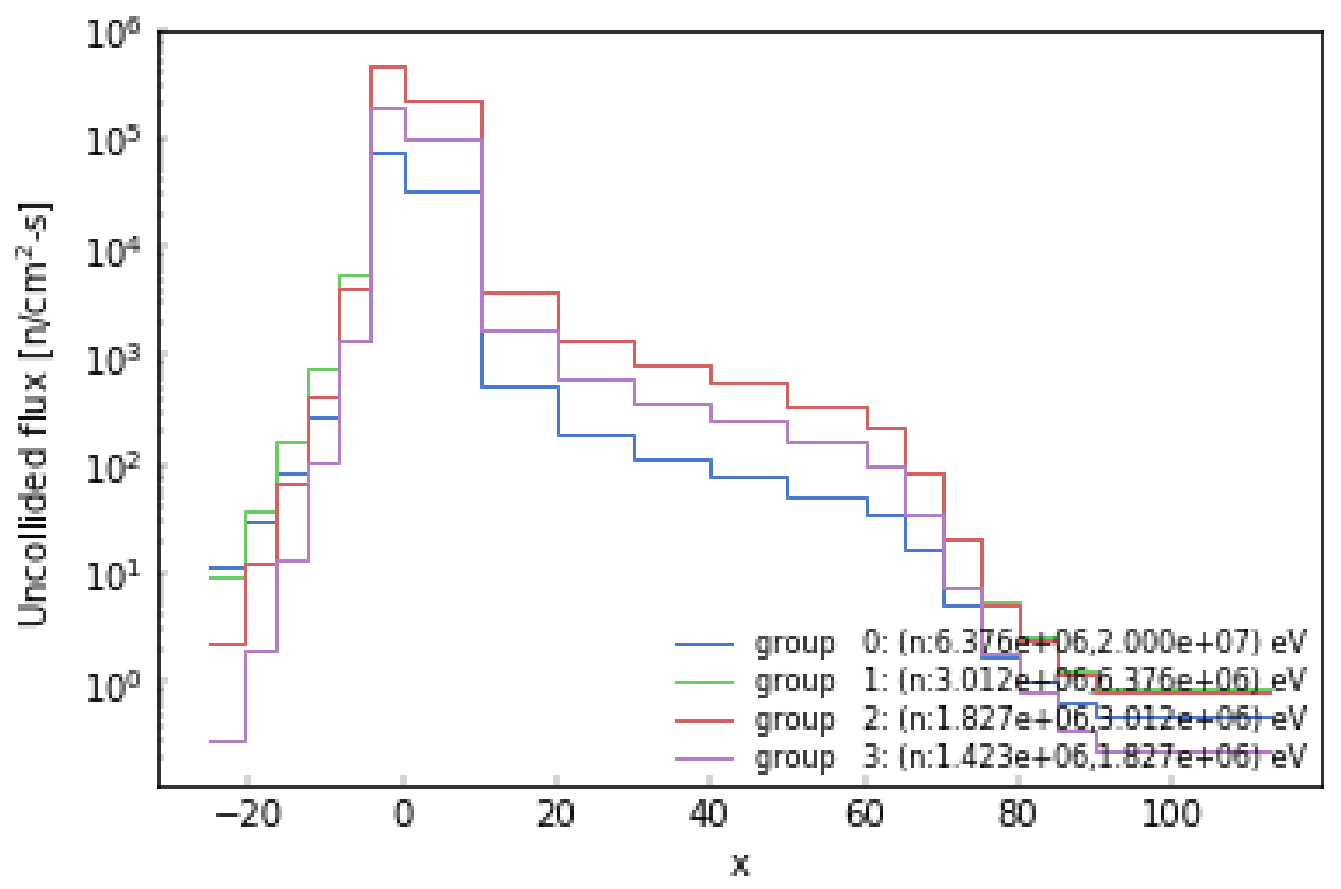

uncflux : group 2: (n:1.827e+06,3.012e+06) eV; $\mathrm{z}$ in $(-0.833333,0.833333)$

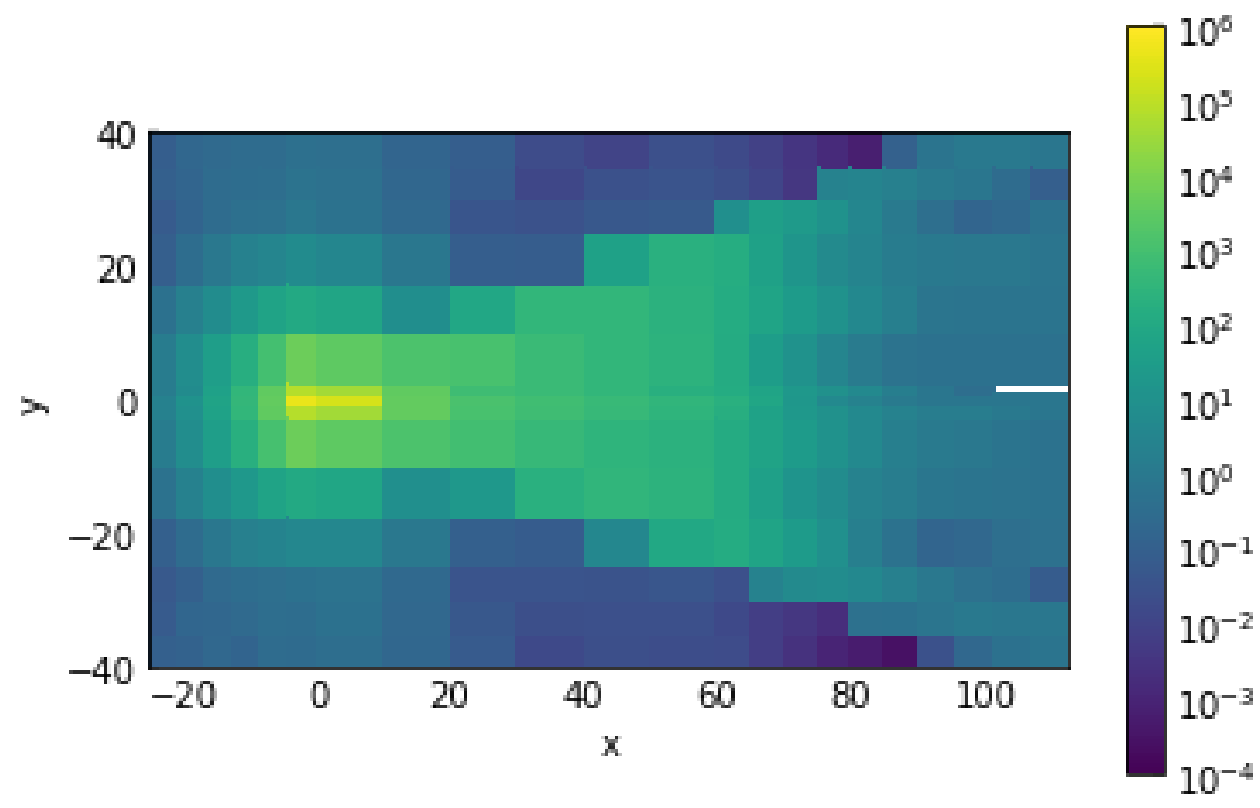

A comparison of the calculated doses for the analytic and Monte Carlo solutions show good agreement away from the source, modulo some statistical noice.

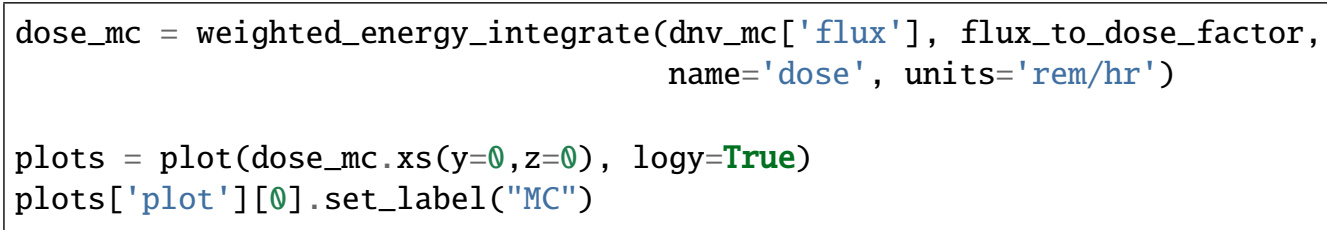



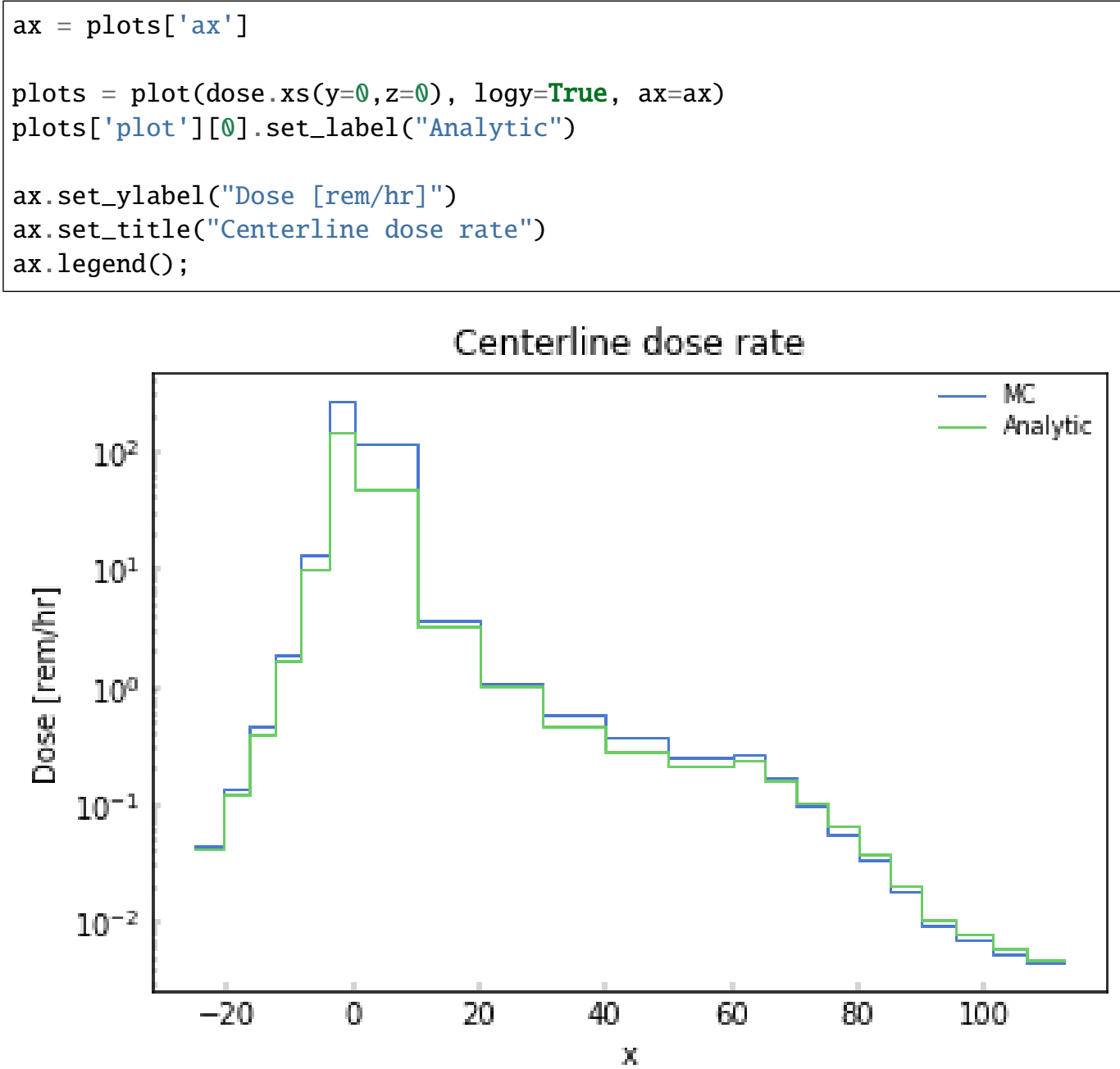

\section{A.2.4.5 Adjoint solutions}

The solution of the adjoint transport equation (adjoint flux) is the expected contribution from the neutron source to the detector. In other words, the adjoint flux is a measurement of the importance of a particle's contribution to the detector tally.

\%cat \{SOURCE_DIR\}/data/denovo-ueki-adj.omn

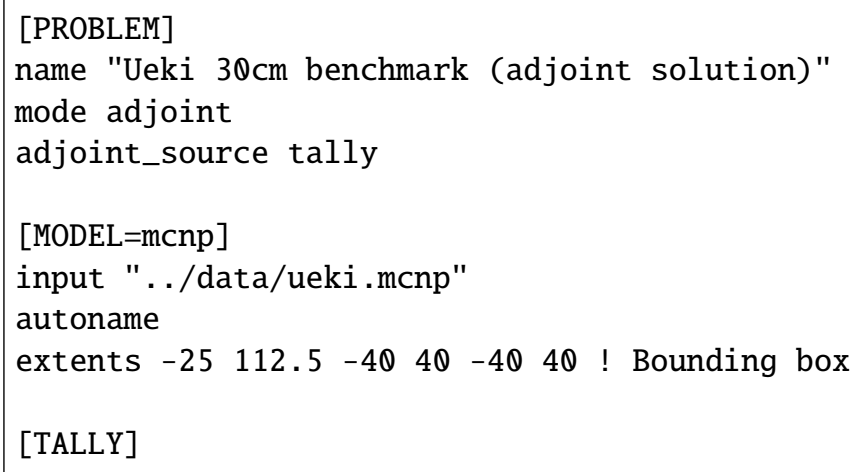




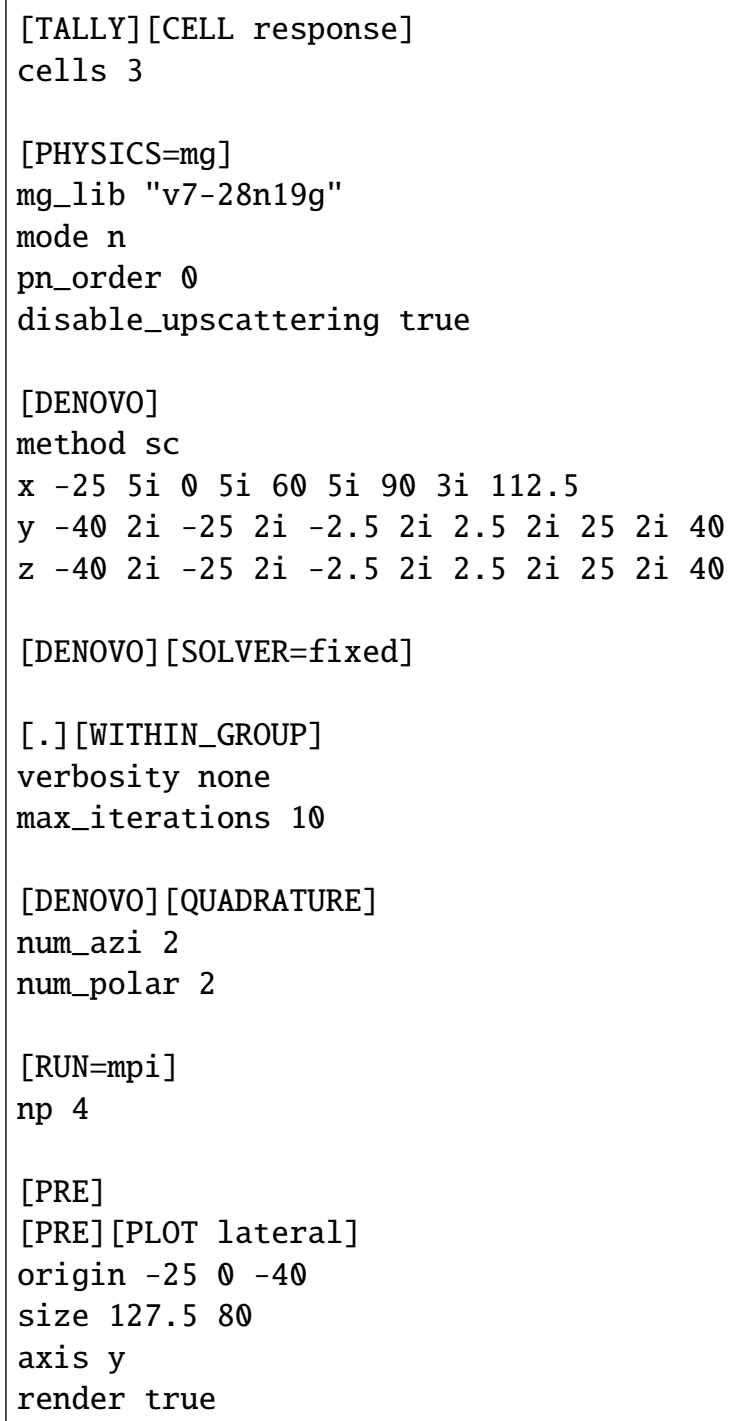

! omnibus-run \{SOURCE_DIR\}/data/denovo-ueki-adj.omn

INF0: Starting Omnibus preprocessor, omnibus version 6.3.pre-b10 (branch 'omnibus-doc' $\hookrightarrow \# 98 \mathrm{~d} 73 \mathrm{c} 8 \mathrm{e}$ on 2020MAR11)

Loading problem db from Omnibus ASCII file...

Loading Omnibus input file at /rnsdhpc/code/src/scale/Exnihilo/packages/Omnibus/driver/

$\hookrightarrow$ example/data/denovo-ueki-adj.omn ...finished loading problem db from Omnibus ASCII file

Loading problem db from Python file... ...finished loading problem db from Python file

INFO: Set default for 'mpiexec_args' to '['-np', '4']' in '/run'

Generating MCNP runtpe file...

...finished generating MCNP runtpe file

$\gg>$ Loading nuclide data from processed MCNP libraries

$\gg>$ Loading compositions from MCNP input

>>> Loading nuclide data from processed MCNP libraries 
(continued from previous page)

>> Loading compositions from MCNP input

INFO: Set default for 'load_scl' to 'True' in '/comp'

INFO: Set default for 'physics' to 'mg' in '/denovo'

WARNING: The 'method' keyword is now 'equations' for SN discretization types. Changing

$\hookrightarrow$ 'method $\mathrm{Sc}$ ' to 'equations $\mathrm{sc}$ '

INFO: Set default for 'boundary' to '\{'_type': 'vacuum'\}' in '/denovo'

INFO: Set default 'x_blocks 2' and 'y_blocks 2'

INFO: Set default for 'z_blocks' to '2' in '/denovo/decomposition'

INFO: Set default for 'quadrature' to 'qr' in '/denovo/quadrature'

INFO: Set default for 'construction' to 'product' in '/denovo/quadrature'

INF0: Set default for 'mat' to 'True' in '/denovo/output'

INFO: Set default for 'source' to 'True' in '/denovo/output'

INFO: Set default for 'uncflux' to 'True' in '/denovo/output'

INFO: Set default for 'flux' to 'True' in '/denovo/output'

INFO: Set default for 'upscatter_groups' to 'thermal' in '/denovo/solver'

INFO: Method: sn

INF0: Equations: $\mathrm{SC}$

INFO: Set default for 'solver' to 'gmres' in '/denovo/solver/within_group'

INF0: Method: sn

INFO: Equations: sc

INFO: Set default for 'tolerance' to ' 0.001 ' in '/denovo/solver/within_group'

INFO: Set default for 'preconditioner' to '\{'_type': 'none'\}' in '/denovo/solver/within_ $\hookrightarrow$ group '

INFO: Global Denovo mesh has 4950 cells

INFO: Renaming existing file denovo-ueki-adj.inp.xml to denovo-ueki-adj.inp-20200311-2236.

$\hookrightarrow \mathrm{Xml}$

INFO: Writing Omnibus input ParameterList to denovo-ueki-adj.inp.xml

〉> Loading SCALE Standard Composition Library from/usr/local/scale/data/scale.rev40.

$\hookrightarrow$ sclib

Generating geometry image...

INFO: Saved geometry image at $y=0$ to 'lateral_y0.png'

...finished generating geometry image

INFO: Renaming existing file denovo-ueki-adj.pp.json to denovo-ueki-adj.pp-20200311-2236. $\hookrightarrow$ json

INFO: Writing preprocessed file to denovo-ueki-adj.pp.json

INFO: Writing processed ASCII input to 'denovo-ueki-adj.inp.omn'

INFO: Launching Omnibus driver on 4 cores

Running Omnibus...

INFO: Renaming existing file omnibus.out to omnibus-20200311-2236a.out

INFO: Renaming existing file omnibus.err to omnibus-20200311-2236a.err

WARNING: The Exnihilo software revision ( $r 540$ ) used to generate the input file differs

$\hookrightarrow$ from this version being used to run it (r539).

WARNING: This could cause internal consistency checks to unexpectedly fail, and it could $\hookrightarrow$ even lead to unexpected database value changes.

WARNING: Please check your output very carefully after this run to make sure the

$\hookrightarrow$ interpreted values match your input values.

Building model

INFO: Loading SCALE Standard Composition Library from/usr/local/scale/data/scale.rev40. $\hookrightarrow$ Sclib

INFO: Loading compositions from MCNP input

INF0: Splitting compound 6000 into at\% $\{6012: 0.9893,6013: 0.0107\}$

INF0: Splitting compound 3006000 into at\% \{3006012: $0.9893,3006013: 0.0107\}$ 
(continued from previous page)

INFO: Creating default boundary mesh from $(-25-40-40)$ to $(112.54040)$ for MCNP $\rightarrow$ geometry

Building physics ' $m g$ '

INFO: Loading AMPX library at '/usr/local/scale/data/scale.rev12.xn28g19v7.1'

INFO: Retained 3 of 455 nuclides on the master AMPX library

INFO: Running XSProc on 2 cells

WARNING: Upscattering has been lumped into within-group scattering in the thermal groups

WARNING: Remapped 4 nuclide IDs: 6012->6000, 6013->6000, 3006012->3006000, 3006013->

$\hookrightarrow 3006000$

INFO: Truncating cross sections to groups $[0,28)$

Building tallies

Building Denovo solver internals

Ray tracing Denovo mesh

WARNING: Number of $\mathrm{Z}$ blocks (2) is not divisible into the number of $\mathrm{z}$ cells 15 ; reducing $\rightarrow$ to 1

Mixing Denovo cross sections

Building Denovo sources

Constructing adjoint sources for Denovo

WARNING: Tally 'response' has no multiplier specified in the HYBRID block; a flat

$\hookrightarrow$ adjoint spectrum will be used

Discretizing adjoint sources (1 cell tallies)

Initializing Denovo solver

INFO: Constructing Denovo state vector with 28 groups, 4950 cells, 1 moments, 1 unknowns $\hookrightarrow$ per cell

Running Denovo transport calculation

Adjoint group 27 finished in 5 Belos Block GMRES iterations.

Adjoint group 26 finished in 5 Belos Block GMRES iterations.

Adjoint group 25 finished in 5 Belos Block GMRES iterations.

Adjoint group 24 finished in 5 Belos Block GMRES iterations.

Adjoint group 23 finished in 4 Belos Block GMRES iterations.

Adjoint group 22 finished in 3 Belos Block GMRES iterations.

Adjoint group 21 finished in 3 Belos Block GMRES iterations.

Adjoint group 20 finished in 3 Belos Block GMRES iterations.

Adjoint group 19 finished in 3 Belos Block GMRES iterations.

Adjoint group 18 finished in 2 Belos Block GMRES iterations.

Adjoint group 17 finished in 2 Belos Block GMRES iterations.

Adjoint group 16 finished in 3 Belos Block GMRES iterations.

Adjoint group 15 finished in 3 Belos Block GMRES iterations.

Adjoint group 14 finished in 3 Belos Block GMRES iterations.

Adjoint group 13 finished in 3 Belos Block GMRES iterations.

Adjoint group 12 finished in 4 Belos Block GMRES iterations.

Adjoint group 11 finished in 4 Belos Block GMRES iterations.

Adjoint group 10 finished in 4 Belos Block GMRES iterations.

Adjoint group 9 finished in 4 Belos Block GMRES iterations.

Adjoint group 8 finished in 4 Belos Block GMRES iterations.

Adjoint group 7 finished in 4 Belos Block GMRES iterations.

Adjoint group 6 finished in 4 Belos Block GMRES iterations.

Adjoint group 5 finished in 3 Belos Block GMRES iterations.

Adjoint group 4 finished in 3 Belos Block GMRES iterations.

Adjoint group 3 finished in 2 Belos Block GMRES iterations.

Adjoint group 2 finished in 3 Belos Block GMRES iterations.

INFO: Writing Silo file to 4 concurrent files using material volume fractions 
INFO: Removing SILO directory denovo_output_silo

Writing Denovo HDF5 output

Run complete

Cleaning up

Adjoint group 1 finished in 3 Belos Block GMRES iterations.

Adjoint group $\theta$ finished in 3 Belos Block GMRES iterations.

...finished running Omnibus in 2.7 seconds

Running Omnibus postprocessing

Loading HDF5 file...

INFO: Loaded Omnibus output data from 'denovo-ueki-adj.out.h5', problem name 'Ueki $30 \mathrm{~cm} \sqcup$

$\hookrightarrow$ benchmark (adjoint solution)', created on 2020MAR11 22:37 using SCALE version 6.3.pre-

$\hookrightarrow$ b10 (branch 'omnibus-doc' \#17579cc6 on 2020MAR10)

...finished loading HDF5 file

Loading HDF5 file...

INFO: Loaded Omnibus output data from 'denovo-ueki-adj.out.h5', problem name 'Ueki $30 \mathrm{~cm} \sqcup$ $\hookrightarrow$ benchmark (adjoint solution)', created on 2020MAR11 22:37 using SCALE version 6.3.pre-

$\hookrightarrow$ b10 (branch 'omnibus-doc' \#17579cc6 on 2020MAR10)

...finished loading HDF5 file

Writing Denovo visualization file...

INFO: Wrote Denovo visualization file to denovo-ueki-adj.denovo.xmf

...finished writing Denovo visualization file

Building RST summary...

INFO: Wrote summary file to denovo-ueki-adj.rst

...finished building RST summary

dnv = load("denovo-ueki-adj.out.h5") ['denovo'].extract()

adj_flux $=\operatorname{dnv}[$ 'flux'].copy()

adj_flux.name ='adjoint_flux'

Loading HDF5 file...

INFO: Loaded Omnibus output data from 'denovo-ueki-adj.out.h5', problem name 'Ueki 30cm ↔benchmark (adjoint solution)', created on 2020MAR11 22:37 using SCALE version 6.3.pre-

$\hookrightarrow$ b10 (branch 'omnibus-doc' \#17579cc6 on 2020MAR10)

... finished loading HDF5 file

Plotting the adjoint flux shows how likely it is for particles at each point in space to contribute to the detector. Denovo predicts that the flux is of lowest importance in the paraffin block and of highest importance behind the graphite shield.

plots $=$ plot (adj_flux.xs $(g=2, z=0.0)$, norm $=$ norm $)$

plots ['ax'].title.set_position([.5, 1.25]); 


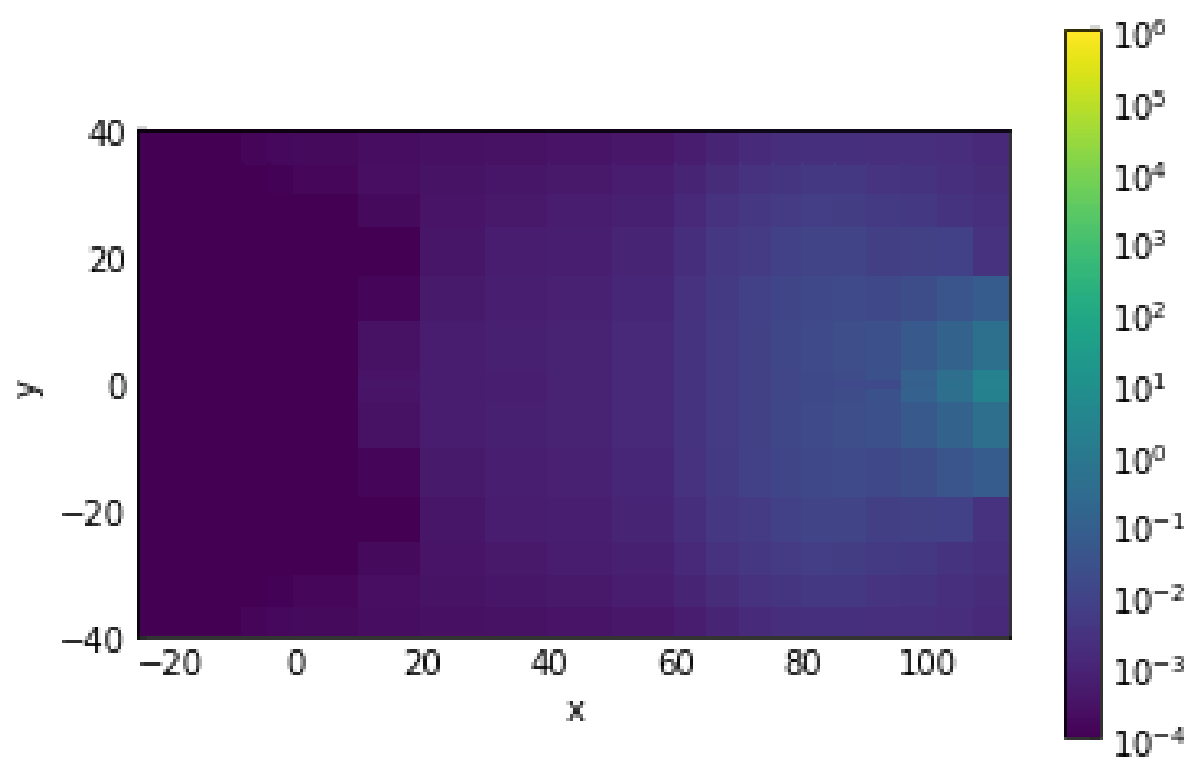

\section{A.2.5 DENOVO/SWORD INTEGRATION}

This example demonstrates how to load and visualize a SWORD input file, create an Omnibus Denovo input for it that writes the angular flux and current, and post-process the output.

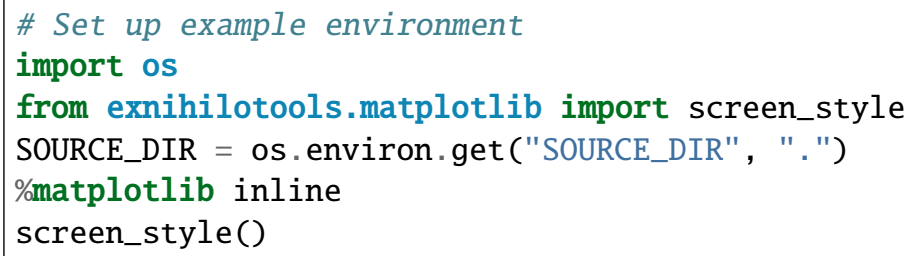

\section{A.2.5.1 Visualize a GDML geometry}

Loading a SWORD geometry reads a set of XML input files and calls Geant4 initialization routines to load a corresponding GDML geometry input.

from omnibus.raytrace. load import load_sword

model = load_sword(os.path.join(SOURCE_DIR, "data", "btest5", "btest5.sword"))

Generating serialized SWORD model...

INF0: Writing packed model to btest5.xdr

...finished generating serialized SWORD model

Omnibus includes tools to create color schemes based on the phyical compositions of the problem. The ColorMap.from_compositions method automatically assigns colors for common real-life materials such as air, carbon-based life forms, and detector materials. The individual compositions from the model can also be explored to validate that Denovo is seeing the right nuclides, weight fractions, etc.

from omnibus.raytrace. colors import ColorMap

colors = ColorMap.from_compositions (model.compositions)

colors.comps [1] - _asdict() 


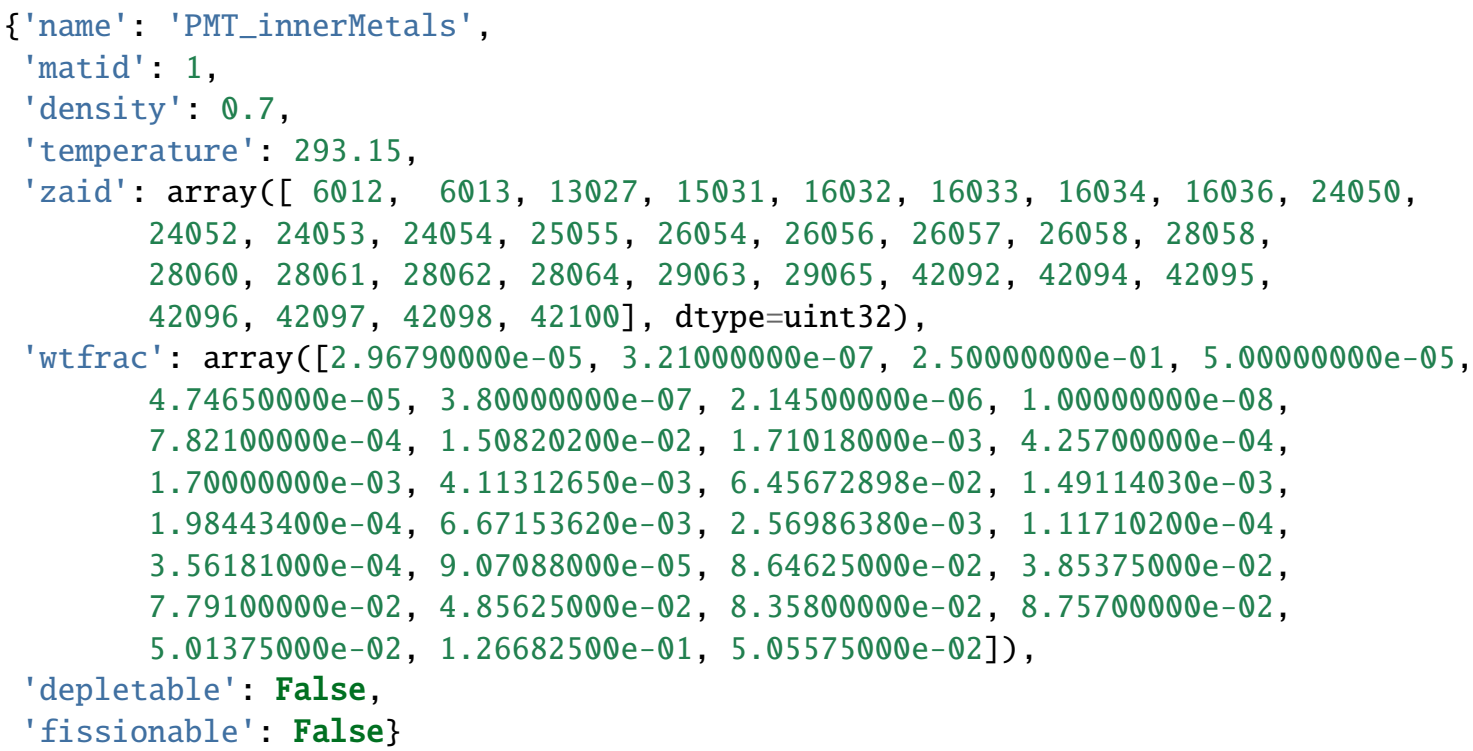

from omnibus.raytrace. imager import Imager

imager $=$ Imager (model.geometry,

lower $=(-100,0,-100)$,

upper $=(250,0,100)$,

basis $=(1,0,0)$,

max_pixels=1024)

imager.names $=$ [c.name for $\mathrm{c}$ in model.compositions]

imager. colors $=$ colors

imager.plot();

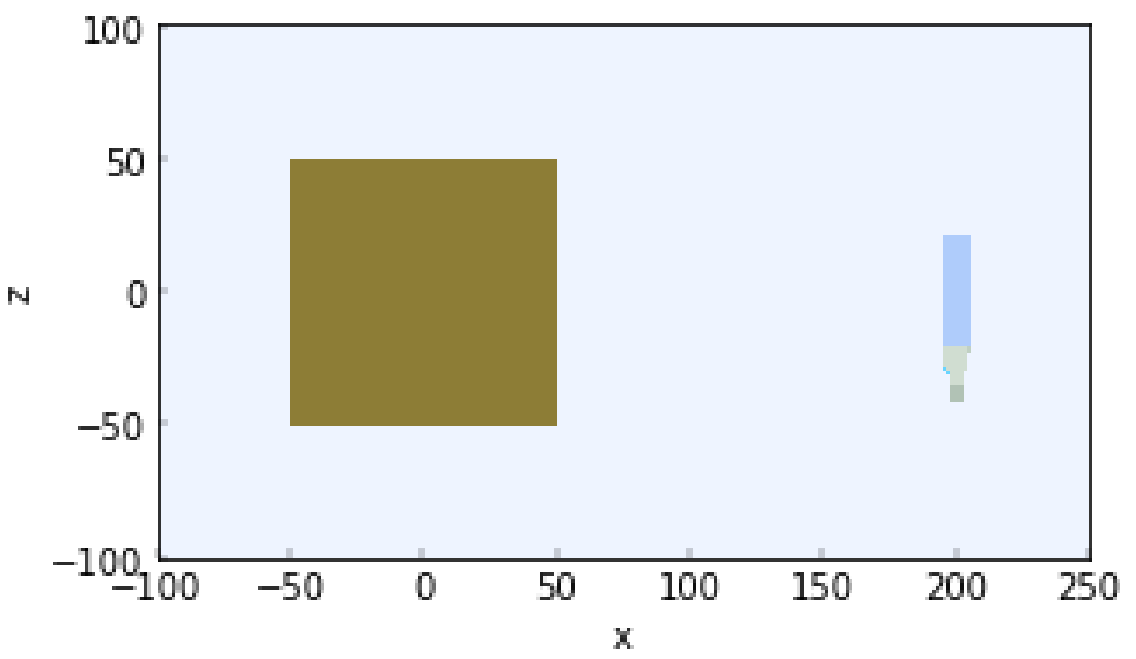

Wood

Air

$\mathrm{Nal}$

Aluminum

Glass

PMT_innerMetals

Aluminum_PMT_Base

Rendering produces a faster, higher-resolution image but doesn't show the extents or material names. 
imager . render ('rendered.png')

from IPython.display import Image

Image ( $f$ ilename $=$ ' rendered . png ')

\section{A.2.5.2 Execute Omnibus}

The Omnibus input to this problem uses ANISN data (the multigroup data distributed with ADVANTG) and the SWORD model loaded above. Inside the Denovo block it specifies to output both the current (first angular moment) and the angular flux (actual discrete ordinates solution at each angle).

\%cat \{SOURCE_DIR $\} /$ data/btest.omn

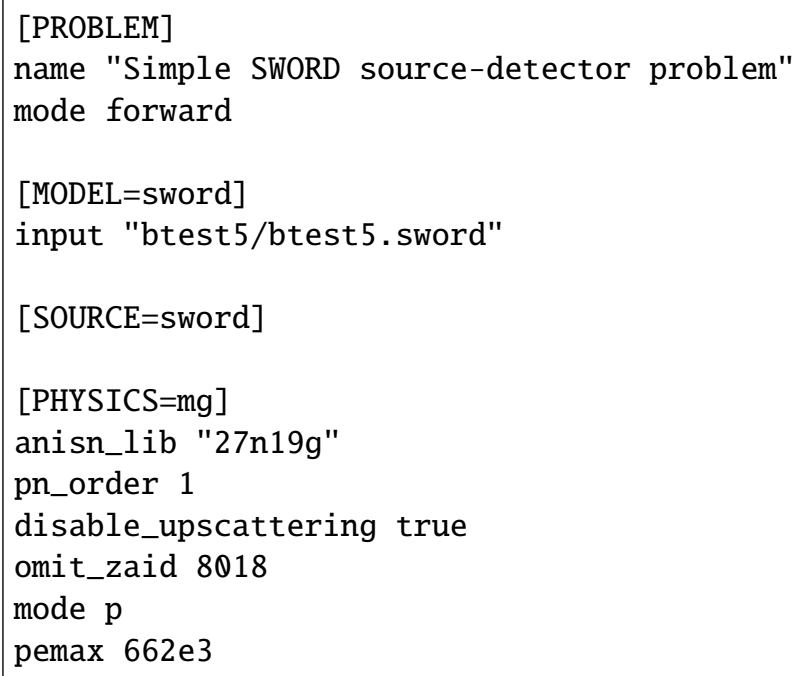


(continued from previous page)

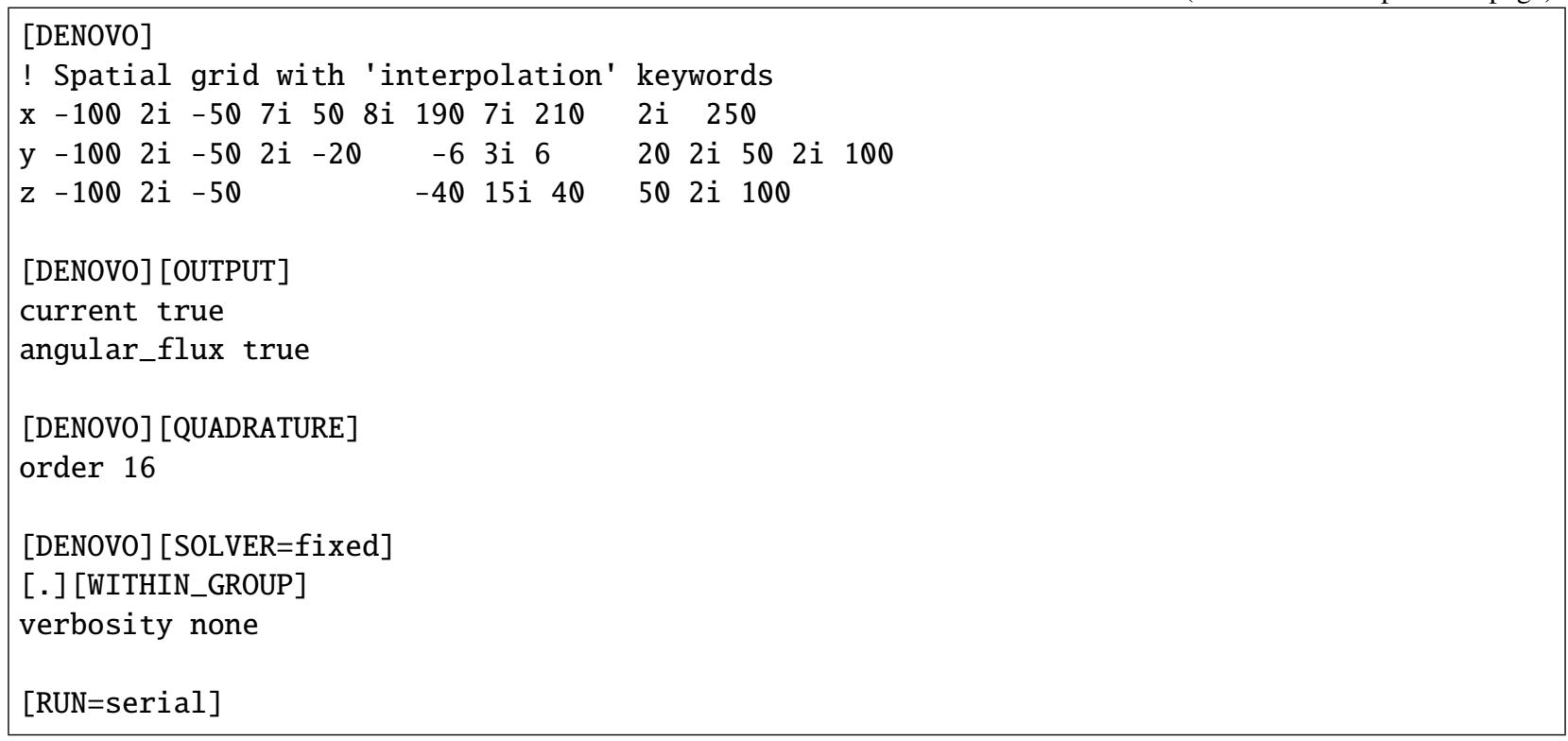

! omnibus-run \{SOURCE_DIR\}/data/btest.omn

INF0: Starting Omnibus preprocessor, omnibus version 6.3.pre-b10 (branch 'omnibus-doc' $\hookrightarrow \# 98 \mathrm{~d} 73 \mathrm{c} 8 \mathrm{e}$ on 2020MAR11)

Loading problem db from Omnibus ASCII file...

Loading Omnibus input file at/rnsdhpc/code/src/scale/Exnihilo/packages/Omnibus/driver/

↔example/data/btest.omn

...finished loading problem db from Omnibus ASCII file

Loading problem db from Python file...

...finished loading problem db from Python file

Generating serialized SWORD model...

...finished generating serialized SWORD model

INFO: Setting ANISN libraries based on data at /usr/local/advantg-data/27n19g.bin

INFO: Set default for 'load_scl' to 'False' in '/comp'

INFO: Set default for 'physics' to ' $\mathrm{mg}$ ' in '/denovo'

INFO: Set default for 'boundary' to '\{'_type': 'vacuum'\}' in '/denovo'

INFO: Set default 'x_blocks 1 ' and 'y_blocks 1 '

INFO: Set default for 'z_blocks' to ' 1 ' in '/denovo/decomposition'

INFO: Set default for 'quadrature' to 'qr' in '/denovo/quadrature'

INFO: Set default for 'construction' to 'levelsym' in '/denovo/quadrature'

INF0: Set default for 'mat' to 'True' in '/denovo/output'

INFO: Set default for 'source' to 'True' in '/denovo/output'

INF0: Set default for 'uncflux' to 'True' in '/denovo/output'

INFO: Set default for 'flux' to 'True' in '/denovo/output'

INFO: Set default for 'upscatter_groups' to 'thermal' in '/denovo/solver'

INF0: Method: sn

INFO: Equations: Sc

INFO: Set default for 'solver' to 'gmres' in '/denovo/solver/within_group'

INF0: Method: sn

INFO: Equations: sc

INFO: Set default for 'tolerance' to ' 0.001 ' in '/denovo/solver/within_group'

INFO: Set default for 'preconditioner' to '\{'_type': 'none'\}' in '/denovo/solver/within_ $\rightarrow$ group' 
(continued from previous page)

INFO: Global Denovo mesh has 13392 cells

INFO: Writing Omnibus input ParameterList to btest.inp.xml

INF0: Writing preprocessed file to btest.pp.json

INFO: Writing processed ASCII input to 'btest.inp.omn'

INFO: Launching Omnibus driver on 1 cores

Running Omnibus...

WARNING: The Exnihilo software revision (r540) used to generate the input file differs

$\hookrightarrow$ from this version being used to run it ( $r 539$ ).

WARNING: This could cause internal consistency checks to unexpectedly fail, and it could $\hookrightarrow$ even lead to unexpected database value changes.

WARNING: Please check your output very carefully after this run to make sure the

$\hookrightarrow$ interpreted values match your input values.

Building model

INFO: Loading Geant problem from GDML file at /rnsdhpc/code/src/scale/Exnihilo/packages/

$\hookrightarrow$ Omnibus/driver/example/data/btest5/sword.gdml

INFO: Loading 19 elements from Geant4

INF0: Loading nuclide data from Geant4 isotope libraries

INFO: Loading 48 isotopes from Geant4

WARNING: Nuclide data for 13027 was already set; ignoring and using existing data

WARNING: Nuclide data for 13027 was already set; ignoring and using existing data

INFO: Loading compositions from Geant4 material data

INFO: Creating default boundary mesh from $\left(\begin{array}{lllll}-12500 & -12500 & -12500\end{array}\right)$ to (12500 1250012500$)$

$\hookrightarrow$ for Geant4 geometry

Building physics 'mg'

INFO: Loading ANISN attributes from /usr/local/advantg-data/27n19g.info

INFO: Loading ANISN zaid map from /usr/local/advantg-data/27n19g.zaid

INFO: Loading ANISN cross sections from/usr/local/advantg-data/27n19g.bin

WARNING: Upscattering has been lumped into within-group scattering in the thermal groups

WARNING: Remapped 2 nuclide IDs: 6012->6000, 6013->6000

WARNING: Omitted 1 nuclide IDs: 8018

INFO: Truncating cross sections to groups $[39,46)$

Building sources

Building Denovo solver internals

Ray tracing Denovo mesh

Mixing Denovo cross sections

Building Denovo sources

Constructing forward sources for Denovo

Discretizing SWORD sources (1 points)

Initializing Denovo solver

INFO: Constructing Denovo state vector with 7 groups, 13392 cells, 4 moments, 1 unknowns $\hookrightarrow$ per cell

Performing analytic first-collision source calculation on 1 sources

Running Denovo transport calculation

Forward group $\theta$ finished in 3 Belos Block GMRES iterations.

Forward group 1 finished in 3 Belos Block GMRES iterations.

Forward group 2 finished in 3 Belos Block GMRES iterations.

Forward group 3 finished in 4 Belos Block GMRES iterations.

Forward group 4 finished in 5 Belos Block GMRES iterations.

Forward group 5 finished in 7 Belos Block GMRES iterations.

INFO: Writing Silo file to 1 concurrent files using material volume fractions

Writing Denovo HDF5 output

Forward group 6 finished in 3 Belos Block GMRES iterations. 
(continued from previous page)

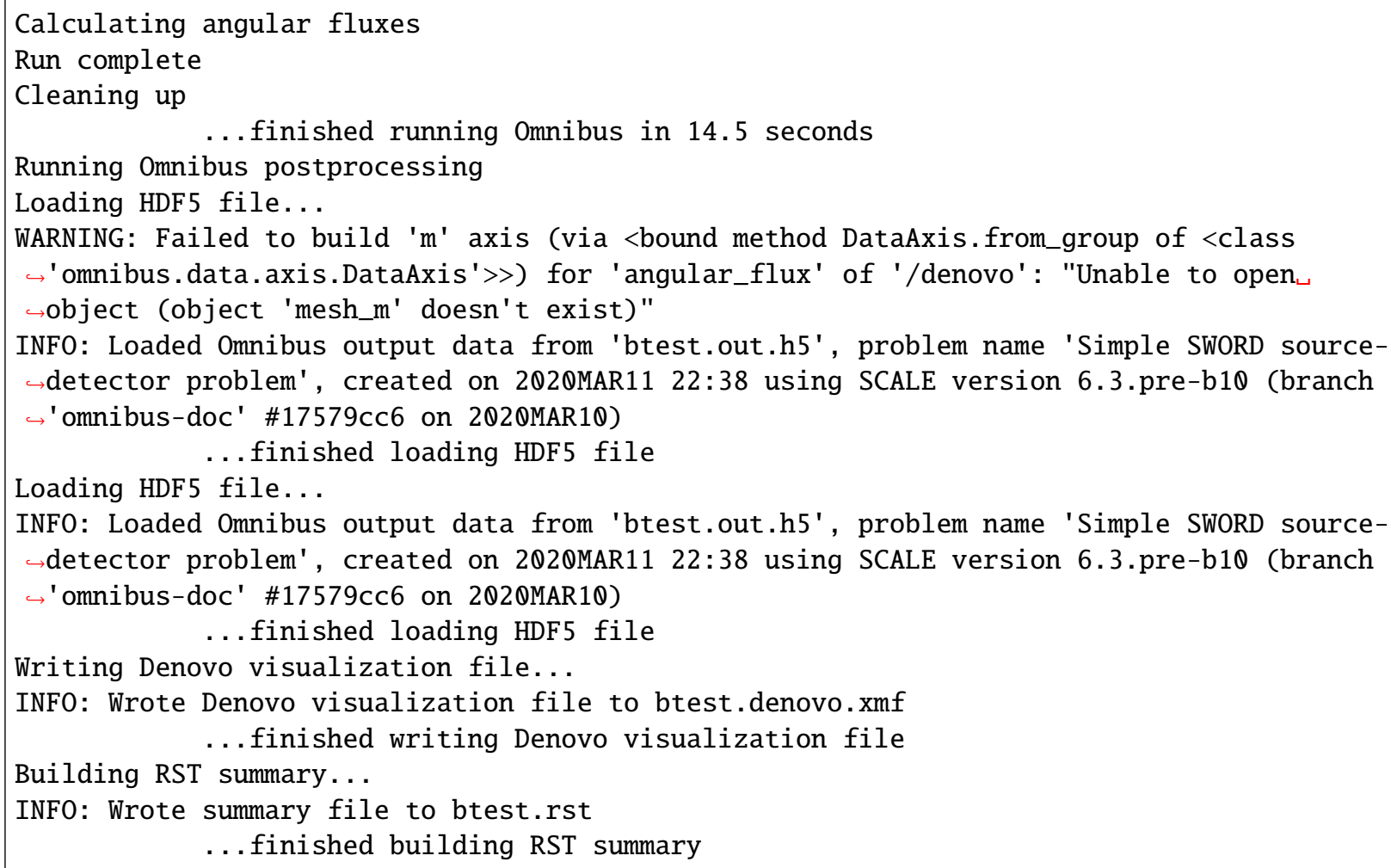

\section{A.2.5.3 Process results}

from omnibus. formats. output import load

dnv = load("btest.out.h5")['denovo'].extract()

Loading HDF5 file...

WARNING: Failed to build ' $m$ ' axis (via <bound method DataAxis.from_group of <class

$\hookrightarrow$ 'omnibus.data.axis.DataAxis'>>) for 'angular_flux' of '/denovo': "Unable to open

$\hookrightarrow$ object (object 'mesh_m' doesn't exist)"

INFO: Loaded Omnibus output data from 'btest.out.h5', problem name 'Simple SWORD source$\rightarrow$ detector problem', created on 2020MAR11 22:38 using SCALE version 6.3.pre-b10 (branch

$\hookrightarrow$ 'omnibus-doc' \#17579cc6 on 2020MAR10)

...finished loading HDF5 file

\section{Uncollided flux}

A good first step in analysis is to verify the source term: in this case, checking that it is nonzero only in the highest group. The xs command slices the data with the group index for a single group, or with a Python slice object to get a range of groups. (a[slice(1,None)] is equivalent to $a[1:]$.)

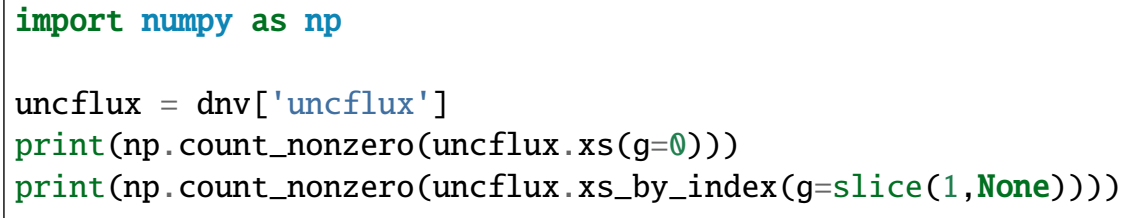


A lineout plot along $y=z=0$ for the source group clearly shows the attenuation through the detector at $x=200$.

from omnibus.data import plot

plots $=$ plot (uncflux.xs $(y=0.0, z=0.0, g=0)$, logy=True);

plots['ax']. set_ylabel(' ');

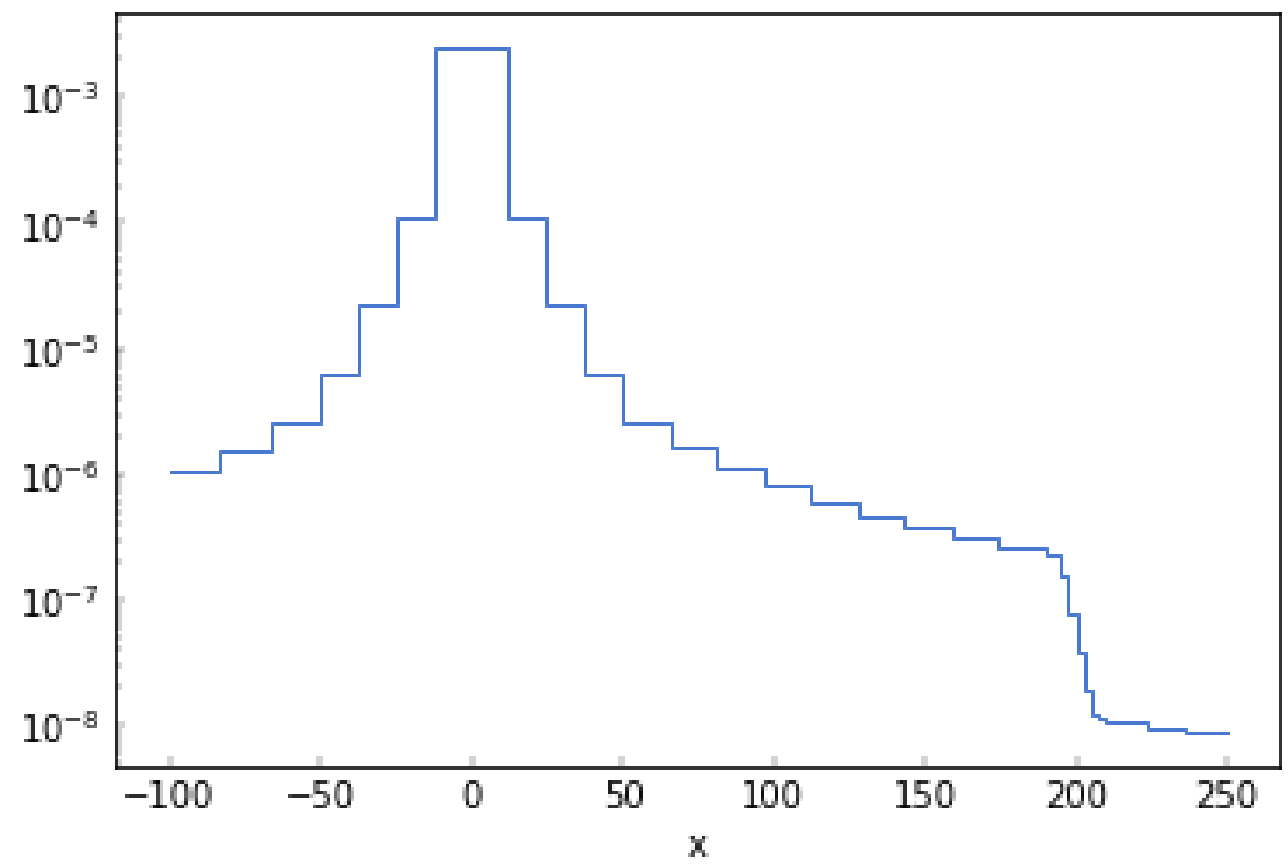

The "scalar flux" is the zeroth angular moment of the angular flux:

$$
\phi=\int_{4 \pi} \psi(\vec{x}, \vec{\Omega}) \mathrm{d} \Omega
$$

It is effectively a measure of particle density, and is used in calculating reaction rates.

The following command extracts the data at the origin. Since the only remaining data axis is energy, the plot function will render a step plot of the group-average fluxes.

\section{Flux}

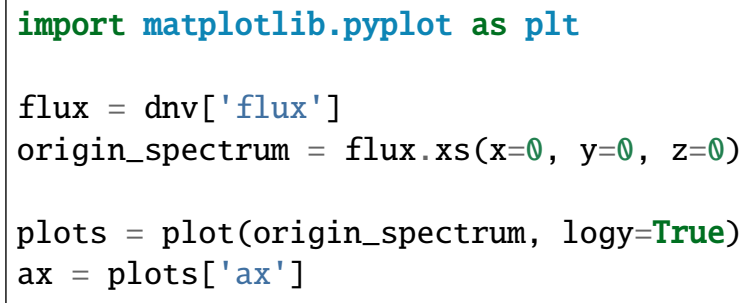


\# Rotate the $X$ tick labels

plt.setp(ax.get_xticklabels(), rotation=90);

ax.set_title(ax.get_ylabel())

ax.set_ylabel("Particle flux $\left(\mathrm{p} / \mathrm{cm}^{\wedge} 2-\mathrm{s}\right)$ ");

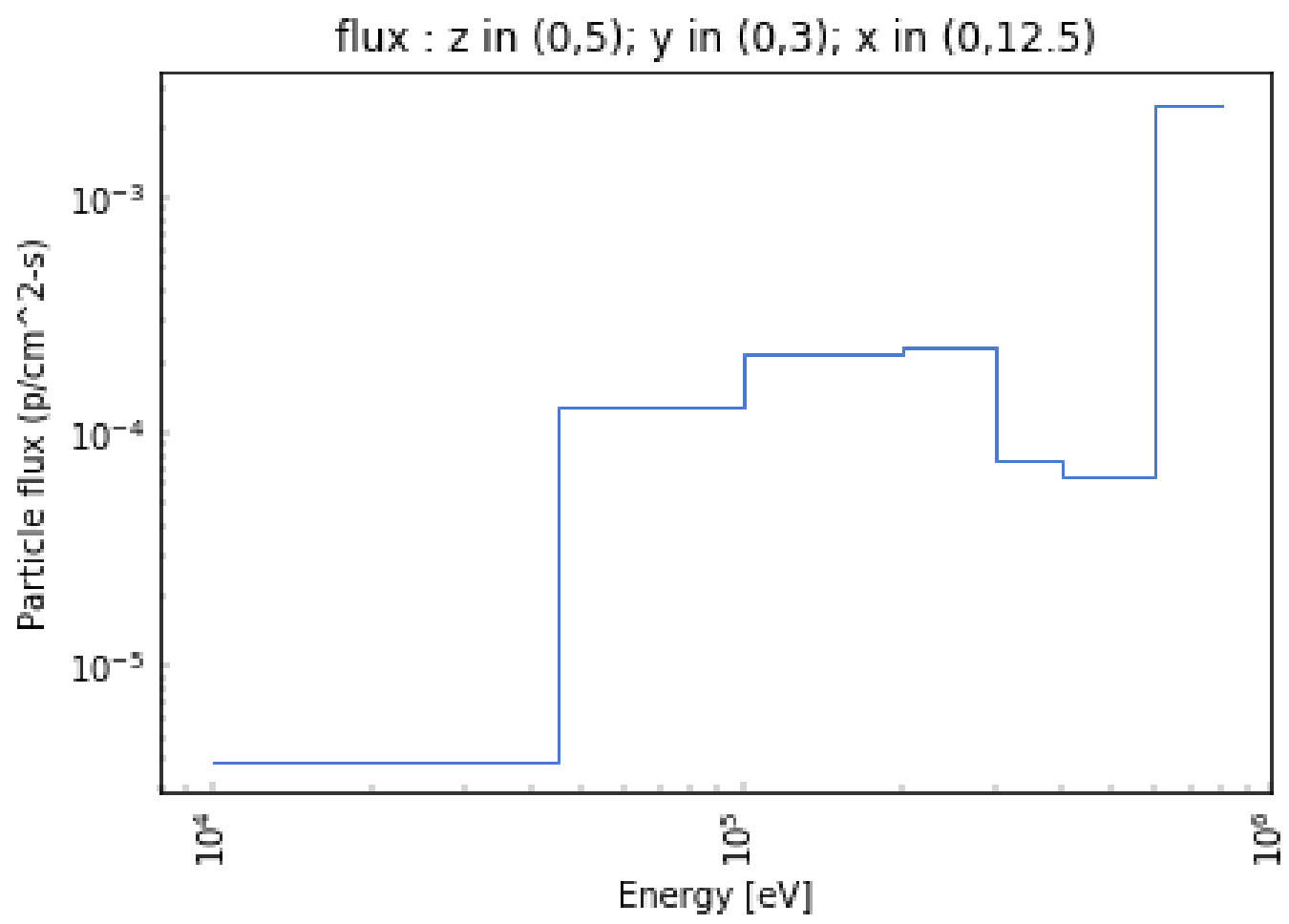

The next command accesses the flux at group index 3 (not $3 \mathrm{eV}$ !) at $z=0$. With the resulting $x-y$ data, the plotter renders a pseudocolor plot. Specifying a special value for norm renders the colors on a logarithmic scale.

from matplotlib.colors import LogNorm

plot (flux.xs(z=0., g=3), norm=LogNorm()); 


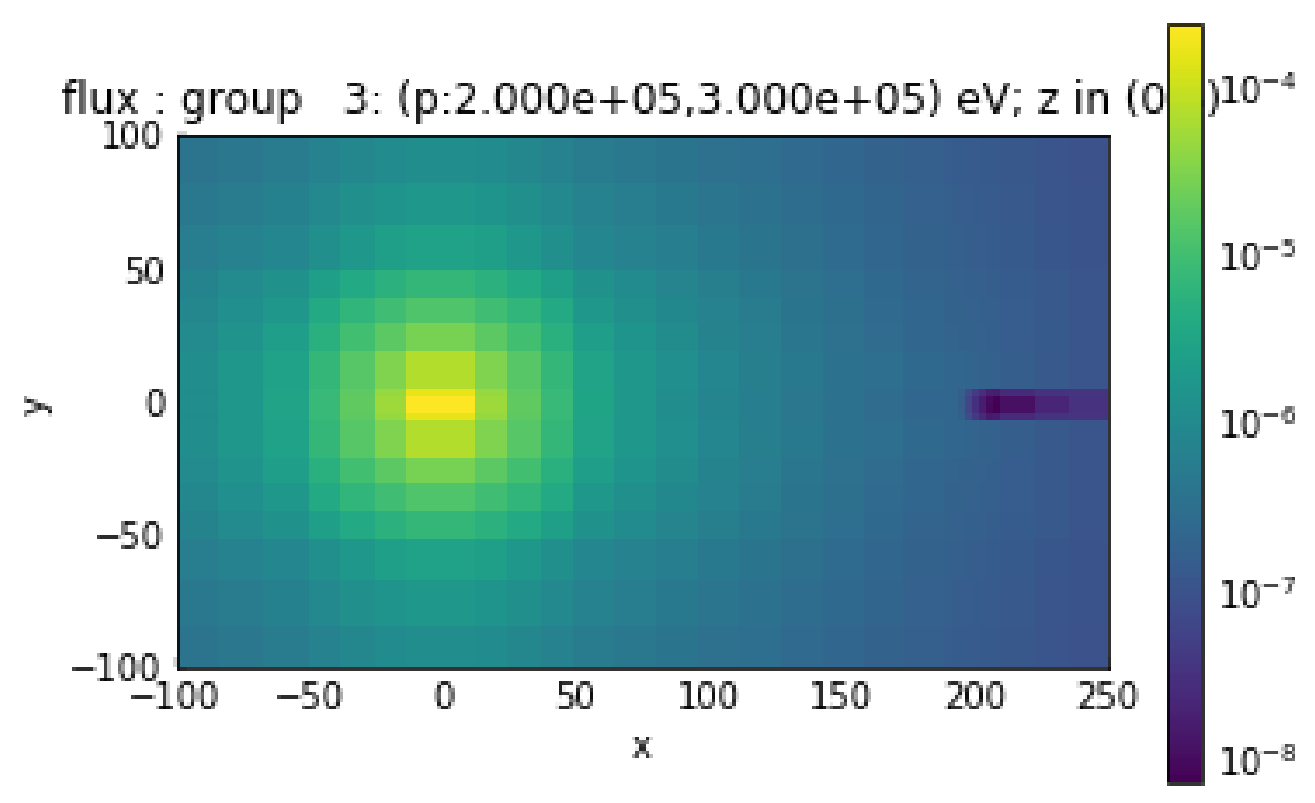

\section{Net current}

The current field is the "net current", the first moment of the angular flux:

$$
\vec{J}=\int_{4 \pi} \vec{\Omega} \psi(\vec{x}, \vec{\Omega}) \mathrm{d} \Omega
$$

It is stored as an energy, space, and direction-dependent field; the data attribute is the pointer to the HDF5 data.

The current describes the net flow rate of particles traveling through a point in space. The following command print the dimensionality of the current:

current $=\operatorname{dnv}[$ 'current']

print (", ".join(" \{\}$=\{\} "$. format $(d, s)$

for $(d, s)$ in zip(current.dims, current.shape)))

$\mathrm{g}=7, \mathrm{z}=24, \mathrm{y}=18, \mathrm{x}=31, \operatorname{dir}=3$

The following command takes a slice at the energy group for photons at $1 \mathrm{e} 5 \mathrm{eV}$, at the $\mathrm{XYZ}$ coordinates of $(7,3,5)$. The resulting vector is the current along the $(x, y, z)$ directions.

sliced = current. $x s\left(x=7.0, y=3.0, z=5.0, g=\left(p^{\prime}, 1 e 5\right)\right)$

print (sliced.mesh('dir'))

sliced

$\left[\begin{array}{lll}\prime x & ' y ' & \prime z\end{array}\right]$

Other slices are of course possible. This command plots the multigroup net current at a particular point:

plot (current. $x s\left(x=7.0, y=3.0, z=5.0, \operatorname{dir}={ }^{\prime} x^{\prime}\right)$, logy=True); 


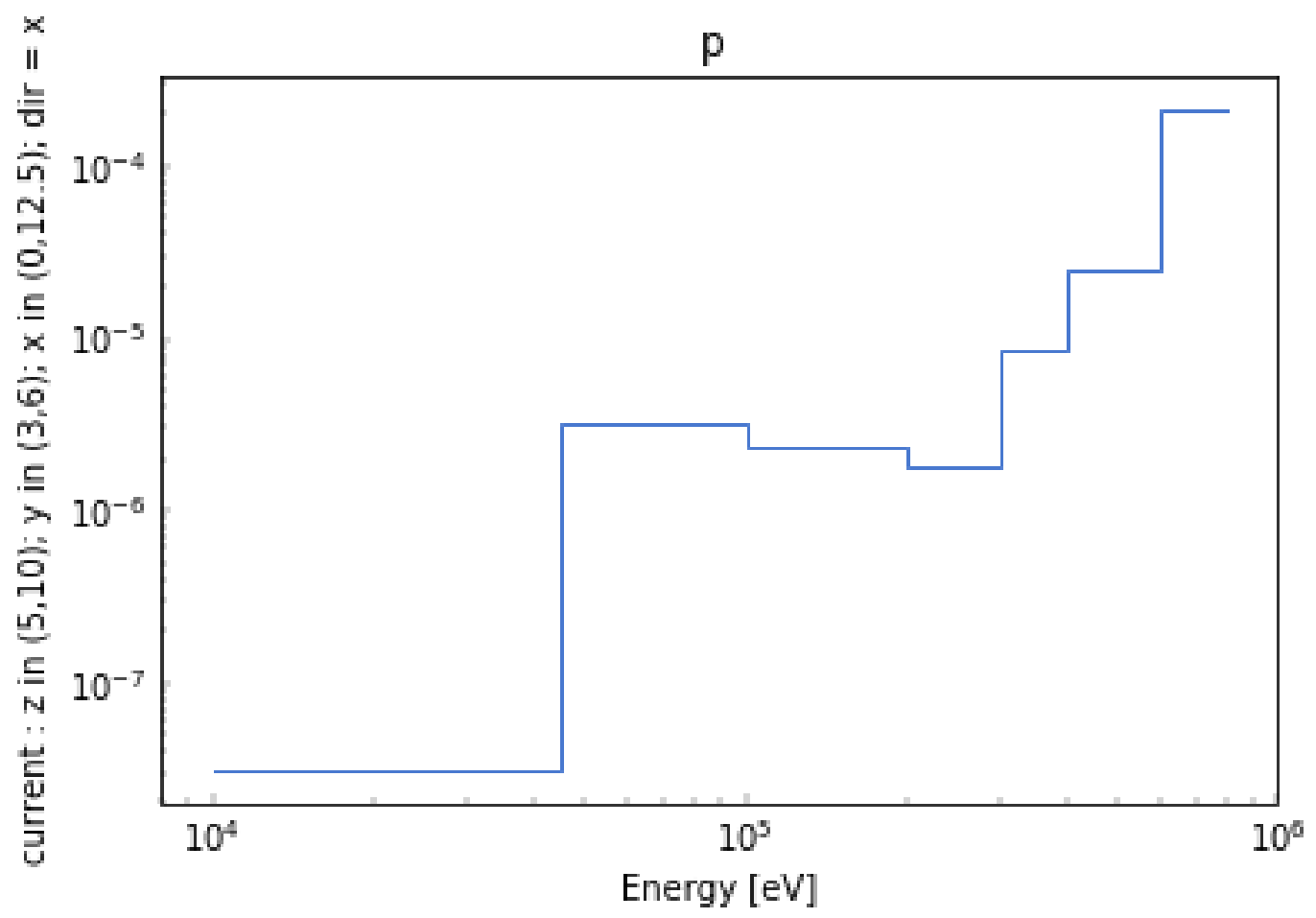

The full angle-dependent particle flux can be approximated by using the angular moments. This is the $P_{N}$ approximation.

$$
\psi(\vec{x}, \vec{\Omega}) \approx \frac{1}{4 \pi}(\phi(\vec{x})+3 \vec{\Omega} \cdot \vec{J}(\vec{x}))
$$

However, a much more accurate representation of the angular flux can be obtained directly from Denovo.

\section{Angular flux}

The full $S_{N}$ flux can be saved by setting angular_flux true inside the [DENOVO] [OUTPUT] block. This option requires careful consideration due to the disk space requirements: for an $S_{16}$ quadrature set, it will be 288 times as large as the scalar flux.

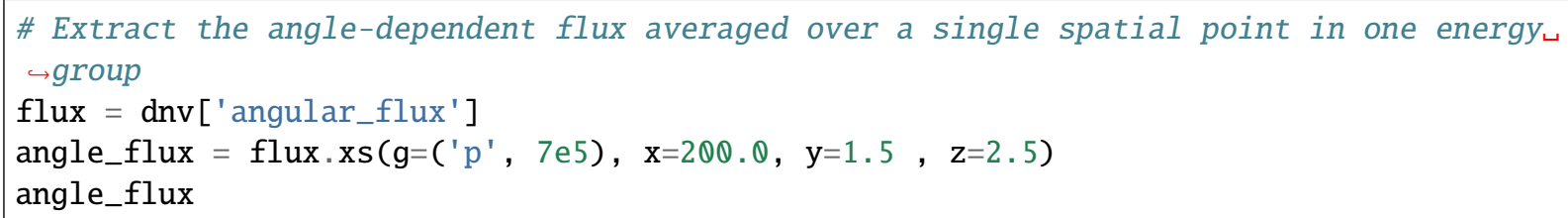

These tools can perform a wide range of functions. For example, numpy logical expressions can determine all the angle indices in the $+z$ direction, and matplotlib can then create a scatter plot of the angluar fluxes projected into the $x y$ plane. 

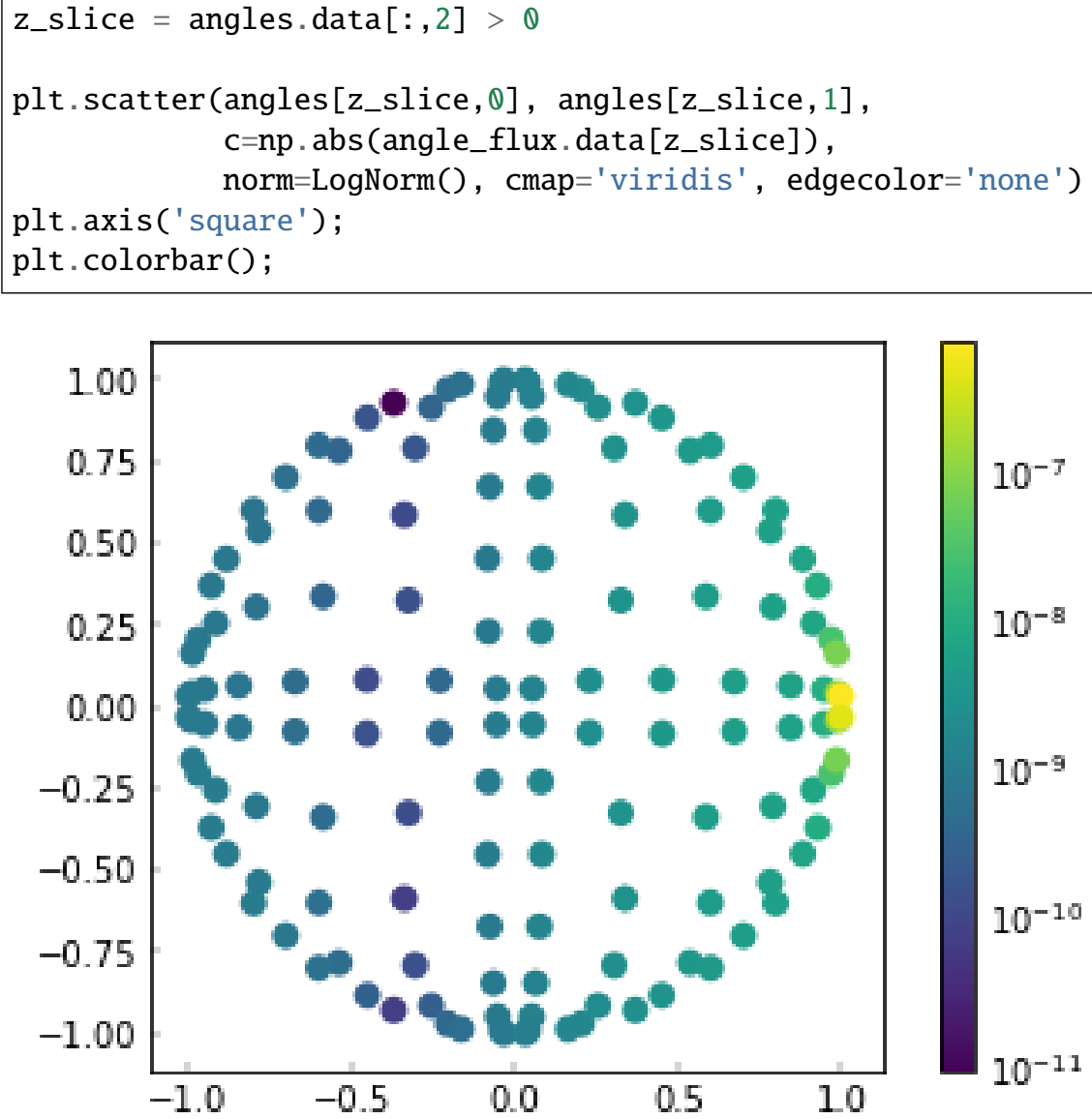

\section{A.2.6 EXNIHILO MESH INPUT DEFINITION}

These small problems are used in the Denovo unit tests. The output files should be saved to Geometria/ mesh/rect/test/data.

\# Set up plotting

\%matplotlib inline

from exnihilotools.matplotlib import screen_style

screen_style()

\section{A.2.6.1 Problem A}

This is a $3 \mathrm{D}$ problem with a volume source.

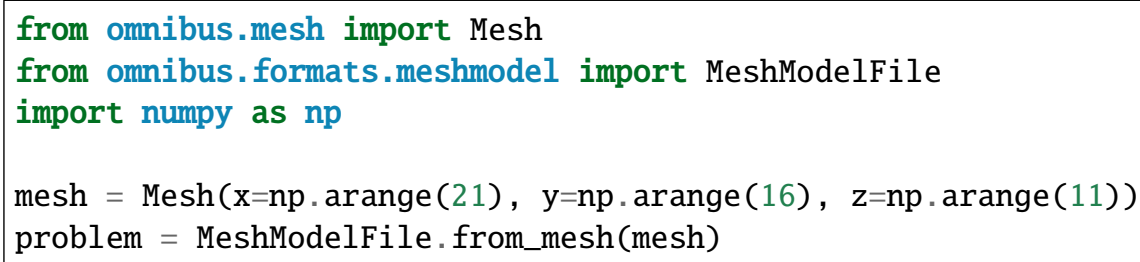

If the mesh axes were irregular, then a snippet of code like the following could be used to 'slice' the matids more easily. In order to select a view of the data to assign matids, we have to use standard python slice objects or integers. 


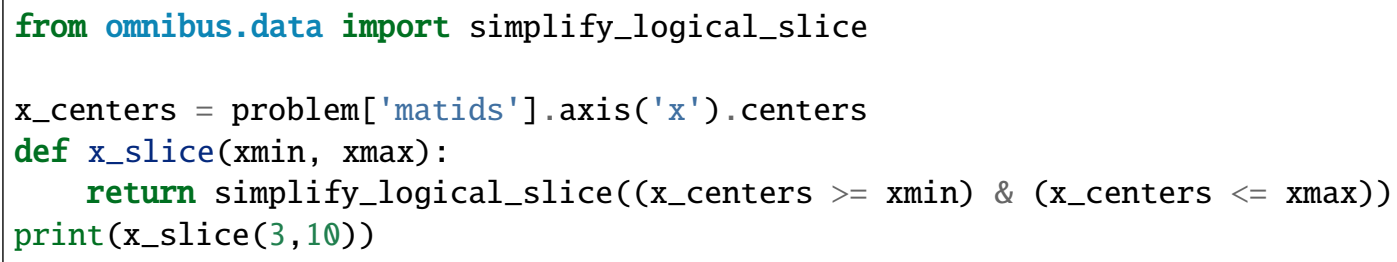

The xs_by_index method selects subsets of the mesh, either multiple elements or a single element, along multiple axes. The following chunks of code are used to assign materials and a source definition.

from omnibus.data import plot

matids $=$ problem ['matids']

zmats $=$ matids.xs_by_index $(z=\operatorname{slice}(1)$,

zmats.xs_by_index $(y=\operatorname{slice}(5,15)) \cdot \operatorname{data}[:]=1$

zmats.xs_by_index $(x=\operatorname{slice}(\theta, 15), y=\operatorname{slice}(10,15)) \cdot \operatorname{data}[:]=2$

zmats.xs_by_index $(y=\operatorname{slice}(3,5)) \cdot \operatorname{data}[:]=2$

plot (matids. $x s(x=0.5)$, edgecolor='gray', linewidth=0.25);
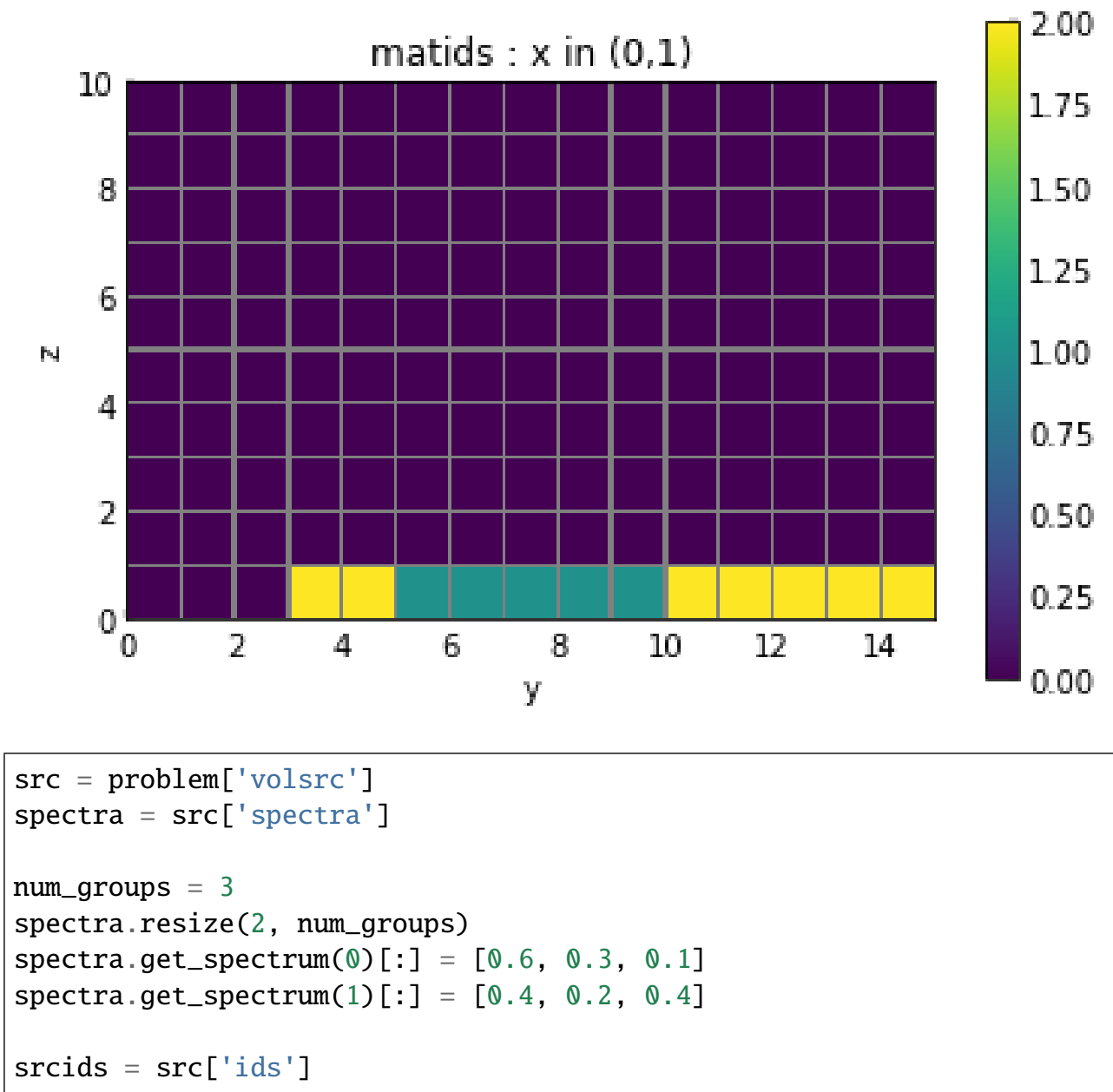
srcids.xs_by_index $(y=\operatorname{slice}(3,5), \mathrm{z}=\operatorname{slice}(1,9)) \cdot \operatorname{data}[:]=1$

plot (srcids.xs $(z=1.0)$ );

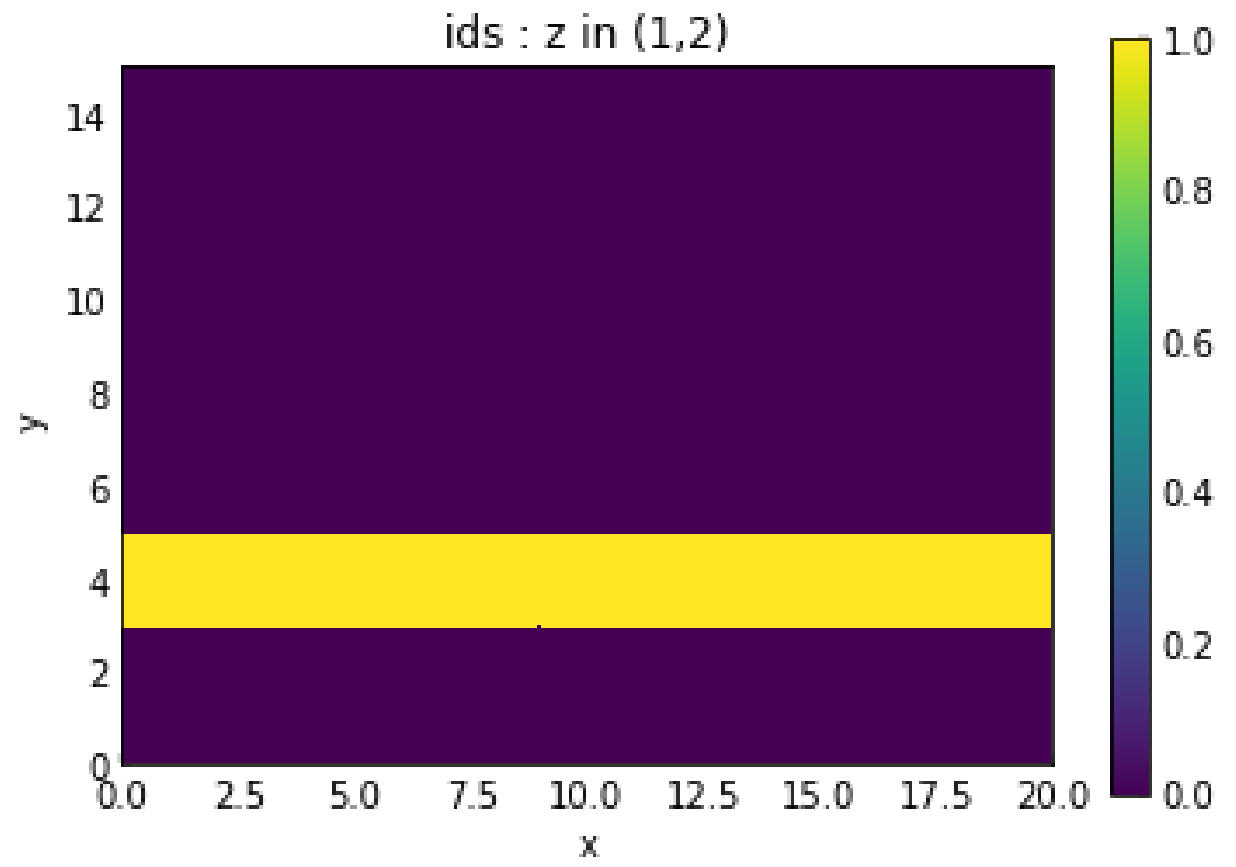

strength $=\operatorname{src}[$ 'strength']

$\mathrm{q} \theta=\mathrm{np} . \operatorname{linspace}(0.5,1.5,15)$

$\mathrm{q} 1=\mathrm{np} . \operatorname{linspace}(0.5,1.5,20)$

strength.xs_by_index $(x=\operatorname{slice}(\theta, 15), y=\operatorname{slice}(10,15), z=\operatorname{slice}(1,10)) \cdot \operatorname{data}[:]=q \theta$

strength.xs_by_index $(y=\operatorname{slice}(3,5), z=\operatorname{slice}(1,10)) \cdot \operatorname{data}[:]=\mathrm{q} 1$

plot (strength.xs $(z=1.0))$; 


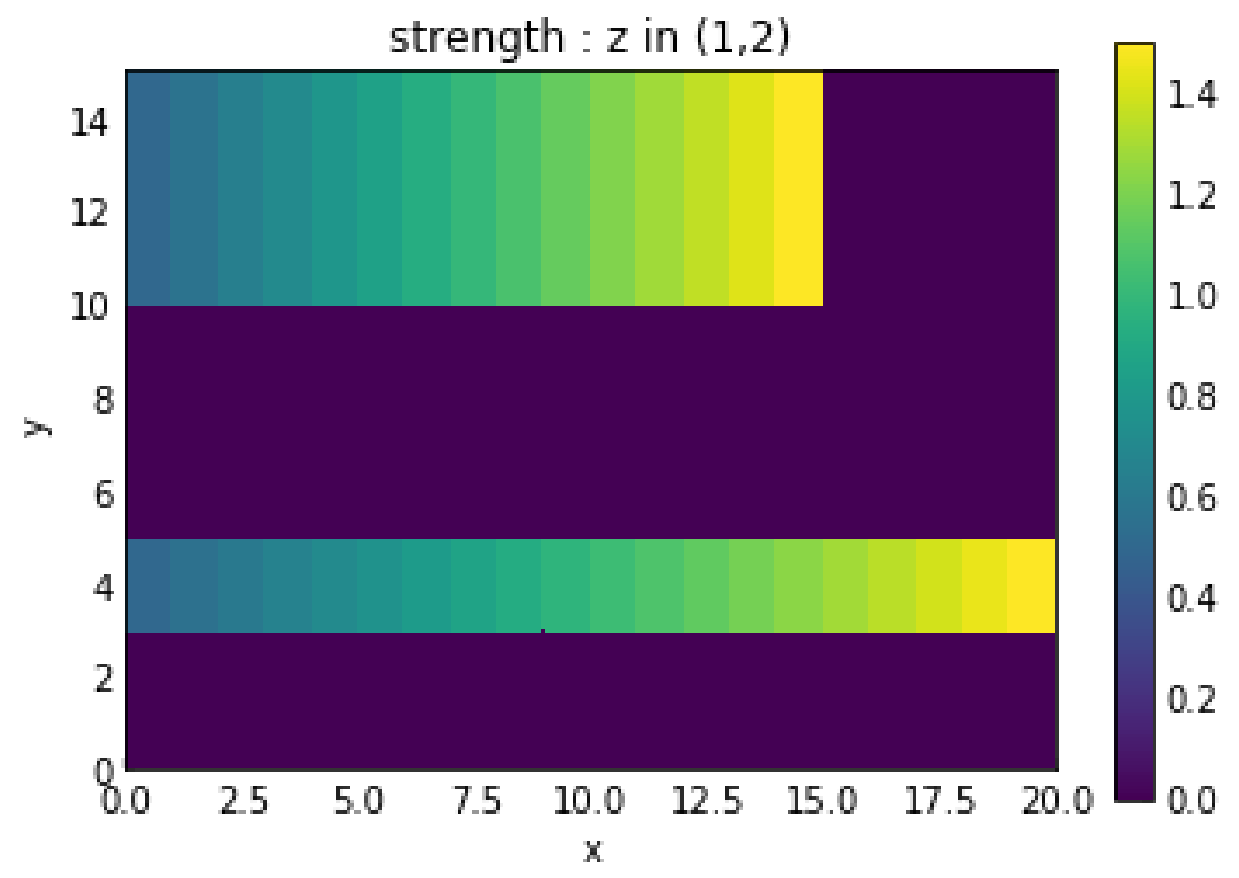

Now that the problem is constructed, it can be written to disk for later use in Omnibus:

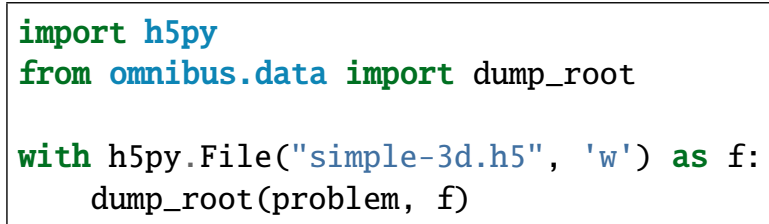

INFO: Omitting empty point source

\section{A.2.6.2 Problem B}

This 2D problem is constructed similarly to the previous one.

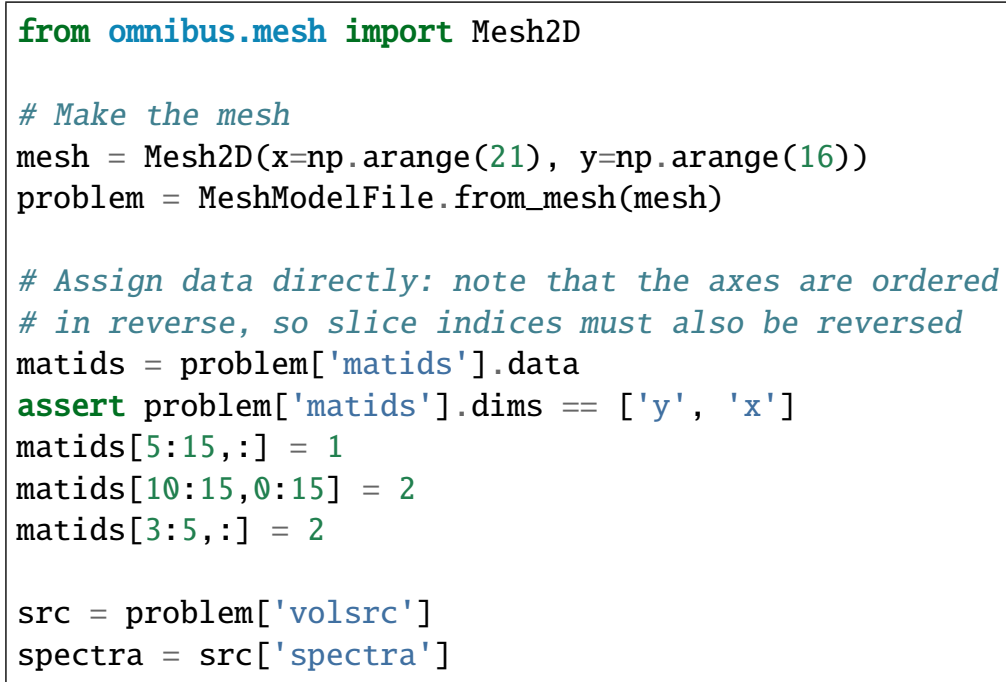




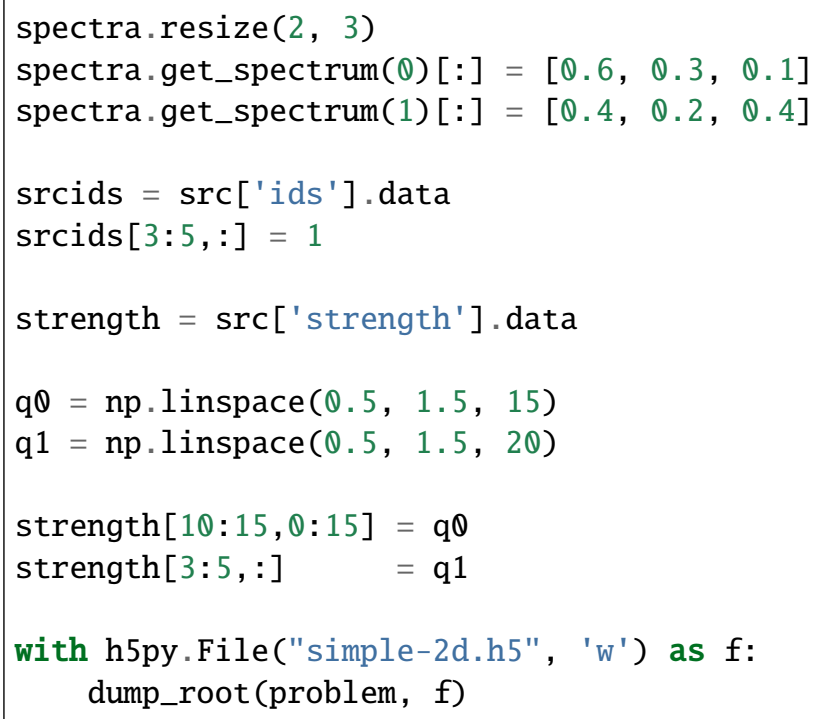

INFO: Omitting empty point source

\section{A.2.6.3 Problem C}

This 3D problem includes mix tables and does not specify a source definition.

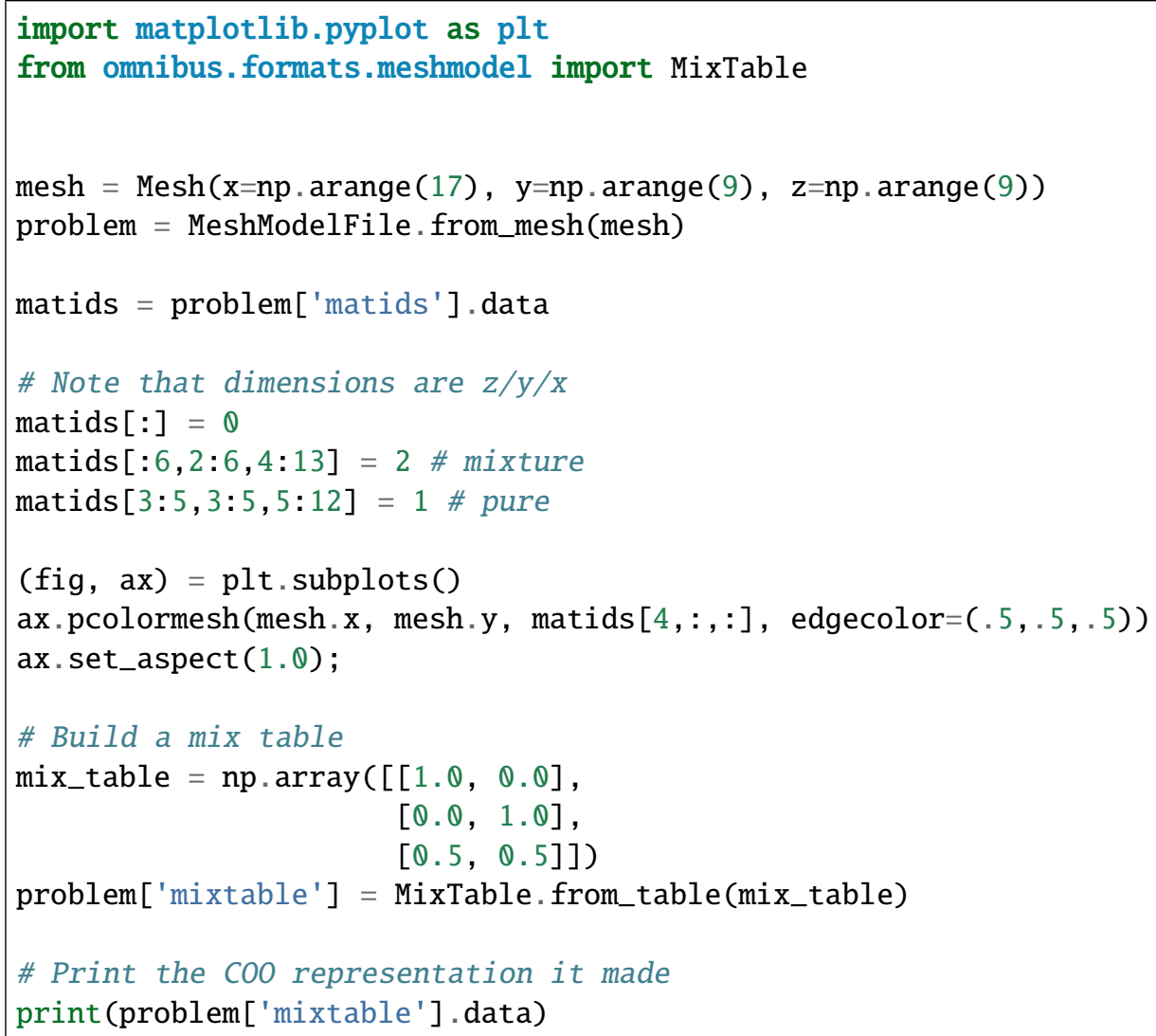


\# Save to disk

with h5py.File("mixed-3d.h5", 'w') as $f$ :

dump_root (problem, f)

INFO: Omitting empty volume source

INFO: Omitting empty point source

$[(\theta, 0,1).(1,1,1).(2,0,0.5)(2,1,0.5)]$

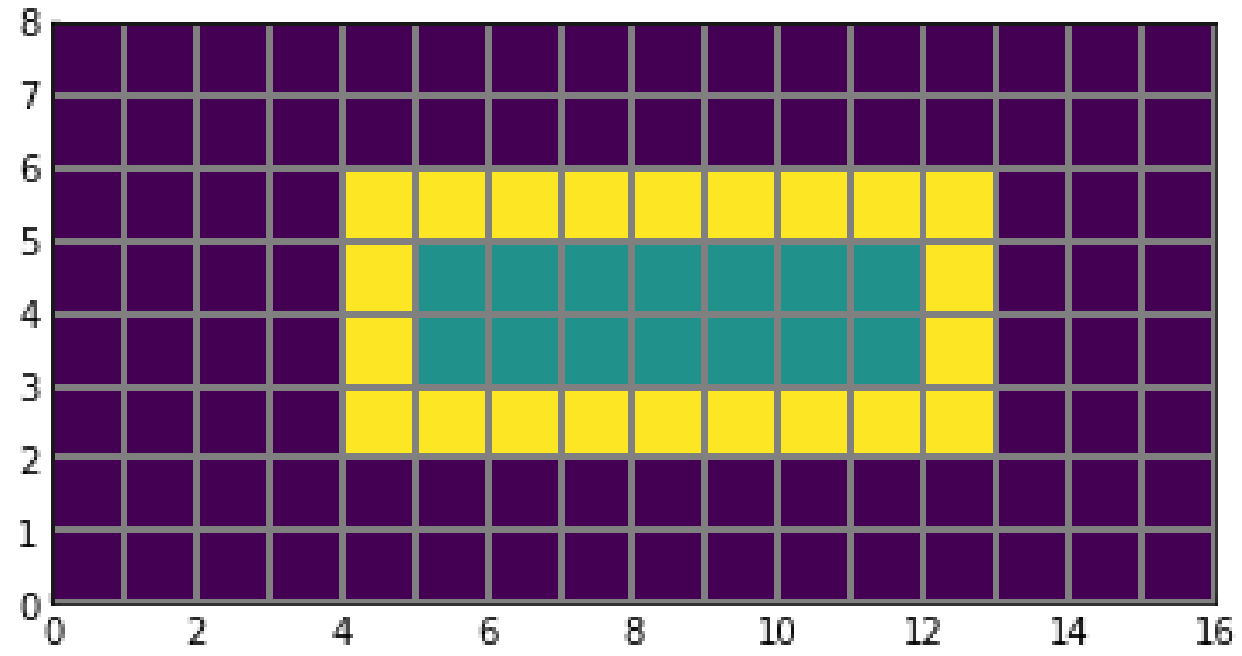

\section{A.2.6.4 Uniform source and material}

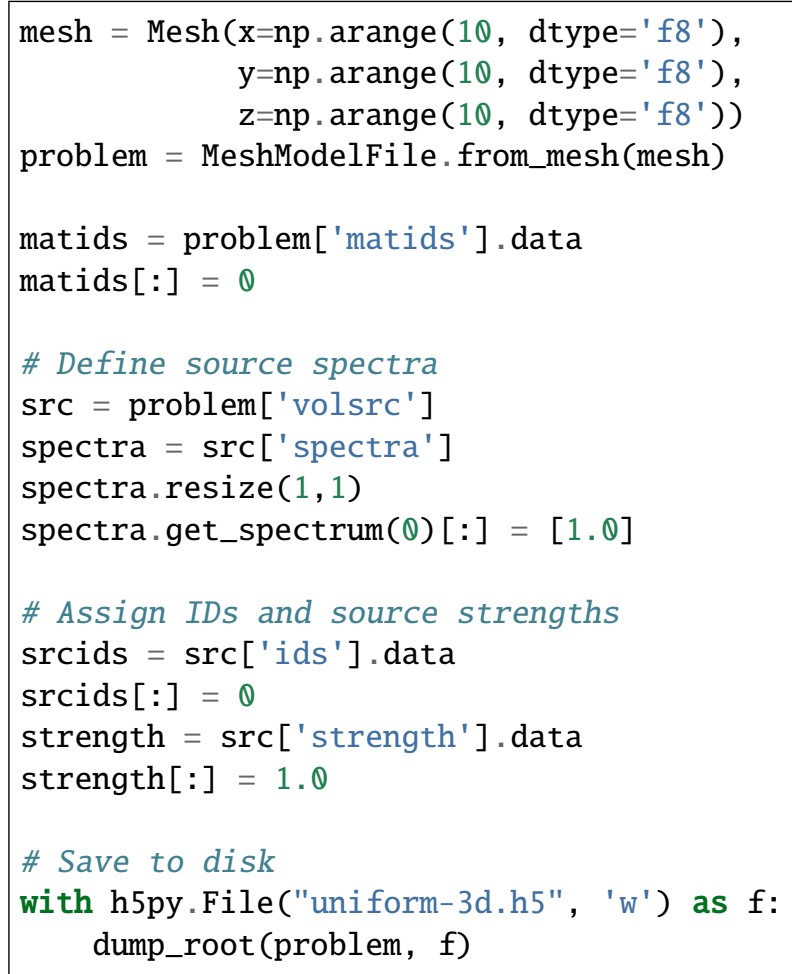


INFO: Omitting empty point source

\section{A.2.6.5 Both point and volume sources}

The input file constructed here will contain both volume and point sources.
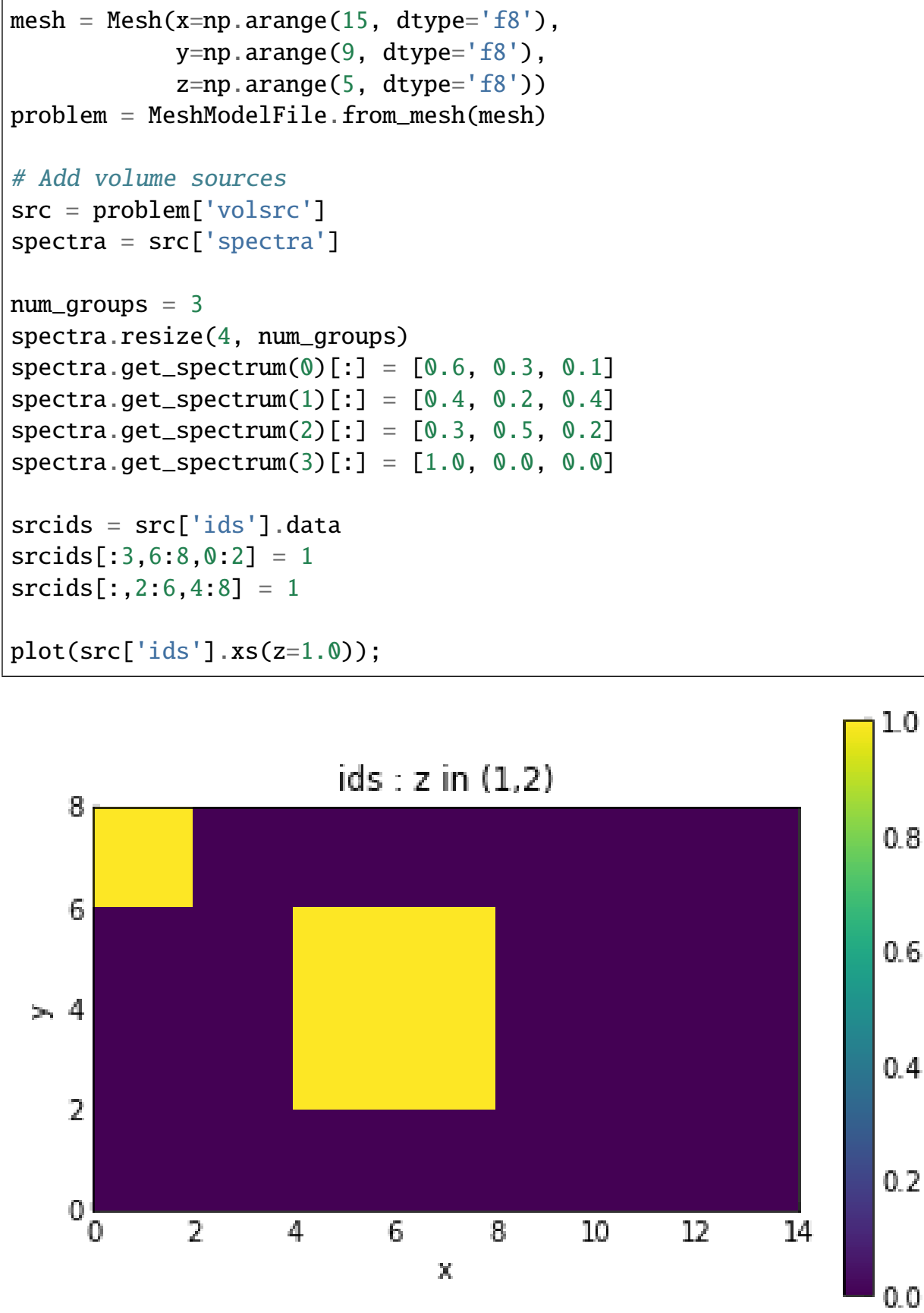

Next, set varying strengths in the different cells.

strength $=\operatorname{src}[$ 'strength']. data

strength $[: 3,6: 8,0: 2]$. flat $=n p . \operatorname{linspace}(0.1,1.1,3 * 2 * 2)$ 
strength $[:, 2: 6,4: 8]$. flat $=$ np.linspace $(0.5,2.5,4 * 4 * 4)$

$\operatorname{plot}(\operatorname{src}[$ 'strength'] $\mathrm{xs}(\mathrm{z}=1.0))$;

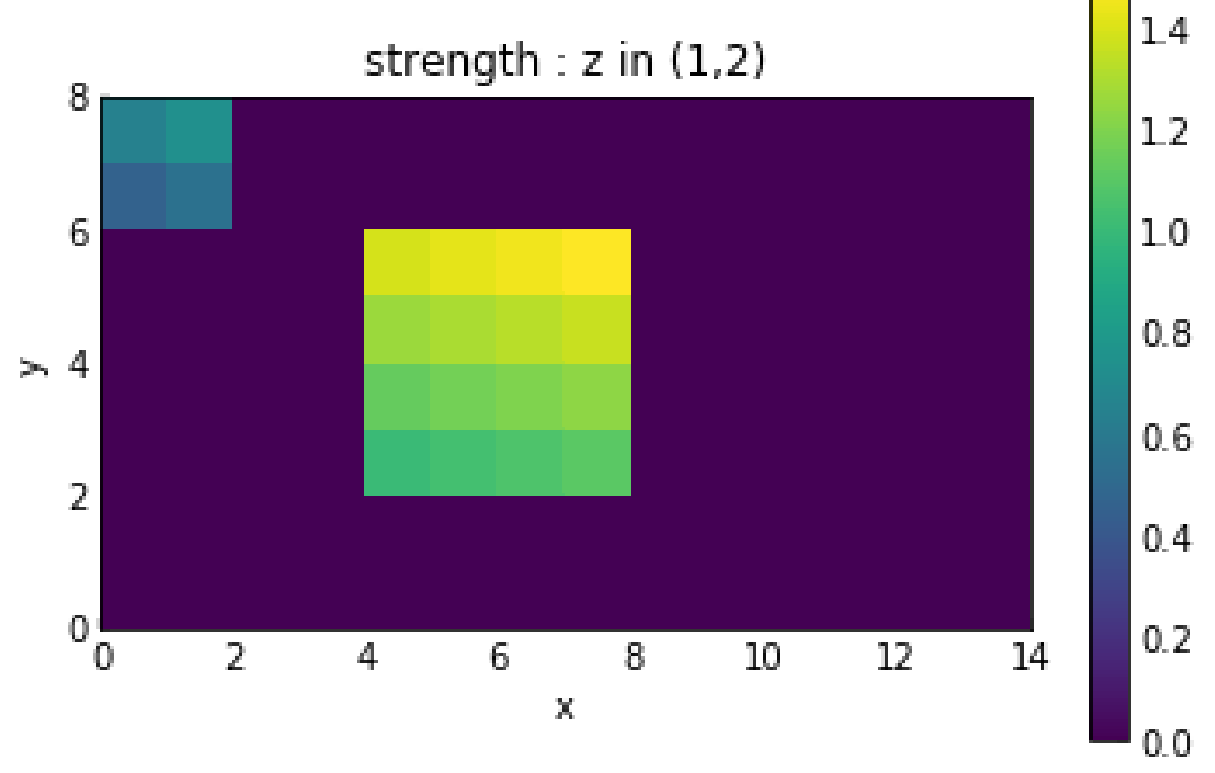

Finally, add the point sources and write the problem to disk.

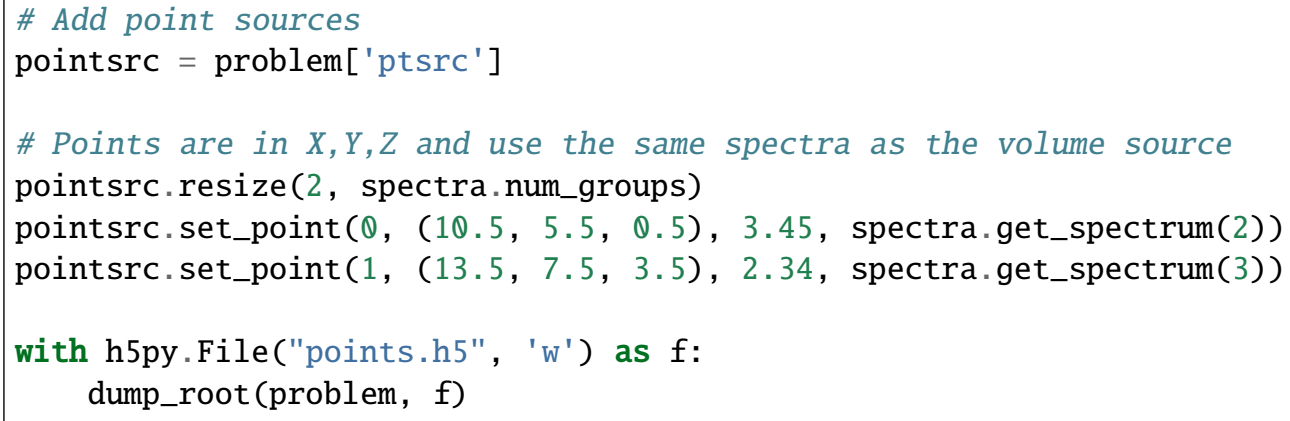

\section{A.3 MULTIGROUP DATA EXPLORATION}

These examples show how to use Exnihilo's Python bindings (page 2) to extract and visualize multigroup and other Denovo-related data.

\section{A.3.1 CROSS SECTION GENERATION}

This example demonstrates generating and visualizing multigroup cross sections using the Exnihilo python interface.

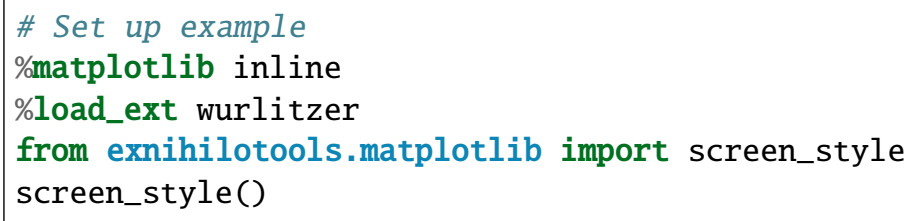


sigma_s = np.asarray (water_xs.sigma_x(SCATTERING))

\#sigma_f $=$ np.asarray (water_xs.sigma_x(FISSION))
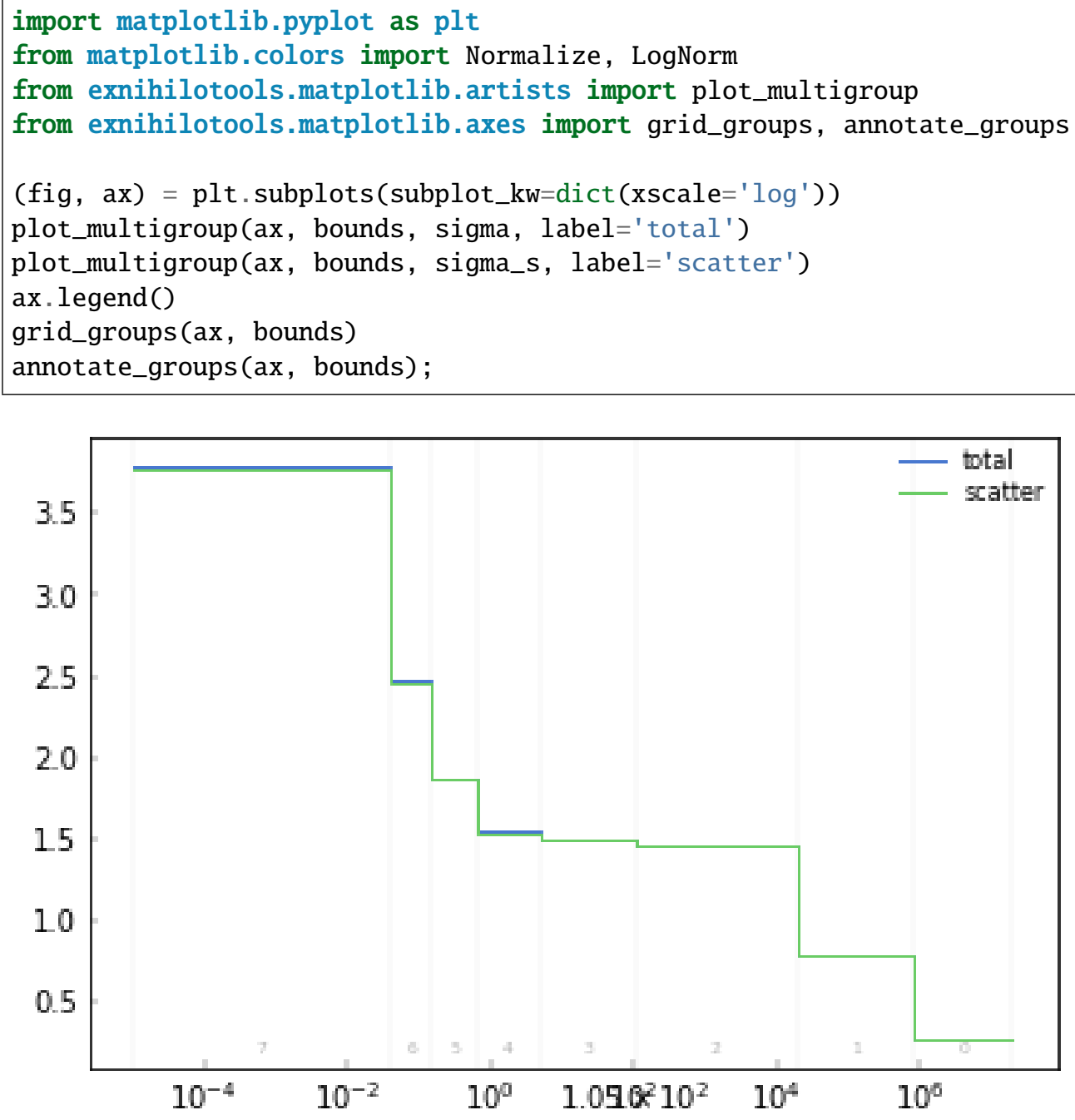

\section{A.3.1.3 Scattering matrix visualization}

The scattering matrix with all the requested angular moments $\left(g, g^{\prime}, l\right)$ can be pulled directly from the cross section container. In this example, there are 4 moments since we requested $P_{3}$ scattering.

scat $=$ water_xs.scatter_matrix

scat. shape

$(8,8,4)$

plt. matshow(scat $[:,:, 0]$, cmap='viridis', norm=LogNorm())

plt.title("\$P_O\$ scattering")

plt. colorbar (); 


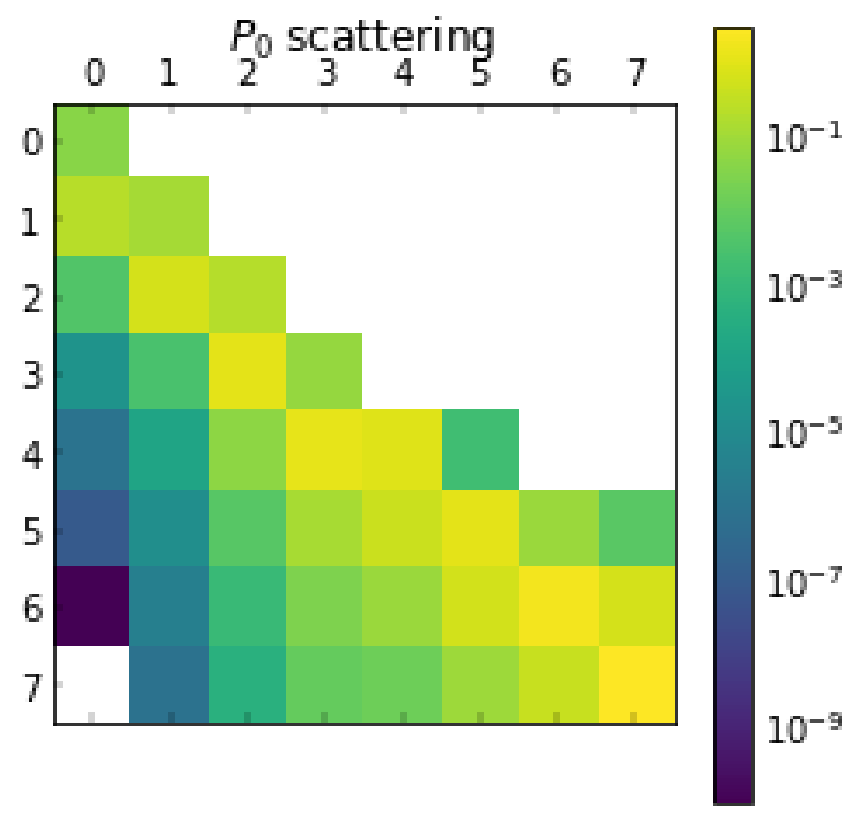

scat $[3,3,:]$

$\operatorname{array}([0.06847651,0.03386217,0.01825174,0.00616768])$

A scattering cross section that's perfectly forward-peaked

$$
\sigma_{s}(\mu)=\delta(\mu-1)
$$

will have $\sigma_{0}=\sigma_{1}=\sigma_{2}=\ldots$.

plt.matshow(scat $[:,:, 1] /$ np.ma.masked_equal (scat $[:,:, 0], 0.0)$, cmap='rb_linear', $\hookrightarrow$ norm=Normalize $(-1,1)$ )

plt.title(r"Forward peakness (\$\sigma_1 / \sigma_O\$)")

plt.colorbar(); 


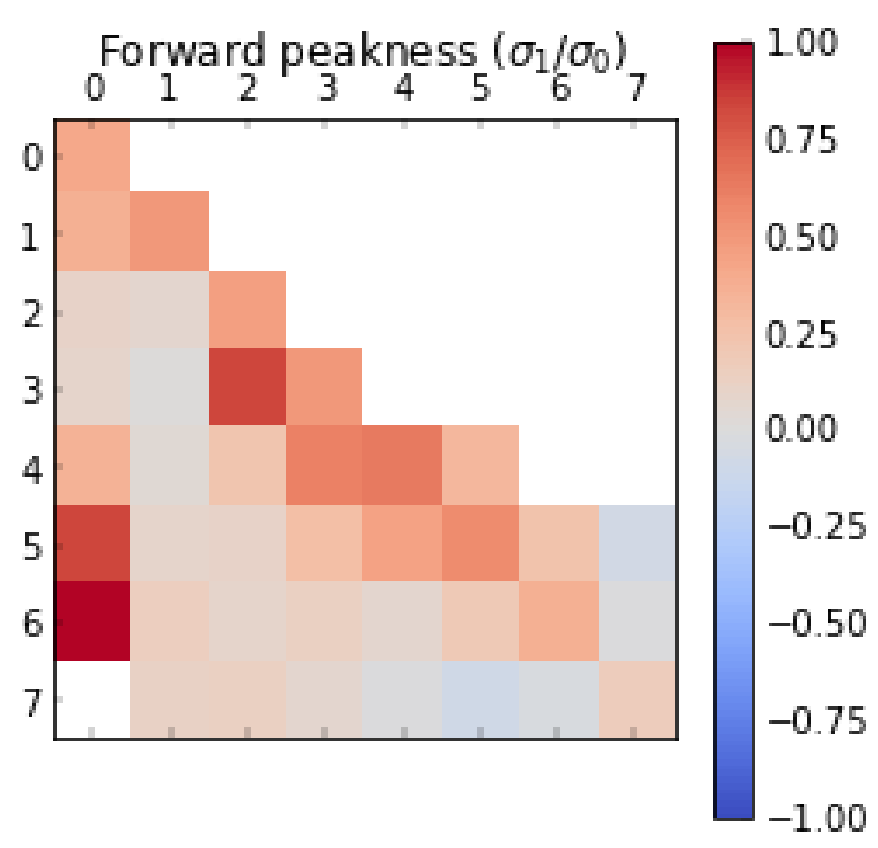

\section{Legendre coefficents}

The numpy package has a function for evaluating a Legendre series at a series of points. This allows us to easily visualize the reconstructed scattering as a function of angle.

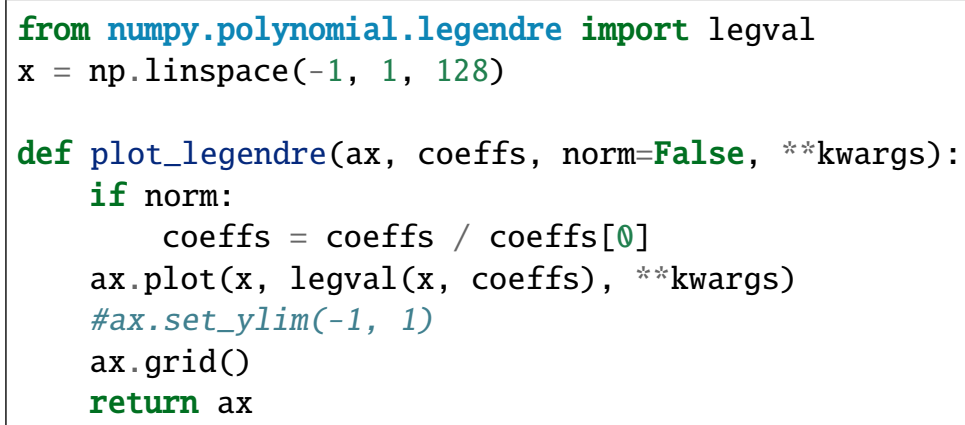

(fig, ax $)=$ plt. $\operatorname{subplots}()$

plot_legendre (ax, $[0,0,0,1])$;

ax.set_title("Legendre function \$P_3\$"); 


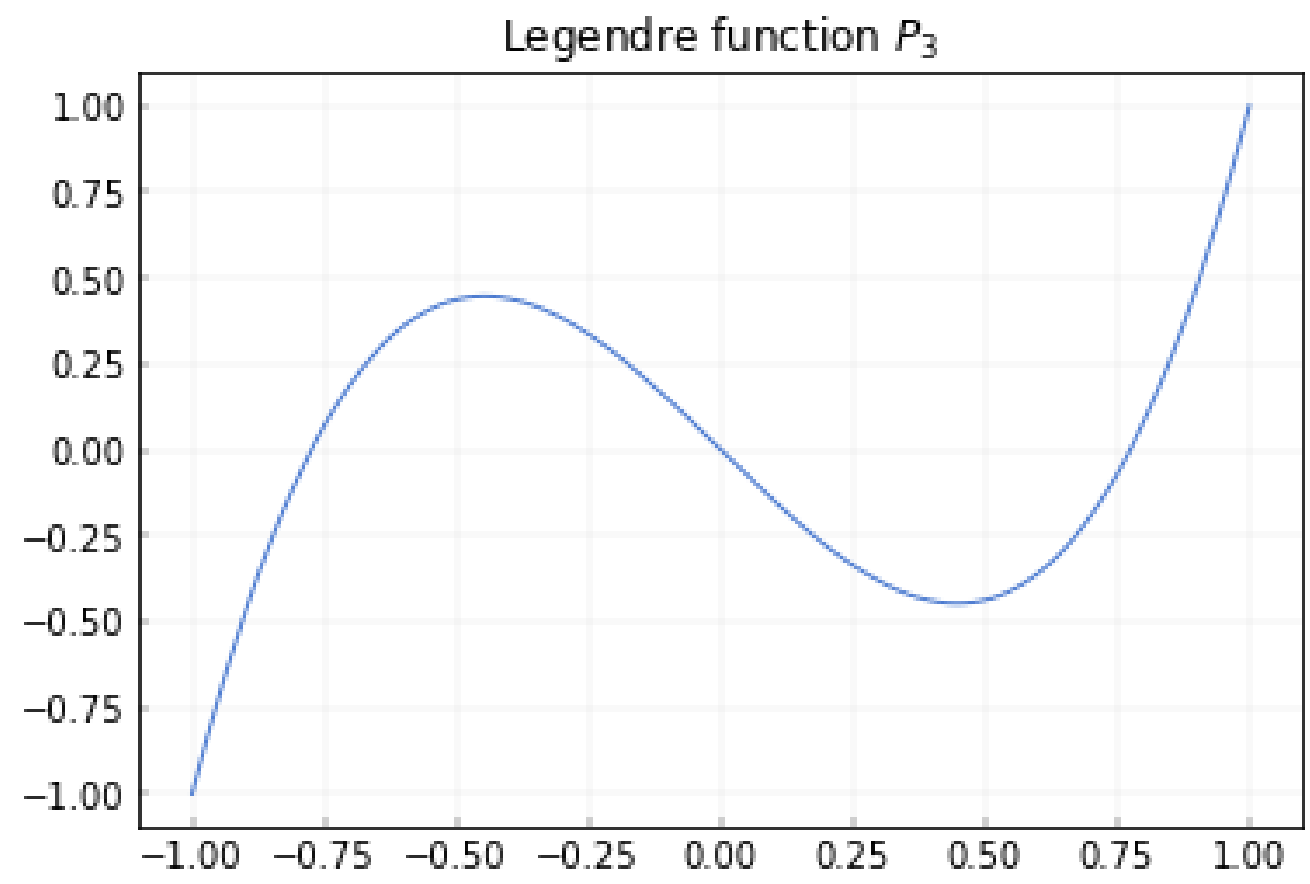

$g=0$

$(\mathrm{fig}, \mathrm{ax})=$ plt. $\operatorname{subplots}()$ \# subplot_kw=dict $\left.\left(\mathrm{yscale=}{ }^{\prime} \log ^{\prime}\right)\right)$

\# Loop through all the outscatter groups for the highest-energy xs

for gp in range(8):

plot_legendre(ax, scat[gp, g, :], label=r'\$\{:d\} \to $\{: d\} \$$ '. format (g,gp)) ax.legend ();

ax.set_title(r'\$\sigma_s( \mu)\$ for the highest-energy group');

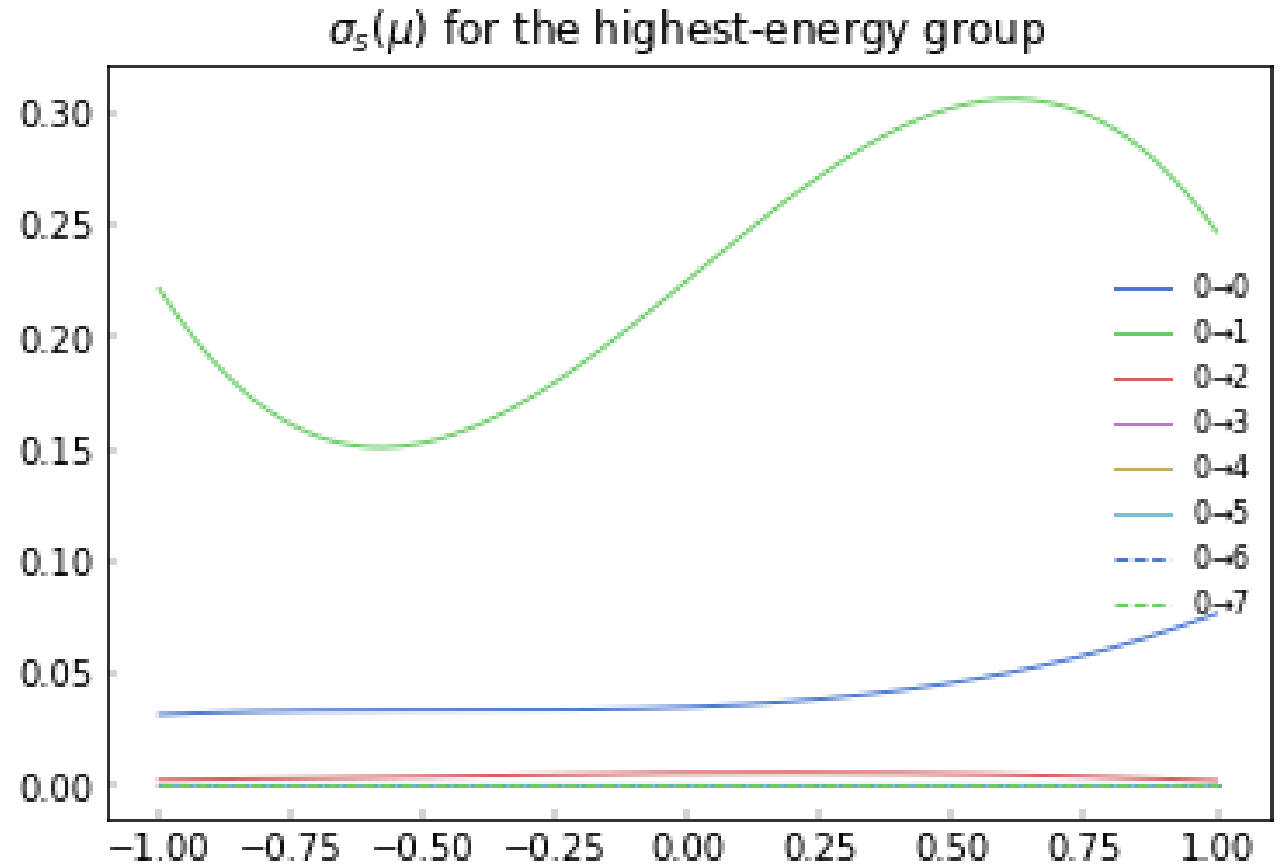




\section{A.3.2 INFINITE-MEDIUM CRITICALITY SEARCH}

Simple linear algebra can solve for the $k_{\text {eff }}$ of an infinite homogeneous medium given multigroup cross sections. This advanced example ties together some low-level Exnihilo utilities (in Robus and Transcore) with python packages numpy and scipy.

This example's objective is to determine a critical homogeneous mixture of $5 \%$-enriched $\mathrm{UO}_{2}$ and water. The final result will be a composition that can then be used for unit testing.

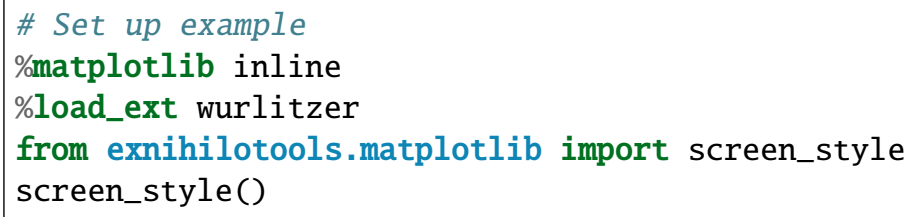

\section{A.3.2.1 Construct compositions}

There are some utility functions in Exnihilo that build $\mathrm{UO}_{2}$ compositions and can calculate mixtures.

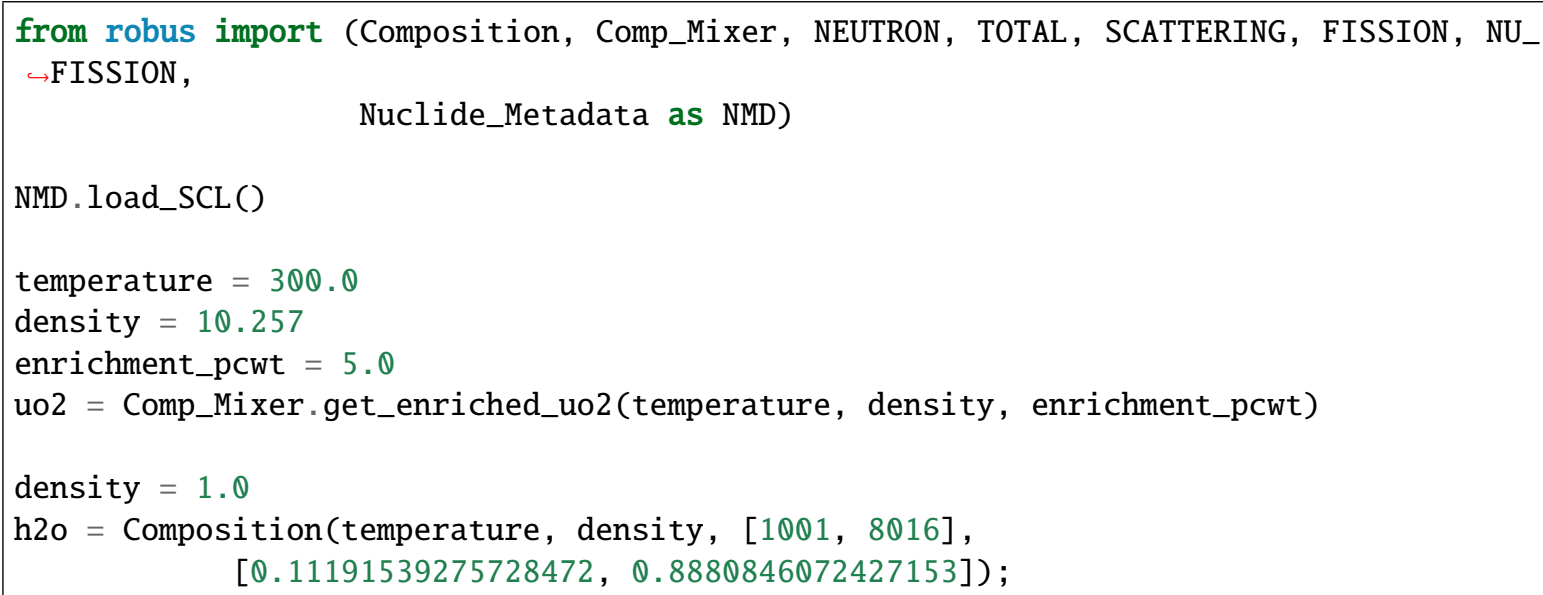




\section{A.3.2.2 Calculating $k$ of a composition}

The multigroup approximation allows k-effective of a homogeneous composition to be calculated straightforwardly by explicitly constructing a matrix whose largest eigenvalue is $k$. The first step of this process is to use SCALE's XSProc code via the XS_Builder_Fulcrum class to collapse an eight-group test library into a homogeneous set of cross sections, which are then converted into native numpy arrays.

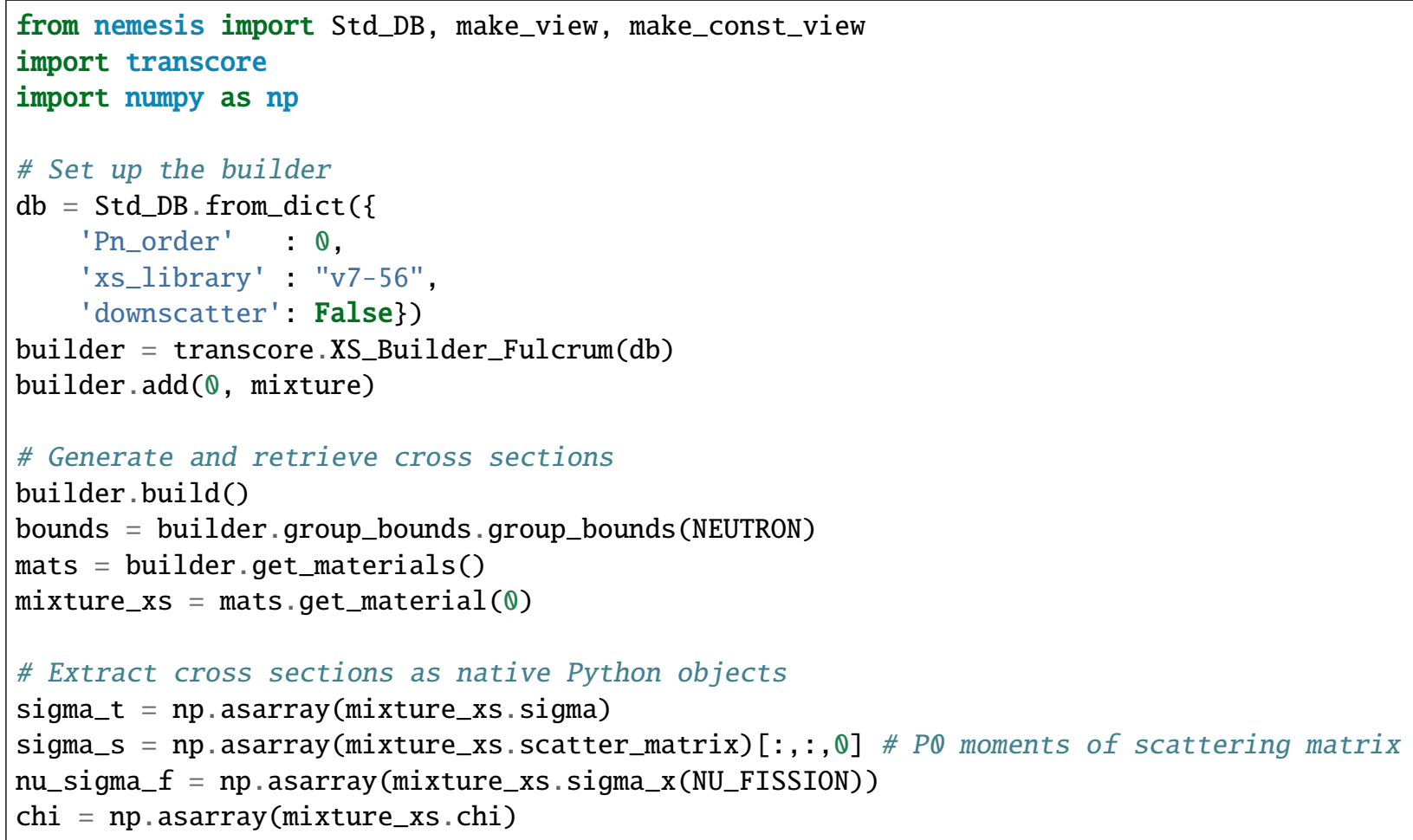

Here is the scattering matrix:

import matplotlib.pyplot as plt

from matplotlib.colors import LogNorm

plt . matshow(sigma_s, norm=LogNorm()); 


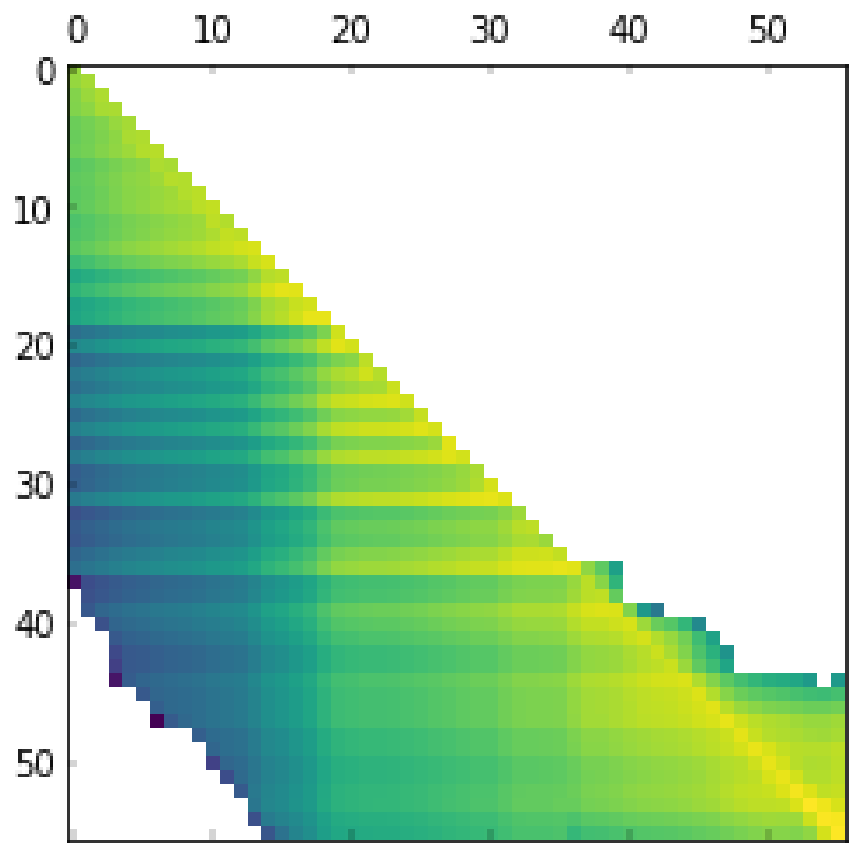

The multigroup neutron balance equation (with fission and no extraneous source) is:

$$
\Sigma_{t, g} \phi_{g}=\sum_{g^{\prime}=0}^{G-1} \Sigma_{s, g^{\prime} \rightarrow g} \phi_{g^{\prime}}+\frac{1}{k} \chi_{g} \sum_{g^{\prime}=0}^{G-1} v \Sigma_{f, g^{\prime}} \phi_{g^{\prime}},
$$

which can be written as the linear algebra equation

$$
T \Phi=S \Phi+\frac{1}{k} \chi f^{T} \Phi
$$

where $f^{T} \Phi$ is the fission source strength density (a scalar), $S$ is the scattering matrix, and $T$ is $\operatorname{diag}\left(\Sigma_{t}\right)$.

Simple manipulations yield an explicit eigenvalue equation

$$
k \Phi=(T-S)^{-1} \chi f^{T} \Phi .
$$

The following code constructs the right-hand-side matrix and solves for its eigenvalues:

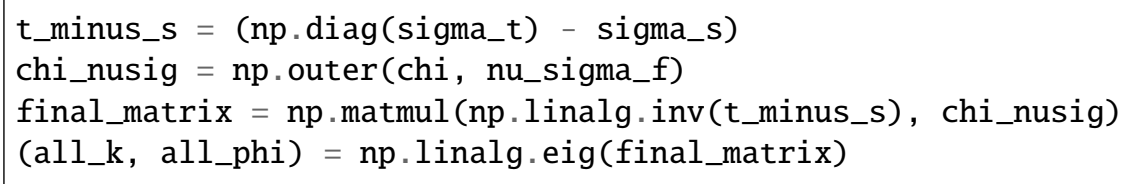


return $(\mathrm{k}, \mathrm{phi})$

$(\mathrm{k}, \mathrm{phi})=$ max_eigenvalue $\left(\mathrm{all} \_\mathrm{k}, \mathrm{all} \_\mathrm{phi}\right)$

print ("K-eff is", k)

K-eff is 1.3696586943136313

delta_lethargy $=-n p \cdot \operatorname{diff}(\mathrm{np} \cdot \log ($ bounds $))$

(fig, ax) = plt. subplots (subplot_kw=dict $\left(\right.$ xscale $\left.={ }^{\prime} \log { }^{\prime}, \quad y s c a l e={ }^{\prime} \log { }^{\prime}\right)$ )

plot_multigroup(ax, bounds, phi / delta_lethargy)

ax.set_xlabel ('Energy (eV)')

ax.set_ylabel ('Flux per unit lethargy')

ax.set_yticks([])

ax.set_yticklabels([]);

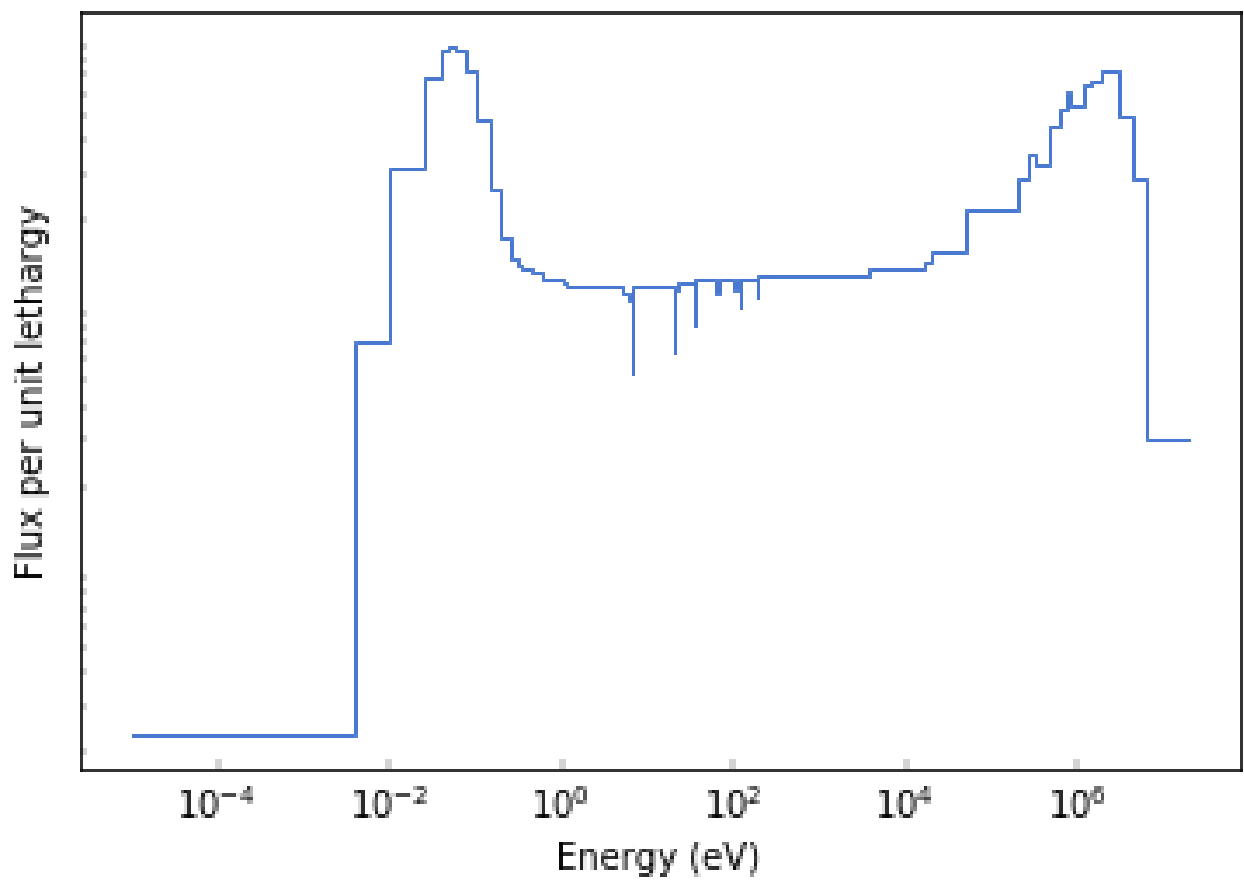

\section{A.3.2.3 Finding criticality}

The above process successfully calculates the eigenvalue $\left(k_{\text {eff }}\right)$ and eigenvector (flux spectrum) given a single scalar, the fuel fraction. It is condensed below into a single function calc_k, which is the workhorse of a function calc_reactivity that gives the reactivity,

$$
\rho=\frac{k-1}{k}
$$

as a function of the fuel fraction. Solving for the single root $\rho=0$ is therefore solving for the critical fuel fraction.

Using an 8-group test library instead of the 56-group library above substantially reduces the calculation time. 


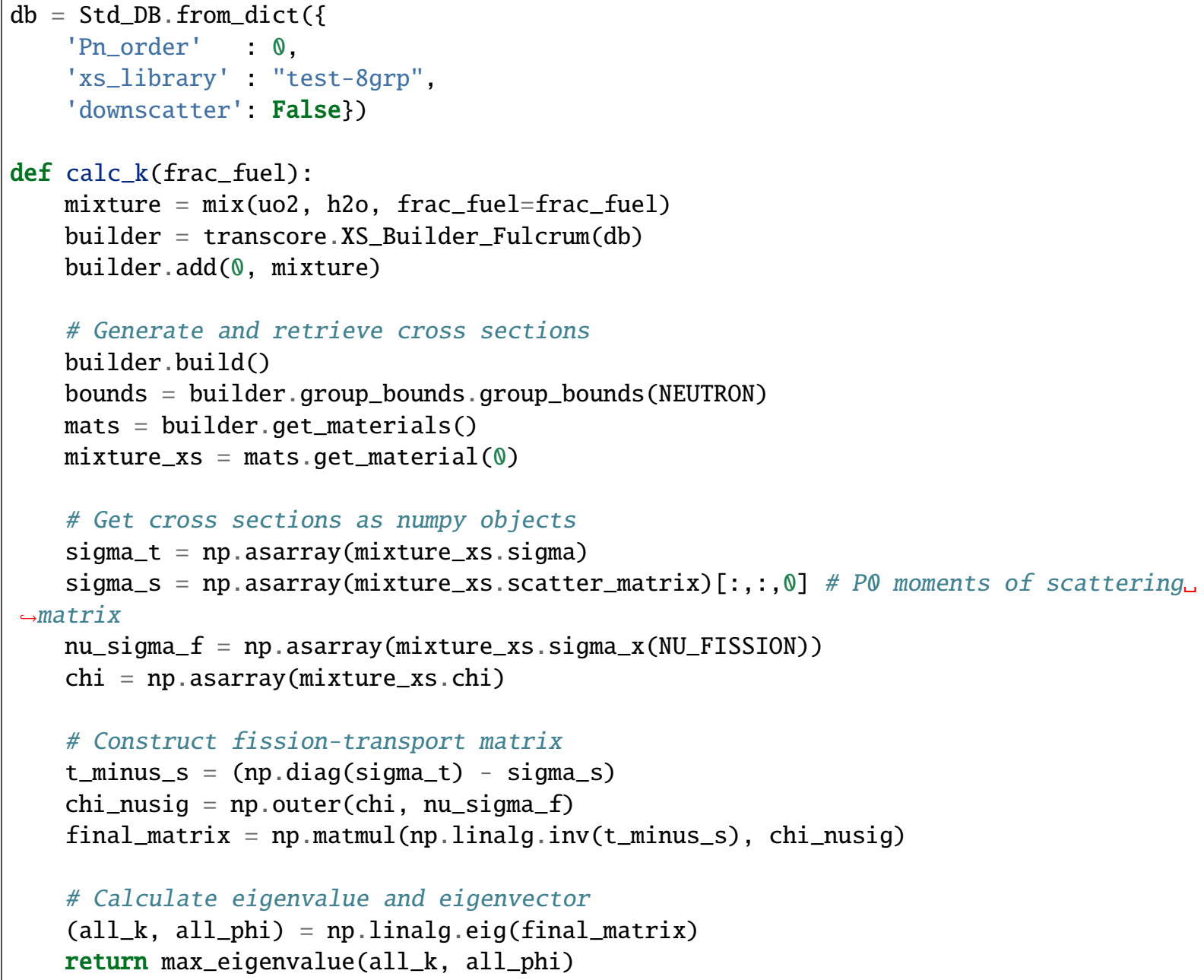

Finally, we use the optimization toolkit from scipy to find the root, giving our final answer.

from scipy.optimize import root_scalar

result $=$ root_scalar (calc_reactivity, method='brentq', bracket $=(\mathbb{\theta}, 1), \mathrm{x} \boldsymbol{\theta}=\mathbf{0} .5, \mathrm{rtol}=1 \mathrm{e}-4)$ print (result)

frac_fuel $=$ result . root 
print("Final fuel fraction:", frac_fuel)

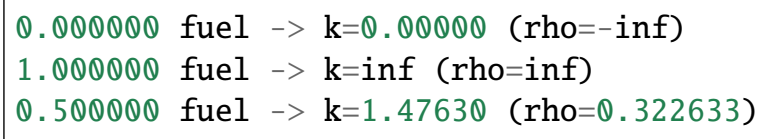

$\gg$ \oading AMPX library at '/usr/local/scale/data/test8g_v7.1'

$\gg>$ Retained 6 of 449 nuclides on the master AMPX library

$\gg$ Running XSProc on 1 cells

〉> Loading AMPX library at '/usr/local/scale/data/test8g_v7.1'

$\gg$ Retained 6 of 449 nuclides on the master AMPX library

〉> Running XSProc on 1 cells

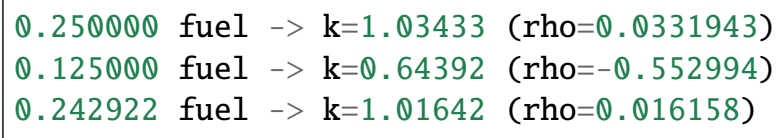

〉> Loading AMPX library at '/usr/local/scale/data/test8g_v7.1'

$\gg$ Retained 6 of 449 nuclides on the master AMPX library

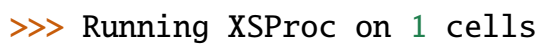

〉> Loading AMPX library at '/usr/local/scale/data/test8g_v7.1'

$\gg>$ Retained 6 of 449 nuclides on the master AMPX library

〉> Running XSProc on 1 cells

〉> Loading AMPX library at '/usr/local/scale/data/test8g_v7.1'

$\gg$ Retained 6 of 449 nuclides on the master AMPX library

〉> Running XSProc on 1 cells

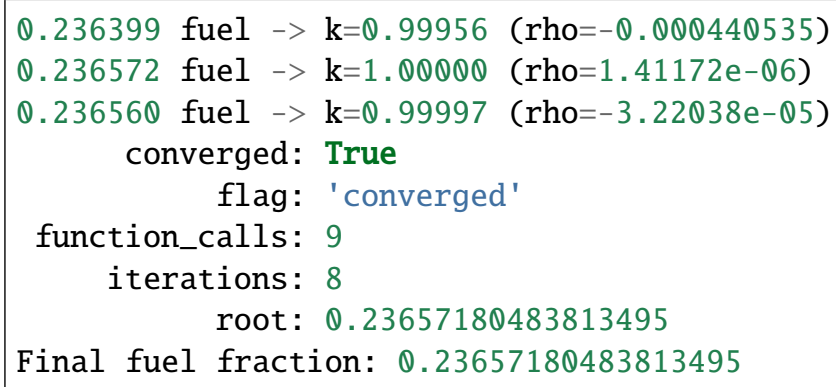

The final answer, the critical fuel mixture, is thus:

$\operatorname{mix}($ uo2, h2o, frac_fuel=frac_fuel)._asdict () 


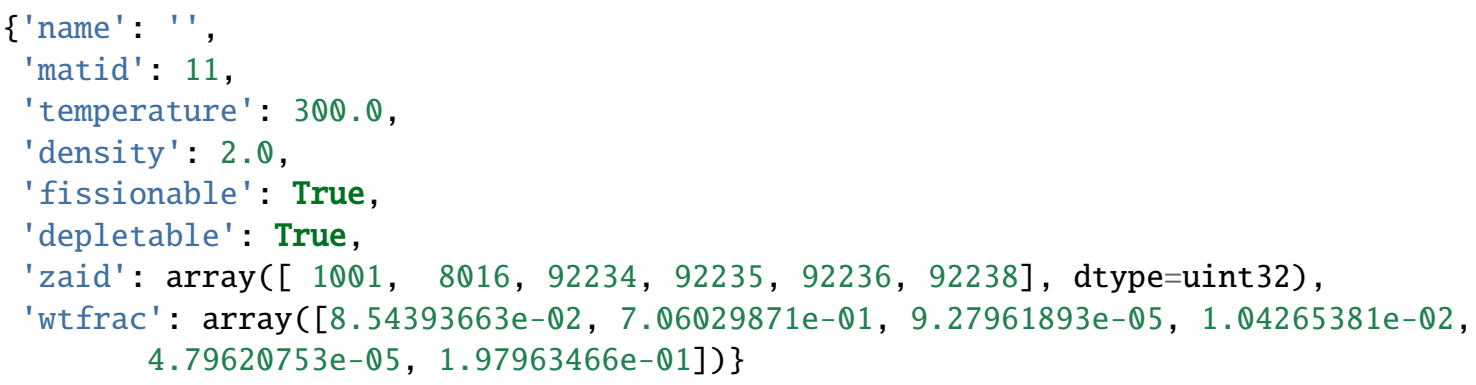

\section{A.4 SHIFT}

\section{A.4.1 DEBUGGING GEOMETRY PROBLEMS IN SHIFT}

It is not unusual to make an error when constructing a geometry model. This example gives a few useful procedures for tracking down geometry errors.

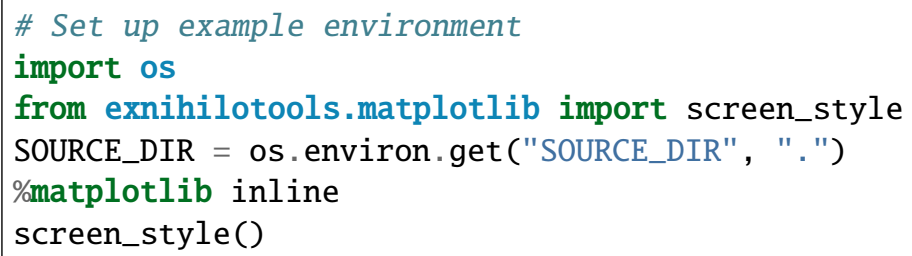

\section{A.4.1.1 Construct compositions via the SCL}

Since this test geometry is a Geometria XML input, it doesn't natively support composition definitions. Here is one of the many ways to define compositions: loading them using the robus Python wrappers from the SCALE standard composition library, and then writing them to an HDF5 file which is then specified in the Omnibus input.

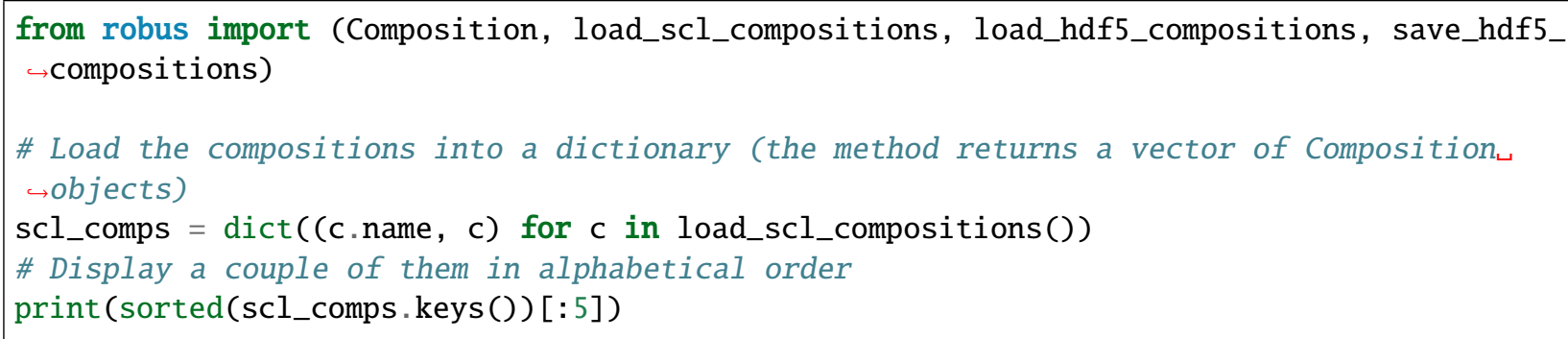

\section{A.4.1.2 Run Shift through Omnibus}

With a pre-constructed (but broken) geometry file, an Omnibus input file, and the set of compositions just generated, Omnibus can be run to see what happens. 
\%cat \{SOURCE_DIR\}/data/buggy.omn

! omnibus-run \{SOURCE_DIR\}/data/buggy.omn

\section{[PROBLEM]}

name "Buggy geometry"

mode forward

[MODEL $=g g]$

input "buggy.gg.omn"

[COMP]

! Note: this file should be generated automatically by the example

input "buggy.comp.h5"

[PHYSICS $=\mathrm{Ce}]$

ce_lib "ce_v7.1_endf"

[SOURCE=separable beam_point]

[SOURCE] [SHAPE=point]

point $-3-20$

[SOURCE] [ANGLE=mono]

$\operatorname{dir} 100$

[SOURCE] [ENERGY=mono]

energy $14 \mathrm{e} 6$

particle_type $n$

[TALLY]

[.] [DIAGNOSTIC=debug_history]

! [...][DIAGNOSTIC=history]

[..][MESH global_mesh]

reactions flux

$\mathrm{x}-4 \quad 7 \mathrm{i} 4$

y $-4 \quad 7 i$

z $-4 \quad 7 i$

[SHIFT]

np 100

[RUN=serial]

INF0: Starting Omnibus preprocessor, omnibus version 6.3.pre-b10 (branch 'omnibus-doc' $\hookrightarrow \# 98 \mathrm{~d} 73 \mathrm{c} 8 \mathrm{e}$ on 2020MAR11)

Loading problem db from Omnibus ASCII file...

Loading Omnibus input file at/rnsdhpc/code/src/scale/Exnihilo/packages/Omnibus/driver/

$\rightarrow$ example/data/buggy.omn

...finished loading problem db from Omnibus ASCII file

Loading problem db from Python file...

...finished loading problem db from Python file

Generating Geometria XML input file from .gg.omn...

INFO: Starting Geometria preprocessor, omnibus version 6.3.pre-b10 (branch 'omnibus-doc' $\hookrightarrow \# 98 \mathrm{~d} 73 \mathrm{c} 8 \mathrm{e}$ on 2020MAR11)

Loading problem db from Omnibus ASCII file...

Loading Omnibus input file at/rnsdhpc/code/src/scale/Exnihilo/packages/Omnibus/driver/

cexample/data/buggy.gg.omn 
(continued from previous page)

...finished loading problem db from Omnibus ASCII file

INFO: Writing Geometria input ParameterList to buggy.gg.xml

...finished generating Geometria XML input file from .gg.omn

INFO: Set default for 'fissionable_only' to 'False' in '/source/beam_point'

INFO: Set default for 'mode' to ' $\mathrm{n}$ ' in '/physics/ce'

INFO: Set default for 'xs_cache' to 'tot' in '/physics/ce'

INFO: Set default for 'xs_accel' to 'True' in '/physics/ce'

INFO: Set default for 'fission_neutrons' to 'True' in '/physics/ce/fission'

INFO: Set default for 'load_scl' to 'True' in '/comp'

INFO: Set default for 'decomposition' to '\{'_type': 'none'\}' in '/shift'

INFO: Set default for 'verbosity' to 'low' in '/shift/transporter'

INFO: Set default for 'method' to 'roulette' in '/shift/vr'

INFO: Writing Omnibus input ParameterList to buggy.inp.xml

INFO: Writing preprocessed file to buggy.pp.json

INFO: Writing processed ASCII input to 'buggy.inp.omn'

INFO: Launching Omnibus driver on 1 cores

Running Omnibus...

WARNING: The Exnihilo software revision ( $(2540)$ used to generate the input file differs

$\rightarrow$ from this version being used to run it ( $r 539)$.

WARNING: This could cause internal consistency checks to unexpectedly fail, and it could $\hookrightarrow$ even lead to unexpected database value changes.

WARNING: Please check your output very carefully after this run to make sure the

$\hookrightarrow$ interpreted values match your input values.

Building model

INFO: Loading SCALE Standard Composition Library from/usr/local/scale/data/scale.rev40. $\hookrightarrow$ Sclib

INFO: Loading compositions from/rnsdhpc/code/src/scale/Exnihilo/packages/Omnibus/driver/

cexample/data/buggy.comp.h5

INFO: Creating default boundary mesh from $\left(\begin{array}{lll}-4 & -4 & -4\end{array}\right)$ to $\left(\begin{array}{lll}4 & 4 & 4\end{array}\right)$ for GG geometry

Building physics 'ce'

INF0: Loading CE library /usr/local/scale/hpcdata/ce_v7.1_endf.h5

WARNING: The following nuclide IDs were remapped due to missing neutron data

WARNING: Remapped 2 nuclide IDs: 6012->6000, 6013->6000

WARNING: The following neutron cross sections were set to zero: 8018

INF0: Corrected thermal xs balance: $1.2 \mathrm{e}-05 \%$ error in $\mathrm{h}-1$ @ $293.6 \mathrm{~K}$

Building tallies

Building sources

Building Shift solver internals

Building Shift sources

Initializing Shift solver

Building Shift tallies

Running Shift transport calculation

ERROR: Geometry error in particle $0: 0$ : In universe 'global': Failed to move from left $\hookrightarrow$ (local volume $\# \mathbb{Q}$ ) across left.s (local surface \#1) at local point $\{\boldsymbol{0} .5,-2, \mathbb{0}\}$ along $\{1$, $\hookrightarrow \mathbb{Q}, \mathbb{Q}\}$ : possible neighbors are $\left\{\right.$ left $(\# \mathbb{Q})$, fill (\#2)\}, possible nearby volumes are $\left\{\right.$ left $_{\sqcup}$ $\hookrightarrow(\# 0)$, right $(\# 1)$, fill (\#2), EXTERIOR (\#3)\}

ERROR: Geometry error in particle $0: 1$ : In universe 'global': Failed to move from left $\hookrightarrow$ (local volume $\# \mathbb{Q}$ ) across left.s (local surface \#1) at local point $\{0.5,-2, \mathbb{Q}\}$ along $\{1$, $\hookrightarrow \mathbb{Q}, \mathbb{Q}\}$ : possible neighbors are $\{$ left $(\# \mathbb{Q})$, fill $(\# 2)\}$, possible nearby volumes are $\left\{\right.$ left $_{\sqcup}$ $\hookrightarrow(\# 0)$, right $(\# 1)$, fill (\#2), EXTERIOR (\#3)\}

FATAL ERROR: Input validation failed:

FATAL ERROR: Lost too many particles (2) locally 
(continued from previous page)

FATAL ERROR: $\wedge \wedge \wedge$ at /rnsdhpc/code/src/scale/Exnihilo/packages/Shift/mc_transport/inst_gg_ $\hookrightarrow$ sce/../DR_Source_Transporter.t.hh:374

Cleaning up

...failed while running Omnibus after 2.0 seconds

INF0: Omnibus run failed; printing log since the last successful status message

INFO:

$* * * * * * * * * * * * * * * * * * * * * * * \quad$ OMNIBUS ERROR LOG $\quad * * * * * * * * * * * * * * * * * * * * * * * * *$

: : : Cleaning up

$* * * * * * * * * * * * * * * * * * * * * * * \quad$ END ERROR LOG $\quad * * * * * * * * * * * * * * * * * * * * * * * * *$

FATAL ERROR: An unexpected error occurred. Please check the log file at omnibus_2020-03$\hookrightarrow$ 11-223747.log for more details.

FATAL ERROR: Command '/rnsdhpc/code/build/Exnihilo-examples/Exnihilo/packages/Omnibus/ $\hookrightarrow$ driver/omnibus buggy.inp.xml' returned non-zero exit status 1 .

\section{A.4.1.3 Understanding the error message}

Oops! Too many particles encountered geometry errors, so the problem aborted. The first thing to do is to try to understand the error message.

!!! Geometry error in particle 0:0: In universe 'global': Could not find the volume connecting volume $\theta$ (left) across surface 2 (right.s) at local point $0.5-20$ along $\left\{\begin{array}{lll}1 & 0 & 0\end{array}\right\}$ : neighbors are $\{0,2\}$, bounding volumes are $\{0,1,2,3\}$

$\wedge \wedge \wedge$ at $\{0.5-20\}$ along $\left\{\begin{array}{lll}1 & 0 & 0\end{array}\right\}$

The first thing to note is the particle identification: 3:15 means the 16th history on the 4th Shift thread being run. Since Omnibus error messages use $\mathrm{C}$ indexing, $0: 0$ means the very first particle to be transported on the only instance of Shift.

Shift indicated a problem with the geometry as opposed to the CE data or physics options that were set. Since this problem uses GG as the geometry model, the error message also provides contextual details of how the error occurred, which can be compared to the provided geometry:

\%cat \{SOURCE_DIR\}/data/buggy.gg.omn

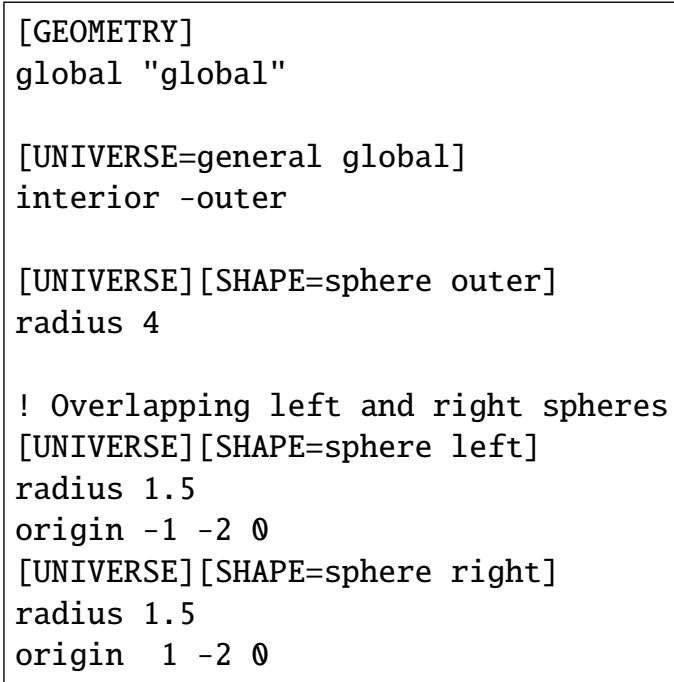




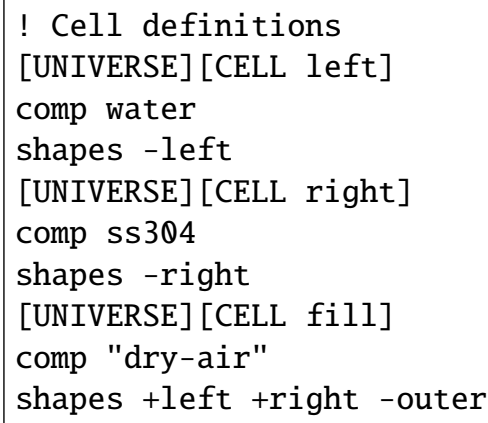

The message says it's a problem connecting the cell named "left" with the surface named "right.s" (which we can guess is the spherical surface created by the "right" sphere shape). It shows the point in space and direction of travel; and if this error had happened inside of a daughter universe (such as an assembly in a reactor), the positions both in local space and global problem space would be presented.

The other information about neighbors and bounding volumes is not immediately useful because it only provides internal IDs for those cells rather than names that you the user input. However, the Omnibus Python front end tools can help translate thse cell IDs. (Note that since the GG input file does not provide a mapping between composition names and internal IDs, an explicit compositions argument must be passed to the load_gg call.)

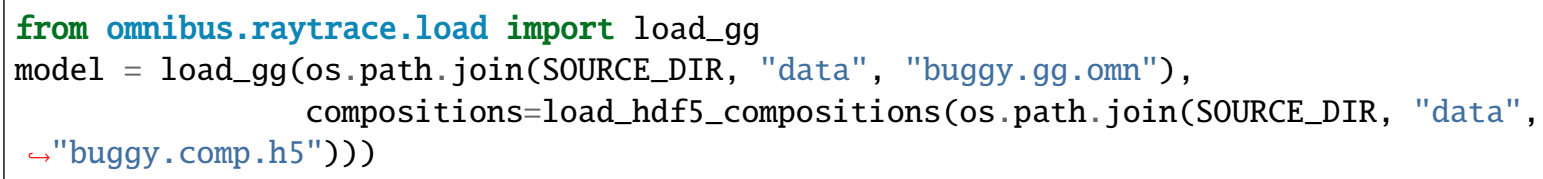

The resulting model contains the geometry, compositions, and (for some geometry/model types) other data such as tallies. In this case, the only concern is the geometry. The generic "describe" function will print a detailed description of the geometry's structure.

print (model . geometry . describe())

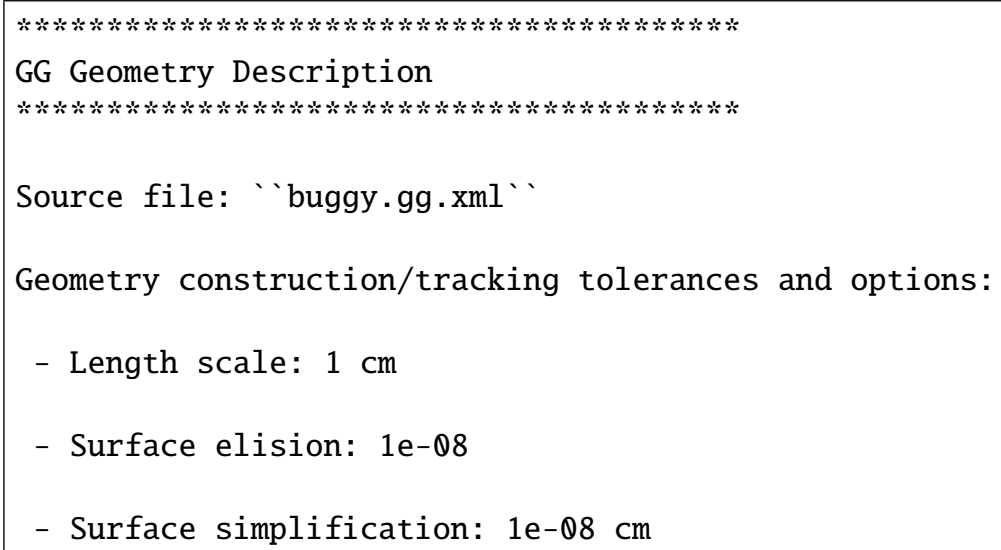


- Shape enclosure: 1e-08

- Bounding box expansion: 1e-07

- Almost-lost particle bump: $1 \mathrm{e}-08 \mathrm{~cm}$

- Fast geometry shortcuts are enabled.

Note that volumes are described in [Reverse Polish Notation] (https://en.wikipedia.org/wiki/Reverse_Polish_notation), where ' $\sim$ ' is the negation operator, ' $\&$ ' is the intersection/and operator, and ' $\mid$ ' is the union/or operator.

$* * * * * * * * * * * * * * * * * * * * * * * * * * * * * * * * * * * * * * * * * * * * * * * *$

global: General universe

$* * * * * * * * * * * * * * * * * * * * * * * * * * * * * * * * * * * * * * * * * * * * * * * *$

:\# Cells: 4 (offset $=0$ )

:\# Surfaces: 3 (offset $=0$ )

:Bounding box: ' $\left\{\begin{array}{lllllll}-4 & -4 & -4 & \text { to } & 4 & 4 & 4\end{array}\right\}^{\cdots}$

Volumes (local indexing):

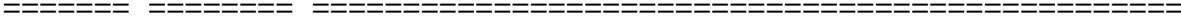

ID Name Surface logic

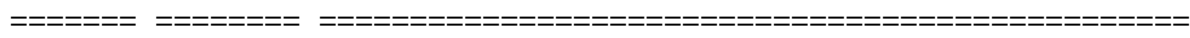

0 left $1 \sim$

1 right $2 \sim$

2 fill $\theta \sim 1 \& 2 \&$

3 EXTERIOR $\theta$

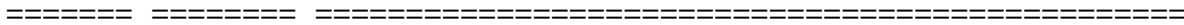

Surfaces:

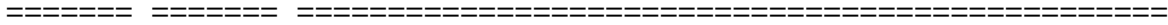

ID Name Description

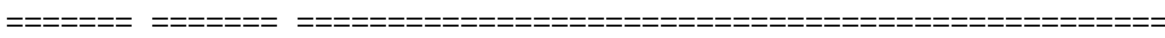

(1) outer.s Sphere: $r=4$

1 left.s Sphere: $r=1.5$ at $-1-20$

2 right.s Sphere: $r=1.5$ at $1-20$

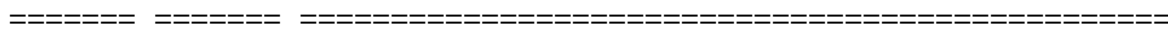

Material fills:

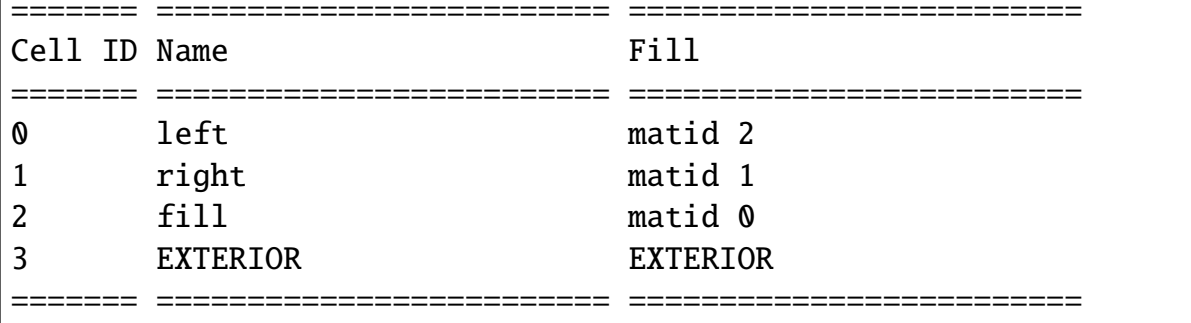


From this diagnostic description, it is clear that the only "neighbor" for volume 0 (left) is volume 2 (fill), but the failed particle tries to cross a surface right.s. In other words, the left and right cells are not connected but the particle crosses the "right" sphere while inside the left. We examine the input and notice that the two spheres overlap. Excluding the right sphere from the left one (or separating the spheres or reducing their size) will fix the problem.

\section{A.4.1.4 Post-mortem analysis of problem run}

For more sinister cases in which the error only occurs after some other sequence of events, or for geometry types that don't provide as much useful debugging information as GG, another diagnostic tool may prove useful: the history tally.

Fortunately, the input already has a debug_history diagnostic although if it did not, it could be simply added and the problem run again to get the same error, since Shift is reproducible on the same number of processors when domain replicated.

The debug_history option keeps a rolling list of all particle events on all processors, and if the particle dies because of an error (usually a geometry error), an HDF5 file will be written with that particle's history. Otherwise, or for successfully transported particles, no history will be output. Thus, for histories where no error occurs, the performance impact of the diagnostic will be minimal: no I/O is occurring, and little to no data is even being allocated.

The erroneous history files are saved to files named debug_history-pNNNN.h5, where NNNN is the process that failed (in this case just zero). The easiest and clearest way to visualize the output is with the convenience function to_frame that returns the data as a Pandas dataframe.

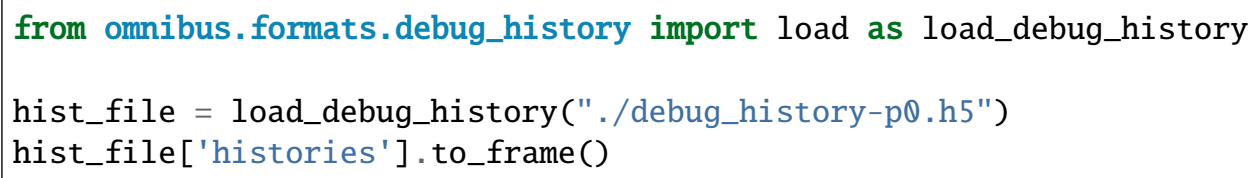

\section{A.4.1.5 2D raytracing through Python}

Visualizing the erroneous part of the geometry is also an extremely useful tool. By default, the ray tracer enables error checking.

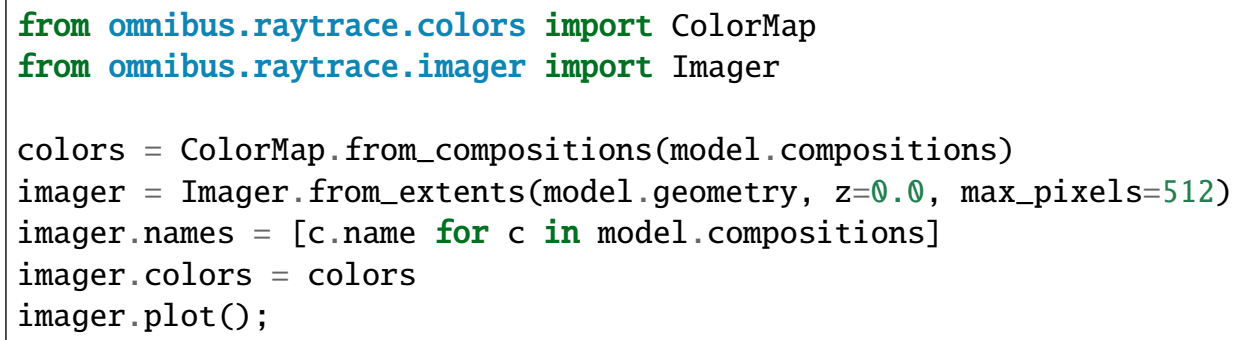

WARNING: Geometry errors were encountered 


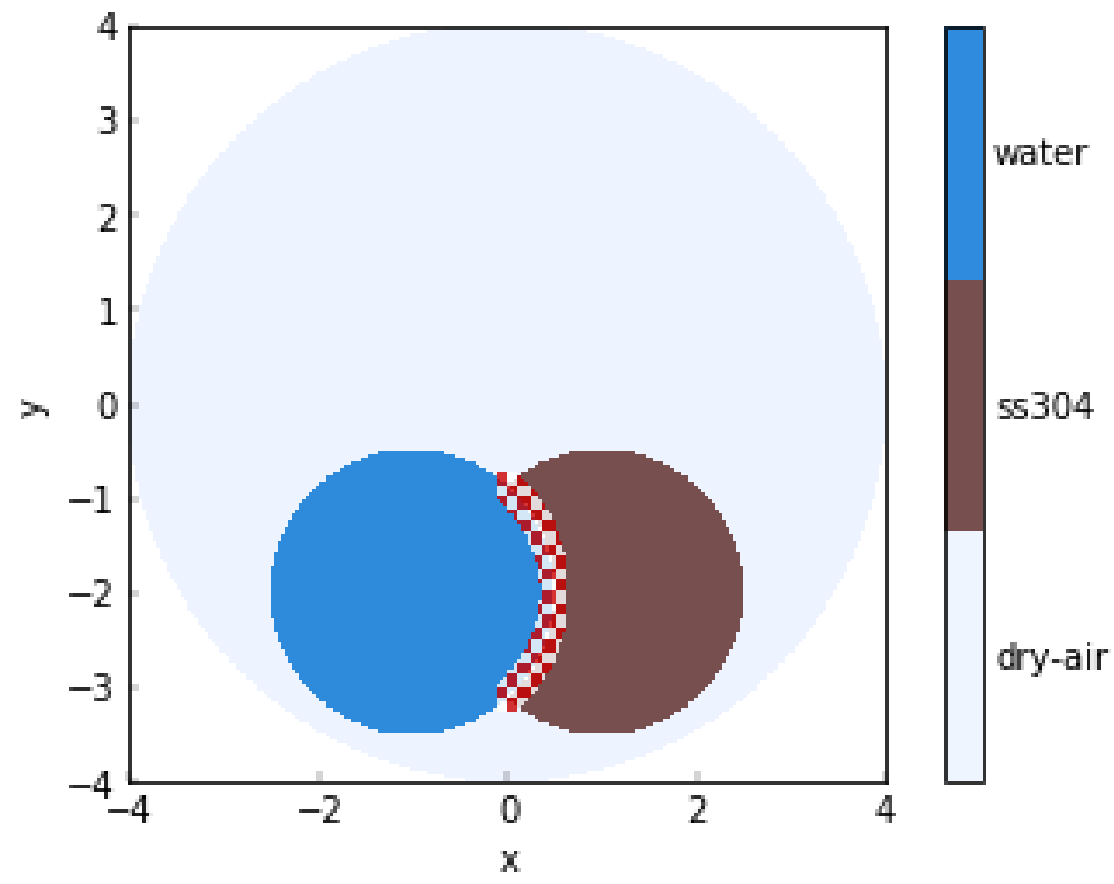

Note the checkered white and red area in the plot indicating the erroneous boundary. The actual exception messages (describing in detail the geometry errors) are printed to the terminal rather than this notebook. However, the error message (similar to the one seen during transport) is visible by disabling error checking on the plotter:

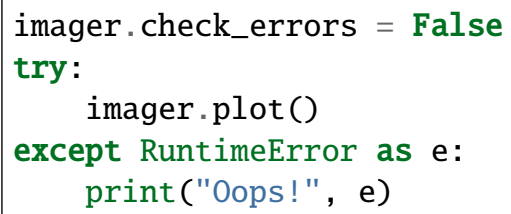

Oops! In universe 'global': Failed to move from left (local volume \#Q) across left.s $\hookrightarrow$ (local surface \#1) at local point $\{0.000945598,-0.882813,0\}$ along $\{1,0,0\}:$ possible $\sqcup$ $\hookrightarrow$ neighbors are $\{$ left $(\# \mathbb{0})$, fill (\#2)\}, possible nearby volumes are \{left (\#0), right ( $\hookrightarrow$ ) , fill (\#2), EXTERIOR (\#3)\}

$\wedge \wedge \wedge$ at $\{0.000945597844243196,-0.882812500000009,0\}$ along $\{1,0,0\}$

More information on the error can be gleaned by seeing how the geometry appears when tracing rays in the opposite direction. To do that, a second Imager is constructed with a basis vector opposite from the default $(1,0,0)$ :

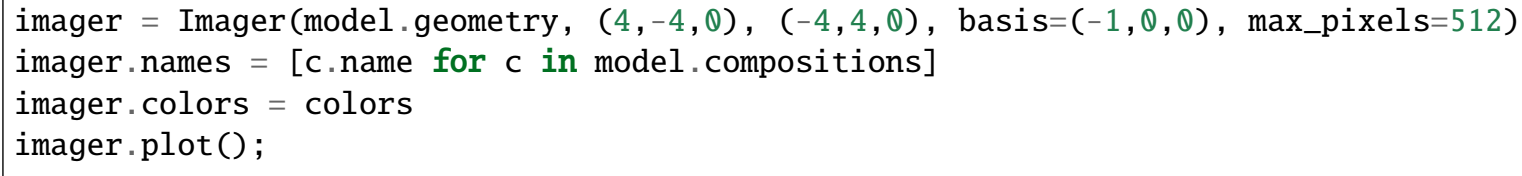

WARNING: Geometry errors were encountered 


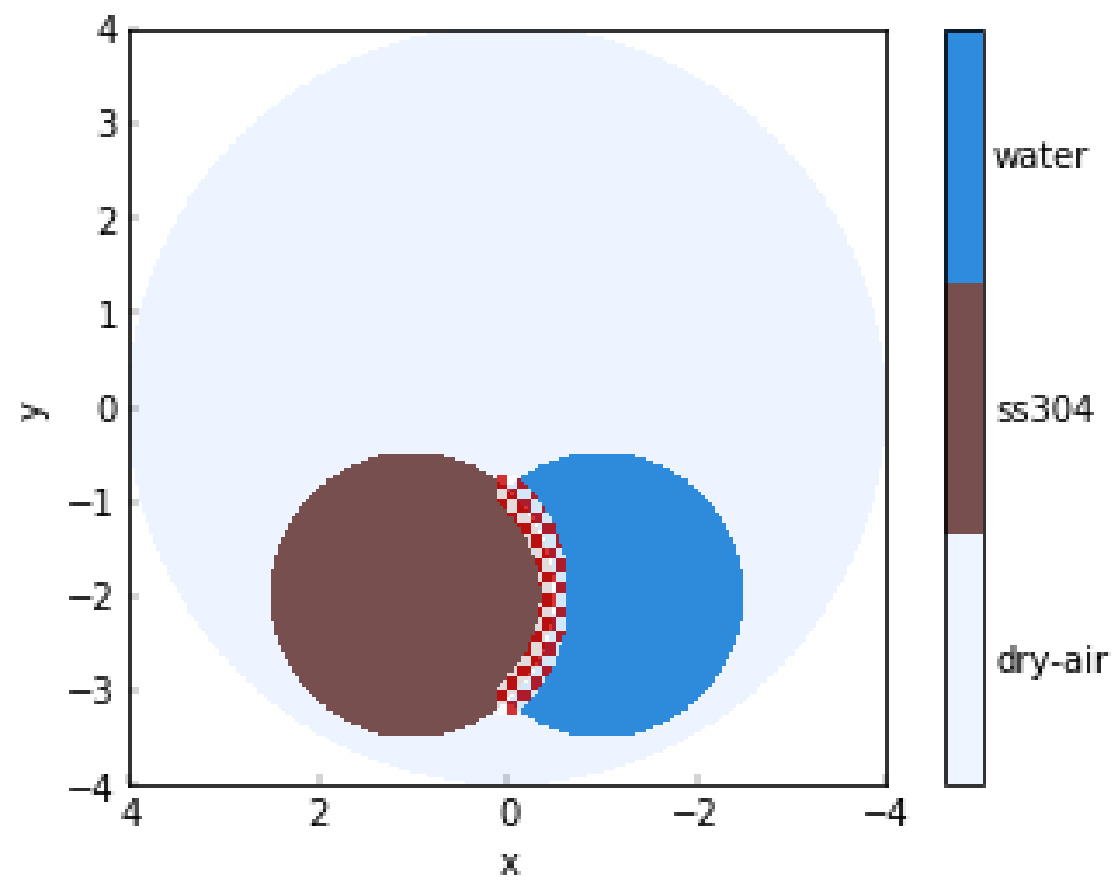

Again the plot shows the erroneous region. Since the basis vector is reversed, so is the $x$ axis. Note that comparing to the previous plot shows that the overlap between the two circles appears to be a different material depending on the particle's direction of travel.

\section{A.4.1.6 Detailed geometry debugging}

One final tool for finding and debugging geometry errors is the GG_Debugger class. (For MCNP geometries, use Lava_Debugger.) Since these tools by default use the C cout/cerr pipes to print, the wurlitzer ${ }^{23}$ tool is needed to redirect terminal output to this notebook.

\%load_ext wurlitzer

from geometria import GG_Debugger

ggd = GG_Debugger (model.geometry)

We can print detailed information about a particle track through the geometry, including the universes and surface names. If an error occurs, the detail particle state is printed.

\section{try:}

ggd.print_track $((-4,-2,0),(1,0,0))$

except RuntimeError as e:

print("Caught error:", e)

Caught error: In universe 'global': Failed to move from left (local volume \#() across $\hookrightarrow$ left.s (local surface \#1) at local point $\{\mathbb{0}, 5,-2, \mathbb{Q}\}$ along $\{1, \mathbb{Q}, \mathbb{Q}\}$ : possible neighbors

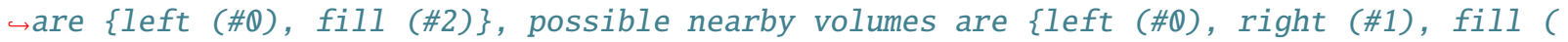
$\hookrightarrow \# 2)$, EXTERIOR (\#3)\}

$\wedge \wedge \wedge$ at $\{\mathbb{0} .5,-2, \mathbb{Q}\}$ along $\{1,0,0\}$

\footnotetext{
${ }^{23}$ https://github.com/minrk/wurlitzer
} 


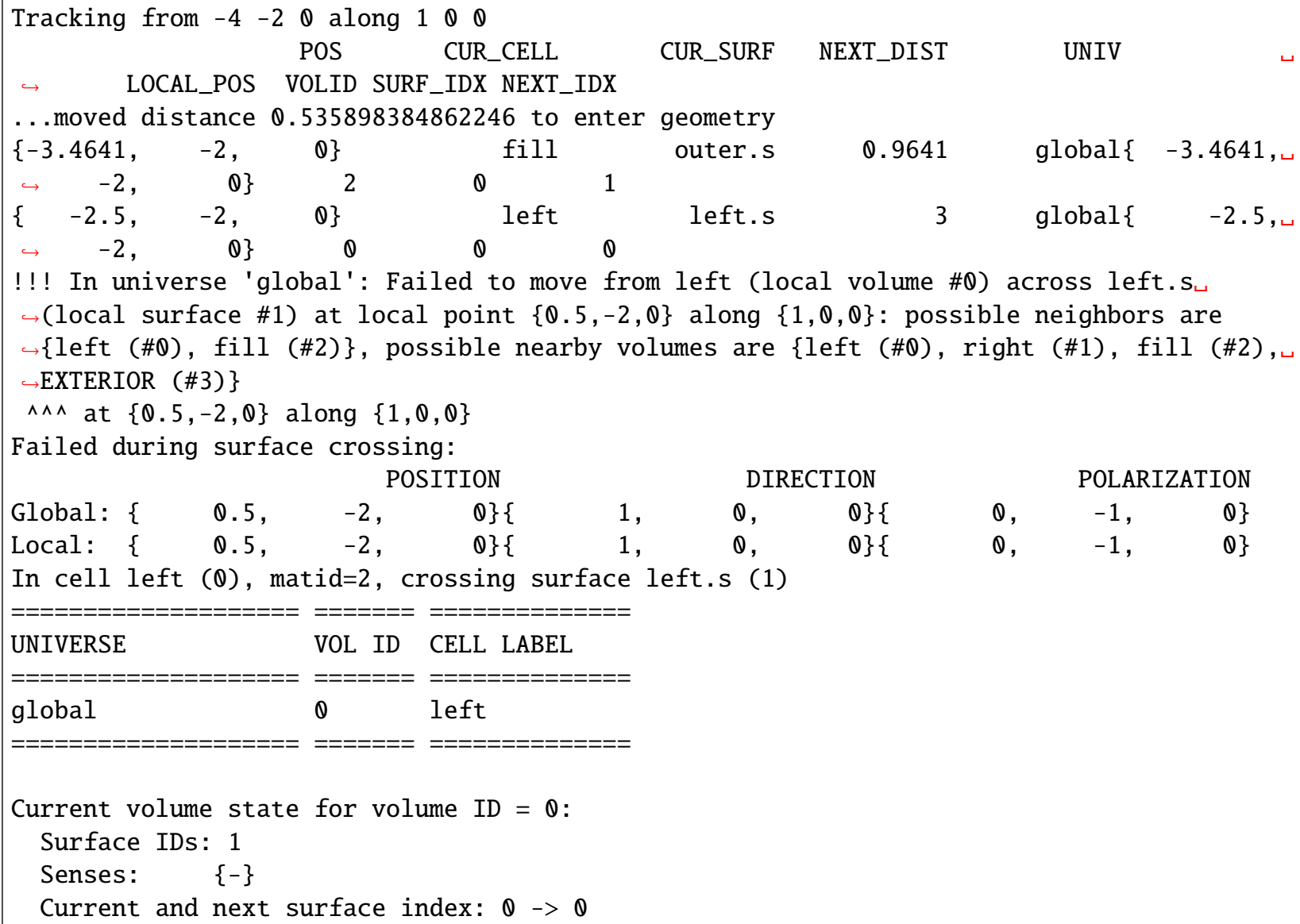

\section{A.4.2 SHIFT DEPLETION WITH MOVABLE CONTROL RODS}

This example demonstrates how to run and post-process a Shift depletion run using an MCNP model with movable elements.

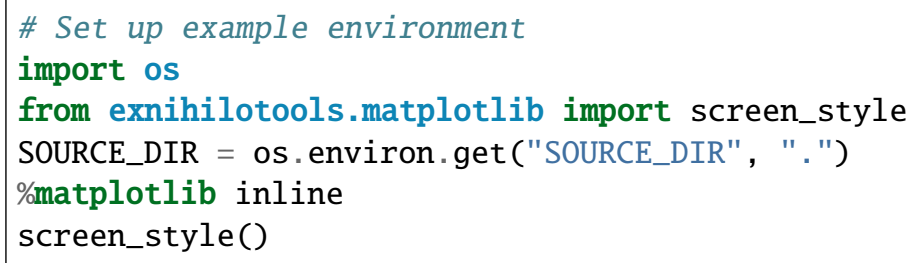

\section{A.4.2.1 Previsualization}

Before running the problem, it is useful to render the input geometry for verification. Note that the different fuel regions (all initially the same composition, but with unique material IDs) are automatically assigned slightly different shades of red by the ColorMap's constructor.

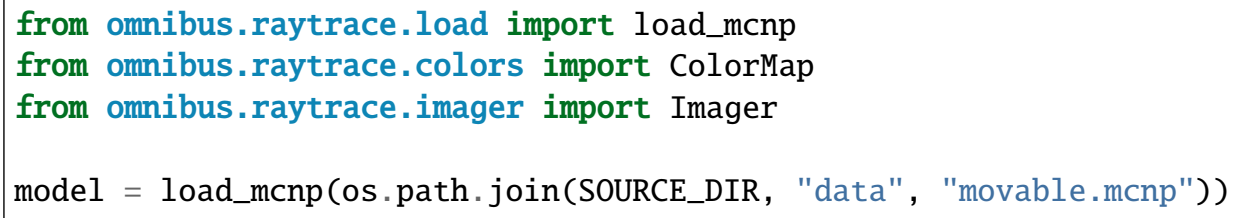




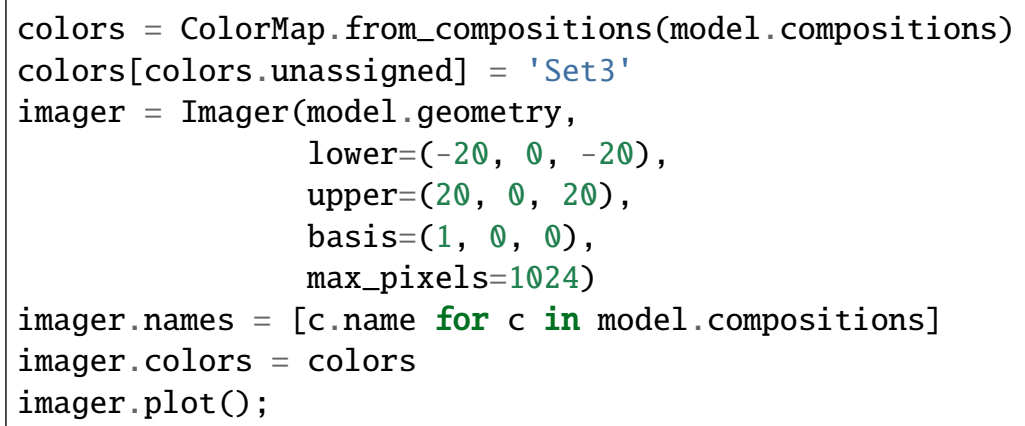

Generating MCNP runtpe file... ...finished generating MCNP runtpe file in 2.8 seconds

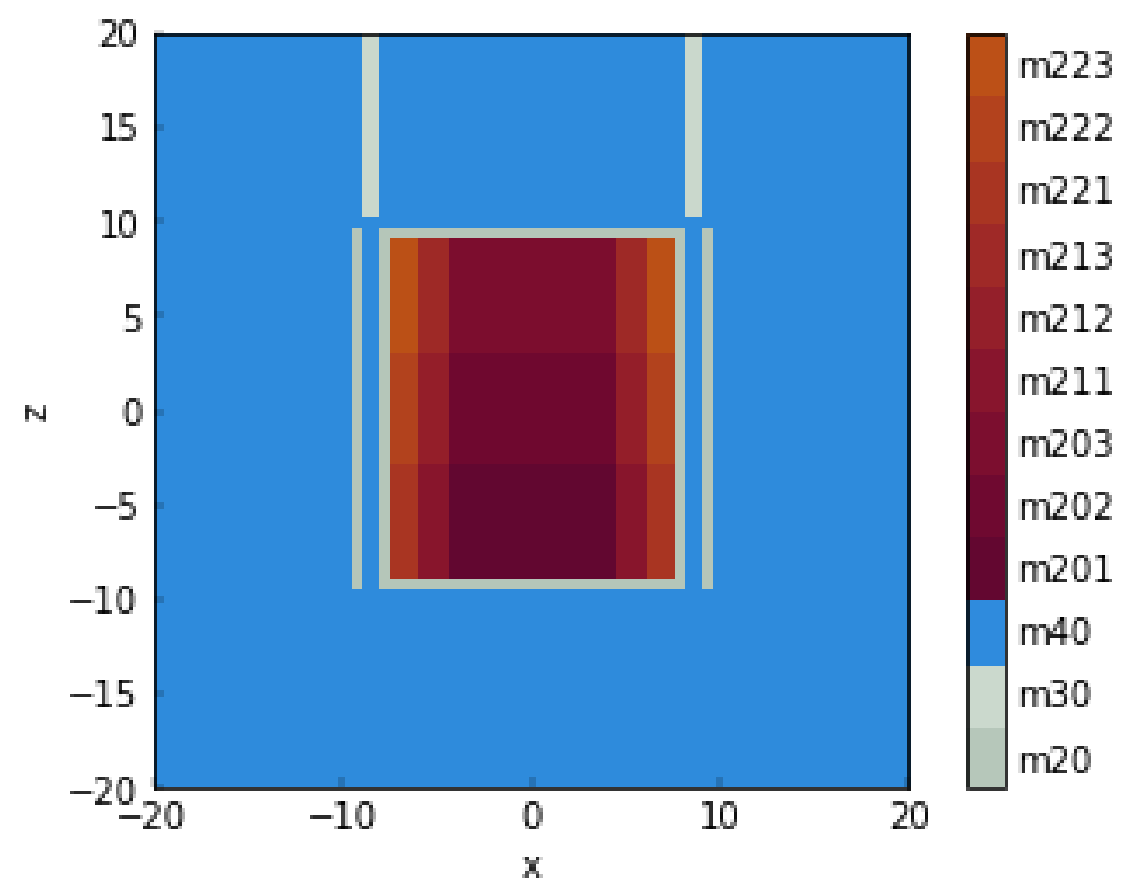

The model's composition data can be used to plot number densities in the reactor. The first step in such a visualization is extracting number densities and their corresponding nuclide IDs from the model's compositions. Note that zaid returns a view (which for efficiency we translate into a numpy array), whereas calc_nd returns a vector that is automatically translated to a native python object.

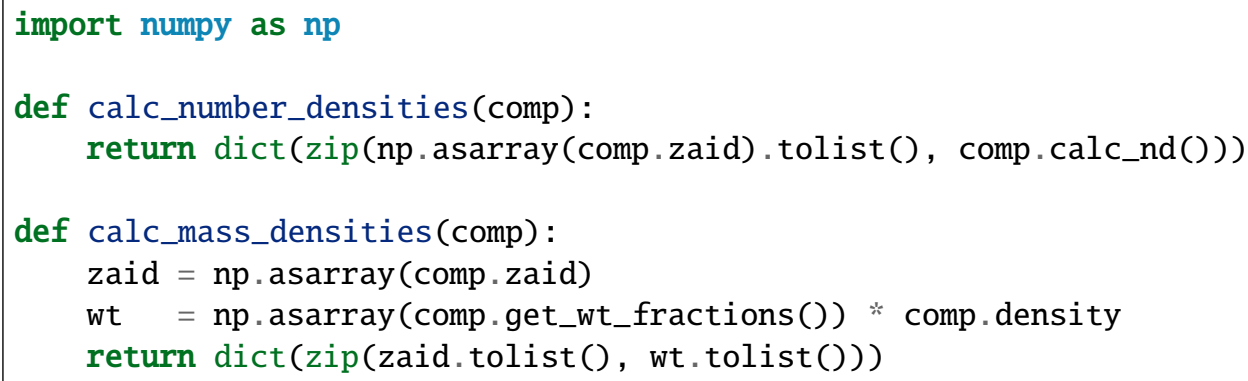




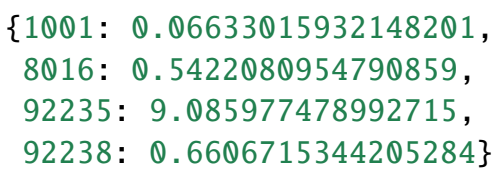

The next step is to extract a particular nuclide's densities for every pixel's matid in the raytrace's view. This requires a Numpy array of values for each matid. Using np. nan instead of 0.0 means the value will be hidden rather than rendered as a zero-value, making it more obvious which parts of the problem have trace nuclides as opposed to none of the nuclide. The pcolor method of the Imager will render an image where the value of each pixel is calculated from the index in the pcolor argument vector corresponding to the material ID at that pixel.
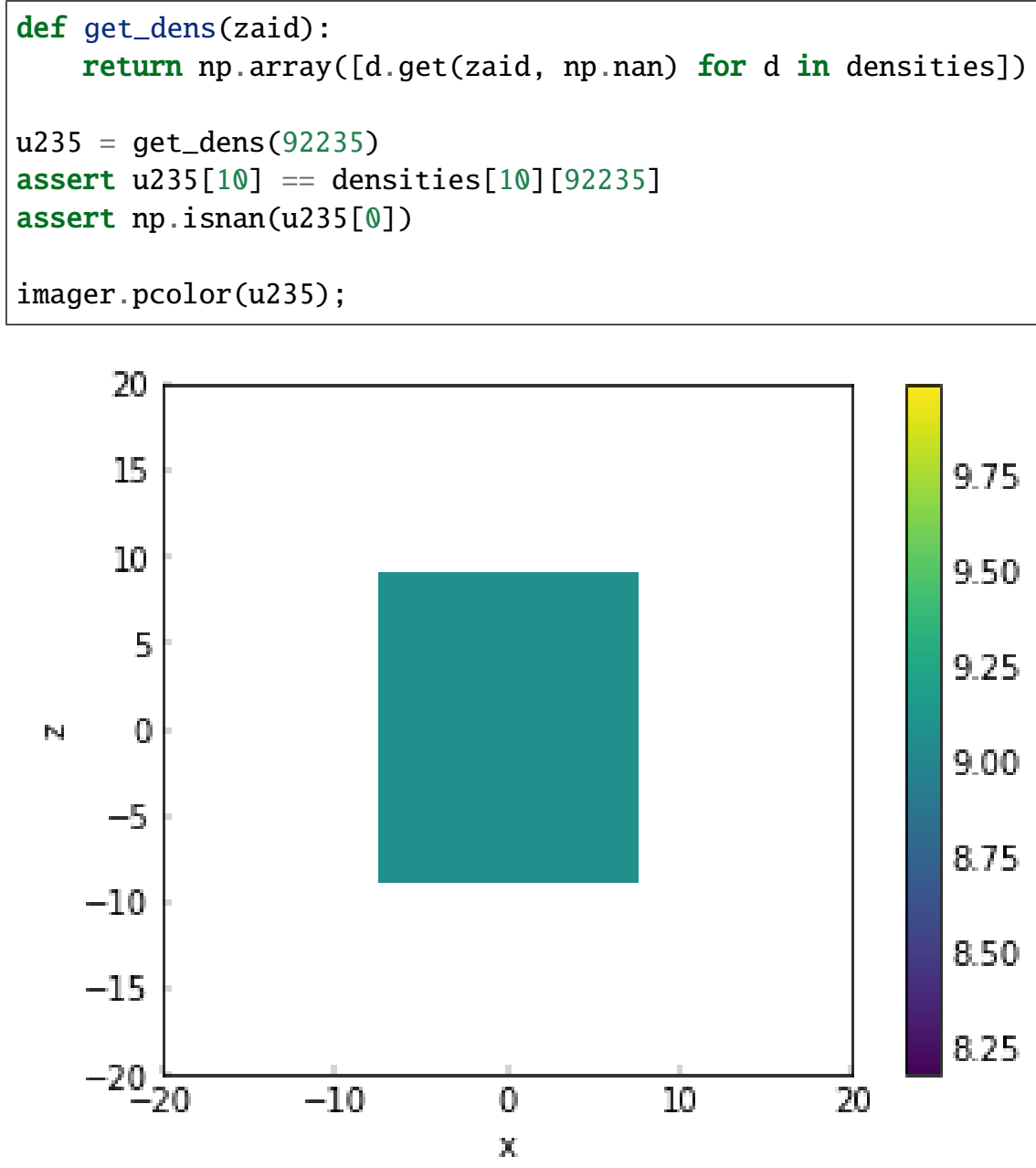

Here is the mass density plot for hydrogen: 
imager . pcolor(get_dens(1001));

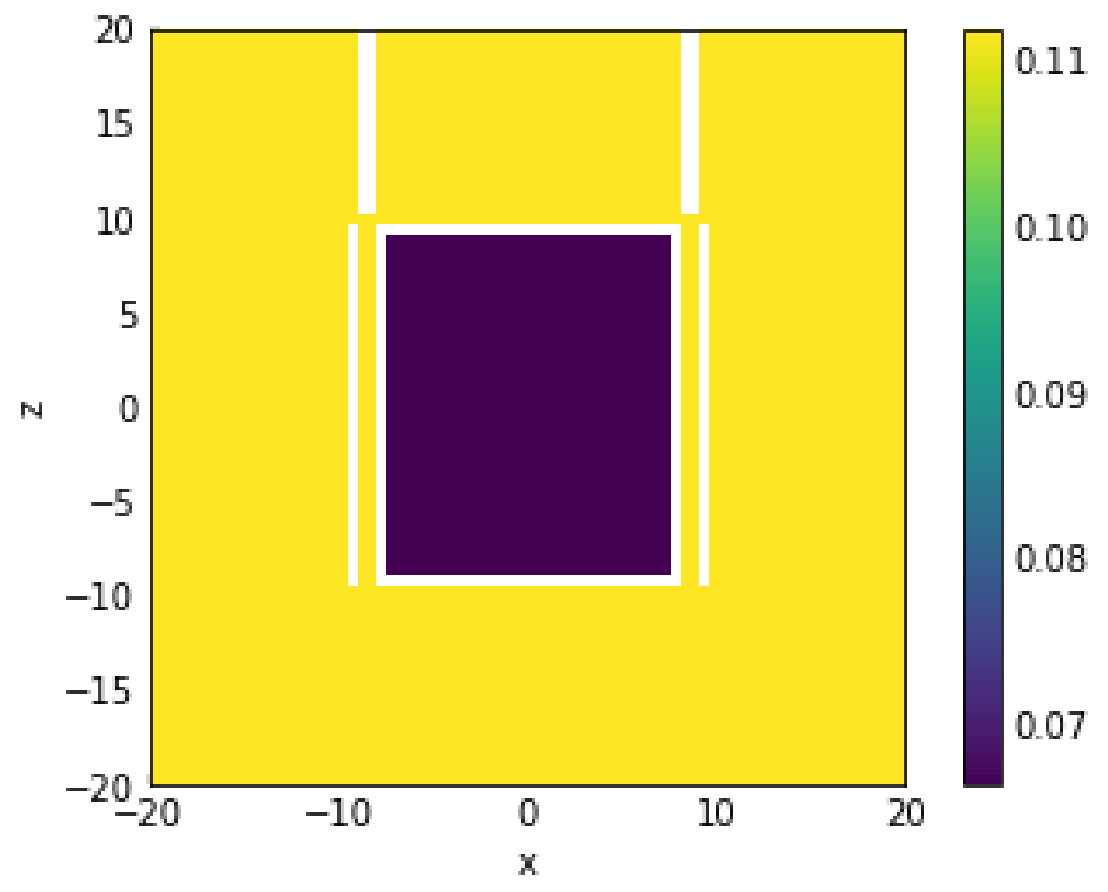

With a little code, it is possible to automate the rendering of all the nuclides in the problem:

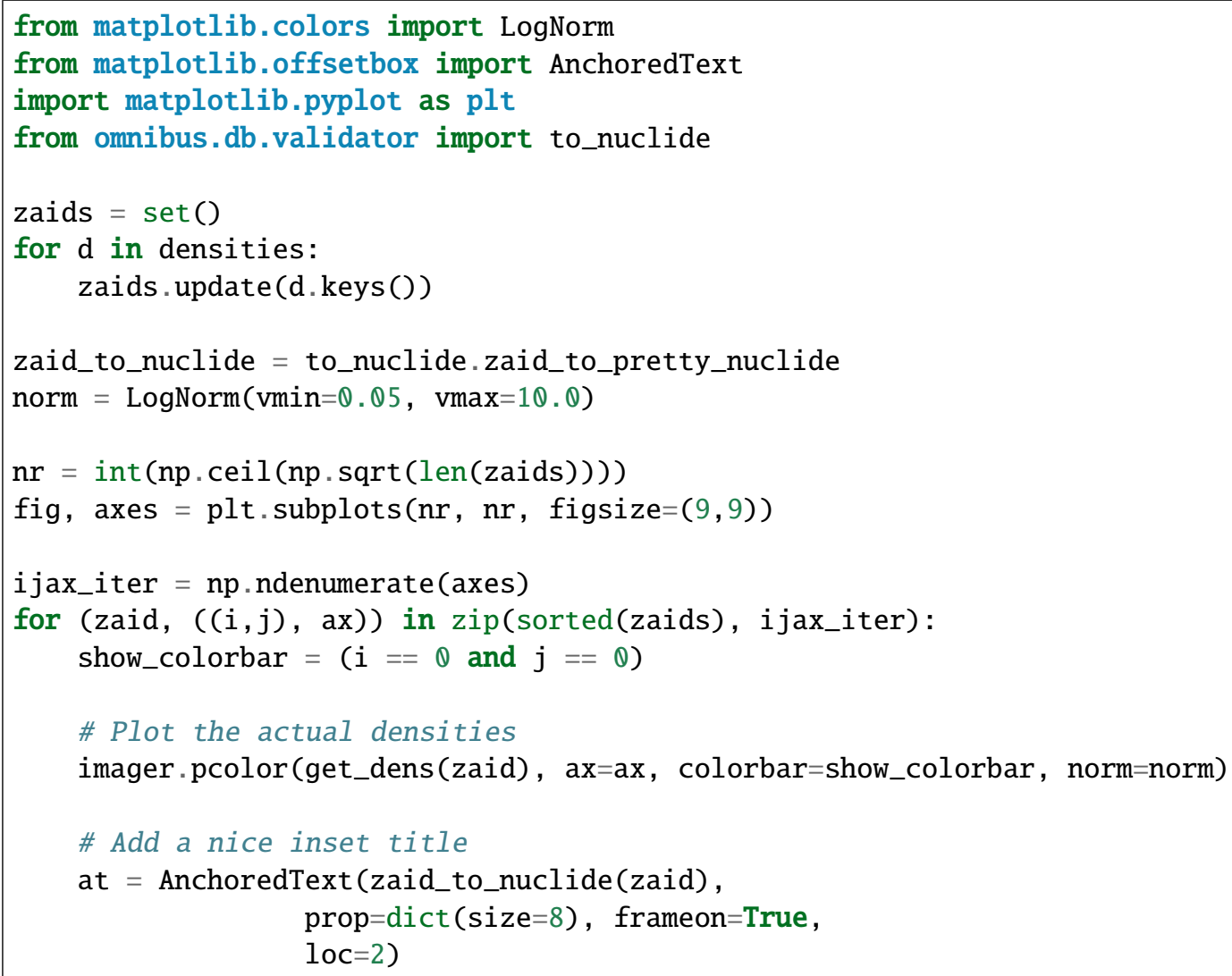


(continued from previous page)

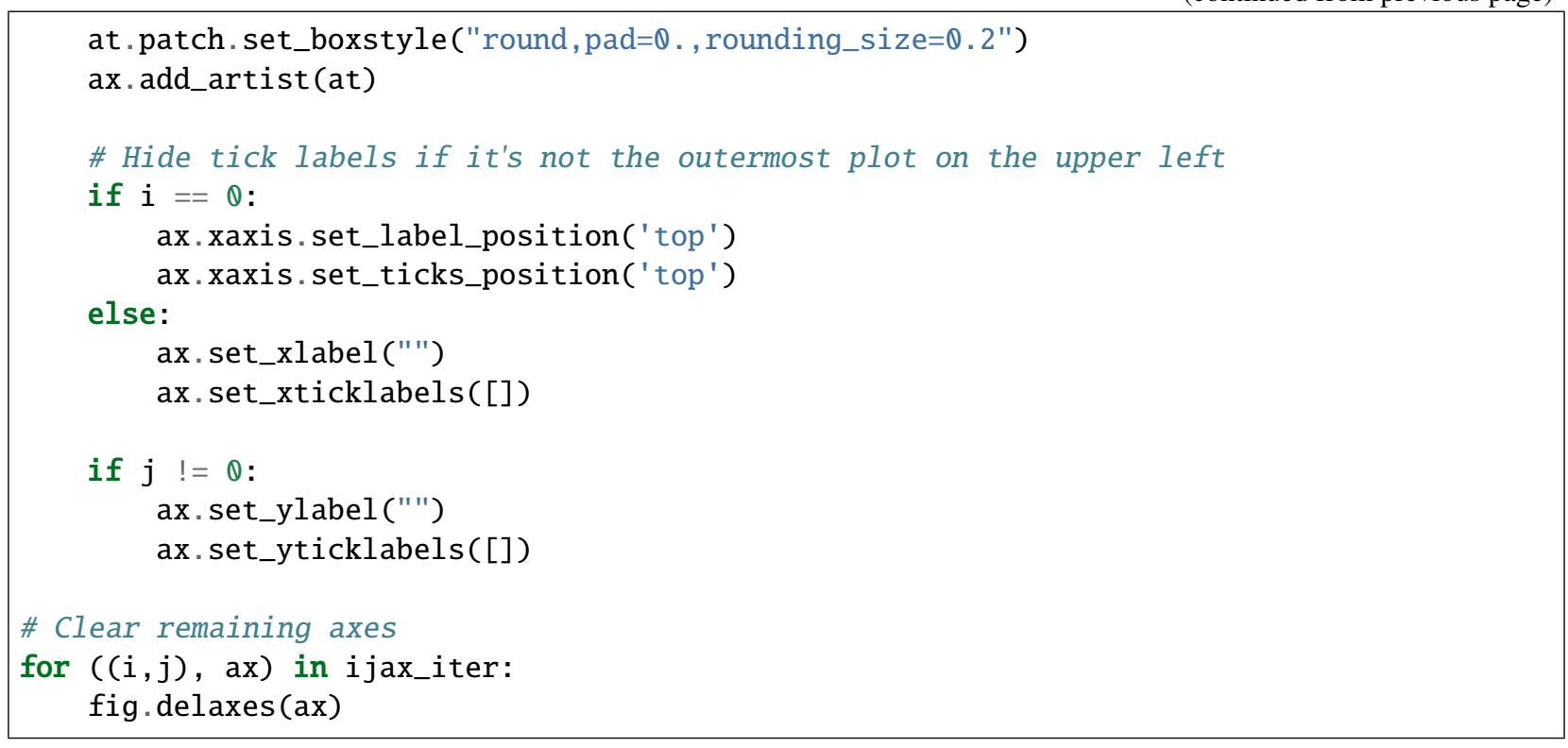

/usr/local/anaconda3/envs/exnihilo/lib/python3.7/site-packages/matplotlib/colors .

$\hookrightarrow$ py:1028: RuntimeWarning: invalid value encountered in less_equal

mask $\mid=$ resdat $<=0$ 

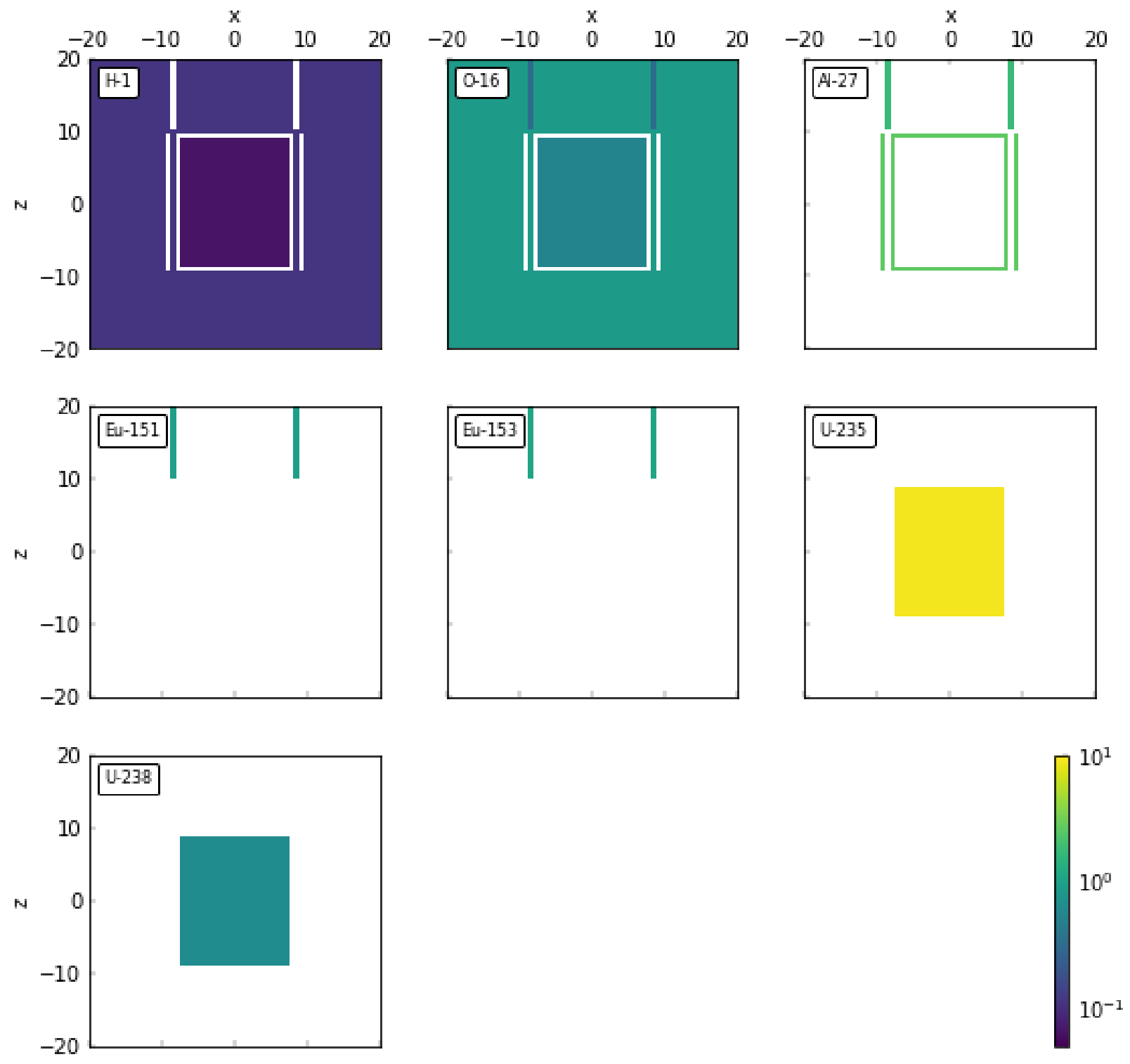

\section{A.4.2.2 Execute Omnibus}

The following Omnibus depletion input transports on this model with four kcode solves, using alternating burn/decay steps. Before the first step, the control rod is moved downward by $10 \mathrm{~cm}$; the next time step it is moved upward by $5 \mathrm{~cm}$.

\%cat \{SOURCE_DIR\}/data/movable.omn

! omnibus-run \{SOURCE_DIR $\} /$ data/movable.omn

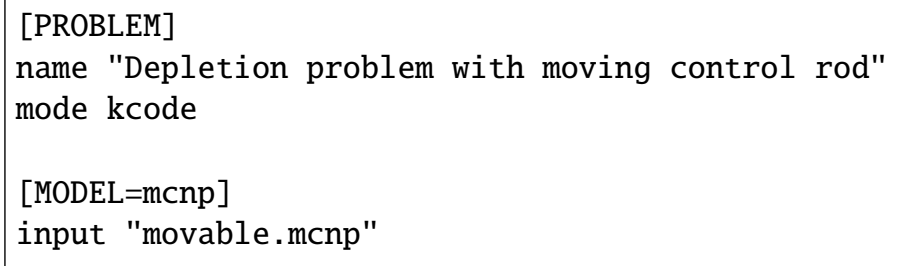


(continued from previous page)

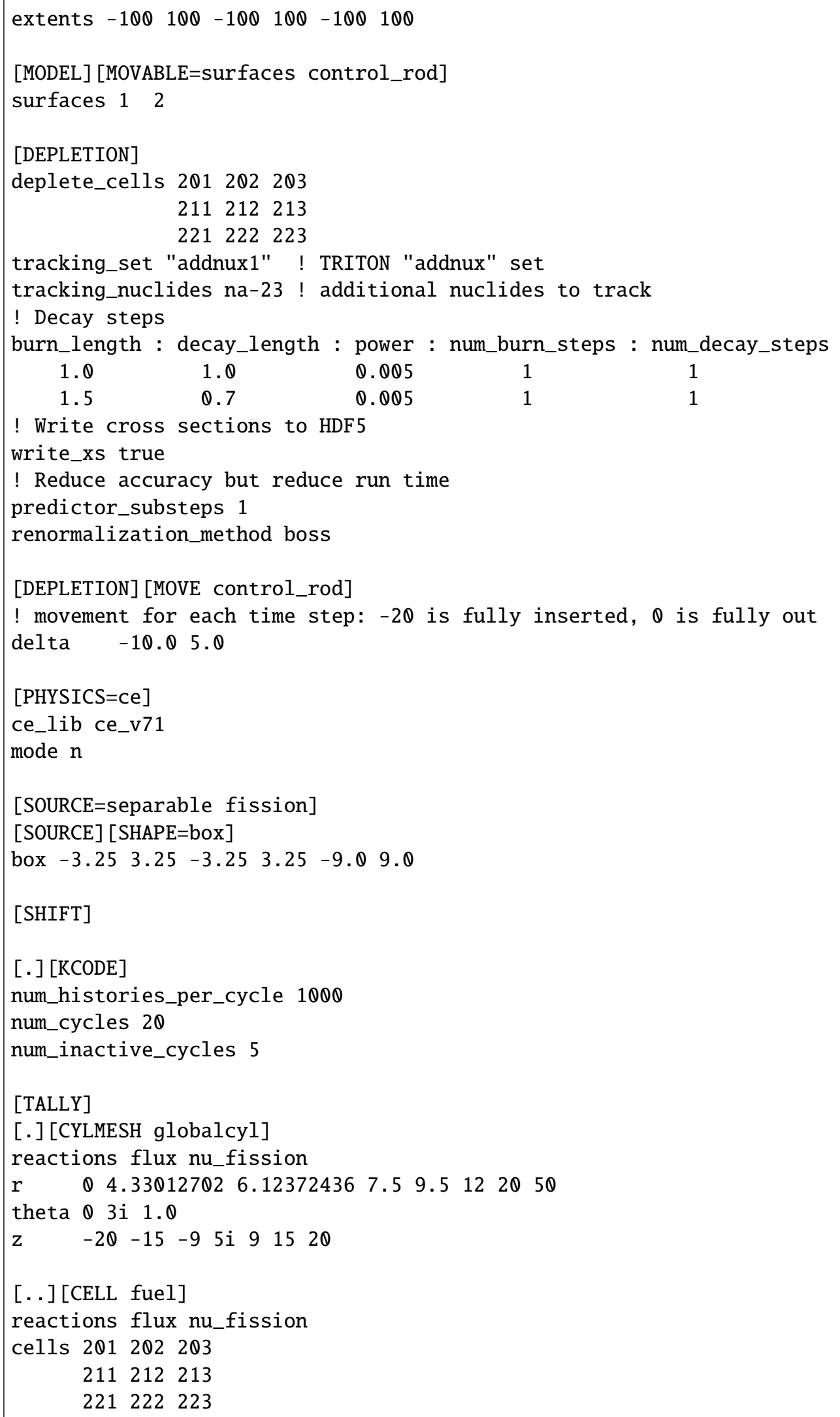


(continued from previous page)

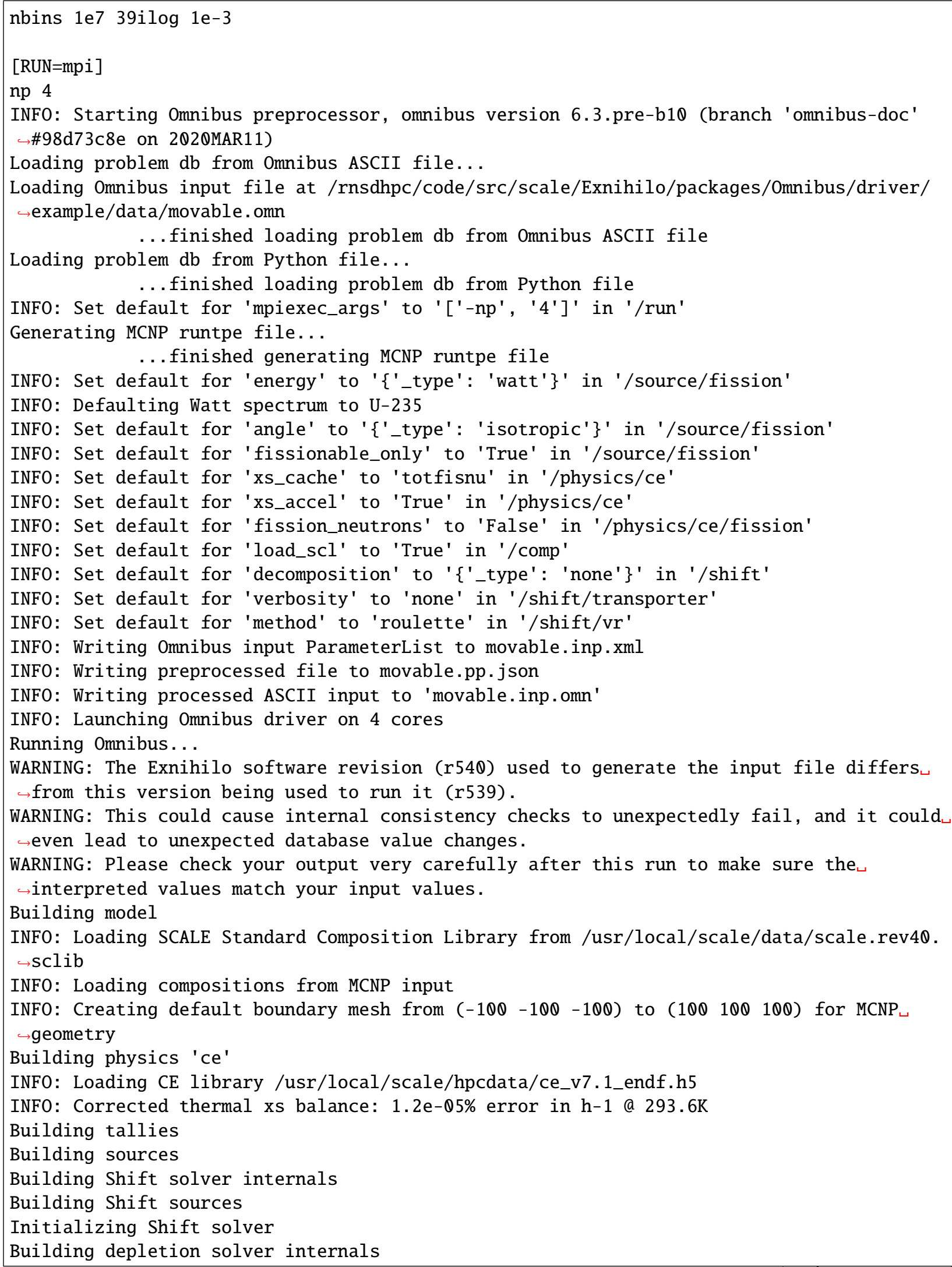


(continued from previous page)

INFO: Using the default VESTA group structure in the depletion tally Building depletion material data

INFO: Loading ORIGEN library/usr/local/scale/data/origen_library/pwr.revQ3.orglib

INFO: Loading fission yield library/usr/local/scale/data/origen_data/origen.rev05.yields. $\hookrightarrow$ data

INFO: Loading JEFF multigroup library /usr/local/scale/data/origen.revQ1.jeff252g

Loading depletion cross sections

INFO: Total initial heavy-metal mass in system: 0.0310027 MT

Building Shift tallies

Solving depletion step $\theta$ from $\theta$ days to 1 days at 0.005 MW

INF0: Moving control_rod by $-10 \mathrm{~cm}$

Running Shift transport calculation

Beginning inactive cycles

INFO: Completed 5 inactive cycles

Beginning active cycles

INF0: Completed 15 active cycles

Collapsing cross sections

Writing tally results

Solving depletion step 1 from 1 days to 2 days at 0 MW

INFO: Deleted delayed fission data because the CE data lacks a delayed fission spectrum:

$\hookrightarrow \mathrm{cm}-243$ @ 293K, am-242m @ 293K

Running Shift transport calculation

Beginning inactive cycles

INFO: Completed 5 inactive cycles

Beginning active cycles

INF0: Completed 15 active cycles

Collapsing cross sections

Writing tally results

Solving depletion step 2 from 2 days to 3.5 days at 0.005 MW

INF0: Moving control_rod by $5 \mathrm{~cm}$

Running Shift transport calculation

Beginning inactive cycles

INFO: Completed 5 inactive cycles

Beginning active cycles

INFO: Completed 15 active cycles

Collapsing cross sections

Writing tally results

Solving depletion step 3 from 3.5 days to 4.2 days at 0 MW

Running Shift transport calculation

Beginning inactive cycles

INFO: Completed 5 inactive cycles

Beginning active cycles

INFO: Completed 15 active cycles

Collapsing cross sections

Writing tally results

Running Shift transport calculation

Beginning inactive cycles

INFO: Completed 5 inactive cycles

Beginning active cycles

INF0: Completed 15 active cycles

Collapsing cross sections

Writing tally results 
(continued from previous page)

WARNING: The first and second halves of the active cycles have statistically different (3. $\hookrightarrow 75514$ sigma) Shannon entropy

Run complete

Cleaning up

...finished running Omnibus in 11.2 seconds

Running Omnibus postprocessing

Loading HDF5 file...

INFO: Loaded Omnibus output data from 'movable.out.h5', problem name 'Depletion problem $\hookrightarrow$ with moving control rod', created on 2020MAR11 22:38 using SCALE version 6.3.pre-b10

$\hookrightarrow$ (branch 'omnibus-doc' \#17579cc6 on 2020MAR10) ...finished loading HDF5 file

Loading HDF5 file...

INF0: Loaded Omnibus output data from 'movable.out.h5', problem name 'Depletion problem. $\hookrightarrow$ with moving control rod', created on 2020MAR11 22:38 using SCALE version 6.3.pre-b10

$\hookrightarrow$ (branch 'omnibus-doc' \#17579cc6 on 2020MAR10)

...finished loading HDF 5 file

Plotting k-effective and related diagnostics...

/rnsdhpc/code/build/Exnihilo-examples/Exnihilo/packages/Omnibus/python/omnibus/formats/

$\hookrightarrow$ tally/field.py:308: FutureWarning: Using a non-tuple sequence for multidimensional

$\hookrightarrow$ indexing is deprecated; use 'arr[tuple(seq)]' instead of 'arr[seq]'. In the future

$\hookrightarrow$ this will be interpreted as an array index, 'arr[np.array(seq)]', which will result

- either in an error or a different result.

mom $=\operatorname{data}[\mathrm{slc}]$

INFO: Saved k-eff plot to movable.keff.pdf

...finished plotting k-effective and related diagnostics in 1.4 seconds

Writing cell tally results to CSV files...

INFO: Wrote cell tally 'fuel' to movable.fuel.tQ.csv

INFO: Wrote cell tally 'fuel' to movable.fuel.t1.csv

INFO: Wrote cell tally 'fuel' to movable.fuel.t2.csv

INF0: Wrote cell tally 'fuel' to movable.fuel.t3.csv

INF0: Wrote cell tally 'fuel' to movable.fuel.t4.csv

...finished writing cell tally results to CSV files

Writing mesh tally visualization file...

...finished writing mesh tally visualization file

Writing depletion number densities...

INFO: Not creating number density CSV file because it would be too large (expected size $_{\sqcup}$ $\hookrightarrow 1289 \mathrm{kB}$ exceeds user-given size 4000000 ); set the 'csv_max' parameter or use Python

$\rightarrow$ tools

...finished writing depletion number densities

Writing depletion cross sections...

Writing cross sections to CSV...

...finished writing cross sections to CSV

INFO: Wrote depletion cross sections to movable.xs.csv

...finished writing depletion cross sections

Building RST summary...

INFO: Wrote summary file to movable.rst

...finished building RST summary

The output file produced includes all the data from the run: debug information, system information, log messages, etc. 


\section{A.4.2.3 Visualize tally results}

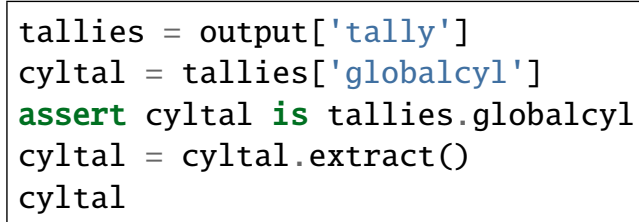

The num_histories field is the number of active histories per depletion step. The total field contains energy-integrated tallies:

cyltal $=$ cyltal $\cdot$ total

cyltal

The tallies can be "sliced" along any of the axes at any of the values. The following code plots the tally estimate of the scalar flux at the initial time step, integrated over all the theta bins.

from omnibus.data import plot

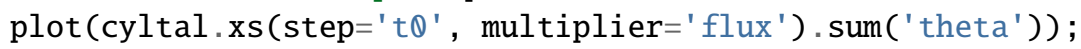

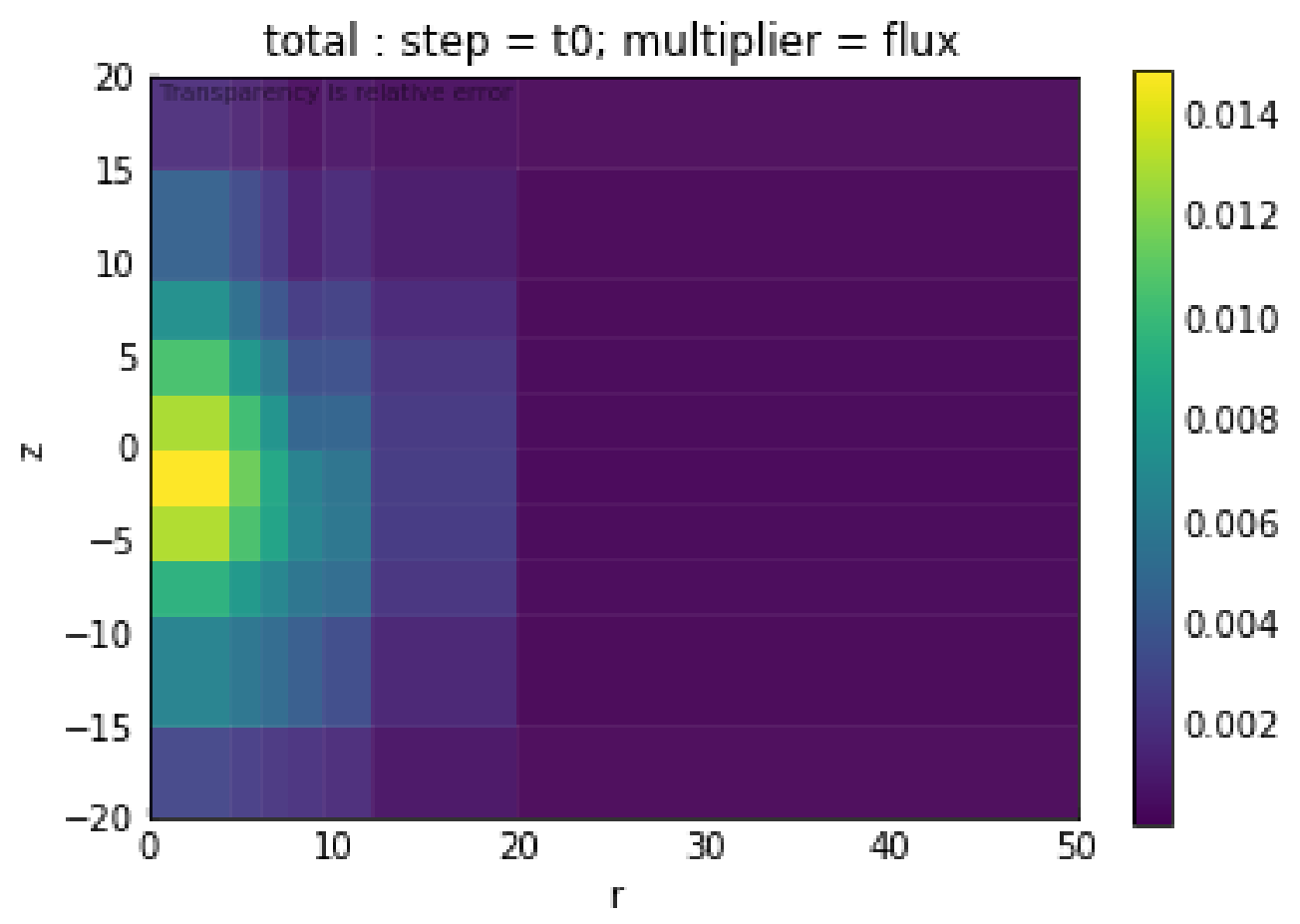

A slice along the $\mathrm{z}$ axis, instead of an integral over the polar axis, yields a polar pseudocolor plot.

flux = cyltal.xs(step='t3', multiplier='flux', z=0.0)

plot (flux.mean)

plot(flux.re); 


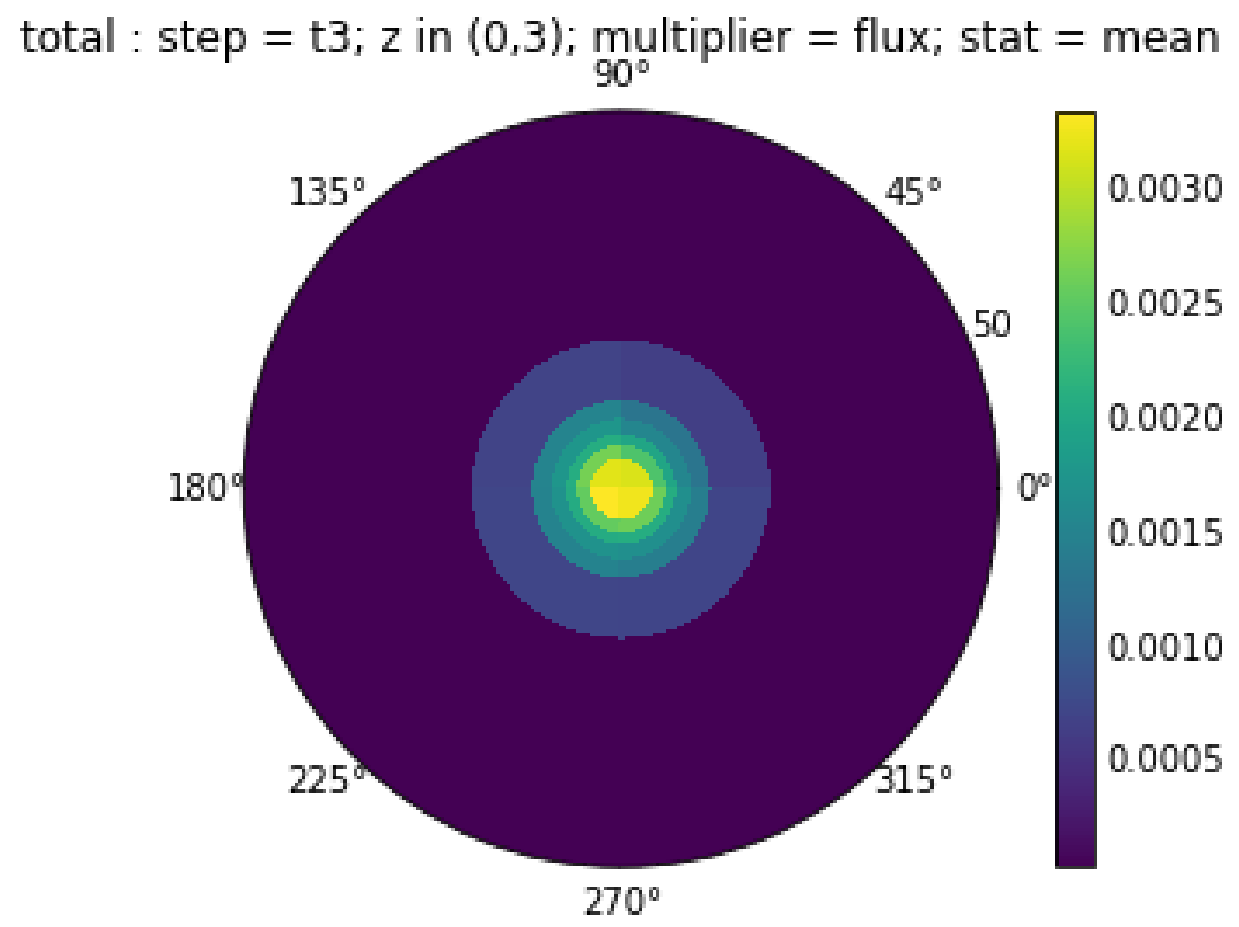

total : step $=\mathrm{t} 3 ; \mathrm{z}$ in $(0,3) ;$ multiplier $=$ flux; stat $=$ re

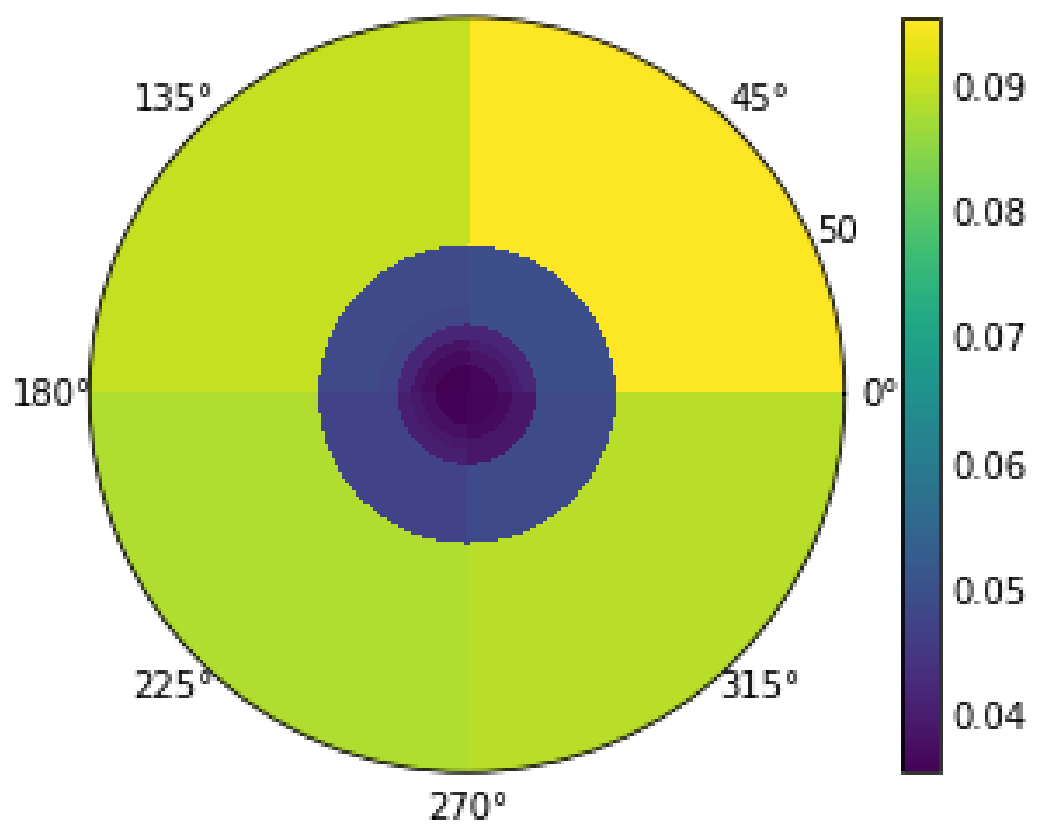

Cell tallies can also be plotted:

celltal = tallies['fuel'].extract ()

binned_tally = celltal ['binned']

print (binned_tally.mesh ('cell'))

binned_tally 
plot (binned_tally.xs(step='tQ', multiplier='flux', stat='mean', cell='213'), logy=True) ;

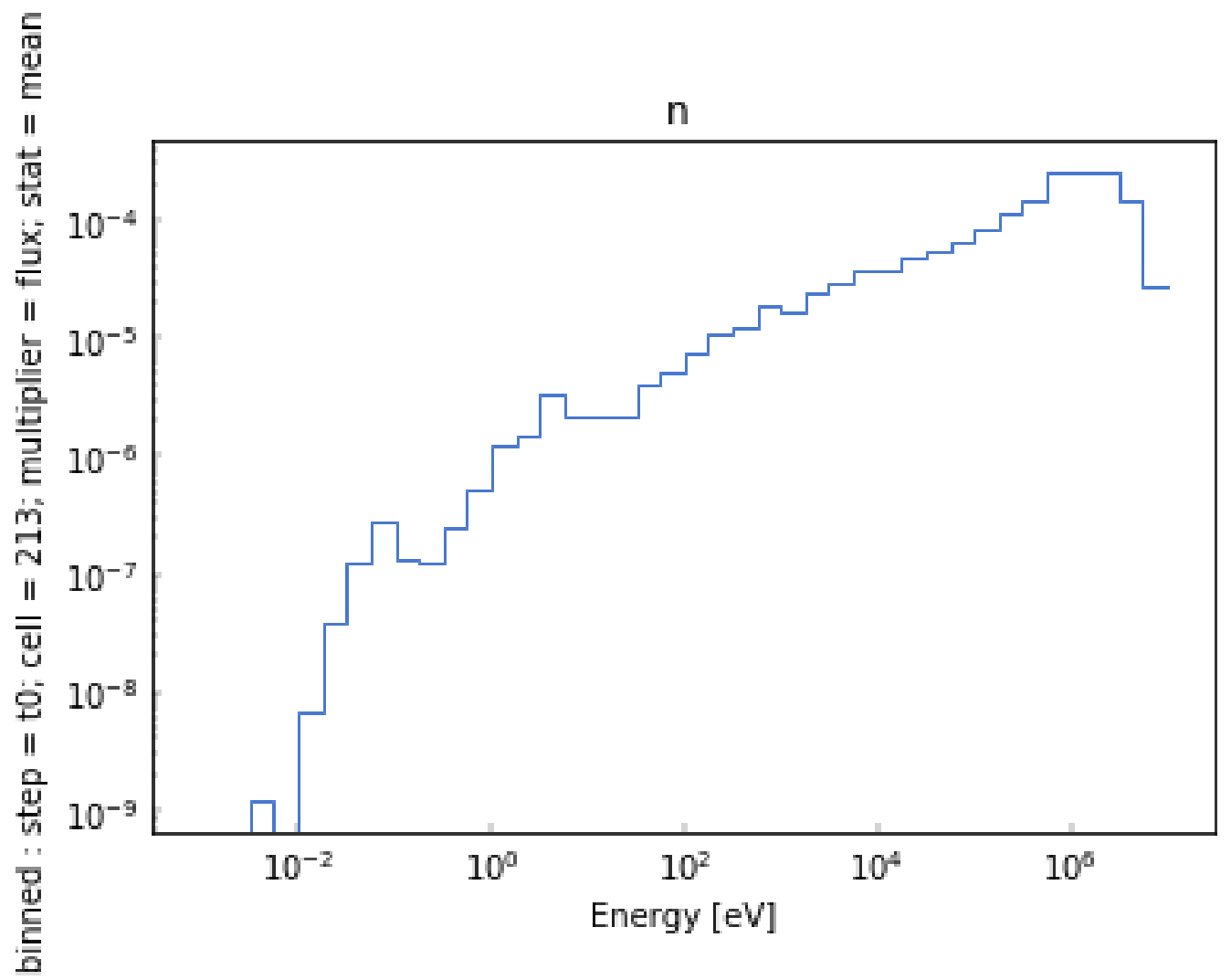

From the spectrum, this is clearly a fast reactor.

In conjunction with the Imager, cell tally results can actually be rendered onto the geometry with a ray trace. Here is a slice of the cells in the geometry at $y=0$ :

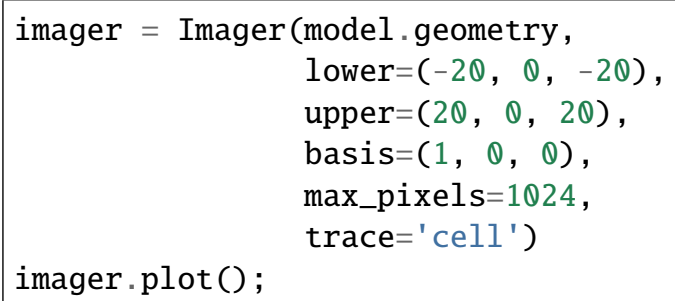




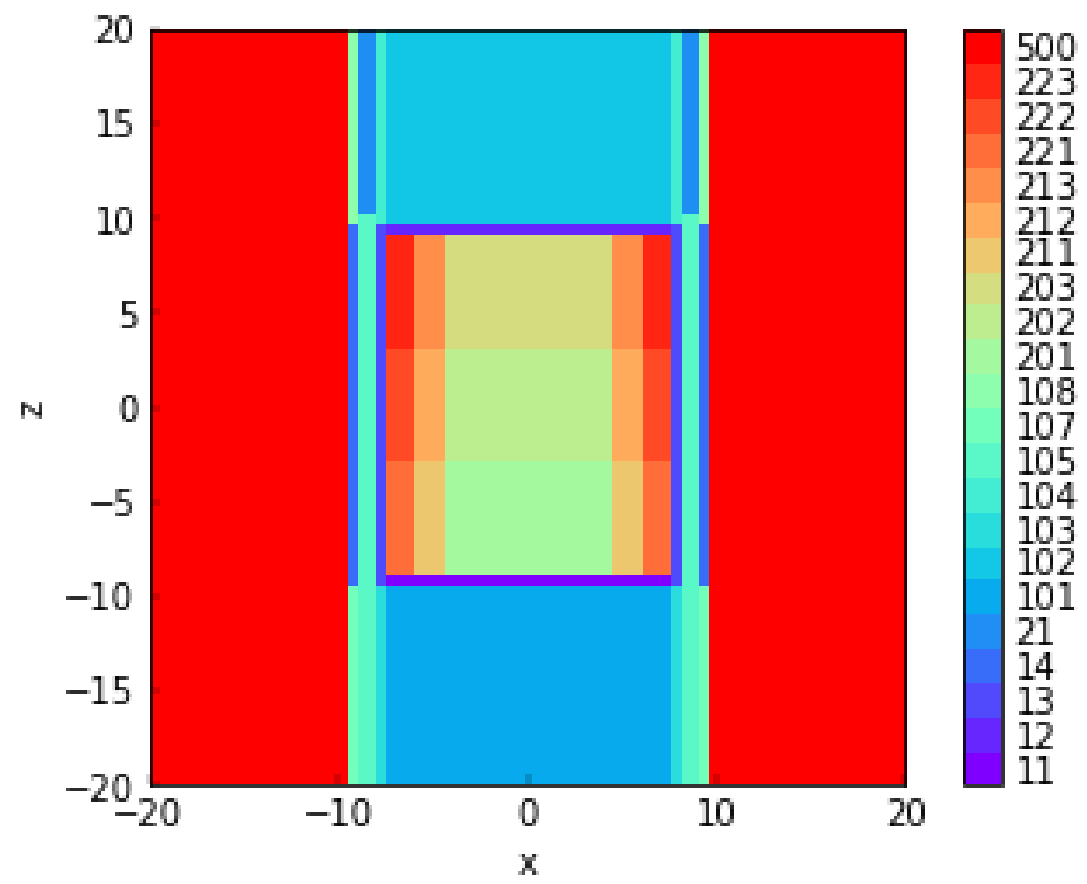

Plotting the reaction rates in each cell requires one-dimensional data (one value per cell), so here are the energy-integrated fluxes at one particular time step.

cell_production $=$ celltal.total.xs(step='t3', stat='mean', multiplier='nu_fission') print(cell_production.selection)

print("Active dimensions:", cell_production.dims)

imager.pcolor(cell_production.data, labels=cell_production.mesh('cell'));

step $=\mathrm{t} 3 ;$ multiplier $=$ nu_fission; stat $=$ mean

Active dimensions: ['cell'] 


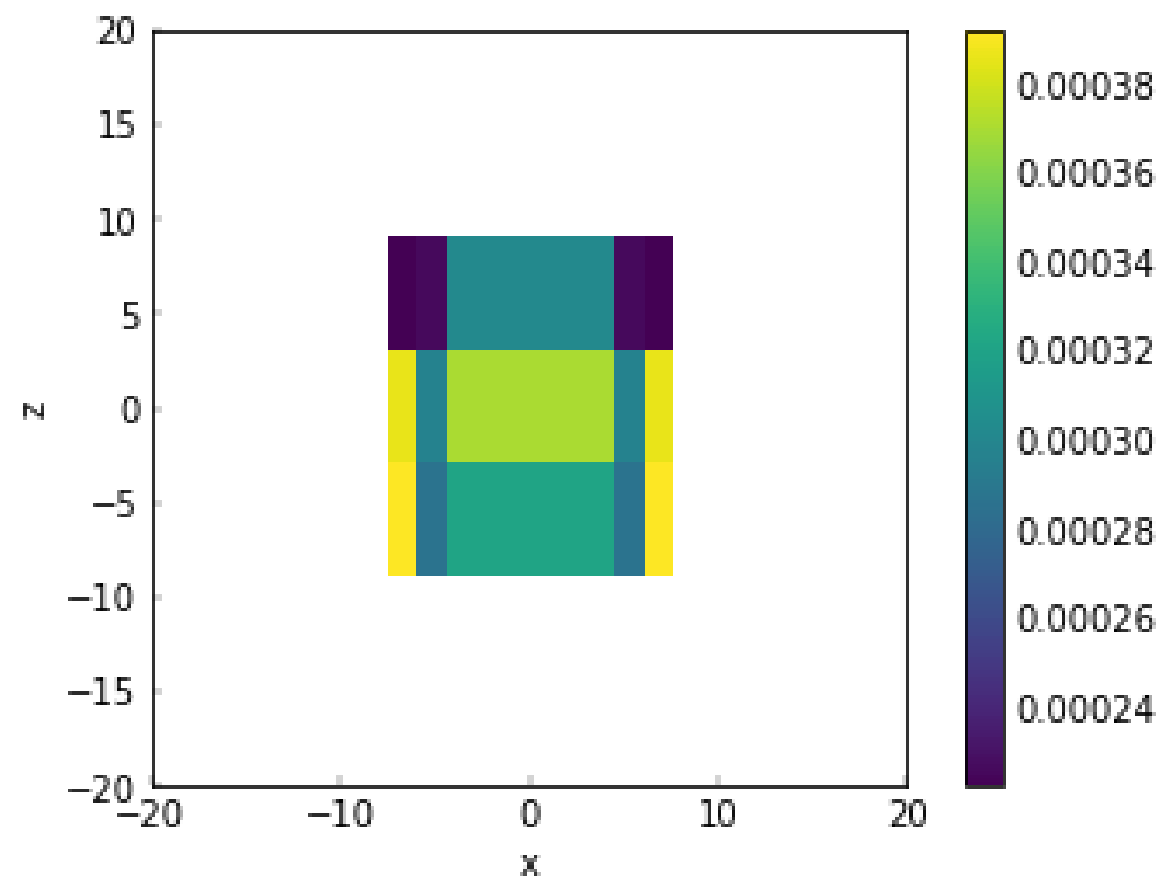

\section{A.4.2.4 Visualize depletion results}

Additional processing and visualization can be peformed from the number densities and one-group cross sections in the depletion output.

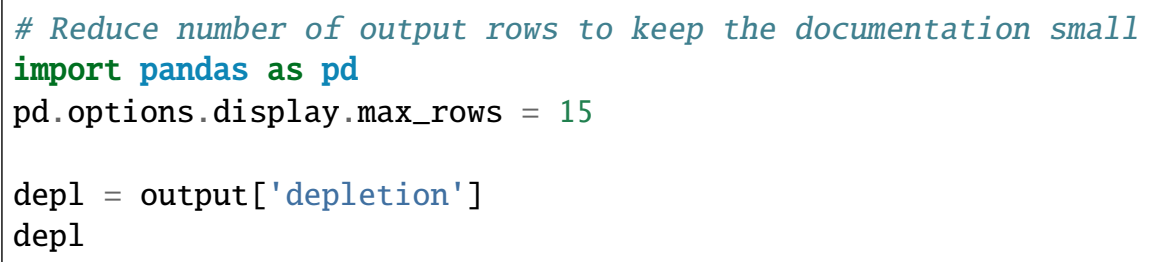

The number density is a function of step, cell, and ZAID. Note that to support restart capability, the "zaid" axis has duplicate entries in it, since ORIGEN tracks some nuclides multiple times. A built-in collapse_nuclides function consolidates these nuclides. Calling this function on a field will pull the entire dataset from disk and return a new field with the collapsed nuclides. This is going to be memory-, disk-, and CPU-intensive, so it is not a bad idea to slice the data first to reduce the amount of work that has to be done if the entire result is not needed.

num_dens = depl.num_dens

num_dens

num_dens = num_dens. collapse_nuclides ()

num_dens

Note that the data entry of the initial num_dens points to an on-disk HDF5 object, whereas after collapsing nuclides the data is an in-memory Numpy array. 
\# Convert xenon densities to a Pandas dataframe

xe_dens = num_dens.xs (zaid=to_nuclide ('xe-135')).to_series ( ).unstack ('cell')

xe_dens

/rnsdhpc/code/build/Exnihilo-examples/Exnihilo/packages/Omnibus/python/omnibus/data/field. $\hookrightarrow$ py:692: FutureWarning: the 'labels' keyword is deprecated, use 'codes' instead index $=$ pd.MultiIndex (levels=levels, labels=labels, names=names)

\# Change indexing from labels to actual simulated time

simtime $=$ depl. simtime $\cdot$ extract ()

xe_dens.index $=$ simtime.data

\# Plot number densities in each cell

$\mathrm{ax}=\mathrm{xe} \_$dens.plot $\left(\right.$marker $={ }^{\prime} \mathrm{x}$ ')

ax.set_ylabel("Xe-135 density ( \{\}$)$ ". format(num_dens.units))

ax.set_xlabel("Time ( \{\}$)$ ".format(simtime.units))

\# Plot power

power $=\operatorname{depl} \cdot$ totpower $\cdot \operatorname{extract}()$

$\operatorname{ax} 2=\operatorname{ax} \cdot \operatorname{twinx}()$

ax2.plot (simtime.data, power.data, drawstyle='steps-post', linewidth=2, $\operatorname{color}=(\mathbb{0}, \mathbb{0}, 0, .5)$ )

ax2.set_ylabel ("Power $(\{\})$ ". format (power.units))

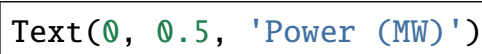

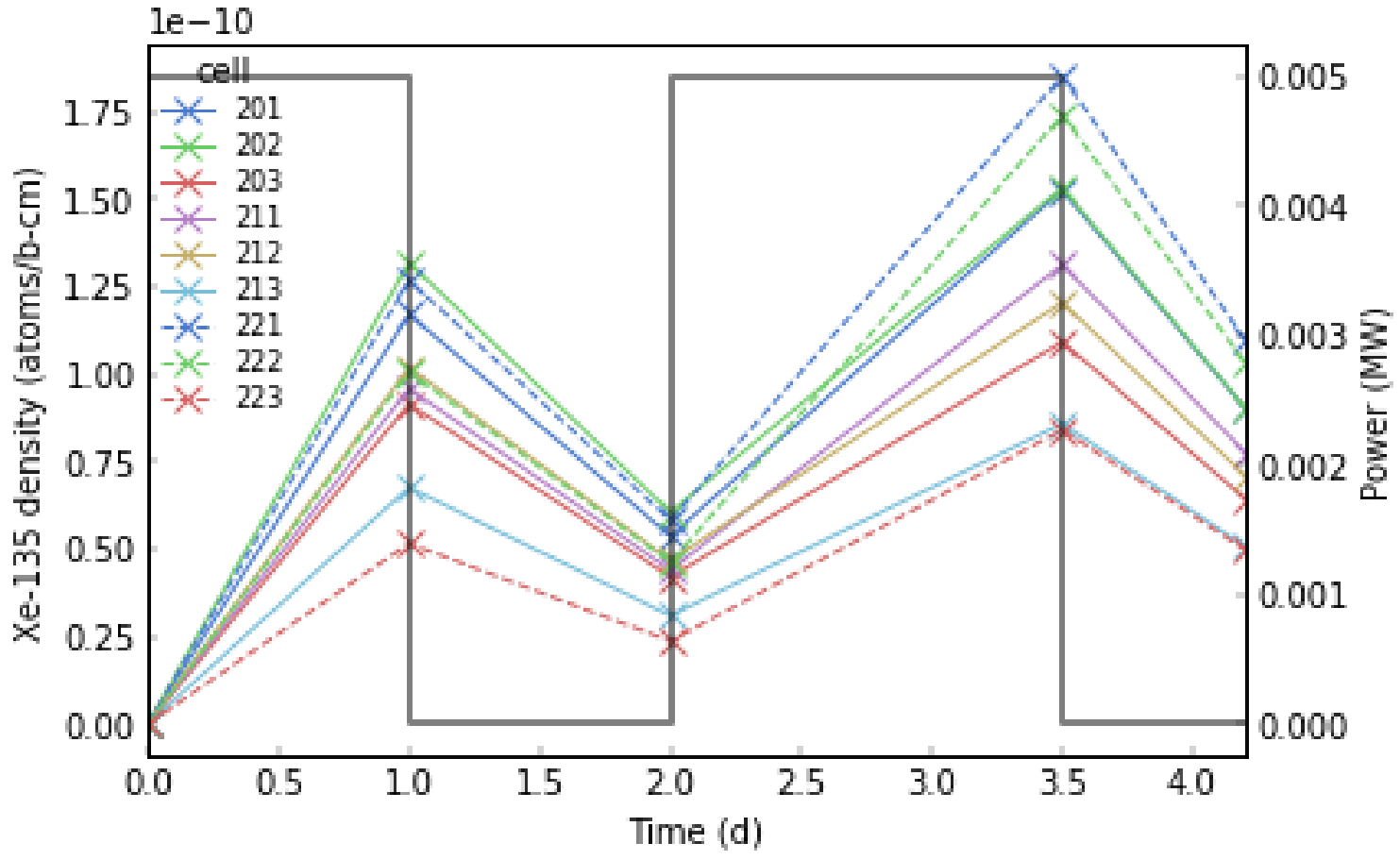

The collapsed cross sections are stored in a single array that holds (ZAID,MT) pairs (since not all nuclides have all reaction types). Because the omnibus. data. Field class is built for only regular multi-dimensional data (no ragged edges), it can be easier to process this field using the Pandas package. The to_series 
function converts a field into a Pandas series. The zaid_mt axis gets expanded into two different "levels" in a pandas. MultiIndex object.

\# Print a table of cross sections, with step/zaid/mt as rows

$\mathrm{xs}=\operatorname{depl}[$ 'xs'].to_series().unstack('cell')

$\mathrm{xS}$

/rnsdhpc/code/build/Exnihilo-examples/Exnihilo/packages/Omnibus/python/omnibus/data/field. $\hookrightarrow$ py:652: FutureWarning: .labels was deprecated in version 0.24.0. Use .codes instead. $\operatorname{lab}[:]=\operatorname{list}(\operatorname{zip}(* \mathrm{mi} .1 \mathrm{abels}))$

\# Tabulate just the fission cross sections

. $\operatorname{xs}(18$, level='mt ')

\# View U-235 cross sections as a function of time in each of the different cells

_. $\operatorname{xs}(92235$, level='zaid') $\cdot \operatorname{plot}()$;

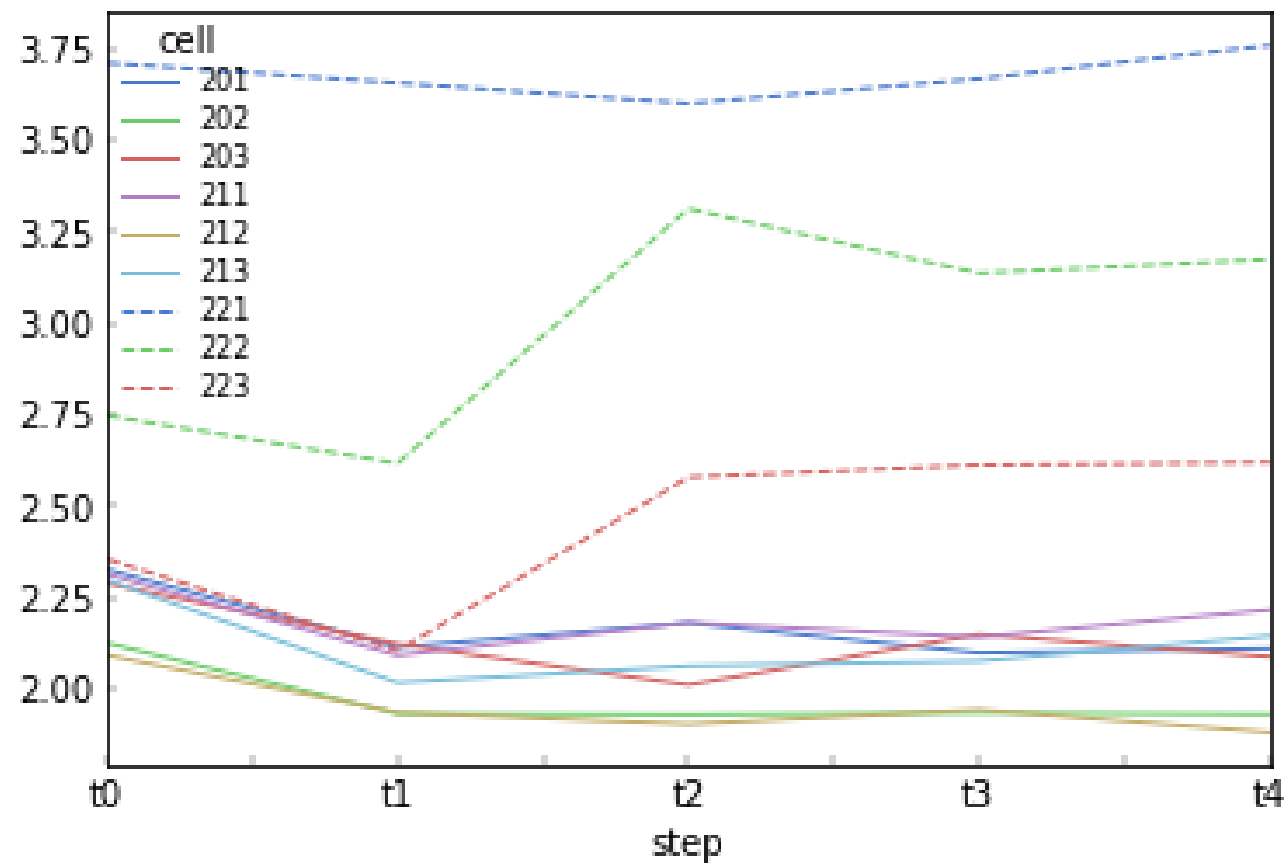

\section{A.4.3 CUSTOM COMPOSITIONS IN OMNIBUS}

The Omnibus [COMP] [MATERIAL] block is convenient for defining small numbers of compositions with few nuclides, but it can be tedious to use for more complex inputs, and especially tedious if the compositions are already defined in another input model. This example demonstrates how to extract compositions from an existing SCALE input and use them in an Omnibus problem with a Geometria-based geometry.

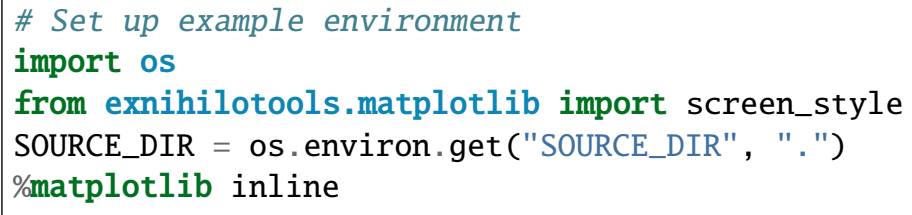




\section{A.4.3.1 Load existing compositions}

Loading the SCALE model provides access to both the geometry definition and the compositions. The _asdict method of a composition object allows it to be rendered as a native Python dictionary for easy perusal.

from omnibus.raytrace.load import load_scale

scale_model = load_scale(os.path.join(SOURCE_DIR, "data", "godiva-keno.inp"))

comps $=$ [c._asdict() for $\mathrm{c}$ in scale_model.compositions]

comps

$\gg$ Using SCALE geometry from 'csas6' sequence.

$\gg$ Loading SCALE Standard Composition Library from/usr/local/scale/data/scale.rev40.

$\hookrightarrow$ sclib

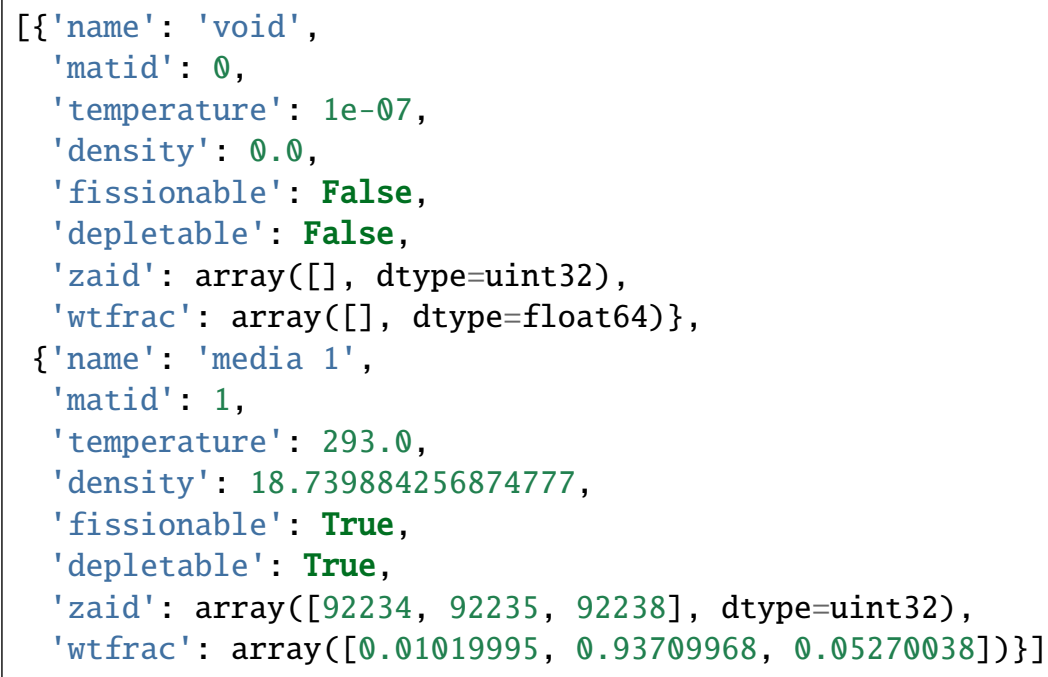

\section{A.4.3.2 Exporting compositions}

The compositions can be immediately saved to disk if they do not need modification:

from robus import save_hdf5_compositions

save_hdf5_compositions("godiva_keno.comp.h5", scale_model.compositions)

Changing the names and ordering of the compositions demonstrate some of the capabilities of the Python representation of the compositions. Writing Python objects to HDF5 requires the Omnibus format wrappers (usually seen when processing existing Omnibus output files).

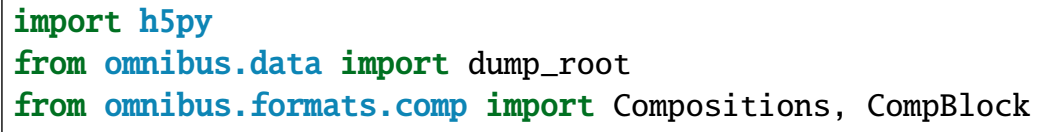


(continued from previous page)

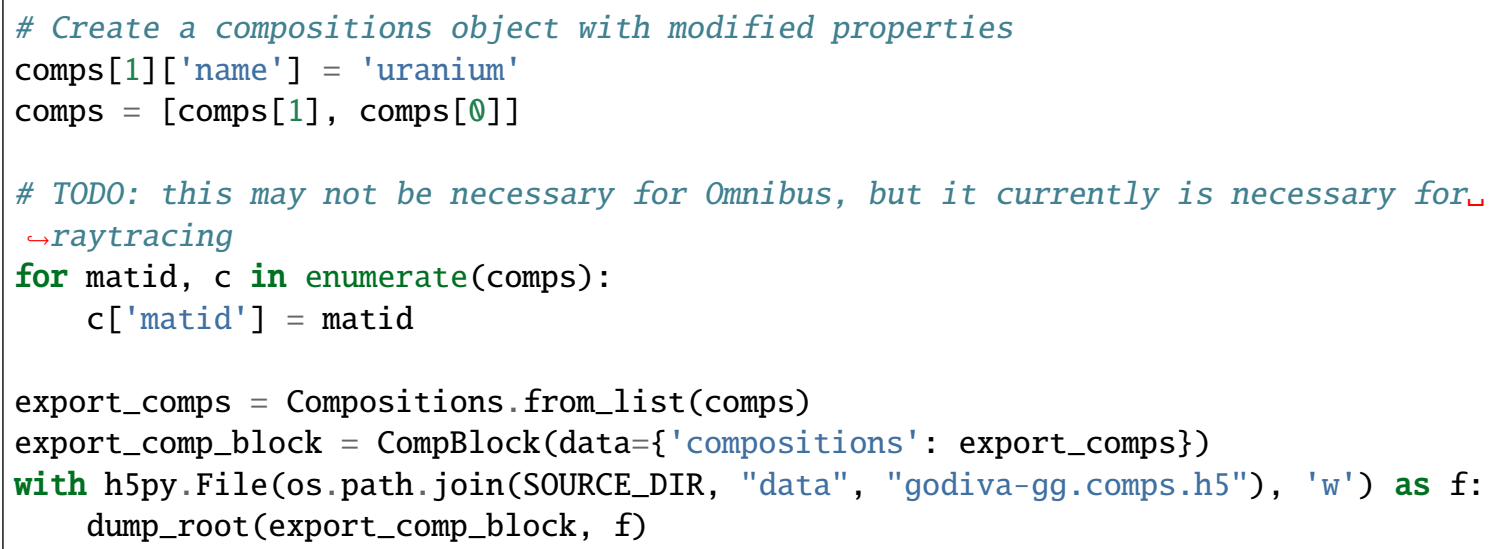

\section{A.4.3.3 Using compositions with GG}

Now that updated compositions have been computed, they can be loaded as part of the Geometria model for raytracing. Although this example uses compositions loaded directly from a SCALE model, it is also possible to directly use compositions loaded from an existing Omnibus HDF5 output file, in which case export_comps here would be replaced by output_file['comp'] ['compositions'].

from omnibus.raytrace.load import load_gg

model = load_gg(os.path.join(SOURCE_DIR, "data", "godiva.gg.omn"), compositions=export_comps)

Generating Geometria XML input file from .gg.omn...

INFO: Starting Geometria preprocessor, omnibus version 6.3.pre-b11 (branch 'comp-example $\hookrightarrow '$ '\#4d64aa1 on 2020APR14)

Loading problem db from Omnibus ASCII file...

Loading Omnibus input file at /rnsdhpc/code/src/scale/Exnihilo/packages/Omnibus/driver/

$\hookrightarrow$ example/data/godiva.gg.omn ...finished loading problem db from Omnibus ASCII file

INFO: Writing Geometria input ParameterList to godiva.gg.xml

...finished generating Geometria XML input file from .gg.omn

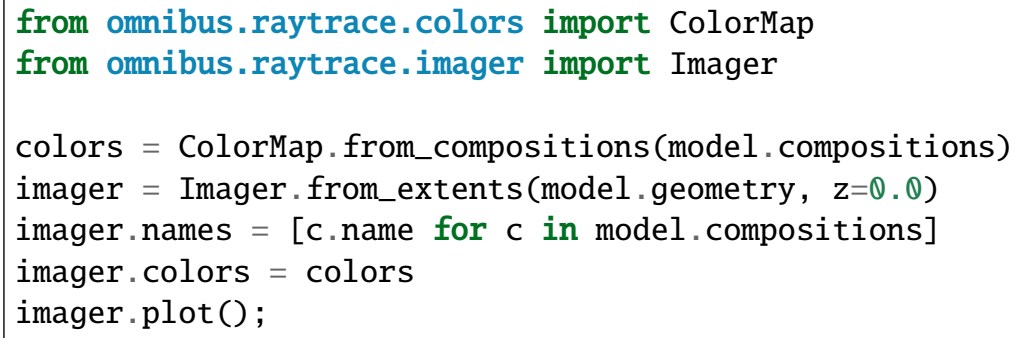




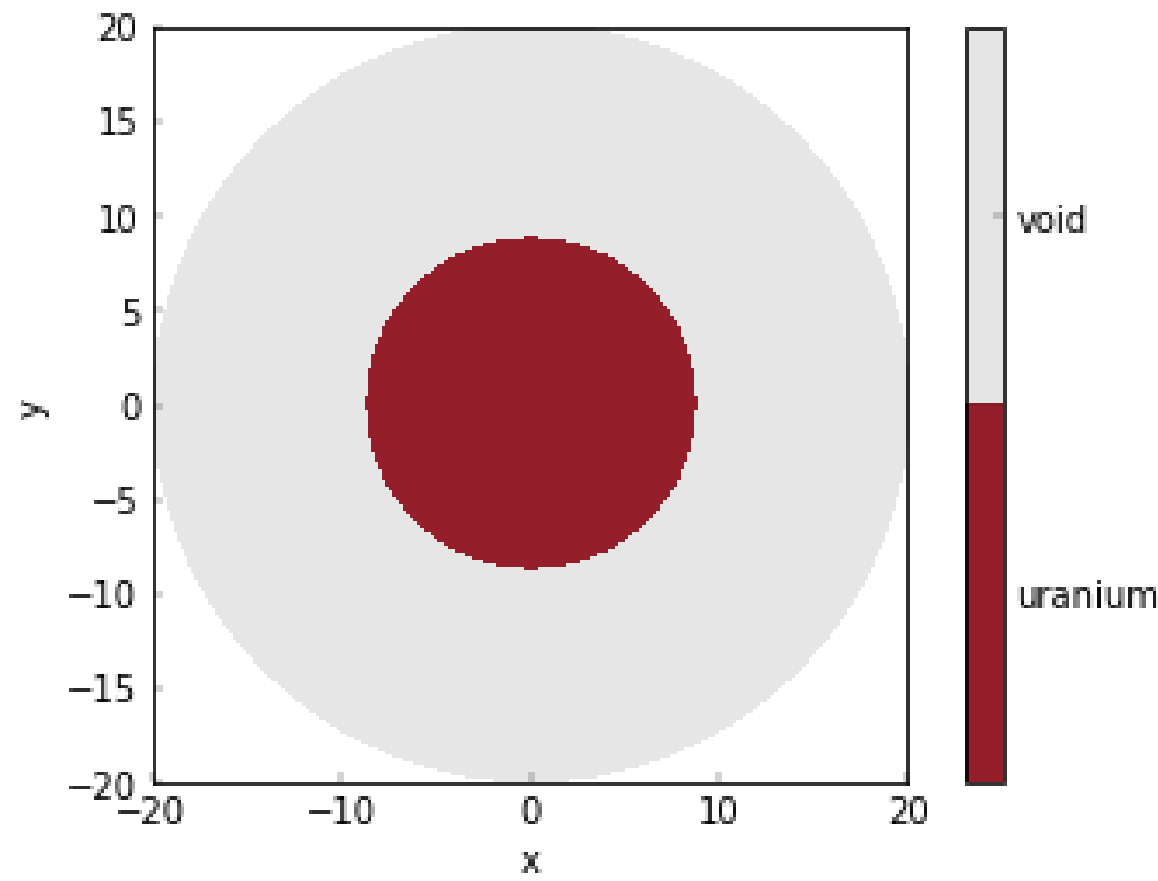

\section{A.5 CE DATA}

\section{A.5.1 INTERACTING WITH CE CROSS SECTION DATA}

This example demonstrates some basic tools for interacting with AMPX-processed continuous energy cross sections.

\# Set up example

\%matplotlib inline

from exnihilotools.matplotlib import screen_style, grid

screen_style()

Providing various options to the Library loader, such as disabling the loading of kinematics data, will reduce data load times and memory consumption. Printing the database after loading shows the defaults for other options not provided in the input database.

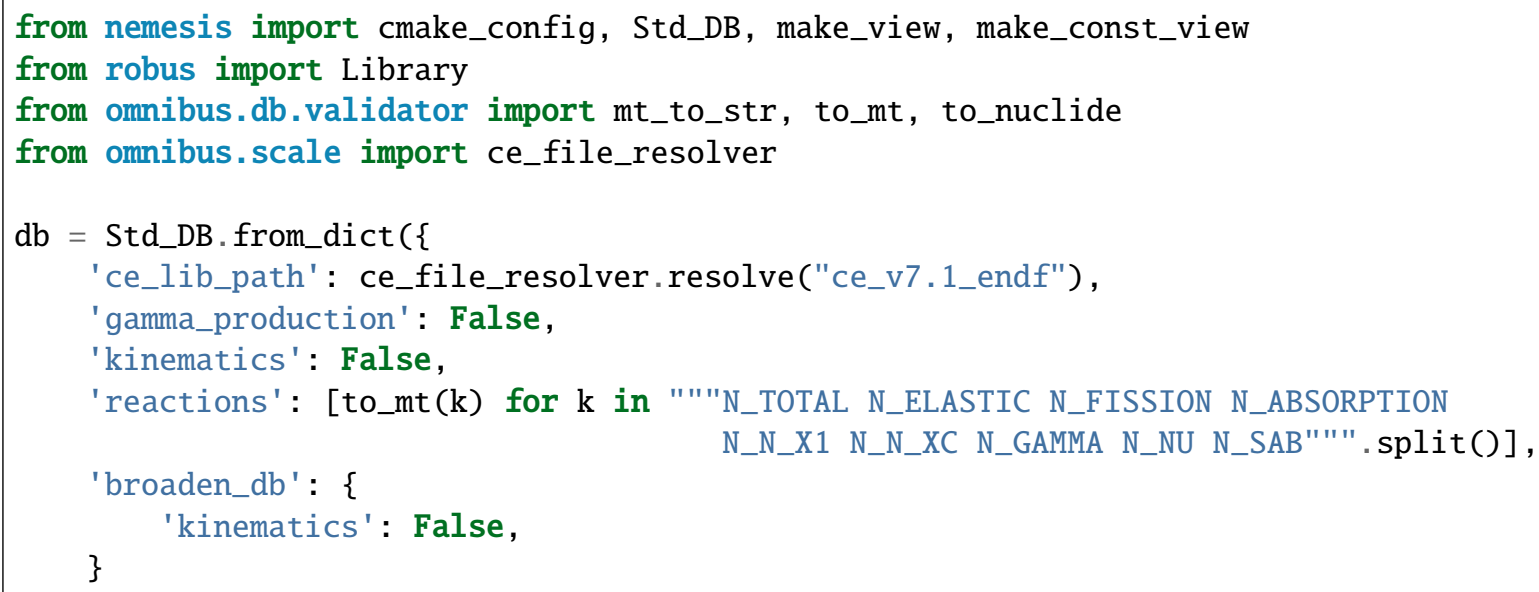


(continued from previous page)

\})

library $=$ Library $(\mathrm{db})$

print $(\mathrm{db})$

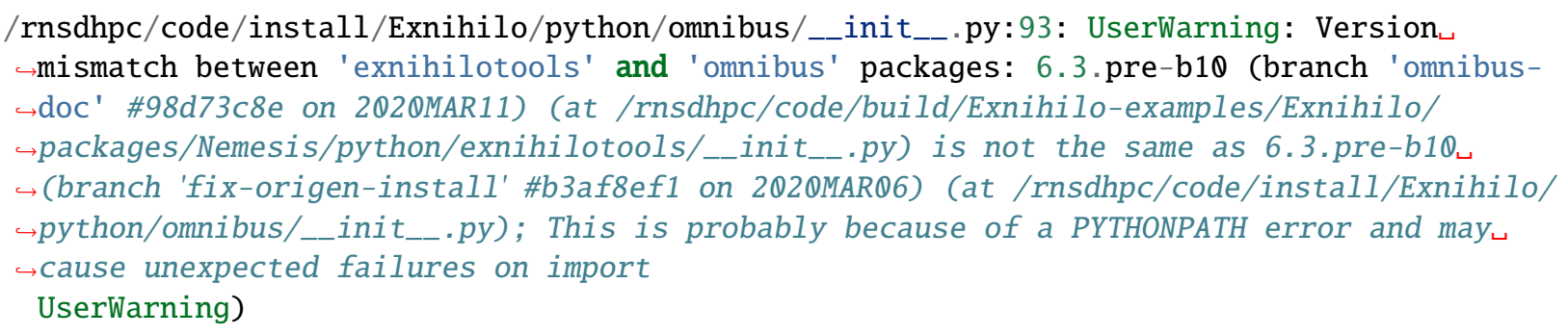


(continued from previous page)

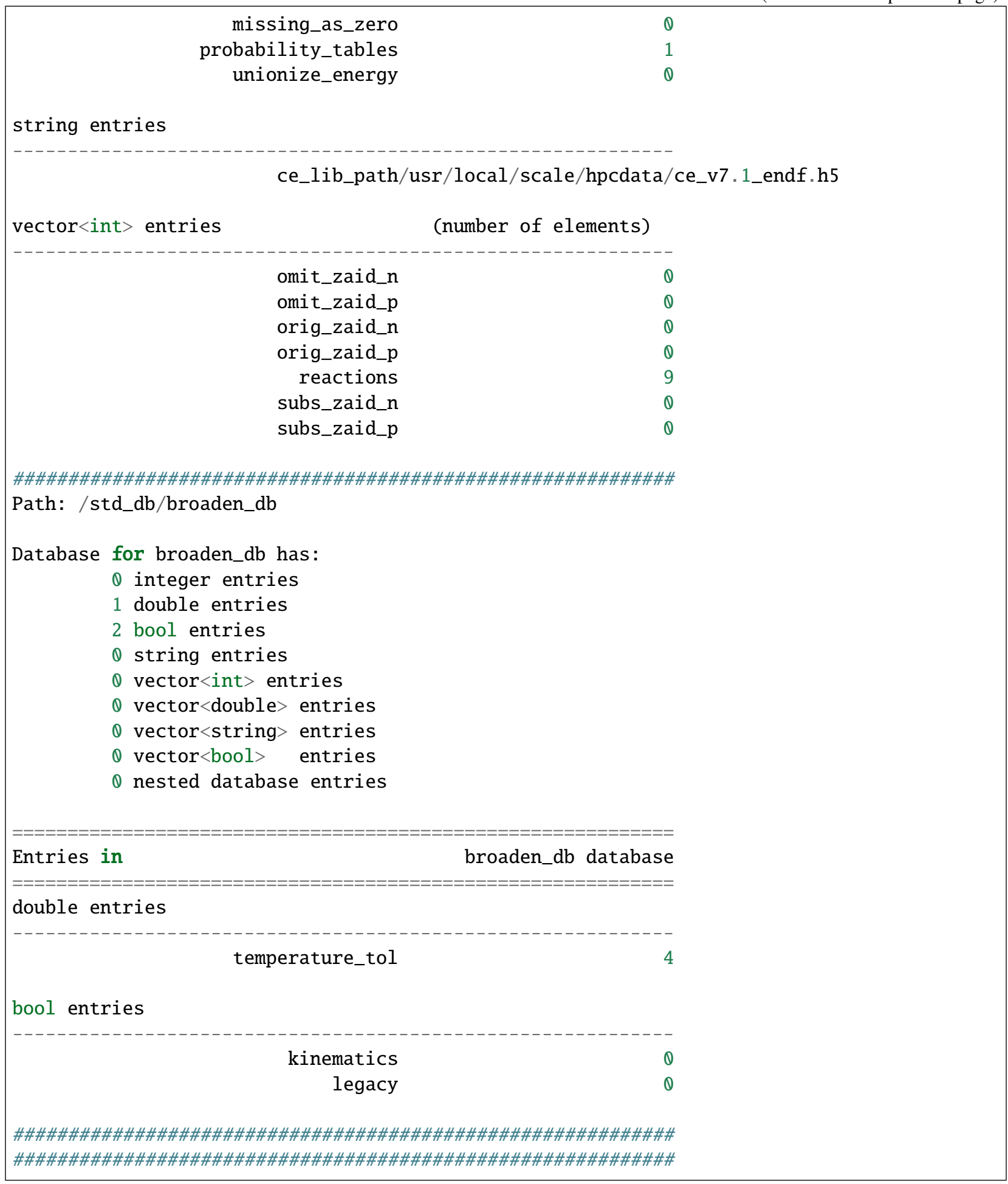

\section{A.5.1.1 Analyze energy grid spacing}

How are the energy grid points distributed for water-bound hydrogen? The Exnihilo python bindings can extract any property of the physics data and manipulate it natively in Python. Here, the ubiquitous Numpy package's powerful math utilities are used to plot a distribution of the relative spacing of the energy grid 
points.
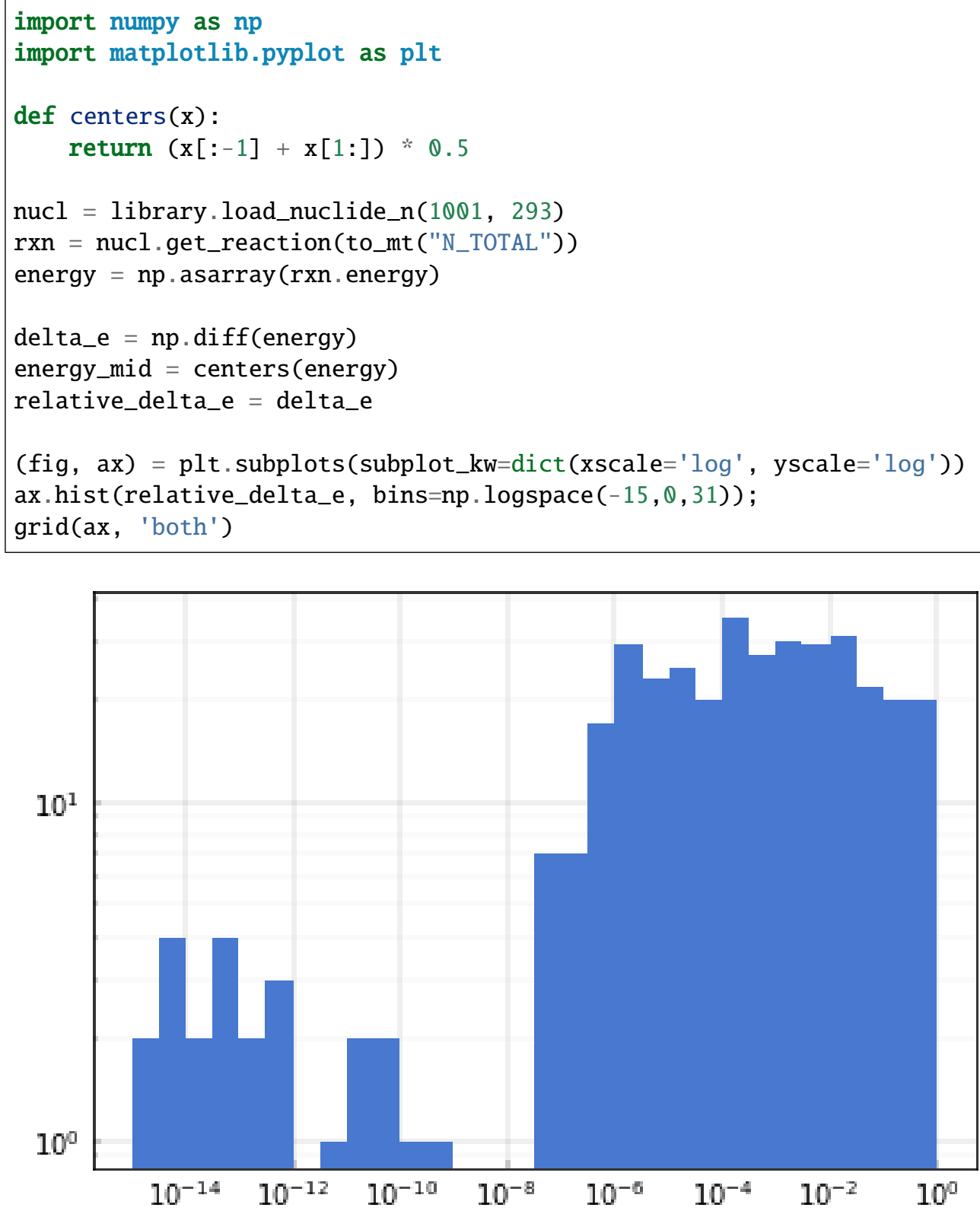

Plotting as a function of energy reveals that the thermal range has lots of micro-sized points, likely a result of AMPX processing of the thermal scattering.

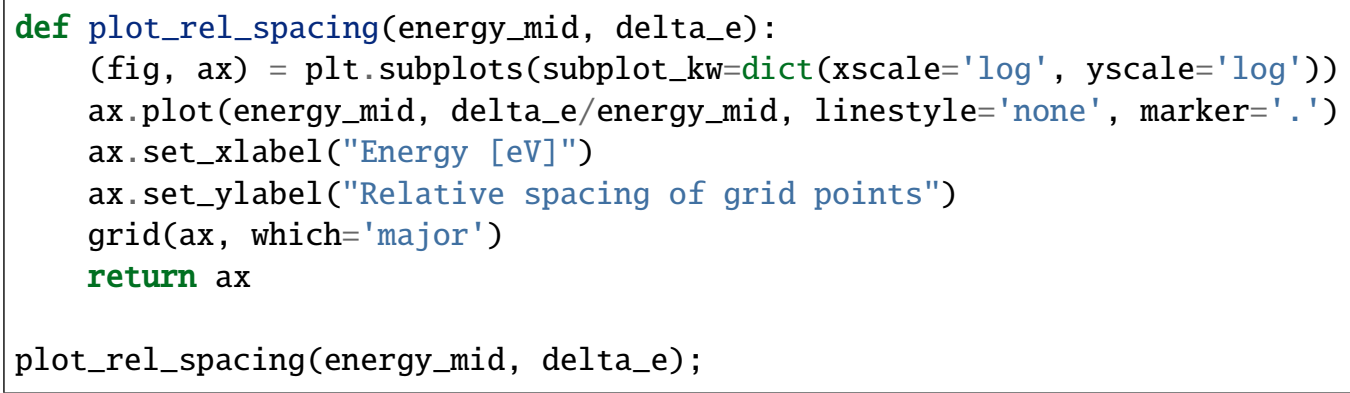




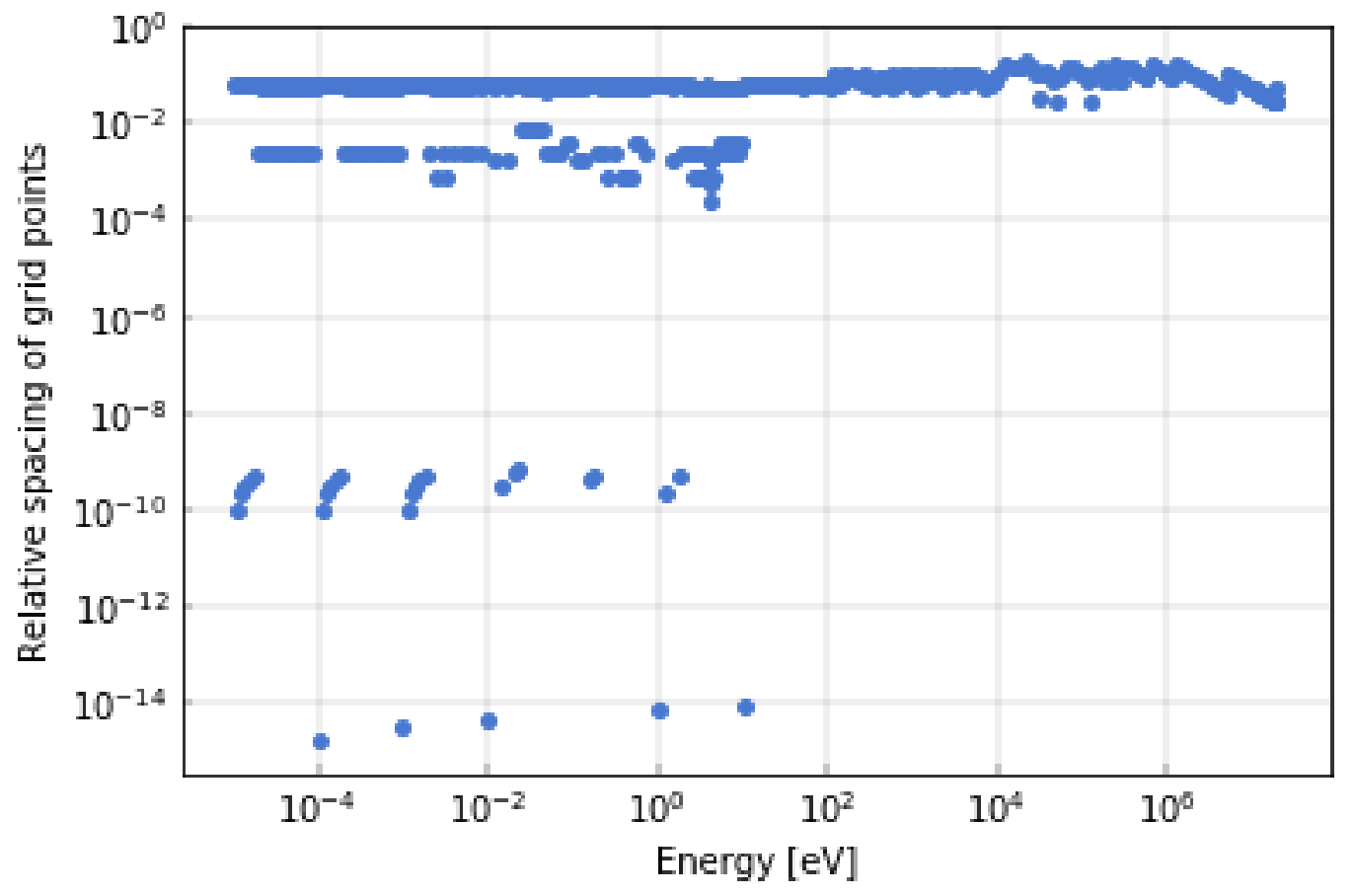

Comparing against free-gas hydrogen confirms that the artifacts are related to the bound cross section treatment:

nucl = library.load_nuclide_n(8001001, 293)

rxn = nucl.get_reaction(to_mt("N_TOTAL"))

energy $=\mathrm{np}$. asarray (rxn.energy)

$\mathrm{ax}=$ plot_rel_spacing(centers(energy), np.diff(energy))

ax.set_ylim(1e-15, 1); 


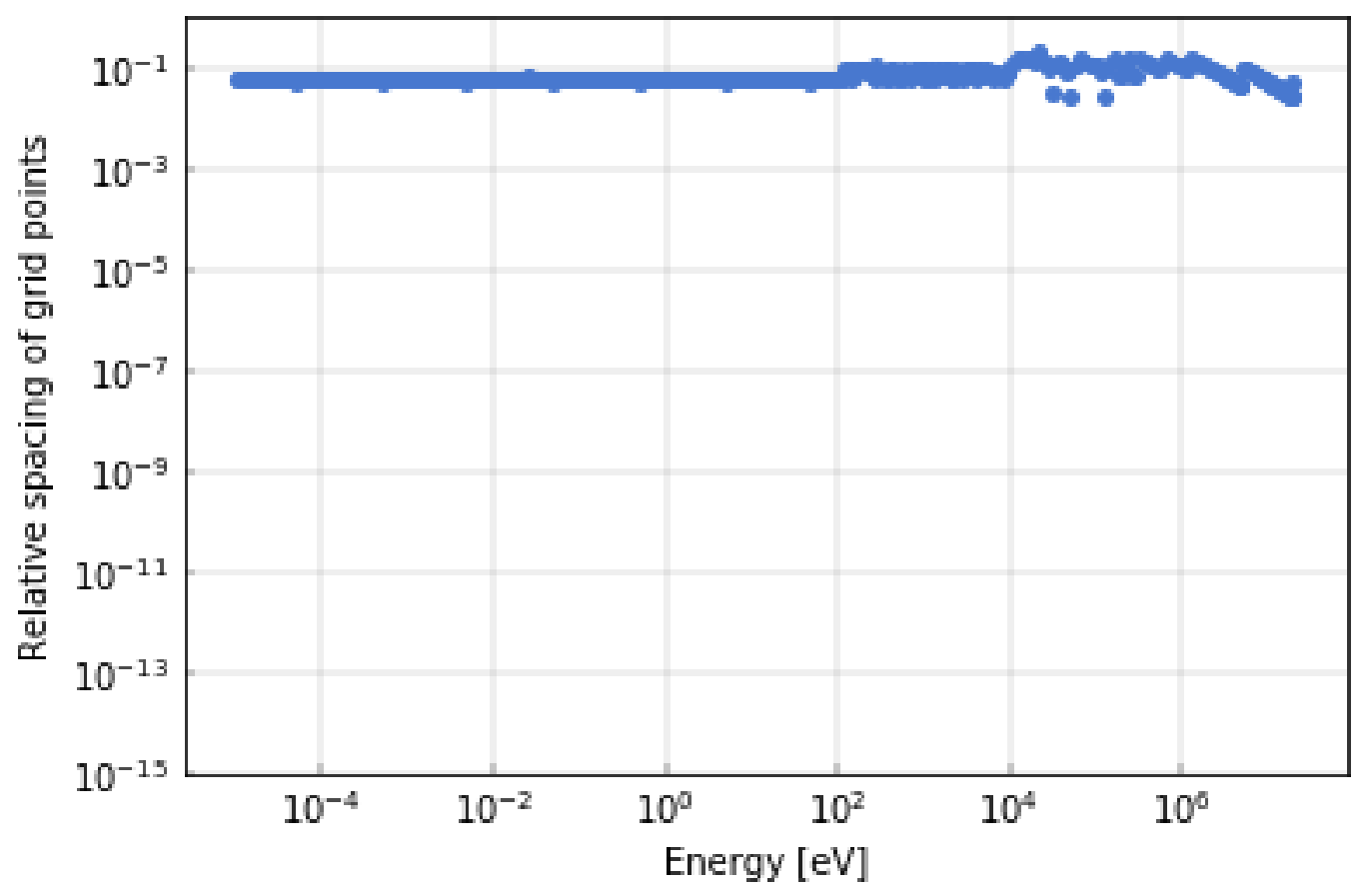

\section{A.5.1.2 Resonance broadening}

This example shows how Doppler broadening affects the cross sections of a large iron resonance. The broaden_db option passed to the Library above enables run-time broadening of cross sections, even if not previously generated for the CE library being used.

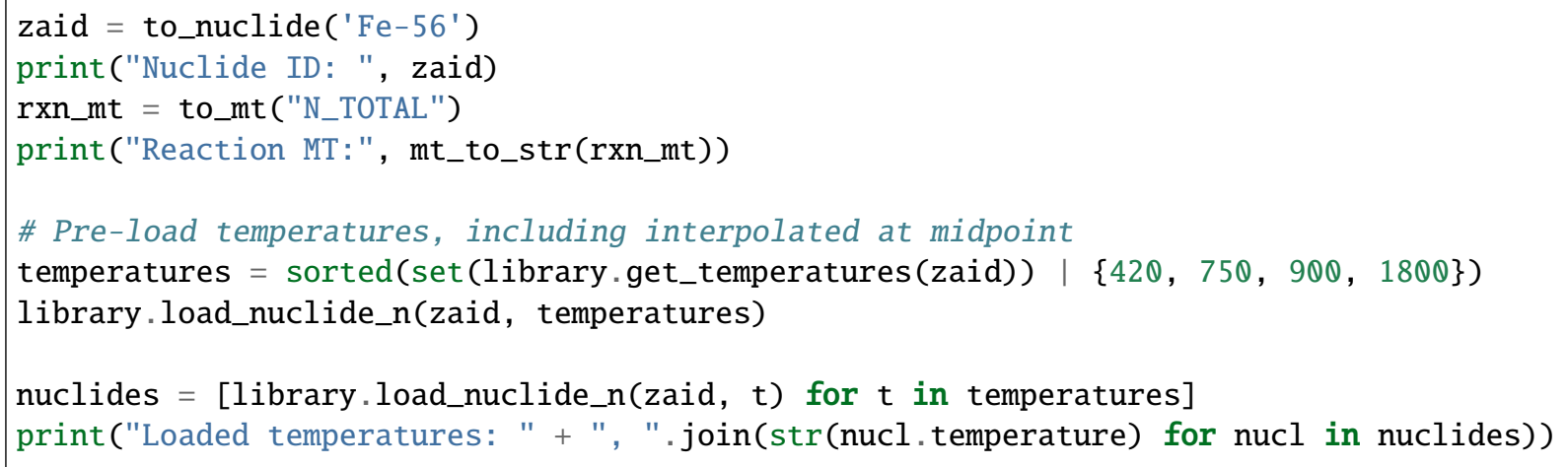

Plot an overall view of the cross sections:

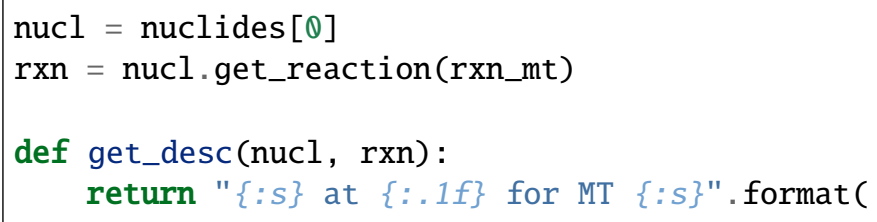


to_nuclide.zaid_to_pretty_nuclide(nucl.zaid), nucl . temperature, to_mt.to_str(rxn.mt))

(fig, ax $)=$ plt. $\operatorname{subplots}()$ ax. $\log \log (r x n . e n e r g y$, rxn.xs, ' - ') ax.set_title(get_desc(nucl, rxn)) $\operatorname{grid}(\mathrm{ax})$

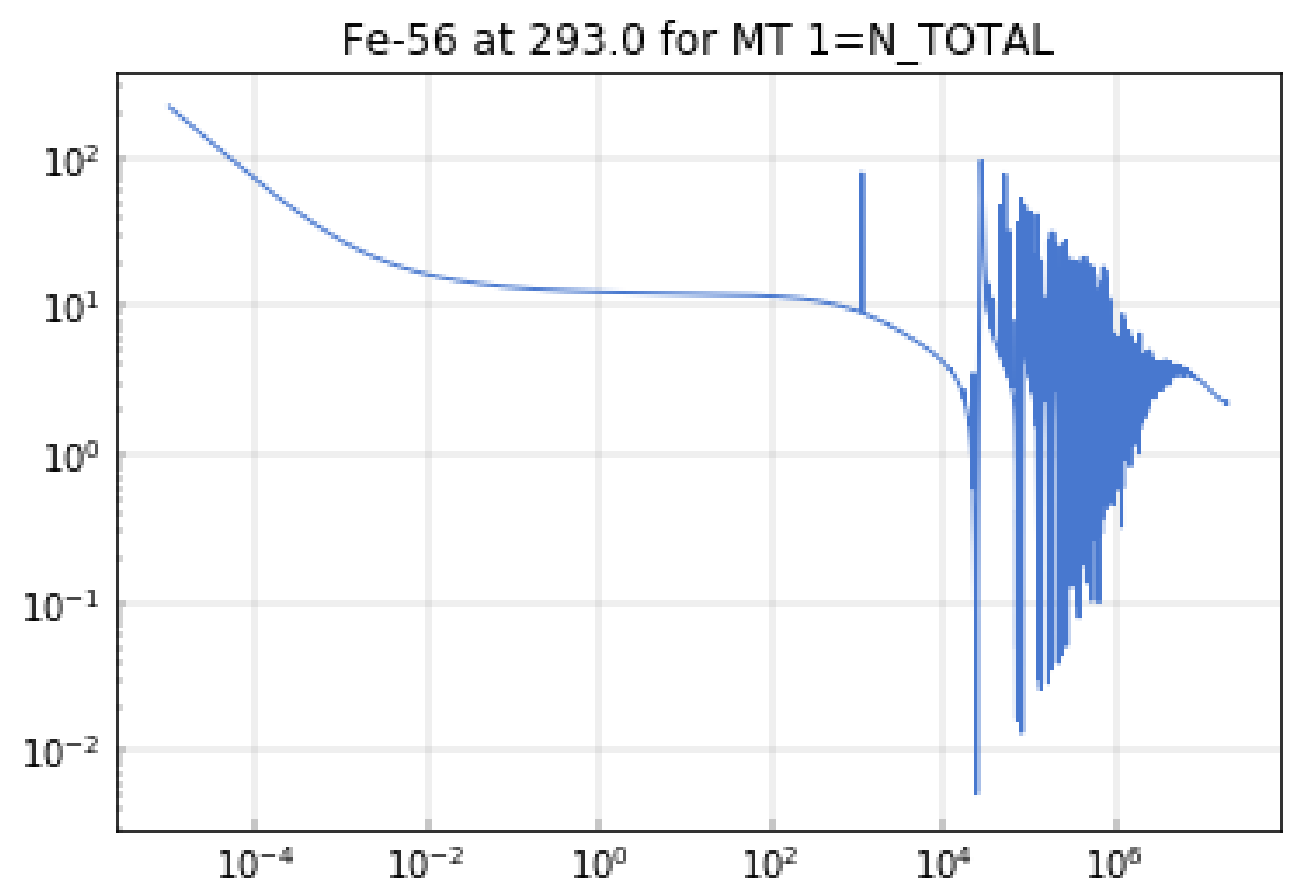

A close-up visualization of the lowest-energy resonance shows how the cross sections vary as a function of temperature:

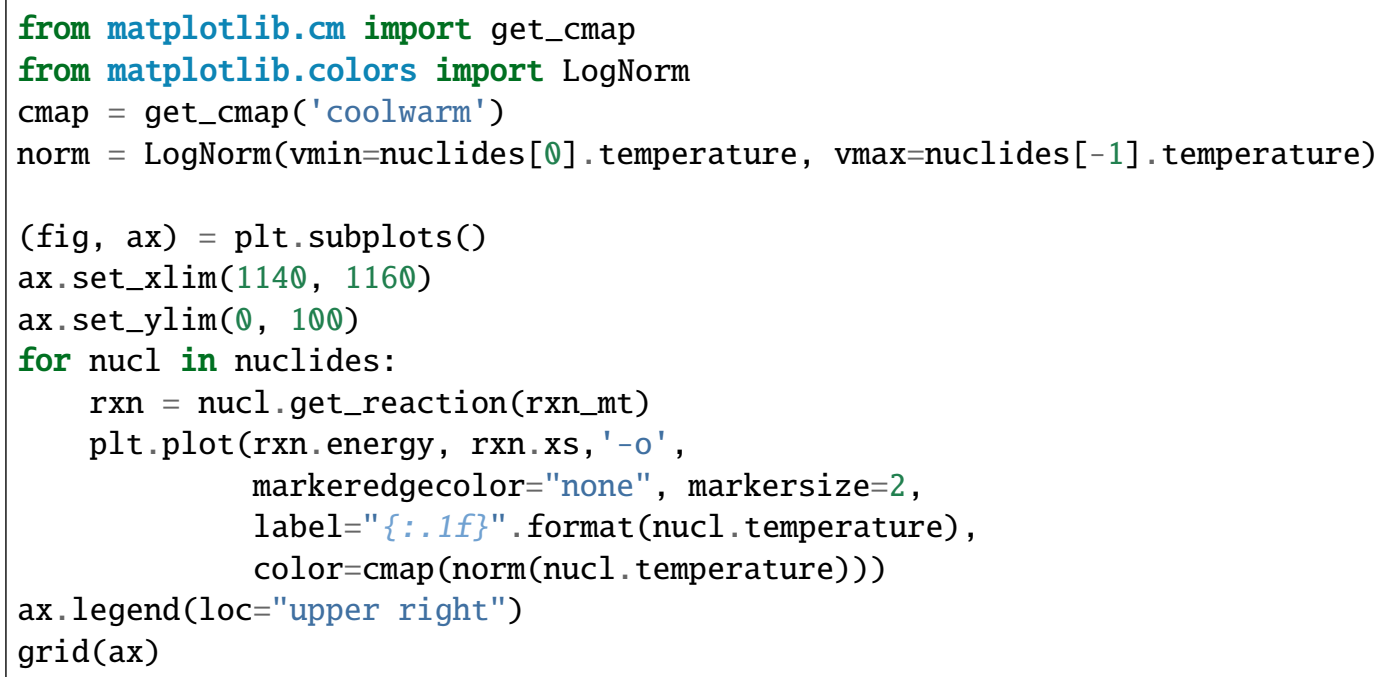




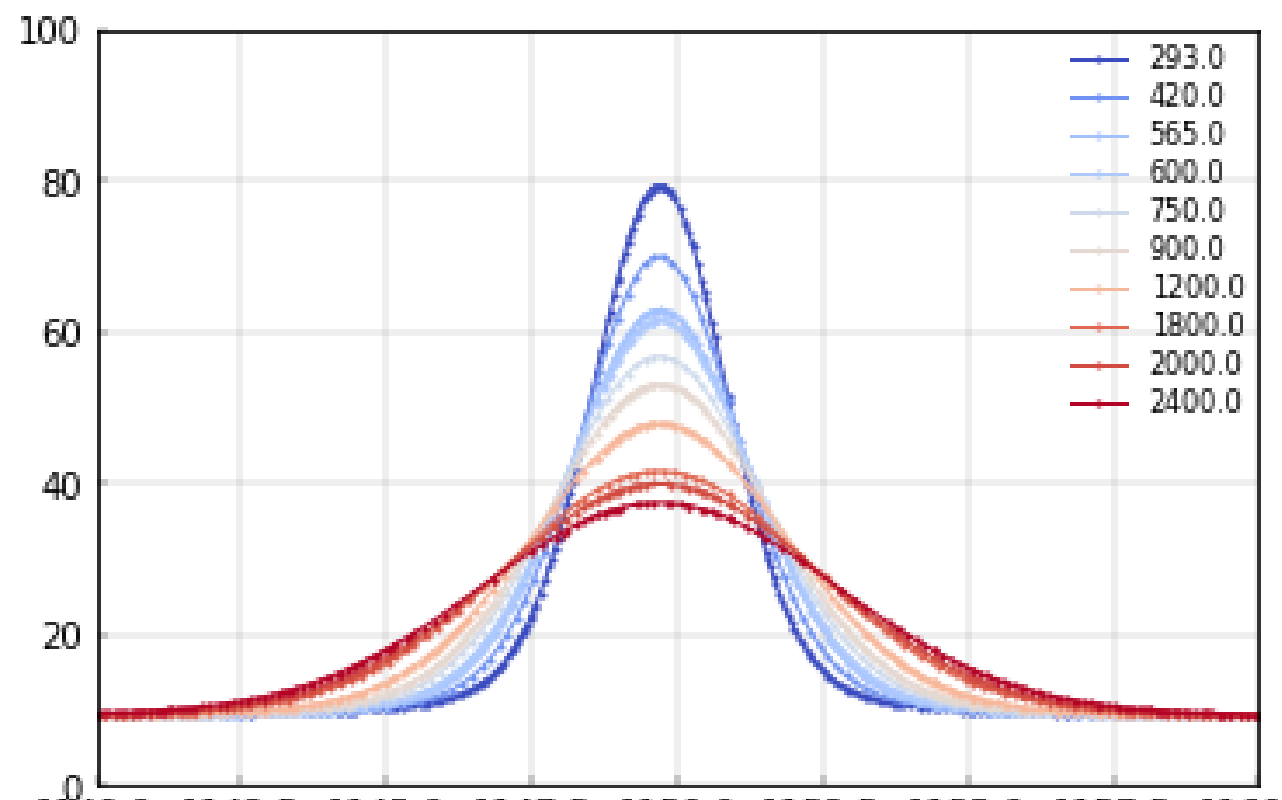

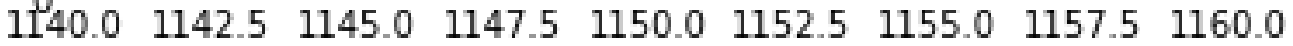

The values at the cross sections at the peaks of these resonances can be found using Numpy:

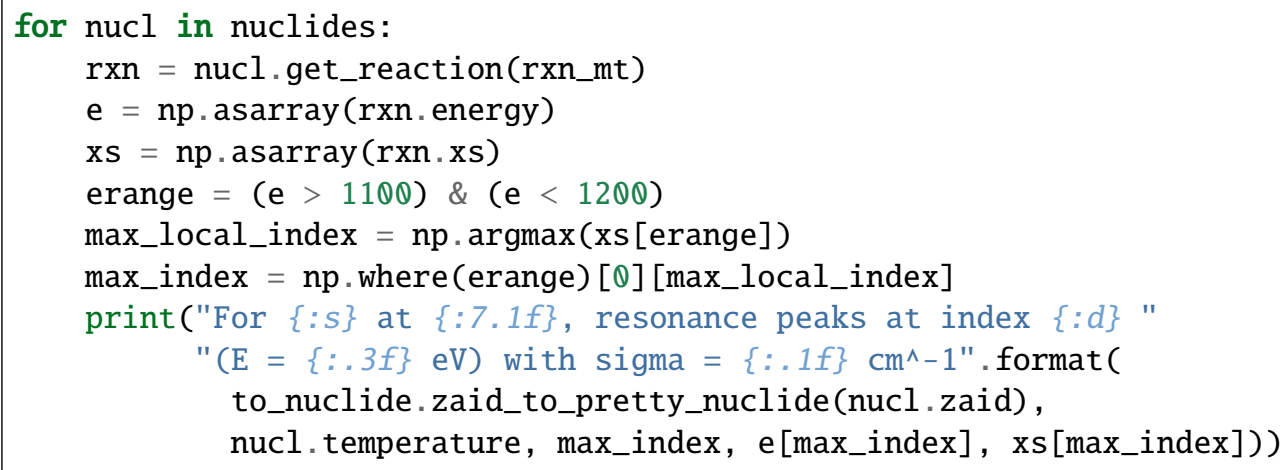

For Fe-56 at 293.0, resonance peaks at index $613(\mathrm{E}=1149.700 \mathrm{eV})$ with sigma $=79.4 \sqcup$ $\hookrightarrow \mathrm{Cm}^{\wedge}-1$

For Fe-56 at 420.0, resonance peaks at index $436(\mathrm{E}=1149.748 \mathrm{eV})$ with $\mathrm{sigma}=70.0_{\sqcup}$ $\hookrightarrow \mathrm{Cm}^{\wedge}-1$

For Fe-56 at 565.0, resonance peaks at index $612(\mathrm{E}=1149.700 \mathrm{eV})$ with $\mathrm{sigma}=62.9 \sqcup$ $\hookrightarrow \mathrm{Cm}^{\wedge}-1$

For $\mathrm{Fe}-56$ at 600.0 , resonance peaks at index $612(\mathrm{E}=1149.700 \mathrm{eV})$ with $\mathrm{sigma}=61.6$ ๖ $\hookrightarrow \mathrm{Cm}^{\wedge}-1$

For Fe-56 at 750.0, resonance peaks at index $436(\mathrm{E}=1149.748 \mathrm{eV})$ with $\mathrm{sigma}=56.8$ ப $\hookrightarrow \mathrm{Cm}^{\wedge}-1$

For $\mathrm{Fe}-56$ at 900.0 , resonance peaks at index $606(\mathrm{E}=1149.700 \mathrm{eV})$ with $\mathrm{sigma}=53.2_{\sqcup}$ $\hookrightarrow \mathrm{Cm}^{\wedge}-1$

For Fe-56 at 1200.0, resonance peaks at index $606(\mathrm{E}=1149.700 \mathrm{eV})$ with sigma $=47.9 \textrm{ }$ $\hookrightarrow \mathrm{Cm}^{\wedge}-1$

For Fe-56 at 1800.0 , resonance peaks at index $436(\mathrm{E}=1149.748 \mathrm{eV})$ with sigma $=41.6 \sqcup$ $\hookrightarrow \mathrm{Cm}^{\wedge}-1$

For Fe-56 at 2000.0, resonance peaks at index $614(\mathrm{E}=1149.597 \mathrm{eV})$ with sigma $=39.9 \sqcup$ $\hookrightarrow \mathrm{Cm}^{\wedge}-1$ 
(continued from previous page)

For Fe-56 at 2400.0, resonance peaks at index 617 ( $E=1149.597 \mathrm{eV})$ with sigma $=37.5 \mathrm{~L}$ $\hookrightarrow \mathrm{Cm}^{\wedge}-1$

\section{A.5.1.3 Cross section interpolation}

The Python methods in this notebook directly access the underlying Shift $\mathrm{C}++$ data using Numpy arrays, with no copying, enabling high performance (speed of $\mathrm{C}$ rather than Python) operations on large data sets. This example demonstrates the performance of interpolating the hydrogen cross section onto a 100k-point energy grid.

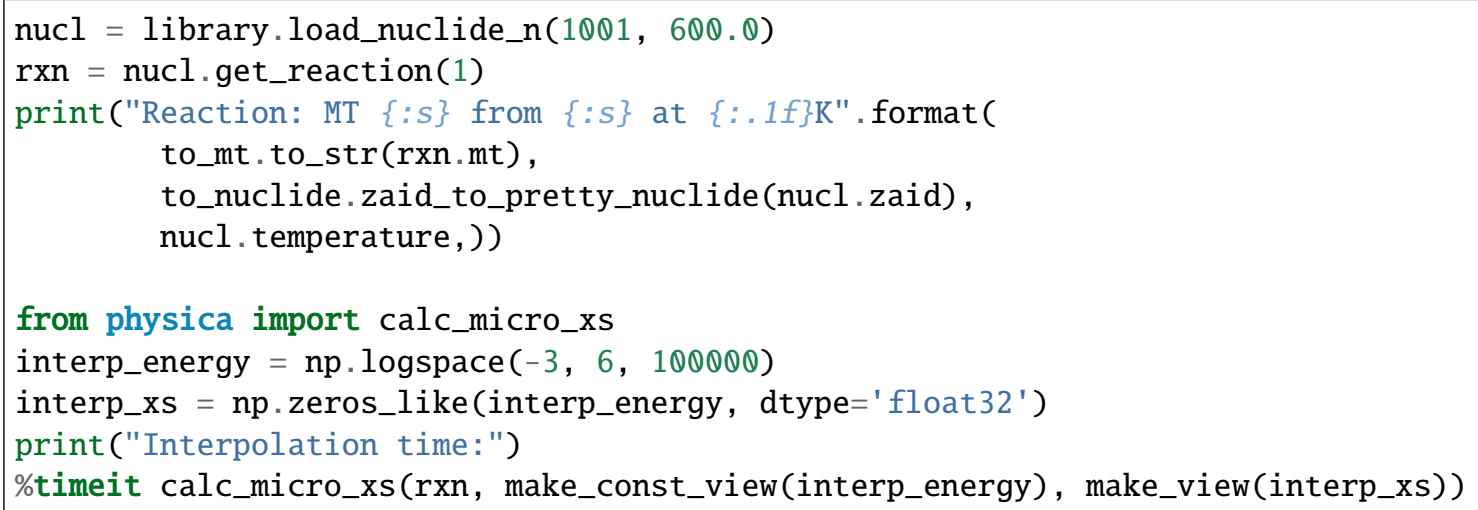

\section{A.5.1.4 Find nonlinear interpolation schemes}

Most of the SCALE cross sections use linear-linear interpolation. To check whether any nonlinear interpolation is used at all, one can loop through all reactions in all nuclides. To reduce load time, gamma production data and kinematics (collision) data are ignored.

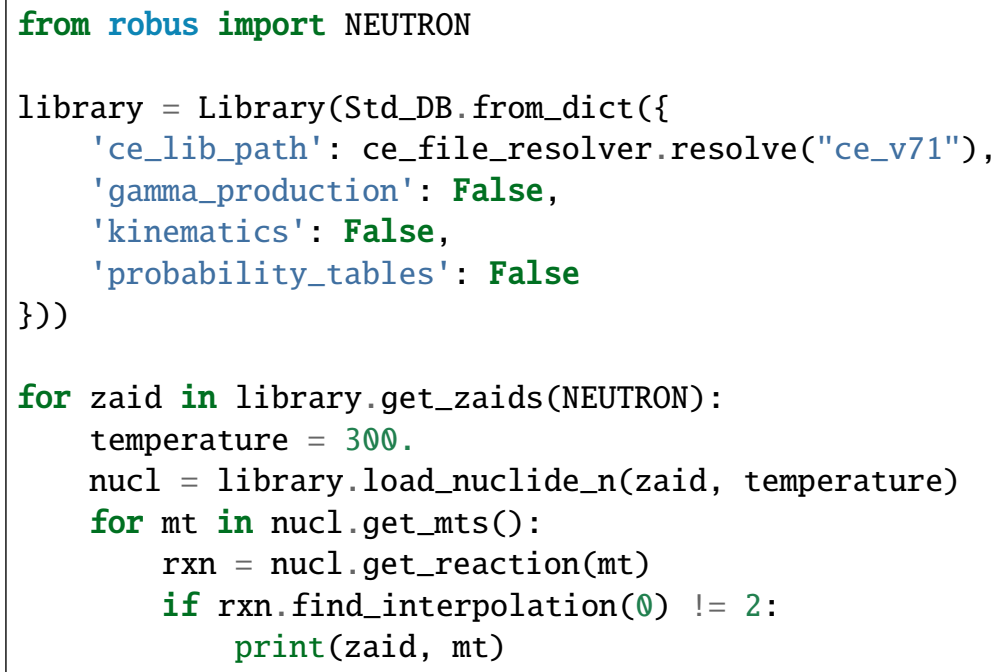


The two nonlinear interpolations are for nu (average neutron yield per fission) in two isotopes of radium!

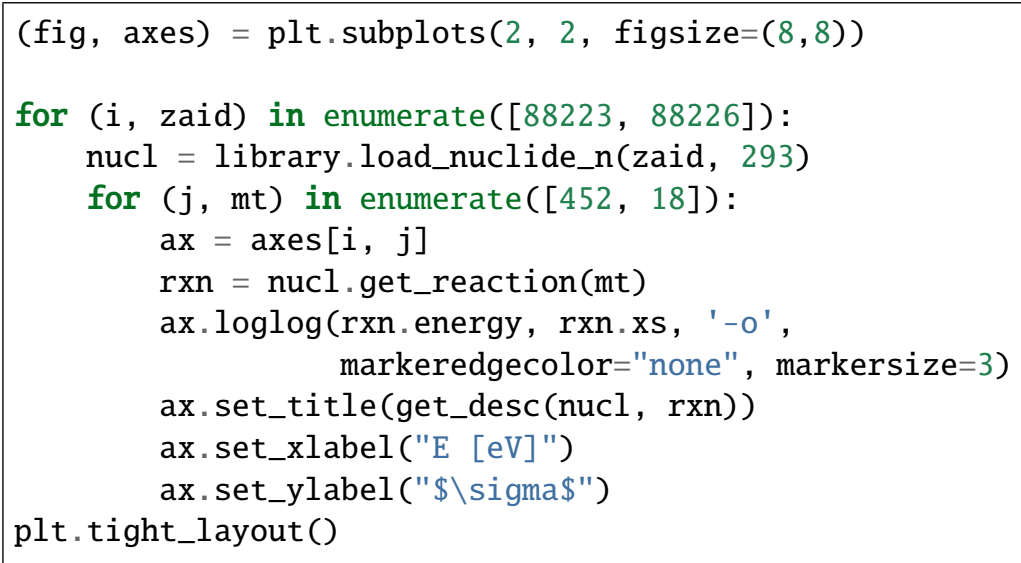



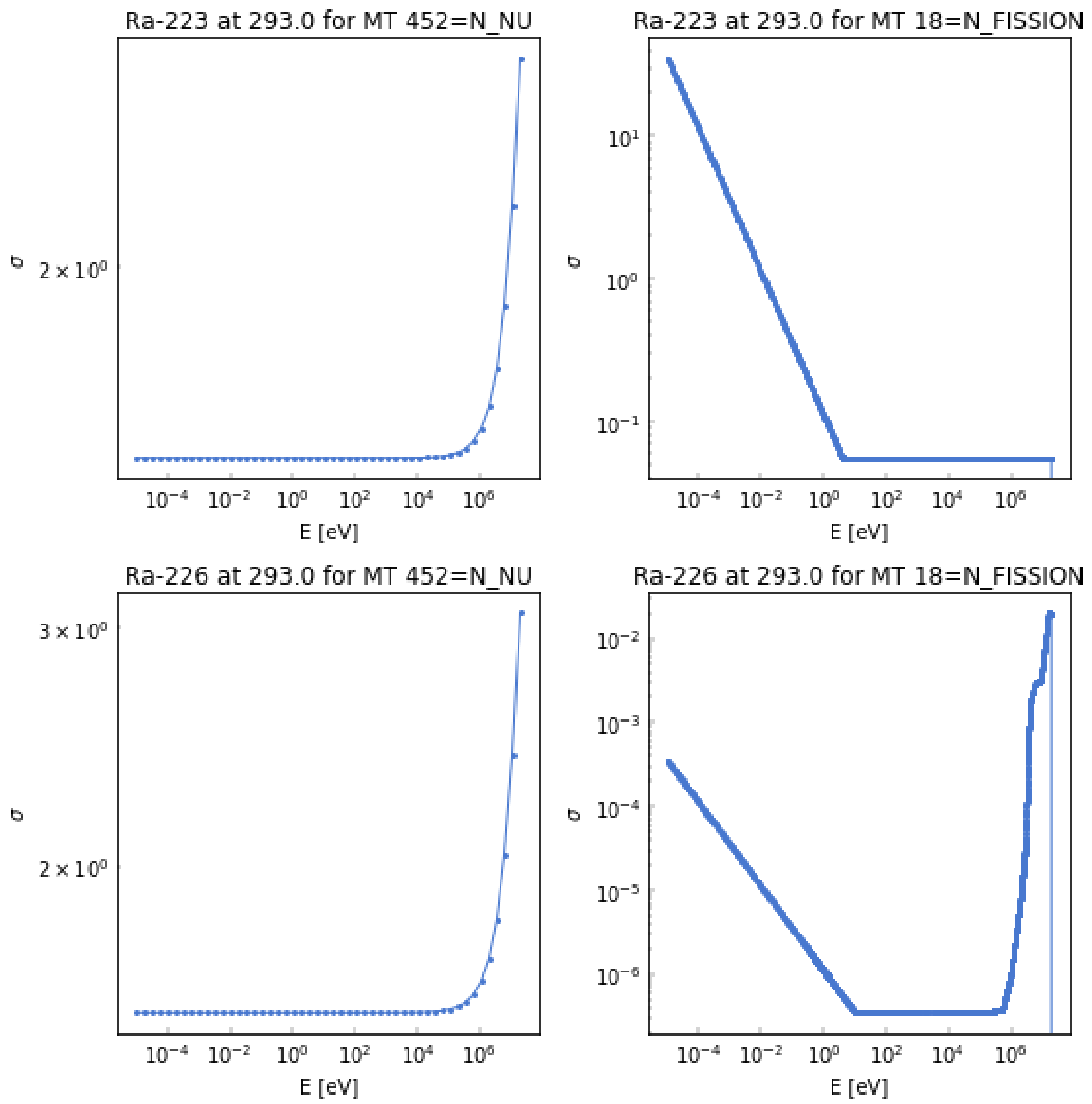

It is exceedingly odd that Radium has fission and production reactions, and that its fission reaction has that obviously nonphysical shape. This has the smell of bad ENDF data.

\section{A.5.1.5 Debug errors during broadening}

During testing of a new feature, Shift developers discovered that the highest few energy grid points in Fe-56 were being broadened strangely, producing large relative errors. Additionally, broadening of MT=5 failed. This section shows some of the steps taken to debug the failure.

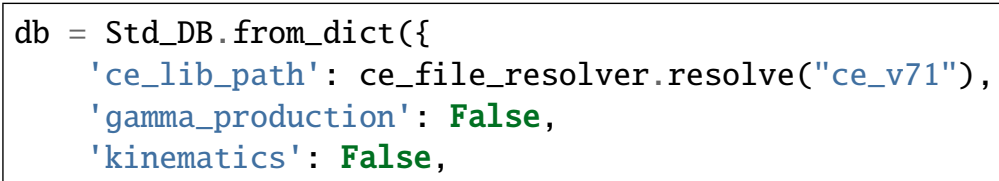


\})

library $=$ Library $(\mathrm{db})$

nucl = library.load_nuclide_n(26056, 300.0)

$\mathrm{rxn}=$ nucl.get_reaction(1)

The cross sections for the highest energies are plotted here:
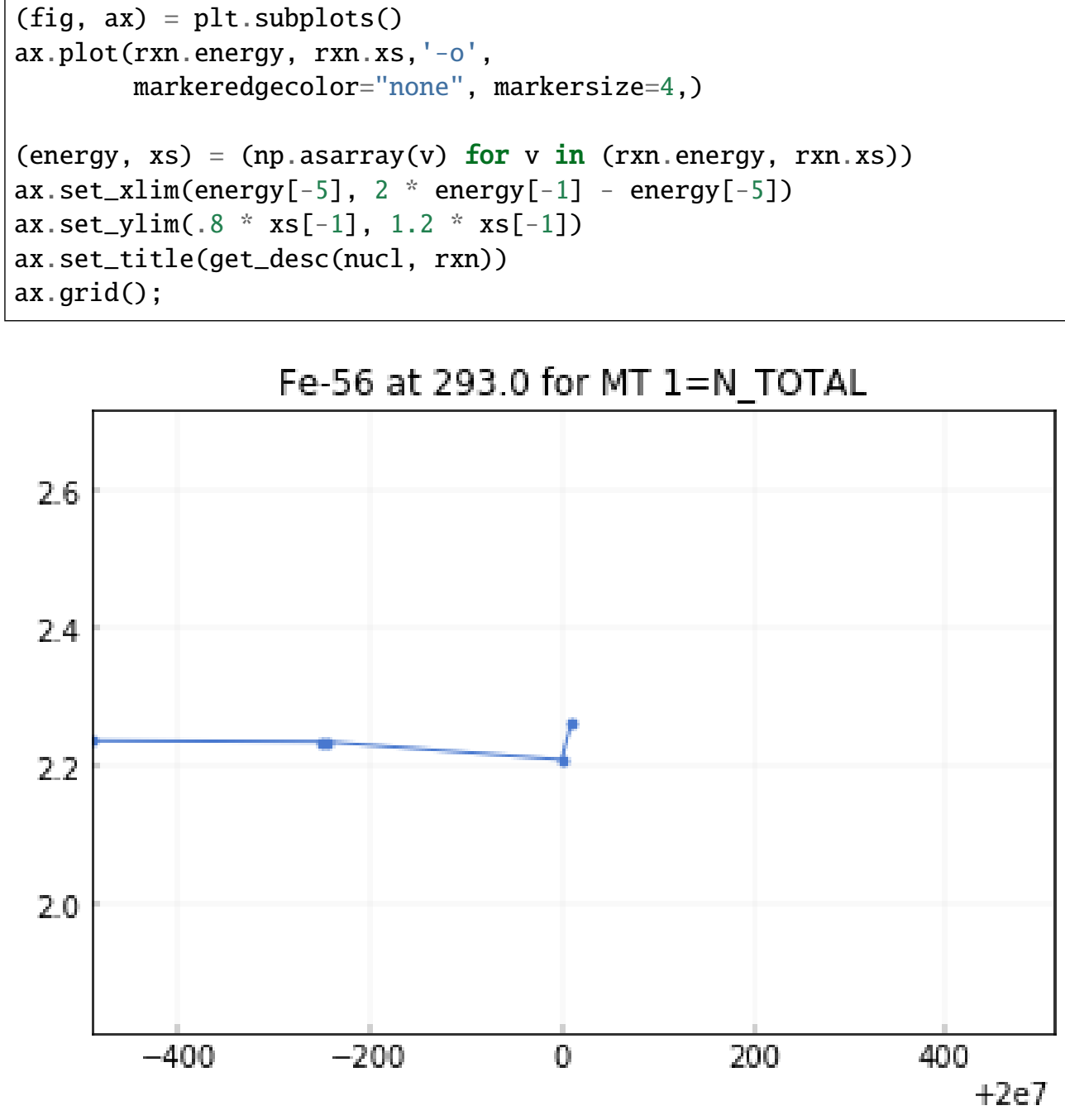

print("Final energy points:", energy[-5:])

Final energy points: [19999508.63125662 19999749.43095107 19999754.3148737920000000. 20000010 . ]

Note that the discontinous point at the end of the cross section is above the maximum requested energy, indicating an error in the CE data processing.

Next let's look at MT 5, which was crashing because it had fewer than $\sim 6$ energy grid points. 


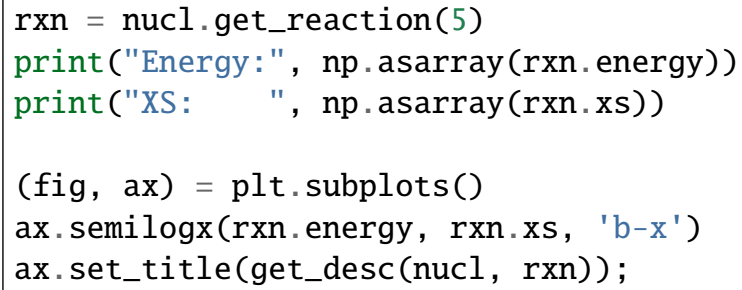

Energy: [1.00000002e+02 2.00000000e+07 2.00000100e+07]

XS: [Q. $0 . \quad 1.29554]$

Fe-56 at 293.0 for MT 5=N_OTHER

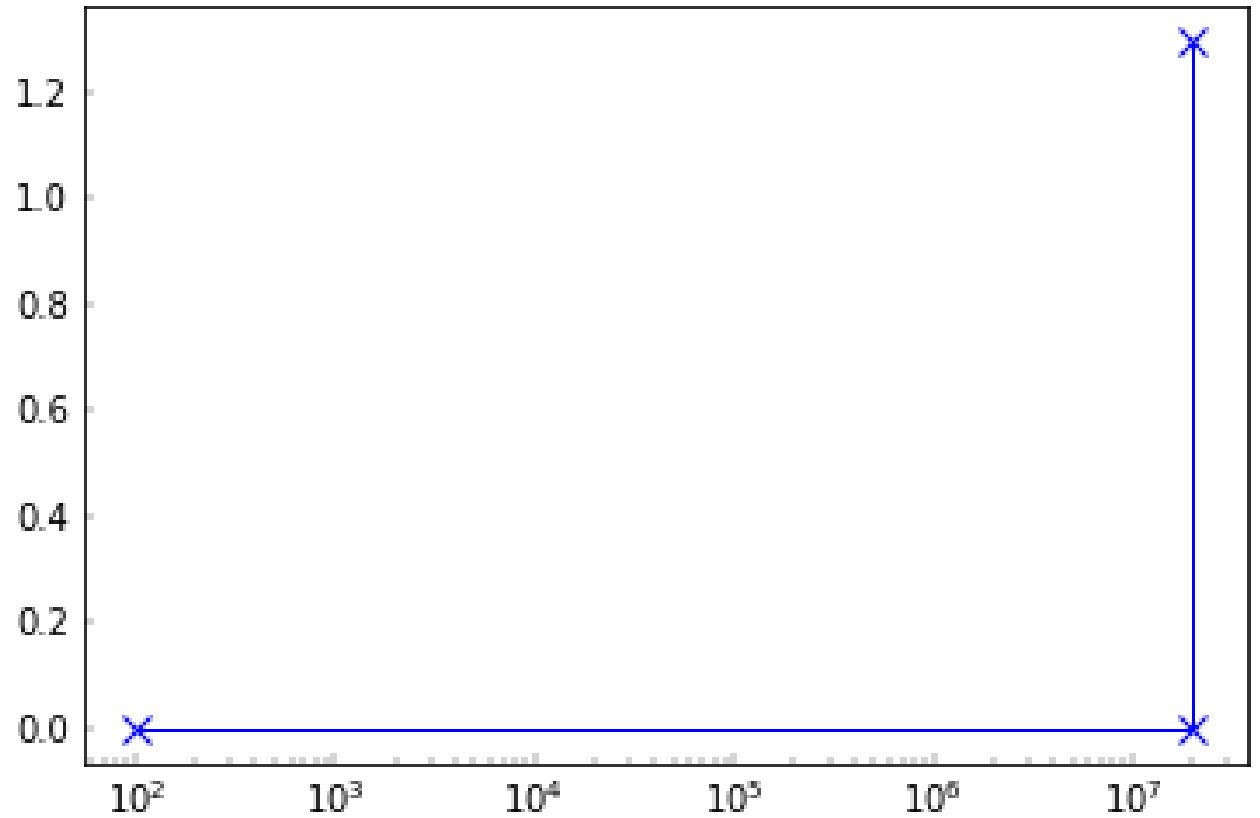

This AMPX internal-use implementation MT is also clearly nonphysical.

\section{A.5.2 UNRESOLVED RESONANCE REGION DATA}

Shift uses AMPX-generated probability table data to evaluate particle cross sections in the unresolved resonance region (URR), where at a single "energy" a particle may encounter a range of cross sections.

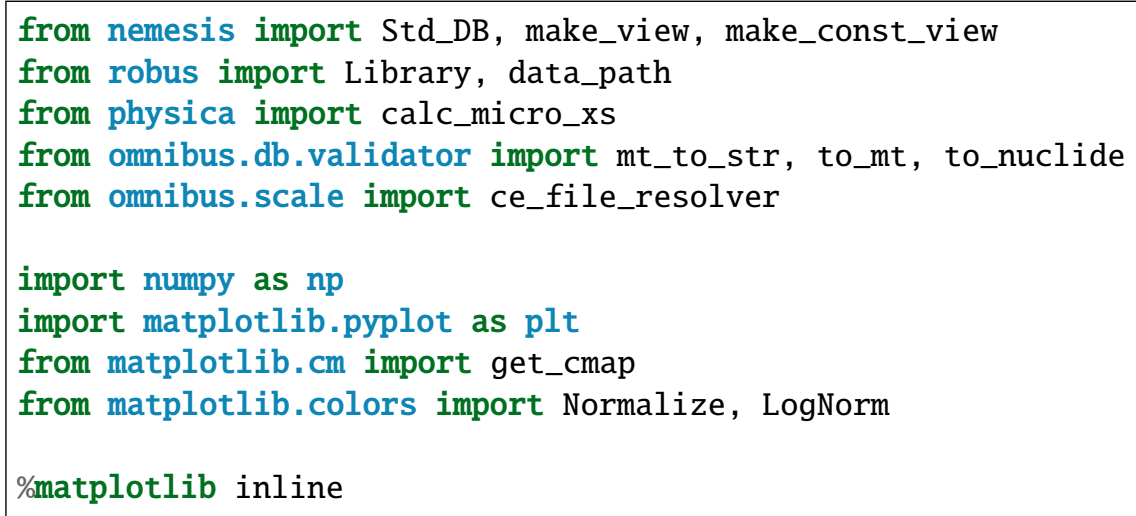




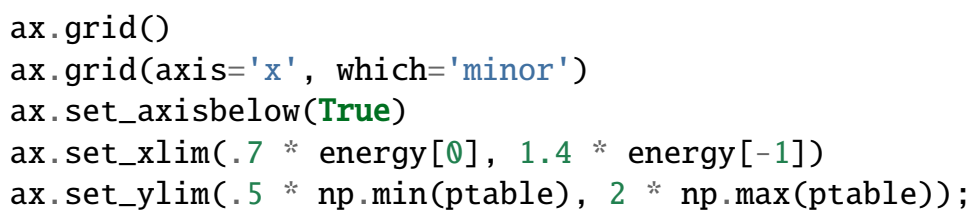

\section{A.5.2.1 Tungsten}

nucl = library.load_nuclide_n(74186, 300)

library. flush_warnings()

print("Reactions with probability table data:",

", ".join(mt_to_str(mt) for mt in which_ptables(nucl)))

Reactions with probability table data: 1=N_TOTAL, 2=N_ELASTIC, 102=N_GAMMA

Print the energy points, as well as the table shape (energy points, cross section bands)

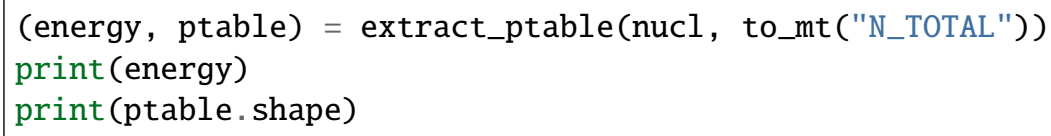

Plot the total cross section at each of the cross section bands and compare it to the mean cross section value. Since the probability table bands at each cross section are discrete and equiprobable, the "mean" cross section provided by the library should equal the mean value across bands:

plot_ptable_and_mean(nucl, mt=1) 


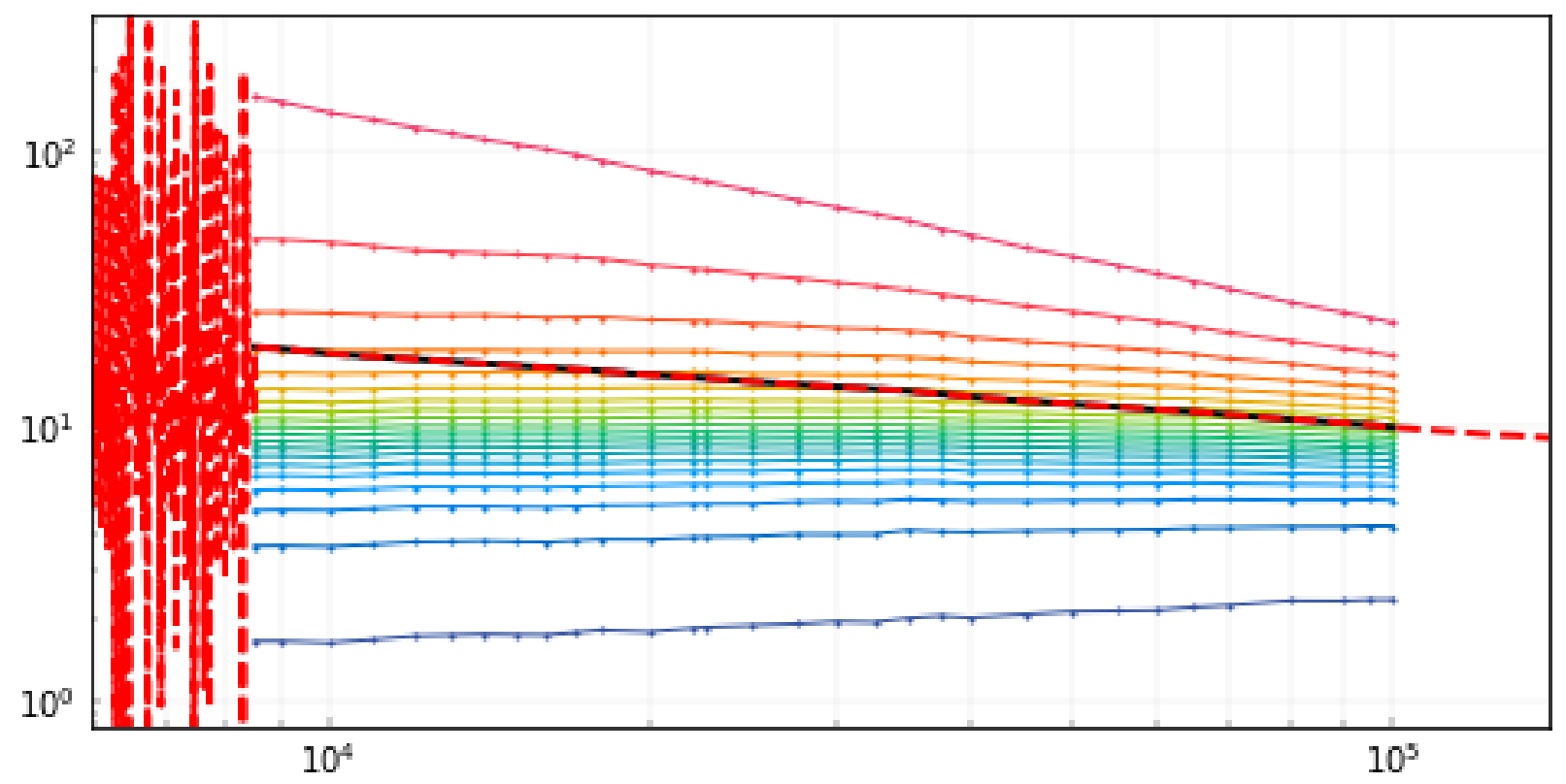

\section{A.5.2.2 Uranium 235}

The MT=1 (total neutron cross section) probability table data stored in AMPX data is defined as the sum of the other probability table reactions, even if other non-probability-table reactions are present. In other words, for every energy point $i$ and every probability band $b$ :

$$
\sigma_{\mathrm{t}, i, b}=\sum_{x \in\{\mathrm{e}, \mathrm{f}, \mathrm{g}\}} \sigma_{x, i, b}
$$

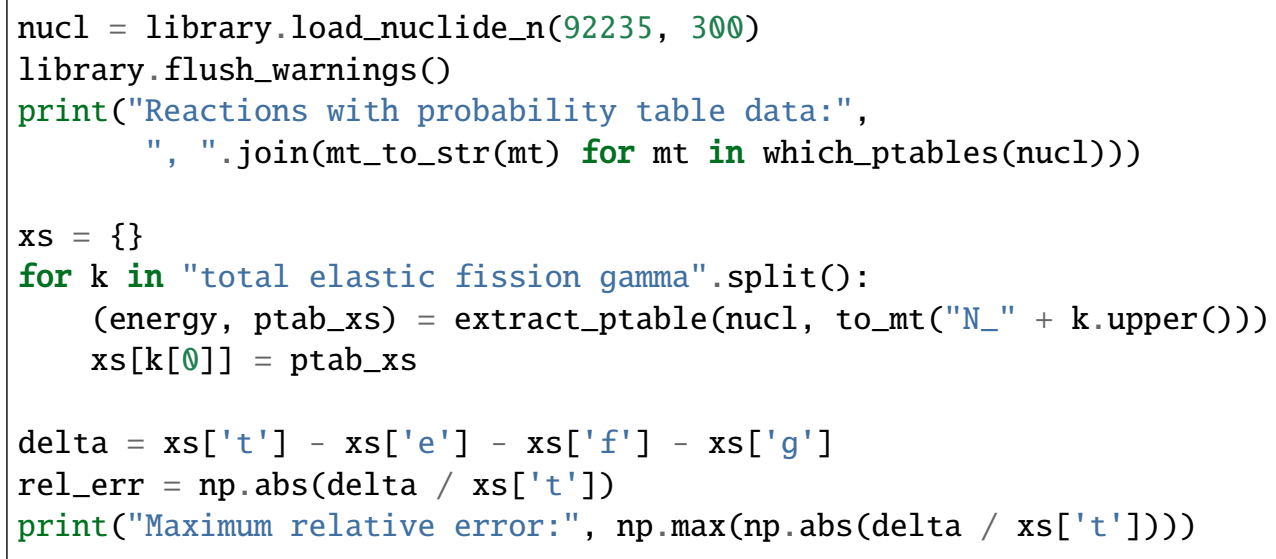

Reactions with probability table data: 1=N_TOTAL, 2=N_ELASTIC, 18=N_FISSION, 102=N_GAMMA Maximum relative error: $8.809924 \mathrm{e}-08$

The error (on the order of single-precision floating point epsilon) confirms this is the case.

\section{A.5.3 FISSION SPECTRUM SAMPLING}

When sampling the energy of a daughter neutron from a fission event, Shift chooses between prompt and delayed spectra. What do these distributions look like? Is the data consistent? 
from nemesis import Std_DB, make_view, make_const_view

from robus import Library, data_path

from physica import calc_micro_xs

from omnibus.db.validator import mt_to_str, to_mt

import matplotlib.pyplot as plt

import numpy as np

\%matplotlib inline

from exnihilotools.matplotlib import screen_style

screen_style()

/rnsdhpc/code/install/Exnihilo/python/omnibus/__init__.py:93: UserWarning: Version

$\hookrightarrow$ mismatch between 'exnihilotools' and 'omnibus' packages: 6.3.pre-b10 (branch 'omnibus-

$\hookrightarrow$ doc' \#98d73c8e on 2020MAR11) (at/rnsdhpc/code/build/Exnihilo-examples/Exnihilo/

$\hookrightarrow$ packages/Nemesis/python/exnihilotools/__init_..py) is not the same as 6.3.pre-b10

$\hookrightarrow$ (branch 'fix-origen-install' \#b3af8ef1 on 2020MAR06) (at/rnsdhpc/code/install/Exnihilo/

$\hookrightarrow$ python/omnibus/__init__.py); This is probably because of a PYTHONPATH error and may

$\rightarrow$ cause unexpected failures on import

UserWarning)

library = Library(Std_DB.from_dict $(\{$

'ce_lib_path' : data_path("ce_v71"),

'gamma_production': True, \}))

nucl = library.load_nuclide_n $(92235,300$.

reactions $=\operatorname{dict}\left(\left[\left(\mathrm{v} . \operatorname{lower}()\right.\right.\right.$, nucl.get_reaction(to_mt $\left.\left.\left(\mathrm{N}_{-}{ }^{\prime}+\mathrm{v}\right)\right)\right)$

for $\mathrm{v}$ in ("NU", "NU_DELAYED", "NU_PROMPT")])

for $(k, r x n)$ in reactions.items():

plt. $\log \log (r \times n . e n e r g y, ~ r x n . x s, ~ l a b e l=k)$

plt. legend (loc=' best'); 


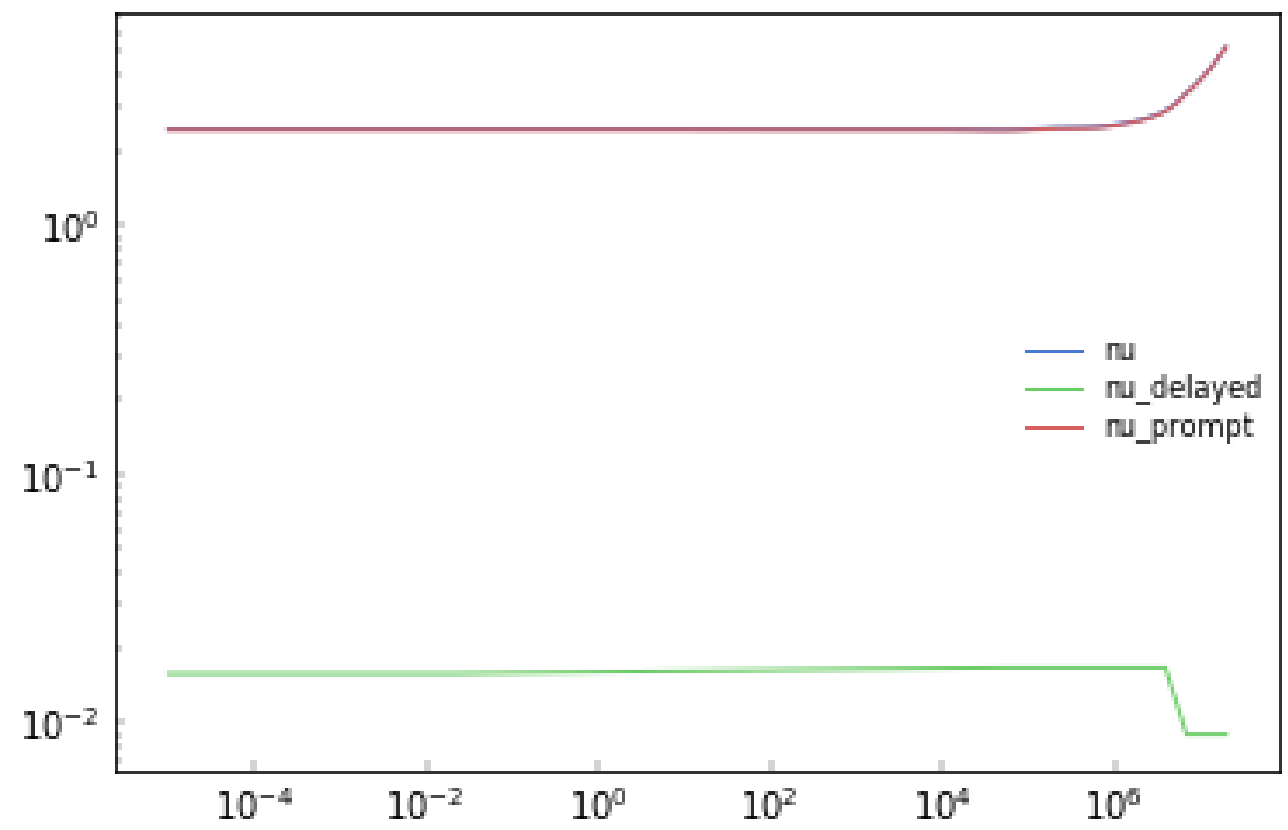

Do the reactions share an energy grid?

print (\{k: len(reactions[k].energy) for $\mathrm{k}$ in sorted(reactions) $\}$ )

print (np.allclose(reactions ['nu'].energy, reactions ['nu_prompt'] .energy))

$\{$ 'nu': 79, 'nu_delayed': 6, 'nu_prompt': 79\}

True

Do the reactions balance? Since the 'delayed' component is on a separate energy grid, it must be interpolated.

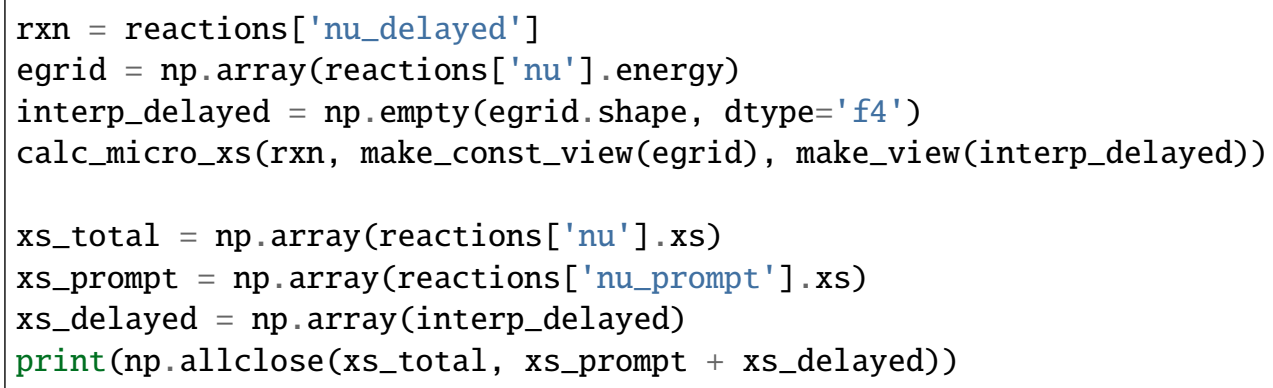

True

\section{A.5.3.1 Examine fission-related reactions}

Which of the reactions have kinematics data?

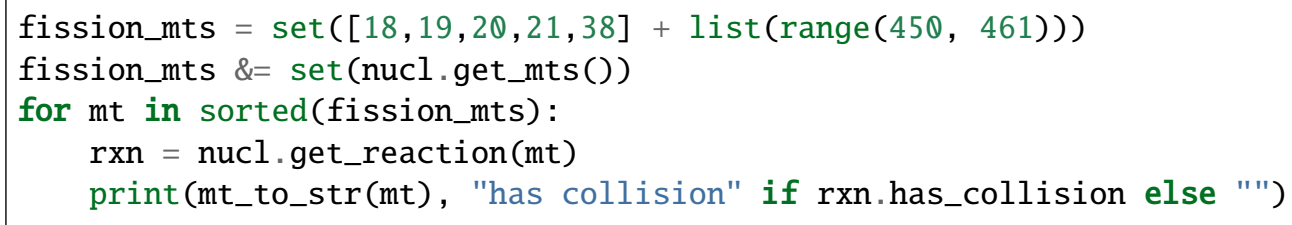


18=N_FISSION has collision

$452=\mathrm{N} \_\mathrm{NU}$

455=N_NU_DELAYED has collision

456=N_NU_PROMPT

$459=$ N_FISS_YIELD

Examine the fission spectrum:

rxn = nucl.get_reaction(459)

plt. $\log \log (r \times n . e n e r g y, ~ r x n . x s, ~ ' . b-')$;

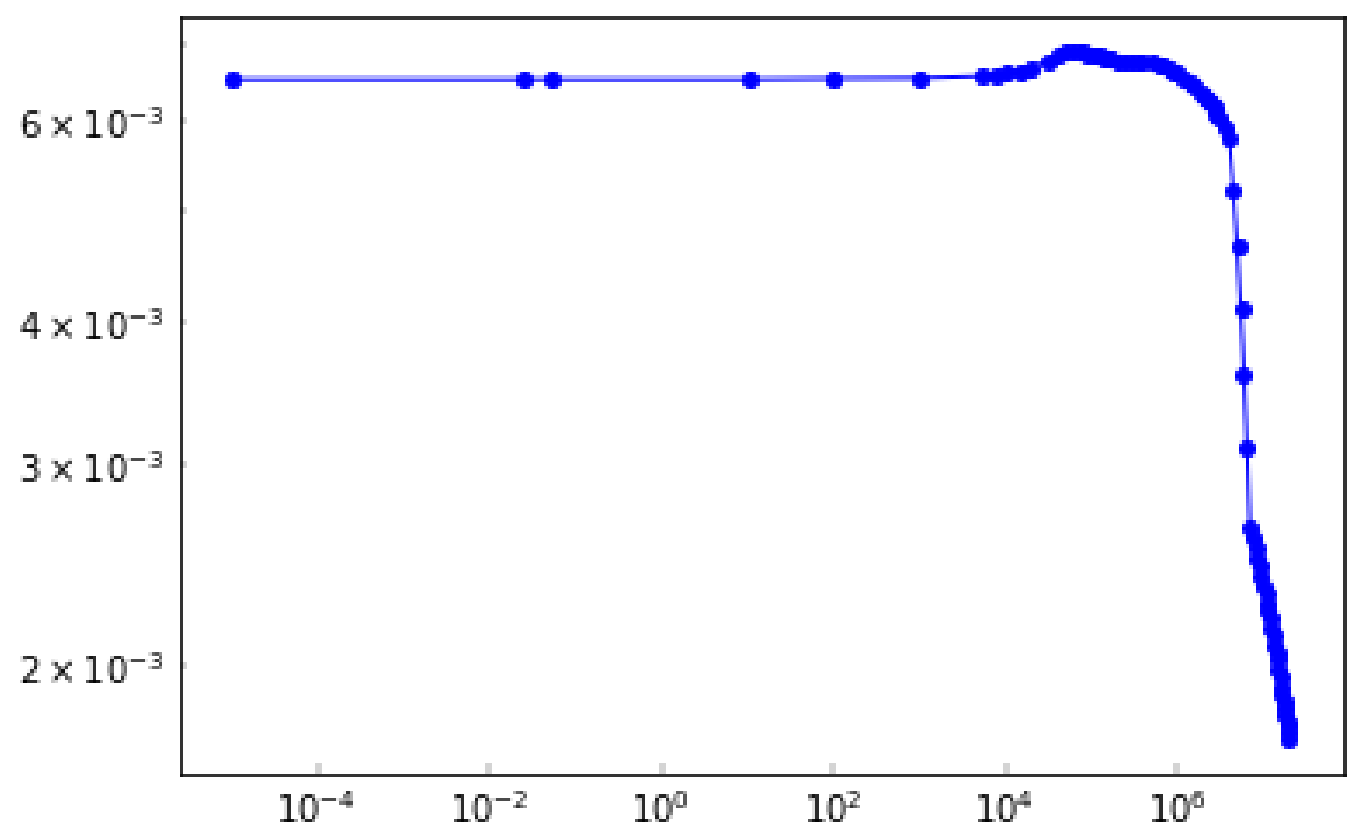

\section{A.5.4 KERMA DATA SPLICING THROUGH AMPX}

Shift supports reading AMPX multigroup files into its CE transport routines for use in reaction rates etc. Note that when splicing AMPX data through the python interface, it is necessary to load the build_library function from physica rather than calling the Library constructor.

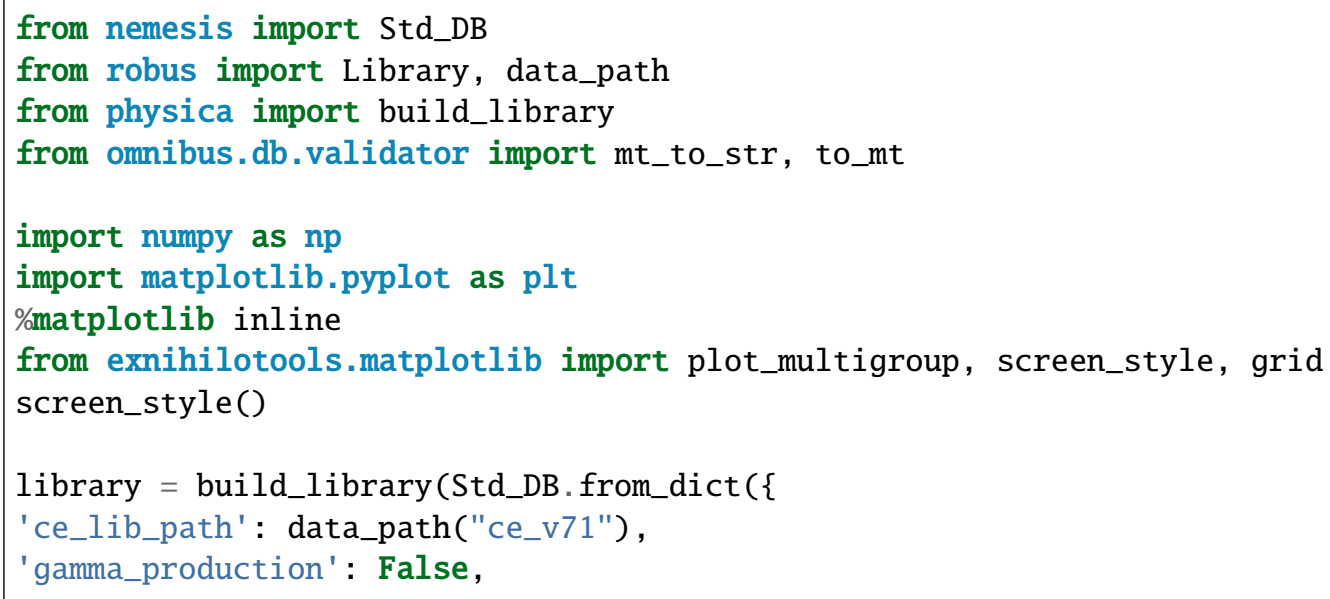




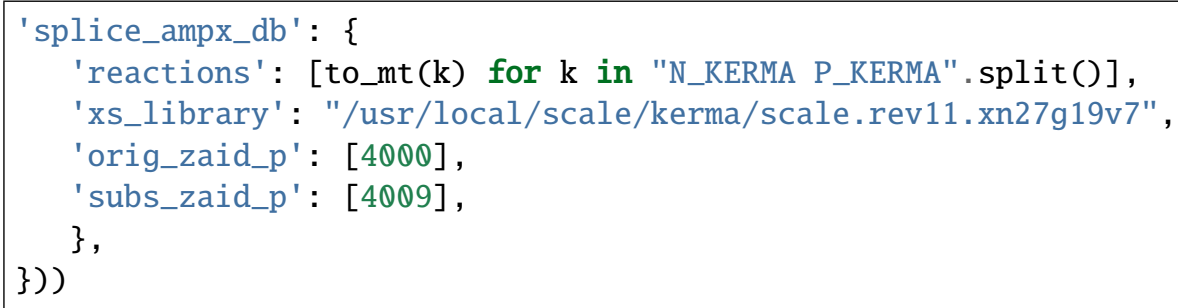

/rnsdhpc/code/install/Exnihilo/python/omnibus/_init__.py:93: UserWarning: Version $\hookrightarrow$ mismatch between 'exnihilotools' and 'omnibus' packages: 6.3.pre-b10 (branch 'omnibus$\hookrightarrow$ doc' \#98d73c8e on 2020MAR11) (at/rnsdhpc/code/build/Exnihilo-examples/Exnihilo/ $\hookrightarrow$ packages/Nemesis/python/exnihilotools/__init__.py) is not the same as 6.3.pre-b10 $\hookrightarrow$ (branch 'fix-origen-install' \#b3af8ef1 on 2020MAR06) (at /rnsdhpc/code/install/Exnihilo/ $\hookrightarrow$ python/omnibus/_init_-.py); This is probably because of a PYTHONPATH error and may $\hookrightarrow$ cause unexpected failures on import

UserWarning)

With the the KERMA data loaded, it can now be plotted:
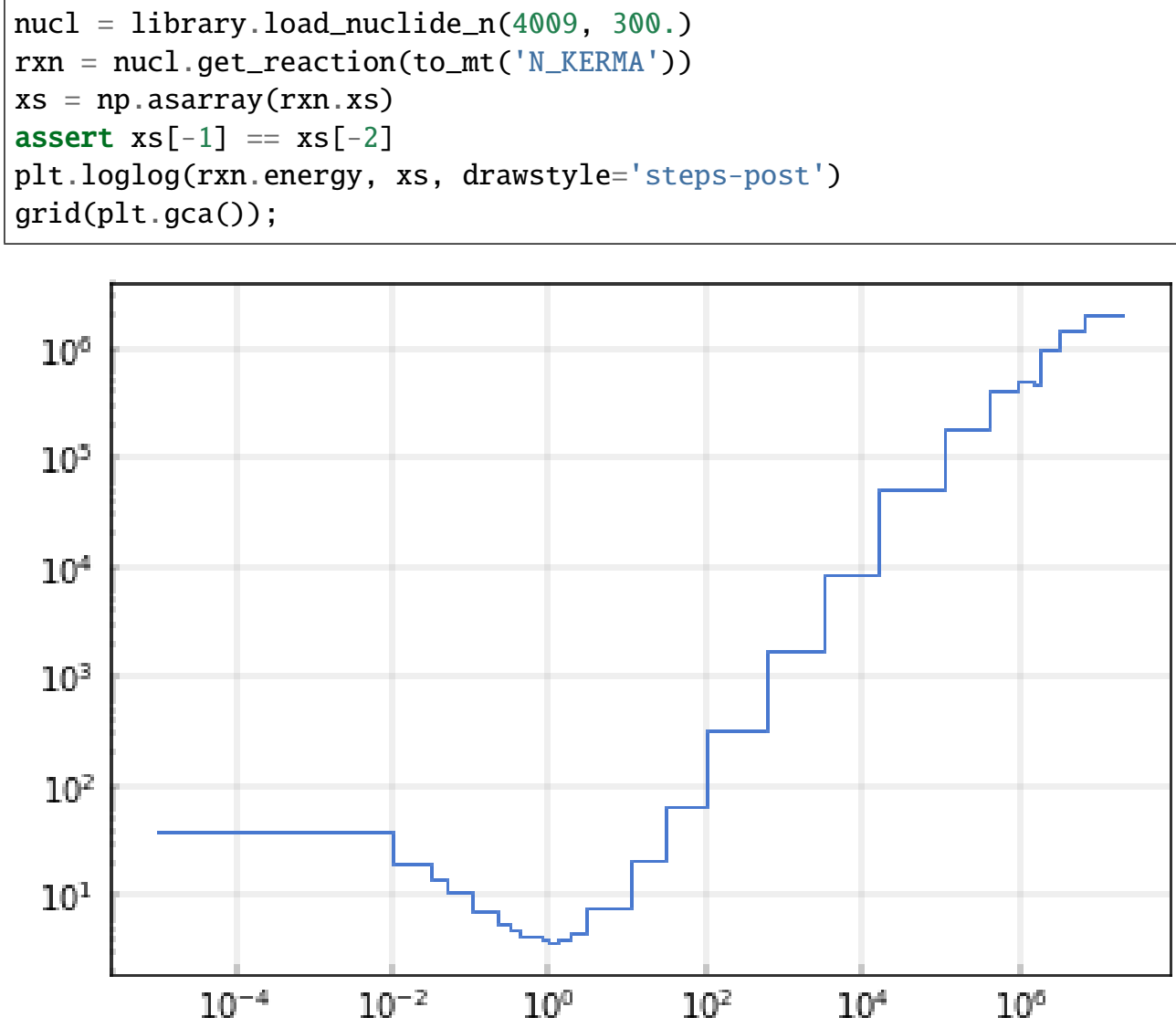

nucl = library.load_nuclide_p(4000)

library.flush_warnings()

rxn = nucl.get_reaction(to_mt ('P_KERMA')) 
$\mathrm{xs}=\mathrm{np} \cdot \operatorname{asarray}(\mathrm{rxn} \cdot \mathrm{xs})$

assert $\mathrm{xs}[-1]==\mathrm{xs}[-2]$

plt. $\log \log (r x n$. energy, xs, drawstyle='steps-post')

$\operatorname{grid}(\mathrm{plt} \cdot \operatorname{gca}())$;

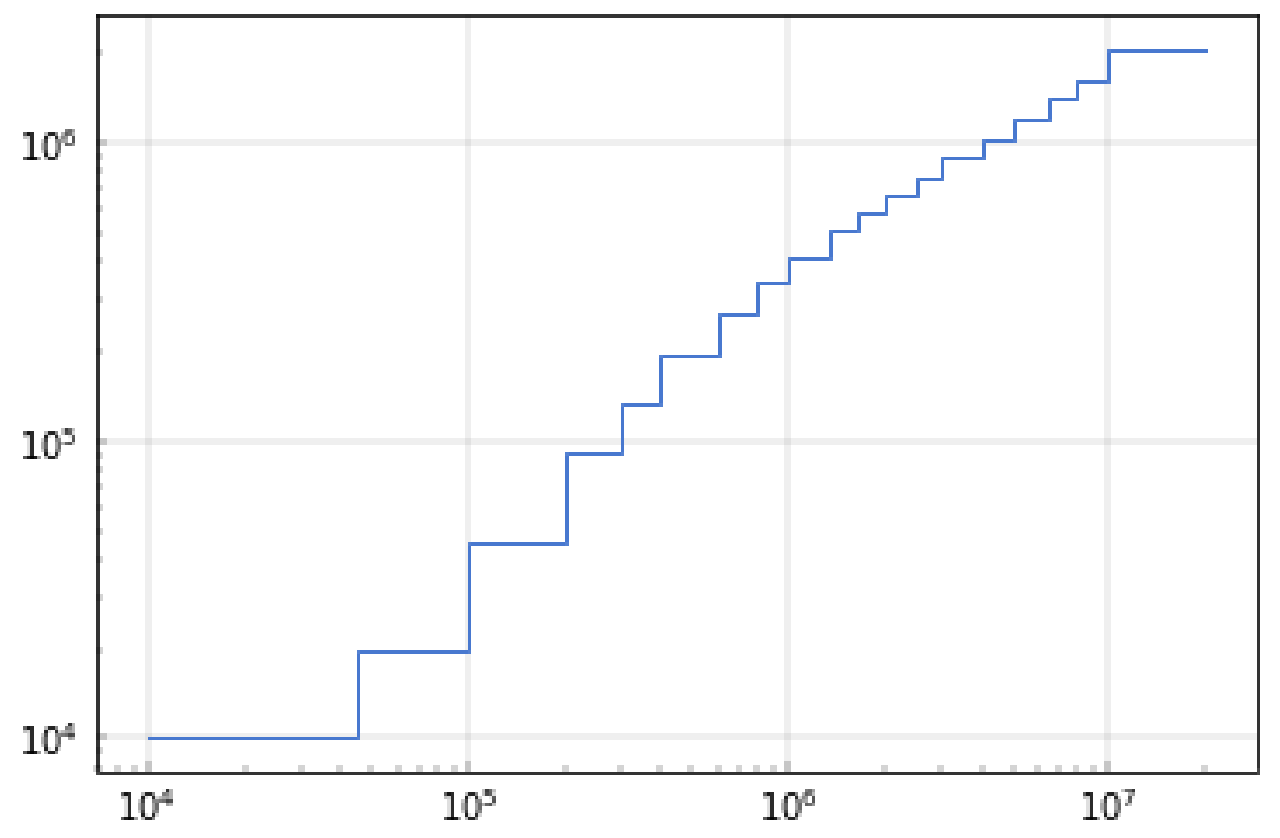

\section{A.6 SUPPLEMENTAL}

\section{A.6.1 USING THE H5SH TOOL}

To enter the h5sh shell, just run h5sh HDF5_FILE. Here is an example of what it looks like:

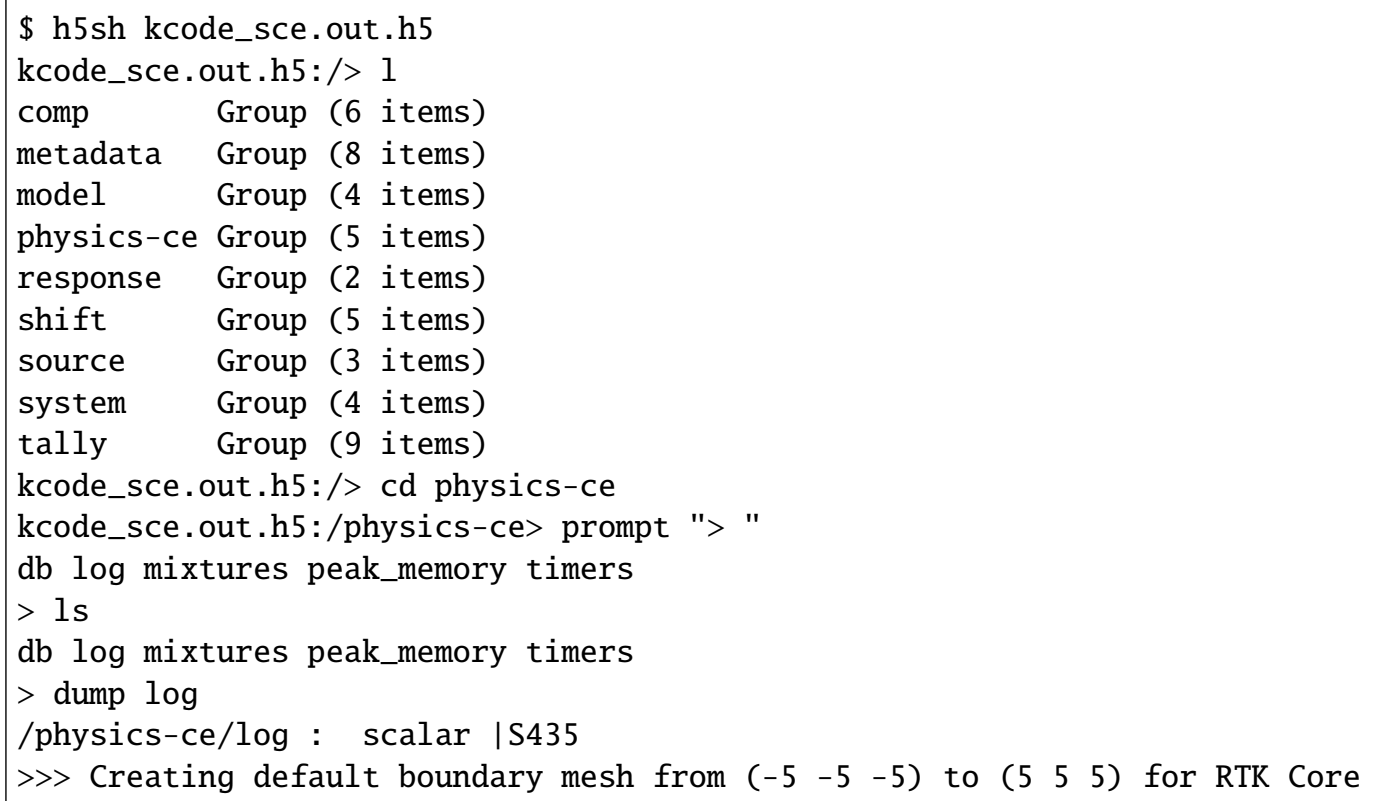


(continued from previous page)

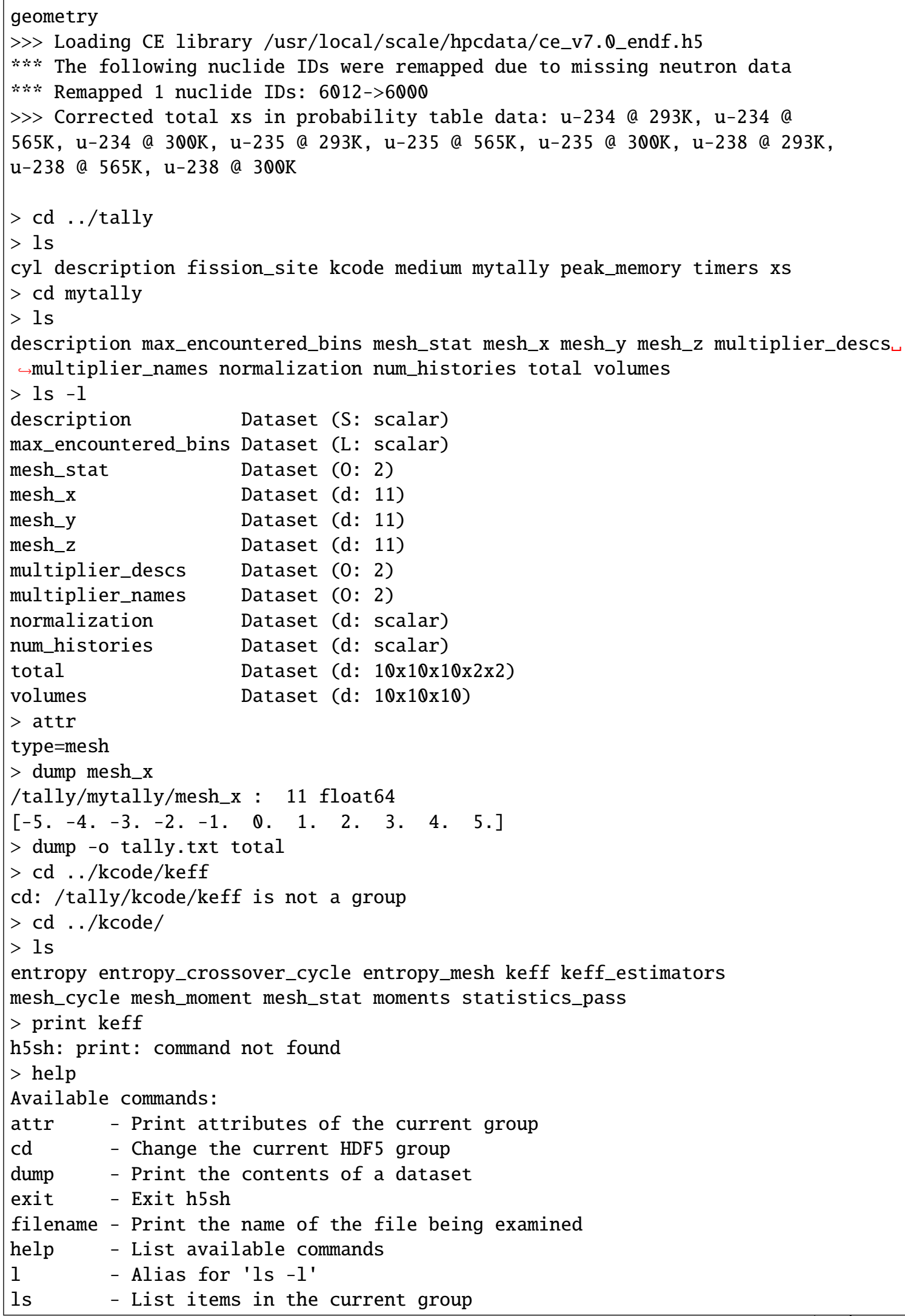




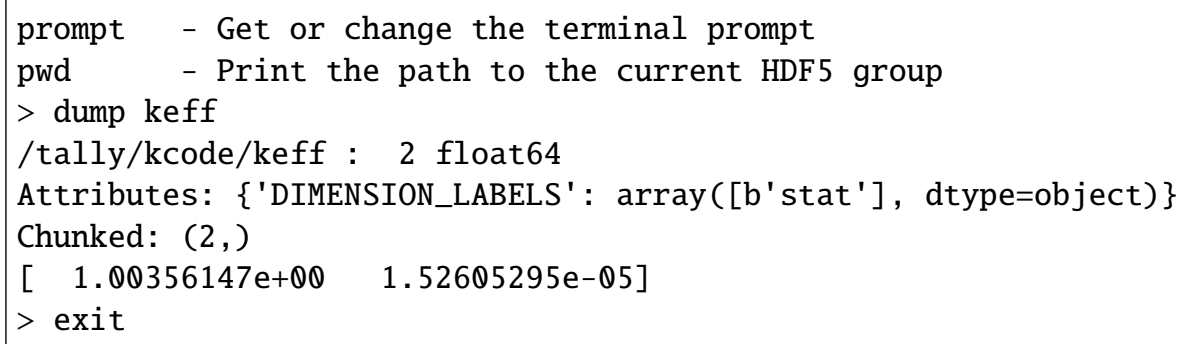

\section{A.6.2 RUNNING OMNIBUS THROUGH PYTHON}

Because the Omnibus front end is simply a Python script, it is possible to drive it through another Python script. For example, the shell command

\$ omnibus-run nyoka.omn

can be written as the following python script:

from omnibus.scripts.omnibus_run import run

run (["nyoka.omn"])

The first argument to run takes a list, because multiple files can be passed to the input just like with the scripted front end. An additional and very useful feature is that a python function can also be passed to modify the database, just like a Python script can be passed through the command line:

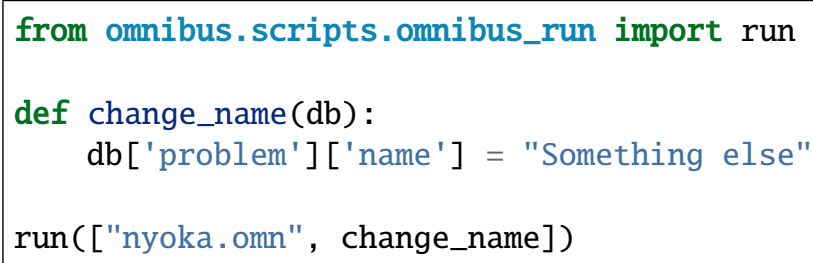

This opens up exciting possibilities of automating scaling studies and parameter studies. To this end, a helper class is available that can drive multiple Omnibus executions with variable parameters. Here is an example that modifies an Omnibus input base-input. omn, loops over different numbers of compute cores and particles per cores, saves the output to subdirectories inside weak-scaling/ppcnnnnnn-npnnnn, and then extracts a few different timers and transport diagnostics and writes them to weak-scaling/results. json.

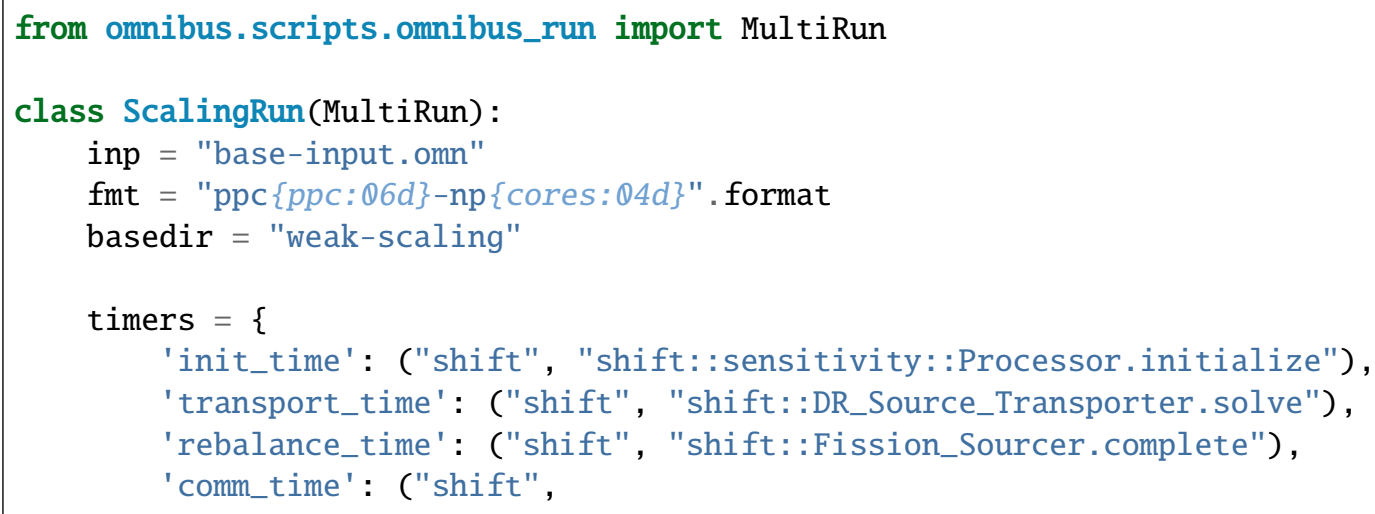




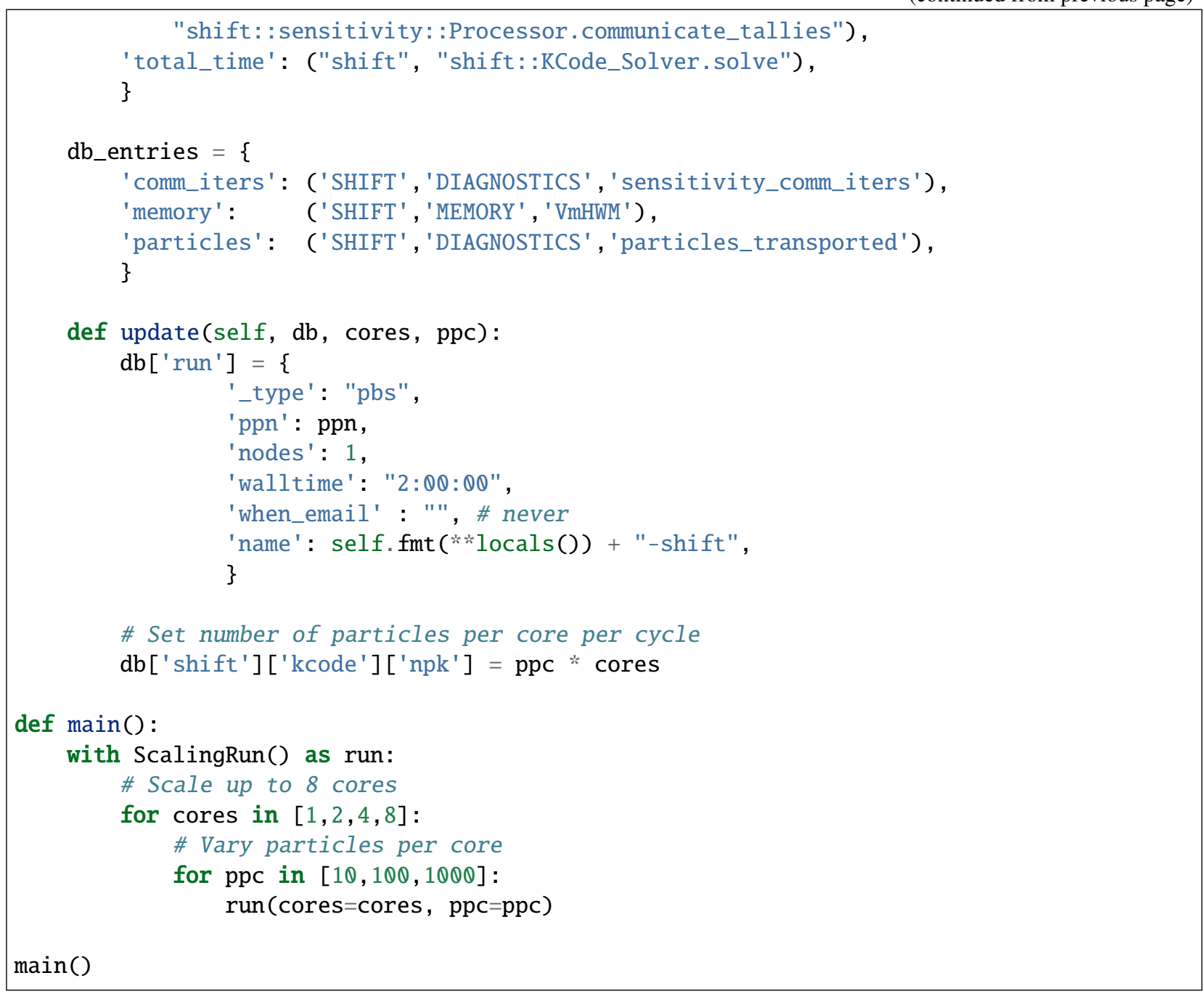

The resulting JSON file looks something like this:

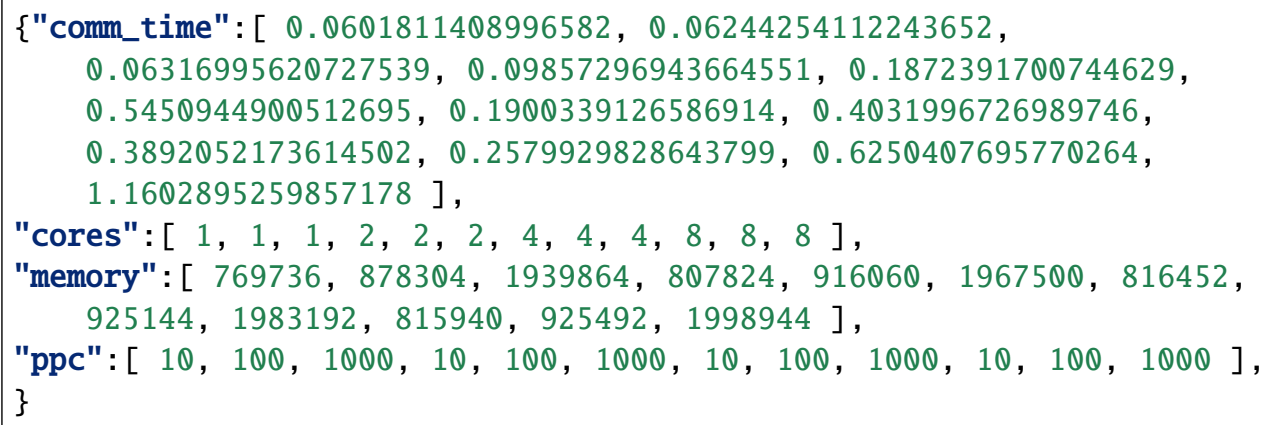

which can be plotted or analyzed easily using Pandas:

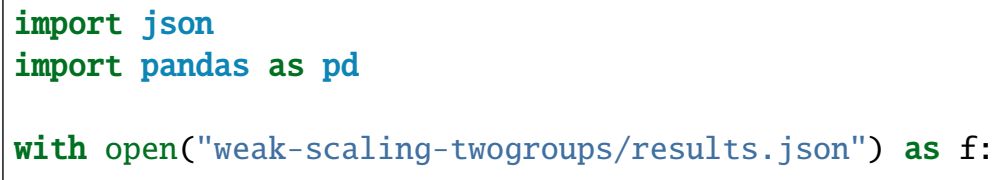




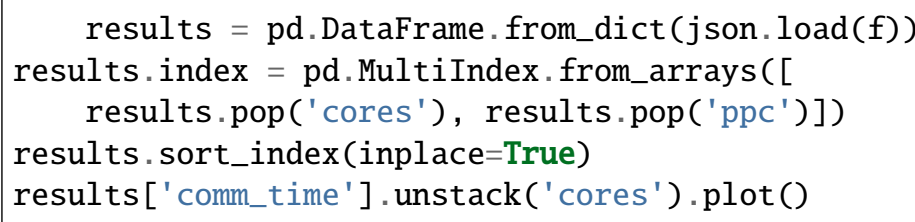

To use this functionality, the user must subclass the MultiRun class and provide:

- a base input filename ("box.omn" in the attached example),

- a formatter for the name of the subdirectories for each file to run (using the new Python-style keyword formatting),

- the root directory of the output files (under which the subdirs will be created, as well as the 'results.json' summary)

- a database of timer names to be recorded from each run,

- a database of $\mathrm{DB}$ entries to be recorded from each run, and

- an update method that modifies the problem input based on the keyword arguments specified. (The first two arguments of this function must be self for the class and $\mathrm{db}$ for the problem database.)

The class is then instantiated as a context manager and called with the keyword arguments specified in the update method.

with WeakScalingRun() as run:

for ppn in range $(1,32+1)$ :

run (instance $=0$, nodes $=1$, ppn $=$ ppn)

It will record all the keywords, the timers, and the DB entries into a JSON file, and will reuse existing output if it is there. If the program aborts from a keyboard interrupt or a failed run, then the context manager will still output all the results it has gathered. 

Appendix B. FILE FORMAT SPECIFICATIONS

B-2 


\section{Appendix B. FILE FORMAT SPECIFICATIONS}

Omnibus uses multiple file formats for input (e.g., geometry definitions and material properties) and output (e.g., Denovo flux HDF5 files).

\section{B.1 DENOVO OUTPUT SPECIFICATION}

Denovo uses parallel HDF5 to write multiple sets of data that correspond to its interpreted input and the solution output. Since it supports both 2D and 3D problem definitions, the mesh cell-based dimensions will change depending on the problem type. Additionally, depending on the problem input parameters, some of the fields may be absent. Notably, if the $P_{N}$ order is zero, then the solution is isotropic, and no current field will be output.

Note: Since Denovo uses column-major ordering for meshes, ' $\mathrm{X}$ ' is always the fastest varying axis. Thus the HDF5 (row-major) dimensions for cells will be (Z,Y,X) or (Y,X).

Tip: Denovo uses the HDF5 Dimension Scale $\mathrm{API}^{24}$ to write dimension labels to its output. If you review these (e.g. with h5dump -A file) you can be sure of the significance of the different dimensions.

\section{B.1.1 ROOT LEVEL DESCRIPTION}

Datasets:

\begin{tabular}{|c|c|c|c|}
\hline Name & Type & Dims & Description \\
\hline mesh_x & double & $X+1$ & Mesh edges along the $\mathrm{x}$ axis. \\
\hline mesh_y & double & $\mathrm{Y}+1$ & Mesh edges along the $y$ axis. \\
\hline mesh_z & double & $Z+1$ & Mesh edges along the $\mathrm{z}$ axis. \\
\hline group_bounds_n & double & $\mathrm{NN}+1$ & $\begin{array}{l}\text { Neutron group boundaries, or empty if no neutron groups } \\
\text { are being solved. }\end{array}$ \\
\hline group_bounds_p & double & $\mathrm{NG}+1$ & $\begin{array}{l}\text { Photon group boundaries, or empty if no photon groups are } \\
\text { being solved. }\end{array}$ \\
\hline flux & double & $\mathrm{G}, \mathrm{Z}, \mathrm{Y}, \mathrm{X}$ & Scalar fluxes for each group in each mesh cell. \\
\hline source & double & $\mathrm{G}, \mathrm{Z}, \mathrm{Y}, \mathrm{X}$ & Volumetric source term if an isotropic fixed source was used. \\
\hline uncflux & double & $\mathrm{G}, \mathrm{Z}, \mathrm{Y}, \mathrm{X}$ & Uncollided flux if used. \\
\hline current & double & $\mathrm{G}, \mathrm{Z}, \mathrm{Y}, \mathrm{X}, 3$ & Currents (first scalar flux moment). \\
\hline block & u short & $\mathrm{Z}, \mathrm{Y}, \mathrm{X}$ & KBA block index for each cell. \\
\hline matids & int & $\mathrm{Z}, \mathrm{Y}, \mathrm{X}$ & Global mixture ID. \\
\hline mixtable & $\mathrm{COO}$ & M & Global mix table. \\
\hline
\end{tabular}

Groups:

\begin{tabular}{ll}
\hline Name & Description \\
\hline metadata & $\begin{array}{l}\text { Metadata about the problem, system, and Exnihilo version } \\
\text { used to create the output file. }\end{array}$ \\
\hline
\end{tabular}

\footnotetext{
${ }^{24}$ https://www.hdfgroup.org/HDF5/doc/HL/RM_H5DS.html
} 
All quantities follow the standard nuclear engineering practice of being averaged over space and integrated in energy and angle.

The mix table stores mixture IDs as rows, and it stores composition IDs/pure matids as columns. For parallel problems, the mix table is essentially the concatenation of all the domain-local mix tables, but with pure materials placed in the beginning rows of the table. Thus, the mix table may have duplicate rows if run in parallel.

\section{B.2 HDF5 MESH MODEL SPECIFICATION}

Denovo accepts an explicit problem model definition via an HDF5 input file. Since it supports both 2D and 3D problem definitions, the mesh cell-based dimensions will change depending on the problem type.

Note: Since Denovo uses column-major ordering for meshes, ' $\mathrm{x}$ ' is always the fastest-varying axis. Thus the HDF5 (row-major) dimensions for cells will be (Z,Y,X) or (Y,X).

\section{B.2.1 ROOT LEVEL DESCRIPTION}

Datasets:

\begin{tabular}{|c|c|c|c|}
\hline Name & Type & Dims & Description \\
\hline mesh_x & double & $X+1$ & Mesh edges along the $\mathrm{x}$ axis. \\
\hline mesh_y & double & $\mathrm{Y}+1$ & Mesh edges along the y axis. \\
\hline mesh_z & double & $Z+1$ & $\begin{array}{l}\text { Mesh edges along the } \mathrm{z} \text { axis. (For a } 2 \mathrm{D} \text { problem, this field } \\
\text { should not be present.) }\end{array}$ \\
\hline matids & int & $\mathrm{Z}, \mathrm{Y}, \mathrm{X}$ & $\begin{array}{l}\text { Material IDs in each mesh cell. (For a 2D problem, dimen- } \\
\text { sions are Y,X.) }\end{array}$ \\
\hline mixtable & $\mathrm{COO}$ & $\mathrm{M}, \mathrm{P}$ & $\begin{array}{l}\text { Sparse matrix representation: } \mathrm{M} \text { is the number of mixtures, } \\
\mathrm{P} \text { the number of "pure" materials (original problem matids) }\end{array}$ \\
\hline source & & $\mathrm{G}, \mathrm{Z}, \mathrm{Y}, \mathrm{X}$ & Optional volumetric source definition. \\
\hline
\end{tabular}

The mix table is stored in Coordinate matrix format, a structure of ('row', 'col', 'val') elements with types (int, int, double), respectively. These datasets are written consistently with the Denovo Output Specification (page B-3) output specification so that it can be used as a restart file.

Groups:

\begin{tabular}{ll}
\hline Name & Description \\
\hline volsrc & Optional cell-based volume source definitions. \\
ptsrc & Optional point source definitions. Only applicable to 3D. \\
metadata & $\begin{array}{l}\text { Optional metadata about the version of Omnibus used to } \\
\text { generate the input file. }\end{array}$ \\
\hline
\end{tabular}




\section{B.2.2 VOLSRC GROUP DESCRIPTION}

Datasets:

\begin{tabular}{llll}
\hline Name & Type & Dims & Description \\
\hline ids & int & Z,Y,X & Spectrum IDs in each mesh cell. \\
strength & double & Z,Y,X & $\begin{array}{l}\text { Source strength in each cell. } \\
\text { spectra }\end{array}$ \\
double & S,G & $\begin{array}{l}\text { The row index (zero-based) in the table is the spectrum ID; } \\
\text { the row itself is a normalized spectrum for all G groups. }\end{array}$ \\
\hline
\end{tabular}

\section{B.2.3 PTSRC GROUP DESCRIPTION}

Datasets:

\begin{tabular}{llll}
\hline Name & Type & Dims & Description \\
\hline $\begin{array}{l}\text { mesh_point } \\
\text { strength }\end{array}$ & $\begin{array}{l}\text { double } \\
\text { double }\end{array}$ & P,3 & P \\
spectra & double & P,G & $\begin{array}{l}\text { Point source locations. Each row is a separate point. } \\
\text { Normalized spectra. Each row is the normalized spectrum } \\
\text { for all G groups corresponding to the point at that row index. }\end{array}$ \\
\hline
\end{tabular}

\section{B.3 RTK XML INPUT SPECIFICATION}

The RTK XML geometry input specification will be documented once the format is finalized.

\section{B.4 XS XML INPUT SPECIFICATION}

The XML cross section input specification will be documented once the format is finalized. 